The Characterization of Cell Surface Receptor Complexes by Affinity Chromatography, Liquid Chromatography and Tandem Mass Spectrometry

\author{
by
}

Jaimie Dufresne

BSc, MSc, Ryerson University

\author{
A dissertation \\ presented to Ryerson University \\ in partial fulfillment of the \\ requirements for the degree of \\ Doctor of Philosophy \\ in the Program of \\ Molecular Science
}

Toronto, Ontario, Canada, 2017

(c) Jaimie Dufresne 2017 


\section{AUTHOR'S DECLARATION FOR ELECTRONIC SUBMISSION OF A DISSERTATION}

I hereby declare that I am the sole author of this dissertation. This is a true copy of the dissertation, including any required final revisions, as accepted by my examiners.

I authorize Ryerson University to lend this dissertation to other institutions or individuals for the purpose of scholarly research.

I further authorize Ryerson University to reproduce this dissertation by photocopying or by other means, in total or in part, at the request of other institutions or individuals for the purpose of scholarly research.

I understand that my dissertation may be made electronically available to the public. 
Cell surface receptors are of critical importance to the treatment of disease but are difficult to isolate and identify by classical approaches. Here, a robust and general method for capturing a receptor complex from the surface of live cells with ligands presented on nanoscopic beads is demonstrated. Two forms of affinity chromatography: the presentation of a biotinylated ligand to the surface of live cells and recovered by classical affinity chromatography was compared to the presentation of the ligand on the surface of nanoscopic chromatography beads for the isolation of the IgG-FcR complex from the surface of live cells. The IgG ligand was first characterized by LC-ESI-MS/MS and compared to controls by classical statistical methods in order to minimize the total error rate of protein identification. The first strategy was to isolate the IgG-FcR complex using biotinylated IgG (IgG-B) compared to IgG with a DTTcleavable biotin spacer arm (IgG-SS-B) to activate and capture the receptor associated supramolecular complex. In this method, live cells were incubated on ice with IgG, IgG-B or IgG-S-S-B, for 30 minutes, washed and disrupted by French press. After washing, the IgG-B and IgG-S-S-B ligands were eluted from a streptavidin-agarose column in DTT followed by mercaptoethanol, $2 \mathrm{M} \mathrm{NH}_{4} \mathrm{OH}$ and then $50 \mathrm{mM}$ biotin in $2 \mathrm{M} \mathrm{NH}_{4} \mathrm{OH}$ and the ligand remaining on the columns was scrubbed with $2 \%$ SDS or digested with trypsin. The analysis of the eluted fractions and SDS scrub by dot blots or Western blots with streptavidin-HRP (SA-HRP) or donkey anti human IgG-HRP (Dk anti-hIgG-HRP) probes agreed with liquid chromatography and tandem mass spectrometry (LC-ESI-MS/MS) of the tryptic peptides that IgG-B or IgG-S-S$\mathrm{B}$ was non-specifically eluted by ammonium hydroxide buffer. LC-ESI-MS/MS of the DTT eluted fraction from the charged streptavidin column (agarose-SA-B-S-S-IgG) to release the S$\mathrm{IgG}$ ligand showed detectable amounts of the $\mathrm{S}-\mathrm{IgG}$ complex and minimal amounts of $\mathrm{Fc}$ and Fcrl proteins. Some of the known components of the Fc mediated phagocytic pathway were enriched in DDT and BME in the Biotin-S-S-IgG-FCGR compared to Biotin-IgG controls. Many variations of the classical ligand affinity chromatography method for the isolation of cell surface receptors were investigated including the use of detergents, heat aggregated ligands, the use of cross linkers and the capture of IgG over streptavidin or protein G chromatography. None of these methods specifically captured Fc receptors however the identified proteins were different from background binding controls In the second strategy, IgG coated PMMA and melamine micro and glass nano beads were presented to live cells, homogenized by French pressing, isolated by ultracentrifugation and then extracted by a salt and acetonitrile step gradient. Laser confocal scanning microscopy, dot blots with Coomassie staining or Western blot all confirm the three nano and micro bead surfaces: silica, melamine and PMMA were coated in IgG and were able to bind RAW 264.7 macrophages. Fc receptors were identified in significant numbers in the silica nano treatments compared to controls as well as members of the FcR mediated phagocytic pathway with very little background binding. In contrast to the strategy of classical affinity chromatography applied to integral membrane receptors, the use of ligand coated micro, and as shown here for the first time, silica nano beads, was effective at binding and capturing a receptor from the surface of a live cell and may serve as a general method for isolating receptor complexes on the nano scale. 


\section{Acknowledgments}

For my beloved husband Daniel who makes life joyous and beautiful. For my sister Billie, and my mother and father Sylvie and Philip who have loved and supported me unconditionally. My heartfelt thanks to my cherished friends and lab mates who are like family to me: Elissa, Kathy, Sabrina, Eun Jee, Thanusi, Pardis, Bobbi, Harvey, John O., Erin, Shelby, Jamie, Lina, Dave, Leslie, Christina, Andy, Regan, Guy, Eric, Susan, Amr, Rasha, and Fiona. Thank you to the many students who have taken part in this work and put forth exceptional efforts: Gar-Way, Trung, Moumita, Eun Jee, Morla and Zhou. Thank you to Angelique and Peter for their mentorship and guidance. My deepest gratitude to my supervisor Dr. John Marshall for his support and encouragement. I will be forever grateful for the opportunity he gave me. 
Table of Contents

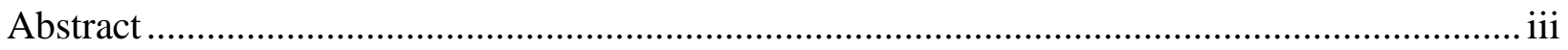

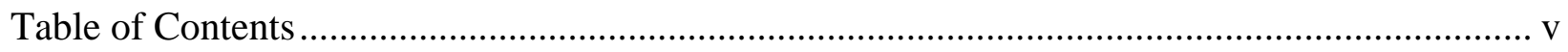

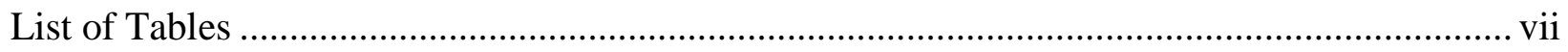

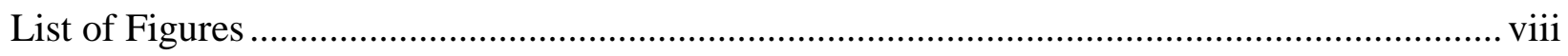

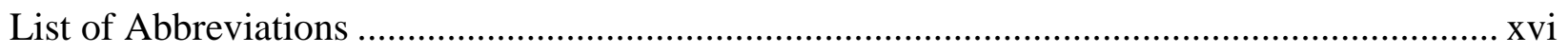

List of Abbreviations for experimental treatments ........................................................ Xviii

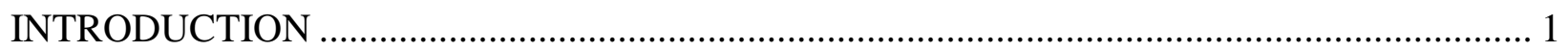

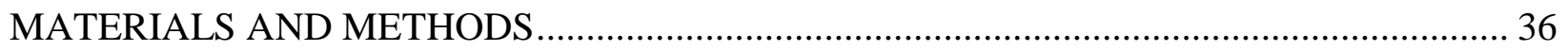

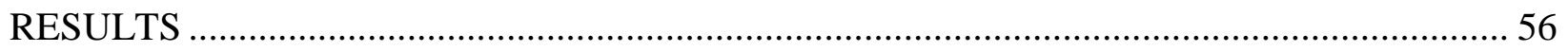

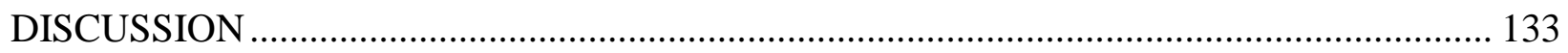

CONCLUSION

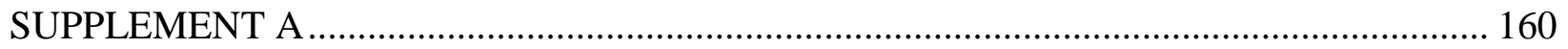

Treatments and ESI-LC-MSMS runs for ligand affinity chromatography experiments ........... 161

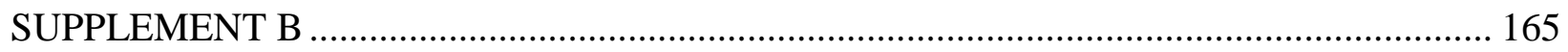

SQL Query Code Used for grouping FC and IgG Tables:.............................................. 166

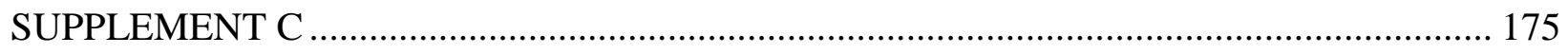

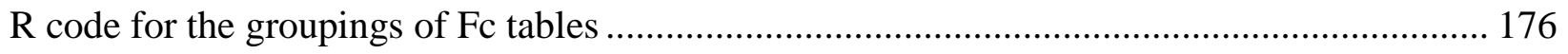

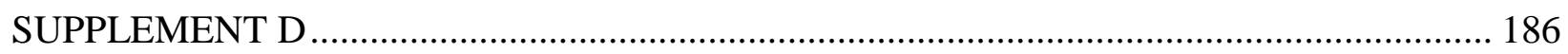

Random and independent sampling of endogenous tryptic peptides from normal human EDTA plasma by liquid chromatography micro electrospray ionization and tandem mass spectrometry

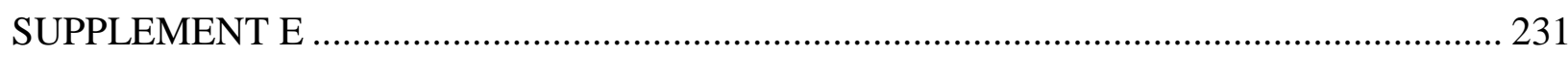

Freeze-dried plasma proteins are stable at room temperature for at least one year................. 232

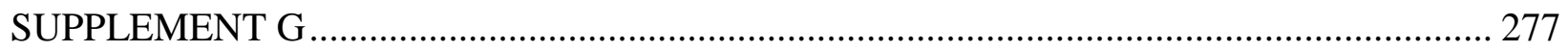


The endogenous tryptic peptides of EDTA plasma from Alzheimer's Dementia, Heart Attack, Sepsis, Ovarian Cancer, Breast Cancer, and Multiple Sclerosis and institution/study matched

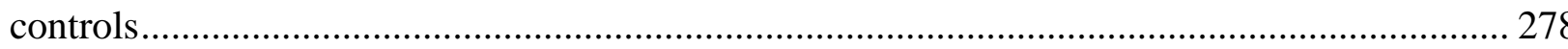




\section{List of Tables}

Table I. The filtering of the cumulative, redundant correlations to protein accession sequences on the basis of charge state and peptide sequence. Bulk IgG, blank, dust and random spectra were searched against a federated human protein library. Myosin was searched against a federated rabbit library. The redundant use of MS/MS spectra to correlate to more than one peptide sequence was eliminated using a composite key in SQL Server.

Table 2. Peptide counts of hIgG from biotinylated monovalent and polyvalent ligands applied to crude or live cells and captured by silica chromatography supports coated with streptavidin, protein G, IgG, polylysine or without coating.

Table 3. Peptide counts of Fc gamma receptors (filter 0) and IgG-Fc KEGG pathway proteins (filter 2) from biotinylated monovalent and polyvalent ligands applied to crude or live cells and captured by silica chromatography supports coated with streptavidin, protein $\mathrm{G}, \mathrm{IgG}$, polylysine or without coating.

Table 4: IgG protein identifications for the classical ligand affinity chromatography method with biotinylated ligands observed across treatments where the DTT, BME, NH4OH and $\mathrm{NH} 4 \mathrm{OH}+$ biotin elutions were grouped by treatment.

Table 5. IgG identifications over treatments for the classical ligand affinity chromatography method with biotinylated ligands.

Table 6: Protein identifications for $\mathrm{Fc}$ and $\mathrm{Fc}$-like receptors by elution buffer using biotinylated ligand affinity chromatography with monovalent ligands.

Table 7: Fc receptors by treatment using biotinylated affinity receptor chromatography using monovalent ligands.

Table 8: Comparison of aggregated versus monovalent ligands (bead digest not included - no bead digests collected for the aggregated ligands)

Table 9: Chi square comparison of peptide to protein ratio for the Ligand biotinylation data compared to Noise (solvents without sample applied) 


\section{List of Figures}

Figure 1: General categories of cell receptors. (A) ion channel-linked receptors, (B) enzyme-linked receptors (C) G-protein-coupled receptors (D) Membrane permeable signaling molecules for intracellular receptors. Image recreated from (Purves, Augustine, \& Fitzpatrick, 2001).

Figure 2. Classes of membrane proteins. (A) Type I are single pass membrane proteins with a cytosolic Cterminus and extracellular $\mathrm{N}$-terminus. (B) Type II are single pass with a cytosolic $\mathrm{N}$-terminus and extracellular C-terminus. (C) Multipass membrane proteins span the plasma membrane multiple times (D) Peripheral membrane proteins interact with the membrane by hydrophobic or ionic interactions (E) Lipid chain anchored proteins are covalently linked to a lipid (F) GPI anchored proteins are covalently linked to a glycolipid.

Figure 3: The KEGG pathway of IgG-FcyR mediated phagocytosis.

Figure 4: The structure of IgG derived from X-ray chrystallography with the carbohydrate moiety essential for binding shown. The PyMOL Molecular Graphics System, Version 1.5.0.4 Schrödinger, LLC.

Figure 5: The structure of interaction the Fc protion of IgG bound to the soluble portion of FcyRIIb derived from X-ray chrystallography The PyMOL Molecular Graphics System, Version 1.5.0.4 Schrödinger, LLC.

Figure 6. Fc receptors in humans and mice. ITAM (black) are activating while ITIM domains (white) inhibit phagocytosis. Recreated from (Bruhns, 2012).

Figure 7: The IgG-Fcy mediated phagocytosis pathway. After ligand activation and receptor clustering the Src family kinases (orange) phosphorylate and/or recruit adaptor proteins (green) that act as a platform for activating lipid modifying enzymes (blue), guanine nucleotide exchange factors (purple) for small GTPases (brown) activating nucleation promoting factors (pink) and the actin nucleation complex. Recreation of the image from (Flannagan, Jaumouille, \& Grinstein, 2012).

Figure 8. Chemical structure of NHS biotin (A) and the cleavable Sulfo-NHS-SS-Biotin tag (B). The cleavable disulphide portion is highlighted.

Figure 9. Ligand affinity receptor chromatography where (A) IgG coated polystyrene beads were presented to live cells, (B) allowed to bind, (C) homogenized by French press, (D) isolated by ultracentrifugation, (E) washed and (F) extracted with salt and AcN buffers prior to digestion and HPLCESI-MSMS.

Figure 10: The chemical structure of melamine (A), Poly(methyl methacrylate) (B) and silica (C).

Figure 11. Coating of silica micro and nano beads.

Figure 12. Electrospray ionization of peptides at the source head in positive mode.

Figure 13. Diagram of a Linear Ion Trap showing the vacuum generated by the roughing and molecular turbo pumps. Sample molecules are ionized at the source then focused into the mass analyzer by the ion optics and then scanned out of the ion trap by the mass to charge ratio where the intensity of the ions is measured by the detectors. 
Figure 14. Diagram of a Linear Ion Trap showing the changes in potential along the ion path.

Figure 15: Ions generated during peptide fragmentation. A, b and c ions extend from the amino terminus and $\mathrm{x}, \mathrm{y}$ and $\mathrm{z}$ the carboxy terminus.

Figure16: Biotinylated ligand affinity chromatography with DTT cleavable biotin linkers Activation of live cells with biotinylated ligands

Figure 17. Summary of treatment and controls for the capture of the IgG-Fc $\gamma$ receptor complex by classic affinity chromatography using SA-agarose and agarose (control) affinity columns and biotinylated ligands applied to live cells and crude homogenates (control).

Figure 18. The effect of sample loading and detection method on the perceived purity of the rabbit myosin standard (Sigma M1636) by SDS-PAGE with Coomassie Brilliant Blue, diamine silver stain and Western blot. Panels: A, 0.1-10 $\mu \mathrm{g}$ Myosin [Lanes: 1, MW marker; 2, $0.1 \mu \mathrm{g}$ myosin; 3, $0.25 \mu \mathrm{g}$ myosin; 4, $0.50 \mu \mathrm{g}$ myosin; Lane 5, $0.75 \mu \mathrm{g}$ myosin; 6, $1.00 \mu \mathrm{g}$ myosin; 7, $2.50 \mu \mathrm{g}$ myosin; 8, $5.00 \mu \mathrm{g}$ myosin; 9 , $7.50 \mu \mathrm{g}$ myosin; 10, $10 \mu \mathrm{g}$ myosin (Symbols *440 kDa myosin complex, @ $220 \mathrm{kDa}$ myosin heavy chain, \# $15 \mathrm{kDa}$ myosin light chain)]; B, A total of 5 micrograms of the rabbit myosin standard was resolved by Tricine SDS-PAGE alongside 10 micrograms total of the SDS-PAGE standards (MW) for staining with Coomasie brilliant blue R-250 (CBBR ) versus diamine silver (AgNO3); C, Western blot of the myosin standard (Lane 1, secondary antibody control. Lane 2 anti actin). The expected migration of myosin heavy chain is approximately $220 \mathrm{kDa}$. The arrow shows the approximate mass of actin, $44 \mathrm{kDa}$.

Figure 19. The box plot of the raw intensity values of the electrospray source noise versus the authentic $\mathrm{IgG}$, dust and myosin proteins samples. The solid line shows the median, the box hinges show the interquantile distance and the wiskers show the $99 \%$ confidence range of the data. The treatments were the affinity purified IgG positive control $(\mathrm{n}=2)$, the source noise from blank runs of water AACN gradients over naïve C18 HPLC columns $(n=36)$, the tryptic peptides of laboratory dust $(n=4)$ and the myosin test standard $(\mathrm{n}=2)$.

Figure 20. The $\log 10$ frequency of non-redundant correlations (Filter 2) of fully tryptic peptides from MASCOT, OMSSA, X!TANDEM and SEQUEST combined per unit MS/MS spectra compared between $\mathrm{IgG}$, myosin, dust, blank and random spectra. The probability that the curves from IgG, myosin, and dust are the same as either the random or blank controls is essentially zero. Loading sufficient sample to ensure the instrument does not sample blank noise during the experimental run essentially prevents contamination of the results by noise spectra. At a cut off of 200 protein accessions only TTN contaminates the $\operatorname{IgG}$ and myosin results that is easily avoided using the controls lists provided in the supplemental data. The two authentic standards bulk human $\mathrm{IgG}$ ( $\mathrm{n}=2$ runs with 6,216 MS/MS spectra) and rabbit myosin standard ( $\mathrm{n}=2$ runs with $17,730 \mathrm{MS} / \mathrm{MS}$ spectra) were compared to laboratory dust ( $\mathrm{n}=4$ runs with 18,387 MS/MS spectra), blank gradient runs on a naïve column with HPLC grade solvents ( $\mathrm{n}=36$ runs with 75,269 MS/MS spectra), and computer generated random MS/MS spectra $(\mathrm{n}=116$ simulated runs with 440,772 spectra).

Figure 21. Affinity purified bulk Human IgG (positive control) results from the X!TANDEM algorithm. The distributions of the fully tryptic peptides identified with total 6,216 MS/MS spectra from the bulk human IgG sample by X!TANDEM against human proteins. Panels: A, the sorted log 10 peptide intensity values; B, the $\log 10$ peptide p-values from X!TANDEM (inset the linear peptide p-values); $\mathrm{C}$, the sorted peptide $[\mathrm{M}+\mathrm{H}]+$ values; $\mathrm{D}$, the sorted peptide delta mass values; E, the $\log 10$ intensity Quantile plot; F, the log peptide p-values Quantile plot (inset the untransformed peptide p-value QQ plot); G, the peptide expected $[\mathrm{M}+\mathrm{H}]+$ Quantile plot; $\mathrm{H}$, the peptide delta mass Quantile plot; I, the Log10 peptide intensity value density plot; J, the log peptide p-value density plot; $\mathrm{K}$, the peptide expected $[\mathrm{M}+\mathrm{H}]+$ density plot; 
$\mathrm{L}$, the peptide delta mass density plot; $\mathrm{M}, \log$ peptide intensity versus $\log [\mathrm{M}+\mathrm{H}]+$ scatter plot; $\mathrm{N}$, the $\log$ peptide $\mathrm{p}$-value versus expected $[\mathrm{M}+\mathrm{H}]+$ scatter plot; $\mathrm{O}, \log$ peptide $\mathrm{p}$-value versus peptide delta mass scatter plot; $\mathrm{P}$, the peptide delta mass versus peptide expected $[\mathrm{M}+\mathrm{H}]+$ scatter plot.

Figure 22. Laboratory dust (contamination control) results from the X!TANDEM algorithm. The distributions of the fully tryptic peptides from proteins identified with 18,387 MS/MS spectra from laboratory dust searched by X!TANDEM against a federated human library. Panels: A, the sorted $\log 10$ peptide intensity values; $B$, the $\log 10$ peptide p-values from X!TANDEM (inset the linear peptide pvalues); $\mathrm{C}$, the sorted peptide $[\mathrm{M}+\mathrm{H}]+$ values; $\mathrm{D}$, the sorted peptide delta mass values; $\mathrm{E}$, the $\log 10$ intensity Quantile plot; F, the log peptide p-values Quantile plot (inset the untransformed peptide p-value QQ plot); G, the peptide expected [M+H]+ Quantile plot; H, the peptide delta mass Quantile plot; I, the $\log 10$ peptide intensity value density plot; $\mathrm{J}$, the $\log$ peptide $\mathrm{p}$-value density plot; $\mathrm{K}$, the peptide expected $[\mathrm{M}+\mathrm{H}]+$ density plot; $\mathrm{L}$, the peptide delta mass density plot; $\mathrm{M}, \log$ peptide intensity versus $\log [\mathrm{M}+\mathrm{H}]+$ scatter plot; $\mathrm{N}$, the log peptide $\mathrm{p}$-value versus expected $[\mathrm{M}+\mathrm{H}]+$ scatter plot; $\mathrm{O}, \log$ peptide $\mathrm{p}$-value versus peptide delta mass scatter plot; $\mathrm{P}$, the peptide delta mass versus peptide expected $[\mathrm{M}+\mathrm{H}]+\mathrm{scatter}$ plot.

Figure 23. The electrospray source noise results (physically generated random spectra) from the X!TANDEM algorithm. The distributions of the fully tryptic peptides and proteins with 75,269 MS/MS spectra of source noise from blank LC-ESI-MS/MS runs on naïve columns computed by X!TANDEM against a human library. Panels: A, the sorted $\log 10$ peptide intensity values; $\mathrm{B}$, the $\log 10$ peptide $\mathrm{p}$ values from X!TANDEM (inset the linear peptide $p$-values); $C$, the sorted peptide $[M+H]+$ values; $D$, the sorted peptide delta mass values; E, the $\log 10$ intensity Quantile plot; F, the log peptide $\mathrm{p}$-values Quantile plot (inset the untransformed peptide p-value QQ plot); G, the peptide expected $[\mathrm{M}+\mathrm{H}]+$ Quantile plot; H, the peptide delta mass Quantile plot; I, the density plot of Log10 peptide intensity value; J, the log peptide p-value density plot; $\mathrm{K}$, the peptide expected $[\mathrm{M}+\mathrm{H}]+$ density plot; $\mathrm{L}$, the peptide delta mass density plot; $\mathrm{M}, \log$ peptide intensity versus $\log [\mathrm{M}+\mathrm{H}]+$ scatter plot; $\mathrm{N}$, the log peptide $\mathrm{p}$ value versus expected $[\mathrm{M}+\mathrm{H}]+$ scatter plot; $\mathrm{O}, \log$ peptide $\mathrm{p}$-value versus peptide delta mass scatter plot; $\mathrm{P}$, the peptide delta mass versus peptide expected $[\mathrm{M}+\mathrm{H}]+$ scatter plot.

Figure 24. The computer generated random spectra results (null random model) from the X!TANDEM algorithm. The distributions of the fully tryptic peptides and proteins identified with 440,772 computer generated random MS/MS spectra fit by X!TANDEM against a human library. Panels: A the $\log 10$ peptide p-values from X!TANDEM (inset the linear peptide p-values); $B$, the sorted peptide $[\mathrm{M}+\mathrm{H}]+$ values; C, the sorted peptide delta mass values; D, the log peptide p-values Quantile plot (inset the untransformed peptide p-value Quantile plot); E, the peptide expected $[\mathrm{M}+\mathrm{H}]+$ Quantile plot; F, the peptide delta mass Quantile plot; G, the log peptide p-value density plot; $\mathrm{H}$, the peptide expected $[\mathrm{M}+\mathrm{H}]+$ density plot; I, the peptide delta mass density plot; J, the log peptide p-value versus expected $[\mathrm{M}+\mathrm{H}]+$ scatter plot; $\mathrm{K}, \log$ peptide $\mathrm{p}$-value versus peptide delta mass scatter plot; $\mathrm{L}$, the peptide delta mass versus peptide expected $[\mathrm{M}+\mathrm{H}]+$ scatter plot.

Figure 25. The rabbit myosin results (experiment test standard) from the X!TANDEM algorithm. The distributions of the fully tryptic peptides identified with 17,730 MS/MS spectra from the rabbit myosin standard searched by X!TANDEM against rabbit proteins. Panels: A, the sorted log 10 peptide intensity values; B, the $\log 10$ peptide p-values from X!TANDEM (inset the linear peptide p-values); $\mathrm{C}$, the sorted peptide $[\mathrm{M}+\mathrm{H}]+$ values; D, the sorted peptide delta mass values; E, the $\log 10$ intensity Quantile plot; F, the log peptide p-values Quantile plot (inset the untransformed peptide p-value QQ plot); G, the peptide expected $[\mathrm{M}+\mathrm{H}]+$ Quantile plot; $\mathrm{H}$, the peptide delta mass Quantile plot; I, the density plot of $\log 10$ peptide intensity value; $J$, the log peptide p-value density plot; $K$, the peptide expected $[\mathrm{M}+\mathrm{H}]+$ density plot; L, the peptide delta mass density plot; $\mathrm{M}, \log$ peptide intensity versus log $[\mathrm{M}+\mathrm{H}]+$ scatter plot; N, 
the log peptide p-value versus expected $[\mathrm{M}+\mathrm{H}]+$ scatter plot; $\mathrm{O}$, log peptide p-value versus peptide delta mass scatter plot; $\mathrm{P}$, the peptide delta mass versus peptide expected $[\mathrm{M}+\mathrm{H}]+$ scatter plot.

Figure 26. The summary of the myosin standard at the levels of peptides, protein accession and gene symbols. Panels: A, the average intensity of precursors per peptides; B, the standard error of the all the precursor intensity values per peptides; C, the cumulative p-value for MS/MS spectra per peptides; D, the $\log 10$ number of peptides correlated to each protein accession number; $\mathrm{E}$, the average intensity of all precursor per protein accession number; $\mathrm{F}$, the standard error of the precursor intensity values per protein accession number; $\mathrm{G}$, the cumulative standard $\mathrm{p}$-value from MS/MS spectra per protein accession; $\mathrm{H}$, the $\log 10$ number of peptides correlated to each protein accession number; I, the average intensity of all precursors per gene symbol; $\mathrm{J}$, the standard error of the all the precursor values per gene symbol; $\mathrm{K}$, the cumulative standard p-value of all peptides for each protein accession; L, the $\log 10$ number of peptides correlated to each protein accession number. After normality testing by Quantile (QQ) plots and ANOVA the probability that the $\log 10$ mean intensity values were the same across peptides, proteins or gene symbols was $\sim \mathrm{E}-16$.

Figure 27. The rabbit myosin protein complex results from the X!TANDEM algorithm analyzed by the STRING algorithm. String data: number of nodes, 326; number of edges, 1032; average node degree, 6.33; clustering coefficient, 0.701; expected number of edges, 462; PPI enrichment p-value, $0(<<0.0001)$.

Figure 28. Streptavidin-agarose beads with IgG (Sigma). 50ul of Steptavidin-agarose resin (SA) or agarose alone (A) was incubated with 10 ug of monovalent IgG, IgG-B, IgG-SS-B alongside a PBS control $(\varnothing)$, washed and eluted in DTT, IgG, $\mathrm{NaOH}$ followed by Biotin, and $\mathrm{NaCl}$ followed by AcN. The remaining protein on the chromatography supports was scrubbed in $2 \%$ SDS. 1 ul was spotted onto PVDF and probed with Dk-anti-hIgG (IgG and IgG-B std at 0,1,10 and 100ug/ml). The second row of blots are the same as the first row but with a longer exposure time. (performed by A. FlorentinusMefailoski)

Figure 29. Streptavidin-agarose beads (Sigma). 50ul of Steptavidin-agarose resin (SA) or agarose alone (A) was incubated with $10 \mathrm{ug}$ monovalent of IgG, IgG-B or IgG-SS-B alongside a PBS control $(\varnothing)$, washed and eluted in DTT, IgG, $\mathrm{NaOH}$ followed by Biotin, and $\mathrm{NaCl}$ followed by $\mathrm{AcN}$. The remaining protein on the chromatography supports was scrubbed in $2 \%$ SDS. 1 ul was spotted onto PVDF and probed with SA-HRP (IgG and IgG-B std at 0, 1, 10 and 100ug/ml). (performed by A. FlorentinusMefailoski)

Figure 30. NUNC Maxisorp high binding plastic. A high binding 96 well microtiter plate was coated with $10 \mathrm{ug}$ proG or Streptavidin vs no coating control. Wells were incubated with 10 ug per well of monovalent IgG, IgG-B, IgG-SS-B alongside a PBS control ( $\varnothing$ ), washed and eluted in DTT, IgG, $\mathrm{NaOH}$, Biotin, $\mathrm{NaCl}, \mathrm{AcN}$ or SDS. 1 ul was spotted onto PVDF and probed with SA-HRP (IgG and IgG-B std at $0,1,10$ and $100 \mathrm{ug} / \mathrm{ml}$ ) (performed by A. Florentinus-Mefailoski)

Figure 31. NUNC Maxisorp high binding plastic. A high binding 96 well microtiter plate was coated with $10 \mathrm{ug}$ proG or streptavidin vs a no coating control. Wells were incubated with $10 \mathrm{ug}$ per well of monovalent IgG, IgG-B, IgG-SS-B alongside a PBS control ( $\varnothing$ ), washed and eluted in DTT, IgG, $\mathrm{NaOH}$, Biotin, $\mathrm{NaCl}, \mathrm{AcN}$ or SDS. 1 ul was spotted onto PVDF and probed with Dk-anti-hIgG (IgG and IgG-B std at $0,1,10$ and $100 \mathrm{ug} / \mathrm{ml}$ ) (performed by A. Florentinus-Mefailoski)

Figure 32: PVDF in 96 well plate. PVDF was coated with 10 ug protein $\mathrm{G}$ (ProG) or streptavidin (SA) vs no coating control. Wells were incubated with 10 ug of monovalent IgG, IgG-B or IgG-SS-B alongside a PBS control $(\varnothing)$. The PVDF membranes were washed and eluted in DTT, IgG, NaOH, Biotin, $\mathrm{NaCl}, \mathrm{AcN}$. The remaining protein was scrubbed in $2 \%$ SDS. 1 ul was spotted onto a new PVDF membrane and 
probed with SA-HRP. (IgG and IgG-B std at 0, 1, 10 and 100ug/ml) (performed by A. FlorentinusMefailoski)

Figure 33: PVDF in 96 well plate. PVDF was coated with 10 ug protein G (ProG) or streptavidin (SA) vs no coating control. Wells were incubated with 10 ug of monovalent IgG, IgG-B or IgG-SS-B alongside a PBS control $(\varnothing)$. The PVDF membranes were washed and eluted in DTT, IgG, NaOH, Biotin, $\mathrm{NaCl}, \mathrm{AcN}$ or SDS. The remaining protein was scrubbed in 2\% SDS. 1 ul was spotted onto a new PVDF membrane and probed with Dk-anti-hIgG. (IgG and IgG-B std at 0, 1, 10 and 100ug/ml) (performed by A. Florentinus-Mefailoski)

Figure 34. Glass cover slips in 96 well plate. Glass was coated with 10 ug protein $\mathrm{G}$ (proG) or streptavidin (SA) vs no coating and poly-Lysine alone controls. Wells were incubated with 10 ug of monovalent IgG, IgG-B, IgG-SS-B alongside a PBS control ( $\varnothing$ ), washed and eluted in DTT, IgG, NaOH, Biotin, $\mathrm{NaCl}, \mathrm{AcN}$ or SDS. The remaining protein was scrubbed in $2 \%$ SDS. 1 ul was spotted onto PVDF and probed with Dk-anti-hIgG. (IgG and IgG-B std at 0, 1, 10 and 100ug/ml) (performed by A. Florentinus-Mefailoski)

Figure 35. Glass cover slips in 96 well plate. Glass was coated with 10 ug protein $\mathrm{G}$ (proG) or streptavidin (SA) vs no coating and poly-Lysine alone controls. Wells were incubated with $10 \mathrm{ug}$ of monovalent IgG, IgG-B, IgG-SS-B alongside a PBS control ( $\varnothing$ ), washed and eluted in DTT, IgG, NaOH followed by biotin, and $\mathrm{NaCl}$ followed by AcN. The remaining protein was scrubbed in 2\% SDS. 1 ul was spotted onto PVDF and probed with SA-HRP. (IgG and IgG-B std at 0, 1, 10 and 100ug/ml) (performed by A. Florentinus-Mefailoski)

Figure 36: The structure of IgG derived from X-ray chrystallography with the all lysine residues indicated in red showing the possible binding sites of NHS Biotin and NHS-SS-Biotin The PyMOL Molecular Graphics System, Version 1.5.0.4 Schrödinger, LLC.

Figure 37. Detection of the Biotin-IgG ligand (B) or the Biotin-S-S-B (B-S-S-IgG) by dot blotting and ECL molar ratio of B-NHS or B-SS- NHS to IgG is shown. Note that the ligand approaches apparent saturation of the ECL detection method by a 1:1 Molar ratio and high amounts of biotin did not result in more intense detection.

Figure 38: Western Blot of (A) IgG-biotin and (B) IgG-sulfo-biotin with Streptavidin-HRP. Legend: lane 1: marker; lane 2: IgG; lane 3: IgG with 0.1 fold NHS-biotin, lane 4: IgG with 0.5 fold NHS-biotin, lane 5: IgG with 1.0 fold NHS-biotin, lane 6: IgG with 2.5 fold NHS-biotin, lane 7: IgG with 5 fold NHSbiotin, Lanes 8: IgG with 7.5 fold NHS-biotin, Lanes 9: IgG with 10 fold NHS-biotin (B) same ratios but with NHS-SS-B

Figure 39. The detection limit of the IgG-B and IgG-S-S-B labeled ligands. The detection limit by dot blot on PVDF and ECL detection was about $10 \mathrm{pg}$ of IgG-B and IgG-SS-B with SA-HRP from Jackdson and 10ng for Dk-anti-hIgG-HRP.

Figure 40. Measure of Sensitivity of Detection Reagents Streptavidin-HRP and DkahmIgG-HRP by Dot blot on PVDF and Western. Stocks of IgG-B and IgG-ss-b where biotinylated at a molar ration of 2 biotins to $1 \mathrm{IgG}$

Figure 41: Comparison of Sigma and homemade streptavidin coated beads. 200ug of IgG, IgG-B and IgG-ss-B was applied to separate 100uL columns of Streptavidin coated agarose beads prepared by Sigma and compared to in house beads. The columns were washed with $3 \mathrm{mLs}$ of 1xPBS followed by the sample 
in $1 \mathrm{~mL} 1 \mathrm{xPBS}$, followed by a $1 \mathrm{~mL}$ clean. The beads were boiled in $1 \mathrm{~mL}$ of $2 \%$ SDS. Dilutions of the boiled beads were made as indicated, spotted on PVDF and detected by SA-HRP

Figure 42: Protein Assay of IgG, IgG-B and IgG-SS-B incubated with live cells and crude homogenates applied to control agarose columns and streptavidin-agarose columns and stained with coomassie brilliant blue. Columns were washed with 1xPBS then sequentially eluted 5mM DTT, 5\% mercaptoethanol, 2M ammonium hydroxide, $2 \mathrm{M}$ ammonium hydroxide $+50 \mathrm{mM}$ biotin.

Figure 43: Western dot blot of IgG, IgG-B and IgG-SS-B incubated with live cells and crude homogenates applied to control agarose and streptavidin-agarose columns and washed with 1xPBS and then eluted sequentially with 5mM DTT, 5\% mercaptoethanol, $2 \mathrm{M}$ ammonium hydroxide, $2 \mathrm{M}$ ammonium hydroxide $+50 \mathrm{mM}$ biotin. The elutions were spotted on a PVDF membrane and visualized with a Dk-anti-hIgG-HRP probe in ECl.

Figure 44: Western Dot Blot of Crude, IgG-B and IgG-SS-B incubated with live cells and and crude homogenates applied to control agarose columns and streptavidin affinity columns (not labelled) with Dkanti-hIgG Antibody. Legend of Elutions buffers: D - 5mM DTT, M - 5\% Beta Mercaproethanol, N 2M Ammonium Hydroxide, B - 2M Ammonium Hydroxide $+50 \mathrm{mM}$ biotin, Y-Spun down cytoskeleton

Figure 45: Treatments of no IgG, IgG, IgG-B and IgG-SS-B were applied to either live cells or crude homogenates and applied to streptavidin columns. The columns were washed and eluted with 5mM DTT, $5 \%$ mercaproethanol, $2 \mathrm{M}$ ammonium hydroxide and $2 \mathrm{M}$ ammonium hydroxide $+50 \mathrm{mM}$ biotin. Live cells were incubated on ice with ligands for 30 minutes and then washed 3 times prior to French pressing. Ligands were added to crude homogenate immediately before French pressing. IgG was detected in each fraction by Western blot with DK anti-hIgG-HRP

Figure 46: Treatments of no IgG, IgG, IgG-B and IgG-SS-B were applied to live cells and applied to streptavidin columns. The columns were washed and eluted with (A) 5mM DTT and 5\% mercaptoethanol, (B) 2M ammonium hydroxide, 2M ammonium hydroxide $+50 \mathrm{mM}$ biotin and (C) scrubbed with $2 \%$ SDS (from both live and crude homogenates only for SDS) Live cells were incubated on ice with ligands for 30 minutes and then washed 3 times prior to French pressing. Ligands were added to crude homogenate immediately before French pressing. (D) Crude homogenates were applied to streptavidin coated beads and naked beads and eluted with the buffers previously listed. IgG was detected in each fraction by Western Blot with DK-anti-hIgG-HRP. PVDF dipped in methanol, blocked for 30 minutes with $5 \%$ skim milk $+1 \%$ Dk serum.

Figure 47. LC-MS/MS TIC and base peak traces of the biotinylated ligand affinity chromatography experiment performed with live RAW macrophages incubated with IgG-SS-B, IgG-B, IgG and crude extracts poured over a streptavidin-agarose column and crude extracts poured over naked beads and eluted with DTT then 5\% B-mercaptoethanol, $\mathrm{NH} 4 \mathrm{OH}$ and $\mathrm{NH} 4 \mathrm{OH}+$ biotin.

Figure 48: LC-MS/MS TIC and base peak traces of the biotinylated ligand affinity chromatography experiment with live RAW macrophages incubated with IgG-SS-B, IgG-B, IgG and crude extracts poured over a streptavidin column and crude extracts poured over naked beads and eluted with $\mathrm{NH} 4 \mathrm{OH}$ followed by $\mathrm{NH} 4 \mathrm{OH}+$ Biotin.

Figure 49: Dumbroff protein assay of IgG boiled off $5 \mathrm{ug}$ of IgG coated glass beads in 200uL of 2\% SDS. Roughly 100ng of IgG eluted from the glass beads when boiled in SDS. 
Figure 50: Dot blot western of IgG boiled off $5 \mathrm{ug}$ of IgG coated glass beads in $200 \mathrm{uL}$ of $2 \%$ SDS using a Dk-anti-hIgG conjugated to HRP. Roughly 100ng of IgG eluted from the glass beads when boiled in SDS.

Figure 51: Coomassie stained BSA coated glass micro beads (left) and nano beads (right) applied to a sucrose gradient.

Figure 52: A. Glass beads without coating (Control) applied to RAW macrophages for 20 minutes in HEPES buffer at $37^{\circ} \mathrm{C}$. Outside beads visualized with Dk anti Human $2^{\circ}$ Ab conjugated to Dy488. B. Glass beads coated with poly D lysine and IgG and applied to RAW macrophages for 20 minutes in HEPES buffer at $37^{\circ} \mathrm{C}$. Outside beads visualized with Dk anti Human $2^{\circ} \mathrm{Ab}$ conjugated to Dy $488 \mathrm{C}$. Glass beads coated with poly L lysine and IgG and applied to RAW macrophages for 20 minutes in HEPES buffer at $37^{\circ} \mathrm{C}$. Outside beads visualized with Dk anti Human $2^{\circ}$ Ab conjugated to Dy 488 .

Figure 53: PMMA microbeads washed in (A) $\mathrm{MeOH}$, (B) isopropanol or (C) 5\% formic acid prior to coating in IgG then washed with serum free media, cooled for $10 \mathrm{~min}$ on ice and applied to cells for 30 min to allow binding

Figure 54: PMMA Nano beads washed in (A) $\mathrm{MeOH}$, (B) isopropanol or (C) 5\% formic acid prior to coating in IgG then washed with serum free media, cooled for $10 \mathrm{~min}$ on ice and applied to cells for 30 min to allow binding

Figure 55: (A) PMMA, (B) Melamine and (C) glass micro beads coated in IgG and applied to cells washed $3 \mathrm{x}$ in serum free media, incubated on ice and ligand coated beads applied for $30 \mathrm{~min}$ on ice

Figure 56: (A) PMMA, (B) Melamine and (C) glass nano beads coated in IgG and applied to cells washed $3 \mathrm{x}$ in serum free media, incubated on ice and ligands applied for $30 \mathrm{~min}$ on ice

Figure 57. Dumbroff dot blot of IgG coated melamine micro beads applied to RAW cells in serum free media, isolated by sucrose gradient and sequentially extracted in increasing salt buffers followed by increasing acetonitrile.

Figure 58. Western dot blot of human IgG coated melamine micro beads applied to RAW cells in serum free media, isolated by a sucrose gradient and sequentially extracted in increasing salt buffers up to 1000 $\mathrm{mM} \mathrm{NaCl}$ followed by $650 \mathrm{mM} \mathrm{NaCl}$ with increasing amounts of acetonitrile. Detected by a Dk anti Human $\operatorname{IgG}(\mathrm{L}+\mathrm{H})$ conjugated to HRP and $\mathrm{ECl}$.

Figure 59. Western dot blot against mouse Fcgr1 from a LARC experiment with IgG coated melamine micro beads applied to RAW cells in serum free media, isolated by a sucrose gradient and sequentially extracted in increasing salt buffers up to $1000 \mathrm{mM} \mathrm{NaCl}$ followed by $650 \mathrm{mM} \mathrm{NaCl}$ with increasing amounts of acetonitrile. Detected by a Rabbit anti Mouse primary Ab and a goat anti Rabbit HRP conjugated secondary $\mathrm{Ab}$ with $\mathrm{ECl}$. The goat anti rabbit antibody looks to be cross reactive with human IgG.

Figure 60. Dumbroff dot blot of IgG coated PMMA microbreads applied to RAW cells in serum free media, isolated by sucrose gradient and sequentially extracted in increasing salt buffers followed by increasing acetonitrile. Table Rt: visual comparison of fraction to standards to determine protein content

Figure 61. Western dot blot of human IgG coated PMMA micro beads applied to RAW cells in serum free media, isolated by a sucrose gradient and sequentially extracted in increasing salt buffers up to 1000 
$\mathrm{mM} \mathrm{NaCl}$ followed by $650 \mathrm{mM} \mathrm{NaCl}$ with increasing amounts of acetonitrile. Detected by a Dk anti Human $\operatorname{IgG}(\mathrm{L}+\mathrm{H})$ conjugated to $\mathrm{HRP}$ and $\mathrm{ECl}$.

Figure 62: Silver stain of LARC RAW IgG alongside crude control. 1ul of MW Std; 10ul sample and 2 ul $\mathrm{IgG}$ as positive control (silver stain performed by Angelique Florentinas) 
List of Abbreviations

A, agarose

aa, amino acids

AcN, acetonitrile

BME, mercaptoethanol

$\mathrm{CH}$, constant heavy

CL, constant light

Dk, donkey

DTT, 1,4-dithiothreitol

Fab. Fragment antigen binding

Fc, fragment crystallizable

$\mathrm{HB}$, homogenization buffer

hIgG-HRP, anti human IgG-HRP

IgG-B, IgG tagged with NHS-biotin

KEGG, Kyoto Encyclopedia of Genes and Genomes

LC-ESI-MS/MS, liquid chromatography electrospray ionization tandem mass spectrometry

LTQ, linear trap quadrupole

NAFLD, nonalcoholic fatty liver disease

$\mathrm{NH}_{4} \mathrm{OH}$, ammonium hydroxide

NHS-Biotin, N-hydroxysulfosuccinimide biotin

PMMA, Poly methyl methacrylate

$\mathrm{RSG}$, random MS/MS spectra generator

ROS, reactive oxygen species

SA, streptavidin

SA, SA-agarose (in treatment tables)

SA-HRP, streptavidin-HRP

SDS, sodium dodecyl sulfate

TTN, Titan 
$\mathrm{VH}$, variable heavy

VL, variable light 
List of Abbreviations for experimental treatments

$\mathrm{Ccb}$, crude homogenates applied to a control agarose column

Csa, crude homogenates applied to a SA-agarose column

Lsa, IgG applied to live cells applied to a SA-agarose column

LBsa, IgG-B applied to live cells applied to a SA-agarose column

LSBsa, IgG-SS-b applied to live cells applied to a SA-agarose column

LABsa, heat aggregated and then NHS biotinylated IgG ligand applied to live cells and captured by a SA-agarose column

LBAsa, NHS biotinylated IgG then heat aggregated ligand applied to live cells and captured by a SAagarose column

LASBsa, heat aggregated and then NHS-SS-biotinylated ligand applied to live cells and captured by a SA-agarose column

LSBAsa - NHS-SS-biotinylated then heat aggregated ligand applied to live cells and captured by a SAagarose column 


\section{INTRODUCTION}

\section{The plasma membrane}

The plasma membrane is an important interface that separates the cell from it's environment. The structure and organization at the plasma membrane is likely very important for the function of receptor complex assembly and function. Typically, plasma membrane proteins make up 50\% of a membrane's total mass and there are roughly 50 phospholipid molecules for every protein molecule on the average mammalian cell membrane (Lodish et al., 2000). Most molecules are unable to passively diffuse through the cell membrane, so they arrive at the cell surface in the form of a chemical or peptide ligand and interact with the extracellular domain of their corresponding cell surface receptor.

\section{Cell surface receptors}

Cell surface receptors play an important role in generating chemical ion gradients; transferring nutrients, metabolites and waste molecules into and out of the cell and sensing and responding to the extracellular environment. Ligand binding at a cell surface receptor often induces a conformational change resulting in activation or signal transduction by post translational modification and/or the recruitment of adaptor proteins or co-receptors. The main types of cell surface receptors include ligand gated ion channels (LGICs), G-protein coupled receptors (GPCRs) and enzyme linked receptors (Fig.1) 
(A)

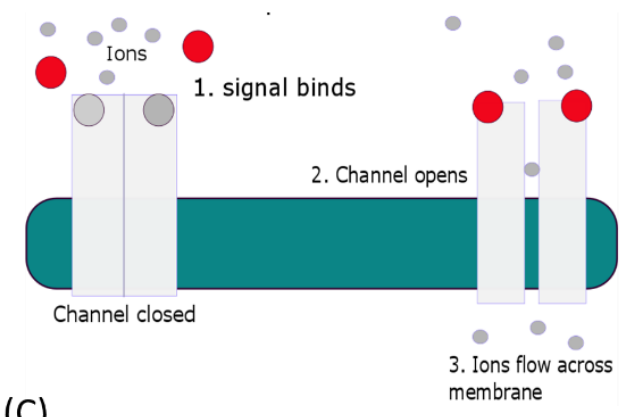

(C)

G-protein coupled receptors

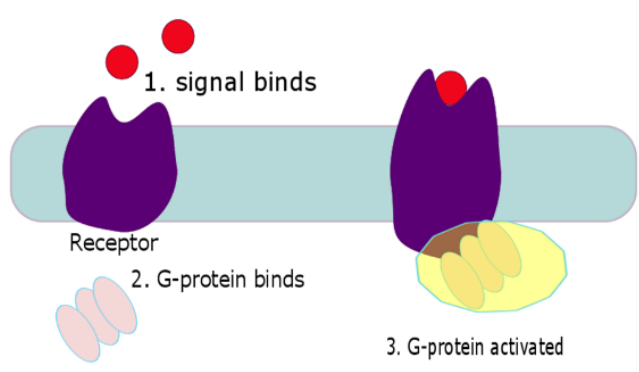

(B)

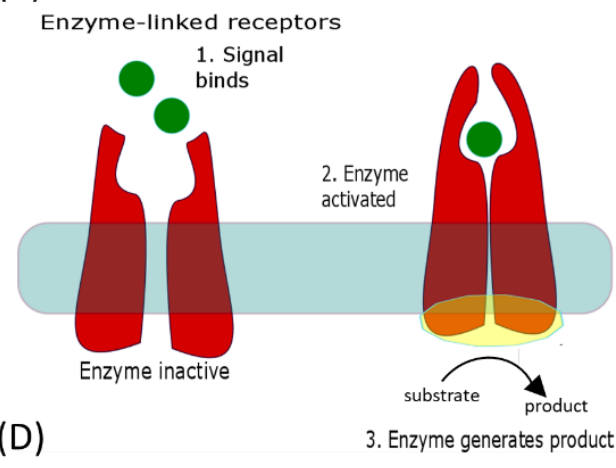

Signaling molecule

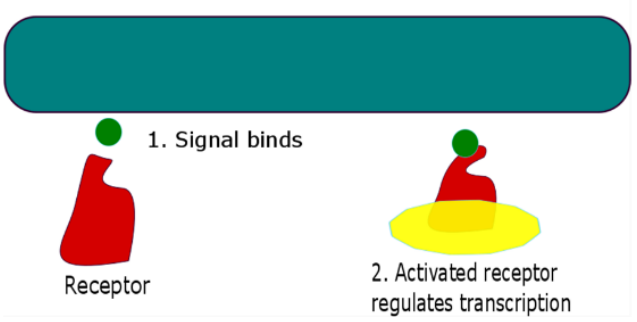

Figure 1: General categories of cell receptors. (A) ion channel-linked receptors, (B) enzymelinked receptors (C) G-protein-coupled receptors (D) Membrane permeable signaling molecules for intracellular receptors. Image recreated from (Purves, Augustine, \& Fitzpatrick, 2001).

LGICs are integral membrane proteins that contain a pore that selectively allows for the passage of ions across the cell membrane. The allosteric binding of the ligand causes a conformational change that opens or closes the pore. GPCRs are a large family of seven-transmembrane receptors that regulate cellular function through their associations with a heterotrimeric Gprotein. Ligand binding causes a conformational change that results in the exchange of GTP for GDP activating downstream signalling. Enzyme linked receptors contain a cytosolic enzymatic domain that becomes active after extracellular ligand binding (Fig.1).

The isolation and characterization of cell surface receptor complexes is of paramount biological importance. It is estimated that approximately $26 \%$ of human protein genes encode integral 
membrane proteins however the structure and function of most of these have not yet been elucidated (Fagerberg, Jonasson, von Heijne, Uhlén, \& Berglund, 2010; Krogh, Larsson, Von Heijne, \& Sonnhammer, 2001; U. Pieper et al., 2013; Wallin \& Heijne, 1998). As early as 1906, John N. Langley predicted that cell function could be manipulated by applying small molecules to cells that would mimic or inhibit their natural ligands (Langley, 1906). Transmembrane proteins currently make up nearly $60 \%$ of all primary drug targets where GPCRs account for 30\% and LGICs for 10\% (Lagerstrom \& Schioth, 2008; Overington, Al-Lazikani, \& Hopkins, 2006). Transmembrane proteins are also important secondary drug targets as they may impact the absorption, distribution, metabolism and excretion of drugs. Stagnation in the identification of new therapeutic targets may be due in large part to the difficult task of isolating and characterizing new ligand-receptor interactions. The identification and characterization of cell surface membrane proteins and their interaction partners will likely reveal important biomarkers and therapeutic targets for the assessment and treatment of disease. While there have been numerous attempts to isolate cell surface receptor complexes by affinity chromatography and mass spectrometry, for the most part, these methods have yielded small numbers of the ligand and target receptor compared to controls. Thus, a new general method for the isolation and characterization of cell surface receptor complexes is needed.

Unlike cytosolic protein complexes, cell surface membrane associated receptor complexes are much more difficult to isolate and characterize. Cell surface receptors are transmembrane proteins with an extracellular domain, a cytosolic domain, and between 1 to 20 membrane spanning domains, each containing on average 15-20 hydrophobic amino acids that typically form an alpha helix (Helbig, Heck, \& Slijper, 2010). Beta barrel folds are also important 
transmembrane domains but are far less common and only found in mitochondrial membranes in humans. These hydrophobic domains are difficult to extract from the plasma membrane and may non-specifically adsorb to hydrophobic chromatography supports. The type of membrane receptor and the number of hydrophobic domains likely affect how easily the protein dissociates from the plasma membrane and how readily it adsorbs to hydrophobic chromatography supports (Fig. 2). However, it is also likely that receptor complex assembly requires the organization of the plasma membrane.

\section{A}

B

C

D

$E$

$\mathrm{F}$

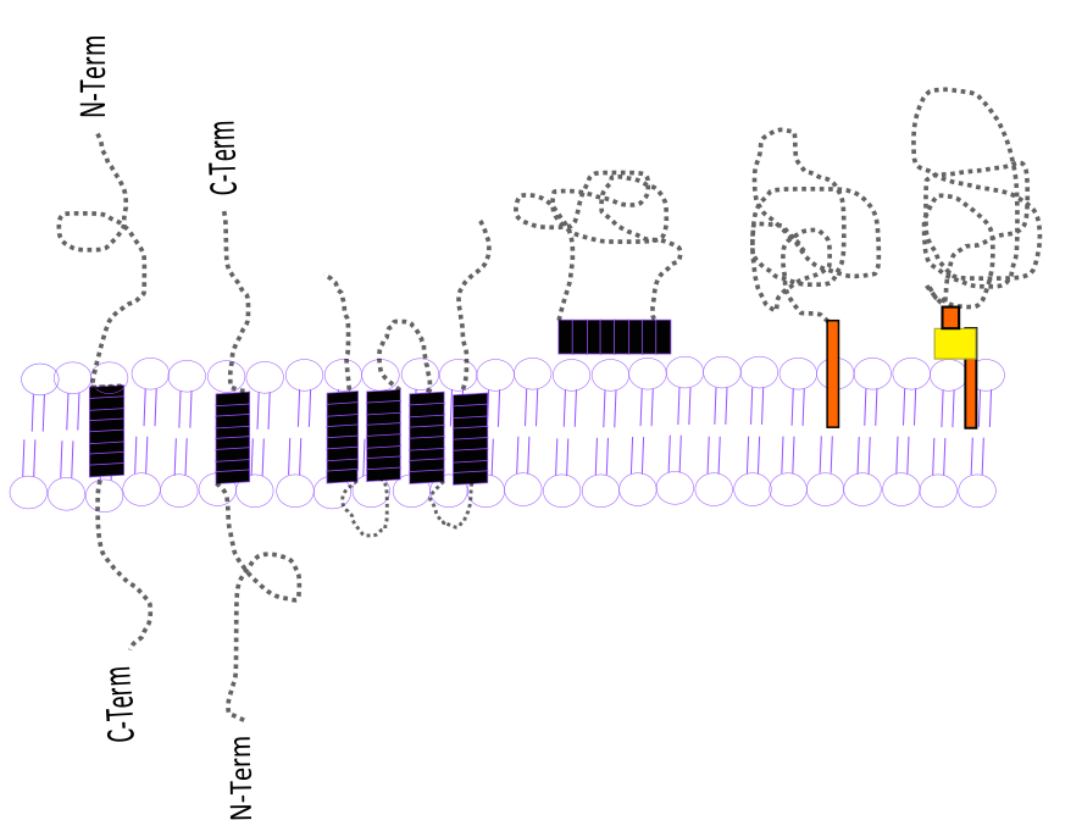

Figure 2. Classes of membrane proteins. (A) Type I are single pass membrane proteins with a cytosolic C-terminus and extracellular N-terminus. (B) Type II are single pass with a cytosolic N-terminus and extracellular C-terminus. (C) Multipass membrane proteins span the plasma membrane multiple times (D) Peripheral membrane proteins interact with the membrane by hydrophobic or ionic interactions (E) Lipid chain anchored proteins are covalently linked to a lipid (F) GPI anchored proteins are covalently linked to a glycolipid. 
The hydrophobic domains of membrane associated cell surface receptor complexes also present additional challenges for detection and identification by high performance liquid chromatography and tandem mass spectrometry (LC-MS/MS). Since there are very few charged amino acids in the hydrophobic transmembrane portions of receptors there is likely less coverage of these proteins and therefore it may be more challenging to make protein identifications as a result. Not only are receptors generally less abundant than cytosolic proteins but they may generate fewer peptides and show less coverage by LC-MS/MS.

\section{The IgG/Fc Receptor Complex}

The IgG-Fc receptor is a suitable model system for the capture and characterization of ligand activated cell surface receptor complexes as affinity purified human IgG1 is readily available and IgG-Fc receptor mediated phagocytosis is a well studied system with 93 known proteins in the Kyoto Encyclopedia of Genes and Genomes (KEGG) pathway (Fig.3). The IgG-Fc receptor complex mediates a number of immune responses in different cell types including the phagocytosis of IgG opsonized pathogens, antibody-dependent cell-mediated cytotoxicity (ADCC), the release of inflammatory cytokines and the production of Reactive Oxygen Species (ROS)(Anderson, Shen, Eicher, Wewers, \& Gill, 1990; Titus, Perez, Kaubisch, Garrido, \& Segal, 1987; Young, Ko, \& Cohn, 1984). Since the IgG-Fc receptor complex is associated with a number of cellular responses a role for identified targets can be shown using simple phagocytosis assays, or the quantification of ROS or inflammatory cytokines in combination with siRNA

knockdowns or drugs (A. K. Florentinus, P. Bowden, V. Barbisan, \& J. Marshall, 2012; Andy Jankowski, Peihong Zhu, \& John G Marshall, 2008; Vance et al., 2016) 


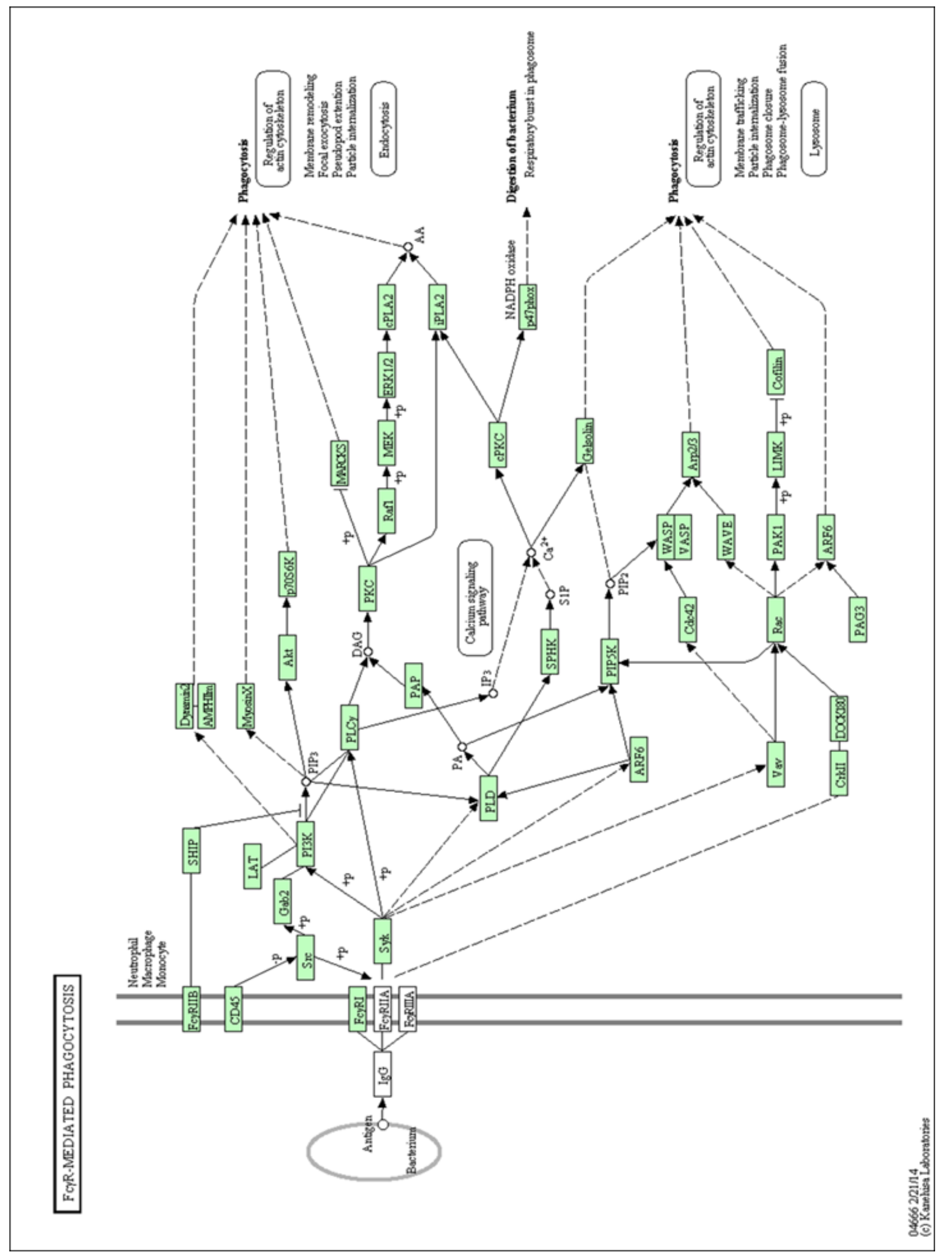

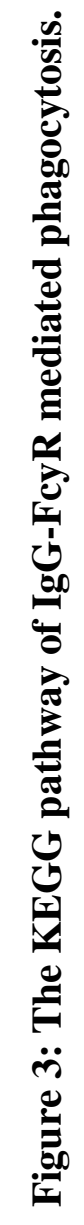


The IgG ligand and Fc receptors are part of the Ig superfamily of molecules that contain immunoglobulin (Ig) folds consisting of two antiparallel beta pleated sheets (Fig.4). This domain is known to play a role in cell adhesion and antigen binding and antigen presentation. There are 5 immunoglobulins in humans including $\operatorname{IgA}, \operatorname{IgD}, \operatorname{IgE}, \operatorname{IgG}$ and $\operatorname{IgM}$. IgG is the most abundant in human serum of which there are four subclasses: IgG1, IgG2, IgG3 and IgG4. The IgG subtypes have highly conserved constant domains with only slight variations at the $\mathrm{CH} 2$ domain and the hinge region which impact binding affinity for the various Fc $\gamma$ receptors.

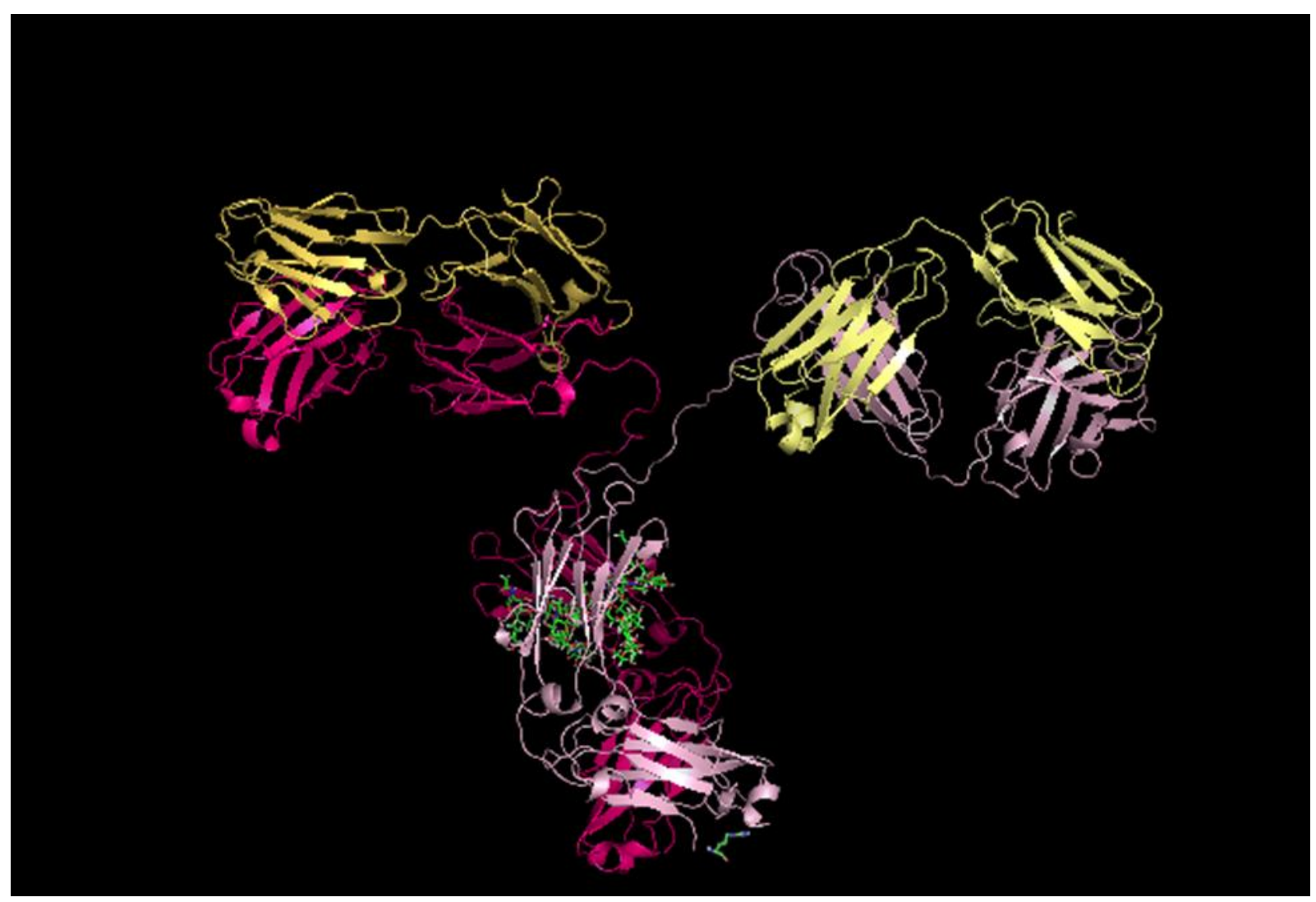

Figure 4: The structure of IgG derived from X-ray chrystallography with the carbohydrate moiety essential for binding shown. The PyMOL Molecular Graphics System, Version 1.5.0.4 Schrödinger, LLC. (Harris, Skaletsky, \& McPherson, 1998) 


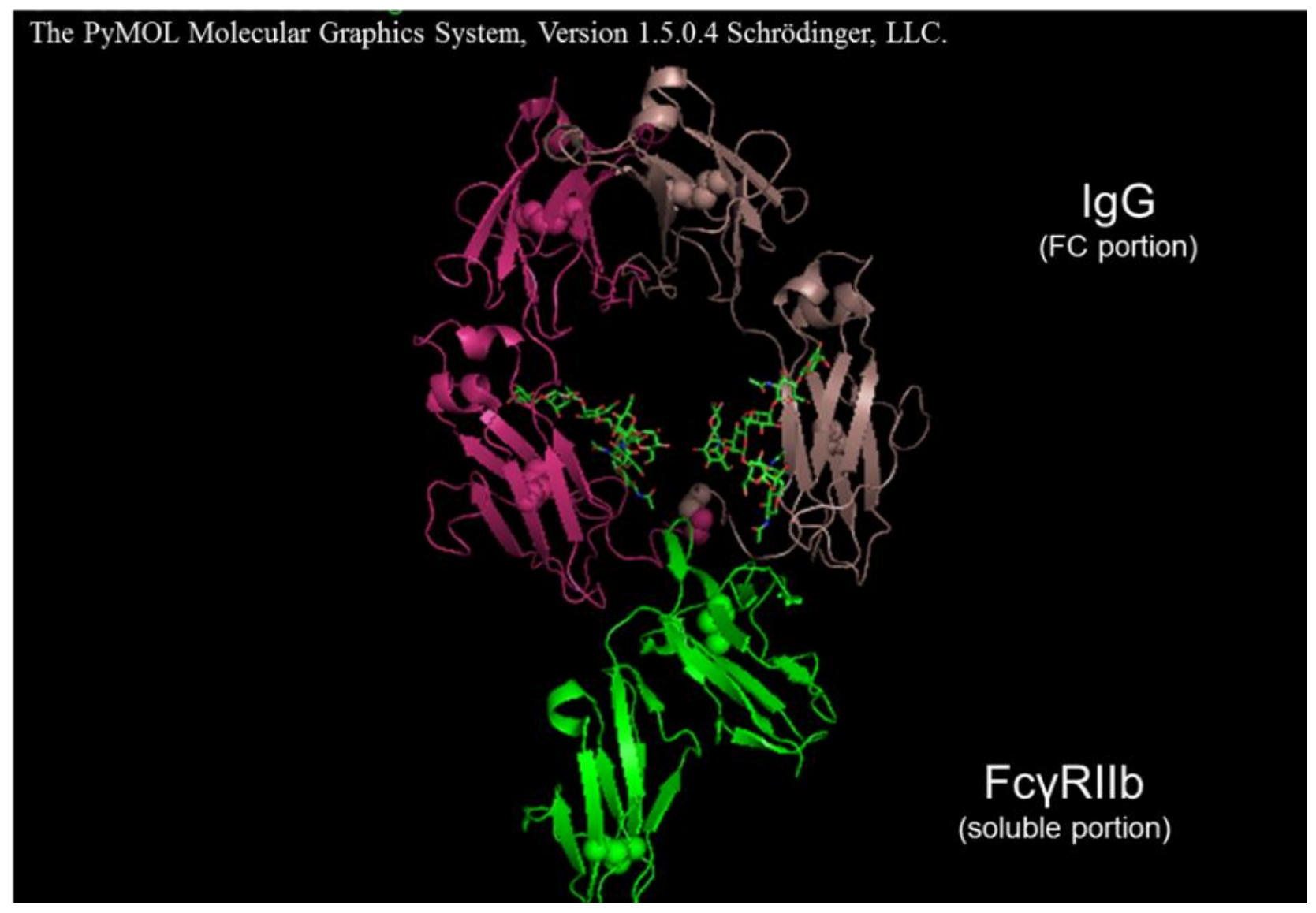

Figure 5: The structure of interaction the Fe protion of IgG bound to the soluble portion of FcyRIIb derived from X-ray chrystallography The PyMOL Molecular Graphics System, Version 1.5.0.4 Schrödinger, LLC (Sondermann, Huber, \& Jacob, 1999)

There are seven Fc $\gamma$ receptors in humans and five in mice. The Fc $\gamma$ receptors are classified as either activating due to the presence of a cytosolic ITAM domain or inhibitory due to an ITIM domain (Fig.6). In the case of activating human Fc $\gamma$ RIIA and Fc $\gamma$ RIIC, the ITAM domain is part of the receptor polypeptide chain whereas in mouse and human Fc $\gamma$ RI and Fc RIIIA and mouse FcyRIV, the ITAM domains are located on an associated subunit (Bolland \& Ravetch, 1999; Daëron, 1997; Ravetch \& Kinet, 1991). Both activating and inhibitory Fcy receptors are typically co-expressed and co-engaged by IgG ligands. The IgG subtypes have been shown to have distinct binding affinities for the various Fc $\gamma$ receptors and also reactivity for certain 
antigen types. For example, $\operatorname{IgG} 2$ has been associated with greater anti carbohydrate reactivities compared to the other subtypes (Ferrante, Beard, \& Feldman, 1990). Also, the Fcy receptor isotypes have been shown to exhibit different functions in the cell. For example, the inhibitory Fc $\gamma$ RIIB2 localizes to clathrin-coated pits while Fc $\gamma$ RIIB1 does not (Miettinen et al, 1989)

(Fig.11). Fc $\gamma \mathrm{R} 1$ is a $72 \mathrm{kD}$ protein and is the highest affinity Fc $\gamma$ receptor for the IgG1 ligand (10$\left.{ }^{9}\right)$ compared to the lower affinity receptors Fc $\gamma$ RIIA and FcyrIIIA $\left(10^{-6}\right)$ (Ravetch \& Kinet, 1991). The Fc $\gamma$ R1, Fc $\gamma r I I A$ and Fc $\gamma r I I I A$ are known to mediate phagocytosis (Joshi, Butchar, \& Tridandapani, 2006).

Human

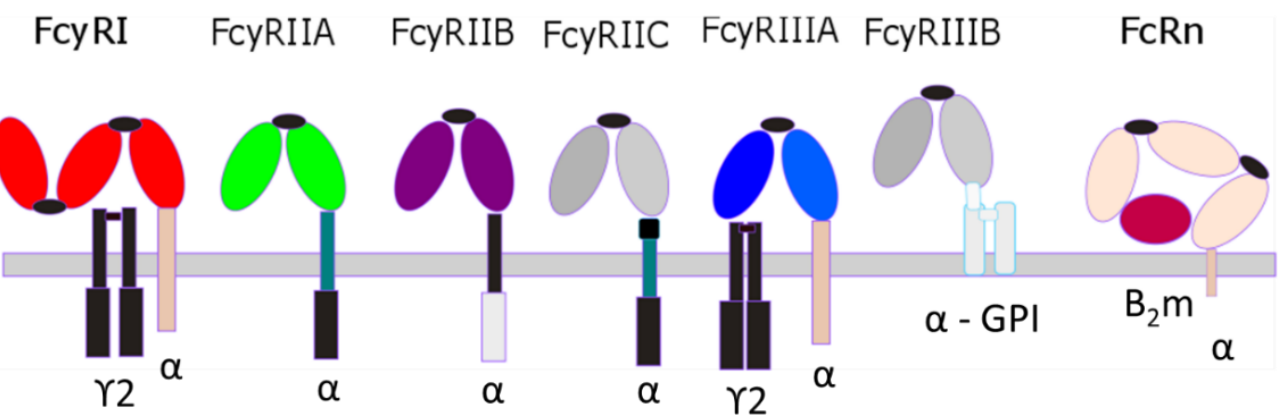

Mouse

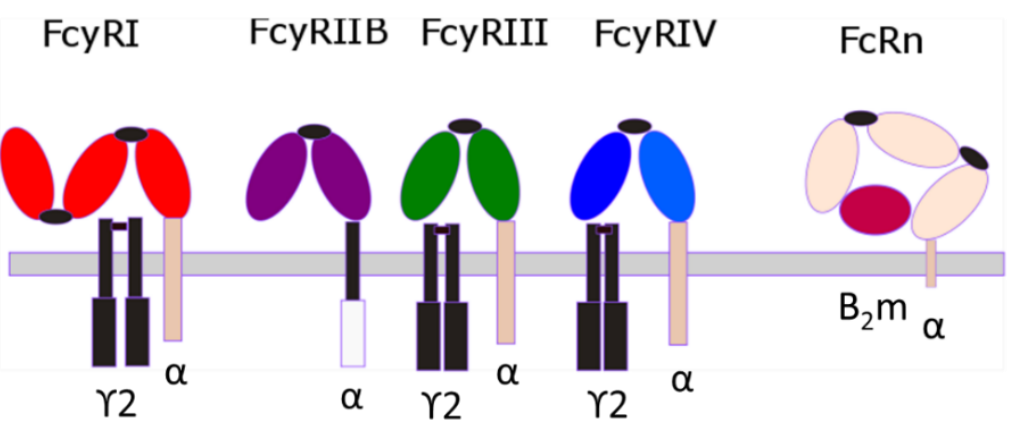

Figure 6. Fc receptors in humans and mice. ITAM (black) are activating while ITIM domains (white) inhibit phagocytosis. Recreated from (Bruhns, 2012). 
IgG-Fc mediated phagocytosis is carried out exclusively by specialized cells including dendritic cells, macrophages and neutrophils and form an important bridge between innate and adaptive immunity (Rabinovitch, 1995). Phagocytosis was first identified by Ilya Metchnikov in 1884 and is described as the receptor mediated engulfment of particles greater than 0.5 um (Metschnikoff, 1884). As the Fc $\gamma$ receptors bind the FC portions of aggregated IgG ligands, signalling is initiated by the clustering of receptors (Fig.7). Multivalent ligands are a prerequisite for downstream cell signalling to occur (Odin, Edberg, Painter, Kimberly, \& Unkeless, 1991). After receptor clustering, the cytosolic ITAM (tandem YxxI/L motifs) domains are tyrosine phosphorylated by Src family kinases (Crowley et al., 1997). Syk binds the phosphorylated ITAM domains via two SH2 domains and recruits and phosphorylates adaptor protein Lat (Tridandapani et al., 2000) which recruits adaptor proteins Grb2 via an SH2 domain and subsequently Gab2. Gab2 associates with PI(3,4,5)P3 by a PH domain and recruits CrK1l (W. L. Lee, Cosio, Ireton, \& Grinstein, 2007). These adaptor proteins activate a number of lipid modifying enzymes and guanine nucleotide exchange factors for Rho family GTPases such as Rac1, Rac2 and Cdc42 that are essential for driving actin assembly and cytoskeleton modification leading to the engulfment of the particle (Lerm, Brodin, Ruishalme, Stendahl, \& Sarndahl, 2007)(Fig.7). 


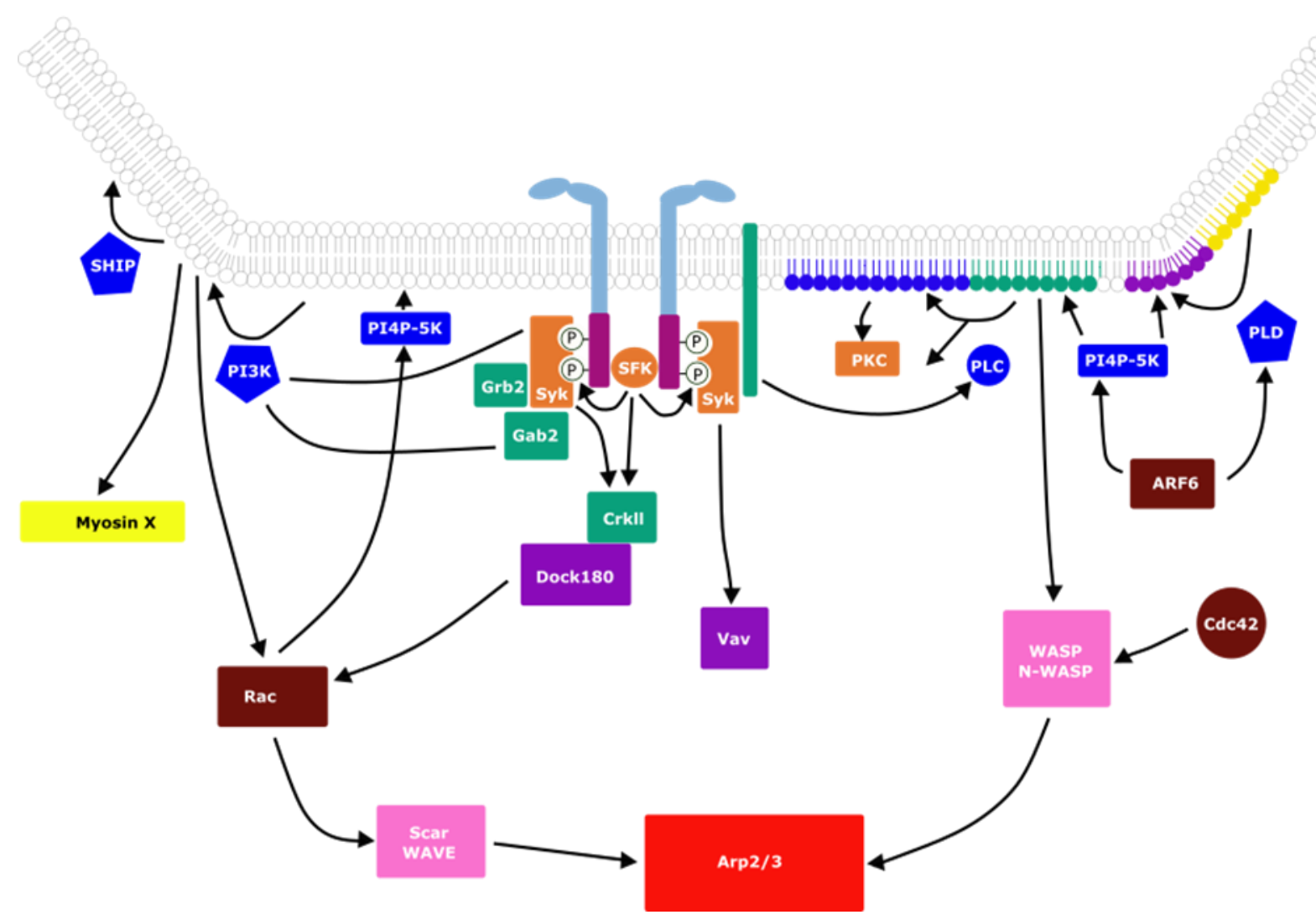

Figure 7: The IgG-Fcy mediated phagocytosis pathway. After ligand activation and receptor clustering the Src family kinases (orange) phosphorylate and/or recruit adaptor proteins (green) that act as a platform for activating lipid modifying enzymes (blue), guanine nucleotide exchange factors (purple) for small GTPases (brown) activating nucleation promoting factors (pink) and the actin nucleation complex. Recreation of the image from (Flannagan, Jaumouille, \& Grinstein, 2012).

In addition to the Fc $\gamma$ receptors, $\operatorname{IgG} 1$ was recently found to bind TRIM21 which is a mediator of innate immunity. Unlike the Fc gamma receptors, Trim21 is a soluble receptor that binds IgG on the conserved Ig domains of the Fc fragment by a PRYSPRY domain (Keeble, Khan, Forster, \& James, 2008). TRIM21 was identified by the immune system as an autoantigen in patients suffering from autoimmune diseases such as rheumatoid arthritis, systemic lupus erythematosus, and Sjögren's syndrome (Ben-Chetrit, Chan, Sullivan, \& Tan, 1988; Moutsopoulos et al., 1985). 


\section{Classical purification of receptors}

Cell surface receptors are usually present in low stoichiometric quantities, on the order of tens of thousands of molecules per cell, and can be difficult to isolate due to their hydrophobic nature (Elschenbroich, Kim, Medin, \& Kislinger, 2010; Helbig et al., 2010; Ravetch \& Kinet, 1991). One strategy for increasing the yield of cell surface receptors is to start with very large amounts of cellular material. Once a receptor ligand interaction is identified; traditionally by gel electrophoresis, recombinant DNA technology can be used to express large quantities of the target receptor so that the kinetics of the ligand receptor interaction can be studied. The use of recombinant DNA technology for isolating and identifying receptor complexes requires previous knowledge of the interaction and overexpression can distort the cell's regular metabolism and function. The hydrophobic nature of membrane proteins also presents multiple challenges for the purification of ligand activated membrane associated supramolecular complexes. The force required to extract a cell surface receptor complex from the plasma membrane may be too great compared to the force generated by one or several receptor ligand interactions. Some groups have attempted to overcome this challenge with the use of cross linkers with apparently little success (Frei et al., 2012). The purification of an active receptor often requires a combination of multiple ultracentrifugation steps in various harsh extraction buffers including a non-ionic detergent often followed by precipitation and centrifugation and/or dialysis to remove the detergent before conducting activity assays with the purified ligand.

Detergents are amphipathic molecules that form micelles at concentrations equal to or greater than the critical micelle concentration. Detergents disrupt hydrophobic interactions in the lipid bilayer by associating with hydrophobic phospholipid tails thus forming detergent-phospholipid mixed micelles. Detergents can help solubilize transmembrane proteins by forming hydrophobic 
interactions with the hydrophobic domains of the receptor leaving the hydrophilic portion of the detergent exposed and the protein more soluble. Some mild detergents such as deoxycholate and octyl glucoside are known to interact with lipid associated membrane proteins reversibly and in a non-denaturing manner where receptors retain some of their biological activity after the detergent is removed by precipitation or dialysis (Makino, Reynolds, \& Tanford, 1973). Homogenization by physical means and/or by detergents may interfere with receptor ligand binding but also likely interferes with binding to and eluting from the affinity chromatography surface (Andy Jankowski, Peihong Zhu, \& John G Marshall, 2008). While detergents can be used to separate and isolate cell surface receptors from the plasma membrane, detergents very likely interfere with the interaction of binding partners and co-receptors with the receptor complex (Von Jagow, Schägger, Riccio, Klingenberg, \& Kolb, 1977).

The difficult task of purifying membrane bound receptors is exemplified by the isolation and characterization of the $\beta$ adrenergic, insulin and the acetylcholine receptors among others in the 1970s and 1980s (Cuatrecasas, 1972; Heinrich, Pilch, \& Czech, 1980; Klett et al., 1973; Lefkowitz, 1973). The acetylcholine receptor for example, was isolated from the electric organ of an eel by homogenization, centrifugation, extraction with a $1 \%$ non-ionic detergent sodium deoxycholate, detergent precipitation first with $\mathrm{MgCl}_{2}$ and then spermine tetrahydrochloride and precipitated detergents removed by centrifugation (Klett et al., 1973). Isolates where pelleted by high speed ultra centrifugation at 100,000 xg for $6 \mathrm{hrs}$. The pellets were then resuspended in 1\% Tween80, dialyzed and centrifuged at 100,000 xg. At this point the supernatant contained only 10-20mg of total protein and only 0.5-1.0 nmoles per $\mathrm{mL}$ of the receptor (Klett et al., 1973).The preparations were further purified by gel filtration and ion exchange chromatography and affinity chromatography using a potent high affinity cobra-[3H] neurotoxin (Klett et al., 1973). The 
extraction resulted in an 8250 fold purification and a yield of only 5\% (Klett et al., 1973). This result underscores the low abundance of cell surface receptors and difficult task of isolating them from the plasma membrane even with a very high affinity ligand (Klett et al., 1973)

Early investigations of insulin showed that radiolabelled $\left[{ }^{125} \mathrm{I}\right]$ insulin could specifically bind fat and liver cells and induce a biological response (Freychet, Roth, \& Neville, 1971; Kono \& Barham, 1971). In subsequent experiments, the insulin receptor was purified from detergent solubilized adipocyte and liver cell membranes using various insulin-sepharose affinity columns and the purification was measured by activity and protein assays (Cuatrecasas, 1972). In a double radiolabelling experiment, radiolabelled $\left[{ }^{131} \mathrm{I}\right]$ insulin was combined with radiolabelled $\left[{ }^{125} \mathrm{I}\right]$ plasma membranes, crosslinked with disuccinimidyl substrate, solubilized in Triton X-100 and incubated with either anti-insulin antibodies or control antibodies and then captured by protein A-sepharose affinity chromatography. $30 \%$ of the labelled insulin and labelled receptor were captured using $3 \mathrm{M}$ potassium thiocyanate where anti insulin was used while only $2 \%$ was recovered using the control antibody. Gel electrophoresis showed a major 125,000 Da labelled band (Heinrich et al., 1980).

The $\beta$ adreneric receptor is another important example of the arduous task of isolating a cell surface receptor and the development a therapeutic drug propranolol to target the receptor (Benovic, Shorr, Caron, \& Lefkowitz, 1984; Lefkowitz, 2004; Stiles, Caron, \& Lefkowitz, 1984). Similar methods are not likely to be effective for the isolation of ligand activated supramolecular receptor complexes as detergents would likely cause the complex to fall apart. 
However, the extraction of the receptor with detergents was useful for biochemical characterization of binding properties.

The Fcy receptor was previously captured by rabbit IgG coupled sepharose from radiolabelled preparations of alveolar macrophages and eluted with $0.5 \mathrm{~N}$ acetic acid and $1 \%$ of the powerful non-ionic detergent NP-40. The receptor was visualized by acrylamide gel electrophoresis (Kulczycki, Krause, Killion, \& Atkinson, 1980). FcrRB1 was first isolated by genetic methods by truncation of the cytoplasmic and transmembrane domains and transfecting the truncated cDNA plasmid into a CHO cell line (Qu, Odin, Glass, \& Unkeless, 1988). A 40-75\% ammonium sulfate precipitation followed by dialysis was used to concentrate the target receptor at lower ionic strength and the truncated receptors were isolated and purified by affinity chromatography using an IgG2BK- agarose-4B column (Qu et al., 1988). To elucidate the function of the Fcy receptors, cell types without endogenous Fc $\gamma$ receptors such as COS-1 have been used as model systems to transfect and study the Fc $\gamma$ isoforms. These studies demonstrated that the Fc receptors encode all of the structural information required to regulate phagocytosis (Indik, Park, Hunter, \& Schreiber, 1995). The Fc $\gamma$ receptor isotypes have several different extracellular domains. FcR $\gamma \mathrm{I}$ has three IgG binding domains compared to the rest of the Fc $\gamma \mathrm{R}$ types and has greater affinity for IgG in general (Russell, 2009).

While affinity chromatography has proven to be a powerful tool for isolating the interaction partners of soluble proteins, the application of classical ligand affinity chromatography to native cell surface receptor complexes such as the insulin receptor and others has proven to be ineffective. The molecular identity of cell surface receptors was not achieved by ligand affinity 
chromatography but rather via gel electrophoresis and/or genetic methods (Allan, Auger, \& Crumpton, 1972; Cuatrecasas \& Parikh, 1974; Cuatrecasas, Wilchek, \& Anfinsen, 1968; Parikh, Sica, Nola, Puca, \& Cuatrecasas, 1974; Qu, Odin, Glass, \& Unkeless, 1988; Shiu \& Friesen, 1974; Sica, Nola, Parikh, Puca, \& Cuatrecasas, 1973; Sica, Parikh, Nola, Puca, \& Cuatrecasas, 1973).

\section{Chemical and genetic tags for affinity chromatography}

Biotin is a vitamin that was first discovered in 1941(Savage et al., 1994) and has become an important biotechnology tool as an affinity chromatography tag due its exceptionally high binding affinity to streptavidin, a tetramer isolated from Streptomyces avidinii with a $\mathrm{Kd}$ of $10^{-}$ ${ }^{15} \mathrm{~mol} / \mathrm{L}$ (Bayer \& Wilchek, 1980)(Fig.8). N-hydroxysulfosuccinimide (NHS) active esters are an effective means to couple biotin to primary amines found on lysine and the $\mathrm{N}$ termini of protein ligands under broad conditions of temperature and pH (Staros, 1982) (Fig.8). Streptavidin coated chromatography surfaces can be used to capture biotinylated cytosolic proteins however, the recovery of biotin from streptavidin without eluting many other non-specific proteins by $\mathrm{pH}$ or salts as chaotropes is a challenge a high binding affinity between under a wide range of conditions.

A

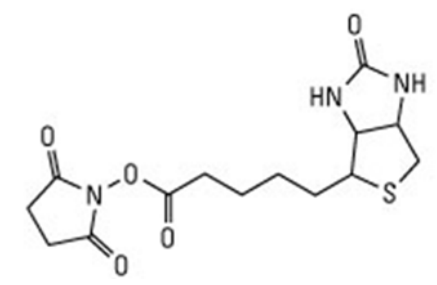

B

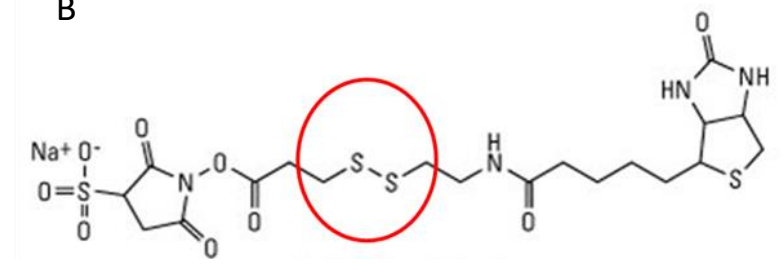

Figure 8. Chemical structure of NHS biotin (A) and the cleavable Sulfo-NHS-SS-Biotin tag (B). The cleavable disulphide portion is highlighted. 
One possible solution for the specific release of biotin tagged ligands without the use of harsh buffers is a cleavable NHS biotin tag. New biotin cleavable linkers have yet to be applied to the challenge of isolating activated cell surface receptor complexes in the native state by ligand affinity chromatography in conjunction with LC-ESI-MSMS.

The Sulfo NHS-SS-Biotin cleavable linker (Fig.8) may allow for more specific elution by reducing agents such as DTT and B-mercaptoethanol. Reducing agents like DTT have been previously used to elute detergent extracted $\operatorname{IgG}$ binding receptors. However, the use of reducing agents to capture activated receptor complexes may have some disadvantages. It is likely that many proteins bind the chromatography support non-specifically as complexes that are held together in part by disulphide bridges and may fall apart in the presence of reducing agents. As well, the reducing environment within the cell may prematurely reduce disulfide bridges after cells are homogenized and before they are applied to a SA-agarose column. The IgG ligand itself may also be sensitive to the reducing environment of cell homogenates as human IgG1 contains 12 intra-chain disulphide bridges which connect the 2 light and heavy chains of the complete molecule (Milstein, 1966). The inter-chain disulphide bridges (between 4 and 7 depending on the $\operatorname{IgG}$ isotype) are especially sensitive as they are highly solvent exposed while the intra chains are buried between two anti-parallel $\beta$-sheets (Kikuchi, Goto, \& Hamaguchi, 1986)(Fig.4). Thus, it may be difficult to determine if the receptor complex is eluted by the cleavage of the sulfo biotin linker or by the disruption if disulfide bonds within the IgG ligand. Also, biotinylation is a common cellular post-translational modification in certain proteins including histones that may be captured by the SA-agarose column and must be accounted for by controls. 
For some of these reasons, traditional biotinylated ligand affinity chromatography methods have not proven successful at isolating target receptors for molecular identification compared to controls when biotin tagged ligands were applied to live cells for affinity chromatography and analysed by mass spectrometry. While the advent of new affinity chromatography tags and technologies to simplify and optimize the isolation of protein-protein interaction complexes has been extremely useful with soluble complexes, a simple and efficient method for isolating membrane bound, native complexes remains elusive. Receptors likely require the organization of the surrounding membrane and cytoskeleton to help recruit adaptors and enzymes to the activated receptor complex (Corbett-Nelson, Mason, Marshall, Collette, \& Grinstein, 2006; Andy Jankowski, Peihong Zhu, \& John G Marshall, 2008; John G Marshall et al., 2001).

\section{Identification of unknown receptors by biotinylated ligand affinity chromatography methods}

A novel biotin linker with three arms: one that conjugates to a primary amine on the ligand by an $\mathrm{N}$-hydroxysuccinimide ester as previously described, a second that binds a glycosylated receptor and a third biotin arm for recovery by SA-agarose has also shown very low yield of the target receptor (Frei, Moest, Novy, \& Wollscheid, 2013). The three-armed ligand tagging system commonly known as ligand-receptor capture-TriCEPS (LRC-TriCEPS) was used to capture unknown receptors for the C1q TNF Related Protein 3 (CTRP1) ligand. CTRP1 is a secreted protein that is a possible therapeutic for non-alcoholic fatty liver disease (NAFLD). Mice treated with CTRP1 on a high fat diet were shown not to develop NAFLD however the receptors that bind CTRP1 in the liver are not yet known (Peterson, Seldin, Wei, Aja, \& Wong, 2013). LRCTriCEPS was used to identify potential receptors of the CTRP1 ligand in a rat hepatoma cell line (Y. Li, Ozment, Wright, \& Peterson, 2016). Two highly expressed glycoproteins LAMP-1 from 
lysosomes and LIMP II were deemed significant compared to an insulin control. However only 4 peptides of LAMP1 and 2 for LIMPII were identified (Y. Li et al., 2016). Thus, a more robust system where greater quantities of the target protein are captured and identified by LC-MS/MS is required.

\section{Ligand coated microbeads as chromatography surfaces}

A more successful strategy that clearly showed an enrichment of both the ligand and receptor compared to controls, was recently achieved by activating and capturing phagocytic receptors by presenting ligand coated polystyrene beads to live cells on the macroscopic spatial scale (A. K. Florentinus et al., 2012; Andy Jankowski, Peihong Zhu, \& John G Marshall, 2008). The micro bead associated receptor complexes were separated by ultracentrifugation in a sucrose gradient and sequentially eluted from the beads in a salt/acetonitrile step gradient and analyzed by high pressure liquid chromatography and tandem mass spectrometry (Fig.9). 


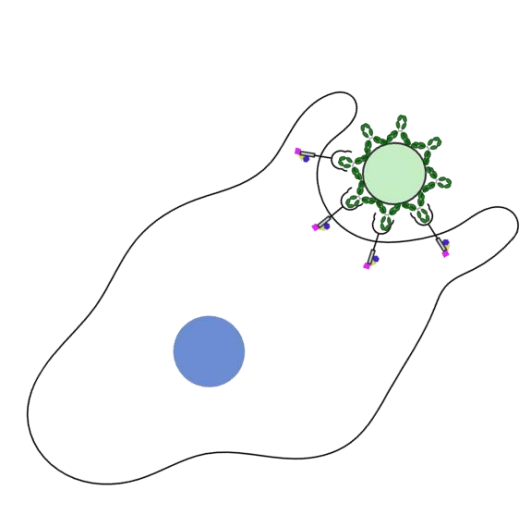

IgG coated beads incubated with live cells then washed
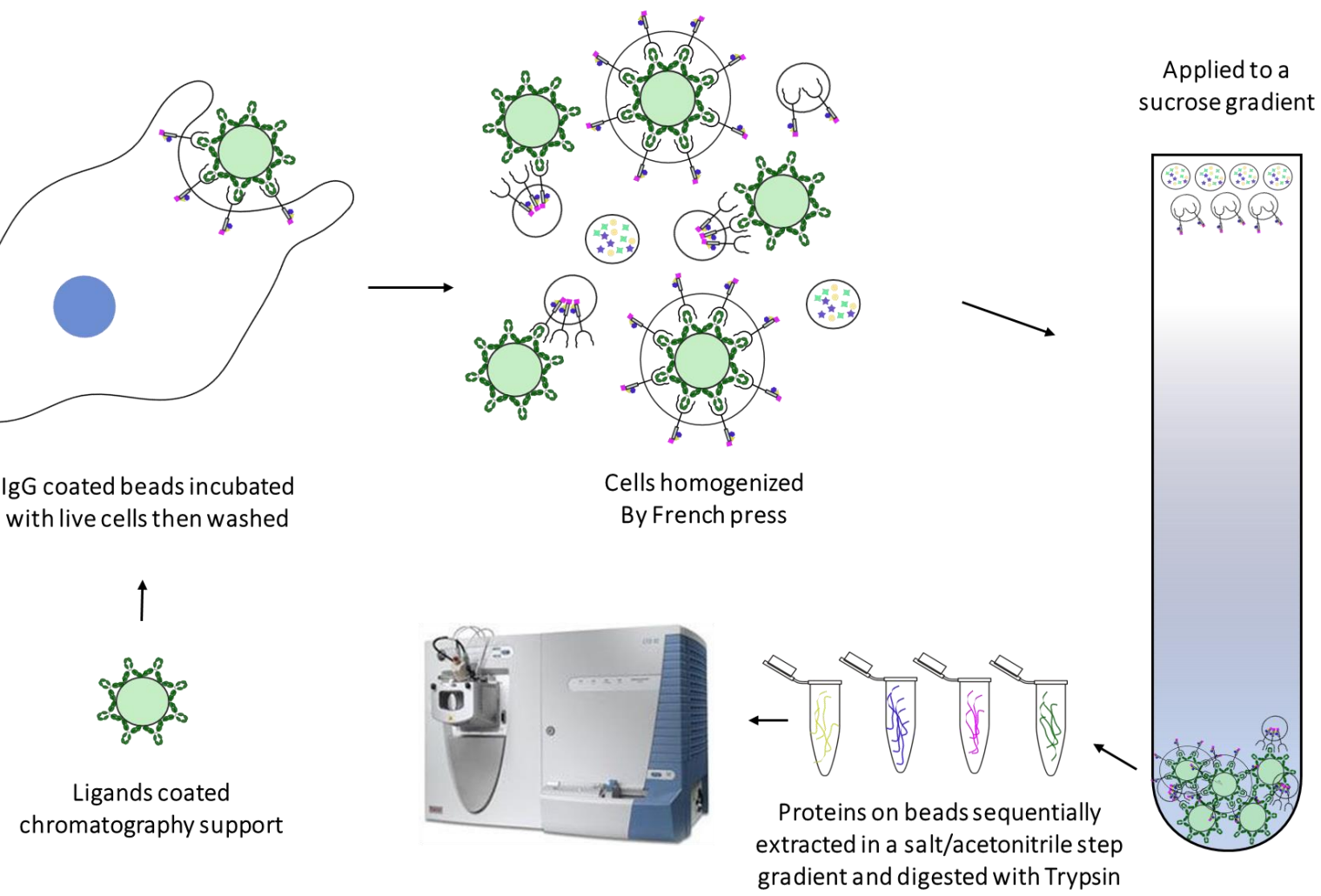

Figure 9. Ligand affinity receptor chromatography where $\mathrm{IgG}$ coated polystyrene beads were presented to live cells, allowed to bind, homogenized by French press, isolated by ultracentrifugation, washed and extracted with salt and AcN buffers prior to digestion and LCESI-MS/MS.

Using this method, receptor associated proteins of the IgG-Fc receptor complex including RHOG, P115 RHOGEF and CRKL, where identified and confirmed by drugs, antibody staining, dominant negative mutants and siRNA using polystyrene coated microbeads (A. Jankowski, P. Zhu, \& J. G. Marshall, 2008) and have been independently confirmed (Tzircotis, Braga, \& Caron, 2011) (Fig.5). Furthermore, the IgG coated polystyrene microbead method was used with different ligands where the Fc-IgG receptor complex was compared to the resulting complexes formed using BSA coated beads, naked beads, IgG coated beads with crude extracts and oxidized LDL coated beads (Jeffrey C Howard et al., 2016). The comparison of IgG to oxidized LDL 
coated polystyrene microbeads identified Syk-L as unique to the engulfment of oxidized LDL versus IgG which may be an extremely useful therapeutic target and may inhibit the engulfment of oxidized LDL by macrophages without compromising immune function (Jeffrey C Howard et al., 2016).

The use of polystyrene micro spheres as a chromatography support presents several challenges in this experiment. Polystyrene has a density of $1.06 \mathrm{~g} / \mathrm{cm} 3$ which makes it difficult for the microbeads to sediment and bind cell surface receptors on cultured adherent cells. As well, polystyrene micro beads have traditionally been difficult to isolate by ultra centrifugation due to inconsistencies in bead shape and density. Most importantly, hydrophobic polystyrene beads may incur a significant amount of background binding confounding the elucidation of the receptor complex and requiring numerous non-specific binding controls (Desjardins \& Griffiths, 2003; Gagnon et al., 2002; Andy Jankowski, Peihong Zhu, \& John G Marshall, 2008; Qin et al., 2016). The application of ligand coated microbeads may be a suitable solution for aggregated ligands as is the case for phagocytic receptors but is not likely a universal solution for the capture of monovalent ligands due to their large size, low density and hydrophobic nature.

A variant of the ligand coated microbead method was applied in a similar study using paramagnetic beads coated in fibronectin and VCAM-1 which are ligands for integrins $\alpha 5 \beta 1$ and $\alpha 4 \beta 1$ respectively. The presence of talin and paxillin were confirmed in bead extracts by western blot and led to the identification of RCC2 in the adhesion signalling pathway (Humphries et al., 2009). Thus, the presentation of ligand coated microbeads to live cells may be able to bind and activate the receptor complex where the organization of the structures associated with the plasma 
membrane are present (A. Jankowski et al., 2008; J G Marshall et al., 2001). Consequently, a robust and universal method for the capture of cell surface receptor complexes from live cells may reside in the application of ligand coated beads on the nanoscopic spatial scale with a chromatography surface that is less susceptible to non-specific binding.

(A)<smiles>CCNc1nc(NC(C)C)nc(NC(C)C)n1</smiles>

(B)

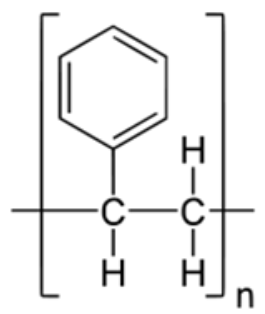

(C)

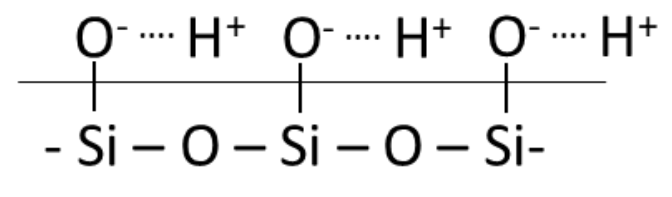

Figure 10: The chemical structure of melamine (A), Poly(methyl methacrylate) (B) and silica (C).

A denser, more hydrophilic ligand coated bead presented on the nanoscopic spatial scale may be a universal solution for the capture of monovalent ligands. It is possible that other surfaces such as glass, melamine and Poly(methyl methacrylate) (PMMA) may have chemical properties that allow for greater ligand binding and/or less background binding (Fig. 6). Also, a smaller deviation in shape and density of beads might ensure a more consistent yield of bead associated receptor complexes. Melamine $(1.51 \mathrm{~g} / \mathrm{cm} 3)$, glass $(1.8 \mathrm{~g} / \mathrm{cm} 3)$ and PMMA $(1.19 \mathrm{~g} / \mathrm{cm} 3)$ are all heavier than polystyrene $(1.06 \mathrm{~g} / \mathrm{cm} 3)$ which may help the beads sediment more readily when binding cells as well as in sucrose, ficcoll or percol gradients for ultra-centrifugation (Fig.10). High density silica nano spheres may incur the least amount of non-specific binding due to its hydrophilic nature and may be a universal solution to the isolation of cell surface receptor 
complexes when presented on the nanoscopic spatial scale (Fig.10). Ligands may be linked to acid washed silica by poly-d-lysine and a paraformaldehyde spacer arm (Fig.11).

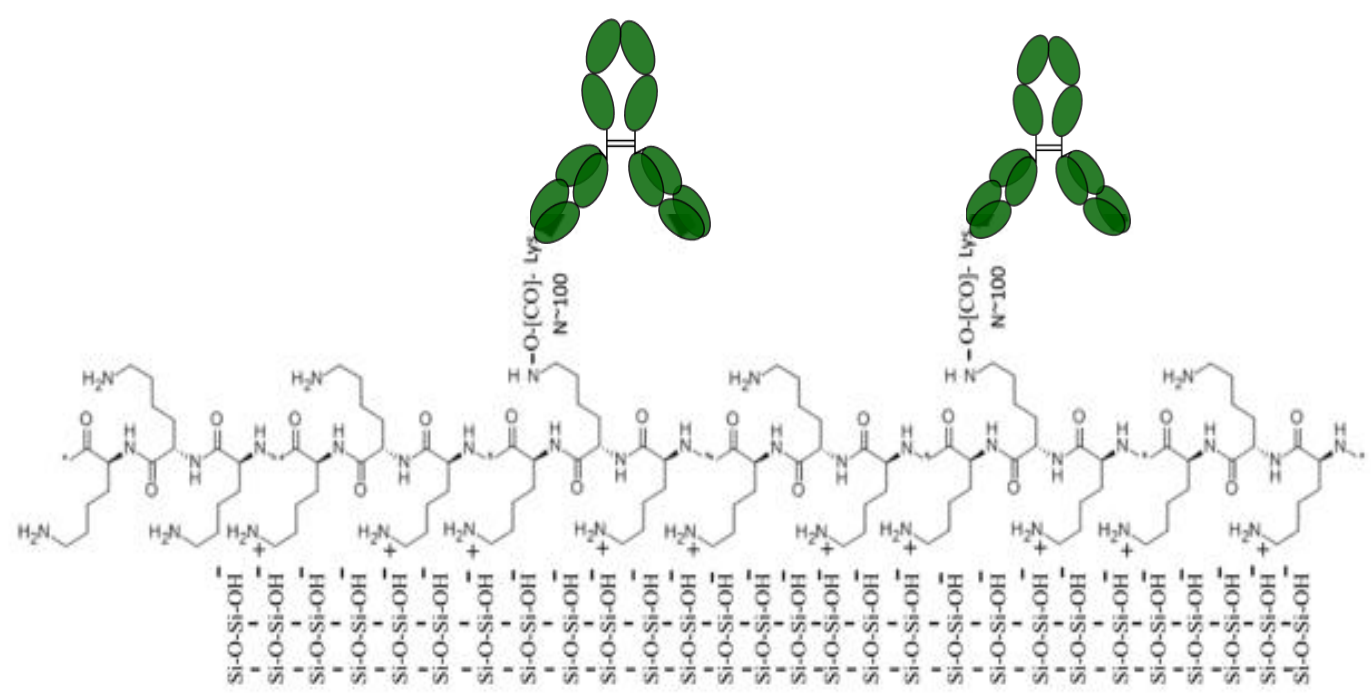

Figure 11. Coating of silica micro and nano beads. Silica washed in $1 \% \mathrm{HCl}$, coated with poly-D-lysine and covalently linked to the ligand by paraformaldehyde.

\section{Tandem mass spectrometry by LCMS and a linear ion trap}

Many of the traditional ligand affinity chromatography approaches were applied to cell surface receptors before the wide spread use of tandem mass spectrometers with limited success.

Complex samples applied to LC-ESI-MSMS are random and independently sampled and show a low type I error rate with Gaussian $\log$ intensity, $\log \mathrm{p}$ values delta mass and $[\mathrm{M}+\mathrm{H}]^{+}$values and so classical statistics can be applied to this data. A common method to identify the proteins from a complex sample is to perform the experiment under various treatment and control conditions followed by digestion with a protease such as trypsin. Trypsin reproducibly cleaves proteins at 
amino acids lysine and arginine where the ionizable fragments with suitable mass to size ratio for LC-MS/MS analysis between 150-2000 m/z units are analyzed. Sample digests are subsequently separated by high pressure liquid chromatography coupled to a tandem mass spectrometer where different peptides elute from the column at the same time and are separated in the gas phase (Fig.12). Peptides are ionized at the electrospray source (Fig.12) were voltage is applied to the peptides and sprayed into a fine mist at atmospheric pressure with heating to prevent condensation.

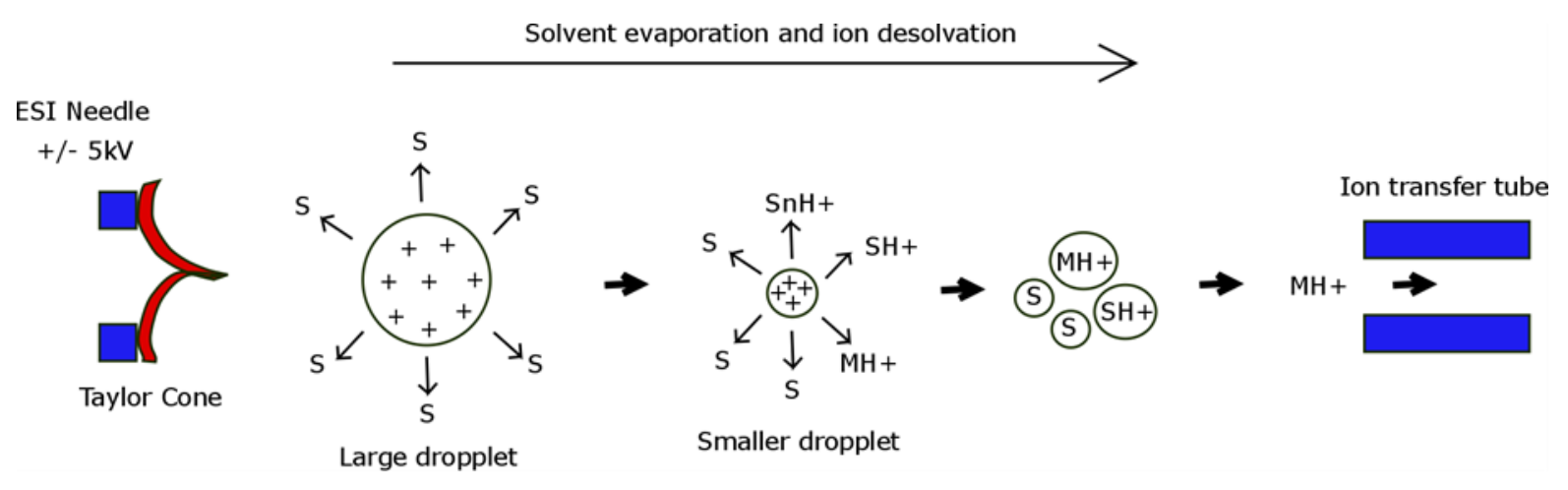

Figure 12. Electrospray ionization of peptides at the source head in positive mode. 


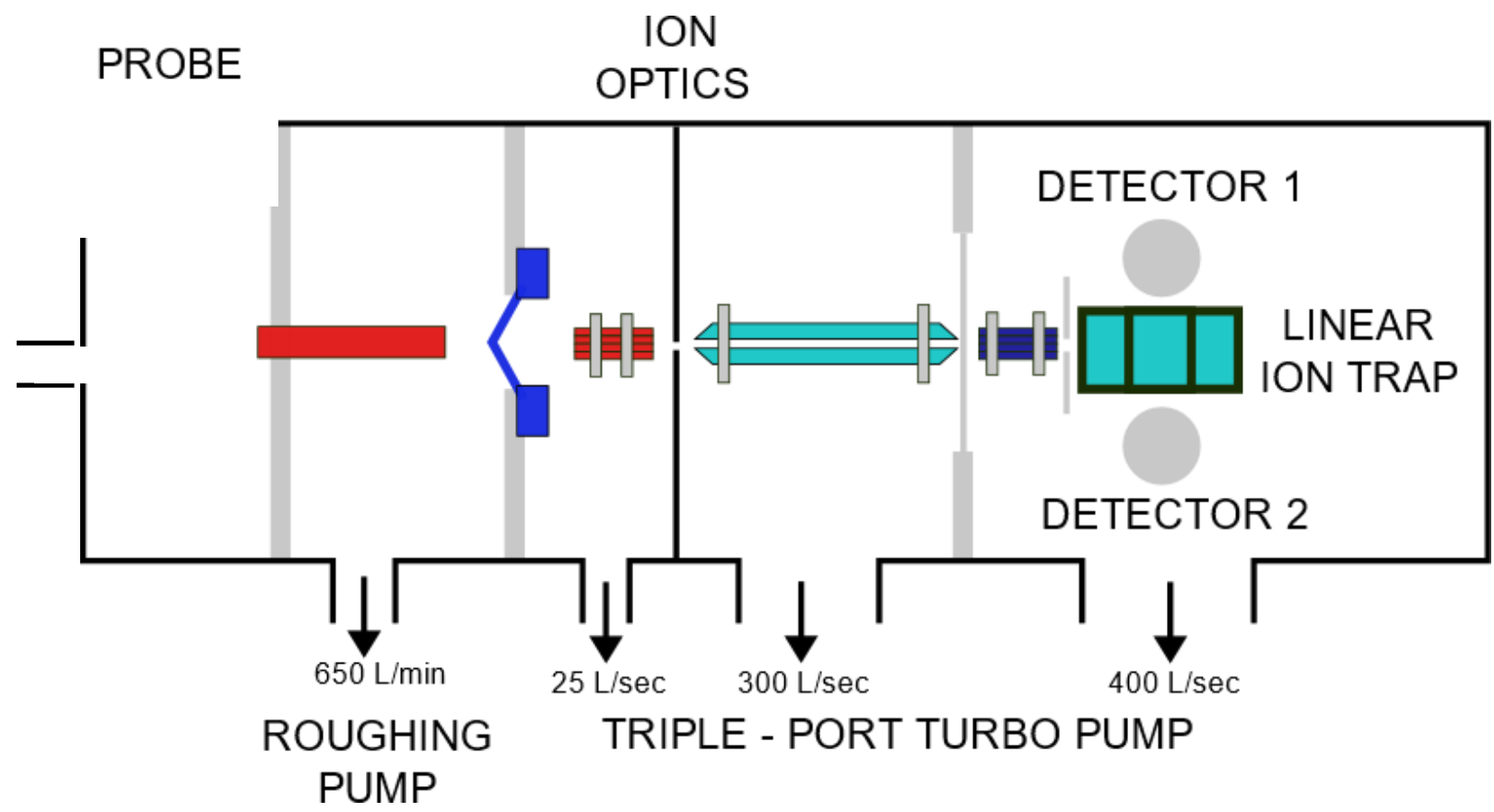

Figure 13. Diagram of a Linear Ion Trap showing the vacuum generated by the roughing and molecular turbo pumps. Sample molecules are ionized at the source then focused into the mass analyzer by the ion optics and then scanned out of the ion trap by the mass to charge ratio where the intensity of the ions is measured by the detectors.

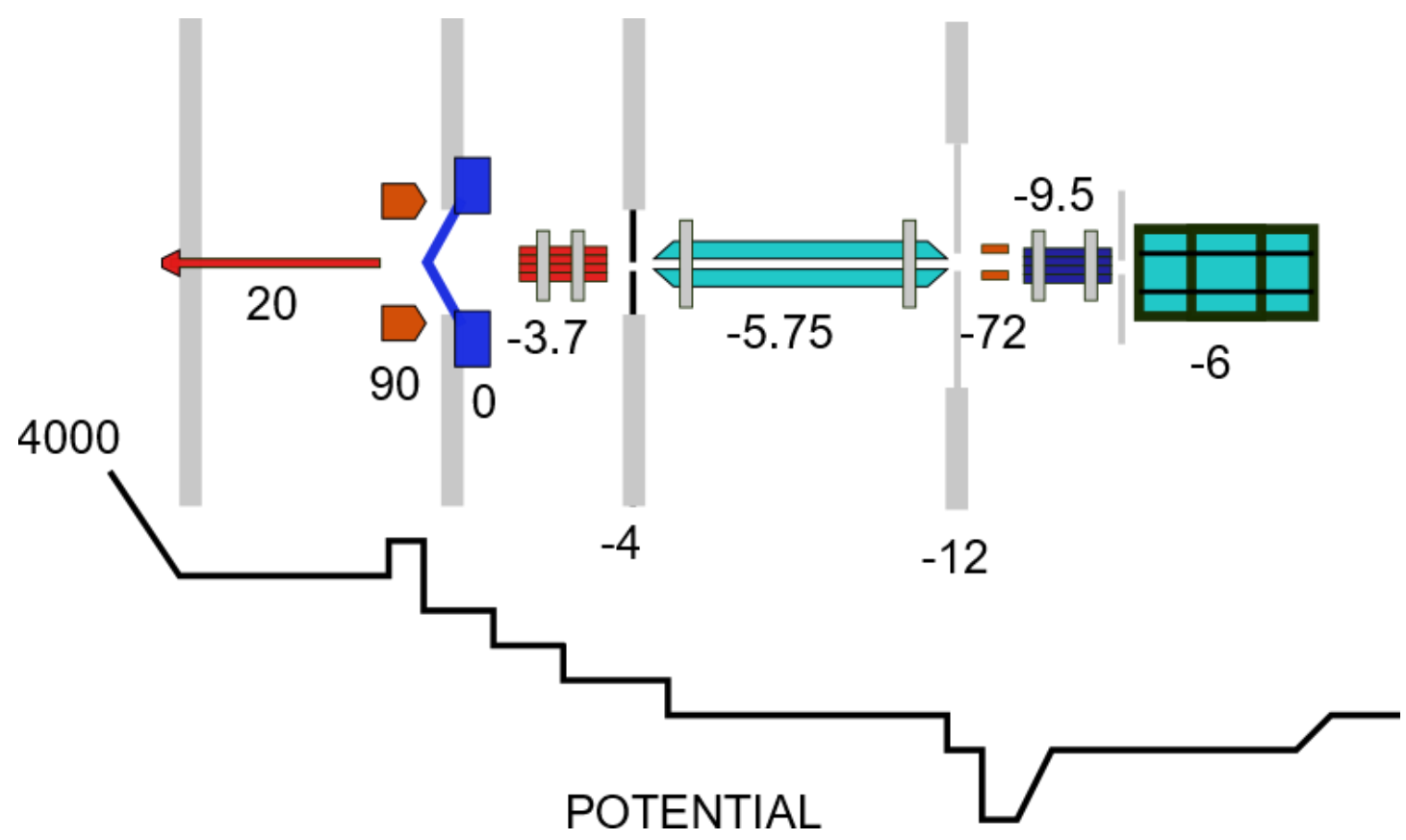

Figure 14. Diagram of a Linear Ion Trap showing the changes in potential along the ion path. 
The ions are guided into the mass spectrometer by the ion optics and by potential and pressure gradients (Fig.13 \& 14). The ion trap randomly and independently samples the ions passing through the aperture via the heated ion transfer tube under vacuum for analysis of a pre-set number of ions and/or for a pre-set time (milliseconds). The first mass analyzer measures the $\mathrm{m} / \mathrm{z}$ and intensity values for the parent masses and scans them out into a detector. The parent ions are fragmented by Collision induced dissociation (CID) where an inert gas is applied (Fig.13). In our method, the five most intense parent ions are sequentially scanned out of the mass analyser and each of the parents is fragmented and the $\mathrm{m} / \mathrm{z}$ values and intensity values measured. The data is converted into parent and fragment $\mathrm{m} / \mathrm{z}$ and intensity tables and that data is searched against a protein library with an algorithm such as SEQUEST or X!Tandem to determine the most likely identity for each MSMS spectra.

\section{Peptide Identification Algorithms for Mass spectrometry}

A number of algorithms have been developed for the identification of peptides based on the correlation of MS/MS spectra to those of predicted peptide fragment spectra (Cottrell \& London, 1999; Robertson Craig \& Ronald C Beavis, 2004; Eng, McCormack, \& Yates, 1994; Lewis Y

Geer et al., 2004). For each MS/MS spectra collected, SEQUEST calls up the closest peptides to masses of the parent ion $+/-3 \mathrm{~m} / \mathrm{z}$ from a curated peptide library. SEQUEST then generates the expected MS/MS for each peptide and compares each of the expected MS/MS fragments to the experimental peptide fragments by cross correlation. A correlation score is computed and ranked for each comparison and an identification is made according to the highest score (Eng et al., 1994). X!Tandem is a peptide identification algorithm based on the hyperscore derived from a goodness of fit algorithm. Unlike SEQUEST, for each of the theoretical MS/MS spectra, $\mathrm{X}$ !Tandem selects only the $\mathrm{b}$ and $\mathrm{y}$ ions that match the experimental MS/MS spectra (Fig.15). 


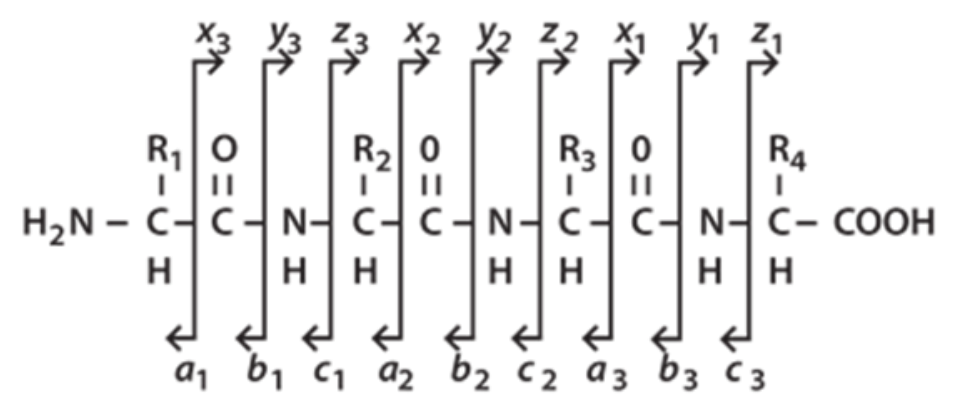

Figure 15: Ions generated during peptide fragmentation. A, b and c ions extend from the amino terminus and $\mathrm{x}, \mathrm{y}$ and $\mathrm{z}$ the carboxy terminus.

$\mathrm{X}$ ! Tandem calculates the goodness of fit as a HyperScore where the sum of each of the matching $b$ and $y$ ion intensities is multiplied by factorial of the number of $b$ ions identified and the factorial of the number of y ions identified as shown in the following equation:

HyperScore $=\left(\sum_{i=0}^{n} I_{i} P_{i}\right) N_{b} ! N_{y} !$

Where I is equal to the spectrum intensity and $\mathrm{P}$ indicates whether or not the $\mathrm{b}$ or $\mathrm{y}$ ion was predicted using a value of 0 or 1 and $\mathrm{Nb}$ is the total number of $\mathrm{b}$ ions identified and $\mathrm{Ny}$ is the total number of y ions identified.

Error in the random and independent sampling of tryptic peptides of a myosin standard by LC-ESI-MS/MS with a linear ion trap

Liquid chromatography electrospray ionization and tandem mass spectrometry (LC-ESI-MS/MS) is a sensitive analytical technique that can identify molecules to $\geq \mathrm{pg} / \mathrm{ml}$ or femtomole on column that may be of great biomedical importance (Shi et al., 2013; Thakur et al., 2011; Monika Tucholska et al., 2009). LC-ESI-MS/MS of peptides with a 3D Paul ion trap showed many peptides correlated to a small number of proteins with a high frequency (Zhu, Bowden, Tucholska, Zhang, \& Marshall, 2011; Zhu, Bowden, Zhang, \& Marshall, 2011) in contrast to 
random spectra or electrospray source noise (chemical and physical noise) that were randomly spread across the peptide database (Cargile, Bundy, \& Stephenson, 2004; Zhu, Bowden, Tucholska, \& Marshall, 2011; Peihong Zhu et al., 2011).

A study with 300,000 synthetic peptides recently demonstrated that unexpected variation in MS/MS fragmentation is not a large source of error and that peptides typically fragment as expected (Zolg et al., 2017) and so peptides can be identified with low type I error by the fit of MS/MS spectra that may be recorded with sufficient accuracy $( \pm 0.5 \mathrm{Da})$ by a simple ion trap (Bowden, Beavis, \& Marshall, 2009; Florentinus, Bowden, Sardana, Diamandis, \& Marshall, 2012; Florentinus, Jankowski, Petrenko, Bowden, \& Marshall, 2011a; P. Zhu, P. Bowden, M. Tucholska, et al., 2011; Peihong Zhu et al., 2011) .

The error rate of LC-ESI-MS/MS has been estimated by the novel statistical procedure of decoy library searching based on the empirical statistical model that assumes proteins standards such as myosin (A. Keller, A. I. Nesvizhskii, E. Kolker, \& R. Aebersold, 2002) are pure and so all other correlations are false positive (Andrew Keller, Samuel Purvine, et al., 2002). For example, the myosin heavy chain may be isolated to high purity by a series of selective extractions and precipitations by centrifugation (Kielley \& Bradley, 1956). The error rate of protein identification of the myosin standard with LC-ESI-MS/MS was previously estimated using the empirical statistical model, widely known as the FDR from decoy library searching, obtained by fusing the protein sequence for rabbit Myosin (Andrew Keller, Samuel Purvine, et al., 2002) to a decoy human protein library, and accepting only correlations to the rabbit Myosin (A. Keller et al., 2002). 
The novel statistical method of decoy library searching using the empirical statistical model indicates that the vast bulk of MS/MS correlations are false positive (Andrew Keller, Alexey I Nesvizhskii, Eugene Kolker, \& Ruedi Aebersold, 2002). In contrast, standard statistical methods such as a null random model (Cargile et al., 2004; Peihong Zhu et al., 2011; P. Zhu, P. Bowden, D. Zhang, et al., 2011), the computation of the goodness of fit of the MS/MS spectra to that of peptides (R. Craig \& R. C. Beavis, 2004) and comparison of the frequency distribution of the results to that of a reference library (von Mering et al., 2007; Zhu et al., 2007) or permutations (S. Kim, Gupta, \& Pevzner, 2008) all agree that LC-ESI-MS/MS has a low error rate as confirmed by immunological methods, drugs or siRNA (J. C. Howard et al., 2016; Andy Jankowski, Peihong Zhu, \& John G. Marshall, 2008; John Marshall et al., 2003; M. Tucholska, Florentinus, Williams, \& Marshall, 2010).

Classical frequency based statistical methods including comparison to random expectation, Chi square test of peptide counts, and transformation of ion intensity, normality testing for ANOVA (Boehm, Putz, Altenhofer, Sickmann, \& Falk, 2007; Florentinus et al., 2011a; John Marshall et al., 2003) may be applied prior to correlation of MS/MS spectra, after making labeled derivatives, or after peptide identification by LC-ESI-MS/MS using SQL/R (Bereman et al., 2014; Boehm et al., 2007; Eckel-Passow, Oberg, Therneau, \& Bergen, 2009; Angelica K Florentinus, Peter Bowden, Girish Sardana, et al., 2012; Florentinus et al., 2011a; John Marshall et al., 2003; Mercier et al., 2009). A direct experimental approach was used to estimate the error in the identification and quantification of peptides by LC-ESI-M/MS with a simple ion trap. 
There are at least six potential sources of type I (false positive) error in the identification of tryptic peptides from one species by LC-ESI-MS/MS: (i) physical and chemical noise from the source of the mass spectrometer; (ii) dust or other contaminants; (iii) cleavage by non-tryptic proteases or exopeptidases; (iv) post translational modifications, oxidations, alkylations or other changes to the expected mass of tryptic peptides. All these sources of error (i-iv) should produce near random mis-correlation and so may be effectively controlled by source noise from blank runs or the random MS/MS spectra distributions (Bowden et al., 2009; Bowden et al., 2012; Angelica K Florentinus, Peter Bowden, Veronica Barbisan, \& John Marshall, 2012; Angelica K Florentinus, Peter Bowden, Girish Sardana, et al., 2012; P. Zhu, P. Bowden, M. Tucholska, et al., 2011; Peihong Zhu et al., 2011). An additional source of error (v), is the re-use of MS/MS spectra for more than one peptide sequence (redundant identifications) that may be rendered distinct by a composite key associated with each MS/MS spectra in an SQL Server database (Bowden et al., 2009; Bowden, Pendrak, Zhu, \& Marshall, 2010; Monika Tucholska et al., 2009). There are two sources of redundancy: the re-use of MS/MS spectra (error v); and the presence of the same peptide sequence in more than one parent protein accession that is sometimes crucially informative (J. C. Howard et al., 2016; Andy Jankowski, Peihong Zhu, \& John G. Marshall, 2008). Human error (vi) can only be addressed by multiple random and independent experiments with replication of the treatments and controls that may be conveniently analyzed using classical statistical in SQL/R (Bowden et al., 2012; Angelica K Florentinus, Peter Bowden, Girish Sardana, et al., 2012; Florentinus et al., 2011a; J. C. Howard et al., 2016). 
In order to maximize utility and reduce total error, it is necessary to minimize both type I (false positive) and type II error (false negative) (Vergeynst, Van Langenhove, \& Demeestere, 2015; P. Zhu, P. Bowden, M. Tucholska, et al., 2011; Peihong Zhu et al., 2011). Here IgG and myosin standards were compared to laboratory dust, chemical and physical noise from blank solvent runs with HPLC grade solvents on naïve C18 chromatography columns, and computer generated random spectra to define the proteins that might be a source of error in the correlation of MS/MS spectra to peptides by a linear ion trap.

The traditional and well established algorithm for generating random numbers from Park and Miller (Park SK, 1988) was harnessed to create a random MS/MS spectra generator (RSG) (Peihong Zhu et al., 2011) that serves, along with noise from blank runs on naïve columns, as a null random model of false positive results. An alternate and completely independent method to test whether proteins have been identified correctly is to compare the frequency distribution of the expected proteins against that of the protein library (von Mering et al., 2005; Zhu et al., 2007). The results clearly demonstrate that MASCOT, OMSSA, X!TANDEM and SEQUEST all agree that actin and many other proteins were identified in the myosin standard. The protein p-values computed from the results of X!TANDEM independently confirmed the low probability of false positive identification.

Here it was shown that erroneous peptides are randomly distributed across the protein library with a low frequency while the authentic hits are concentrated on a small set of peptides and proteins with a high frequency and can be easily resolved from erroneous correlations by classical statistical methods (P. Zhu, P. Bowden, M. Tucholska, et al., 2011; Peihong Zhu et al., 
2011). It was possible to identify and quantify thousands of peptides from hundreds of proteins simultaneously with a demonstrated low probability of type I error by LC-ESI-MS/MS with a linear ion trap. 


\section{OBJECTIVES}

The overarching goal of this work was to create a robust and universal method for the capture and characterization of cell surface receptor complexes by presenting ligand coated silica nanobeads to live cells using the IgG-Fc receptor complex as a model system and to compare this method to classical ligand affinity chromatography using DTT cleavable biotin linkers coupled with LC-ESI-MS/MS.

\section{Classical ligand affinity chromatography with DTT cleavable biotin linkers}

1. To identify a specific classical affinity chromatography system by applying IgG, IgGB and IgG-SS-B to chromatography supports: PVDF, high binding plastic, agarose or glass, coated in either protein $\mathrm{G}$ or streptavidin and eluted in $5 \mathrm{mM}$ DTT in 1x PBS, $5 \mathrm{uM}$ IgG in $1 \mathrm{XPBS}, \mathrm{NH} 4 \mathrm{OH}$ in $\mathrm{H} 2 \mathrm{O}$ followed by $\mathrm{NH} 4 \mathrm{OH}+50 \mathrm{mM}$ Biotin in $\mathrm{H} 2 \mathrm{O}$ and $300 \mathrm{mM} \mathrm{NaCl}$ followed by $650 \mathrm{mM} \mathrm{NaCl}+30 \% \mathrm{AcN}$. Chromatography supports without coatings will be used to assess whether the IgG ligand specifically elutes from the affinity chromatography system. The specific elution of the IgG ligand will be assessed by Dumbroff dot blot onto PVDF membrane and visualized a donkey anti human IgG secondary antibody conjugated to HRP.

2. To use the most specific affinity chromatography strategy to capture the ligand activated receptor complex from live cells. Treatments and controls will be compared by the number of ligands and Fc receptors identified by LC-ESI-MS/MS.

3. To determine if the addition of detergents deoxycolate and octyl glucoside to the cell homogenization buffer increases the number of ligands and Fc receptors recovered by classical affinity chromatography and tandem mass spectrometry. 
4. To determine heat aggregated IgG, IgG-B and IgG-SS-B with biotinylation both before and after heating increases the number of ligands and Fc receptors recovered by classical affinity chromatography and tandem mass spectrometry.

5. To determine if the application of cross linkers before and after ligand binding increases the number of ligands and Fc receptors recovered by classical affinity chromatography and tandem mass spectrometry.

6. To determine if blocking the affinity chromatography surface with bovine serum albumin increases the number of ligands and Fc receptors recovered by classical affinity chromatography and tandem mass spectrometry.

\section{Ligand coated nano silica beads presented to live cells}

1. To determine if silica, melamine and PMMA beads can be densely coated with IgG ligand by Dumbroff dotblot (Sibdas Ghosh, Shimon Gepstein, John J Heikkila, \& Erwin B Dumbroff, 1988) with Coomassie staining and by western blot onto a PVDF membrane and visualized by a donkey anti human IgG secondary antibody conjugated to HRP as well as laser scanning confocal microscopy.

2. To determine whether the nano and micro formats of these ligand coated beads bind to RAW 264.7 cells by laser scanning confocal microscopy.

3. To determine if IgG can be detected by Dumbroff dot blot (Sibdas Ghosh et al., 1988) with Coomassie staining and by Western dot blot after ligand coated PMMA, melamine and glass microbeads are presented to live cells, allowed to bind, are homogenized and separated by ultra-centrifugation in a sucrose gradient and extracted by an acetonitrile/salt step gradient. 
4. Lastly, we will identify the Fc receptors in IgG-Fc mediated phagocytosis using the entirely novel method where $\mathrm{IgG}$ coated silica nano beads are presented to live cells. 


\section{MATERIALS AND METHODS}

The linear trap quadrupole (LTQ) XL tandem mass spectrometers were obtained from Thermo Electron Corporation (Waltham, MA,USA). The random spectra generator was slightly modified from that provided by Zhu et al. (P. Zhu, P. Bowden, M. Tucholska, et al., 2011). The C18 with 5 micron particle size and 300 Angstrom pore size was from VYDAK (Hesperia, CA, USA). The 1100 HPLC system was from Agilent (Santa Clara, CA, USA). The HPLC grade water and acetonitrile was from Caledon laboratories and the formic acid was from Fluka (Georgetown, ON, Canada). Sequencing grade trypsin was from Roche (Basel, Switzerland). The $\mathrm{C} 18$ zip tips were from Millipore (Billerica, MA, USA). The beta Actin Monoclonal Antibody (AC-15) was obtained from Thermo Fisher Scientific (Waltham, MA USA) and the Dk anti mouse secondary antibody was obtained from Jackson Immuno Research (West Grove, PA, USA). The bulk human IgG and rabbit myosin standards were obtained from Sigma Aldrich and except where indicated other reagents buffers and salts were obtained from Sigma Aldrich (St Louis, MO USA). Dust samples were collected from Kerr Hall East room 215 of Ryerson University.

\section{SDS-PAGE and Western Blot}

The rabbit myosin standard was separated over $9 \%$ polyacrylamide tris gels with tricine electrophoresis buffer (Schagger \& von Jagow, 1987) and either stained in 1\% Coomassie Brilliant Blue R (CBBR) in 40\% methanol and 5\% acetic acid, reduced in glutaraldehyde and stained by $\mathrm{AgNO}_{3}$ (Dionne, Forest, Moutquin, De Grandpre, \& Masse, 1994) or transferred to

PVDF and probed with anti actin antibody (1/500) and washed before detection with anti-mouse secondary antibody (1/2000) with detection by ECL (Haan \& Behrmann, 2007). 


\section{Calibration, tuning, conditioning and sensitivity}

The linear ion trap instrument (Schwartz, Senko, \& Syka, 2002) was cleaned and calibrated with the manufacturers' standards. The instrument was tuned and mass accuracy confirmed with the direct infusion of angiotensin and glu-fibrinogen immediately prior to the experiments. The electrospray voltage was typically about $4.5 \mathrm{kV}$ with $\sim 10 \mathrm{~L} \mathrm{~N}_{2}$ per minute and the transfer capillary was heated to $200{ }^{\circ} \mathrm{C}$. The instrument was set at 20 milli seconds for the wide scan (150 to $2000 \mathrm{~m} / \mathrm{z}$ ) and to randomly and independently sample the 5 most intense peptides eluting without replacement every few moments for up to 200 milli seconds to fill the trap with up to 250,000 ions, and to continue collecting MS/MS spectra for up to 4 independent MS/MS fragment scans that were averaged. The columns were conditioned and quality control tested with a digested mixture of alcohol dehydrogenase, cytochrome $\mathrm{c}$ and glycogen phosphorylase to confirm the system was working normally versus historical benchmarks (Bowden et al., 2012). The sensitivity of the system was tested with a BSA digest on the conditioned columns that showed a sensitivity for automatic identification by SEQUEST to $\sim 1 \mathrm{fmol}$ on column.

\section{Blank water acetonitrile solvent runs}

Three LC-ESI-MS/MS systems were fitted with naive 300-micron ID silica C18 columns with $20 \mu \mathrm{L}$ sample loops and blank samples of 5\% formic acid and 5\% acetonitrile were injected into a flow of $5 \%$ acetonitrile and $0.1 \%$ acetic acid. The gradient of acetonitrile was commenced after 12 minutes from $15 \%$ to $45 \%$ ACN over the course of 60 minutes and then to $65 \% \mathrm{ACN}$ over 30 minutes, cleaning at $65 \%$ for 5 minutes before returning to $5 \%$ ACN. A total of 36 
replicate blank recordings with some 75,269 MS/MS spectra of any intensity were recorded without excluding precursor ions of less than 1000 counts.

\section{IgG, Myosin and Dust tryptic protein digestion and analysis}

Only precursor values of greater than 10,000 (E4) arbitrary counts were accepted for the $\mathrm{IgG}$, myosin and dust samples in order to avoid the collection of chemical or physical source noise spectra in these treatments. Dust immediately proximal to the LC-ESI-MS/MS instruments were sampled at 4 locations in the Ryerson Analytical Biochemistry Laboratory (RABL). The IgG, Myosin and dust were digested with a 1/100 ratio (g/g) of trypsin for $8 \mathrm{~h}$, reduced in $2 \mathrm{mM}$ DTT and digested in another 1/100 ratio of trypsin in $200 \mathrm{mM}$ urea and 5\% ACN in $50 \mathrm{mM}$ Tris $\mathrm{pH}$ 8.85 prior to collection over a preparative C18 zip tip, elution in $2 \mu \mathrm{L}$ of $65 \% \mathrm{ACN}$ and $5 \%$ formic acid and diluted in $18 \mu \mathrm{L}$ of $5 \%$ formic acid prior to manual injection with a Rheodyne injector. IgG and Myosin standards were analyzed in duplicate while 4 replicate dust digests were recorded with MS/MS spectra from precursor ions of greater than 1000 arbitrary counts. A fresh column was created and used for each experimental treatment to prevent cross contamination. The peptides of $\operatorname{IgG}$, myosin and human dust were randomly and independently sampled without replacement from the effluent of the HPLC C18 gradient from water to acetonitrile.

\section{Random Spectra}

The Random Spectra Generator (RSG) was used to generate a total of 440,772 MS/MS spectra from 166 simulated LC-ESI-MS/MS runs. The random spectra generator (RSG) uses the random number generator of Park and Miller (Park SK, 1988) to create the MS and MS/MS spectra as 
previously described (P. Zhu, P. Bowden, M. Tucholska, et al., 2011; Peihong Zhu et al., 2011). First the mass to charge of the precursor ion was randomly generated from 150 to $2000 \mathrm{~m} / \mathrm{z}$. The number of +1 fragment ions ( $\geq 35$ ), and then the $\mathrm{m} / \mathrm{z}$ values from 150 to $2000 \mathrm{~m} / \mathrm{z}$ were then randomly generated.

\section{MS/MS correlation}

The MS/MS spectra were correlated to the proteins of a federated human or rabbit library with Gene Symbol annotation as previously described (Bowden et al., 2009; Bowden et al., 2012; Angelica K Florentinus, Peter Bowden, Girish Sardana, et al., 2012). The MASCOT, OMSSA, X!TANDEM and SEQUEST algorithms were applied with the correlation setting of $2+$ or $3+$ precursor within $\pm 3 \mathrm{~m} / \mathrm{z}$ and with fragments within $\pm 0.5 \mathrm{Da}$ (Bowden et al., 2009; Bowden et al., 2012; Angelica K Florentinus, Peter Bowden, Girish Sardana, et al., 2012). The MS/MS spectra were correlated to the peptides while considering the loss of water, ammonia, or acetylation, or methionine oxidation. Only the highest scoring combination of charge state (Filter 1) and peptide sequence (Filter 2) was accepted for each MS/MS spectra that were assigned to only one peptide using a composite identification key in the SQL database. MASCOT and OMSSA rely on fitting the distribution of an empirical score to the gamma function (L. Y. Geer et al., 2004; Perkins, Pappin, Creasy, \& Cottrell, 1999). The SEQUEST algorithm relies in part on the cross correlation analysis of the experimental versus predicted MS/MS spectra (Yates, Eng, McCormack, \& Schieltz, 1995). The MS/MS spectra to peptide sequence matching algorithm X!TANDEM that directly to estimates the p-value that the fit was merely random chance (R. Craig \& R. C. Beavis, 2004). 


\section{Computation and Statistical analysis}

The results of the LC-ESI-MS/MS and the correlation analysis were collected in Structured Query Language (SQL-Sever) and analyzed using the R generic statistical analysis system. The results were summarized at the level of peptides, protein accessions or gene symbols using the $\mathrm{R}$ statistical system.

TESTING AFFINITY SYSTEMS WITH IgG, IgG-B and IgG-SS-B

Affinity chromatography surfaces: glass, PVDF, high binding plastic and agarose were coated with streptavidin and Proteins $\mathrm{G}$ and used to capture bait ligands IgG, IgG-B and IgG-SS-B. Glass coverslips (mm) were tested in a low binding 96 well dish, PVDF in a low binding 96 well dish and a high binding plastic using a high binding 96 well dish. Streptavidin-agarose beads were purchased pre-coated.

\section{Coating Glass cover slips with SA and Protein G}

Small glass coverslips $(3 \mathrm{~mm})$ were placed in a low binding untreated 96 well dish and washed three times in $100 \%$ ethanol and allowed to dry. The glass coverslips were activated with $1 \%$ $\mathrm{HCl}$ for five minutes prior to washing three times with $100 \%$ ethanol and left to air dry. Coverslips were washed in 1xPBS and then coated in $0.1 \%(\mathrm{w} / \mathrm{v})$ poly-L-lysine for 5 minutes and air dried overnight. In the morning, coverslips were washed 5 times in 1xPBS and then crosslinked with $1 \%$ paraformaldehyde for 30 minutes at RT followed by 3 washes in $1 \times$ PBS. Coverslips were coated in $1 \mathrm{mg} / \mathrm{mL}$ of either streptavidin or protein $\mathrm{G}$ and the glass control was 
incubated in PBS at 4 degrees overnight or for 30 minutes at RT. Coverslips were rinsed $3 \mathrm{x}$ in 1xPBS and quench in $2 \%$ glycine in $1 x$ PBS for 5 min at RT and washed 3 times in 1x PBS.

\section{Coating high binding plastic with SA and Protein G}

A high binding plastic 96 well dish was coated with $1 \mathrm{mg} / \mathrm{mL} \mathrm{SA}$ or Protein G or nothing (Ctrl) in $0.05 \mathrm{M}$ carbonate/bicarbonate buffer overnight at 4 degrees covered and washed five times in 1 $x$ PBS before use.

\section{Coating PVDF membrane with SA and Protein G}

PVDF membranes were cut into x $3 \mathrm{~mm}$ circles and placed in a low binding plastic 96 well dish. The wells were washed and the PVDF activated in 100\% methanol. The wells were subsequently washed in $\mathrm{H} 2 \mathrm{O}$ three times and then coated in $1 \mathrm{mg} / \mathrm{mL}$ SA or Protein $\mathrm{G}$ or nothing (Ctrl) in 1xPBS buffer overnight at 4 degrees covered. In the morning, the wells were washed 5 times with 1X PBS.

\section{Preparation of ligands}

Ligands were either prepared fresh on the day of the experiment or the day before the experiment and left on ice in the fridge overnight. $10 \mathrm{mg}$ of human $\mathrm{IgG}$ was dissolved in $1 \mathrm{~mL}$ of $1 \mathrm{xPBS}$ and biotinylated at a molar ratio of 2-5:1 biotin to IgG with NHS-B and NHS-S-S-B on ice for two hours. The reaction was terminated with a ratio of 20:1 glycine to biotin. Aliquots of 200ug were dried down in a lapholizer and stored at $-20^{\circ} \mathrm{C}$.

Comparison of surfaces, coatings, biotin linkers and elution buffers for the capture of IgG 
The ligands: IgG, IgG-B and IgG-SS-B, were applied to each surface coating, washed five times in $1 x P B S$ and eluted in 200uL of the elution buffers (some sequentially). Elution buffers included: 1) $5 \mathrm{mM}$ DTT in 1x PBS 2) $5 \mathrm{uM}$ IgG in $1 \mathrm{XPBS}$ 3) $\mathrm{NH}_{4} \mathrm{OH}$ in $\mathrm{H} 2 \mathrm{O}$ followed by $\mathrm{NH}_{4} \mathrm{OH}+50 \mathrm{mM}$ Biotin in $\mathrm{H} 2 \mathrm{O}$, and 5) $300 \mathrm{mM} \mathrm{NaCl}$ followed by $650 \mathrm{mM} \mathrm{NaCl}+30 \% \mathrm{AcN}$. The elution buffers were collected, dried down and resuspended in 2 x sample buffer. The wells were scrubbed with $2 \%$ SDS and all recovered materials were dotted on a PVDF membrane.

\section{Western ECI dot blot of elution buffers}

A stack of 5-6 filter papers were placed into a flat dish. A similarly sized piece of pvdf membrane was placed over the filter papers. Methanol was added to the bottom of the flat dish but only enough for the methanol wet the pvdf without soaking it. Standard serial dilutions IgG for the Dk anti human conjugated HRP probe were made and IgG-B for the streptavidin-HRP probe. Standards and samples were spotted of the PVDF membrane, left to dry, dipped in methanol for 30 seconds with shaking and washed in water for 1 minute with shaking. The membrane was blocked for 30 minutes in $5 \%$ w/v skim milk (+1\% Dk serum for Dk anti hIgG probe) and TBST, followed by three five minute washes in TBST. The membrane was incubated for 30 min in TBST with 4:10,000 Dk anti human conjugated HRP or 1: 20,000 streptavidin HRP and then washed in TBST 3 times for five minutes with rocking followed by a one minute incubation with ECL solution and detection by gel doc using the high chemiluminescent setting. 


\section{TESTING GLASS SUPPORTS AND AFFINITY SYSTEMS WITH CELLS}

RAW macrophages were seeded in 10mLs of DMEM with $5 \%$ fetal calf serum and incubated at 37 degrees and 5\% CO2 for 24-48 hrs until 70\% confluent. Glass microbeads were coated as previously described however the glass beads were prepared in microcentrifuge tubes and centrifugation at 13,400 rpm was used to pellet the beads in order to remove washes and buffers. Cells were washed 10 times with HEPES buffer and incubated for 50 minutes at 37 degrees in the last HEPES wash. After 50 minutes, $5 \mathrm{~mL}$ of fresh HEPES buffer was added to cells and the flasks were incubated on ice for 10 minutes, IgG, IgG-B or IgG-SS-B ligands were added to the flasks and were incubated for a further 30 minutes on ice. To remove unbound ligands, cells were then washed with 1xPBS seven times and $10 \mathrm{mLs}$ of homogenization buffer $(\mathrm{HB})$ was applied, the cells were scraped and applied to a French press twice. The homogenates were kept on ice till applied to resins. The homogenates from live RAW 264.7 macrophages incubated with either IgG, IgG-B or IgG-SS-B ligands were applied to the coated silica supports and incubated for 10 minutes and allowed to bind the streptavidin or protein $\mathrm{G}$ coated silica. The beads were then washed 5 times to remove unbound proteins and the elution buffers previously mentioned were applied and collected for digestion and analysis by LC-MSMS.

\section{Sample digestion for LC-MS/MS}

Samples were diluted in 10x digestion buffer $(50 \% \mathrm{AcN}, 2 \mathrm{M}$ Urea, $0.5 \mathrm{M}$ Tris $\mathrm{pH} 8.8)$ to a final concentration of $1 \mathrm{x}$ digestion buffer and $1 \mathrm{ug}$ of trypsin per 100ug of protein was added prior to incubation at 37 degrees overnight. In the morning samples were brought to a final concentration $2 \mathrm{mM}$ DTT and heated at $50^{\circ} \mathrm{C}$ for 30 minutes or 37 degrees for $1 \mathrm{hr}$. After cooling, another $1 \mathrm{ug}$ 
of trypsin per $100 \mathrm{~g}$ of protein was added and incubated for one hour at $37^{\circ} \mathrm{C}$. Formic acid was added to the sample to a final concentration of 5\% and the samples dried down and stored at $20^{\circ} \mathrm{C}$ until application to LC-MSMS.

CLASSICAL AFFINITY CHROMATOGRAPHY METHODS TO CAPTURE RECEPTOR COMPLEXES FROM LIVE CELLS

\section{Preparation of homemade Streptavidin-agarose}

SA-agarose was purchased from Sigma Aldrich or produced in house in subsequent experiments. The preparation of home-made SA-agarose was based on previous methods by Cuatrecasas and Porath. $50 \mathrm{mLs}$ of agarose was gently mixed in an equal volume of water. $5000 \mathrm{mg}$ of $\mathrm{CNBr}(100 \mathrm{mg} / \mathrm{mL}$ of settled agarose $)$ was brought to a final volume of $100 \mathrm{mLs}$ with water and gently added to the agarose mixture and titrated to a $\mathrm{pH}$ of 11 with $\mathrm{NaOH}$. After ten minutes, the mixture was applied to a Buchner funnel under suction and the agarose washed with 20 volumes of cold $0.1 \mathrm{M} \mathrm{NaHCO} 3$ over a period of 2 minutes. The agarose was suspended in $50 \mathrm{mls}$ of $0.1 \mathrm{M} \mathrm{NaHCO} 3$ at $\mathrm{pH} 9$ and streptavidin was added to $5 \%$ of the final volume. The streptavidin agarose mixture was mixed gently for $24 \mathrm{hrs}$, applied to the Buchner funnel under suction and washed again with water and stored at $4^{\circ} \mathrm{C}$ (Axen, Porath, \& Ernback, 1967; Cuatrecasas, Wilchek, \& Anfinsen, 1968; Porath, Axén, \& Ernback, 1967)(Diamandis \& Christopoulos, 1991) 
Biotinylated ligands applied to live cells and captured using a traditional streptavidinagarose affinity column

Classical ligand affinity chromatography using IgG-SS-biotin were applied to live cells, homogenized and applied to SA-agarose affinity columns and sequentially eluted in 2-5mM DTT, 5\% mercaptoethanol, 2M NH $4{ }_{4} \mathrm{OH}$ and 2M NH $4 \mathrm{OH}+50 \mathrm{mM}$ biotin (Staros, 1982)(Fig.16).

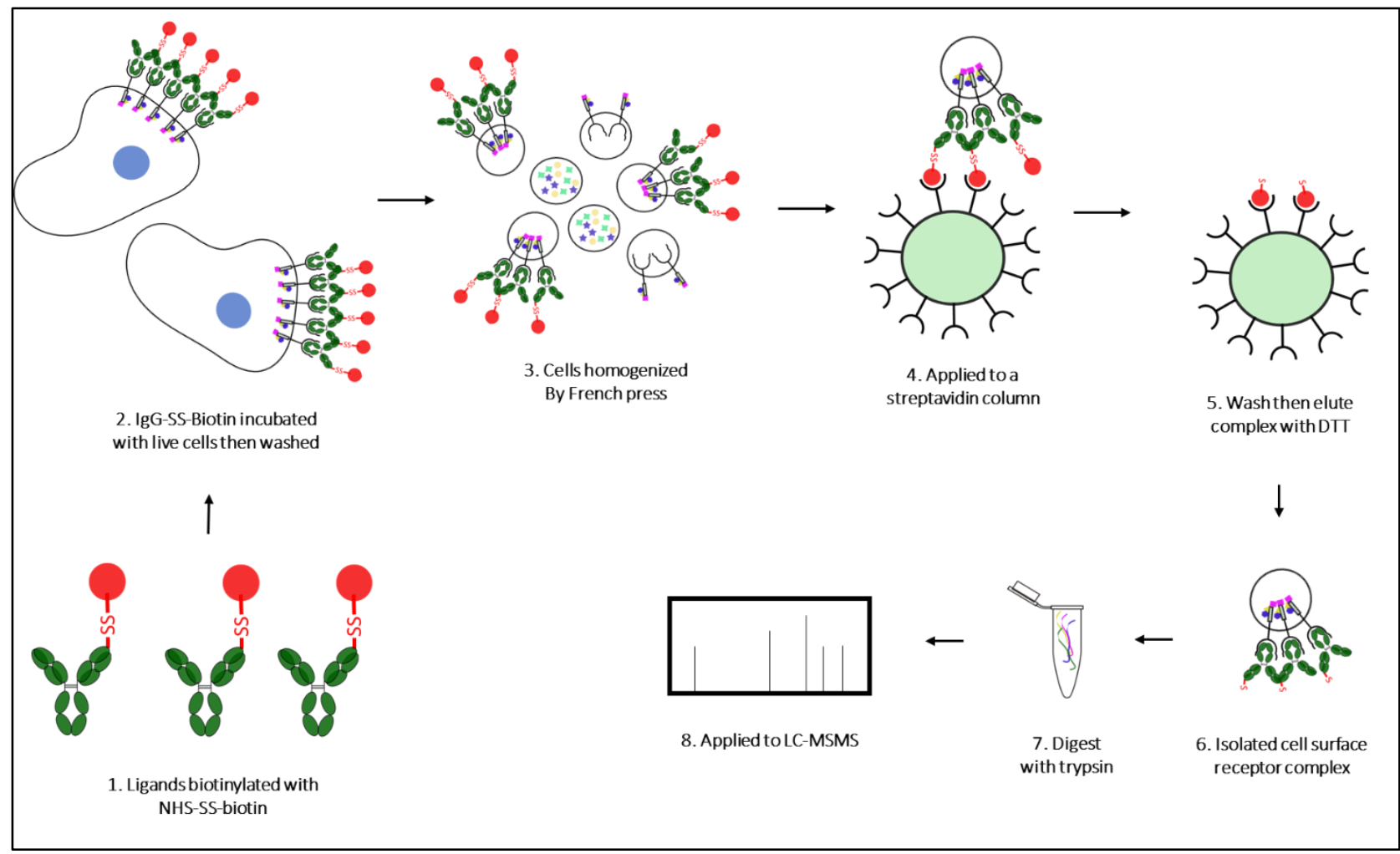

Figure16: Biotinylated ligand affinity chromatography with DTT cleavable biotin linkers Activation of live cells with biotinylated ligands

Raw 264.7 cells were seeded and grown to $70 \%$ confluency in DMEM media supplemented with $5 \%$ fetal bovine serum. Cells were washed 10 times with serum free media and incubated at $37^{\circ} \mathrm{C}$ for 50 minutes and placed on ice for a further 10 minutes. Two hundred ug aliquots of $\operatorname{IgG}$, IgG-B and IgG-SS-B were re-suspended in 200ul of serum free media as well as two controls without ligand. Each ligand was applied to a separate flask. Cells were incubated on ice for 30 
minutes. After incubation, cells were washed three times with ice cold 1xPBS with the flask still on ice and scraped in $10 \mathrm{mLs}$ of HB. The cells were physically disrupted by French press twice (Fig.16).

\section{Capture of Activated Receptor Complex by Biotinylated Ligand Affinity Chromatography}

Gravity micro preparative chromatography columns were prepared in $10 \mathrm{ml}$ transfer pipettes as previously described by Tucholska et al. (Tucholska et al., 2009) by pushing a small amount of glass wool to the bottom of a transfer pipette with half the bulb cut horizontally across. A total of $200 \mathrm{ul}$ of a $50 \%$ slurry was applied to each column resulting in a $100 \mathrm{uL}$ column bed of CL-4B agarose (control) or streptavidin conjugated CL-4B agarose. Each column was washed with $4 \mathrm{mLs}$ of 1xPBS. Seven mLs of each of the samples where ligands were incubated with live cells or applied to crude homogenates immediately after French pressing was applied to agarose (control) or SA-agarose columns (Fig.17). The sample flow through was collected and the column washed with $2 \mathrm{~mL}$ of ice cold 1xPBS. Each column was then sequentially eluted with the following buffers: 2mM DTT (2nd \& 3rd rep with 5mM DTT) in 1xPBS, 5\% mercaptoethanol in 1xPBS, 2M NH4OH, and lastly 2M NH4OH with 50mM biotin (Fig.16). Ten uL of each elution was set aside for Dumbroff dot blots stained with Coomassie brilliant blue and Western dot blots on PVDF membrane and probed with Dk anti hIgG and SA-HRP. The beads were collected and resuspended in $400 \mathrm{uL}$ of $1 \mathrm{xPBS}$. The beads along with the collected elutions were digested and analyzed by LC-MS/MS as described below. 
Ligands incubated with Live Cells

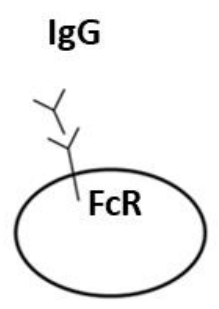

$\lg G$

Samples applied to control beads and streptavidin affinity columns
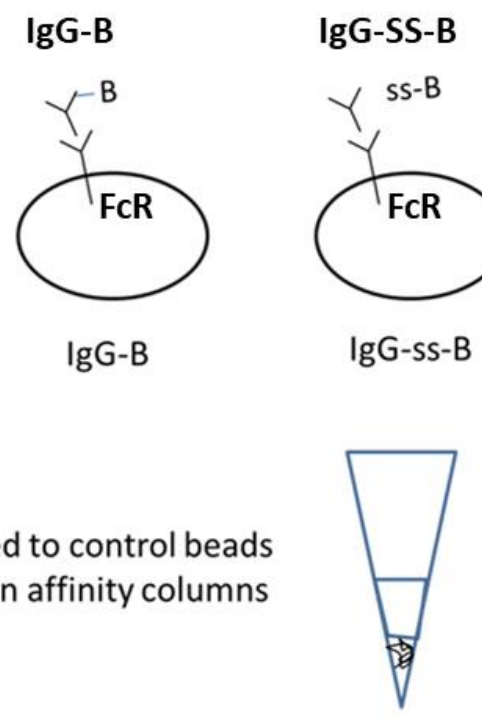

Control

Agarose Beads

$$
\text { IgG-SS-B }
$$

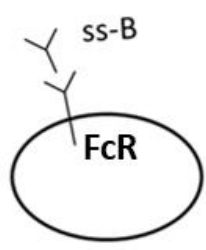

IgG-ss-B
Ligands applied to crude homogenates

IgG

IgG-B IgG-SS-B

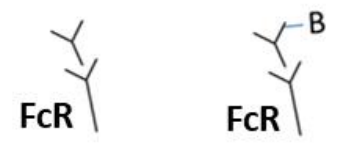

FCR

Figure 17. Summary of treatment and controls for the capture of the IgG-Fc $\gamma$ receptor complex by classic affinity chromatography using SA-agarose and agarose (control) affinity columns and biotinylated ligands applied to live cells and crude homogenates (control).

\section{Dumbroff Protein Assay}

A serial dilution of IgG was prepared in $2 \%$ SDS. The standard IgG dilution series and samples were boiled for 10 minutes at $100^{\circ} \mathrm{C}$ and spotted on Whatmann paper (1), air dried, stained in Coomassie Blue with acetic acid for 15 minutes and then destained with 5\% $\mathrm{MeOH}, 7 \% \mathrm{HAc}$ and $88 \% \mathrm{H}_{2} \mathrm{O}$ till a high the signal to noise was achieved. 


\section{Western ECI dot blot of elution buffers}

A stack of 5-6 filter papers were placed into a flat dish. A similarly sized piece of PVDF membrane was placed over the filter papers. Methanol was added to the bottom of the flat dish but only enough so the methanol permeated up to the pvdf without soaking it. The serial IgG dilutions of IgG and samples were spotted of the PVDF membrane, left to dry, dipped in methanol for 30 seconds with shaking and washed in water for 1 minute with shaking. The membrane was block for 30 minutes in 5\% w/v skim milk and TBST, followed by three 5 minute washes in TBST. The membrane was incubated for $30 \mathrm{~min}$ in TBST with 1:10,000 Dk anti human conjugated HRP and then washed in TBST 3 times for five minutes with rocking followed by a 1 minute incubation with ECL solution and detection by gel doc using the high chemiluminescent setting.

\section{Biotinylated ligand affinity chromatography (modified protocol)}

The biotinylated affinity chromatography experiments were carried out as described above with several variations. Only DTT was used as an elution buffer. Aggregated ligands biotinylated by NHS-B and NHS-S-S-B before and after aggregation were applied to live cells. In addition, monovalent ligands with the SS cleavable linker were used in combination with HB containing detergents deoxycholate or octyl glucoside. To determine the effect of crosslinkers on the isolation of receptor complexes, paraformaldehyde was applied before (ctrl) and after ligands were allowed to bind to cells. 


\section{Preparation of aggregated ligands by heating}

Where aggregated IgG was used, the ligand was heated at $63^{\circ} \mathrm{C}$ for 30 minutes and then cooled on ice and then biotinylated with either the NHS-biotin or NHS-ss-biotin linker at a ratio of 5:1. The same protocol was used to biotinylate the ligands first and aggregate by heat afterward.

\section{Biotinylated ligand affinity chromatography media controls}

To control for the possibility that $\operatorname{IgG}$ may form soluble complexes in the media and not on the surfaces of live cells, the ligands were applied to media that had previously been incubated with live cells. Flasks of $70 \%$ confluent RAW 264.6 cells were wash ten times with serum free media (DMEM) and incubated at $37^{\circ} \mathrm{C}$. After 4 hours, the serum free media was removed and applied to a fresh flask without cells. No ligand, IgG-B and IgG-SS-B were applied to separate flasks and incubated overnight at $37^{\circ} \mathrm{C}$. In the morning protease inhibitors ABSF, PMSF, and eukaryotic protease inhibitor were applied to each sample before French pressing twice in the following order: no ligand, IgG-B, IgG-SS-B. The French press was washed with ice cold water between each sample and kept on ice. The samples were kept on ice till applied to a column. The SAagarose affinity chromatography was set up as previously described. Each type of ligand was applied to it's own SA-agarose column, washed with $4 \mathrm{~mL}$ of $1 \mathrm{xPBS}$ and eluted with 500uL of 5mM DTT. The beads were collected in 400uL of 1xPBS, digested and analyzed by LC-MS/MS as described below. 
APPLICATION OF LIGAND COATED AFFINITY CHROMATOGRAPHY MICRO AND NANO PARTICLES TO CAPTURE RECEPTOR COMPLEXES FROM LIVE CELLS

\section{Overview}

In this study, we dramatically advance a previously established method where polystyrene beads were applied to live cells to capture the IgG-FcR complex. However, polystyrene microparticles do not sediment easily for binding to adherent cells, show high background binding and are difficult to isolate by ultra-centrifugation. Thus, we have attempted to capture the IgG-FcR complex from live cells using melamine and acrylate (PMMA) micro beads and compared this method to traditional affinity chromatography methods. In addition, we used an entirely novel method where ligand coated silica nano beads were applied to live cells as a potential universal method for eluting monovalent versus polyvalent receptor complexes.

\section{Coating PMMA micro and nano particles in IgG}

PMMA nano and micro beads were pre-washed three times in either $\mathrm{MeOH}$, isopropanol or 5\% formic acid using a low binding eppendorf tube for high speeds and spun down with an ultracentrifuge after each wash at 4 degrees $C$, with acceleration level 9 and deceleration level 9 and 35,000 rpm for ten minutes. Beads were washed 3 times in $\mathrm{H}_{2} \mathrm{O}$ and incubated in $10 \mathrm{mg} / \mathrm{mL} \mathrm{IgG}$ for 1 hour. Unbound ligands were washed away in 1x PBS three times and sonicated 10 times using 3 second bursts. 


\section{Coating melamine micro and nano particles in IgG}

Melamine micro and nano particles were washed three times in 1x PBS and incubated in $10 \mathrm{mg} / \mathrm{mL} \operatorname{IgG}$ for 30 minutes at room temperature on a ferris wheel. Unbound ligands were washed off the beads with 1xPBS 5 times.

\section{Coating silica nano particles in IgG}

Silica micro and nano particles were coated with IgG using the same protocol for coating glass slides as previously described.

\section{Immuno-fluorescence of IgG coated beads binding to RAW 264.7 cells}

To determine whether RAW 264.7 macrophages would bind melamine, PMMA, and silica beads in micro and nano format, beads were incubated with cells, washed and then probed with a $\mathrm{Dk}$ anti hIgG and visualized by confocal microscopy. Cells were washed on ice with ice cold PBS + $1 \mathrm{mM} \mathrm{MgCl} 2$. After the final wash was removed $1.5 \mathrm{mLs}$ of ice cold HEPES buffer was applied to cells with just enough liquid to cover cells in order to increase the likelihood of binding. Forty $\mathrm{uL}$ of each bead preparation was applied to a separate well and incubated for either 5, 10, 15, 30 or 60 minutes at $37^{\circ} \mathrm{C}$. After each incubation period, cells were put on ice and washed $5 \mathrm{x}$ with ice cold PBS (2mL/wash) and fixed with $4 \%$ paraformaldehyde for 20 minutes on a rocker plate at RT. Fixing was terminated with PBS $+5 \%$ glycine at room temperature with rocking for 5 minutes followed by three washes in 1xPBS and a 30 minute blocking step with PBS $+5 \%(\mathrm{w} / \mathrm{v})$ skim milk powder and $1 \%$ serum Dk serum. Cells were then washed three times in $1 \mathrm{x}$ PBS followed by incubation with Dk anti human heavy and light chain IgG Dy488 (7.5:20,000) against the IgG coated beads for $30 \mathrm{~min}$ in the dark at room temperature on a rocker plate. After 
5 washes in 1x PBS the coverslips were mounted on glass slides with Dako and left to harden overnight at RT in the DARK (results not shown).

\section{Ligand affinity receptor chromatography with PMMA and melamine micro beads and silica nano by LC-MS/MS}

RAW 264.7 macrophages were seeded onto a $75 \mathrm{~cm} 2$ culture dish in $10 \mathrm{mLs}$ of DMEM with $5 \%$ fetal calf serum and grown to $70 \%$ confluence. Glass beads were prepared the night before the experiment as well as a sucrose gradient by filling a $1.7 \mathrm{~mL}$ ultracentrifuge tube with $60 \%(\mathrm{w} / \mathrm{v})$ sucrose and centrifuging for $1 \mathrm{hr}$ at $4 \mathrm{C}$ at $85,000 \mathrm{xg}$ in a TLA110 rotor. The gradient was stored

undisturbed on ice overnight. For each treatment, five flasks were sequentially placed on ice at 5 minute intervals and washed with $5 \mathrm{mLs}$ of ice cold serum free media and left in the final wash. The ligand coated beads were applied to each of 5 flasks at 5 minute intervals. After 15 minutes of incubation on ice, each flask was incubated at $37^{\circ} \mathrm{C}$ for either $0,5,10,15$ or 20 minutes. After the incubation period, the flasks were placed on ice. The first flask was washed five times in ice cold PBS with $1 \mathrm{mM} \mathrm{MgCl} 2$ and then $10 \mathrm{mLs}$ of $\mathrm{HB}$ was applied. The cells were scraped and transferred to the next flask after washing 5 times with $1 \mathrm{mM} \mathrm{MgCl}_{2}$ in $1 \times \mathrm{PBS}$. The $\mathrm{HB}$ and cells were transferred and scraped from each flask until all 5 time points were combined and applied to a French press twice and transferred to a $15 \mathrm{~mL}$ falcon tube on ice.

\section{Crude Control}

A crude control was prepared by washing one flask five times with $1 \mathrm{xPBS}+1 \mathrm{mM} \mathrm{MgCl} 2$ and adding $10 \mathrm{mLs}$ of HB. The cells were scraped and applied to a French press twice. IgG coated beads were applied to the crude homogenate immediately after French pressing and stored on ice in a $15 \mathrm{~mL}$ falcon tube on ice. 


\section{Bead recovery by sucrose gradient and salt/acetonitrile step gradient elution of peptides}

PMMA micro, melamine micro and silica nano beads were centrifuged at $4000 \mathrm{xg}$ for 15 minutes at $4^{\circ} \mathrm{C}$. The supernatant was collected and saved as the "HB" (homogenization buffer) fraction. The beads were resuspended in 200uL of ice cold 1xPBS and layered on top of the pre-prepared sucrose gradient. A $200 \mathrm{uL}$ wash of the flacon tube was also gently layered over top of the sucrose gradient and centrifuge at $85,000 \mathrm{xg}$ for 10 minutes at $4^{\circ} \mathrm{C}$. The beads were withdrawn from the gradient and resuspend in $1 \mathrm{~mL}$ of ice cold $1 \mathrm{XPBS}$ and centrifuged at 14,800 $\mathrm{xg}$ for 3 minutes. The supernatant was collected and labelled 'wash'. The proteins and peptides were then sequentially eluted from the beads by a salt/acetonitrile step gradient. The eluted fractions and remaining complexes on the chromatography resin were digested as previously described with 1 $\mu \mathrm{g}$ of trypsin per $100 \mathrm{ug}$ of protein overnight at $37^{\circ} \mathrm{C}$ in $50 \mathrm{mM}$ Tris, $\mathrm{pH} 8.85$, containing 200 $\mathrm{mM}$ urea and $5 \%$ acetonitrile followed by reduction in $2 \mathrm{mM}$ DTT and re-digestion with trypsin for $2 \mathrm{hrs}$ at $37^{\circ} \mathrm{C}$ and acidified to a final concentration of $5 \%$ formic acid. Samples were dried down and stored at $-20^{\circ} \mathrm{C}$ and analyzed by LC-MS/MS as described below.

\section{Dumbroff Protein assay, Western ECl dot blot and silver staining of elutions}

A Dumbroff protein assay was performed on all fractions as previously described to determine the total protein content in each of the fractions. A Western dot blot on PVDF with detection by a Dk anti hIgG - HRP probe was used to detect the amount of IgG eluted in each fraction. The IgG coated melamine micro bead fractions were separate by 9\% Tricine SDS PAGE with silver staining. 


\section{Peptide collection by ZipTip}

A C18 ZipTip was cleaned by pipetting 10 times up and down in $65 \%$ Acetonitrile and $0.01 \%$ Acetic Acid (Buffer B) using a pipetter set at $20 \mu$ l. Once wet, the ziptip is kept submerged in liquid and is never depressed passed the top of the resin. Buffer B is expelled down to the top of the ziptip and then pipetted up and down five times with 5\% acetonitrile and $0.01 \%$ Acetic Acid (Buffer A). Buffer A is expelled to the top of the resin and then the sample is pipetted up and down 20 times without depressing passed the resin. The sample is expelled down to the resin and the ziptip is washed once with buffer A and expelled down to the top of the resin. $2 \mathrm{uL}$ of buffer $\mathrm{B}$ is is drawn up to the first mark on the ziptip above the resin and eluted into a new Eppendorf tube. $18 \mathrm{uL}$ of buffer $\mathrm{A}$ is added to the Eppendorf tube and injected onto a clean $20 \mathrm{uL}$ loop.

\section{LC-MS/MS}

The samples were applied to a 150 ID C18 reverse phase column with a flow of approximately $2 \mathrm{uL} / \mathrm{min}$ of HPLC water and $0.01 \%$ acetic acid. The samples were resolved over an 80 minute gradient of aqueous (water and $0.01 \%$ acetic acid) and organic solvent (acetonitrile and $0.01 \%$ acetic acid) starting at 5\% acetonitrile and $0.01 \%$ HAc up to $65 \% \mathrm{ACN}$ and $0.01 \%$ HAc and then back down to 5\%. The gradient was applied using an Agilent 1100 HPLC pump. The samples were ionized with a nano spray source, separated by HPLC and detected by electrospray ionization into a Thermo LTQ ion trap and identified by the SEQUEST search algorithm against a mouse library. The results were parsed into an SQL database according to treatment for comparison (Bowden et al., 2009). The sample was introduced onto the analytical HPLC column $(150 \mathrm{~mm} \times 150 \mu \mathrm{m})$ via a $20 \mu \mathrm{l}$ loop with a Rheodyne injector. The samples were resolved over analytical C18 reverse phase column with a flow of approximately $2 \mathrm{uL} / \mathrm{min}$ of HPLC water and 
$0.01 \%$ acetic acid. The samples were resolved over an 80 minute gradient of aqueous (water and $0.01 \%$ acetic acid) and organic solvent (acetonitrile and $0.01 \%$ acetic acid) starting at $5 \%$ acetonitrile and 0.01 acetic acid up to $65 \%$ acetonitrile and $0.01 \%$ acetic acid and then back down to 5\%. The captured FcR's were compared for each of the affinity chromatography combinations of ligand, affinity surface and elution buffer. 


\section{RESULTS}

Error in the random and independent sampling of tryptic peptides of a myosin standard by LC-ESI-MS/MS with a linear ion trap

\section{SDS-PAGE with CBBR, AgNO3 and anti-actin Western blot}

If the central assumption of the Empirical Statistical Model is true, i.e. correlations to rabbit myosin are true positive, while all other correlations are false positive, then analysis of SDSPAGE should show a single band by staining with Coomassie Brilliant blue (CBBR) or diamine silver (AgNO3), and Western blot against other members of the functional myosin protein complex such as Actin should show no reaction. The rabbit myosin standard showed a single band at low protein loadings but at higher loadings many bands were detectable including a CBBR visible band at $44 \mathrm{kDa}$ - corresponding to actin (Fig.18A). At least 50 additional protein bands were detectable by SDS-PAGE with diamine silver staining (Fig.18B). Western blot confirmed the strong presence of actin in the myosin standard (Fig.18C) in agreement with LCESI-MS/MS but in contrast to the central assumption of the empirical statistical model (Keller, Nesvizhskii, Kolker, \& Aebersold, 2002). Thus, peptides that correlate to actin and other common proteins in the myosin standard may be true positive. 

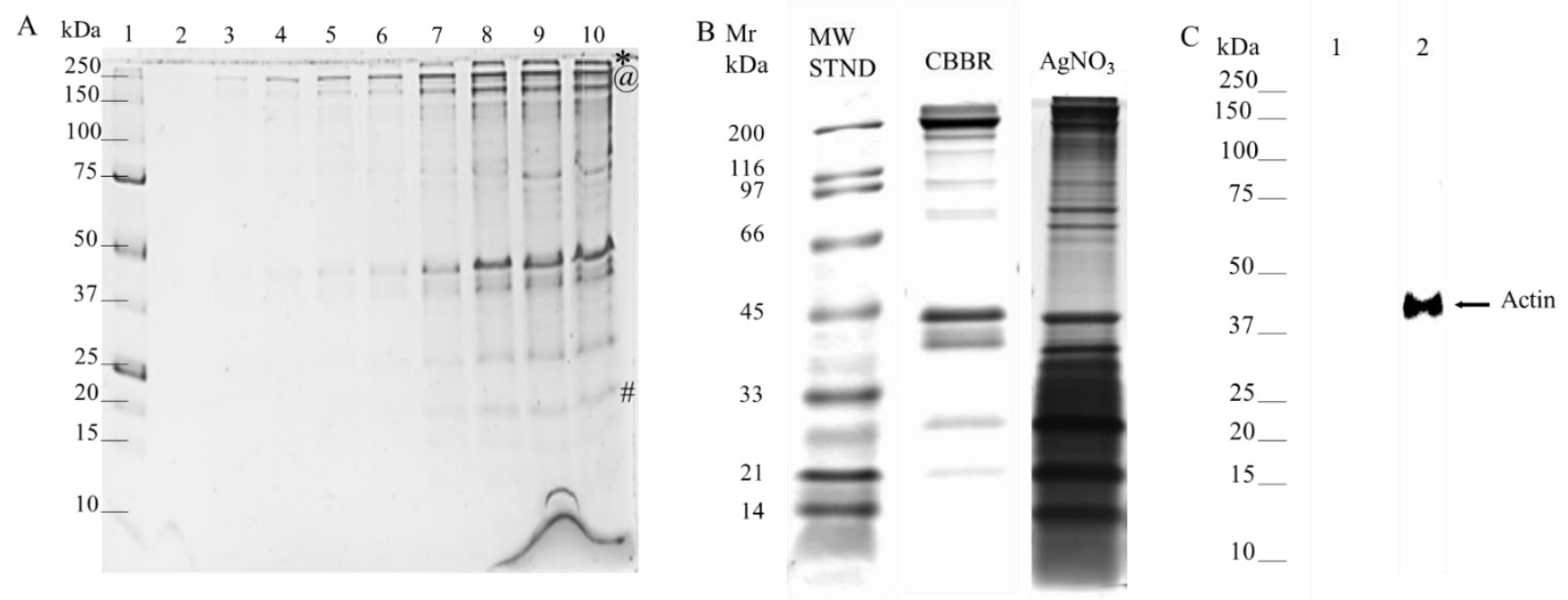

Figure 18. The effect of sample loading and detection method on the perceived purity of the rabbit myosin standard (Sigma M1636) by SDS-PAGE with Coomassie Brilliant Blue, diamine silver stain and Western blot. Panels: A, 0.1-10 $\mu$ g Myosin [Lanes: 1, MW marker; 2, $0.1 \mu \mathrm{g}$ myosin; 3, $0.25 \mu \mathrm{g}$ myosin; 4, $0.50 \mu \mathrm{g}$ myosin; Lane 5, $0.75 \mu \mathrm{g}$ myosin; $6,1.00 \mu \mathrm{g}$ myosin; 7, $2.50 \mu \mathrm{g}$ myosin; 8, $5.00 \mu \mathrm{g}$ myosin; 9, 7.50 $\mu \mathrm{g}$ myosin; 10, $10 \mu \mathrm{g}$ myosin (Symbols *440 kDa myosin complex, @ $220 \mathrm{kDa}$ myosin heavy chain, \# $15 \mathrm{kDa}$ myosin light chain)]; B, A total of 5 micrograms of the rabbit myosin standard was resolved by Tricine SDS-PAGE alongside 10 micrograms total of the SDS-PAGE standards (MW) for staining with Coomasie brilliant blue $\mathrm{R}-250$ (CBBR ) versus diamine silver $\left(\mathrm{AgNO}_{3}\right)$; $\mathrm{C}$, Western blot of the myosin standard (Lane 1, secondary antibody control. Lane 2 anti actin). The expected migration of myosin heavy chain is approximately $220 \mathrm{kDa}$. The arrow shows the approximate mass of actin, $44 \mathrm{kDa}$.

\section{Removal of noise and redundant MS/MS spectra correlations by SQL}

Here the peptide-to-protein distributions of tandem mass spectra from IgG, myosin, dust, noise from blank solvents and random spectra serve as a control for MASCOT, OMSSA, SEQUEST and X!TANDEM for a linear ion trap. The affinity purified standard bulk human $\operatorname{IgG}(\mathrm{n}=2$ runs with 6,216 MS/MS spectra), rabbit myosin standard obtained from differential centrifugation ( $\mathrm{n}=2$ runs17,730 MS/MS spectra) were compared to laboratory dust ( $\mathrm{n}=4$ runs with 18,387 MS/MS spectra), blank gradient runs on a naïve column with HPLC grade solvents ( $\mathrm{n}=36$ runs with 75,269 correlated MS/MS spectra), and computer generated random MS/MS spectra ( $n=116$ 
simulated runs with 440,772 MS/MS spectra). The authentic IgG, myosin and dust samples all showed intensity values $\geq \mathrm{E} 4$ counts while the blank noise recordings showed intensity values typically $\leq$ E4 counts (Fig.19).

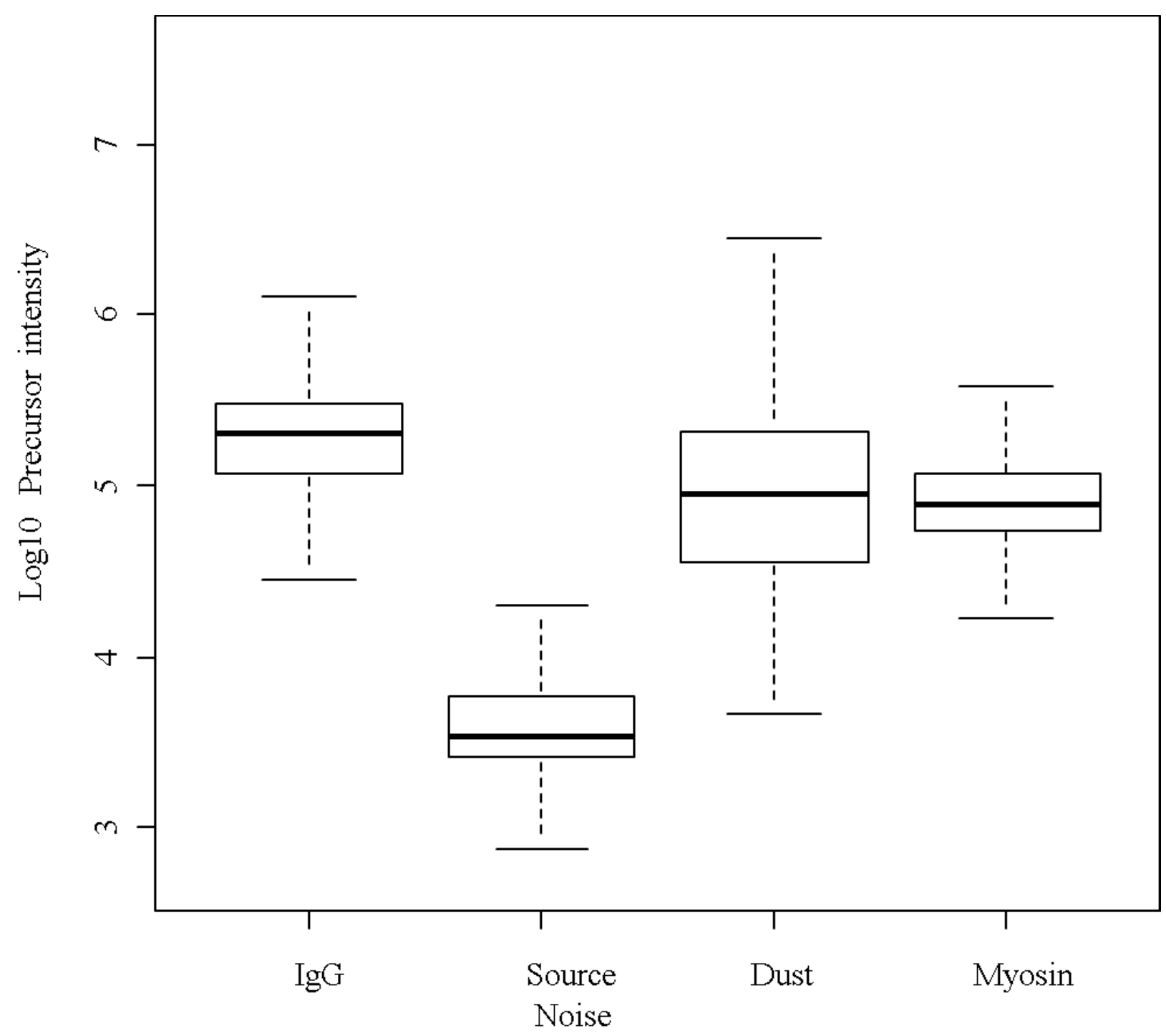

Figure 19. The box plot of the raw intensity values of the electrospray source noise versus the authentic IgG, dust and myosin proteins samples. The solid line shows the median, the box hinges show the interquantile distance and the wiskers show the $99 \%$ confidence range of the data. The treatments were the affinity purified $\operatorname{IgG}$ positive control $(n=2)$, the source noise from blank runs of water AACN gradients over naïve C18 HPLC columns ( $\mathrm{n}=36)$, the tryptic peptides of laboratory dust $(n=4)$ and the myosin test standard $(n=2)$. 
The full list of all the proteins identified in all samples by each algorithm are provided in the supplemental data for dust, source noise from blank solvents and random spectra as a reference for MASCOT, SEQUEST, OMSSA and X!TANDEM. The use of SQL server to store the results of LC-ESI-MS/MS and peptide correlations together for classical statistical analysis with generic data analysis systems (e.g. S Plus, SAS or R) permits the analysis of peptide and protein confidence, intensity and other attributes based on their distributions with respect to random expectation (Bowden et al., 2012; Florentinus, Bowden, Sardana, Diamandis, \& Marshall, 2012; Florentinus, Jankowski, Petrenko, Bowden, \& Marshall, 2011; P. Zhu, Bowden, Tucholska, \& Marshall, 2011; Peihong Zhu, Bowden, Tucholska, Zhang, \& Marshall, 2011). Correlation algorithms may attempt to model the fit of an MS/MS spectra to more than one charge state or peptide sequence and so it is important to ensure that each MS/MS spectra is only used once to implicate a single peptide sequence and charge state combination that is the best fit of the data and the simplest model. The combination of the precursor ID and file ID was used to create a unique, composite-key in SQL that may be applied to MS/MS spectra to ensure that redundant use of the same mass spectral data is efficiently removed from the results on the basis of best charge state and then within the best charge state the selection of the best peptide sequence (Table I). Thus analysis in SQL/R can be used to removed noise and redundant correlations to controls type I error rate. 
Table I. The filtering of the cumulative, redundant correlations to protein accession sequences on the basis of charge state and peptide sequence. Bulk IgG, blank, dust and random spectra were searched against a federated human protein library. Myosin was searched against a federated rabbit library. The redundant use of MS/MS spectra to correlate to more than one peptide sequence was eliminated using a composite key in SQL Server.

\begin{tabular}{lrrrrr} 
& Bulk IgG & Myosin & Dust & \multicolumn{1}{l}{ Blank } & \multicolumn{1}{l}{ Random } \\
Species & Human & Rabbit & Human & Human & \multicolumn{1}{l}{ Human } \\
No Filter (0) & 36786 & 19647 & 117177 & 227396 & 2745779 \\
Charge State (1) & 9189 & 6762 & 36314 & 45044 & 1101897 \\
Peptide Sequence (2) & 9183 & 6762 & 29337 & 44605 & 1101305 \\
MS/MS Spectra & 6216 & 17730 & 18387 & 75,269 & 440772
\end{tabular}

\section{Peptide-to-protein distributions of authentic versus noise and random controls in $\mathbf{R}$}

The MS/MS spectra were correlated with the MASCOT, OMSSA, SEQUEST and X!TANDEM algorithms separately and the results combined as an arbitrary figure of merit (Fig. 20). The SEQUEST algorithm showed $\mathrm{a} \geq 10$-fold increase in the number of correlations to each protein compared to the other algorithms and made by far the greatest contribution to the experiment (see supplemental data). The number of successful peptide identifications was corrected by the non-redundant number of MS/MS records written into the system from the Mascot Generic Format (mgf) files to compute the observed frequency. After accepting only best fit correlations in terms of charge state and peptides sequence the authentic $\mathrm{IgG}$, and myosin or dust (i.e. authentic samples) showed a much higher frequency (E-1 to E-2) concentrated in a 100 proteins or so compared to that of source noise and random MS/MS (E-3 to E-4) that showed a random or flat distribution concentrated on the giant protein Titan (TTN) . The authentic standards and samples show a 10 to 100 -fold greater frequency highly concentrated on a discrete set of proteins, compared to the noise and random controls (Fig. 20). In contrast, source noise or 
random MS/MS spectra showed peptides correlated randomly across the entire protein database with a low frequency (Bowden et al., 2012; P. Zhu et al., 2011; Peihong Zhu et al., 2011).

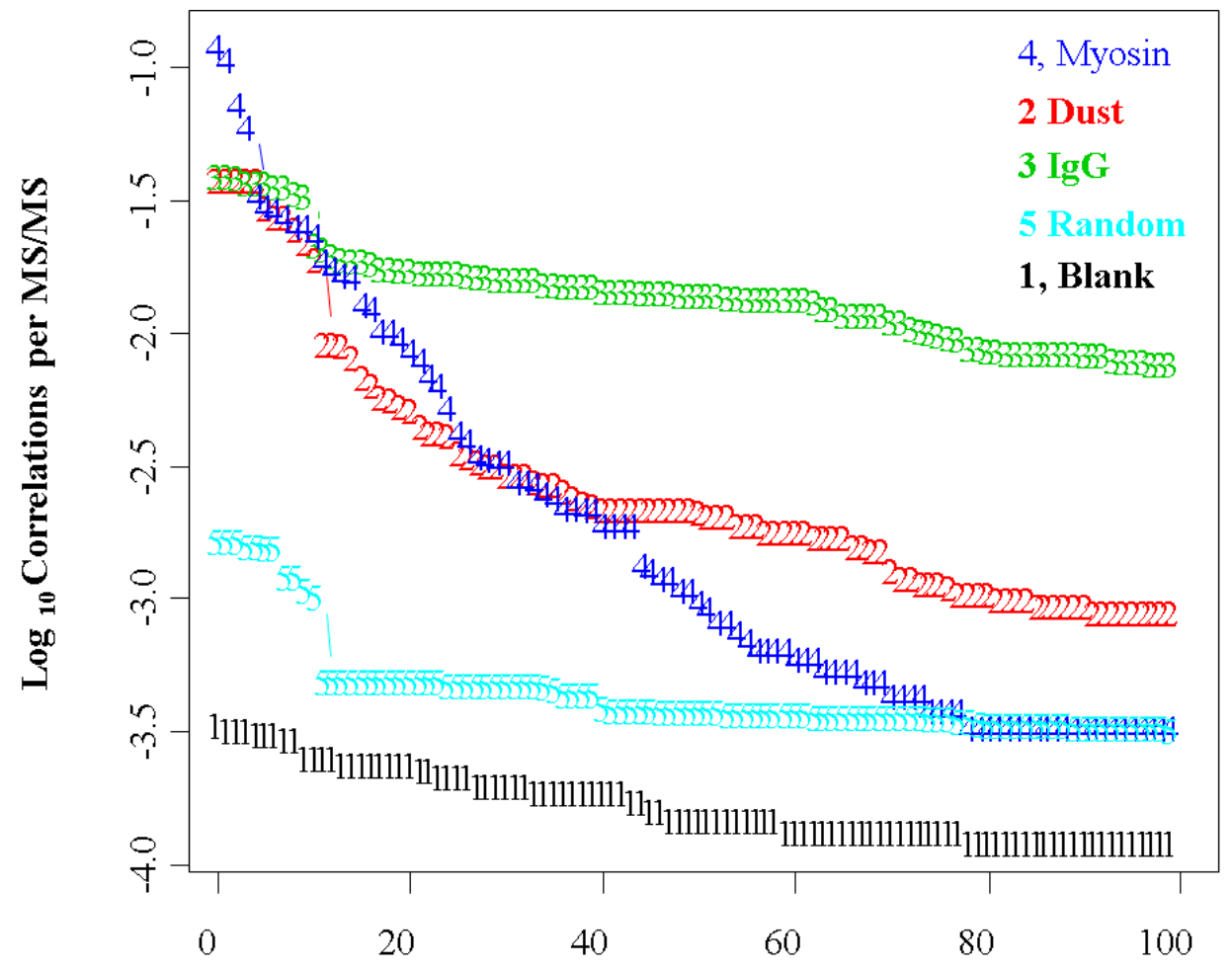

Protein Accession

Figure 20. The $\log _{10}$ frequency of non-redundant correlations (Filter 2 ) of fully tryptic peptides from MASCOT, OMSSA, X!TANDEM and SEQUEST combined per unit MS/MS spectra compared between IgG, myosin, dust, blank and random spectra. The probability that the curves from IgG, myosin, and dust are the same as either the random or blank controls is essentially zero. Loading sufficient sample to ensure the instrument does not sample blank noise during the experimental run essentially prevents contamination of the results by noise spectra. At a cut off of 200 protein accessions only TTN contaminates the IgG and myosin results that is easily avoided using the controls lists provided in the supplemental data. The two authentic standards bulk human IgG ( $\mathrm{n}=2$ runs with 6,216 MS/MS spectra) and rabbit myosin standard ( $\mathrm{n}=2$ runs with $17,730 \mathrm{MS} / \mathrm{MS}$ spectra) were compared to laboratory dust ( $\mathrm{n}=4$ runs with 18,387 MS/MS spectra), blank gradient runs on a naïve column with HPLC grade solvents ( $\mathrm{n}=36$ runs with 75,269 MS/MS spectra), and computer generated random MS/MS spectra ( $n=116$ simulated runs with 440,772 spectra). 


\section{Human IgG positive control}

The bulk human IgG sample was searched against the federated human library and found to contain Immunoglobulin heavy and light chain, kappa variable domain, and even peptides from specific known VDJ sequences were observed and few other cellular factors. Some 12,000 redundant peptide correlations from 800 protein accession numbers collapsed to no less than 257 gene symbols were identified and quantified by X!TANDEM in the bulk human IgG sample (Fig. 20). Many of the proteins identified in bulk human IgG share peptide sequences in the federated human library since IgG is part of the Ig Superfamily that contains some highly conserved sequences found in hundreds of protein accessions, but may also have contained some proteins bound to the Fc-, variable or other domains.

\section{Laboratory dust contamination control}

In order to determine the proteins that are likely to result from laboratory contaminants, laboratory dust was collected and digested with trypsin prior to LC-ESI-MS/MS that identified many keratins and other proteins (Fig. 20). Many keratins (KRT1, KRT10, KRT2, KRT6A, KRT5, KRT9, KRT6BC, KRT13), actins (ACT), albumin (ALB), ankyrin (POTE), and a few other types of proteins were identified. No food, plants, bacterial cultures, or animals (other than the staff of the RABL) were permitted in the mass spectrometry room that contains only equipment and human beings. Thus, the only source of proteins such as keratin in dust was the human occupants of the Ryerson Analytical Biochemistry Laboratory (RABL). Titan was observed at 1/35th the frequency of keratin in the laboratory dust sample, at about the same frequency of random. However, nesprin (SYNE), nebulin (NEB) and other giant proteins from random MS/MS spectra were not observed in dust. After discounting the giant protein TTN (see 
below), the identification of keratins (KRT), actins (ACT), albumin (ALB), ankyrin (POTE), caspase 14 (CASP14) showed $p$-values that ranged from E-215 to E-2 (i.e. $p \leq 0.01$ ). Caspase 14 that was automatically identified 7 times is associated with the expression of keratin and terminal differentiation in skin cells leading to apoptosis (Denecker, Ovaere, Vandenabeele, \& Declercq, 2008).

\section{Blank source noise null random model}

Running blank gradients with HPLC grade solvents over naive C18 columns for LC-ESI-MS/MS to create source noise also resulted in the identification of TTN as the major protein at a frequency about 100 fold below that of the IgG, myosin, or keratin. Most source noise shows intensity values of less than E4 counts but collecting 36 blank gradients eventually identified TTN (LeWinter \& Granzier, 2013) and to a lesser degree ZNF503, VWCE, API5, TMEM199, SYT7, TPSG1, USP9X, DACT3, SUGP2, CXorf31, MYO3A, KLRC4, CYCS, RGP1, SYN1, DSPP, IMMP1L CAMK1, ASPM, AHNAK and several others at very low frequencies ( 2000 peptides /75,000 MS/MS spectra) compared to authentic samples (Fig. 3). Little keratin or other known dust proteins were observed in the blank runs indicating there was little contamination of the LC-ESI-MS/MS system.

\section{Computer random spectra null random model}

Computer generated random spectra confirmed that the TTN observed in source noise from blank runs results from the near random distribution of mis-correlations. The results indicate that correlating $\sim 440,772$ random MS/MS spectra against a protein library with some $\sim 110,000$ distinct proteins resulted in a low frequency of peptide-to-protein correlations to the largest 
protein Titin (TTN). In addition to TTN (34,350 aa), also observed from blank runs, other large proteins including NEB (8560 aa), CCDC168 (7081 aa), CCDC168 (7081 aa), SYNE1 (8797 aa), OBSCN (6620 aa), TROPH (6907 aa), SYNE2 (6885), MACF1 (7388 aa), AHNAK (5890 aa), DST (3685) as well as LRP1, XIRP2, MUC16, FSIP2, LRP1B, PLEC were observed with a low frequency from computer generated random spectra (P. Zhu et al., 2011). It was apparent that random spectra were randomly correlated to all peptides at a low frequency and so the distribution was strongly skewed towards giant proteins like titin (TTN). The low frequency and random distribution of computer generated random MS/MS peptides may serve as an internal statistical control for LC-ESI-MS/MS (Bowden et al., 2012; Florentinus et al., 2012; Florentinus et al., 2011). A large number of random MS/MS spectra $(440,772)$ resulted in sufficient correlations to robustly define the distribution that showed much lower average and total peptide correlation that were apparently randomly distributed across the protein library (Fig. 20).

\section{Myosin test standard}

The myosin standard purified from rabbit muscle by differential centrifugation (Kielley \& Bradley, 1956) previously used to set false positive error rates using the empirical statistical model (Andrew Keller et al., 2002) was searched against a rabbit library and found to contain myosin, tropomyosin, actin, collagen, actinin, microtubule and other known protein interactors (MYH4, ACTA1, MYH2, MYH1, ACTB, MYH3, MYH7, ACTC1, ACTA2, ACTG2, MYH13, MYH6, NEB, GAPDH, TNNT3, ACTBL2, TPM1) with a high frequency (Fig. 20). There was agreement on the set myosin proteins by no-enzyme versus fully-tryptic peptide correlation (not shown). In contrast to the central assumption of the empirical statistical model that only correlations to myosin were true positive (A. Keller et al., 2002; Andrew Keller et al., 2002), the 
LC-ESI-MS/MS results indicated that the myosin standard contained many bona fide proteins. Hence, the empirical statistical model, i.e. decoy library searching, suffered from a large Type II error and thus a large total error.

\section{Statistical distribution of peptides and proteins from X!TANDEM in SQL-R}

In addition to the peptide-to-protein frequency with respect to random expectation, another independent method is to compute the random expectation value ( $\mathrm{p}$-value) of the fit of

experimental MS/MS spectra to the expected peptide spectra. The peptide intensity, $[\mathrm{M}+\mathrm{H}]+$, delta mass and p-values results of the IgG, myosin, dust, from X!TANDEM were parsed into SQL and plotted with R to illustrate the peptide intensity and p-value distributions, normality, and relationship to $[\mathrm{M}+\mathrm{H}]+($ Figs. $4-8)$. The probability that proteins were false positive identifications was independently calculated using X!TANDEM by taking the product of the peptide p-values for each protein (Bowden, Beavis, \& Marshall, 2009). The p-values of the IgG, myosin and dust proteins clearly agree with the comparison of the peptide frequency to both source noise or computer-generated random spectra that indicate many proteins were correctly identified from the IgG or myosin standards and the authentic laboratory dust samples. The authentic myosin standard showed far lower cumulative $\mathrm{p}$-values proteins, from a much small number of spectra compared to those of source noise or random MS/MS. The low p-values clearly indicated that LC-ESI-MS/MS with a linear ion trap (Schwartz, Senko, \& Syka, 2002) can identify thousands of peptides from hundreds of proteins in the rabbit myosin protein complex. From 17,730 MS/MS spectra of greater than E4 intensity X!TANDEM made 6829 correlations to a set of 638 peptides (i.e. on average $\sim 10$ independent observations of each peptide) found in 606 protein accessions that reduced to 409 Gene Symbols. The relative peptide 
counts and/or intensity values directly distinguish the results of authentic MS/MS spectra of $\operatorname{IgG}$, myosin or dust samples from the false positive random and noise distributions. The intensity results of random and independent sampling of peptides and proteins from LC-ESI-MS/MS may be log transformed, tested for normality and analyzed by ANOVA (Florentinus et al., 2012; Florentinus et al., 2011; John Marshall et al., 2003). The distribution of precursor ion intensity of the peptides, the p-values of peptide fits, $\mathrm{M}+\mathrm{H}$ and delta mass values in the authentic samples and were qualitatively different from those of noise or random negative controls. In particular, the p-value and delta mass distributions showed a marked difference between authentic versus negative controls spectra. For example, authentic spectra show masses with a Gaussian distribution around the expected value but the noise and random spectra showed a random, i.e. flat, distribution (Fig. 21-25). Random and noise p-values show a Gaussian distribution compared to authentic data that did not (Fig. 21-25). 

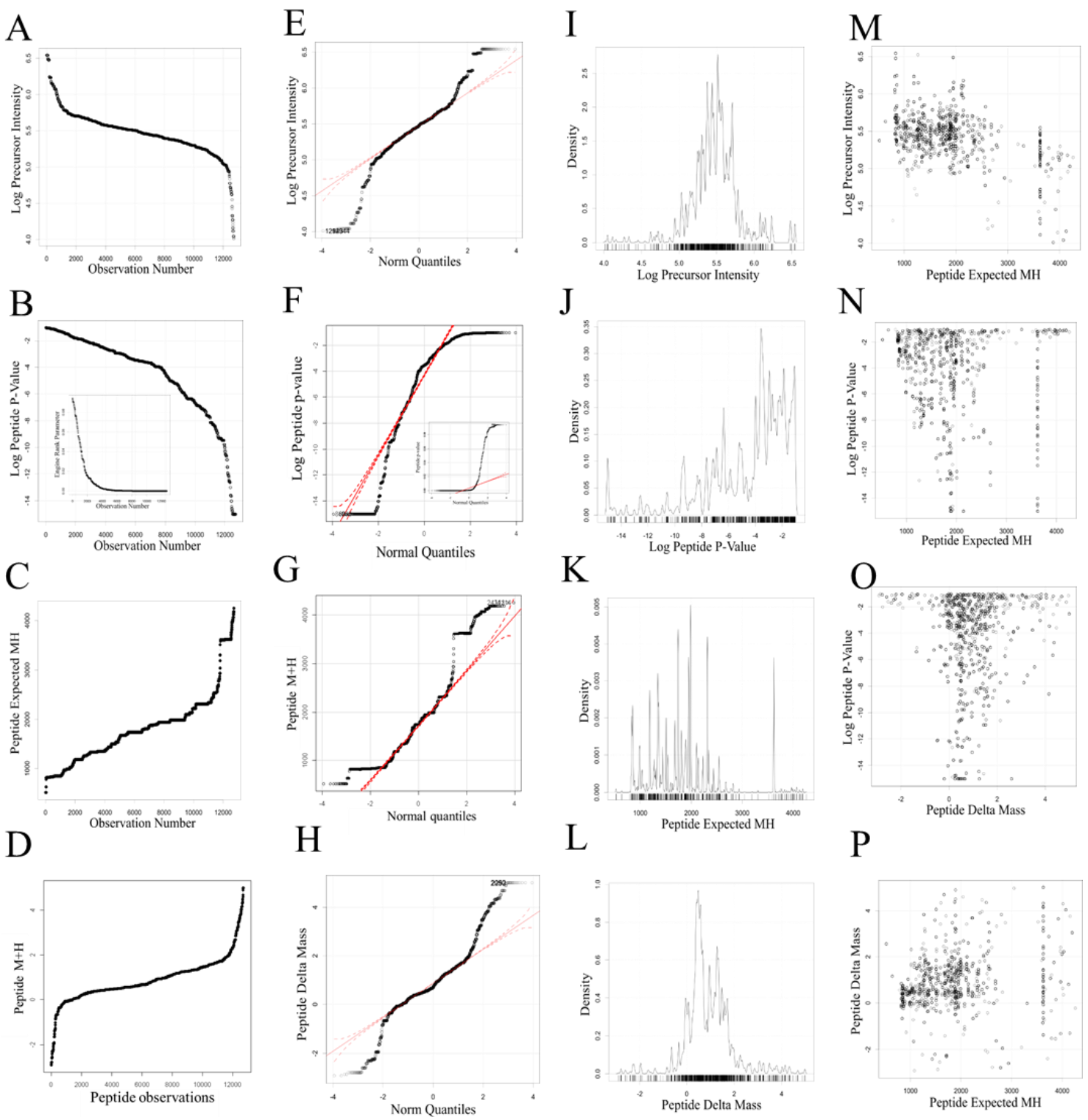

Figure 21. Affinity purified bulk Human IgG (positive control) results from the X!TANDEM algorithm. The distributions of the fully tryptic peptides identified with total 6,216 MS/MS spectra from the bulk human IgG sample by X!TANDEM against human proteins. Panels: A, the sorted $\log _{10}$ peptide intensity values; $\mathrm{B}$, the $\log _{10}$ peptide $\mathrm{p}$-values from $\mathrm{X}$ !TANDEM (inset the linear peptide $\mathrm{p}$-values); $\mathrm{C}$, the sorted peptide $[\mathrm{M}+\mathrm{H}]+$ values; $\mathrm{D}$, the sorted peptide delta mass values; E, the $\log 10$ intensity Quantile plot; F, the log peptide p-values Quantile plot (inset the untransformed peptide p-value QQ plot); G, the peptide expected $[\mathrm{M}+\mathrm{H}]^{+}$Quantile plot; $\mathrm{H}$, the peptide delta mass Quantile plot; I, the $\mathrm{Log}_{10}$ peptide intensity value density plot; $J$, the log peptide $p$-value density plot; $K$, the peptide expected $[\mathrm{M}+\mathrm{H}]^{+}$ density plot; L, the peptide delta mass density plot; $\mathrm{M}$, log peptide intensity versus $\log [\mathrm{M}+\mathrm{H}]^{+}$ scatter plot; $\mathrm{N}$, the log peptide $\mathrm{p}$-value versus expected $[\mathrm{M}+\mathrm{H}]^{+}$scatter plot; $\mathrm{O}$, log peptide $\mathrm{p}$ value versus peptide delta mass scatter plot; $\mathrm{P}$, the peptide delta mass versus peptide expected $[\mathrm{M}+\mathrm{H}]+$ scatter plot. 

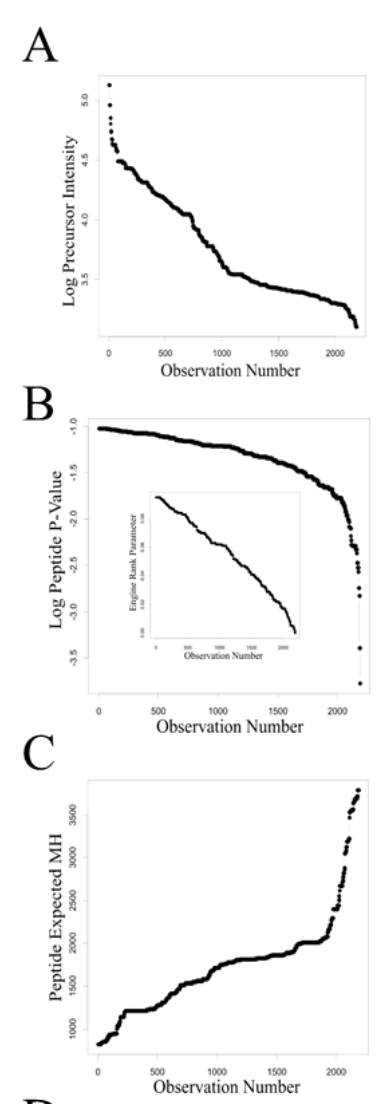

$\mathrm{D}$

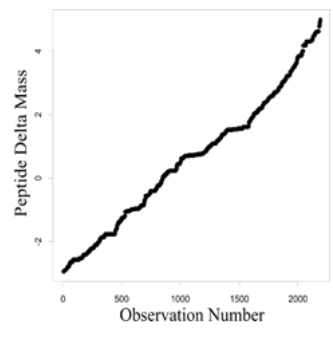

$\mathrm{E}$

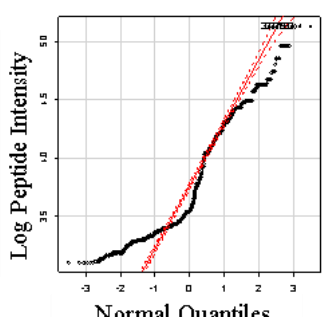

F

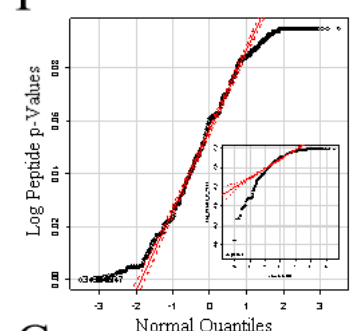

G

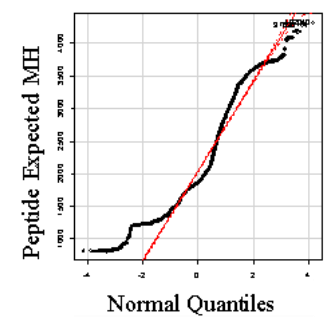

$\mathrm{H}$

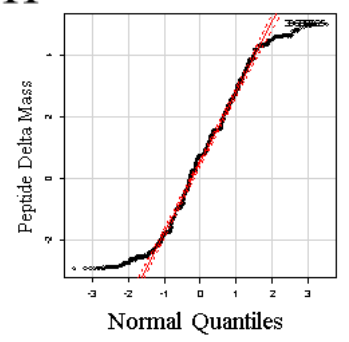

I

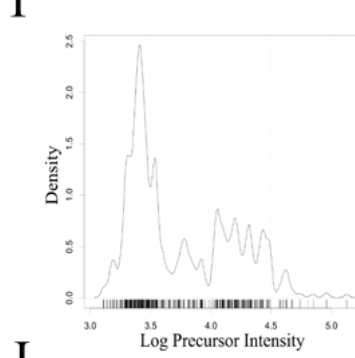

J

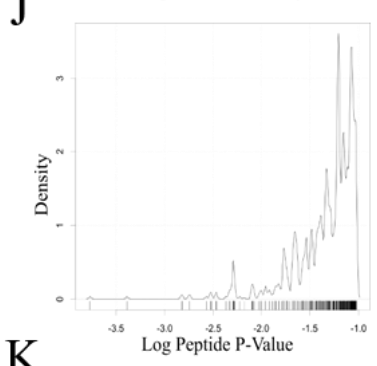

$\mathrm{K}$

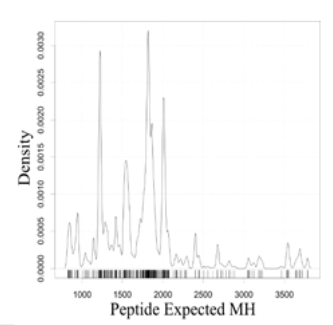

$\mathrm{L}$

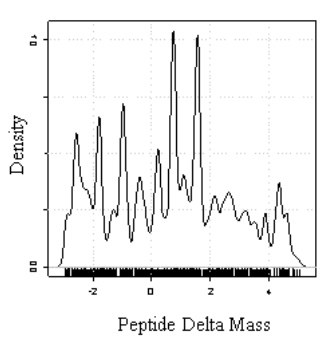

M

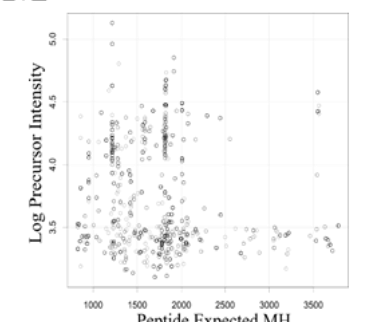

N

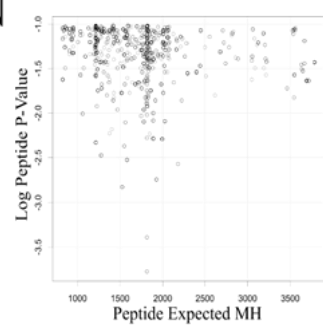

O

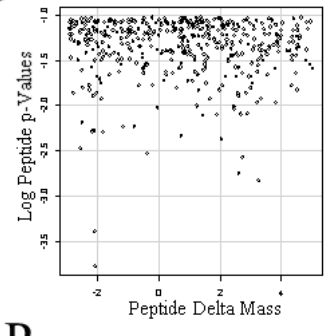

P

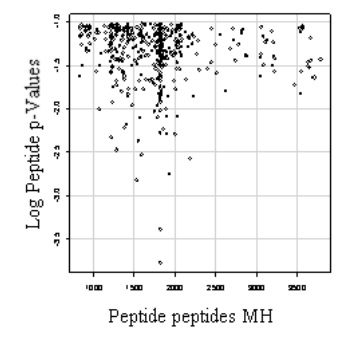

Figure 22. Laboratory dust (contamination control) results from the X!TANDEM algorithm. The distributions of the fully tryptic peptides from proteins identified with $\mathbf{1 8 , 3 8 7} \mathrm{MS} / \mathrm{MS}$ spectra from laboratory dust searched by X!TANDEM against a federated human library. Panels: A, the sorted $\log _{10}$ peptide intensity values; $\mathrm{B}$, the $\log _{10}$ peptide $\mathrm{p}$ values from X!TANDEM (inset the linear peptide p-values); $\mathrm{C}$, the sorted peptide $[\mathrm{M}+\mathrm{H}]+$ values; D, the sorted peptide delta mass values; E, the $\log 10$ intensity Quantile plot; F, the $\log$ peptide p-values Quantile plot (inset the untransformed peptide p-value QQ plot); G, the peptide expected $[\mathrm{M}+\mathrm{H}]^{+}$Quantile plot; $\mathrm{H}$, the peptide delta mass Quantile plot; I, the $\log _{10}$ peptide intensity value density plot; $\mathrm{J}$, the $\log$ peptide $\mathrm{p}$-value density plot; $\mathrm{K}$, the peptide expected $[\mathrm{M}+\mathrm{H}]^{+}$density plot; $\mathrm{L}$, the peptide delta mass density plot; $\mathrm{M}, \log$ peptide intensity versus $\log$ $[\mathrm{M}+\mathrm{H}]^{+}$scatter plot; $\mathrm{N}$, the log peptide $\mathrm{p}$-value versus expected $[\mathrm{M}+\mathrm{H}]^{+}$scatter plot; $\mathrm{O}, \log$ peptide $\mathrm{p}$-value versus peptide delta mass scatter plot; $\mathrm{P}$, the peptide delta mass versus peptide expected $[\mathrm{M}+\mathrm{H}]+$ scatter plot. 

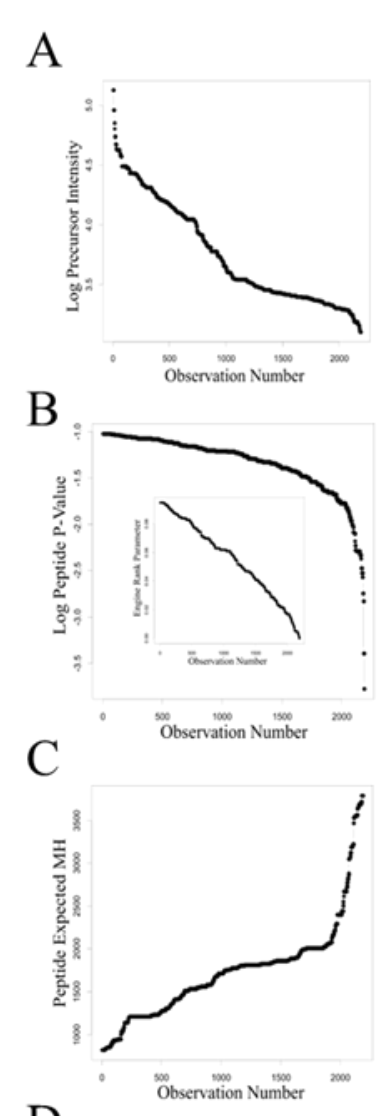

$\mathrm{D}$

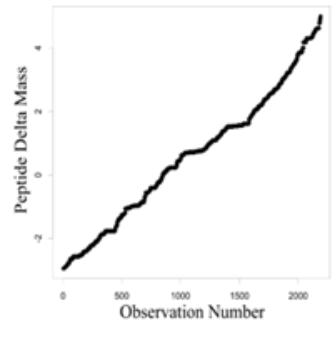

$\mathrm{E}$

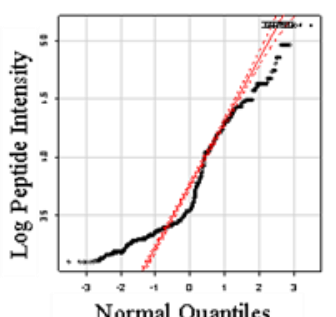

$\mathrm{F}$

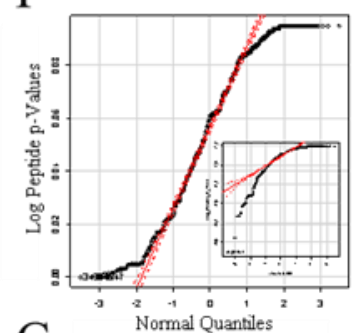

$\mathrm{G}$

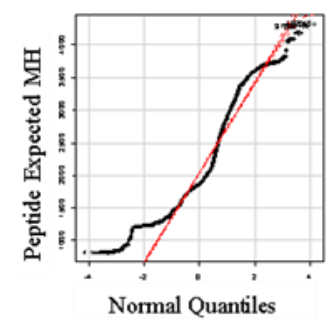

$\mathrm{H}$

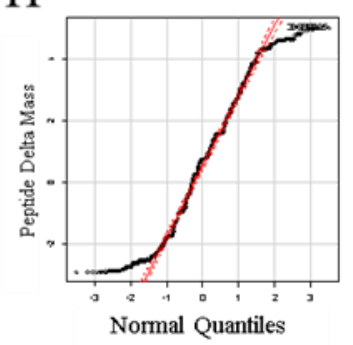

I
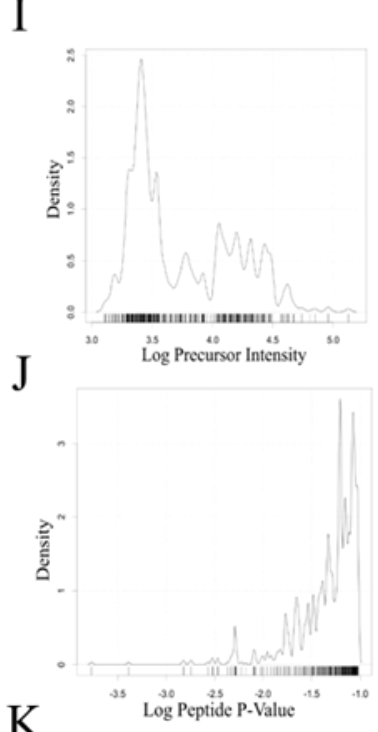

M
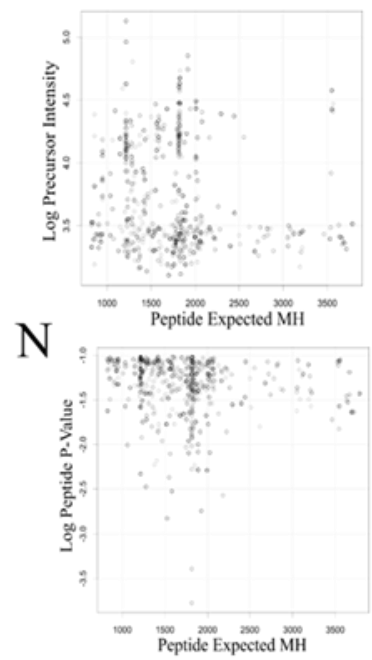

$\mathrm{O}$

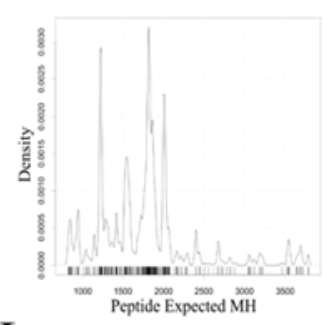

$\mathrm{L}$

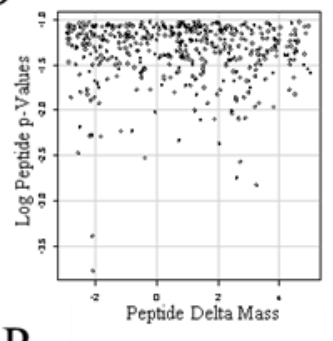

$\mathrm{P}$

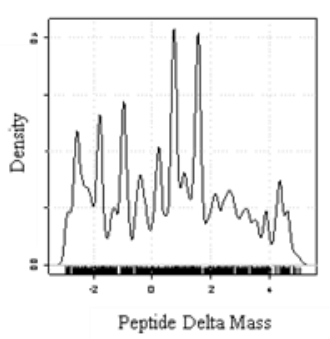

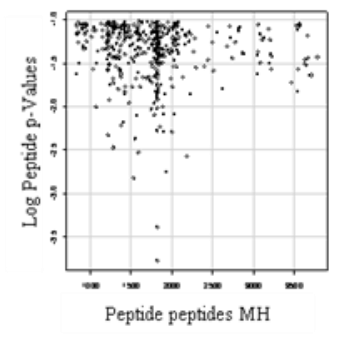

Figure 23. The electrospray source noise results (physically generated random spectra) from the X!TANDEM algorithm. The distributions of the fully tryptic peptides and proteins with 75,269 MS/MS spectra of source noise from blank LC-ESI-MS/MS runs on naïve columns computed by X!TANDEM against a human library. Panels: A, the sorted log10 peptide intensity values; $\mathrm{B}$, the $\log 10$ peptide $\mathrm{p}$-values from X!TANDEM (inset the linear peptide $\mathrm{p}$ values); $\mathrm{C}$, the sorted peptide $[\mathrm{M}+\mathrm{H}]+$ values; $\mathrm{D}$, the sorted peptide delta mass values; $\mathrm{E}$, the $\log 10$ intensity Quantile plot; F, the log peptide p-values Quantile plot (inset the untransformed peptide p-value QQ plot); G, the peptide expected $[\mathrm{M}+\mathrm{H}]+$ Quantile plot; H, the peptide delta mass Quantile plot; I, the density plot of Log10 peptide intensity value; J, the log peptide pvalue density plot; $\mathrm{K}$, the peptide expected $[\mathrm{M}+\mathrm{H}]+$ density plot; $\mathrm{L}$, the peptide delta mass density plot; $\mathrm{M}, \log$ peptide intensity versus $\log [\mathrm{M}+\mathrm{H}]+$ scatter plot; $\mathrm{N}$, the log peptide p-value versus expected $[\mathrm{M}+\mathrm{H}]+$ scatter plot; $\mathrm{O}, \log$ peptide $\mathrm{p}$-value versus peptide delta mass scatter plot; $\mathrm{P}$, the peptide delta mass versus peptide expected $[\mathrm{M}+\mathrm{H}]+$ scatter plot. 
A
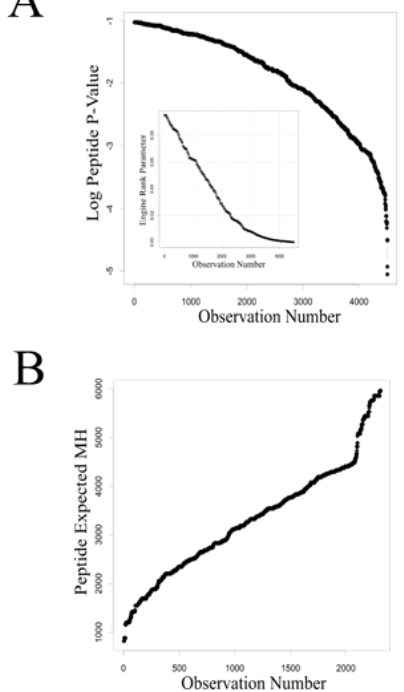

C

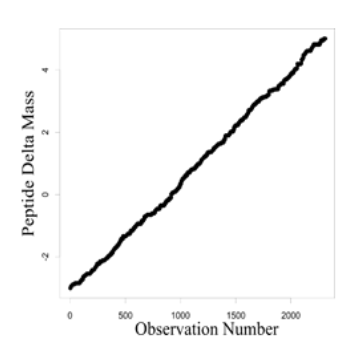

$\mathrm{D}$

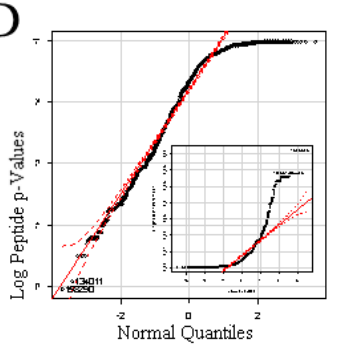

$\mathrm{E}$

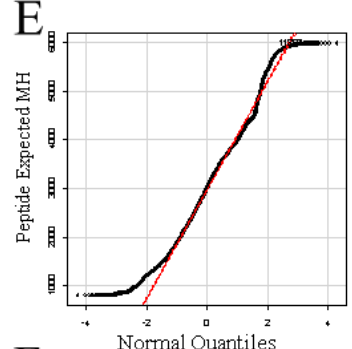

$\mathrm{F}$

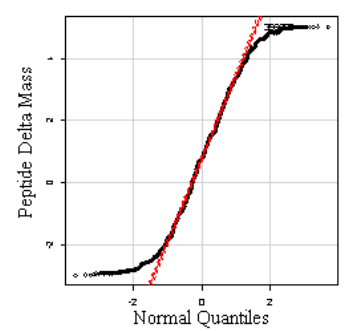

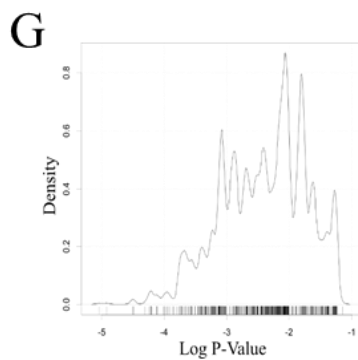

$\mathrm{H}$

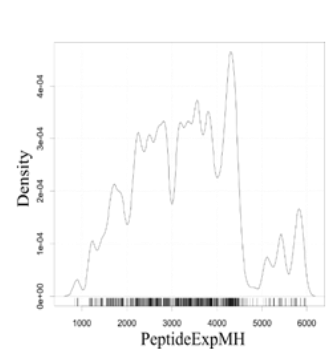

I

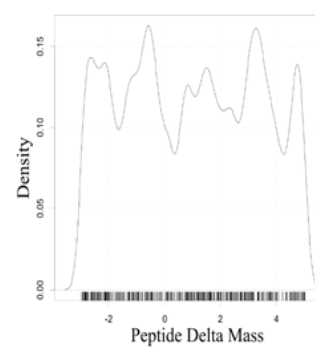

$\mathrm{J}$

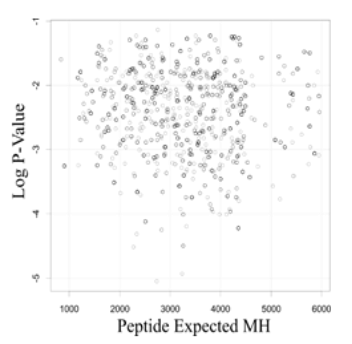

$\mathrm{K}$

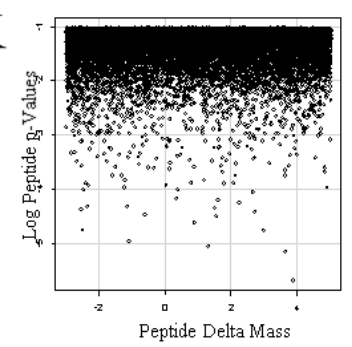

$\mathrm{L}$

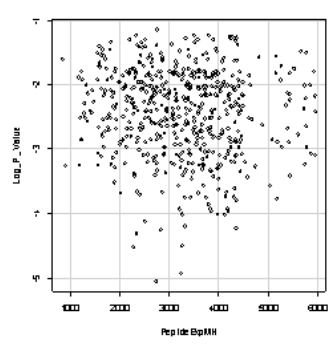

Figure 24. The computer generated random spectra results (null random model) from the X!TANDEM algorithm. The distributions of the fully tryptic peptides and proteins identified with 440,772 computer generated random MS/MS spectra fit by X!TANDEM against a human library. Panels: A the $\log _{10}$ peptide p-values from X!TANDEM (inset the linear peptide $\mathrm{p}$ values); $\mathrm{B}$, the sorted peptide $[\mathrm{M}+\mathrm{H}]+$ values; $\mathrm{C}$, the sorted peptide delta mass values; $\mathrm{D}$, the $\log$ peptide p-values Quantile plot (inset the untransformed peptide p-value Quantile plot); E, the peptide expected $[\mathrm{M}+\mathrm{H}]^{+}$Quantile plot; F, the peptide delta mass Quantile plot; $\mathrm{G}$, the log peptide $\mathrm{p}$-value density plot; $\mathrm{H}$, the peptide expected $[\mathrm{M}+\mathrm{H}]^{+}$density plot; $\mathrm{I}$, the peptide delta mass density plot; $\mathrm{J}$, the $\log$ peptide $\mathrm{p}$-value versus expected $[\mathrm{M}+\mathrm{H}]^{+}$scatter plot; $\mathrm{K}, \log$ peptide p-value versus peptide delta mass scatter plot; $\mathrm{L}$, the peptide delta mass versus peptide expected $[\mathrm{M}+\mathrm{H}]+$ scatter plot. 

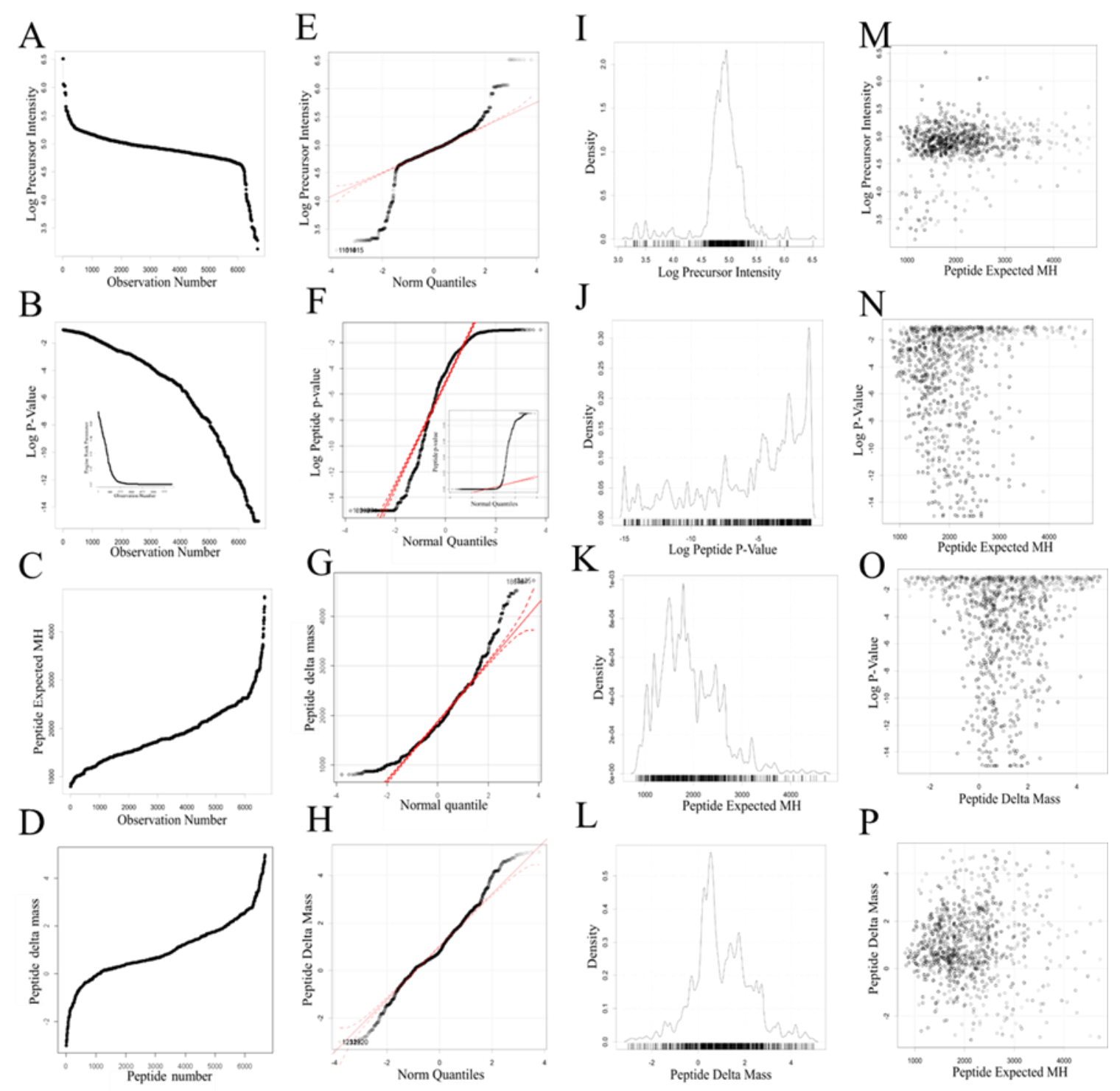

Figure 25. The rabbit myosin results (experiment test standard) from the X!TANDEM algorithm. The distributions of the fully tryptic peptides identified with $17,730 \mathrm{MS} / \mathrm{MS}$ spectra from the rabbit myosin standard searched by X!TANDEM against rabbit proteins. Panels: A, the sorted $\log _{10}$ peptide intensity values; $B$, the $\log _{10}$ peptide $p$-values from X!TANDEM (inset the linear peptide $\mathrm{p}$-values); $\mathrm{C}$, the sorted peptide $[\mathrm{M}+\mathrm{H}]+$ values; $\mathrm{D}$, the sorted peptide delta mass values; E, the $\log 10$ intensity Quantile plot; F, the log peptide p-values Quantile plot (inset the untransformed peptide p-value QQ plot); $\mathrm{G}$, the peptide expected $[\mathrm{M}+\mathrm{H}]^{+}$Quantile plot; $\mathrm{H}$, the peptide delta mass Quantile plot; I, the density plot of $\log _{10}$ peptide intensity value; J, the $\log$ peptide $\mathrm{p}$-value density plot; $\mathrm{K}$, the peptide expected $[\mathrm{M}+\mathrm{H}]^{+}$density plot; $\mathrm{L}$, the peptide delta mass density plot; $\mathrm{M}, \log$ peptide intensity versus $\log [\mathrm{M}+\mathrm{H}]^{+}$scatter plot; $\mathrm{N}$, the $\log$ peptide $\mathrm{p}$ value versus expected $[\mathrm{M}+\mathrm{H}]^{+}$scatter plot; $\mathrm{O}, \log$ peptide $\mathrm{p}$-value versus peptide delta mass scatter plot; $\mathrm{P}$, the peptide delta mass versus peptide expected $[\mathrm{M}+\mathrm{H}]+$ scatter plot. 


\section{Computation on the basis of peptides, proteins or Gene Symbols by SQL/R}

The relative quantification of the various $\mathrm{IgG}$, myosin and keratins ion intensity values from random and independent sampling are shown with the standard error that was typically on the order of less than $10 \%$ of the peptide mean for all the myosin and IgG proteins with 3 or more peptides and often much lower (Fig. 26). The mean peptide $\log 10$ precursor intensity can be

directly computed on the basis of peptide sequence, protein accession or available gene symbols using the SQL/R system with no proteomic specific software. 

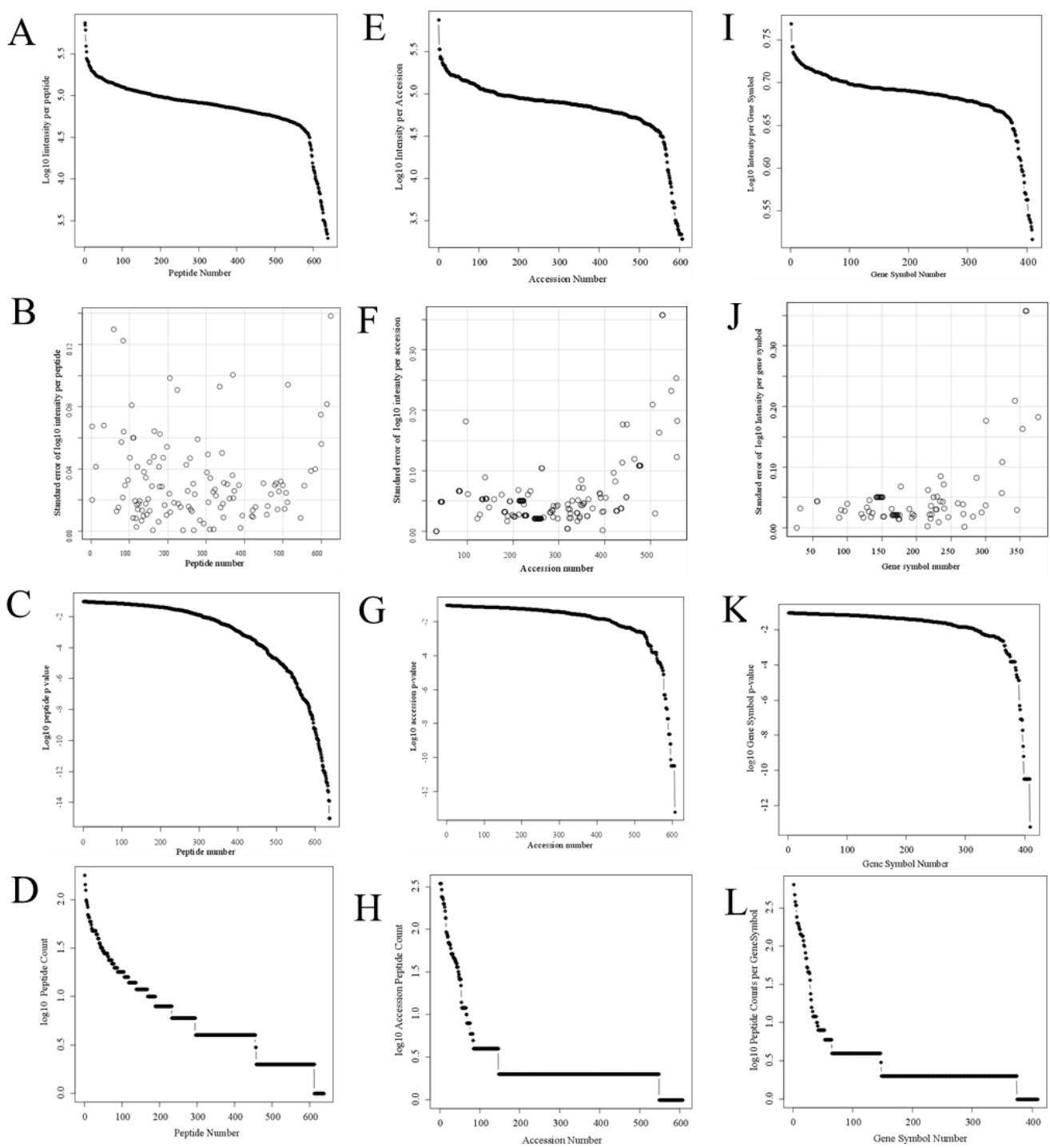

Figure 26. The summary of the myosin standard at the levels of peptides, protein accession and gene symbols. Panels: A, the average intensity of precursors per peptides; $\mathrm{B}$, the standard error of the all the precursor intensity values per peptides; $\mathrm{C}$, the cumulative $\mathrm{p}$-value for MS/MS spectra per peptides; D, the log 10 number of peptides correlated to each protein accession number; E, the average intensity of all precursor per protein accession number; $\mathrm{F}$, the standard error of the precursor intensity values per protein accession number; $G$, the cumulative standard p-value from MS/MS spectra per protein accession; $\mathrm{H}$, the $\log 10$ number of peptides correlated to each protein accession number; I, the average intensity of all precursors per gene symbol; J, the standard error of the all the precursor values per gene symbol; $\mathrm{K}$, the cumulative standard $\mathrm{p}$ value of all peptides for each protein accession; L, the $\log 10$ number of peptides correlated to each protein accession number. After normality testing by Quantile (QQ) plots and ANOVA the probability that the $\log 10$ mean intensity values were the same across peptides, proteins or gene symbols was $\sim$ E-16. 


\section{STRING analysis of the myosin gene symbols}

Analysis of the gene symbols from the rabbit myosin samples using the STRING algorithm provided a further independent means to confirm the validity of the many proteins observed in the myosin preparation. The myosin sample also showed some 1032 known interactions between the identified proteins compared to the expected value of 462 that showed a $\mathrm{p}$ value $\sim 0$ ( $\mathrm{p}<\mathrm{E}-16)$ indicating that the myosin was purified as a complex with other proteins by network analysis with STRING (see supplemental). The network analysis in STRING confirmed that the myosin standard is not a pure protein but rather was a supramolecular complex with many other proteins. The sample was very highly enriched in muscle proteins, proteins with contractile or nucleotide binding functions and actin cytoskeleton components as expected (Fig. 27). Thus, in contrast to the central assumption of the Empirical Statistical model, the myosin standard is not pure and the many peptides and proteins observed are true positive. 


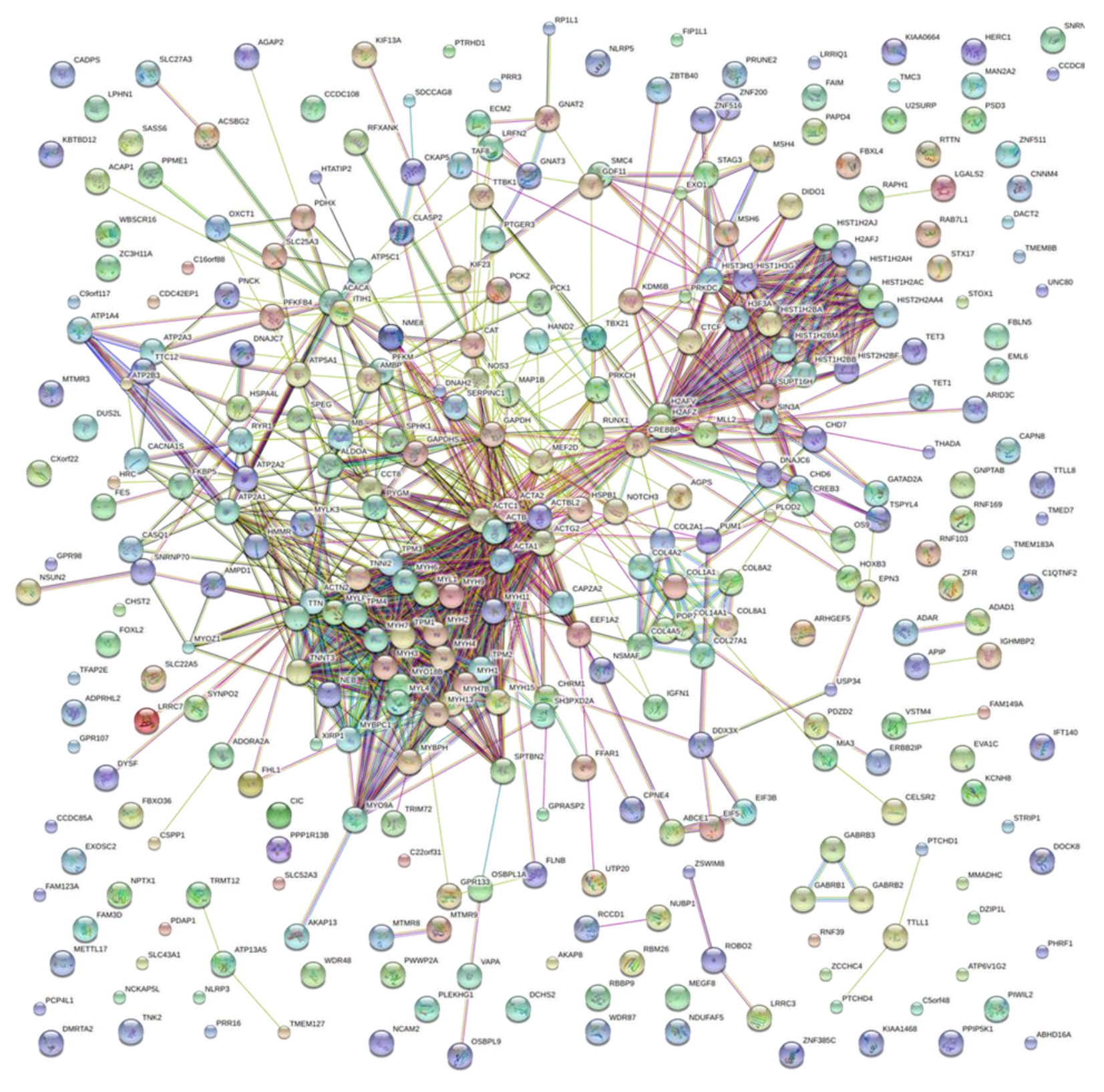

Figure 27. The rabbit myosin protein complex results from the X!TANDEM algorithm analyzed by the STRING algorithm. String data: number of nodes, 326; number of edges, 1032; average node degree, 6.33; clustering coefficient, 0.701; expected number of edges, 462; PPI enrichment p-value, $0(<<0.0001)$. 


\section{Comparison of affinity chromatography surfaces}

Prior to the classical affinity chromatography experiments, various combination of surfaces, coatings, ligands and elution buffers were tested to determine which combination would selectively elute the most ligand compared to controls.

\section{Biotinylated ligands on Streptavidin-Agarose}

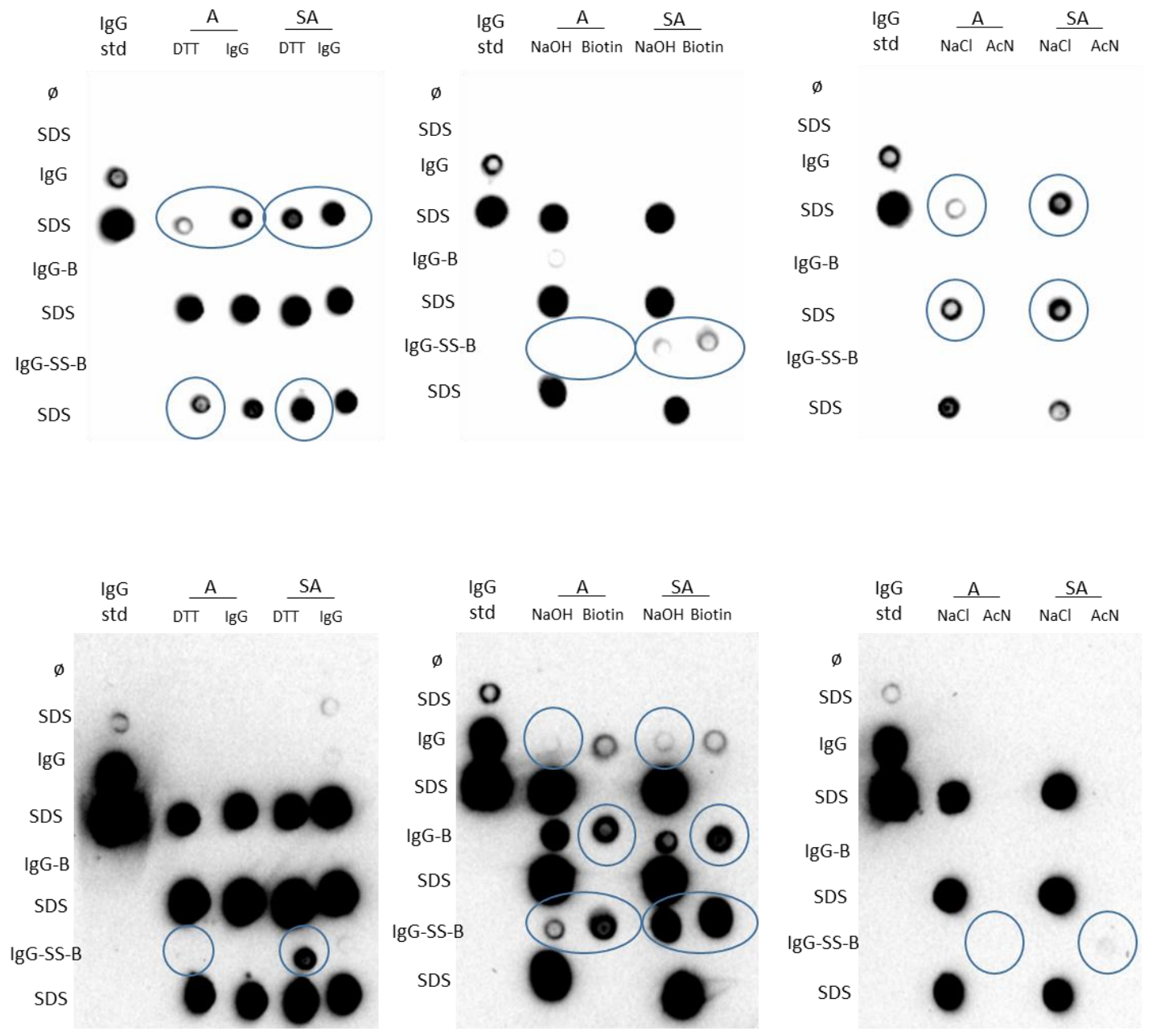

Figure 28. Streptavidin-agarose beads with IgG (Sigma). 50ul of Steptavidin-agarose resin (SA) or agarose alone (A) was incubated with 10 ug of monovalent IgG, IgG-B, IgG-SS-B alongside a PBS control (ø), washed and eluted in DTT, IgG, $\mathrm{NaOH}$ followed by Biotin, and $\mathrm{NaCl}$ followed by AcN. The remaining protein on the chromatography supports was scrubbed in $2 \%$ SDS. 1 ul was spotted onto PVDF and probed with Dk-anti-hIgG (IgG and IgG-B std at 0, $1,10$ and $100 \mathrm{ug} / \mathrm{ml})$. The second row of blots are the same as the first row but with a longer exposure time. (performed by A. Florentinus-Mefailoski) 
Monovalent IgG, IgG-B and IgG-SS-B were applied to tstreptavidin-agarose resin or agarose alone, washed and eluted in DTT, IgG, $\mathrm{NaOH}$ followed by biotin, and $\mathrm{NaCl}$ followed by $\mathrm{AcN}$. After the elutions, the remaining protein on the SA-agarose and agarose control beads were scrubbed in $2 \%$ SDS. The elutions and the $2 \%$ SDS scrubs were all spotted onto a PVDF membrane and probed with Dk-anti-hIgG and SA-HRP.

Streptavidin coated agarose in combination with the IgG-SS-B ligand showed the greatest specificity in the DTT elution buffer compared to all other ligands, coatings and buffers (Fig. 28). IgG and IgG-B applied to SA-agarose did not elute in the DTT elution as indicated by the detection of IgG with Dk anti hIgG-HRP by ECl. No ligands applied to the agarose control and eluted in DTT were detected (Fig. 28). The DTT elution of the IgG-SS-B ligand applied to SAagarose beads showed no signal when probed with the SA-HRP indicating that the agarosestreptavidin-biotin-s remained intact while the IgG-S (Fig. 29) was eluted as expected (Fig. 28).

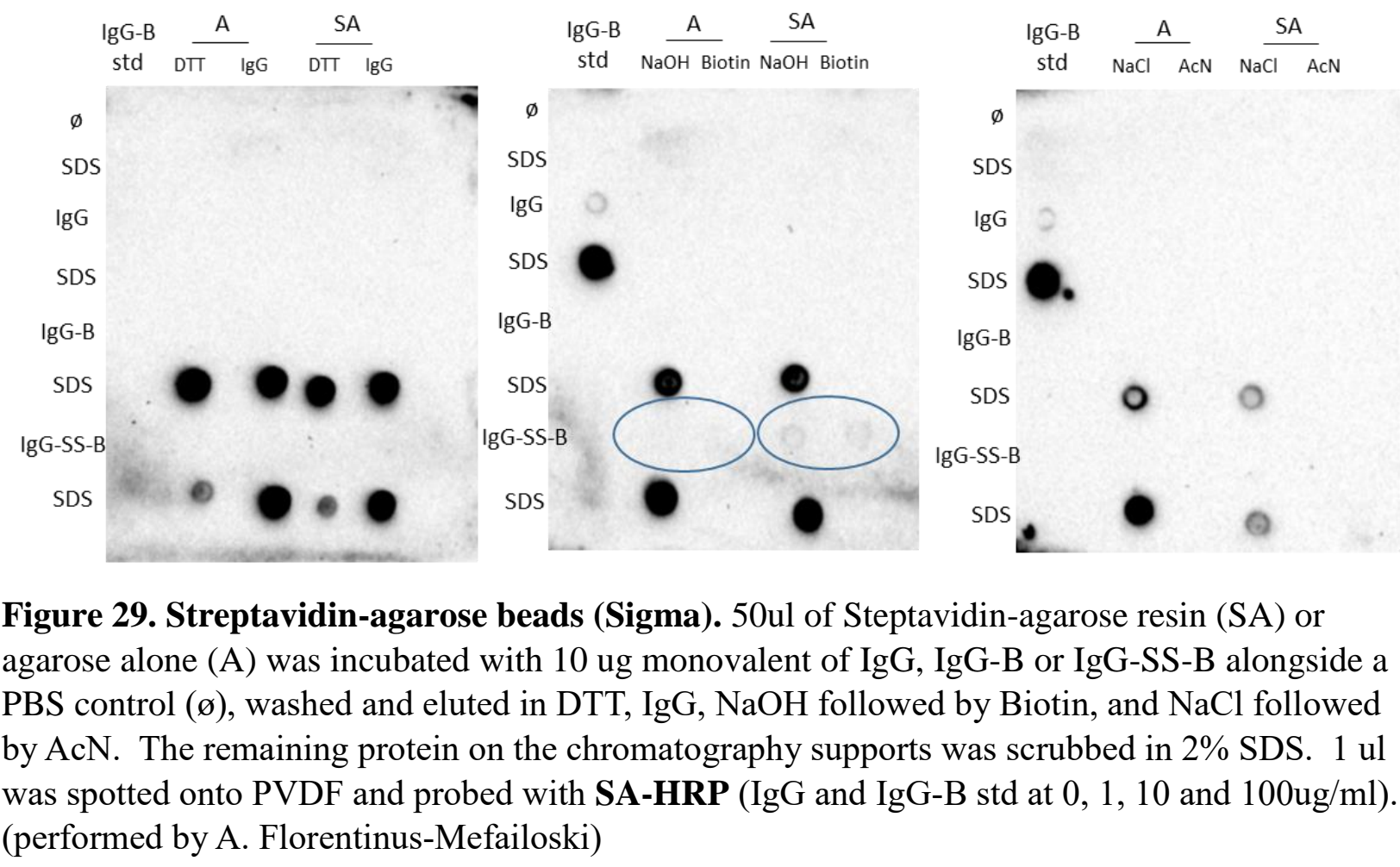


$\mathrm{NaOH}$ and $\mathrm{NaOH}+$ biotin non-specifically eluted all ligands detected by Dk anti-hIgG-HRP from both the agarose and SA-agarose beads (Fig 28). Only trace amounts of IgG-SS-B eluted in the acetonitrile elution from SA-agarose (Fig. 28). No IgG or IgG-B was detected in $\mathrm{NaCl}$ or acetonitrile buffers from either the SA-agarose or the agarose control beads (Fig 28). The $\mathrm{NaOH}$ and biotin buffers did elute biotin from the SA-agarose column as shown by detection with SAHRP, indicating that biotin was removed from the agarose bead and not specifically eluted by cleaving the S-S linker (Fig. 29).

\section{The capture of biotinylated ligands from high binding plastic coated in SA or protein G}

Monovalent IgG, IgG-B and IgG-SS-B were applied to high binding plastic 96 well dish coated in either protein G, streptavidin or without coating. The wells were washed and eluted in DTT, $\mathrm{IgG}, \mathrm{NaOH}$ followed by biotin, and $\mathrm{NaCl}$ followed by $\mathrm{AcN}$. After the elutions, the remaining protein in the high binding plastic 96 well dish was scrubbed in $2 \%$ SDS. The elutions and the 2\% SDS scrubs were all spotted onto a PVDF membrane and probed with Dk-anti-hIgG and SAHRP.

High binding plastic coated in streptavidin or protein $\mathrm{G}$ showed no detection of the biotin by SAHRP (Fig. 30 \& Fig. 31). No biotin was detected by SA-HRP in the NH4OH+biotin elution for any of the ligands indicating that biotinylated ligands bound to streptavidin are not likely released by free biotin (Fig. 30). IgG, IgG-B and IgG-SS-B were eluted non-specifically in $\mathrm{NaOH}$ and $\mathrm{NaOH}+$ biotin from $\mathrm{SA}$ and Protein $\mathrm{G}$ coated high binding plastic as well as uncoated high binding plastic Dk anti-hIgG (Fig. 31). Salt eluted a small amount of only the IgG ligand and not IgG-B or IgG-SS-B from the uncoated and SA coated high binding plastic by Dk- 
anti-hIgG (Fig. 31). DTT was not shown to specifically elute IgG-SS-B from high binding plastic coated in streptavidin as it was shown using SA-agarose (Fig. 31 \& Fig. 28).

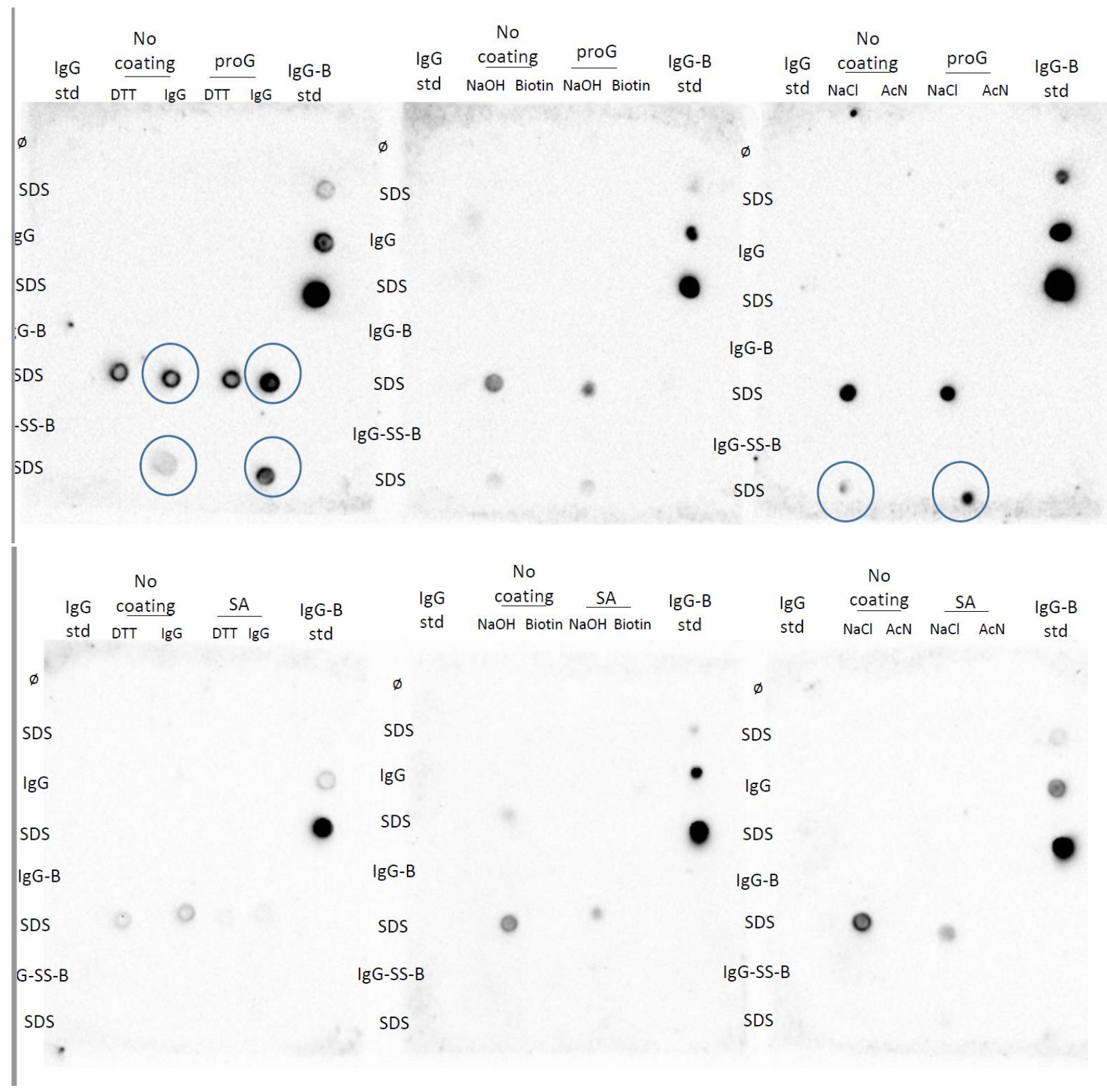

Figure 30. NUNC Maxisorp high binding plastic. A high binding 96 well microtiter plate was coated with $10 \mathrm{ug}$ proG or Streptavidin vs no coating control. Wells were incubated with $10 \mathrm{ug}$ per well of monovalent IgG, IgG-B, IgG-SS-B alongside a PBS control (ø), washed and eluted in DTT, IgG, NaOH, Biotin, $\mathrm{NaCl}$, AcN or SDS. 1 ul was spotted onto PVDF and probed with SAHRP (IgG and IgG-B std at 0, 1, 10 and 100ug/ml) (performed by A. Florentinus-Mefailoski) 


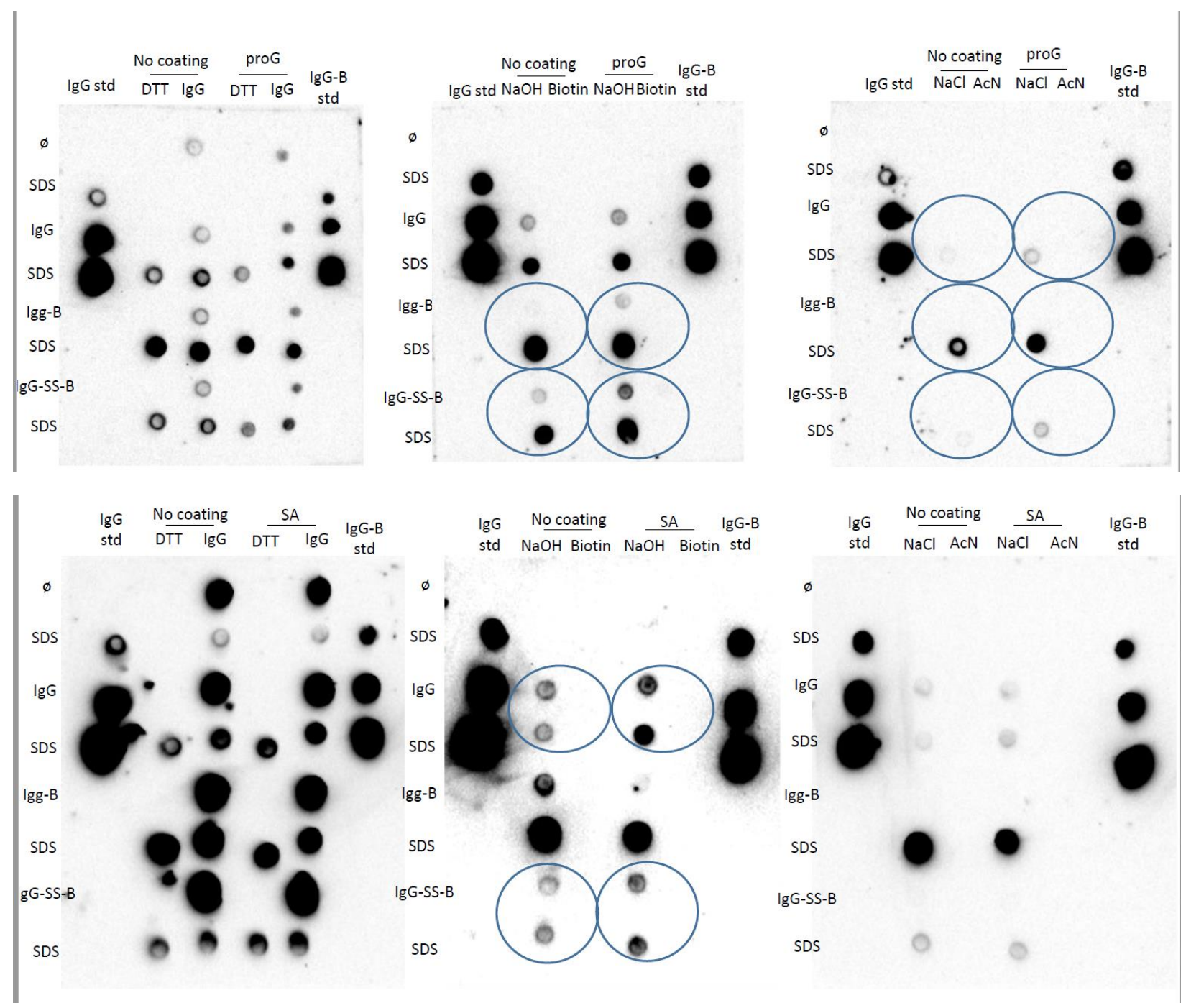

Figure 31. NUNC Maxisorp high binding plastic. A high binding 96 well microtiter plate was coated with $10 \mathrm{ug}$ proG or streptavidin vs a no coating control. Wells were incubated with $10 \mathrm{ug}$ per well of monovalent IgG, IgG-B, IgG-SS-B alongside a PBS control (ø), washed and eluted in DTT, IgG, $\mathrm{NaOH}$, Biotin, $\mathrm{NaCl}$, AcN or SDS. 1 ul was spotted onto PVDF and probed with Dkanti-hIgG (IgG and IgG-B std at 0, 1, 10 and 100ug/ml) (performed by A. FlorentinusMefailoski) 
The capture of biotinylated ligands by PVDF with SA or protein G coating

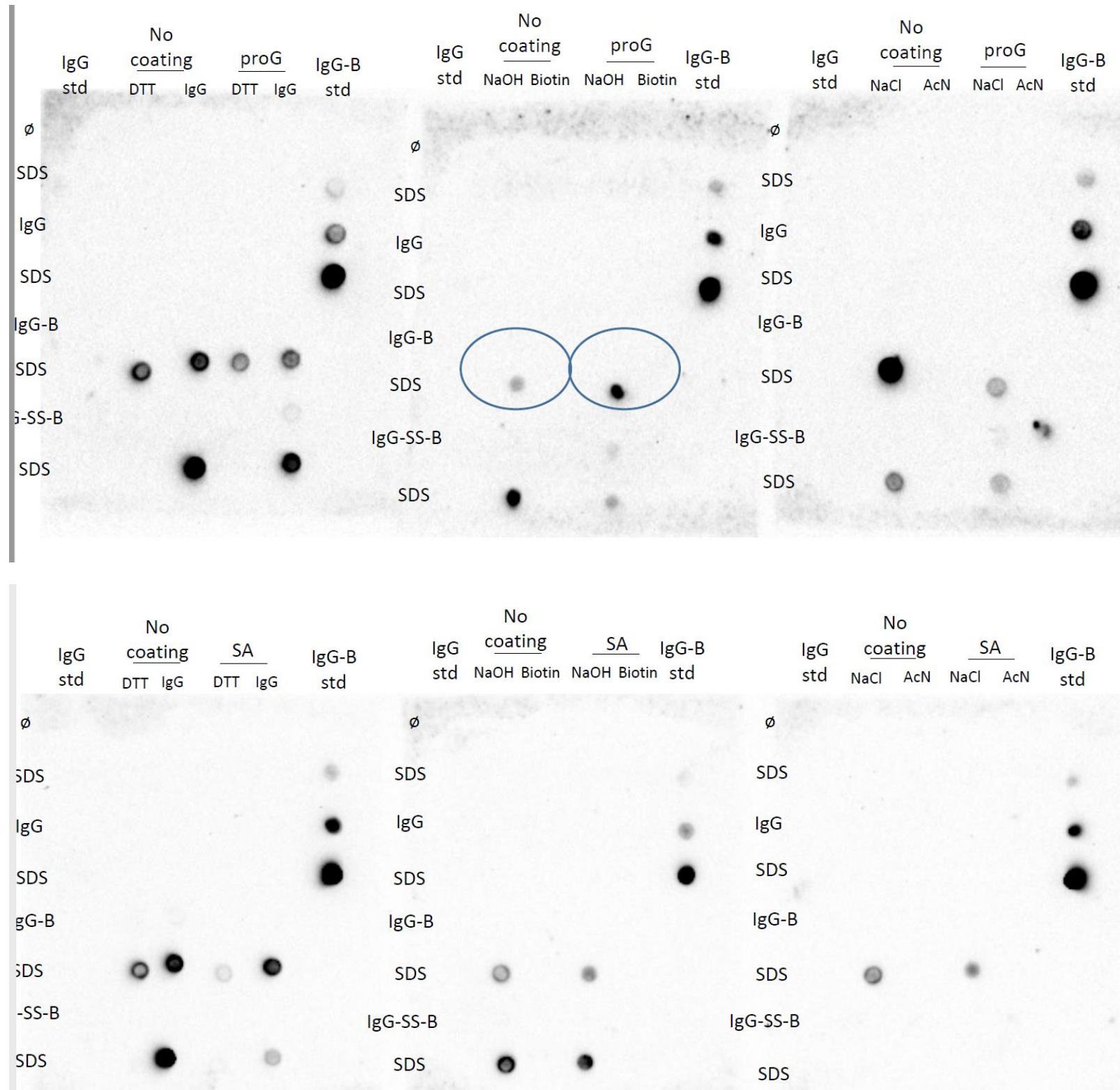

Figure 32: PVDF in 96 well plate. PVDF was coated with 10 ug protein G (ProG) or streptavidin (SA) vs no coating control. Wells were incubated with 10 ug of monovalent IgG, IgG-B or IgG-SS-B alongside a PBS control (ø). The PVDF membranes were washed and eluted in DTT, $\mathrm{IgG}, \mathrm{NaOH}$, Biotin, $\mathrm{NaCl}, \mathrm{AcN}$. The remaining protein was scrubbed in 2\% SDS. 1 ul was spotted onto a new PVDF membrane and probed with SA-HRP. (IgG and IgG-B std at 0, 1, 10 and $100 \mathrm{ug} / \mathrm{ml}$ ) (performed by A. Florentinus-Mefailoski) 


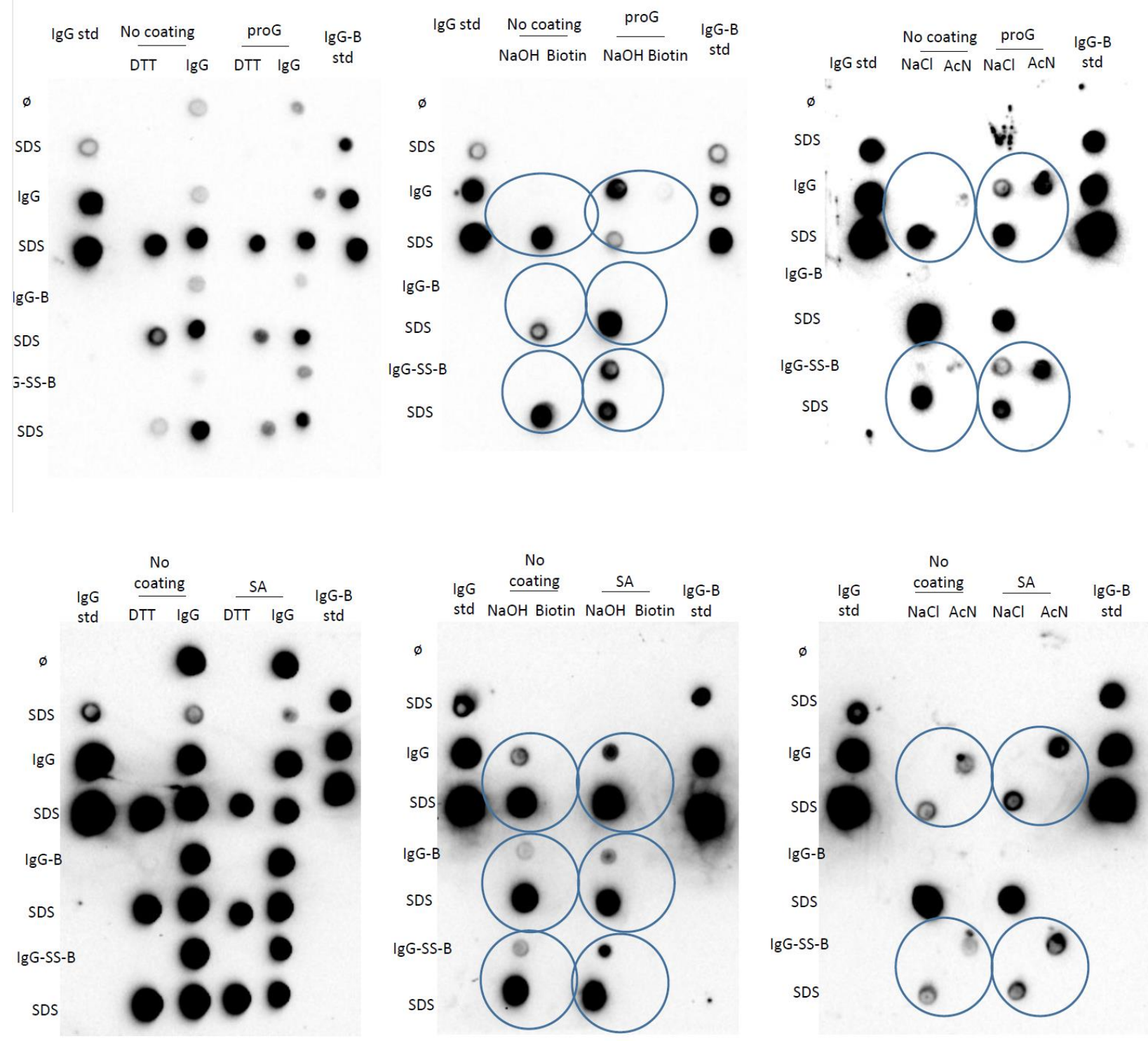

Figure 33: PVDF in 96 well plate. PVDF was coated with 10 ug protein $G$ (ProG) or streptavidin (SA) vs no coating control. Wells were incubated with $10 \mathrm{ug}$ of monovalent $\mathrm{IgG}$, IgG-B or IgG-SS-B alongside a PBS control (ø). The PVDF membranes were washed and eluted in DTT, IgG, $\mathrm{NaOH}$, Biotin, $\mathrm{NaCl}, \mathrm{AcN}$ or SDS. The remaining protein was scrubbed in $2 \%$ SDS. 1 ul was spotted onto a new PVDF membrane and probed with Dk-anti-hIgG. (IgG and IgG-B std at 0, 1, 10 and 100ug/ml) (performed by A. Florentinus-Mefailoski)

Monovalent IgG, IgG-B and IgG-SS-B were applied to a PVDF membrane coated in either protein $\mathrm{G}$, streptavidin or no coating, washed and eluted in DTT, IgG, $\mathrm{NaOH}$ followed by biotin, and $\mathrm{NaCl}$ followed by $\mathrm{AcN}$. After the elutions, the remaining protein on the PVDF membrane 
was scrubbed in $2 \%$ SDS. The elutions and the $2 \%$ SDS scrubs were all spotted onto PVDF and probed with Dk-anti-hIgG and SA-HRP.

No specific elution of the IgG-SS-B ligands was observed in DTT on either the protein G or streptavidin coated PVDF membranes by Dk-anti-hIgG (Fig. 33). Acetonitrile eluted IgG and IgG-SS-B from SA-PVDF but also PVDF alone (Fig. 33). $\mathrm{NaCl}$ and $\mathrm{AcN}$ were both shown to elute IgG and IgG-SS-B ligands from protein G coated pvdf membranes and not the PVDF control by Dk-anti-hIgG (Fig. 33). NaOH non-specifically eluted all of the IgG ligands from both SA coated and uncoated PVDF(Fig. 33). Salt and AcN were also shown to elute IgG and IgG-SS-B ligands from Protein G-PVDF and not PVDF alone (Fig. 33). 
The capture of biotinylated on Glass with SA or protein G coating
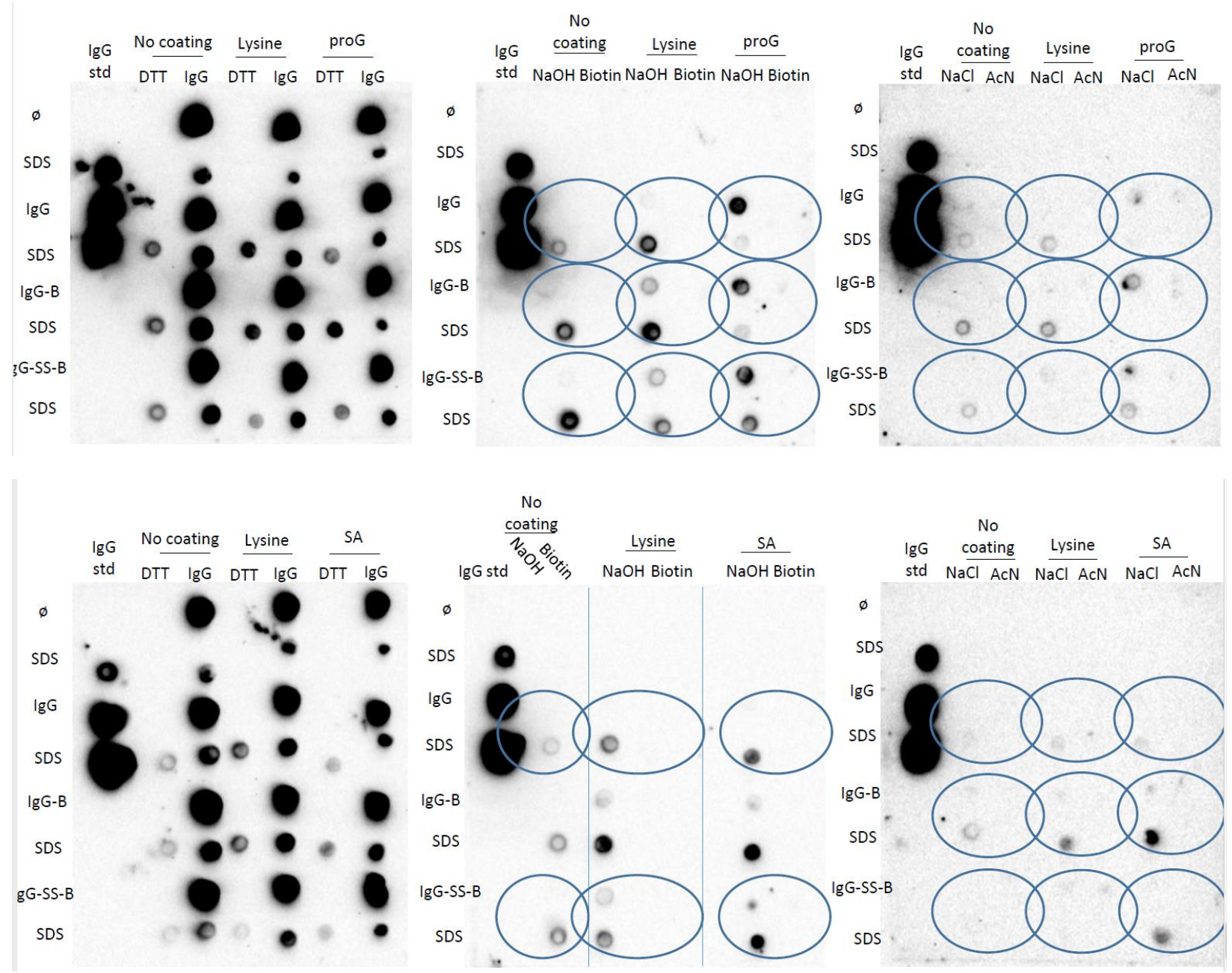

Figure 34. Glass cover slips in 96 well plate. Glass was coated with 10 ug protein $\mathrm{G}$ (proG) or streptavidin (SA) vs no coating and poly-Lysine alone controls. Wells were incubated with 10 ug of monovalent IgG, IgG-B, IgG-SS-B alongside a PBS control (ø), washed and eluted in DTT, IgG, $\mathrm{NaOH}$, Biotin, $\mathrm{NaCl}, \mathrm{AcN}$ or SDS. The remaining protein was scrubbed in $2 \%$ SDS. 1 ul was spotted onto PVDF and probed with Dk-anti-hIgG. (IgG and IgG-B std at 0, 1, 10 and $100 \mathrm{ug} / \mathrm{ml}$ ) (performed by A. Florentinus-Mefailoski) 

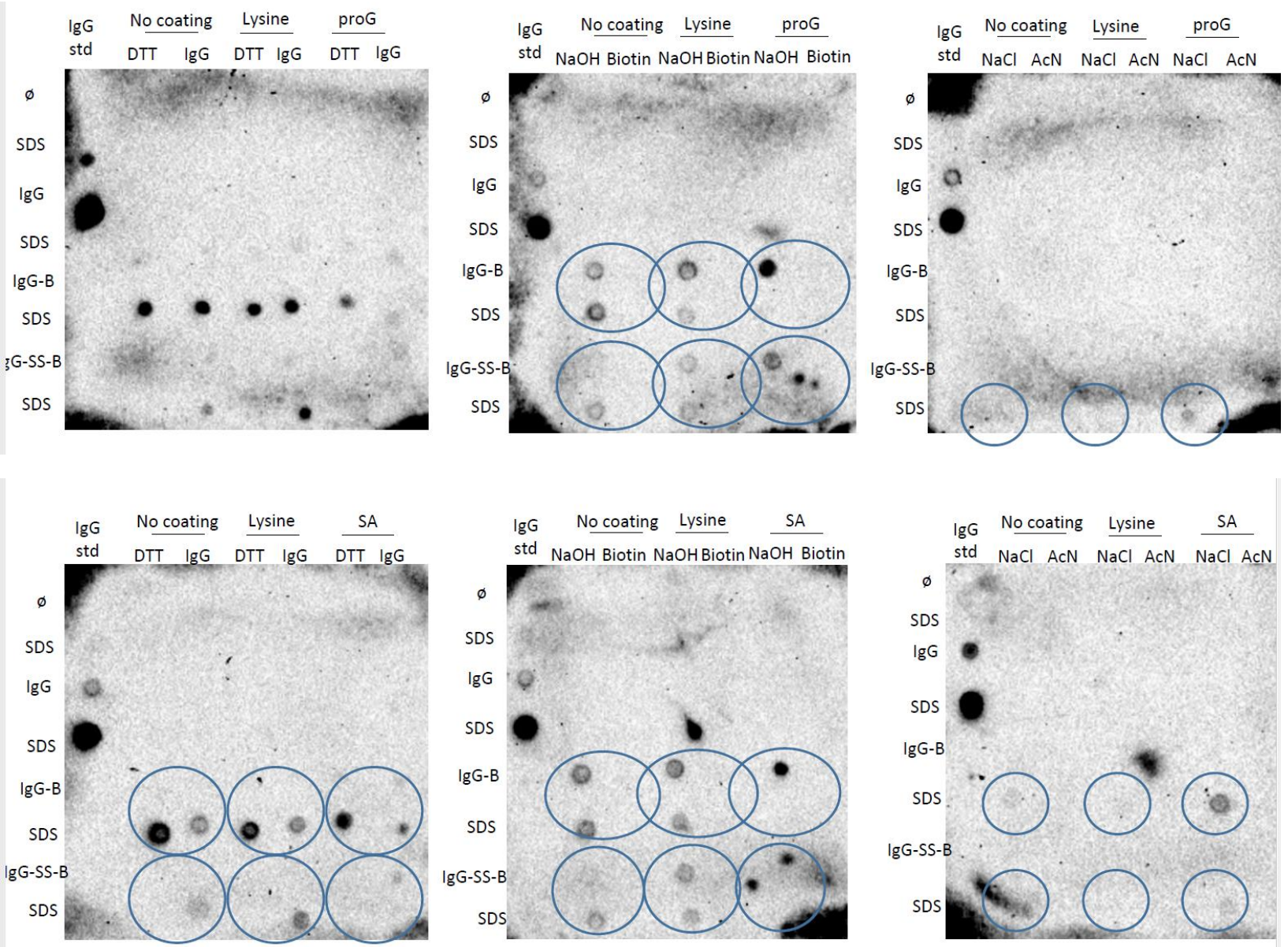

Figure 35. Glass cover slips in 96 well plate. Glass was coated with 10 ug protein $\mathrm{G}$ (proG) or streptavidin (SA) vs no coating and poly-Lysine alone controls. Wells were incubated with $10 \mathrm{ug}$ of monovalent IgG, IgG-B, IgG-SS-B alongside a PBS control (ø), washed and eluted in DTT, $\mathrm{IgG}, \mathrm{NaOH}$ followed by biotin, and $\mathrm{NaCl}$ followed by $\mathrm{AcN}$. The remaining protein was scrubbed in 2\% SDS. 1 ul was spotted onto PVDF and probed with SA-HRP. (IgG and IgG-B std at 0, 1, 10 and $100 \mathrm{ug} / \mathrm{ml}$ ) (performed by A. Florentinus-Mefailoski)

Monovalent ligands were applied to glass coverslips coated in either protein G, streptavidin, poly-Lysine or no coating, washed and eluted in DTT, IgG, $\mathrm{NaOH}$ followed by biotin, and $\mathrm{NaCl}$ followed by AcN. After the elutions, the remaining protein on the PVDF membrane was scrubbed in 2\% SDS. The elutions and the 2\% SDS scrubs were all spotted onto PVDF and probed with Dk-anti-hIgG and SA-HRP. 
No clear enrichment was detectable in the glass beads in treatments compared to controls with either the Dk-a-hIgG-HRP or the SA-HRP probes (Fig. 34 \& 35). However, glass showed the least amount of non-specific binding when scrubbed with $2 \%$ SDS. The system with the best hope for specific elution for a classical affinity chromatography experiment based on these results was apparently the IgG-SS-B ligand in combination with a streptavidin-agarose affinity column and DTT as an elution buffer. The SA-agarose IgG-SS-B approach was replicated several times and the beads were subsequently digested to estimate non-specific binding. In general, there was no good way to elute the IgG-B, IgG-SS-B or IgG ligand from affinity surfaces that was clearly better than controls.

\section{TESTING GLASS SUPPORTS AND AFFINITY SYSTEMS WITH CELLS}

While glass coverslips showed considerably lower signals for the capture and elution of $\operatorname{IgG}$ compared to high binding plastic, PVDF and SA-agarose by Dk-anti-hIgG with ECl, silica may be an advantageous chromatography support for capturing cell surface receptor complexes as silica is more hydrophilic compared to melamine and PMMA and may result in less non-specific binding. Thus, we tested silica supports in microbead format to increase the surface area for binding compared to $2 \mathrm{D}$ coverslips. Cells were used in this experiment instead of ligands alone to better assess non-specific binding and to determine if there was an enrichment of $\mathrm{IgG}, \mathrm{Fc}$ receptors or proteins part the IgG-Fc receptor mediated phagocytosis KEGG pathway compared to controls. The glass microbead supports were activated in acid and then coated in polylysine, paraformaldehyde and then protein $\mathrm{G}$ or streptavidin. Monomeric $\mathrm{IgG}$ and biotinylated $\mathrm{IgG}$ 
ligands were applied to live cells and crude homogenates. In the case of live cells, unbound ligands were washed prior to application to the silica chromatography beads. Ligands applied to the crude homogenates were applied shortly after French pressing. The live cell and crude homogenate preparations were then applied to the glass microbead chromatography supports, washed and eluted in either 1) $5 \mathrm{mM}$ DTT in $1 \mathrm{x}$ PBS, 2) $5 \mathrm{uM} \mathrm{IgG}$ in $1 \mathrm{XPBS}$, 3) $2 \mathrm{MNH}_{4} \mathrm{OH}$ in $\mathrm{H}_{2} \mathrm{O}$ followed by $\mathrm{NH}_{4} \mathrm{OH}+50 \mathrm{mM}$ Biotin in $\mathrm{H}_{2} \mathrm{O}$, and 5) $300 \mathrm{mM} \mathrm{NaCl}$ followed by $650 \mathrm{mM}$ $\mathrm{NaCl}+30 \% \mathrm{AcN}$.

Table 2. Peptide counts of hIgG from biotinylated monovalent and polyvalent ligands applied to crude or live cells and captured by silica chromatography supports coated with streptavidin, protein G, IgG, polylysine or without coating.

$\begin{array}{llllccc}\text { Ligand } & \text { Applied to } & \text { Chromatography } & \text { Elution Buffer } & \text { Filter } & \text { Filter } & \text { Filter } \\ \text { No ligand } & \text { Support Coating } & & \mathbf{0} & \mathbf{1} & \mathbf{2} \\ \text { No ligand } & \text { HEPES } & \text { No Coating } & \text { Acetonitrile } & 0 & 0 & 0 \\ \text { No ligand } & \text { HEPES } & \text { Poly D lysine } & \text { Acetonitrile } & 0 & 0 & 0 \\ \text { No ligand } & \text { HEPES } & \text { Protein G } & \text { Acetonitrile } & 0 & 0 & 0 \\ \text { No ligand } & \text { HEPES } & \text { Streptavidin } & \text { Acetonitrile } & 0 & 0 & 0 \\ \text { No ligand } & \text { HEPES } & \text { IgG } & \text { Acetonitrile } & 0 & 0 & 0 \\ \text { No ligand } & \text { CRUDE } & \text { No Coating } & \text { Acetonitrile } & 2 & 2 & 2 \\ \text { No ligand } & \text { CRUDE } & \text { Poly L Lysine } & \text { Acetonitrile } & 1 & 1 & 1 \\ \text { No ligand } & \text { CRUDE } & \text { IgG } & \text { Acetonitrile } & 1 & 1 & 1 \\ \text { IgG } & \text { CRUDE } & \text { Streptavidin } & \text { Acetonitrile } & 2 & 0 & 0 \\ \text { IgG-B } & \text { CRUDE } & \text { Streptavidin } & \text { Acetonitrile } & 0 & 0 & 0 \\ \text { IgG-SS-B } & \text { CRUDE } & \text { Streptavidin } & \text { Acetonitrile } & 0 & 0 & 0 \\ \text { Aggregated hIgG } & \text { CRUDE } & \text { Streptavidin } & \text { Acetonitrile } & 3 & 0 & 0 \\ \text { Aggregated IgG-B } & \text { CRUDE } & \text { Protein G } & \text { Acetonitrile } & 2 & 0 & 0 \\ \text { Aggregated IgG-B } & \text { CRUDE } & \text { Protein G } & \text { Acetonitrile } & 4 & 3 & 3 \\ \text { Aggregated IgG-SS-B } & \text { CRUDE } & \text { Protein G } & \text { Acetonitrile } & 1 & 0 & 0 \\ \text { IgG } & \text { CRUDE } & \text { Protein G } & \text { Acetonitrile } & 0 & 0 & 0 \\ \text { IgG-B } & \text { LIVE } & \text { Streptavidin } & \text { Acetonitrile } & 0 & 0 & 0 \\ \text { IgG-SS-B } & \text { LIVE } & \text { Streptavidin } & \text { Acetonitrile } & 2 & 0 & 0 \\ \text { Aggregated IgG } & \text { LIVE } & \text { Streptavidin } & \text { Acetonitrile } & 0 & 0 & 0 \\ \text { Aggregated IgG-B } & \text { LIVE } & \text { Protein G } & \text { Acetonitrile } & 0 & 0 & 0 \\ \text { Aggregated IgG-SS-B } & \text { LIVE } & \text { Protein G } & \text { Acetonitrile } & 0 & 0 & 0 \\ \text { No ligand } & \text { HEPES } & \text { No Coating } & \text { Biotin } & 0 & 0 & 0 \\ & & & & 0 & 0\end{array}$




\begin{tabular}{|c|c|c|c|c|c|c|}
\hline No ligand & HEPES & Poly D lysine & Biotin & 0 & 0 & 0 \\
\hline No ligand & HEPES & Protein G & Biotin & 0 & 0 & 0 \\
\hline No ligand & HEPES & Streptavidin & Biotin & 0 & 0 & 0 \\
\hline No ligand & HEPES & $\lg G$ & Biotin & 0 & 0 & 0 \\
\hline No ligand & CRUDE & No Coating & Biotin & 0 & 0 & 0 \\
\hline No ligand & CRUDE & Poly L Lysine & Biotin & 0 & 0 & 0 \\
\hline No ligand & CRUDE & $\operatorname{lgG}$ & Biotin & 0 & 0 & 0 \\
\hline No ligand & CRUDE & Streptavidin & Biotin & 0 & 0 & 0 \\
\hline $\operatorname{lgG}$ & CRUDE & Streptavidin & Biotin & 0 & 0 & 0 \\
\hline IgG-B & CRUDE & Streptavidin & Biotin & 0 & 0 & 0 \\
\hline IgG-SS-B & CRUDE & Streptavidin & Biotin & 0 & 0 & 0 \\
\hline Aggregated hlgG & CRUDE & Protein G & Biotin & 0 & 0 & 0 \\
\hline Aggregated IgG-B & CRUDE & Protein G & Biotin & 0 & 0 & 0 \\
\hline Aggregated IgG-B & CRUDE & Protein G & Biotin & 0 & 0 & 0 \\
\hline Aggregated IgG-SS-B & CRUDE & Protein G & Biotin & 0 & 0 & 0 \\
\hline $\lg G$ & LIVE & Streptavidin & Biotin & 0 & 0 & 0 \\
\hline IgG-B & LIVE & Streptavidin & Biotin & 0 & 0 & 0 \\
\hline IgG-SS-B & LIVE & Streptavidin & Biotin & 0 & 0 & 0 \\
\hline Aggregated IgG & LIVE & Protein G & Biotin & 0 & 0 & 0 \\
\hline Aggregated IgG-B & LIVE & Protein G & Biotin & 0 & 0 & 0 \\
\hline Aggregated IgG-SS-B & LIVE & Protein G & Biotin & 0 & 0 & 0 \\
\hline No ligand & HEPES & No Coating & DTT & 1 & 0 & 0 \\
\hline No ligand & HEPES & Poly D lysine & DTT & 1 & 0 & 0 \\
\hline No ligand & HEPES & Protein G & DTT & 1 & 0 & 0 \\
\hline No ligand & HEPES & Streptavidin & DTT & 1 & 0 & 0 \\
\hline No ligand & HEPES & $\lg G$ & DTT & 1 & 0 & 0 \\
\hline No ligand & CRUDE & No Coating & DTT & 1 & 0 & 0 \\
\hline No ligand & CRUDE & Poly L Lysine & DTT & 14 & 14 & 14 \\
\hline No ligand & CRUDE & $\lg G$ & DTT & 0 & 0 & 0 \\
\hline No ligand & CRUDE & Streptavidin & DTT & 0 & 0 & 0 \\
\hline $\operatorname{lgG}$ & CRUDE & Streptavidin & DTT & 0 & 0 & 0 \\
\hline IgG-B & CRUDE & Streptavidin & DTT & 0 & 0 & 0 \\
\hline IgG-SS-B & CRUDE & Streptavidin & DTT & 0 & 0 & 0 \\
\hline Aggregated hlgG & CRUDE & Protein G & DTT & 1 & 0 & 0 \\
\hline Aggregated IgG-B & CRUDE & Protein G & DTT & 1 & 0 & 0 \\
\hline Aggregated IgG-B & CRUDE & Protein G & DTT & 0 & 0 & 0 \\
\hline Aggregated IgG-SS-B & CRUDE & Protein G & DTT & 1 & 0 & 0 \\
\hline $\lg G$ & LIVE & Streptavidin & DTT & 1 & 0 & 0 \\
\hline IgG-B & LIVE & Streptavidin & DTT & 1 & 0 & 0 \\
\hline IgG-SS-B & LIVE & Streptavidin & DTT & 1 & 0 & 0 \\
\hline Aggregated IgG & LIVE & Protein G & DTT & 1 & 0 & 0 \\
\hline Aggregated IgG-B & LIVE & Protein G & DTT & 1 & 1 & 1 \\
\hline Aggregated IgG-SS-B & LIVE & Protein G & DTT & 2 & 0 & 0 \\
\hline No ligand & HEPES & No Coating & $\lg G$ & 460 & 444 & 344 \\
\hline No ligand & HEPES & Poly D lysine & $\lg G$ & 362 & 344 & 253 \\
\hline
\end{tabular}




\begin{tabular}{|c|c|c|c|c|c|c|}
\hline No ligand & HEPES & Protein G & $\lg G$ & 1218 & 1188 & 822 \\
\hline No ligand & HEPES & Streptavidin & $\lg G$ & 783 & 756 & 487 \\
\hline No ligand & HEPES & $\lg G$ & $\lg G$ & 89 & 83 & 63 \\
\hline No ligand & CRUDE & No Coating & $\lg G$ & 517 & 502 & 371 \\
\hline No ligand & CRUDE & Poly L Lysine & $\lg G$ & 581 & 568 & 418 \\
\hline No ligand & CRUDE & IgG & $\lg G$ & 900 & 873 & 603 \\
\hline No ligand & CRUDE & Streptavidin & $\lg G$ & 949 & 928 & 671 \\
\hline $\lg G$ & CRUDE & Streptavidin & $\lg G$ & 819 & 778 & 558 \\
\hline $\lg G-B$ & CRUDE & Streptavidin & $\lg G$ & 177 & 169 & 121 \\
\hline IgG-SS-B & CRUDE & Streptavidin & $\lg G$ & 366 & 350 & 261 \\
\hline Aggregated hlgG & CRUDE & Protein G & $\lg G$ & 674 & 649 & 449 \\
\hline Aggregated IgG-B & CRUDE & Protein G & $\lg G$ & 699 & 679 & 498 \\
\hline Aggregated IgG-B & CRUDE & Protein G & $\lg G$ & 269 & 256 & 188 \\
\hline Aggregated IgG-SS-B & CRUDE & Protein G & $\lg G$ & 517 & 498 & 379 \\
\hline $\lg G$ & LIVE & Streptavidin & $\lg G$ & 166 & 144 & 118 \\
\hline IgG-B & LIVE & Streptavidin & $\lg G$ & 857 & 820 & 610 \\
\hline IgG-SS-B & LIVE & Streptavidin & $\lg G$ & 731 & 704 & 513 \\
\hline Aggregated IgG & LIVE & Protein G & $\lg G$ & 750 & 702 & 480 \\
\hline Aggregated IgG-B & LIVE & Protein G & $\lg G$ & 465 & 413 & 322 \\
\hline Aggregated IgG-SS-B & LIVE & Protein G & $\lg G$ & 85 & 75 & 60 \\
\hline No ligand & HEPES & No Coating & $\mathrm{NH} 4 \mathrm{OH}$ & 0 & 0 & 0 \\
\hline No ligand & HEPES & Poly D lysine & $\mathrm{NH} 4 \mathrm{OH}$ & 3 & 2 & 2 \\
\hline No ligand & HEPES & Protein G & $\mathrm{NH} 4 \mathrm{OH}$ & 1 & 0 & 0 \\
\hline No ligand & HEPES & Streptavidin & $\mathrm{NH} 4 \mathrm{OH}$ & 0 & 0 & 0 \\
\hline No ligand & HEPES & $\lg G$ & $\mathrm{NH} 4 \mathrm{OH}$ & 0 & 0 & 0 \\
\hline No ligand & CRUDE & No Coating & $\mathrm{NH} 4 \mathrm{OH}$ & 7 & 3 & 3 \\
\hline No ligand & CRUDE & Poly L Lysine & $\mathrm{NH} 4 \mathrm{OH}$ & 6 & 1 & 1 \\
\hline No ligand & CRUDE & $\lg G$ & $\mathrm{NH} 4 \mathrm{OH}$ & 1 & 0 & 0 \\
\hline No ligand & CRUDE & Streptavidin & $\mathrm{NH} 4 \mathrm{OH}$ & 2 & 0 & 0 \\
\hline $\lg$ & CRUDE & Streptavidin & $\mathrm{NH} 4 \mathrm{OH}$ & 1 & 0 & 0 \\
\hline $\lg G-B$ & CRUDE & Streptavidin & $\mathrm{NH} 4 \mathrm{OH}$ & 0 & 0 & 0 \\
\hline IgG-SS-B & CRUDE & Streptavidin & $\mathrm{NH} 4 \mathrm{OH}$ & 5 & 0 & 0 \\
\hline Aggregated hIgG & CRUDE & Protein G & $\mathrm{NH} 4 \mathrm{OH}$ & 1 & 0 & 0 \\
\hline Aggregated IgG-B & CRUDE & Protein G & $\mathrm{NH} 4 \mathrm{OH}$ & 3 & 2 & 2 \\
\hline Aggregated IgG-B & CRUDE & Protein G & $\mathrm{NH} 4 \mathrm{OH}$ & 8 & 3 & 3 \\
\hline Aggregated IgG-SS-B & CRUDE & Protein G & $\mathrm{NH} 4 \mathrm{OH}$ & 3 & 1 & 1 \\
\hline $\lg G$ & LIVE & Streptavidin & $\mathrm{NH} 4 \mathrm{OH}$ & 5 & 0 & 0 \\
\hline IgG-B & LIVE & Streptavidin & $\mathrm{NH} 4 \mathrm{OH}$ & 1 & 0 & 0 \\
\hline IgG-SS-B & LIVE & Streptavidin & $\mathrm{NH} 4 \mathrm{OH}$ & 2 & 1 & 1 \\
\hline Aggregated IgG & LIVE & Protein G & $\mathrm{NH} 4 \mathrm{OH}$ & 6 & 0 & 0 \\
\hline Aggregated IgG-B & LIVE & Protein G & $\mathrm{NH} 4 \mathrm{OH}$ & 6 & 2 & 2 \\
\hline Aggregated IgG-SS-B & LIVE & Protein G & $\mathrm{NH} 4 \mathrm{OH}$ & 5 & 0 & 0 \\
\hline No ligand & HEPES & No Coating & Salt & 4 & 0 & 0 \\
\hline No ligand & HEPES & Poly D lysine & Salt & 0 & 0 & 0 \\
\hline No ligand & HEPES & Protein G & Salt & 0 & 0 & 0 \\
\hline
\end{tabular}




\begin{tabular}{|c|c|c|c|c|c|}
\hline No ligand & HEPES & Streptavidin & Salt & 0 & 0 \\
\hline No ligand & HEPES & $\lg G$ & Salt & 0 & 0 \\
\hline No ligand & CRUDE & No Coating & Salt & 1 & 0 \\
\hline No ligand & CRUDE & Poly L Lysine & Salt & 0 & 0 \\
\hline No ligand & CRUDE & $\lg G$ & Salt & 3 & 0 \\
\hline No ligand & CRUDE & Streptavidin & Salt & 0 & 0 \\
\hline $\lg G$ & CRUDE & Streptavidin & Salt & 0 & 0 \\
\hline $\operatorname{lgG}-B$ & CRUDE & Streptavidin & Salt & 0 & 0 \\
\hline IgG-SS-B & CRUDE & Streptavidin & Salt & 0 & 0 \\
\hline Aggregated hlgG & CRUDE & Protein G & Salt & 0 & 0 \\
\hline Aggregated IgG-B & CRUDE & Protein G & Salt & 0 & 0 \\
\hline Aggregated IgG-B & CRUDE & Protein G & Salt & 1 & 0 \\
\hline Aggregated IgG-SS-B & CRUDE & Protein G & Salt & 0 & 0 \\
\hline $\lg G$ & LIVE & Streptavidin & Salt & 0 & 0 \\
\hline IgG-B & LIVE & Streptavidin & Salt & 0 & 0 \\
\hline IgG-SS-B & LIVE & Streptavidin & Salt & 0 & 0 \\
\hline Aggregated IgG & LIVE & Protein G & Salt & 0 & 0 \\
\hline Aggregated IgG-B & LIVE & Protein G & Salt & 5 & 0 \\
\hline Aggregated IgG-SS-B & LIVE & Protein G & Salt & 0 & 0 \\
\hline
\end{tabular}

Table 3. Peptide counts of Fc gamma receptors (filter 0) and IgG-Fc KEGG pathway proteins (filter 2) from biotinylated monovalent and polyvalent ligands applied to crude or live cells and captured by silica chromatography supports coated with streptavidin, protein G, IgG, polylysine or without coating.

$\begin{array}{llllcc}\text { Ligand } & \begin{array}{l}\text { Applied } \\ \text { To }\end{array} & \begin{array}{l}\text { Chromatography } \\ \text { Support Coating }\end{array} & \begin{array}{l}\text { Elution } \\ \text { Buffer }\end{array} & \begin{array}{l}\text { Sum of FCGRs } \\ \text { (filter 0) }\end{array} & \begin{array}{l}\text { Sum of IgG-Fc } \\ \text { KEGG Pathway } \\ \text { Proteins (filter 2) }\end{array} \\ \text { Aggregated IgG } & \text { CRUDE } & \text { Protein G } & \text { Acetonitrile } & 0 & 20 \\ \text { Aggregated IgG } & \text { CRUDE } & \text { Protein G } & \text { Biotin } & 0 & 0 \\ \text { Aggregated IgG } & \text { CRUDE } & \text { Protein G } & \text { DTT } & 0 & 11 \\ \text { Aggregated IgG } & \text { CRUDE } & \text { Protein G } & \text { IgG } & 0 & 2 \\ \text { Aggregated IgG } & \text { CRUDE } & \text { Protein G } & \text { NH4OH } & 2 & 26 \\ \text { Aggregated IgG } & \text { CRUDE } & \text { Protein G } & \text { Salt } & 0 & 21 \\ \text { Aggregated IgG_B } & \text { CRUDE } & \text { Protein G } & \text { Acetonitrile } & 0 & 9 \\ \text { Aggregated IgG_B } & \text { CRUDE } & \text { Protein G } & \text { Biotin } & 0 & 0 \\ \text { Aggregated IgG_B } & \text { CRUDE } & \text { Protein G } & \text { DTT } & 0 & 28 \\ \text { Aggregated IgG_B } & \text { CRUDE } & \text { Protein G } & \text { IgG } & 0 & 0 \\ \text { Aggregated IgG_B } & \text { CRUDE } & \text { Protein G } & \text { NH4OH } & 3 & 22 \\ \text { Aggregated IgG_B } & \text { CRUDE } & \text { Protein G } & \text { Salt } & 0 & 10 \\ \text { No ligand } & \text { CRUDE } & \text { Protein G } & \text { Acetonitrile } & 0 & 22 \\ \text { No ligand } & \text { CRUDE } & \text { Protein G } & \text { Biotin } & 0 & 0\end{array}$




\begin{tabular}{|c|c|c|c|c|c|}
\hline No ligand & CRUDE & Protein G & DTT & 0 & 13 \\
\hline No ligand & CRUDE & Protein G & $\lg G$ & 0 & 5 \\
\hline No ligand & CRUDE & Protein G & $\mathrm{NH} 4 \mathrm{OH}$ & 9 & 125 \\
\hline No ligand & CRUDE & Protein G & Salt & 0 & 16 \\
\hline Aggregated IgG-SS-B & CRUDE & Protein G & Acetonitrile & 1 & 10 \\
\hline Aggregated IgG-SS-B & CRUDE & Protein G & Biotin & 0 & 0 \\
\hline Aggregated IgG-SS-B & CRUDE & Protein G & DTT & 0 & 31 \\
\hline Aggregated IgG-SS-B & CRUDE & Protein G & $\lg G$ & 0 & 6 \\
\hline Aggregated IgG-SS-B & CRUDE & Protein G & $\mathrm{NH} 4 \mathrm{OH}$ & 0 & 52 \\
\hline Aggregated IgG-SS-B & CRUDE & Protein G & Salt & 0 & 0 \\
\hline No ligand & CRUDE & $\lg G$ & Acetonitrile & 4 & 12 \\
\hline No ligand & CRUDE & $\lg G$ & Biotin & 0 & 0 \\
\hline No ligand & CRUDE & $\lg G$ & DTT & 0 & 6 \\
\hline No ligand & CRUDE & $\lg G$ & $\lg G$ & 0 & 44 \\
\hline No ligand & CRUDE & $\lg G$ & $\mathrm{NH} 4 \mathrm{OH}$ & 3 & 50 \\
\hline No ligand & CRUDE & $\lg G$ & Salt & 0 & 9 \\
\hline No ligand & CRUDE & No coating & Acetonitrile & 0 & 17 \\
\hline No ligand & CRUDE & No coating & Biotin & 0 & 0 \\
\hline No ligand & CRUDE & No coating & DTT & 0 & 10 \\
\hline No ligand & CRUDE & No coating & $\lg G$ & 0 & 3 \\
\hline No ligand & CRUDE & No coating & $\mathrm{NH} 4 \mathrm{OH}$ & 2 & 57 \\
\hline No ligand & CRUDE & No coating & Salt & 0 & 5 \\
\hline No ligand & CRUDE & Polylysine & Acetonitrile & 0 & 7 \\
\hline No ligand & CRUDE & Polylysine & Biotin & 0 & 0 \\
\hline No ligand & CRUDE & Polylysine & DTT & 2 & 3 \\
\hline No ligand & CRUDE & Polylysine & $\lg G$ & 0 & 22 \\
\hline No ligand & CRUDE & Polylysine & $\mathrm{NH} 4 \mathrm{OH}$ & 0 & 97 \\
\hline No ligand & CRUDE & Polylysine & Salt & 0 & 1 \\
\hline IgG-B & CRUDE & Streptavidin & Acetonitrile & 0 & 25 \\
\hline IgG-B & CRUDE & Streptavidin & Biotin & 0 & 0 \\
\hline IgG-B & CRUDE & Streptavidin & DTT & 0 & 16 \\
\hline $\operatorname{lgG}-B$ & CRUDE & Streptavidin & $\lg G$ & 0 & 3 \\
\hline IgG-B & CRUDE & Streptavidin & $\mathrm{NH} 4 \mathrm{OH}$ & 5 & 14 \\
\hline $\operatorname{lgG}-B$ & CRUDE & Streptavidin & Salt & 0 & 0 \\
\hline $\lg G$ & CRUDE & Streptavidin & Acetonitrile & 0 & 0 \\
\hline $\lg G$ & CRUDE & Streptavidin & Biotin & 0 & 0 \\
\hline $\lg G$ & CRUDE & Streptavidin & DTT & 0 & 6 \\
\hline $\lg G$ & CRUDE & Streptavidin & $\lg G$ & 1 & 5 \\
\hline $\lg G$ & CRUDE & Streptavidin & $\mathrm{NH} 4 \mathrm{OH}$ & 0 & 29 \\
\hline $\lg G$ & CRUDE & Streptavidin & Salt & 0 & 1 \\
\hline No ligand & CRUDE & Streptavidin & Acetonitrile & 0 & 18 \\
\hline No ligand & CRUDE & Streptavidin & Biotin & 5 & 0 \\
\hline No ligand & CRUDE & Streptavidin & DTT & 0 & 12 \\
\hline No ligand & CRUDE & Streptavidin & $\lg G$ & 0 & 5 \\
\hline No ligand & CRUDE & Streptavidin & $\mathrm{NH} 4 \mathrm{OH}$ & 0 & 48 \\
\hline
\end{tabular}




\begin{tabular}{|c|c|c|c|c|c|}
\hline No ligand & CRUDE & Streptavidin & Salt & 0 & 6 \\
\hline IgG-SS-B & CRUDE & Streptavidin & Acetonitrile & 2 & 17 \\
\hline IgG-SS-B & CRUDE & Streptavidin & Biotin & 0 & 0 \\
\hline IgG-SS-B & CRUDE & Streptavidin & DTT & 0 & 6 \\
\hline IgG-SS-B & CRUDE & Streptavidin & $\operatorname{lgG}$ & 0 & 21 \\
\hline IgG-SS-B & CRUDE & Streptavidin & $\mathrm{NH} 4 \mathrm{OH}$ & 8 & 45 \\
\hline IgG-SS-B & CRUDE & Streptavidin & Salt & 0 & 8 \\
\hline No ligand & HEPES & Protein G & Acetonitrile & 0 & 5 \\
\hline No ligand & HEPES & Protein G & Biotin & 0 & 4 \\
\hline No ligand & HEPES & Protein G & DTT & 0 & 2 \\
\hline No ligand & HEPES & Protein G & $\lg G$ & 3 & 8 \\
\hline No ligand & HEPES & Protein G & $\mathrm{NH} 4 \mathrm{OH}$ & 0 & 0 \\
\hline No ligand & HEPES & Protein G & Salt & 0 & 0 \\
\hline No ligand & HEPES & $\lg G$ & Acetonitrile & 0 & 0 \\
\hline No ligand & HEPES & $\lg G$ & Biotin & 0 & 0 \\
\hline No ligand & HEPES & $\lg G$ & DTT & 0 & 6 \\
\hline No ligand & HEPES & IgG & $\lg G$ & 0 & 5 \\
\hline No ligand & HEPES & $\lg G$ & $\mathrm{NH} 4 \mathrm{OH}$ & 0 & 3 \\
\hline No ligand & HEPES & $\lg G$ & Salt & 0 & 0 \\
\hline No ligand & HEPES & No coating & Acetonitrile & 0 & 3 \\
\hline No ligand & HEPES & No coating & Biotin & 0 & 0 \\
\hline No ligand & HEPES & No coating & DTT & 0 & 2 \\
\hline No ligand & HEPES & No coating & $\lg G$ & 0 & 5 \\
\hline No ligand & HEPES & No coating & $\mathrm{NH} 4 \mathrm{OH}$ & 0 & 0 \\
\hline No ligand & HEPES & No coating & Salt & 0 & 0 \\
\hline No ligand & HEPES & Polylysine & Acetonitrile & 0 & 14 \\
\hline No ligand & HEPES & Polylysine & Biotin & 0 & 0 \\
\hline No ligand & HEPES & Polylysine & DTT & 0 & 2 \\
\hline No ligand & HEPES & Polylysine & $\lg G$ & 0 & 0 \\
\hline No ligand & HEPES & Polylysine & $\mathrm{NH} 4 \mathrm{OH}$ & 0 & 3 \\
\hline No ligand & HEPES & Polylysine & Salt & 2 & 7 \\
\hline No ligand & HEPES & Streptavidin & Acetonitrile & 0 & 1 \\
\hline No ligand & HEPES & Streptavidin & Biotin & 0 & 0 \\
\hline No ligand & HEPES & Streptavidin & DTT & 0 & 6 \\
\hline No ligand & HEPES & Streptavidin & $\lg G$ & 0 & 2 \\
\hline No ligand & HEPES & Streptavidin & $\mathrm{NH} 4 \mathrm{OH}$ & 0 & 0 \\
\hline No ligand & HEPES & Streptavidin & Salt & 0 & 0 \\
\hline Aggregated IgG & LIVE & Protein G & Acetonitrile & 7 & 29 \\
\hline Aggregated IgG & LIVE & Protein G & Biotin & 0 & 0 \\
\hline Aggregated IgG & LIVE & Protein G & DTT & 0 & 16 \\
\hline Aggregated IgG & LIVE & Protein G & $\lg G$ & 0 & 1 \\
\hline Aggregated IgG & LIVE & Protein G & $\mathrm{NH} 4 \mathrm{OH}$ & 0 & 129 \\
\hline Aggregated IgG & LIVE & Protein G & Salt & 0 & 6 \\
\hline $\lg G-B$ & LIVE & Protein G & Acetonitrile & 2 & 31 \\
\hline IgG-B & LIVE & Protein G & Biotin & 0 & 0 \\
\hline
\end{tabular}




\begin{tabular}{|c|c|c|c|c|c|}
\hline IgG-B & LIVE & Protein G & DTT & 0 & 18 \\
\hline IgG-B & LIVE & Protein G & IgG & 1 & 18 \\
\hline IgG-B & LIVE & Protein G & $\mathrm{NH} 4 \mathrm{OH}$ & 0 & 38 \\
\hline IgG-B & LIVE & Protein G & Salt & 1 & 10 \\
\hline IgG-SS-B & LIVE & Protein G & Acetonitrile & 0 & 1 \\
\hline IgG-SS-B & LIVE & Protein G & Biotin & 0 & 0 \\
\hline IgG-SS-B & LIVE & Protein G & DTT & 1 & 24 \\
\hline IgG-SS-B & LIVE & Protein G & $\lg G$ & 2 & 0 \\
\hline IgG-SS-B & LIVE & Protein G & $\mathrm{NH} 4 \mathrm{OH}$ & 0 & 19 \\
\hline IgG-SS-B & LIVE & Protein G & Salt & 2 & 1 \\
\hline $\lg G-B$ & LIVE & Streptavidin & Acetonitrile & 2 & 15 \\
\hline IgG-B & LIVE & Streptavidin & Biotin & 0 & 0 \\
\hline IgG-B & LIVE & Streptavidin & DTT & 0 & 4 \\
\hline IgG-B & LIVE & Streptavidin & $\lg G$ & 0 & 28 \\
\hline IgG-B & LIVE & Streptavidin & $\mathrm{NH} 4 \mathrm{OH}$ & 2 & 81 \\
\hline IgG-B & LIVE & Streptavidin & Salt & 0 & 21 \\
\hline $\lg G$ & LIVE & Streptavidin & Acetonitrile & 0 & 16 \\
\hline $\lg G$ & LIVE & Streptavidin & Biotin & 0 & 0 \\
\hline $\lg G$ & LIVE & Streptavidin & DTT & 0 & 6 \\
\hline $\lg G$ & LIVE & Streptavidin & $\operatorname{IgG}$ & 0 & 7 \\
\hline $\lg G$ & LIVE & Streptavidin & $\mathrm{NH} 4 \mathrm{OH}$ & 3 & 87 \\
\hline $\lg G$ & LIVE & Streptavidin & Salt & 0 & 10 \\
\hline IgG-SS-B & LIVE & Streptavidin & Acetonitrile & 0 & 25 \\
\hline IgG-SS-B & LIVE & Streptavidin & Biotin & 0 & 0 \\
\hline IgG-SS-B & LIVE & Streptavidin & DTT & 0 & 8 \\
\hline IgG-SS-B & LIVE & Streptavidin & $\operatorname{IgG}$ & 3 & 15 \\
\hline IgG-SS-B & LIVE & Streptavidin & $\mathrm{NH} 4 \mathrm{OH}$ & 1 & 47 \\
\hline IgG-SS-B & LIVE & Streptavidin & Salt & 4 & 11 \\
\hline
\end{tabular}

No clear enrichment of IgG, Fc receptors and KEGG proteins for IgG-Fc mediated phagocytosis was observed in any combination of ligand, chromatography bead coating and elution buffer attempted where ligands were applied to live cells compared to crude controls.

\section{Biotinylated ligand affinity receptor chromatography of the Fc Receptor}

A classical ligand affinity chromatography approached where IgG, IgG-B and IgG-SS-B ligands were applied to live cells, allow for bind, and then homogenized by French press and isolated using a SA-agarose affinity column. We attempted many variations of the classical ligand 
affinity chromatography method including applying the ligand alone, the biotinylated ligand, and the biotinylated ligand with SS cleavable linker in addition to detergents as well as biotinylated heat formed IgG aggregates.

\section{Biotinylation of IgG}

Immunoglobulin $\mathrm{G}$ contains 28 lysines which are the targets of the NHS moiety (Fig. 36). To determine the optimum molar ratio of biotin to $\operatorname{IgG}$ for biotinylation, $\operatorname{IgG}$ was biotinylated with both NHS-biotin and NHS-ss-biotin at molar ratios of 1:1 up to 100:1 (Fig.37).

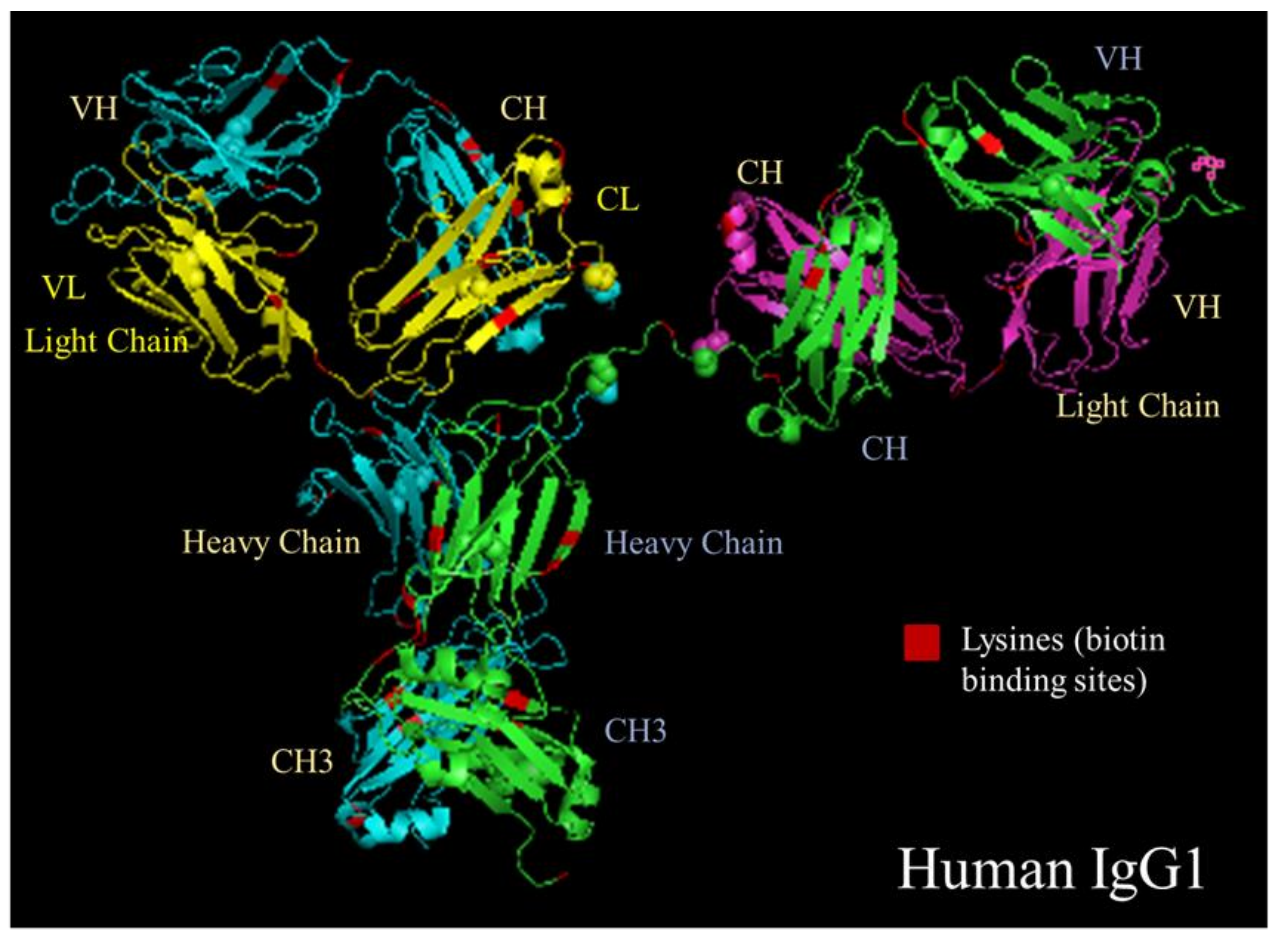

Figure 36: The structure of IgG derived from X-ray chrystallography with the all lysine residues indicated in red showing the possible binding sites of NHS Biotin and NHS-SSBiotin The PyMOL Molecular Graphics System, Version 1.5.0.4 Schrödinger, LLC. (Creutz \& Harrison, 1984) 


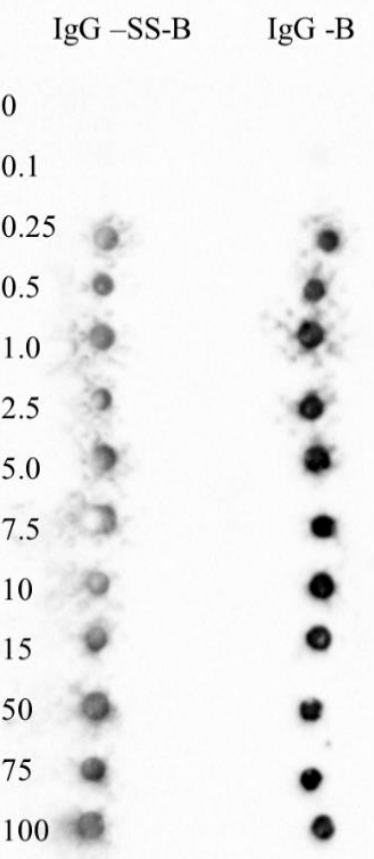

SA-HRP

Figure 37. Detection of the Biotin-IgG ligand (B) or the Biotin-S-S-B (B-S-S-IgG) by dot blotting and ECL molar ratio of B-NHS or B-SS- NHS to IgG is shown. Note that the ligand approaches apparent saturation of the ECL detection method by a 1:1 Molar ratio and high amounts of biotin did not result in more intense detection. 
A

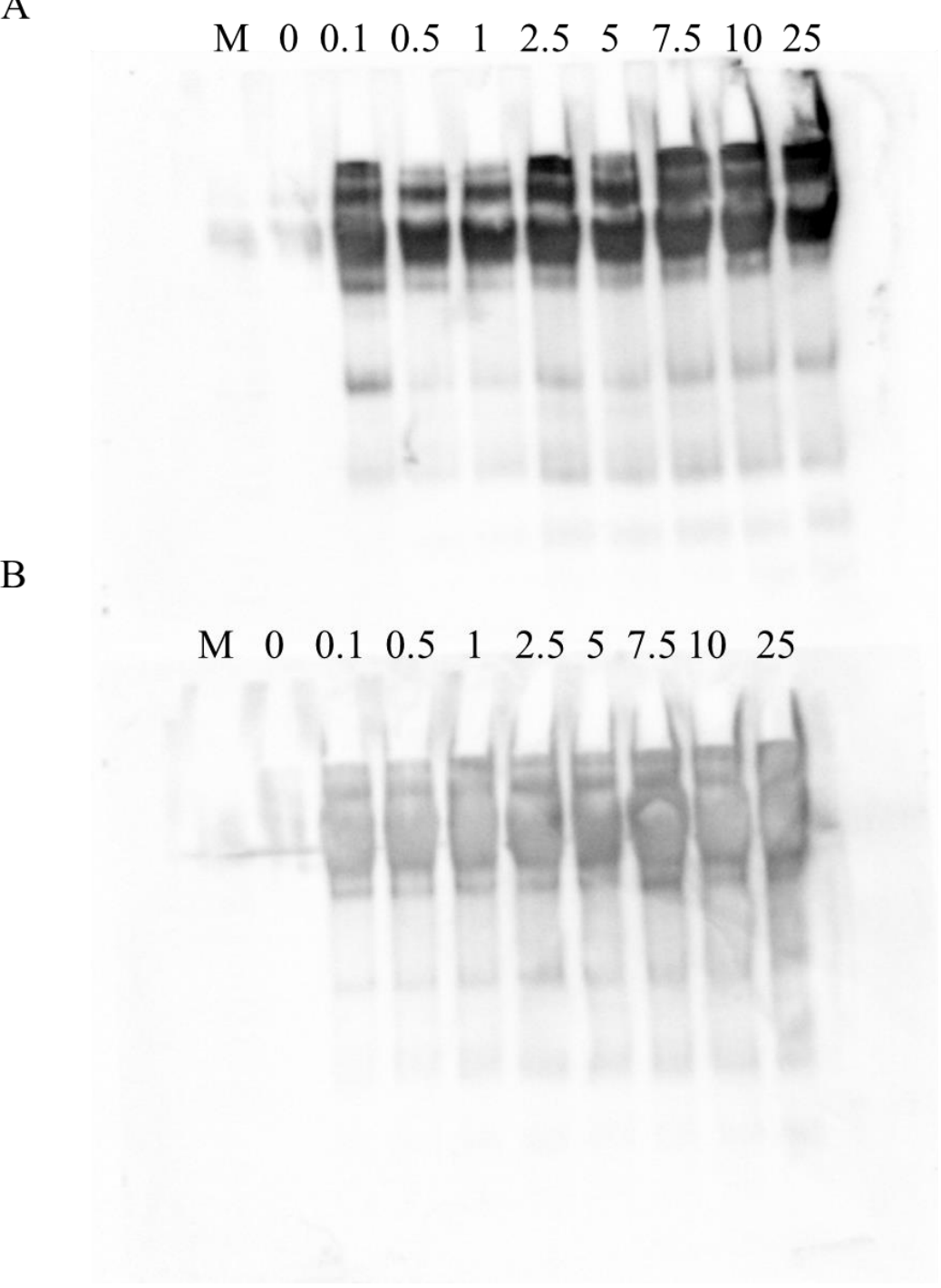

Figure 38: Western Blot of (A) IgG-biotin and (B) IgG-sulfo-biotin with Streptavidin-HRP. Legend: lane 1: marker; lane 2: IgG; lane 3: IgG with 0.1 fold NHS-biotin, lane 4: IgG with 0.5 fold NHS-biotin, lane 5: IgG with 1.0 fold NHS-biotin, lane 6: IgG with 2.5 fold NHS-biotin, lane 7: IgG with 5 fold NHS-biotin, Lanes 8: IgG with 7.5 fold NHS-biotin, Lanes 9: IgG with 10 fold NHS-biotin (B) same ratios but with NHS-SS-B

The streptavidin-HRP probe was used to determine the saturation by ECL detection of NHSbiotin and NHS-SS-biotin binding which was apparently a 1:1 molar ratio of biotin to IgG (Fig. $37 \& 38)$. Since IgG contains a large number or lysines it is possible that more than one NHS- 
B/NHS-SS-B bound to each IgG leaving some IgG ligands without biotin tags. To avoid producing IgG molecules without tags we increased the binding ratio from 1:1 to 2:1 and 5:1 for subsequent experiments to increase the likelihood of at least 1 biotinylation per IgG molecule. The NHS-biotinylation reaction was quenched with glycine to prevent subsequent non-specific biotinylation reactions and after IgG-B incubation with cells all unbounding molecules where washed several times prior to homogenization. Another concern with regard to over-biotinylation is that too many biotinylations can make the molecule hydrophobic causing aggregation. Also, an excess of NHS-B binding can lead to unintended tyrosine biotinylations.

\section{Detection reagents}

Biotinylated ligands IgG-B and IgG-SS-B at a molar ratio of 2:1 were clearly detected to the 10ng range by the Streptavidin-HRP and 10ng range by the Dk anti hIgG-HRP probe and barely visible at 1 ng (Fig. $39 \& 40)$.

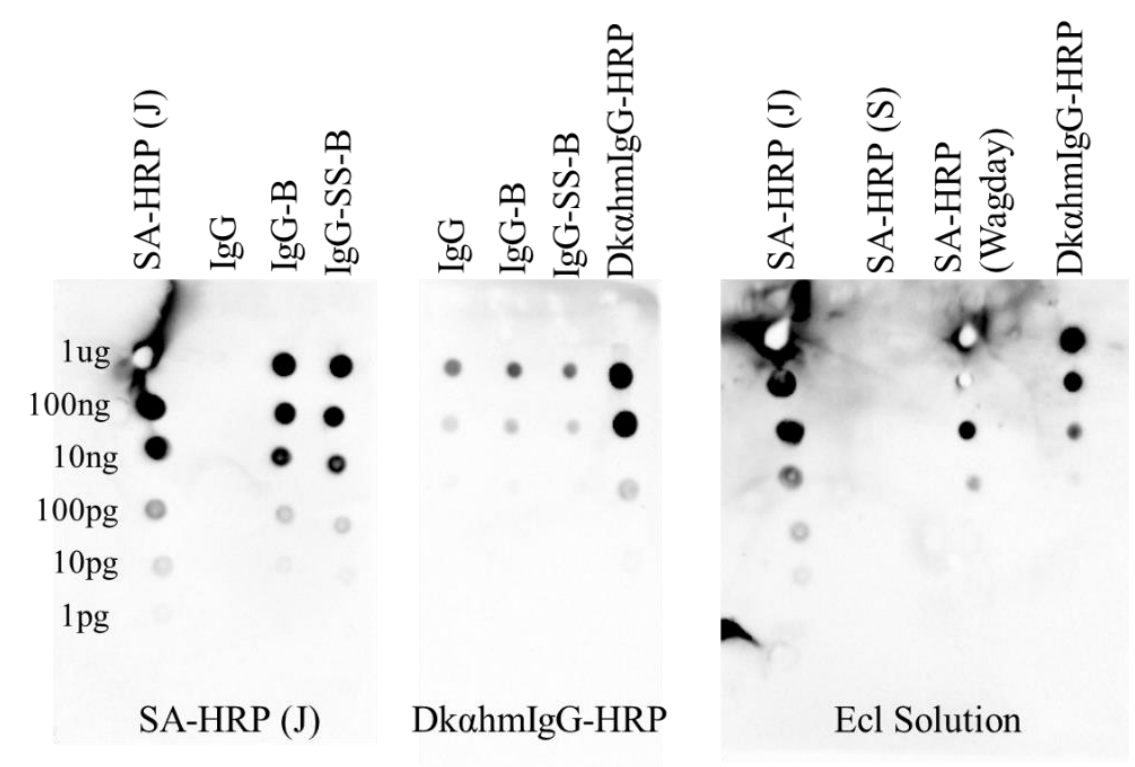

Figure 39. The detection limit of the IgG-B and IgG-S-S-B labeled ligands. The detection limit by dot blot on PVDF and ECL detection was about $10 \mathrm{pg}$ of IgG-B and IgG-SS-B with SAHRP from Jackdson and 10ng for Dk-anti-hIgG-HRP. 


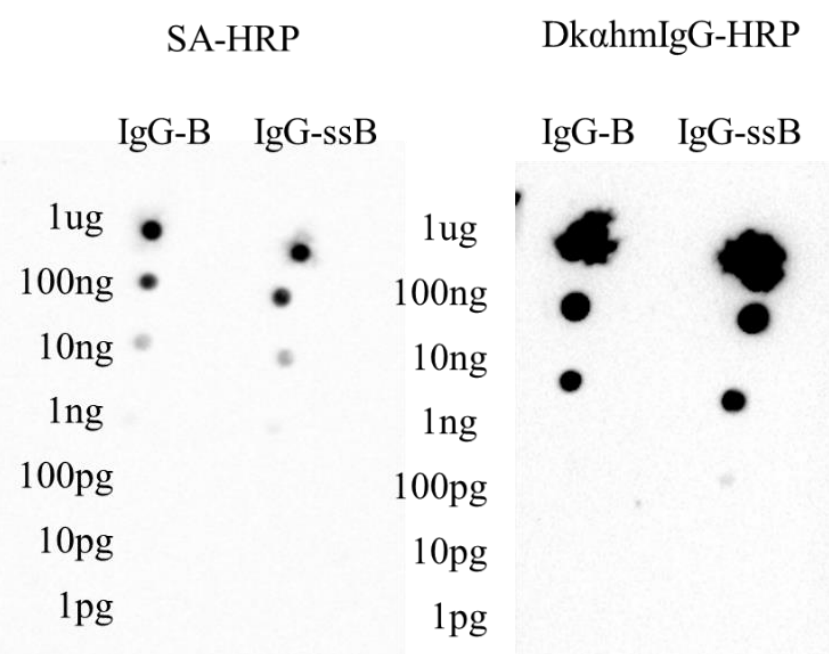

Figure 40. Measure of Sensitivity of Detection Reagents Streptavidin-HRP and DkahmIgGHRP by Dot blot on PVDF and Western. Stocks of IgG-B and IgG-ss-b where biotinylated at a molar ration of 2 biotins to $1 \mathrm{IgG}$

\section{Comparison of affinity chromatography supports by dot blot with SA-HRP or DahmIgG}

Chromatography surfaces included naked agarose beads and streptavidin conjugated agarose beads (SA-agarose). Home made beads were prepared using the $\mathrm{CNBr}$ method and compared to beads purchased from Sigma (Axen, Porath, \& Ernback, 1967; Cuatrecasas, Wilchek, \& Anfinsen, 1968; Porath, Axén, \& Ernback, 1967) (Diamandis \& Christopoulos, 1991). To compare homemade and store-bought beads, 200 ug of IgG, IgG-B and IgG-SS-B ligands were applied to 100uL columns of SA-agarose beads, washed in 1x PBS and then scrubbed with $2 \%$ SDS, spotted on PVDF and detected with the SA-HRP probe with ECL. In house beads performed similarly to sigma beads where IgG-SS-B ligands were applied to SA-agarose (Fig. $41)$. 


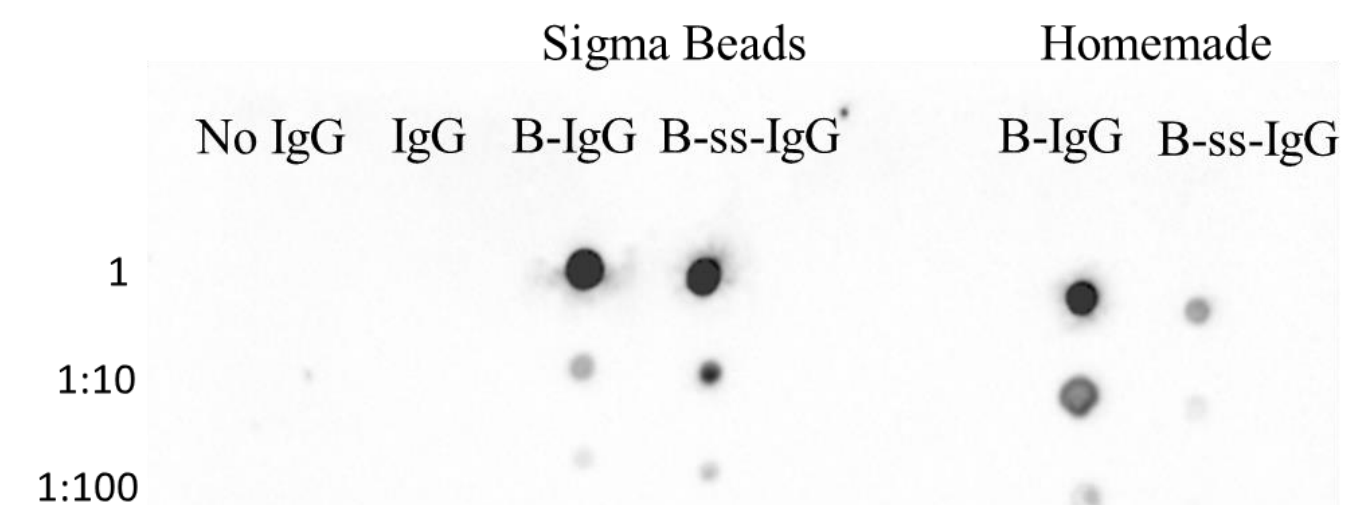

$1: 1,000$

Figure 41: Comparison of Sigma and homemade streptavidin coated beads. 200ug of IgG, IgG-B and IgG-ss-B was applied to separate $100 \mathrm{uL}$ columns of Streptavidin coated agarose beads prepared by Sigma and compared to in house beads. The columns were washed with $3 \mathrm{mLs}$ of 1xPBS followed by the sample in $1 \mathrm{~mL} 1 \mathrm{xPBS}$, followed by a $1 \mathrm{~mL}$ clean. The beads were boiled in $1 \mathrm{~mL}$ of $2 \%$ SDS. Dilutions of the boiled beads were made as indicated, spotted on PVDF and detected by SA-HRP

\section{Recovery of IgG and biotin after biotinylated affinity chromatography experiment with SA-agarose}

IgG, IgG-B and IgG-SS-B ligands were applied to live cells and crude homogenates (Fig. 17).

The chromatography surfaces included naked agarose beads (non-specific binding control) and SA-agarose. The crude extract with no ligand, IgG-B and IgG-SS-B was used as an additional control to uncoated agarose beads and streptavidin coated agarose (Fig. 17). After sample loading and washing with $1 \mathrm{xPBS}$ we attempted to elute the receptor complex sequentially with the following buffers: 5mM DDT, 5\% $\beta$-mercaptoethanol (BME), 2M ammonium hydroxide and $2 \mathrm{M}$ ammonium hydroxide with $50 \mathrm{mM}$ biotin. The total protein concentration of each elution was measured by Dumbroff protein assay by applying $1 \mathrm{uL}$ aliquots onto Whattmann paper 1 and staining with coomassie brilliant blue (Fig. 42). 

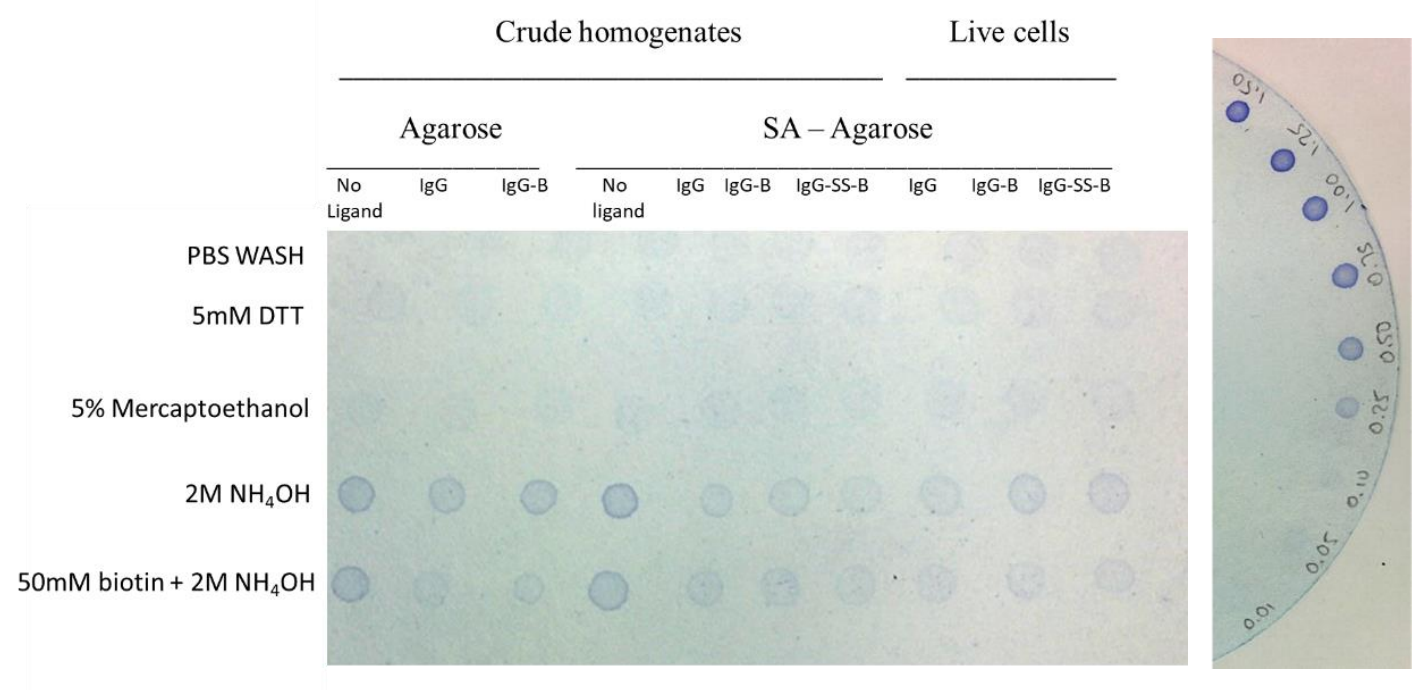

Figure 42: Protein Assay of IgG, IgG-B and IgG-SS-B incubated with live cells and crude homogenates applied to control agarose columns and streptavidin-agarose columns and stained with coomassie brilliant blue. Columns were washed with 1xPBS then sequentially eluted 5mM DTT, 5\% mercaptoethanol, 2M ammonium hydroxide, 2M ammonium hydroxide + $50 \mathrm{mM}$ biotin.

DTT and mecaptoethanol elutions showed concentrations of approximately $0.01 \mathrm{ug} / \mathrm{uL}$ regardless of treatment. Ammonium hydroxide and ammonium hydroxide + biotin elutions contained between 0.1 and $0.3 \mathrm{ug} / \mathrm{mL}$ of protein. The treatments yielding the highest concentration of protein were from crude homogenates without ligand applied to SA-agarose beads and naked agarose beads demonstrating the potential for non-specific adsorption. 


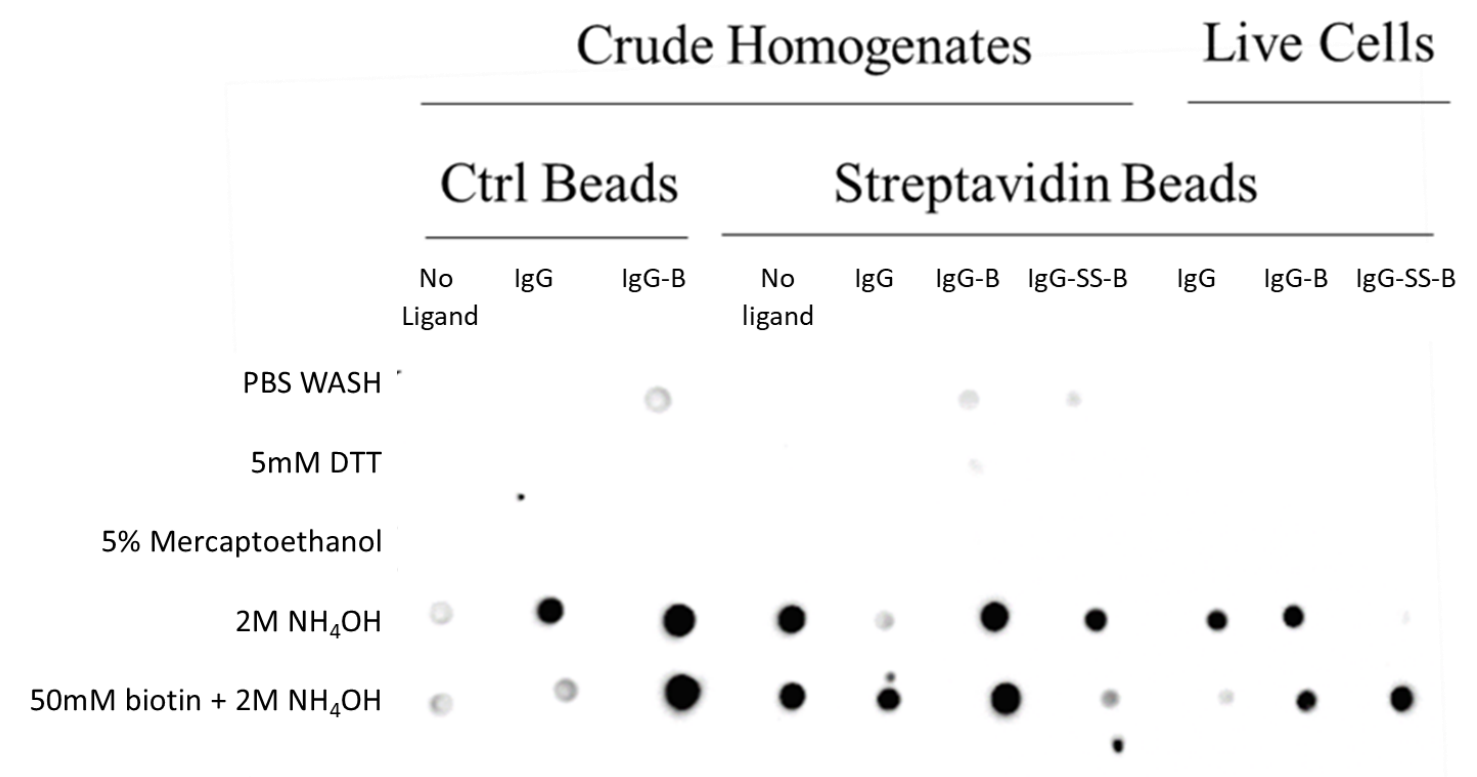

Figure 43: Western dot blot of IgG, IgG-B and IgG-SS-B incubated with live cells and crude homogenates applied to control agarose and streptavidin-agarose columns and washed with 1xPBS and then eluted sequentially with $5 \mathrm{mM}$ DTT, $5 \%$ mercaptoethanol, $2 \mathrm{M}$ ammonium hydroxide, $2 \mathrm{M}$ ammonium hydroxide $+50 \mathrm{mM}$ biotin. The elutions were spotted on a PVDF membrane and visualized with a Dk-anti-hIgG-HRP probe in ECl.

The relative quantities of $\operatorname{IgG}$ for the micro affinity experiment were measured by spotting $1 \mathrm{uL}$ of the $1 \mathrm{~mL}$ samples on PVDF membrane and was detected by a Dk-anti-hIgG-HRP probe and $\mathrm{ECl}$ (Fig. 43). IgG was only very faintly detectable in one of the DDT elutions where IgG-B was added to crude homogenates and applied to an SA-agarose column. At least 10ng of IgG was recovered from live cells from $1 \mathrm{uL}$ test samples.

IgG was detectable in all elutions with $\mathrm{NH}_{4} \mathrm{OH}$ and $\mathrm{NH}_{4} \mathrm{OH}+$ biotin except where IgG-SS-B added to live cells was applied to SA-agarose beads. If DTT and/or BME were able to cleave the SS biotin linker they did so below the detection limit of the Western dotblot between 10ng of IgG to $100 \mathrm{fmol}$ (Fig. 43). IgG-B showed a stronger signal in the ammonium hydroxide buffers when applied to control beads compared to SA-agarose beads (Fig. 43). Agarose beads seem to 
have a significant binding capacity in general compared to beads coated in streptavidin by the $\mathrm{CNBr}$ method which may also change the binding properties of the support surface. A higher IgG detection was expected in the crude plus ligand treatments since the crude plus IgG treatment suffered from an excess of $\operatorname{IgG}$ that was not bound to a receptor compared to $\operatorname{IgG}$, $\operatorname{IgG}$ B and IgG-S-S-B treatments bound to live cells that were thoroughly washed prior to homogenization.

Ammonium hydroxide has been shown to break disulphide bridges however it does not seem to be eluting the IgG-S from the S-biotin-SA-agarose column specifically compared to IgG-biotinSA-agarose since there is greater detection of $\mathrm{IgG}$ in the IgG-B treatment compared to IgG-SS-B for the crude homogenates applied to SA beads. The ammonium hydroxide was apparently nonspecifically eluting proteins from the column possibly due to the highly basic conditions.

Biotin replacement was not expected to elute biotinylated ligands from SA columns due to the high binding affinity of biotin for streptavidin and low off rate. IgG was detected in most treatments where biotinylated ligands were used. IgG was only detected in the ammonium hydroxide + biotin fraction where IgG-S-S-B was applied to live cells (Fig. 43).

In a separate Western dot blot assay, the spun down cytoskeleton from the crude and live homogenates were spotted and showed much of the $\operatorname{IgG}$ to be associated with the actin cytoskeleton (Fig. 44). 
Crude homogenates

Agarose

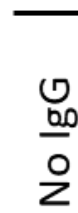

SA- Agarose

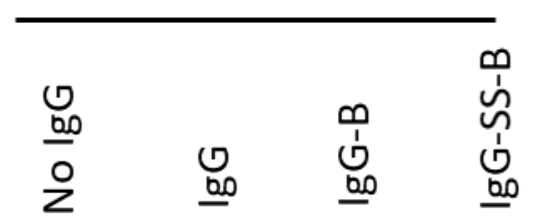

Agarose

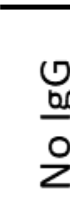

Live cells
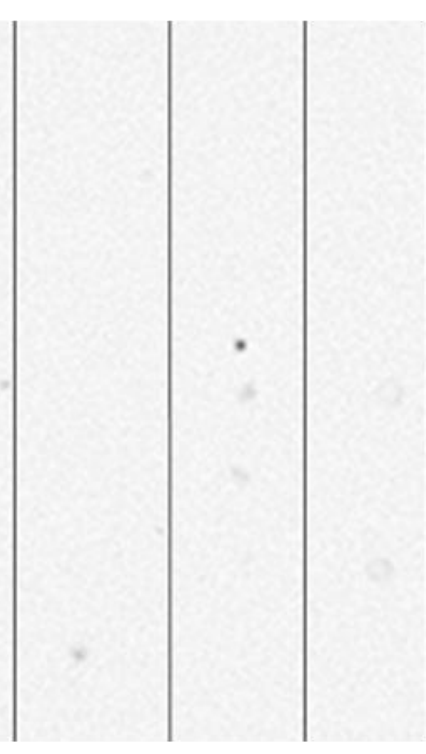

SA- Agarose

$\begin{array}{lll}0 & 0 \\ 0 & 0 \\ 0 & 0 & 0 \\ 2 & 0\end{array}$

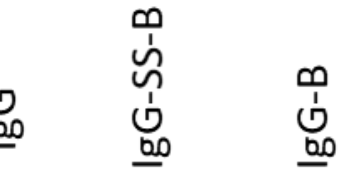

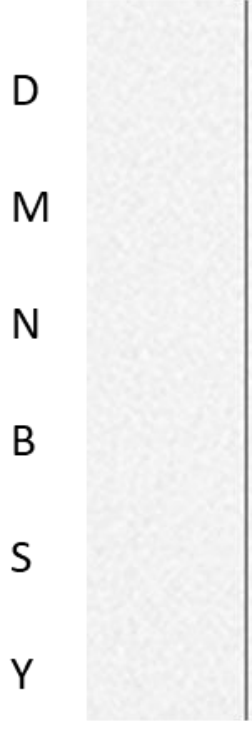
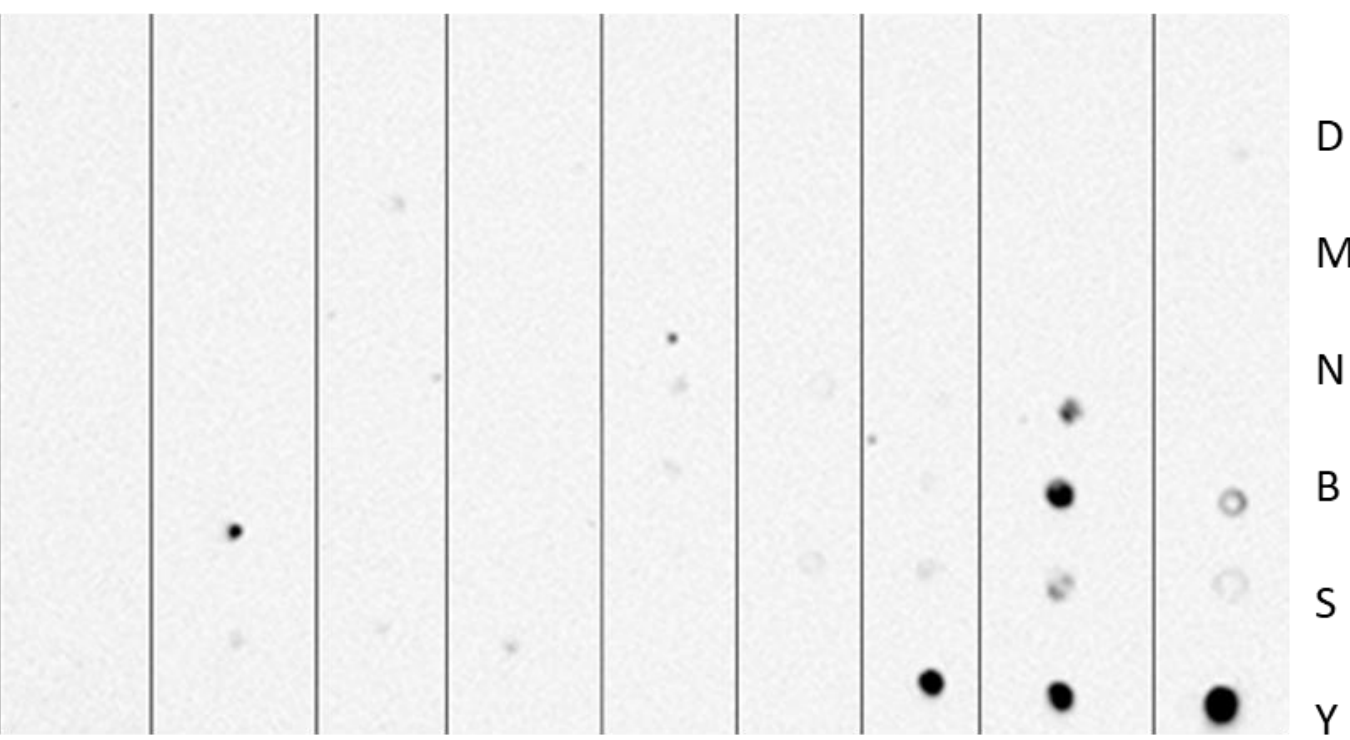

Figure 44: Western Dot Blot of Crude, IgG-B and IgG-SS-B incubated with live cells and and crude homogenates applied to control agarose columns and streptavidin affinity columns (not labelled) with Dk-anti-hIgG Antibody. Legend of Elutions buffers: D - 5mM DTT, $\mathrm{M}-5 \%$ Beta Mercaproethanol, N - 2M Ammonium Hydroxide, B - 2M Ammonium Hydroxide + $50 \mathrm{mM}$ biotin, Y-Spun down cytoskeleton

\section{Streptavidin-HRP and anti-human IgG-HRP detection of Western blots}

We observed that affinity ligands IgG-B and IgG-SS-B were both efficiently captured by the SAagarose micro columns when $50 \mathrm{ul}$ of the resin was mixed with an equal volume of $2 \mathrm{x}$ sample buffer for tricine SDS-PAGE with a 7\% gel followed by transfer to PVDF and probing with both the SA-HRP probe or the anti-human IgG antibody (Fig. 45). 


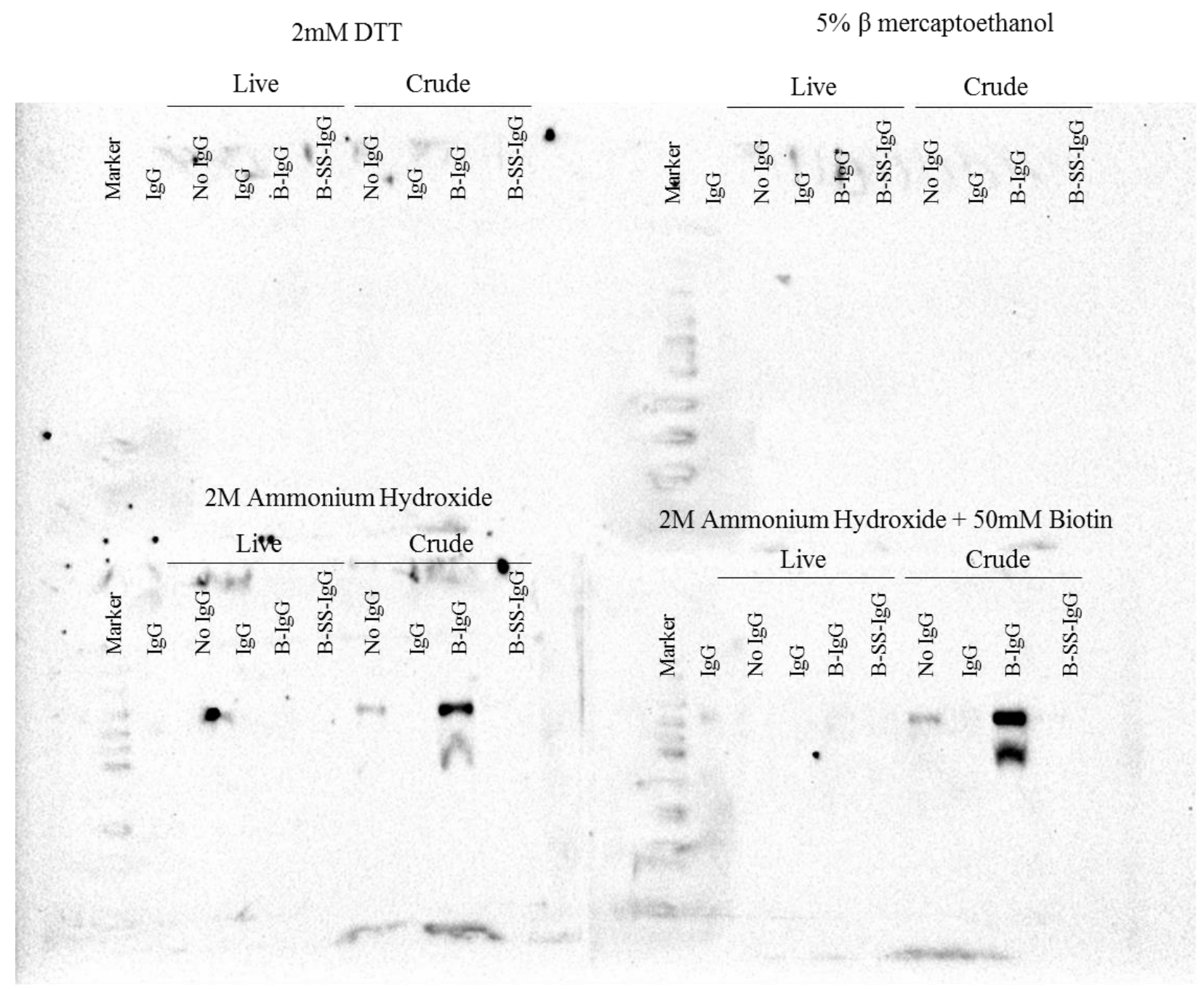

Figure 45: Treatments of no IgG, IgG, IgG-B and IgG-SS-B were applied to either live cells or crude homogenates and applied to streptavidin columns. The columns were washed and eluted with 5mM DTT, 5\% mercaproethanol, 2M ammonium hydroxide and 2M ammonium hydroxide $+50 \mathrm{mM}$ biotin. Live cells were incubated on ice with ligands for 30 minutes and then washed 3 times prior to French pressing. Ligands were added to crude homogenate immediately before French pressing. IgG was detected in each fraction by Western blot with DK anti-hIgGHRP

The IgG ligands were not detectable in from the Western blots in the eluates of the DTT or BME treatments in live cells. Trace amounts of $\mathrm{IgG}$ was detected in the $\mathrm{NH} 4 \mathrm{OH}$ elution and were more clearly detected in the $\mathrm{NH} 4 \mathrm{OH}+$ biotin sample (Fig. 45). 
A
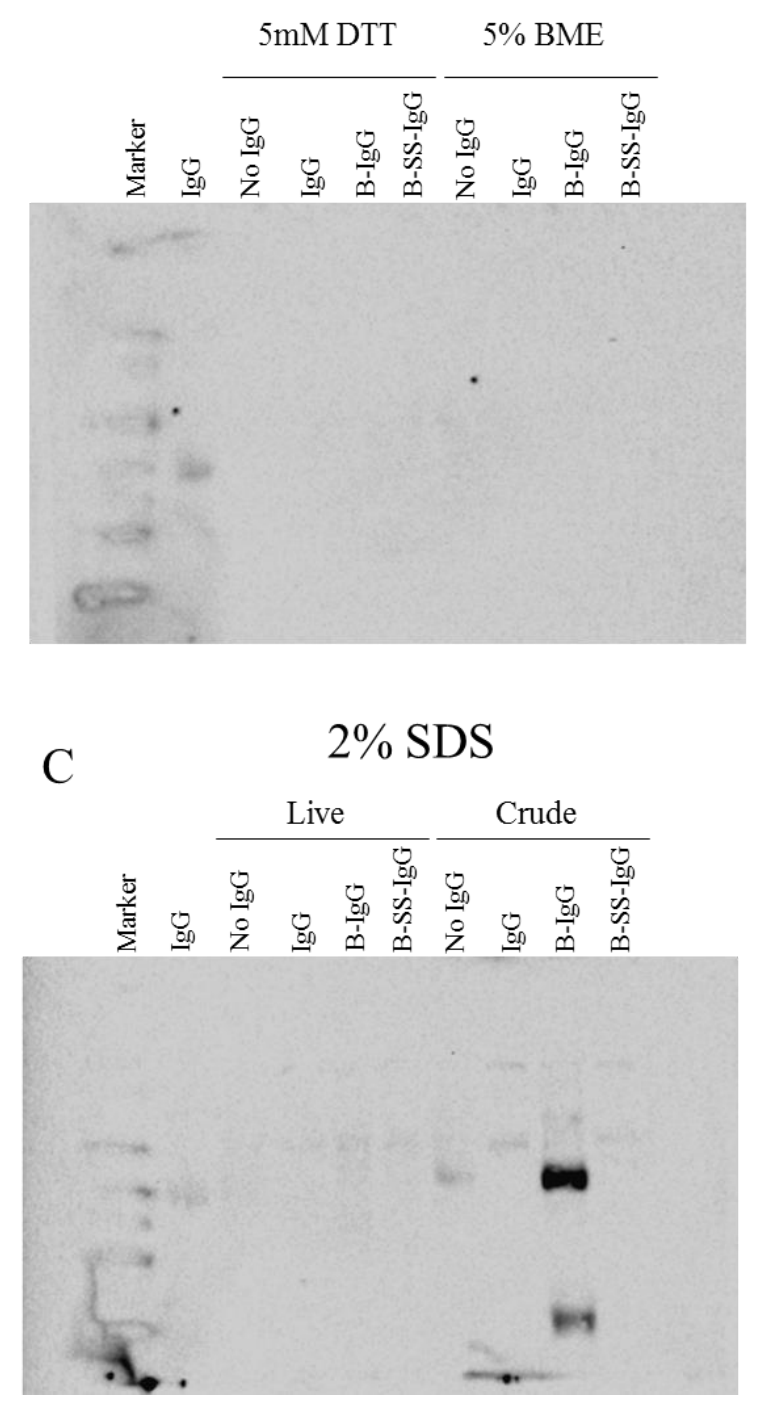

B

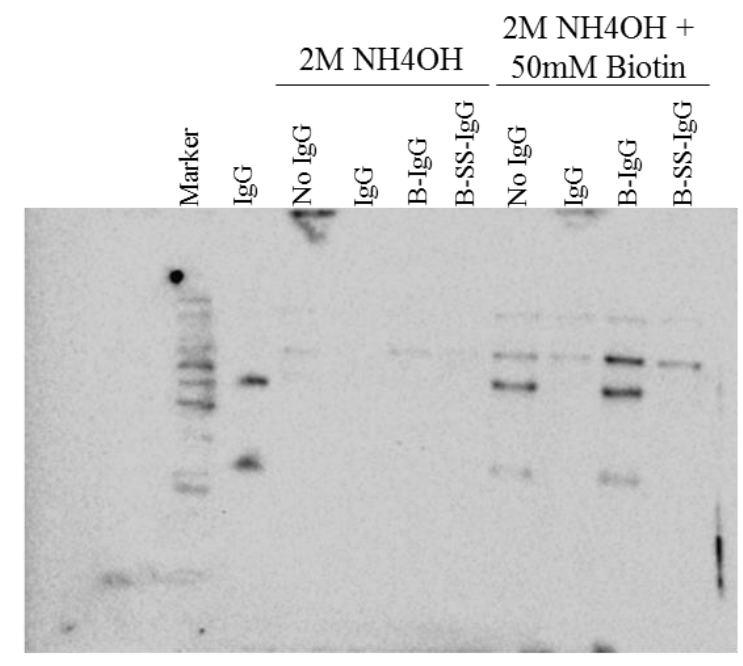

D $\frac{\begin{array}{c}\text { Crude on } \\ \text { SA beads }\end{array}}{\text { Naked beads }} \begin{gathered}\text { Crude on } \\ \text { Nats }\end{gathered}$

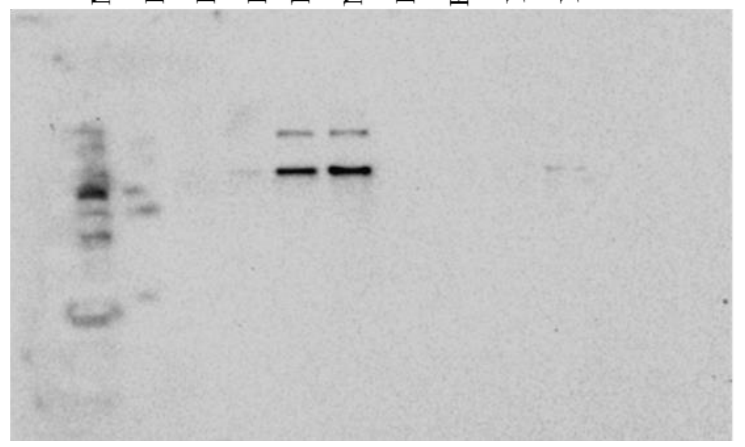

Figure 46: Treatments of no IgG, IgG, IgG-B and IgG-SS-B were applied to live cells and applied to streptavidin columns. The columns were washed and eluted with (A) 5mM DTT and $5 \%$ mercaptoethanol, (B) 2M ammonium hydroxide, 2M ammonium hydroxide $+50 \mathrm{mM}$ biotin and (C) scrubbed with 2\% SDS (from both live and crude homogenates only for SDS) Live cells were incubated on ice with ligands for 30 minutes and then washed 3 times prior to French pressing. Ligands were added to crude homogenate immediately before French pressing. (D) Crude homogenates were applied to streptavidin coated beads and naked beads and eluted with the buffers previously listed. IgG was detected in each fraction by Western Blot with DK-antihIgG-HRP. PVDF dipped in methanol, blocked for 30 minutes with $5 \%$ skim milk $+1 \%$ Dk serum. 
The IgG ligands added to live cells and applied to SA-agarose showed trace amounts of ligands scrubbed from beads with $2 \%$ SDS and higher amounts in the crude samples as indicated by the Western dot blots (Fig. 46). The 2\% SDS scub showed much of the IgG-B remained on the SAagarose beads despite previous washes of $\mathrm{NH}_{4} \mathrm{OH}$ and $\mathrm{NH}_{4} \mathrm{OH}+$ biotin (Fig. 46).

\section{Liquid chromatography tandem mass spectrometry (LC-ESI-MS/MS)}

The entire volume of the $1.0 \mathrm{ml}$ elutions from the biotinylated affinity chromatography experiment on SA-agarose beads were digested with trypsin and then the peptides collected over C18 for LC ESI-MS/MS with a linear quadrupole ion trap. Peptides were detectable from each of the fractions by the LC-ESI-MS/MS analysis. A comparison of the based peak traces seemed to indicate that some selectivity was achieved by the affinity chromatography treatments employed compared to crude extracts incubated with agarose or SA-agarose (Fig 47).

We observed that the ligand IgG may be applied to live cells and crude homogenates, captured by agarose or SA-agarose and eluted by $\mathrm{NH} 4 \mathrm{OH}$ by a non-specific chromatography mechanism. 


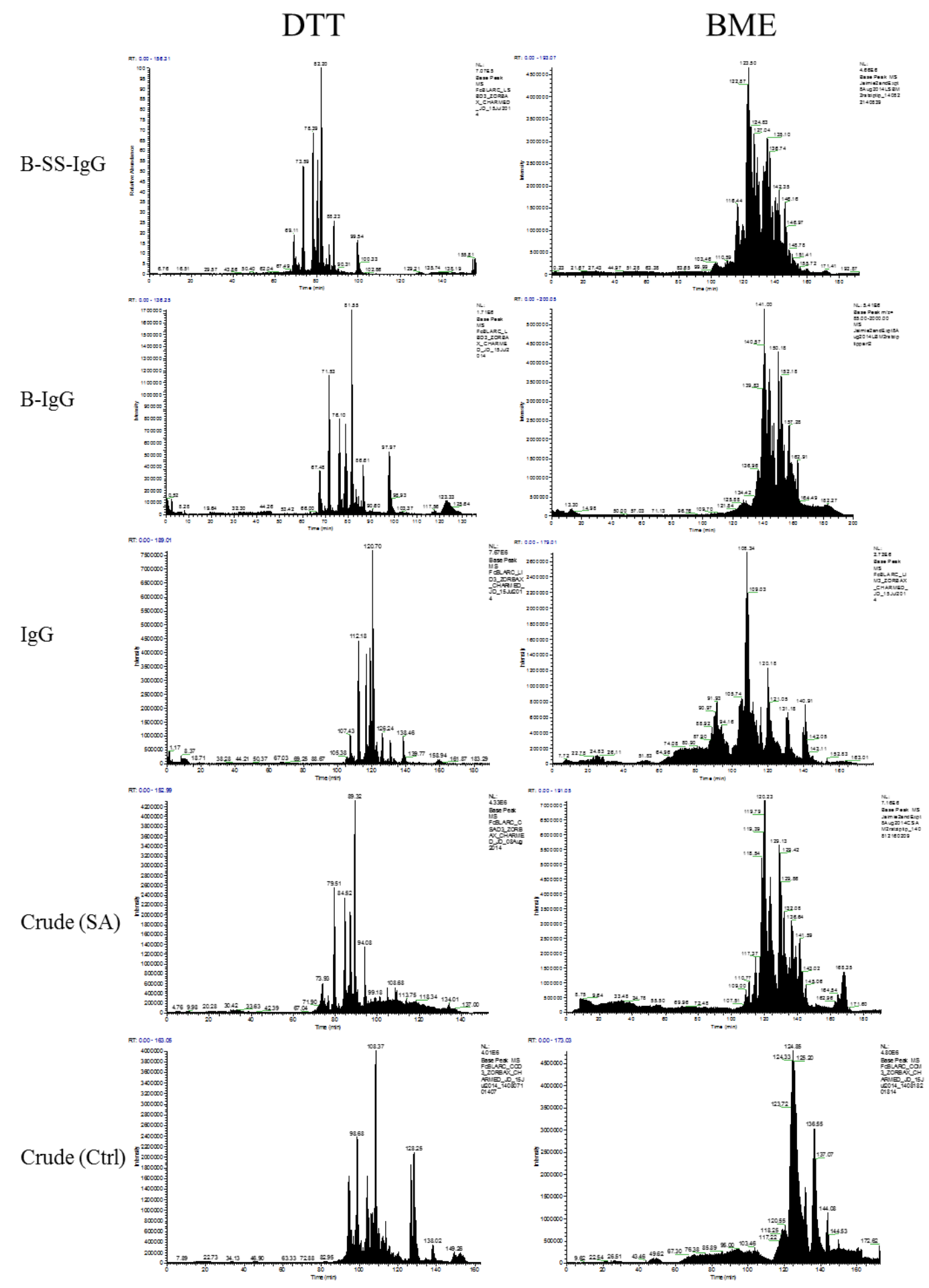

Figure 47. LC-MS/MS TIC and base peak traces of the biotinylated ligand affinity chromatography experiment performed with live RAW macrophages incubated with IgGSS-B, IgG-B, IgG and crude extracts poured over a streptavidin-agarose column and crude extracts poured over naked beads and eluted with DTT then $5 \%$ B-mercaptoethanol, $\mathrm{NH}_{4} \mathrm{OH}$ and $\mathrm{NH}_{4} \mathrm{OH}+$ biotin. 


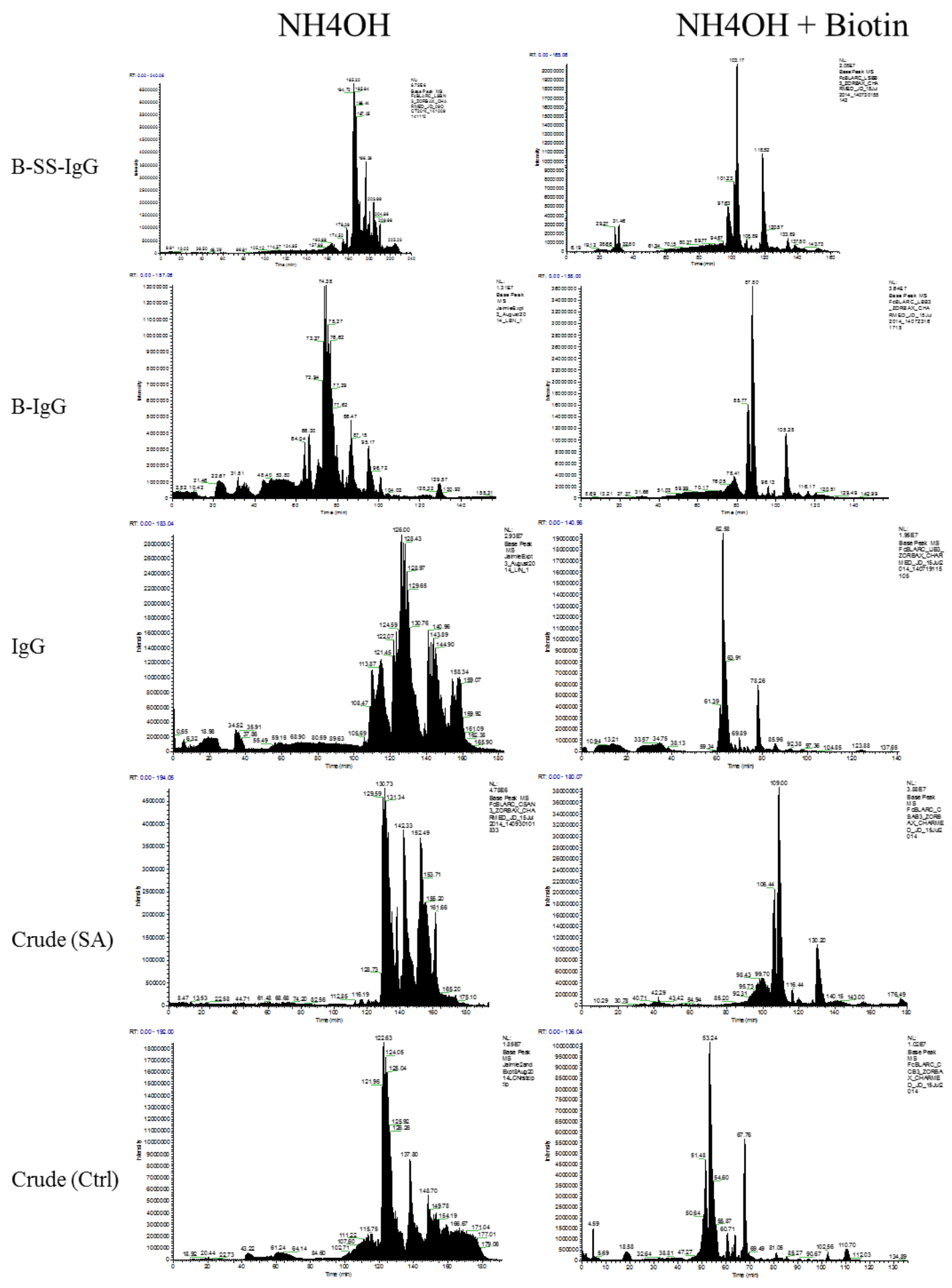

Figure 48: LC-MS/MS TIC and base peak traces of the biotinylated ligand affinity chromatography experiment with live RAW macrophages incubated with IgG-SS-B, IgGB, IgG and crude extracts poured over a streptavidin column and crude extracts poured over naked beads and eluted with $\mathrm{NH}_{4} \mathrm{OH}$ followed by $\mathrm{NH}_{4} \mathrm{OH}+$ Biotin. 
Comparing base peak chromatograms from the DTT eluted fraction to the crude extract mixed with agarose as a control showed that some selectivity was obtained from the DTT elution experiments showing multiple sharp clearly separated peaks (Fig 47). A comparison of the peptide to protein distribution of the data compared to random spectra or noise from by Chi Square test showed the experimental data had more proteins with many peptides compared to that of random expectation and few proteins with 3 or less peptide that showed $p$ value for the entire experiment of $\mathrm{P}<0.001$ or $1 / 10,000$ chance the data was the same as random expectation. As previously described, peptide searches against blank solvent runs and randomly generated MSMS spectra result in peptide IDs that are randomly spread across the peptide database and skewed to the larger proteins.

\section{Detection of human IgG and the Fc gamma receptors by classical affinity chromatography}

The classical affinity chromatography capture of the $\mathrm{Fc}$ receptor using SA-agarose beads and biotinylated IgG results were searched against a curated human library to identify the human $\operatorname{IgG}$ ligand and against a mouse library with SEQUEST and X!TANDEM to identify mouse peptides. As described earlier filter method 2 is the highest scoring combination of best charge state and sequence and filter 1 is the highest scoring charge state. Filter 2 is the most stringent, reducing the likelihood of obtaining false positive identifications. A plot of peptide to proteins for digested IgG compared to the null models of noise and random using filter method 2 showed that type one error can be effectively minimized by imposing a filter of a minimum of 3 peptide identifications per protein at filter 2. Given that $\operatorname{IgG}$ is the ligand and $\mathrm{Fc}$ is the intended target of the experiment an argument can be made to use filter 0 when searching for IgG or the Fc gamma receptors. 
The eluted and retained fractions digested and analyzed by LC-ESI-MS/MS revealed that hIgG was released in the greatest quantities when captured from live cells using the IgG-SS-B ligand (125 protein identifications) compared to IgG-B (75) and IgG (77) captured by an SA-agarose column where the protein identifications from all elution buffers were summed (Table 2). The most number of protein identifications for hIgG were identified in the DTT elution where the IgG-SS-B (44) ligand was used compared to IgG-B (36) and IgG (31) where an SA-agarose column was used (Table 3). 
Table 4: IgG protein identifications for the classical ligand affinity chromatography method with biotinylated ligands observed across treatments where the DTT, BME, NH4OH and NH4OH + biotin elutions were grouped by treatment.

\begin{tabular}{|c|c|c|c|c|c|c|c|c|c|c|}
\hline $\begin{array}{l}\text { Description } \\
\text { Database }\end{array}$ & $\begin{array}{l}\text { LIVE } \\
\text { SA } \\
\text { IgG-SS-B } \\
\mathrm{N}=48\end{array}$ & $\begin{array}{l}\text { LIVE } \\
\text { SA } \\
\text { IgG-SS-B } \\
\text { STYP } \\
\text { N=48 }\end{array}$ & $\begin{array}{l}\text { LIVE } \\
\text { SA } \\
\text { IgG-B } \\
\mathrm{N}=48\end{array}$ & $\begin{array}{l}\text { LIVE } \\
\text { SA } \\
\text { IgG-B } \\
\text { STYP } \\
\mathrm{N}=48\end{array}$ & $\begin{array}{l}\text { LIVE } \\
\text { SA } \\
\text { IgG } \\
\mathrm{N}=36\end{array}$ & $\begin{array}{l}\text { LIVE } \\
\text { SA } \\
\text { IgG } \\
\text { STYP } \\
\mathrm{N}=36\end{array}$ & $\begin{array}{l}\text { Crude } \\
S A \\
N=40\end{array}$ & $\begin{array}{l}\text { Crude } \\
\text { SA } \\
\text { STYP } \\
\mathrm{N}=40\end{array}$ & $\begin{array}{l}\text { Crude } \\
A \\
N=40\end{array}$ & $\begin{array}{l}\text { Crude } \\
\text { A } \\
\text { STYP } \\
\mathrm{N}=40\end{array}$ \\
\hline IgG L chain & 3 & 21 & 1 & 9 & 0 & 3 & 6 & 12 & 6 & 6 \\
\hline IgG H chain & 5 & 4 & 7 & 2 & 5 & 1 & 12 & 5 & 13 & 4 \\
\hline IgG H chain & 4 & 6 & 3 & 2 & 1 & 5 & 4 & 11 & 5 & 23 \\
\hline IgG H chain & 4 & 7 & 5 & 4 & 8 & 1 & 5 & 6 & 11 & 2 \\
\hline IgG H chain & 4 & 6 & 2 & 1 & 4 & 0 & 1 & 5 & 6 & 4 \\
\hline IgG H chain & 5 & 6 & 2 & 2 & 1 & 0 & 2 & 5 & 5 & 2 \\
\hline IgG H chain & 3 & 4 & 1 & 2 & 9 & 3 & 6 & 0 & 5 & 2 \\
\hline IgG H chain & 6 & 5 & 2 & 5 & 3 & 3 & 4 & 1 & 1 & 2 \\
\hline IgG H chain & 4 & 6 & 2 & 2 & 1 & 0 & 2 & 5 & 4 & 4 \\
\hline IgG H chain & 3 & 7 & 2 & 1 & 3 & 1 & 1 & 6 & 2 & 2 \\
\hline $\operatorname{lgG} \mathrm{L}$ chain & 2 & 1 & 1 & 5 & 1 & 1 & 4 & 2 & 6 & 4 \\
\hline IgG L chain & 0 & 4 & 0 & 5 & 3 & 5 & 2 & 2 & 4 & 0 \\
\hline IgG H chain & 5 & 3 & 3 & 0 & 3 & 3 & 5 & 2 & 1 & 1 \\
\hline IgG H chain & 5 & 2 & 7 & 1 & 1 & 0 & 4 & 0 & 4 & 0 \\
\hline IgG L chain & 1 & 4 & 0 & 1 & 0 & 1 & 1 & 4 & 4 & 4 \\
\hline IgG L chain & 0 & 3 & 0 & 4 & 1 & 1 & 4 & 2 & 4 & 0 \\
\hline IgG L chain & 6 & 1 & 3 & 0 & 3 & 1 & 6 & 1 & 5 & 2 \\
\hline IgG L chain & 0 & 3 & 0 & 4 & 1 & 1 & 4 & 2 & 4 & 0 \\
\hline IgG VH & 4 & 5 & 2 & 1 & 3 & 0 & 1 & 5 & 1 & 2 \\
\hline IgG L chain & 5 & 0 & 2 & 2 & 1 & 3 & 6 & 1 & 7 & 0 \\
\hline IgG H chain & 5 & 1 & 1 & 1 & 3 & 3 & 2 & 1 & 4 & 0 \\
\hline IgG H chain & 3 & 1 & 3 & 0 & 4 & 0 & 5 & 0 & 4 & 1 \\
\hline IgG L chain & 0 & 2 & 0 & 3 & 0 & 0 & 1 & 4 & 4 & 1 \\
\hline IgG H chain & 3 & 1 & 1 & 1 & 1 & 0 & 2 & 2 & 4 & 2 \\
\hline IgG L chain & 1 & 2 & 1 & 1 & 0 & 0 & 1 & 4 & 2 & 1 \\
\hline IgG L chain & 6 & 0 & 3 & 0 & 1 & 3 & 4 & 1 & 6 & 0 \\
\hline IgG H chain & 1 & 3 & 2 & 1 & 1 & 1 & 0 & 0 & 6 & 0 \\
\hline IgG H chain & 3 & 1 & 2 & 0 & 3 & 0 & 2 & 0 & 1 & 0 \\
\hline IgG L chain & 0 & 2 & 0 & 1 & 0 & 0 & 1 & 2 & 4 & 1 \\
\hline IgG L chain & 3 & 1 & 3 & 2 & 1 & 1 & 4 & 2 & 2 & 0 \\
\hline IgG L chain & 5 & 1 & 1 & 1 & 0 & 1 & 2 & 1 & 6 & 0 \\
\hline IgG L chain & 5 & 2 & 1 & 1 & 0 & 3 & 2 & 2 & 4 & 0 \\
\hline IgG L chain & 3 & 0 & 2 & 1 & 3 & 1 & 5 & 1 & 4 & 0 \\
\hline IgG L chain & 3 & 2 & 1 & 1 & 0 & 0 & 1 & 1 & 7 & 0 \\
\hline IgG L chain & 4 & 0 & 2 & 0 & 0 & 1 & 4 & 1 & 4 & 1 \\
\hline
\end{tabular}




$\begin{array}{lllllllllll}\text { IgG L chain } & 4 & 0 & 1 & 1 & 0 & 1 & 4 & 1 & 4 & 1 \\ \operatorname{IgG~H} \text { chain } & 1 & 1 & 1 & 0 & 1 & 0 & 0 & 0 & 5 & 0 \\ \text { IgG H chain } & 2 & 0 & 2 & 0 & 3 & 0 & 0 & 0 & 1 & 0 \\ \text { IgG L chain } & 3 & 0 & 1 & 1 & 1 & 1 & 1 & 1 & 4 & 0 \\ \text { IgG H chain } & 1 & 0 & 2 & 0 & 1 & 0 & 0 & 0 & 1 & 0 \\ \text { TOTAL } & 125 & 118 & 75 & 69 & 77 & 52 & 121 & 104 & 170 & 74\end{array}$

These are the protein identifications where all elution buffers where grouped according to treatment.

([DiscriptionDatabase] like ' IgG H chain\%' and [identificationfiltermethod]=0) OR

([DescriptionDatabase] like ' IgG L chain\%' and [identificationfiltermethod] $=0$ ) OR

([DescriptionDatabase] like ' IgG VH protein\%' and [identificationfiltermethod] $=0$ ) and Experiments 1 and 2 combined ("NO MODS" for experiment 3 where not put into the SQL database so experiment 3 is not included in human Tables) Corrected for total number of runs

Table 5. IgG identifications over treatments for the classical ligand affinity chromatography method with biotinylated ligands.

\begin{tabular}{|c|c|c|c|c|c|}
\hline Elution Buffer & IGG-SS-B & IGG-B & IGG & $\begin{array}{l}\text { CRUDE ON } \\
\text { STREPTAVIDIN } \\
\text { BEADS }\end{array}$ & $\begin{array}{l}\text { CRUDE ON } \\
\text { CONTROL BEADS }\end{array}$ \\
\hline DTT & 44 & 36 & 31 & 14 & 27 \\
\hline Mercaptoethanol & 26 & 6 & 7 & 14 & 34 \\
\hline Ammonium & 8 & 6 & 5 & 16 & 21 \\
\hline Biotin & 9 & 12 & 15 & 38 & 49 \\
\hline Beads $\left(1 / 9^{\text {th }}\right.$ the & 4 & 37 & 170 & 8 & 8 \\
\hline
\end{tabular}

Data was obtained from [FcBlark_Pass11_human].[dbo].[DistinctProteinMatrix] where [identificationfiltermethod] $=0$ and (([DescriptionFasta] like 'IgG L\%') or ([DescriptionFasta] like 'IgG H\%') or ([DescriptionFasta] like 'IgG VH\%'))

The identification of $\mathrm{Fc}$ gamma receptors at filter 2 was the highest where IgG-SS-B was applied to live cells and eluted with mercaptoethanol (Table 4). Searches by filter 0 showed that Fcgr1 was eluted from the bead digest (140) in the highest quantities closely followed by DTT (136), biotin $+\mathrm{NaOH}$ (66), $\mathrm{NaOH}$ alone (50) and lastly mercaptoethanol (38) (Table 4). At filter 2 Fcgr1 protein identifications were highest on the bead digests (49) followed by biotin and $\mathrm{NH} 4 \mathrm{OH}$ (20), DDT (8), and $\mathrm{NH} 4 \mathrm{OH}$ (3) and no identifications with mecaptoethanol (Table 4). 
The presence of Fcgr1 in the residual bead digest seems to indicate a binding and elution mechanism other than ligand affinity.

Table 6: Protein identifications for Fc and Fc-like receptors by elution buffer using biotinylated ligand affinity chromatography with monovalent ligands.

\begin{tabular}{|c|c|c|c|c|c|c|c|c|c|c|c|}
\hline $\begin{array}{l}\text { Gene } \\
\text { Symbol }\end{array}$ & Filter & DDT & $\begin{array}{l}\text { DTT } \\
\text { STYP }\end{array}$ & BME & $\begin{array}{l}\text { BME } \\
\text { STYP }\end{array}$ & $\mathrm{NH} 4 \mathrm{OH}$ & $\begin{array}{l}\mathrm{NH} 4 \mathrm{OH} \\
\text { STYP }\end{array}$ & BIOTIN & $\begin{array}{l}\text { BIOTIN } \\
\text { STYP }\end{array}$ & $\begin{array}{l}\text { BEAD } \\
\text { DIGEST }\end{array}$ & $\begin{array}{l}\text { BEADS } \\
\text { DIGEST } \\
\text { STYP }\end{array}$ \\
\hline Fcamr & 0 & 81 & 57 & 126 & 34 & 43 & 50 & 105 & 38 & 147 & 40 \\
\hline Fcer1a & 0 & 26 & 14 & 33 & 4 & 12 & 8 & 21 & 14 & 5 & 0 \\
\hline Fcer1g & 0 & 3 & 30 & 12 & 6 & 7 & 26 & 9 & 38 & 11 & 21 \\
\hline Fcer2 & 0 & 4 & 12 & 13 & 15 & 7 & 15 & 1 & 3 & 10 & 11 \\
\hline Fcer2a & 0 & 32 & 72 & 82 & 85 & 46 & 98 & 13 & 21 & 68 & 75 \\
\hline Fcgr1 & 0 & 44 & 18 & 20 & 4 & 26 & 1 & 31 & 16 & 41 & 16 \\
\hline Fcgr2 & 0 & 6 & 10 & 6 & 9 & 4 & 6 & 7 & 11 & 8 & 19 \\
\hline Fcgr2b & 0 & 15 & 24 & 22 & 26 & 14 & 24 & 27 & 34 & 17 & 48 \\
\hline Fcgr3 & 0 & 49 & 59 & 56 & 17 & 13 & 20 & 44 & 32 & 48 & 43 \\
\hline Fcgr4 & 0 & 37 & 22 & 6 & 32 & 40 & 31 & 8 & 3 & 50 & 0 \\
\hline Fcrl1 & 0 & 43 & 34 & 31 & 5 & 44 & 14 & 130 & 27 & 46 & 32 \\
\hline Fcrl5 & 0 & 147 & 39 & 73 & 73 & 61 & 74 & 86 & 70 & 528 & 128 \\
\hline Fcrl6 & 0 & 19 & 10 & 22 & 3 & 20 & 3 & 26 & 12 & 77 & 11 \\
\hline TOTAL & & 505 & 400 & 501 & 313 & 337 & 370 & 509 & 318 & 1056 & 443 \\
\hline Fcamr & 1 & 9 & 12 & 5 & 16 & 8 & 9 & 28 & 9 & 26 & 0 \\
\hline Fcer1a & 1 & 6 & 0 & 0 & 1 & 3 & 3 & 3 & 5 & 0 & 0 \\
\hline Fcer1g & 1 & 0 & 3 & 1 & 3 & 0 & 6 & 2 & 0 & 2 & 8 \\
\hline Fcer2 & 1 & 1 & 3 & 1 & 6 & 2 & 4 & 0 & 0 & 0 & 0 \\
\hline Fcer2a & 1 & 6 & 23 & 9 & 35 & 11 & 31 & 0 & 0 & 0 & 0 \\
\hline Fcgr1 & 1 & 4 & 3 & 0 & 1 & 1 & 1 & 10 & 4 & 14 & 4 \\
\hline Fcgr2 & 1 & 2 & 3 & 0 & 1 & 3 & 2 & 1 & 1 & 0 & 0 \\
\hline Fcgr2b & 1 & 4 & 10 & 0 & 4 & 8 & 6 & 6 & 6 & 0 & 0 \\
\hline Fcgr3 & 1 & 0 & 15 & 0 & 1 & 0 & 3 & 0 & 9 & 0 & 0 \\
\hline Fcgr4 & 1 & 1 & 1 & 0 & 6 & 4 & 10 & 0 & 0 & 0 & 0 \\
\hline Fcrl1 & 1 & 0 & 0 & 20 & 0 & 20 & 2 & 29 & 3 & 0 & 0 \\
\hline Fcrl5 & 1 & 51 & 14 & 22 & 15 & 1 & 10 & 17 & 1 & 368 & 0 \\
\hline Fcrl6 & 1 & 0 & 0 & 4 & 0 & 5 & 0 & 0 & 3 & 0 & 11 \\
\hline TOTAL & & 85 & 88 & 64 & 91 & 67 & 87 & 95 & 41 & 411 & 23 \\
\hline Fcamr & 2 & 9 & 12 & 5 & 16 & 8 & 9 & 28 & 9 & 26 & 0 \\
\hline Fcer1a & 2 & 6 & 0 & 0 & 1 & 3 & 3 & 3 & 5 & 0 & 0 \\
\hline Fcer1g & 2 & 0 & 3 & 1 & 3 & 0 & 6 & 2 & 0 & 2 & 8 \\
\hline Fcer2 & 2 & 1 & 3 & 1 & 6 & 2 & 4 & 0 & 0 & 0 & 0 \\
\hline
\end{tabular}




\begin{tabular}{|c|c|c|c|c|c|c|c|c|c|c|c|}
\hline Fcer2a & 2 & 6 & 23 & 9 & 35 & 11 & 31 & 0 & 0 & 0 & 0 \\
\hline Fcgr1 & 2 & 4 & 3 & 0 & 1 & 1 & 1 & 10 & 4 & 14 & 4 \\
\hline Fcgr2 & 2 & 1 & 3 & 0 & 1 & 3 & 2 & 1 & 1 & 0 & 0 \\
\hline Fcgr2b & 2 & 2 & 10 & 0 & 4 & 8 & 6 & 6 & 6 & 0 & 0 \\
\hline Fcgr3 & 2 & 0 & 15 & 0 & 1 & 0 & 3 & 0 & 9 & 0 & 0 \\
\hline Fcgr4 & 2 & 1 & 1 & 0 & 6 & 4 & 10 & 0 & 0 & 0 & 0 \\
\hline Fcrl1 & 2 & 0 & 0 & 20 & 0 & 20 & 2 & 29 & 3 & 0 & 0 \\
\hline Fcrl5 & 2 & 51 & 14 & 22 & 15 & 1 & 10 & 17 & 1 & 368 & 0 \\
\hline Fcrl6 & 2 & 0 & 0 & 4 & 0 & 5 & 0 & 0 & 3 & 0 & 11 \\
\hline TOTAL & & 82 & 88 & 64 & 91 & 67 & 87 & 95 & 41 & 411 & 23 \\
\hline
\end{tabular}

DTT - 5mM DDT, BME - b mercaptoethanol, NH4OH - 2M ammonium hydroxide, biotin - biotin + 2M ammonium hydroxide, beads - bead digests. STYP indicates the search was conducted considering tyrosine phosphorylations post translational modification. Values shown in the table are the sum of all treatments eluted with each buffer including Ccb, Csa, Lsa, LBsa, LSBsa.

The largest number of Fc gamma receptors identified at filter 2 from the mercaptoethanol elution with comparatively large amounts of Fcgr2 (30), Fcgr2b (121), Fcgr3 (151) (Table 4). The eluted and retained fractions from live cells were digested and analyzed by LC-ESI-MS/MS and revealed the Fc receptors were detected using the IgG-B, IgG-SS-B and IgG ligands. 
Table 7: Fc receptors by treatment using biotinylated affinity receptor chromatography using monovalent ligands.

\begin{tabular}{|c|c|c|c|c|c|c|c|c|c|c|c|}
\hline $\begin{array}{l}\text { Gene } \\
\text { Symbol }\end{array}$ & Filter & $\mathrm{Ccb}$ & $\begin{array}{l}\text { Ccb } \\
\text { STYP }\end{array}$ & Csa & $\begin{array}{l}\text { Csa } \\
\text { STYP }\end{array}$ & Lsa & $\begin{array}{l}\text { Lsa } \\
\text { STYP }\end{array}$ & LBsa & $\begin{array}{l}\text { LBsa } \\
\text { STYP }\end{array}$ & LSBsa & $\begin{array}{l}\text { LSBsa } \\
\text { STYP }\end{array}$ \\
\hline Fcamr & 0 & 81 & 31 & 143 & 60 & 100 & 46 & 103 & 27 & 86 & 55 \\
\hline Fcer1a & 0 & 12 & 15 & 18 & 12 & 23 & 0 & 11 & 3 & 33 & 11 \\
\hline Fcer1g & 0 & 12 & 27 & 22 & 18 & 8 & 19 & 4 & 30 & 4 & 27 \\
\hline Fcer2 & 0 & 4 & 19 & 3 & 13 & 10 & 10 & 7 & 6 & 5 & 7 \\
\hline Fcer2a & 0 & 27 & 118 & 19 & 86 & 66 & 67 & 41 & 35 & 35 & 45 \\
\hline Fcgr1 & 0 & 35 & 17 & 27 & 10 & 28 & 11 & 27 & 11 & 30 & 7 \\
\hline Fcgr2 & 0 & 11 & 6 & 6 & 18 & 2 & 5 & 1 & 7 & 11 & 19 \\
\hline Fcgr2b & 0 & 36 & 15 & 16 & 59 & 9 & 14 & 3 & 14 & 30 & 53 \\
\hline Fcgr3 & 0 & 112 & 20 & 65 & 51 & 20 & 30 & 40 & 29 & 22 & 40 \\
\hline Fcgr4 & 0 & 11 & 17 & 25 & 39 & 37 & 12 & 18 & 1 & 35 & 18 \\
\hline Fcrl1 & 0 & 43 & 4 & 90 & 59 & 36 & 18 & 115 & 1 & 21 & 30 \\
\hline Fcrl5 & 0 & 132 & 60 & 169 & 81 & 164 & 67 & 221 & 93 & 209 & 83 \\
\hline Fcrl6 & 0 & 42 & 9 & 50 & 7 & 51 & 11 & 6 & 0 & 27 & 11 \\
\hline TOTAL & & 556 & 356 & 654 & 512 & 554 & 311 & 597 & 257 & 547 & 407 \\
\hline Fcamr & 1 & 14 & 15 & 20 & 8 & 18 & 0 & 4 & 4 & 27 & 19 \\
\hline Fcer1a & 1 & 0 & 3 & 3 & 3 & 0 & 0 & 2 & 3 & 7 & 0 \\
\hline Fcer1g & 1 & 2 & 11 & 4 & 0 & 0 & 3 & 1 & 3 & 0 & 3 \\
\hline Fcer2 & 1 & 0 & 4 & 0 & 3 & 2 & 2 & 1 & 3 & 1 & 1 \\
\hline Fcer2a & 1 & 0 & 31 & 0 & 17 & 11 & 12 & 9 & 17 & 6 & 10 \\
\hline Fcgr1 & 1 & 1 & 10 & 5 & 3 & 6 & 0 & 12 & 1 & 1 & 0 \\
\hline Fcgr2 & 1 & 1 & 1 & 2 & 3 & 0 & 0 & 0 & 0 & 3 & 3 \\
\hline Fcgr2b & 1 & 2 & 4 & 6 & 11 & 0 & 0 & 0 & 0 & 10 & 12 \\
\hline Fcgr3 & 1 & 0 & 0 & 0 & 5 & 0 & 0 & 0 & 6 & 0 & 17 \\
\hline Fcgr4 & 1 & 0 & 0 & 1 & 6 & 0 & 2 & 4 & 0 & 0 & 10 \\
\hline Fcrl1 & 1 & 0 & 1 & 31 & 2 & 0 & 0 & 38 & 1 & 0 & 0 \\
\hline Fcrl5 & 1 & 69 & 1 & 82 & 23 & 64 & 0 & 142 & 0 & 121 & 16 \\
\hline Fcrl6 & 1 & 2 & 3 & 8 & 0 & 0 & 11 & 0 & 0 & 0 & 0 \\
\hline TOTAL & & 92 & 85 & 161 & 83 & 100 & 30 & 215 & 39 & 176 & 91 \\
\hline Fcamr & 2 & 14 & 15 & 20 & 8 & 18 & 0 & 4 & 4 & 27 & 19 \\
\hline Fcer1a & 2 & 0 & 3 & 3 & 3 & 0 & 0 & 2 & 3 & 7 & 0 \\
\hline Fcer1g & 2 & 2 & 11 & 4 & 0 & 0 & 3 & 1 & 3 & 0 & 3 \\
\hline Fcer2 & 2 & 0 & 4 & 0 & 3 & 2 & 2 & 1 & 3 & 1 & 1 \\
\hline Fcer2a & 2 & 0 & 31 & 0 & 17 & 11 & 12 & 9 & 17 & 6 & 10 \\
\hline Fcgr1 & 2 & 1 & 10 & 5 & 3 & 6 & 0 & 12 & 1 & 1 & 0 \\
\hline Fcgr2 & 2 & 1 & 1 & 2 & 3 & 0 & 0 & 0 & 0 & 2 & 3 \\
\hline Fcgr2b & 2 & 2 & 4 & 6 & 11 & 0 & 0 & 0 & 0 & 8 & 12 \\
\hline Fcgr3 & 2 & 0 & 0 & 0 & 5 & 0 & 0 & 0 & 6 & 0 & 17 \\
\hline Fcgr4 & 2 & 0 & 0 & 1 & 6 & 0 & 2 & 4 & 0 & 0 & 10 \\
\hline Fcrl1 & 2 & 0 & 1 & 31 & 2 & 0 & 0 & 38 & 1 & 0 & 0 \\
\hline Fcrl5 & 2 & 69 & 1 & 82 & 23 & 64 & 0 & 142 & 0 & 121 & 16 \\
\hline
\end{tabular}




\begin{tabular}{|lccccccccccc|} 
Fcrl6 & 2 & 2 & 3 & 8 & 0 & 0 & 11 & 0 & 0 & 0 & 0 \\
\hline TOTAL & 92 & 85 & 161 & 83 & 100 & 30 & 215 & 39 & 173 & 91 \\
\hline
\end{tabular}

For each treatment Ccb- crude homogenates applied to control agarose beads, Csa - crude homogenated applied to SA-agarose beads, Lsa - IgG incubated with live cells applied to SA-agarose, LBsa- IgG-b incubated with live cells applied to SA-agarose, LSBsa - IgG-ss-b incubated with live cells applied to SA-agarose. STYP indicates the search was conducted considering tyrosine phosphorylations post translational modification. Values in the table are the sum of all the elution buffers for the treatment indicated (DTT, BME, NH4OH, NH4OH+Biotin, Bead digests and were corrected by the largest number of runs)

\section{KEGG Fc receptor pathway}

The Kyoto Encyclopedia of Genes and Genomes (KEGG) lists the expected members of the Fc receptor complex. An additional way to compare the efficacy of the various affinity techniques was to determine the number of known Fc pathway proteins isolated with the $\mathrm{IgG}, \mathrm{B}-\mathrm{IgG}$ and BS-S-IgG ligands. The reducing agent DTT should cleave the disulphide linker and thus release the biotinylated $\mathrm{IgG}$ with its cognate receptor (S-IgG-FcRs) and might also be complexed with some of the Fc associated receptor complex. The Kegg pathway obtained from the ratio of (IgGSS-B applied to SA-agarose and eluted by DTT and BME)/(IgG-B applied to SA-agarose and eluted by DTT and BME) does show a highly interconnected network (supplements table) by STRING.

\section{Monomeric versus aggregated}

In this study, monovalent IgG was compared to polyvalent $\operatorname{IgG}$ that was aggregated with heat treatment at $50^{\circ} \mathrm{C}$ for 30 minutes with shaking (Frøland, Natvig, \& Michaelsen, 1974). The biotinylation of IgG with both NHS-biotin and NHS-ss-biotin was performed both before and after heat aggregation and captured and eluted as previously described with similar results. 
Table 8: Comparison of aggregated versus monovalent ligands (bead digest not included - no bead digests collected for the aggregated ligands)

\begin{tabular}{|c|c|c|c|c|c|c|c|c|c|c|c|c|c|}
\hline $\begin{array}{l}\text { Gene } \\
\text { Symbol }\end{array}$ & Filter & LBsa & $\begin{array}{l}\text { LBsa } \\
\text { STYP }\end{array}$ & LABsa & $\begin{array}{l}\text { LABsa } \\
\text { STYP }\end{array}$ & LBAsa & $\begin{array}{l}\text { LBAsa } \\
\text { STYP }\end{array}$ & LSBsa & $\begin{array}{l}\text { LSBsa } \\
\text { STYP }\end{array}$ & LASBsa & $\begin{array}{l}\text { LASBsa } \\
\text { STYP }\end{array}$ & LSBAsa & $\begin{array}{l}\text { LSBAsa } \\
\text { STYP }\end{array}$ \\
\hline Fcamr & 0 & 79 & 15 & 0 & 0 & 48 & 0 & 62 & 43 & 64 & 16 & 48 & 0 \\
\hline Fcer1a & 0 & 11 & 3 & 0 & 32 & 16 & 0 & 33 & 11 & 64 & 0 & 32 & 0 \\
\hline Fcer1g & 0 & 4 & 26 & 0 & 0 & 0 & 0 & 4 & 23 & 0 & 0 & 0 & 0 \\
\hline Fcer2 & 0 & 7 & 6 & 0 & 0 & 0 & 0 & 5 & 7 & 16 & 0 & 0 & 0 \\
\hline Fcer2a & 0 & 41 & 35 & 0 & 16 & 0 & 0 & 35 & 45 & 112 & 0 & 0 & 0 \\
\hline Fcgr1 & 0 & 19 & 7 & 16 & 16 & 0 & 32 & 26 & 3 & 32 & 0 & 0 & 0 \\
\hline Fcgr2 & 0 & 1 & 3 & 0 & 0 & 16 & 0 & 7 & 15 & 0 & 0 & 0 & 0 \\
\hline Fcgr2b & 0 & 3 & 6 & 0 & 0 & 64 & 0 & 22 & 45 & 0 & 0 & 0 & 0 \\
\hline Fcgr3 & 0 & 40 & 29 & 32 & 0 & 80 & 80 & 22 & 40 & 0 & 0 & 0 & 0 \\
\hline Fcgr4 & 0 & 18 & 1 & 0 & 0 & 0 & 0 & 35 & 18 & 0 & 0 & 0 & 0 \\
\hline Fcrl1 & 0 & 87 & 1 & 0 & 0 & 0 & 0 & 21 & 30 & 16 & 0 & 0 & 0 \\
\hline Fcrl5 & 0 & 81 & 45 & 0 & 256 & 16 & 0 & 105 & 35 & 0 & 0 & 0 & 16 \\
\hline Fcrl6 & 0 & 6 & 0 & 0 & 0 & 0 & 0 & 11 & 11 & 0 & 0 & 0 & 0 \\
\hline TOTAL & & 397 & 177 & 48 & 320 & 240 & 112 & 387 & 327 & 304 & 16 & 80 & 16 \\
\hline Fcamr & 1 & 4 & 4 & 0 & 0 & 0 & 0 & 15 & 19 & 16 & 0 & 48 & 0 \\
\hline Fcer1a & 1 & 2 & 3 & 0 & 0 & 0 & 0 & 7 & 0 & 0 & 0 & 0 & 0 \\
\hline Fcer1g & 1 & 1 & 3 & 0 & 0 & 0 & 0 & 0 & 3 & 0 & 0 & 0 & 0 \\
\hline Fcer2 & 1 & 1 & 3 & 0 & 0 & 0 & 0 & 1 & 1 & 0 & 0 & 0 & 0 \\
\hline Fcer2a & 1 & 9 & 17 & 0 & 16 & 0 & 0 & 6 & 10 & 0 & 0 & 0 & 0 \\
\hline Fcgr1 & 1 & 4 & 1 & 0 & 0 & 0 & 0 & 1 & 0 & 0 & 0 & 0 & 0 \\
\hline Fcgr2 & 1 & 0 & 0 & 0 & 0 & 16 & 0 & 3 & 3 & 0 & 0 & 0 & 0 \\
\hline Fcgr2b & 1 & 0 & 0 & 0 & 0 & 64 & 0 & 10 & 12 & 0 & 0 & 0 & 0 \\
\hline Fcgr3 & 1 & 0 & 6 & 32 & 0 & 80 & 0 & 0 & 17 & 0 & 0 & 0 & 0 \\
\hline Fcgr4 & 1 & 4 & 0 & 0 & 0 & 0 & 0 & 0 & 10 & 0 & 0 & 0 & 0 \\
\hline Fcrl1 & 1 & 38 & 1 & 0 & 0 & 0 & 0 & 0 & 0 & 0 & 0 & 0 & 0 \\
\hline Fcrl5 & 1 & 22 & 0 & 0 & 0 & 0 & 0 & 37 & 16 & 0 & 0 & 0 & 0 \\
\hline Fcrl6 & 1 & 0 & 0 & 0 & 0 & 0 & 0 & 0 & 0 & 0 & 0 & 0 & 0 \\
\hline TOTAL & & 87 & 39 & 32 & 16 & 160 & 0 & 80 & 91 & 16 & 0 & 48 & 0 \\
\hline Fcamr & 2 & 4 & 4 & 0 & 0 & 0 & 0 & 15 & 19 & 16 & 0 & 48 & 0 \\
\hline Fcer1a & 2 & 2 & 3 & 0 & 0 & 0 & 0 & 7 & 0 & 0 & 0 & 0 & 0 \\
\hline Fcer1g & 2 & 1 & 3 & 0 & 0 & 0 & 0 & 0 & 3 & 0 & 0 & 0 & 0 \\
\hline Fcer2 & 2 & 1 & 3 & 0 & 0 & 0 & 0 & 1 & 1 & 0 & 0 & 0 & 0 \\
\hline Fcer2a & 2 & 9 & 17 & 0 & 16 & 0 & 0 & 6 & 10 & 0 & 0 & 0 & 0 \\
\hline Fcgr1 & 2 & 4 & 1 & 0 & 0 & 0 & 0 & 1 & 0 & 0 & 0 & 0 & 0 \\
\hline Fcgr2 & 2 & 0 & 0 & 0 & 0 & 16 & 0 & 2 & 3 & 0 & 0 & 0 & 0 \\
\hline Fcgr2b & 2 & 0 & 0 & 0 & 0 & 64 & 0 & 8 & 12 & 0 & 0 & 0 & 0 \\
\hline Fcgr3 & 2 & 0 & 6 & 32 & 0 & 80 & 0 & 0 & 17 & 0 & 0 & 0 & 0 \\
\hline Fcgr4 & 2 & 4 & 0 & 0 & 0 & 0 & 0 & 0 & 10 & 0 & 0 & 0 & 0 \\
\hline Fcrl1 & 2 & 38 & 1 & 0 & 0 & 0 & 0 & 0 & 0 & 0 & 0 & 0 & 0 \\
\hline Fcrl5 & 2 & 22 & 0 & 0 & 0 & 0 & 0 & 37 & 16 & 0 & 0 & 0 & 0 \\
\hline
\end{tabular}




\begin{tabular}{|lccccccccccccc|} 
Fcrl6 & 2 & 0 & 0 & 0 & 0 & 0 & 0 & 0 & 0 & 0 & 0 & 0 & 0 \\
\hline TOTAL & 87 & 39 & 32 & 16 & 160 & 0 & 77 & 91 & 16 & 0 & 48 & 0 \\
\hline
\end{tabular}

For each treatment LBsa - IgG-B applied to live cells and captured by a SA-agarose column, LABsa heat aggregated and then NHS biotinylated IgG ligand applied to live cells and captured by a SA-agarose column, LBAsa - NHS biotinylated IgG then heat aggregated ligand applied to live cells and captured by a SA-agarose column, LSBsa - IgG-SS-B applied to live cells and captured by a SA-agarose column, LASBsa - heat aggregated and then NHS-SS-biotinylated ligand applied to live cells and captured by a SA-agarose column, LSBAsa - NHS-SS-biotinylated then heat aggregated ligand applied to live cells and captured by a SA-agarose column. STYP indicates the search was conducted considering tyrosine phosphorylations post translational modification. Values in the table are the sum of all the elution buffers for the treatment indicated (DTT, BME, $\mathrm{NH}_{4} \mathrm{OH}, \mathrm{NH}_{4} \mathrm{OH}+$ Biotin). The results were compared between treatments after correction for the number of LC-ESI runs.

NHS-biotinylated then heat activated IgG (LBAsa) applied to agarose resulted in no Fcgr1, 16 Fcgr2, 64 Fcgr2b and 80 Fcgr3 protein identifications using filter method 2 (these were corrected by the largest number of runs) (Table 6). Heat activated and then biotinylated IgG (LABsa) resulted in no Fcgr1 and 32 Fcgr3 identifications at FM 2 (Table 6). NHS-SS-biotinylation after heat aggregated IgG yielded 32 Fcgr1 identifications at filter 0 (Table 6). NHS-SS-biotinylation after heat aggregated IgG yielded 40 Fcgr1 identifications at filter 2 (Table 6). In contrast, NHSSS-biotinylation of IgG followed by aggregation captured no Fc gamma receptors even at filter 0. Thus, we observed that aggregation first, followed by biotinylation second was more effective in capturing Fcgr1.

\section{Chi square test}

The peptide to protein ratio for this set of experimental data was compared to that of random expectation generated by noise using the Chi square test to determine the probability that these experimental results occurred by chance (Zhu et al., 2011). As expected, the data from the classical affinity chromatography experiment with biotinylated ligands shows a greater number of proteins with high peptide counts and a reduced number of proteins with low peptide counts compared to noise (Table. 7) 
Table 9: Chi square comparison of peptide to protein ratio for the Ligand biotinylation data compared to Noise (solvents without sample applied)

\begin{tabular}{|l|l|l|l|l|l|l|}
\hline bins & Noise & Noise $\mathrm{Hz}$ & FcBLARC & FcBLARC Hz & Noise expect & NoiseChi Square \\
\hline $19-5061$ & 16133 & 0.157174311 & 50087 & 0.48197652 & 16333.55442 & 69751.81636 \\
\hline 18 & 1594 & 0.015529403 & 1955 & 0.018812548 & 1613.815518 & 72.13144853 \\
\hline 17 & 1822 & 0.017750672 & 2023 & 0.019466898 & 1844.649858 & 17.24380001 \\
\hline 16 & 1941 & 0.018910019 & 2202 & 0.021189376 & 1965.129184 & 28.55170222 \\
\hline 15 & 2089 & 0.020351896 & 2231 & 0.021468437 & 2114.969019 & 6.365667014 \\
\hline 14 & 2365 & 0.023040801 & 2385 & 0.022950346 & 2394.400062 & 0.036903262 \\
\hline 13 & 2722 & 0.026518842 & 2305 & 0.022180523 & 2755.838042 & 73.75431254 \\
\hline 12 & 2996 & 0.029188262 & 2588 & 0.024903772 & 3033.244223 & 65.35656325 \\
\hline 11 & 3325 & 0.032393515 & 2622 & 0.025230947 & 3366.334126 & 164.5806001 \\
\hline 10 & 3757 & 0.036602237 & 2829 & 0.027222864 & 3803.704454 & 249.7693458 \\
\hline 9 & 4286 & 0.041755972 & 2851 & 0.027434565 & 4339.28062 & 510.4484819 \\
\hline 8 & 4863 & 0.047377343 & 3137 & 0.030186682 & 4923.45349 & 648.206808 \\
\hline 7 & 5524 & 0.053817076 & 3094 & 0.029772902 & 5592.67059 & 1116.34587 \\
\hline 6 & 6170 & 0.060110674 & 3278 & 0.031543495 & 6246.70122 & 1410.854565 \\
\hline 5 & 6652 & 0.064806516 & 3271 & 0.031476135 & 6734.693114 & 1781.39817 \\
\hline 4 & 7230 & 0.070437629 & 3318 & 0.031928406 & 7319.878415 & 2187.882085 \\
\hline 3 & 7948 & 0.07743268 & 3607 & 0.034709392 & 8046.8041 & 2449.650843 \\
\hline 2 & 8224 & 0.080121585 & 3506 & 0.03373749 & 8326.235143 & 2790.536951 \\
\hline 1 & 8011 & 0.078046452 & 3558 & 0.034237875 & 8110.587273 & 2555.431583 \\
\hline 0 & 4992 & 0.048634114 & 3073 & 0.029570824 & 5054.057129 & 776.5221583 \\
\hline SUM & 102644 & & 103920 & & 103920 & 86656.88422 \\
\hline
\end{tabular}


Ligand affinity receptor chromatography on micro melamine, micro PMMA and Glass nano beads

In order to reduce the non-specific binding observed with previous polystyrene microbeads the ligand, background and receptor binding were compared on more polar surfaces including silica, melamine and PMMA. The binding of IgG alone to silica beads was confirmed by Dumbroff protein assay and a dot blot on PVDF and Western by Dk -anti-hIgG conjugated to HRP (Fig. 49 $\& 50)$.

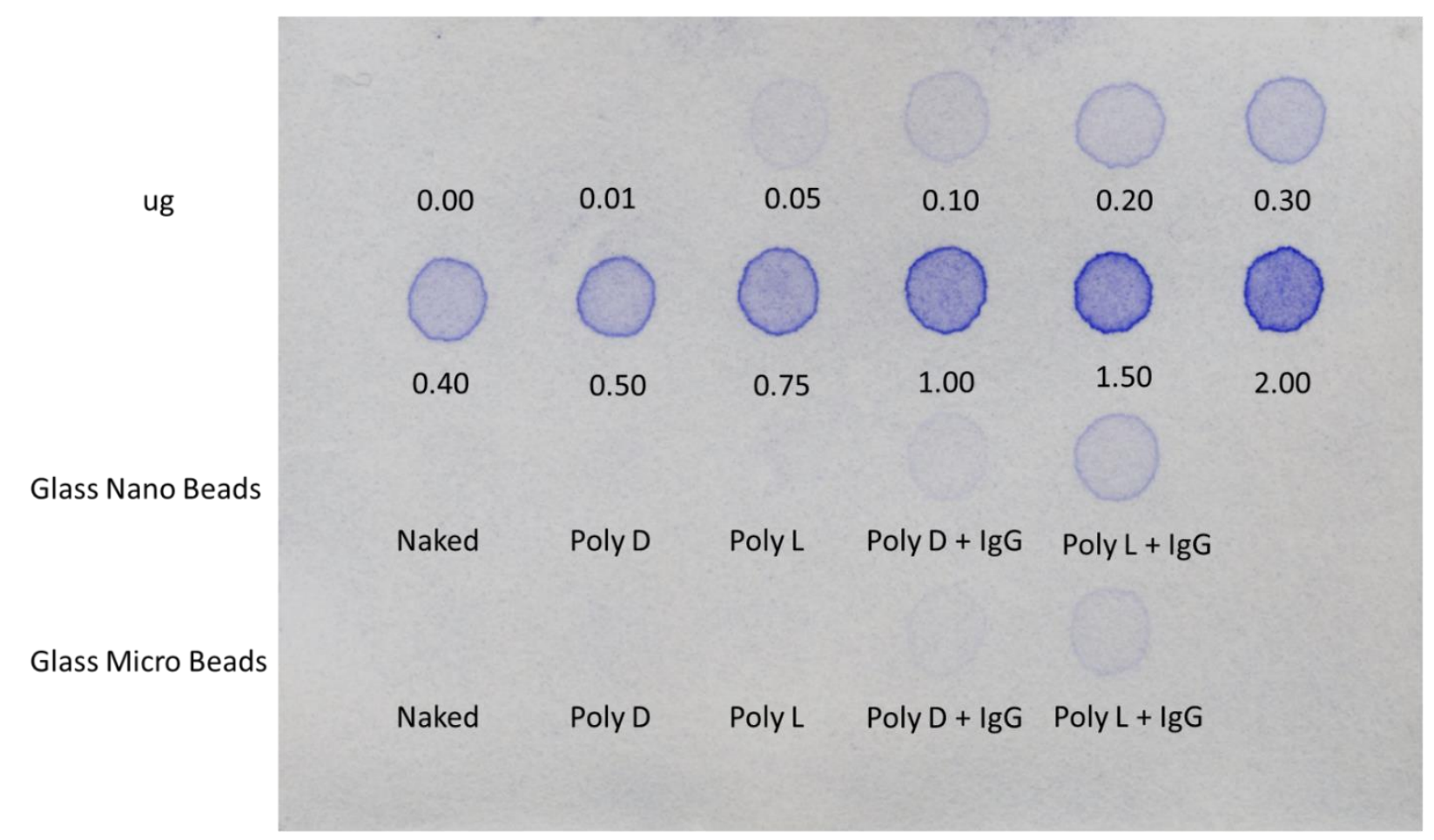

Figure 49: Dumbroff protein assay of IgG boiled off 5ug of IgG coated glass beads in 200uL of $2 \%$ SDS. Roughly 100ng of IgG eluted from the glass beads when boiled in SDS. 


\section{$\begin{array}{lllllll}0 & 1 & 10 & 100 & 1 & 10 & 100\end{array}$ \\ pg pg pg ng ng ng}

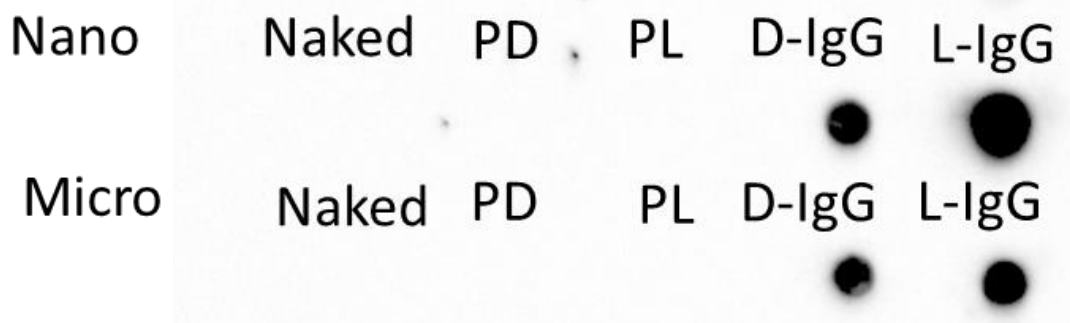

Figure 50: Dot blot western of IgG boiled off $5 \mathrm{ug}$ of IgG coated glass beads in 200uL of $2 \%$ SDS using a Dk-anti-hIgG conjugated to HRP. Roughly 100ng of IgG eluted from the glass beads when boiled in SDS.
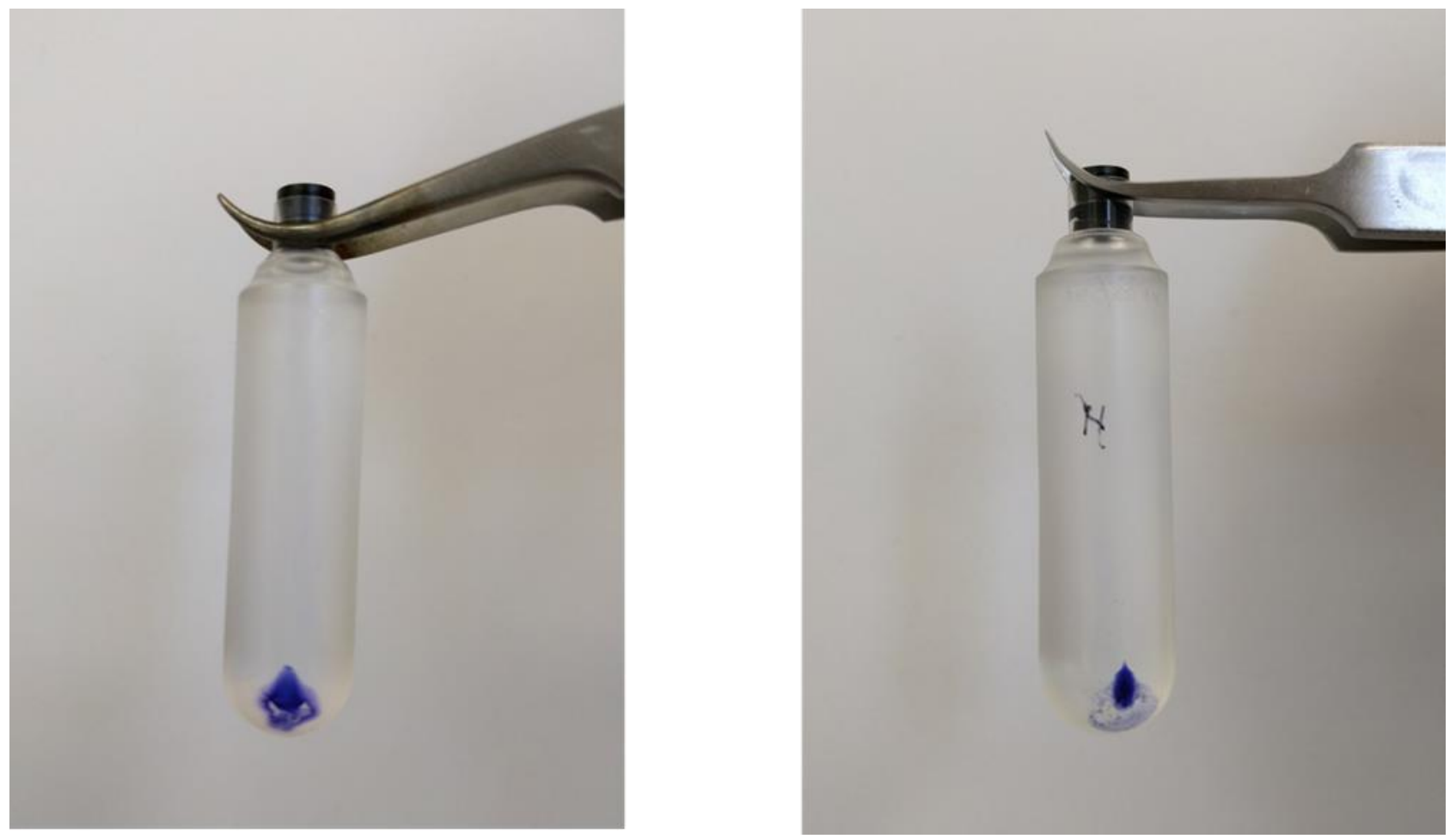

Figure 51: Coomassie stained BSA coated glass micro beads (left) and nano beads (right) applied to a sucrose gradient. 
A
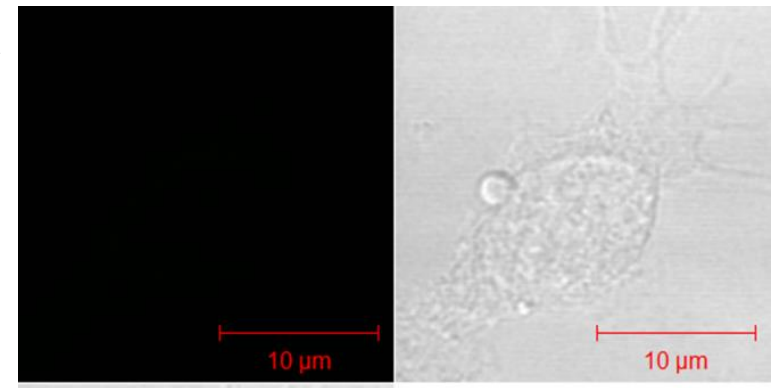

C

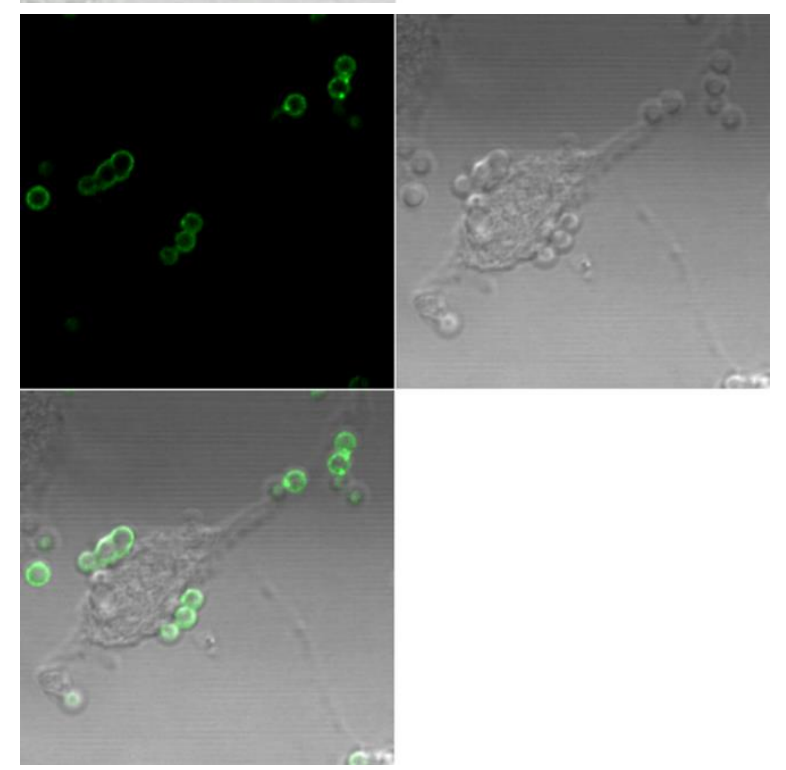

B
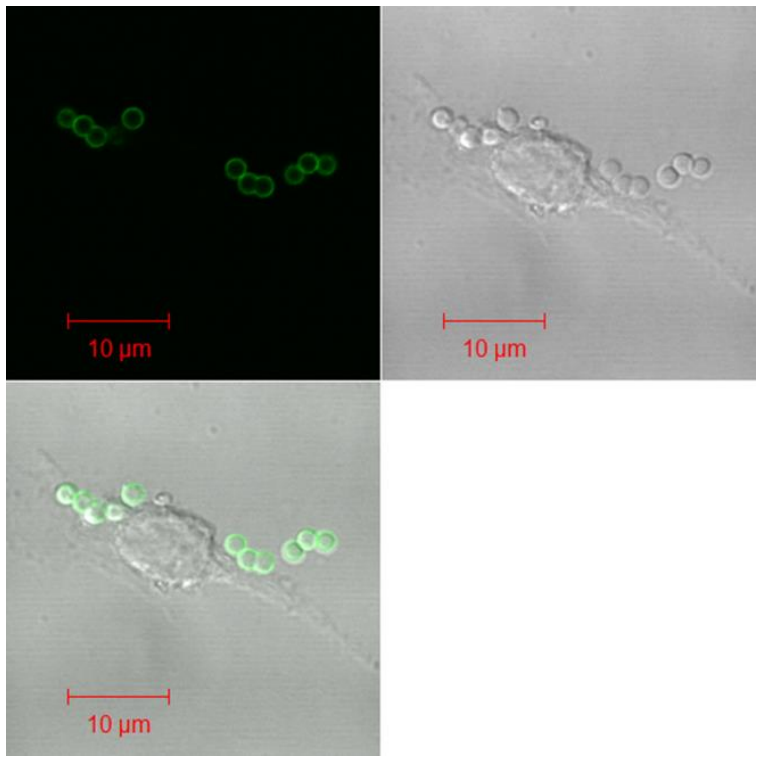

Figure 52: A. Glass beads without coating (Control) applied to RAW macrophages for 20 minutes in HEPES buffer at $37^{\circ} \mathrm{C}$. Outside beads visualized with Dk anti Human $2^{\circ} \mathrm{Ab}$ conjugated to Dy488. B. Glass beads coated with poly D lysine and IgG and applied to RAW macrophages for 20 minutes in HEPES buffer at $37^{\circ} \mathrm{C}$. Outside beads visualized with Dk anti Human $2^{\circ} \mathrm{Ab}$ conjugated to Dy488 C. Glass beads coated with poly-D-lysine and IgG and applied to RAW macrophages for 20 minutes in HEPES buffer at $37^{\circ} \mathrm{C}$. Outside beads visualized with Dk anti Human $2^{\circ}$ Ab conjugated to Dy488. 


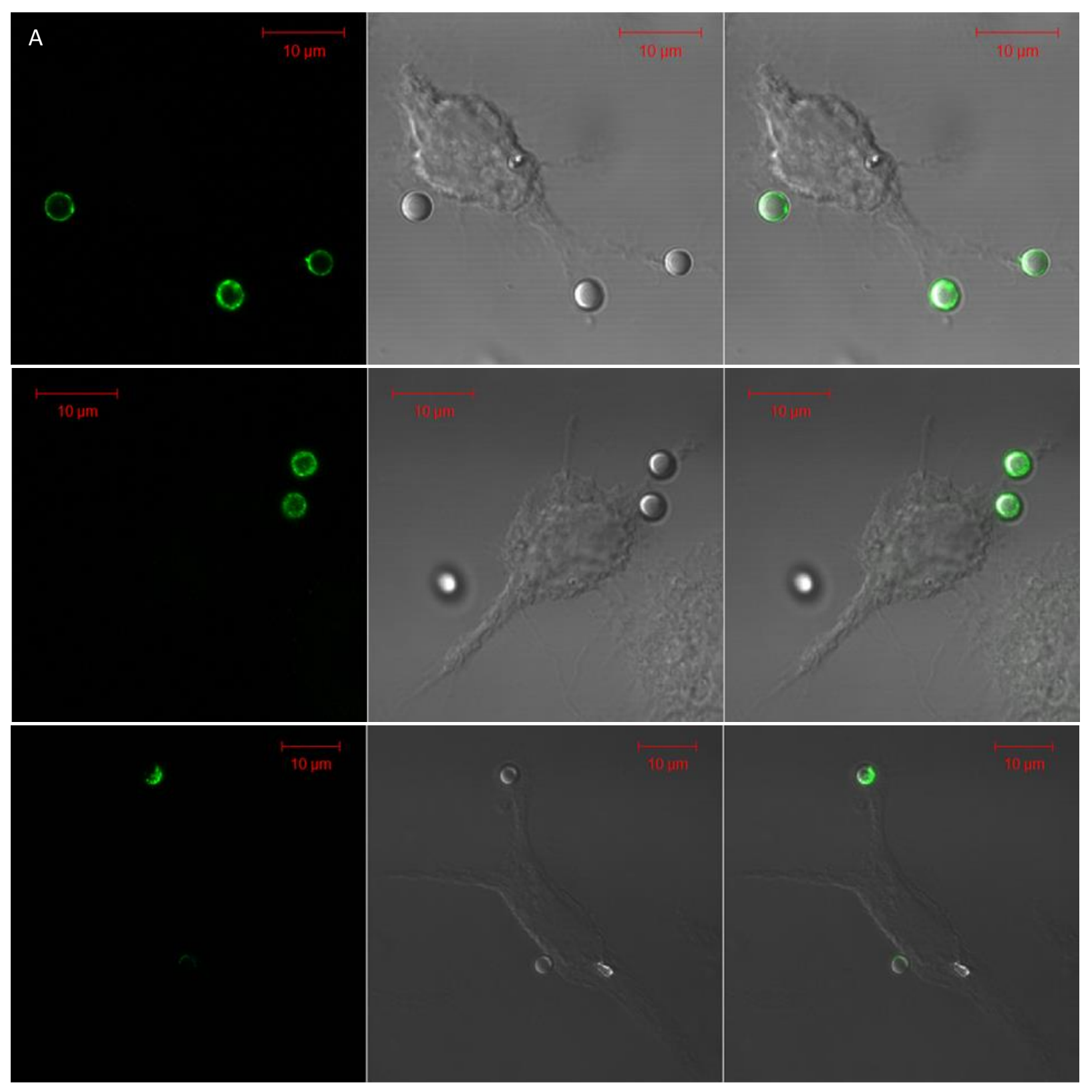

Figure 53: PMMA microbeads washed in (A) MeOH, (B) isopropanol or (C) $5 \%$ formic acid prior to coating in IgG then washed with serum free media, cooled for $10 \mathrm{~min}$ on ice and applied to cells for 30 min to allow binding. Outside beads visualized with Dk anti Human $2^{\circ} \mathrm{Ab}$ conjugated to Dy488. 


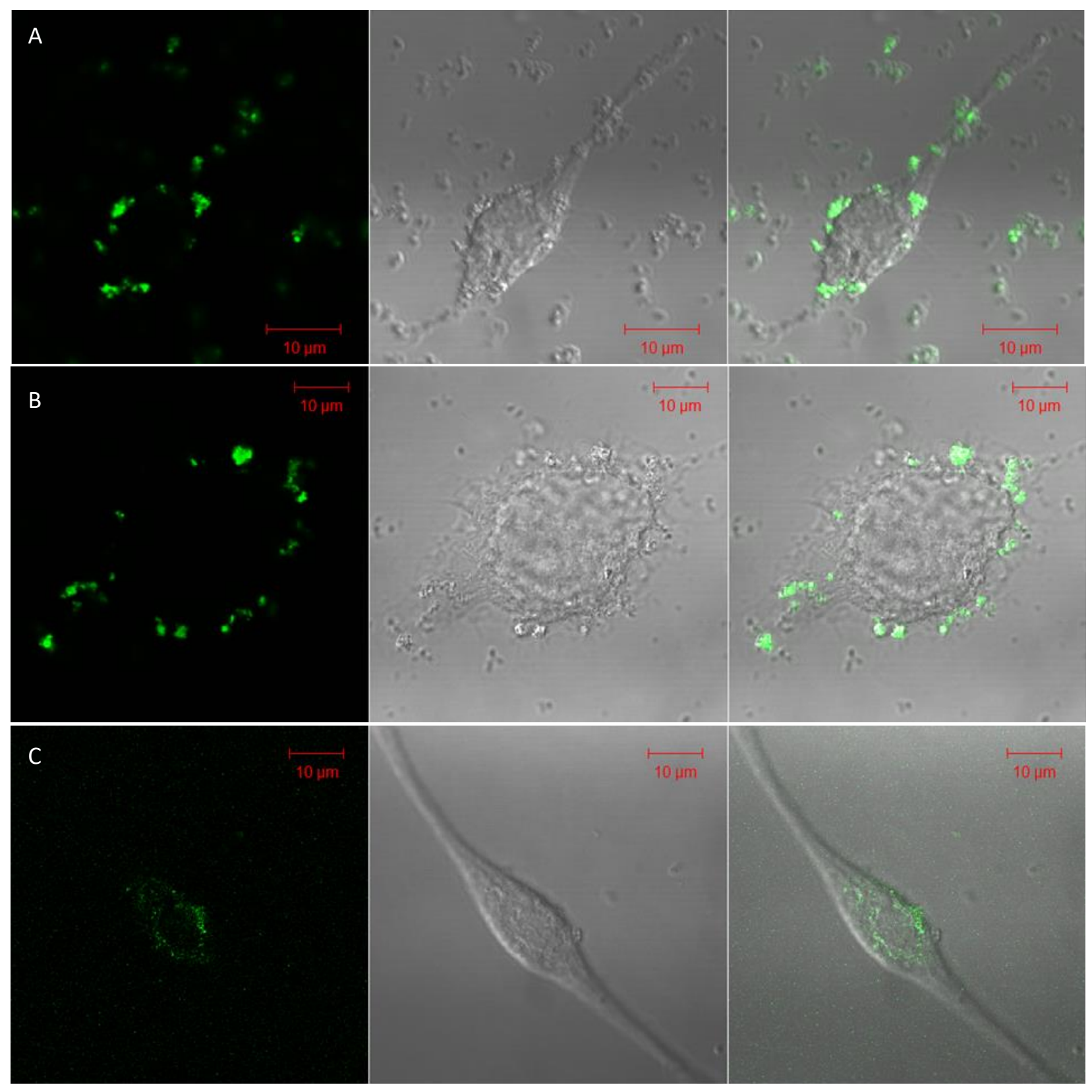

Figure 54: PMMA Nano beads washed in (A) MeOH, (B) isopropanol or (C) $5 \%$ formic acid prior to coating in IgG then washed with serum free media, cooled for 10 min on ice and applied to cells for $\mathbf{3 0}$ min to allow binding. Outside beads visualized with Dk anti Human $2^{\circ} \mathrm{Ab}$ conjugated to Dy488. 


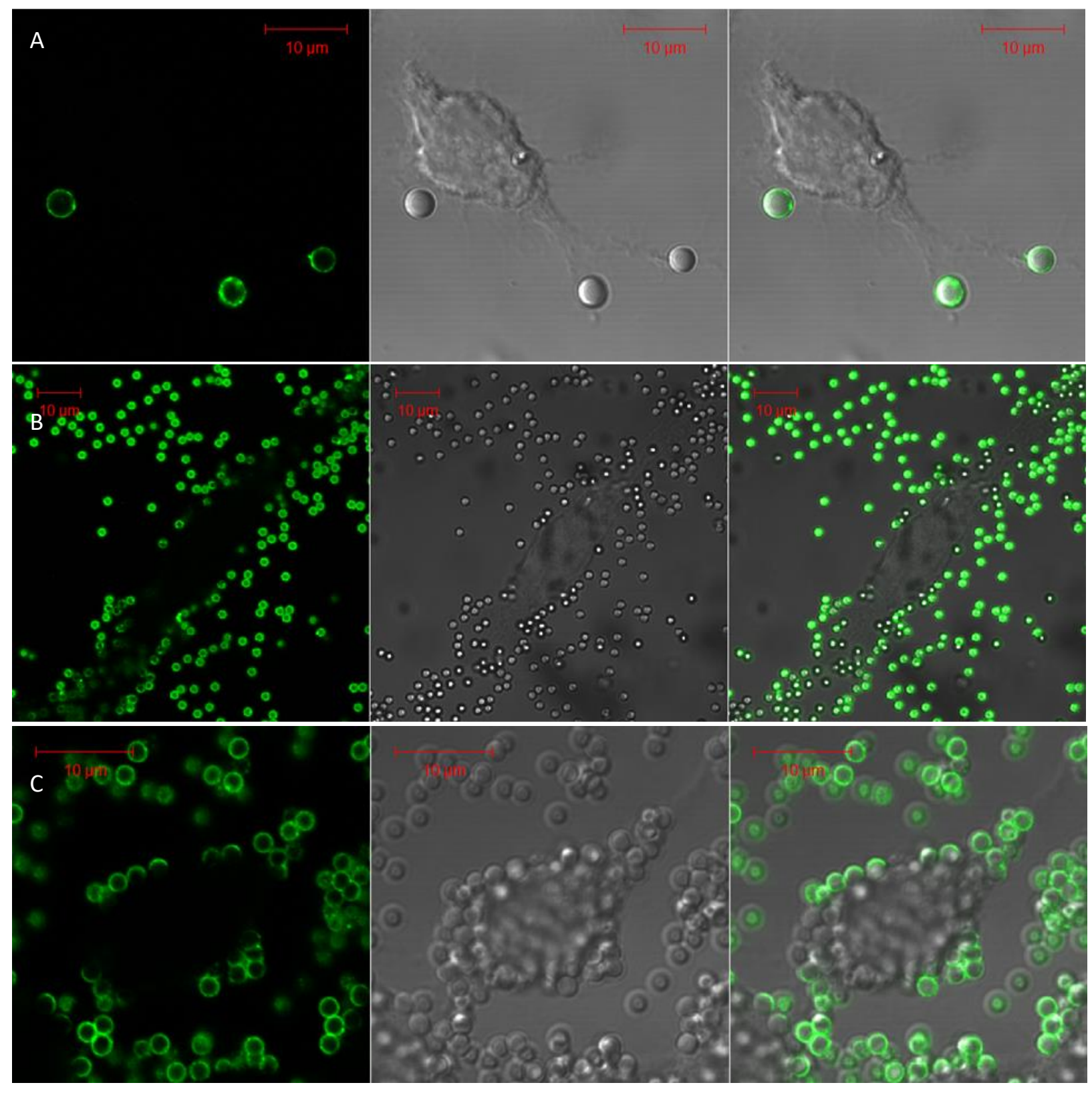

Figure 55: (A) PMMA, (B) Melamine and (C) glass micro beads coated in IgG and applied to cells washed $3 x$ in serum free media, incubated on ice and ligand coated beads applied for $30 \mathrm{~min}$ on ice. Outside beads visualized with Dk anti Human $2^{\circ} \mathrm{Ab}$ conjugated to Dy488. 


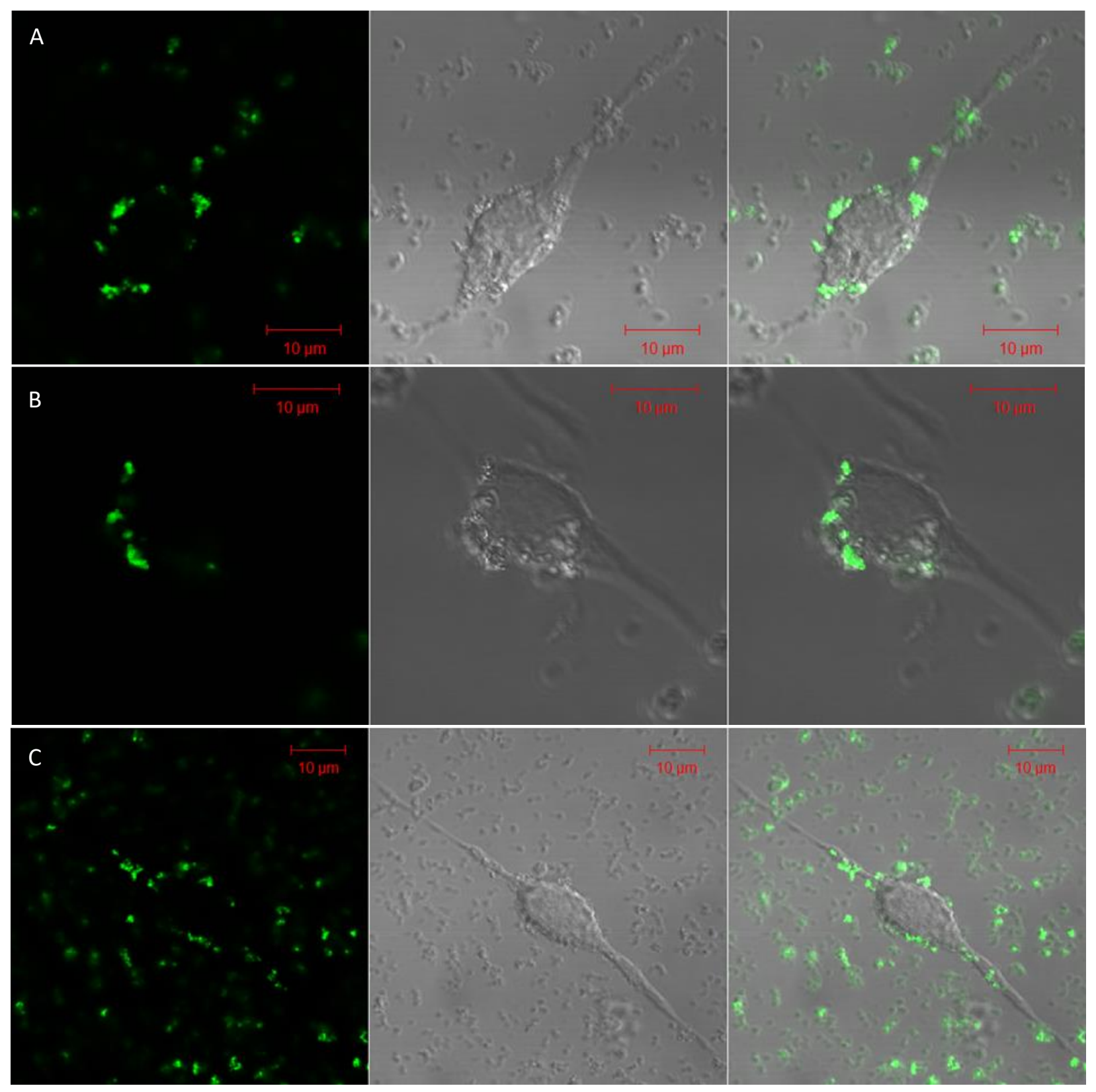

Figure 56: (A) PMMA, (B) Melamine and (C) glass nano beads coated in IgG and applied to cells washed $3 x$ in serum free media, incubated on ice and ligands applied for 30 min on ice. Outside beads visualized with Dk anti Human $2^{\circ} \mathrm{Ab}$ conjugated to Dy488.

Scanning confocal laser microscopy was used to show that human IgG1 was able to coat silica, melamine and PMMA beads in addition to polystyrene beads as previously shown. Each of these 
ligand coated beads in both micro and nano format was able to bind live Raw 264.7 macrophages as shown in figures 52 to 56 . PMMA prewashed in methanol seemed to show the strongest signal compared to prewashing in isopropanol or $5 \%$ formic acid by laser scanning confocal microscopy (Fig.53).

IgG coated melamine micro beads, PMMA micro beads and glass nano beads were applied to live cells, homogenized by French pressing, isolated by ultracentrifugation and sequentially separated into 26 fractions of salt in 1xPBS with an additional $50-1000 \mathrm{mM}$ of $\mathrm{NaCl}$ followed by PBS $+650 \mathrm{mM} \mathrm{NaCl}$ plus a step gradient of acetonitrile from 10 to $70 \%$ for Dumbroff protein assays and Western dot blots probed by Dk anti-hIgG-HRP (Fig. 57 - Fig 61).

On average, roughly $0.2 \mathrm{ug} / \mathrm{uL}$ was extracted per fraction using the IgG coated PMMA and melamine beads for a total of ( $1 \mathrm{uL}$ per dot x $0.2 \mathrm{ug} / \mathrm{ul}$ x $200 \mathrm{uL}$ per fraction $\mathrm{x} 26$ fractions) 1,040 ug of protein extracted per experiment compared to roughly 50ug in the classical biotinylated ligand affinity chromatography experiments with SA-agarose beads. 


\begin{tabular}{|cccccccc|}
\hline & 0 & 0.05 & 0.10 & 0.20 & 0.30 & 0.40 \\
\hline IgG standard (ug/uL) & 0 & 0 & 0 & 0 & 0 & 0 \\
& 0.50 & 0.75 & 1.00 & 1.25 & 1.50 & 2.00 \\
\hline & PBS NaCl & 50 & 100 & 150 & 200 & 250 \\
& 300 & 350 & 400 & 450 & 500 & 550 \\
& & & & & & & \\
& 600 & 650 & 700 & 800 & 900 & 1000 \\
& $10 \%$ & $20 \%$ & $30 \%$ & $40 \%$ & $50 \%$ & $60 \%$ \\
& & & & & & & \\
\hline
\end{tabular}

Figure 57. Dumbroff dot blot of IgG coated melamine micro beads applied to RAW cells in serum free media, isolated by sucrose gradient and sequentially extracted in increasing salt buffers followed by increasing acetonitrile. 


\begin{tabular}{|c|c|c|c|c|c|c|c|}
\hline IgG Standard & $0 \mathrm{pg}$ & 1pg & 10pg & $\begin{array}{c}\text { 100pg } \\
8\end{array}$ & 1ng & 10ng & 100ng \\
\hline \multirow[t]{6}{*}{$1 \times \mathrm{PBS}+\mathrm{mM} \mathrm{NaCl}$} & PBS & 50 & 100 & 150 & 200 & 250 & 300 \\
\hline & 0 & 8 & $\bullet$ & $\wedge$ & , & $\because$ & \\
\hline & 350 & 400 & 450 & 500 & 550 & 600 & 650 \\
\hline & * & • & $\bullet$ & $n$ & 4 & & \\
\hline & 700 & 800 & 900 & 1000 & $10 \%$ & $20 \%$ & $30 \%$ \\
\hline & $40 \%$ & $50 \%$ & $60 \%$ & $70 \%$ & & & \\
\hline
\end{tabular}

Figure 58. Western dot blot of human IgG coated melamine micro beads applied to RAW cells in serum free media, isolated by a sucrose gradient and sequentially extracted in increasing salt buffers up to $1000 \mathrm{mM} \mathrm{NaCl}$ followed by $650 \mathrm{mM} \mathrm{NaCl}$ with increasing amounts of acetonitrile. Detected by a Dk anti Human IgG $(\mathrm{L}+\mathrm{H})$ conjugated to HRP and ECl.

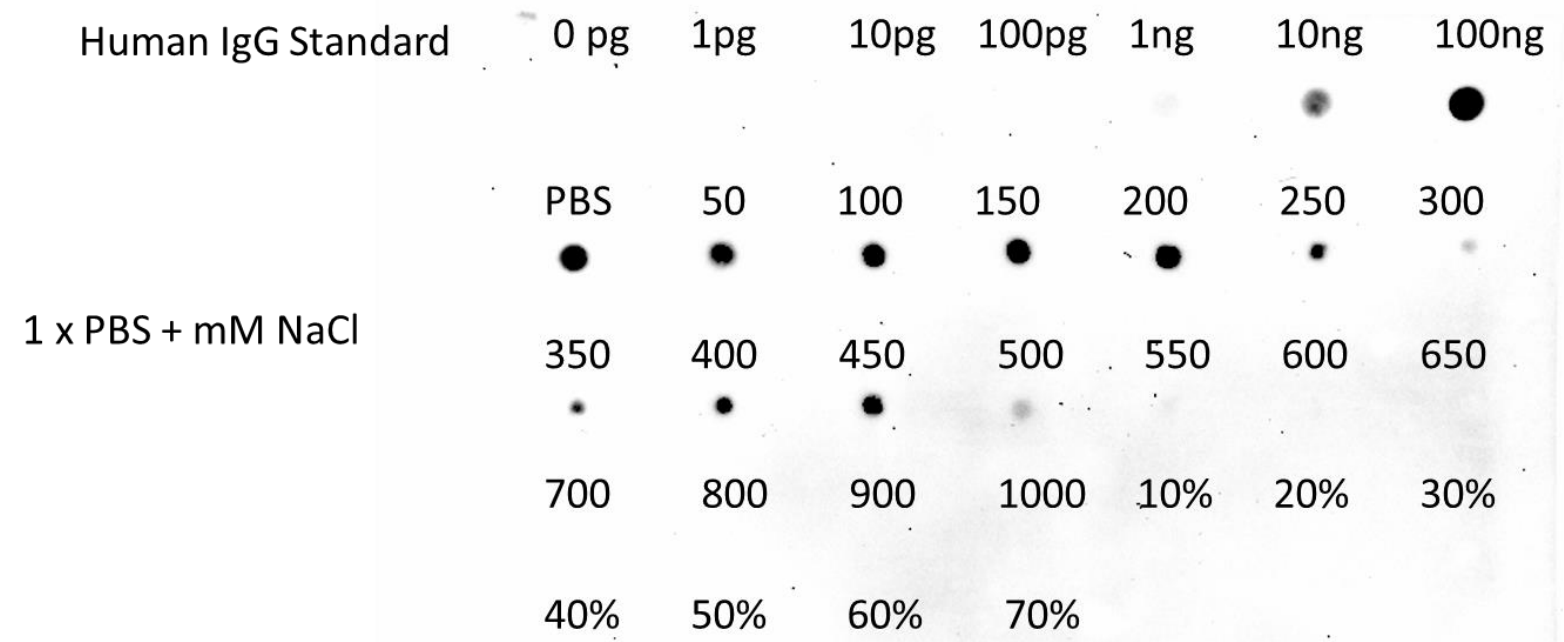

Figure 59. Western dot blot against mouse Fcgr1 from a LARC experiment with IgG coated melamine micro beads applied to RAW cells in serum free media, isolated by a sucrose gradient and sequentially extracted in increasing salt buffers up to $1000 \mathrm{mM} \mathrm{NaCl}$ followed by $650 \mathrm{mM} \mathrm{NaCl}$ with increasing amounts of acetonitrile. Detected by a Rabbit 
anti Mouse primary Ab and a goat anti Rabbit HRP conjugated secondary Ab with ECl. The goat anti rabbit antibody looks to be cross reactive with human IgG.

\begin{tabular}{|c|c|c|c|c|c|c|}
\hline IgG standard (ug/uL) & $\begin{array}{c}0 \\
0 \\
0.50\end{array}$ & $\begin{array}{c}0.05 \\
0.75\end{array}$ & $\begin{array}{c}0.10 \\
1.00\end{array}$ & $\begin{array}{c}0.20 \\
0 \\
1.25\end{array}$ & 0.30 & $\begin{array}{c}0.40 \\
0 \\
2.00\end{array}$ \\
\hline \multirow[t]{3}{*}{$\mathrm{mM} \mathrm{NaCl}$} & PBS & 50 & 100 & 150 & 200 & 250 \\
\hline & 300 & 350 & 400 & 450 & 500 & 550 \\
\hline & 600 & 650 & 700 & 800 & 900 & 1000 \\
\hline $650 \mathrm{mM} \mathrm{NaCl}+\%$ Acetonitrile & $10 \%$ & $20 \%$ & $30 \%$ & $40 \%$ & $50 \%$ & $60 \%$ \\
\hline & $70 \%$ & Beads & & & & \\
\hline
\end{tabular}

Figure 60. Dumbroff dot blot of IgG coated PMMA microbreads applied to RAW cells in serum free media, isolated by sucrose gradient and sequentially extracted in increasing salt buffers followed by increasing acetonitrile. Table Rt: visual comparison of fraction to standards to determine protein content 


\begin{tabular}{llllllll}
\hline pg & 1 pg & $10 p g$ & $100 p g$ & 1 ng & 10 ng & 100 ng \\
& & & $*$ & - & & \\
PBS & 50 & 100 & 150 & 200 & 250 & 300 \\
350 & 400 & 450 & 500 & 550 & 600 & 650 \\
700 & 800 & 900 & 1000 & $10 \%$ & $20 \%$ & $30 \%$ \\
$40 \%$ & $50 \%$ & $60 \%$ & $70 \%$ & & &
\end{tabular}

Figure 61. Western dot blot of human IgG coated PMMA micro beads applied to RAW cells in serum free media, isolated by a sucrose gradient and sequentially extracted in increasing salt buffers up to $1000 \mathrm{mM} \mathrm{NaCl}$ followed by $650 \mathrm{mM} \mathrm{NaCl}$ with increasing amounts of acetonitrile. Detected by a Dk anti Human IgG $(\mathrm{L}+\mathrm{H})$ conjugated to HRP and ECl.

The salt and acetonitrile fractions eluted from IgG melamine coated beads applied to live cells where separated by polyacrylamide gel electrophoresis and compared to uncoated bead controls. IgG coated melamine showed a distinct band pattern compared to controls with much higher signals especially in the salt fractions with greater than $300 \mathrm{mM} \mathrm{NaCl}$ and up to $50 \% \mathrm{AcN}$ (Fig. $62)$. 

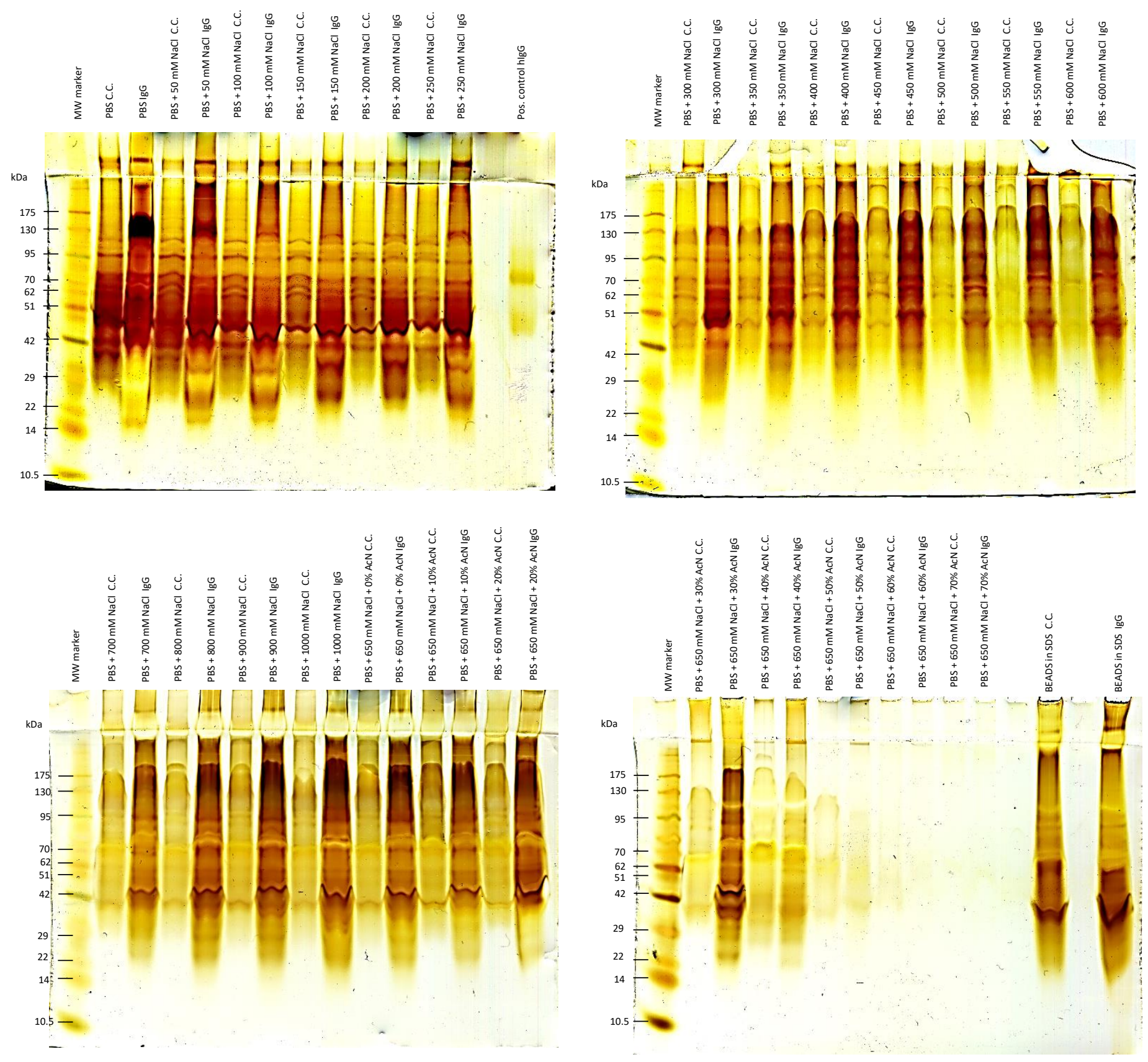

Figure 62: Silver stain of LARC RAW IgG alongside crude control. 1ul of MW Std; 10ul sample and 2 ul IgG as positive control (silver stain performed by Angelique Florentinas) 


\section{DISCUSSION}

After testing a number of chromatography surfaces, it was determined that nano-sized glass beads coated in ligand may be a simple and robust general method to activate and isolate receptor complexes from the surface of live cells by presenting the cognate receptor ligand at the nanoscopic spatial scale.

The classical form of affinity chromatography was compared to a novel approach where the nano chromatography bead itself was coated by the natural ligand and directly applied to live cells. After binding ligand coated nanobeads, the cells were homogenized, disrupted in a French press and the nanobeads isolated by ultracentrifugation over a sucrose density gradient. The protein coated beads were sequentially extracted using a salt and acetonitrile step gradient. In this strategy, the ligand is aggregated on the bead forming many receptor-ligand interactions which may produce enough force to keep the receptor-ligand interaction intact during disruption. After French pressing, the sheared membrane may reform a thermodynamically favorable bilipid vesicle configuration. A bilipid vesicle reforms either around the bead or remains adsorbed to the bead where the receptor is still associated with the ligand and the membrane is enriched in the activated receptor complex. In the case of phagocytic receptors, the partial engulfment of the bead would capture the relevant portion of the membrane dedicated to phagocytosis.

\section{Error from noise and redundant MS/MS correlation}

Imposing a signal intensity cut off of $\geq E 4$ counts did not entirely eliminate noise spectra (see below). Thus, it is important to run blank noise controls and to statistically analyze the distributions of blank source noise to control error. Another source of error (v) in correlation of 
MS/MS spectra to tryptic peptides may be the redundant use of an MS/MS spectra to implicate more than one peptide amino acid sequence. The composite key in the SQL Server system completely eliminated the re-use of MS/MS spectra for more than one peptide sequence leaving only the simplest interpretation of the data with the most stringent fit parameter.

\section{Direct experimental strategy for controlling error}

A direct experimental approach to address the effects of physical/chemical noise, the near random-distribution of mis-correlations spectra, and contamination is to run multiple blank solvent gradients on naïve columns to record source noise, to create random MS and MS/MS spectra, and to purposefully digest laboratory dust to make a list of contaminants that might be stored in an SQL database and compared to experimental results. After removing redundant use of the same MS/MS spectra (error v) with SQL Server, the other sources of error (i-iv) may be addressed by direct experimentation. Chemical or physical noise, as well as unexpected cleavages, or post-translational modifications, should result in the near-random correlation of precursor and fragments to peptides (P. Zhu, P. Bowden, M. Tucholska, et al., 2011; Peihong Zhu et al., 2011) that show a low frequency distribution sharply skewed to giant proteins such as TTN (Bowden et al., 2010) that is easily discounted based on frequency. The use of blank runs with physical and chemical noise, dust or contaminant controls, and random spectra is a direct method to control for sources of error. The experimental design of the decoy library experiment does not actually define the scoring distribution of true positive and false positive correlations (A. Keller et al., 2002; Andrew Keller, Samuel Purvine, et al., 2002) since true positive peptide correlation may have high or low p-values and yet be correct but the in contrast the peptide-toprotein distribution of true positive correlation shows a marked difference from that of the false 
positive distribution that is a simple and efficient method to separate authentic from erroneous results using only the existing laws of statistics shared with all other branches of science (Bowden et al., 2012; P. Zhu, P. Bowden, M. Tucholska, et al., 2011; Peihong Zhu et al., 2011). The analysis of LC-ESI-MSMS data from affinity experiments was greatly enriched in high peptide counts $(50,000$ versus 16,000$)$ and showed fewer proteins with low counts.

\section{Error from noise in blank runs on naïve columns}

The vast bulk of source noise ions show intensity values less than E4 counts and only about 2000 of the $\sim 75,000$ noise MS/MS spectra showed intensity greater than E4 and correlated to a peptide in error. There may be a background, i.e noise, MS "peak" (spectral line) at every possible $\mathrm{m} / \mathrm{z}$ value and so measuring low intensity peptides from unfractionated samples that are not safely separated from noise with great mass-accuracy using high resolution instruments may not provide authentic confidence (Cooper, 2012). Compiling many blank runs with a naïve column was a direct way to control for source noise from low-intensity samples that might lead to nearrandom correlations. The production of random noise spectra at low intensity values from the electrospray source is somewhat alarming, but the effect can be quantified and effectively utilized as an internal control distribution. One interpretation of the TTN observed in blank gradients, was that these proteins are from dust and laboratory contamination, but the conspicuous absence of much keratin in the blank runs compared to the giant protein TTN seems to indicate that laboratory contaminants are not the major source of spurious results from LCESI-MS/MS. TTN (LeWinter \& Granzier, 2013) was observed both experimental blank run noise or computer derived random spectra controls and thus represents random mis-correlations

to the largest proteins in the library (P. Zhu, P. Bowden, M. Tucholska, et al., 2011; Peihong Zhu 
et al., 2011). The blank solvent runs indicated that there is a random component of physical chemical noise in MS/MS spectra using HPLC grade water and organic solvents over naïve C18 analytical columns. The simplest way to avoid confounding noise spectra, avoid co-elution, and thus provide accurate results while achieving a high sensitivity with a simple ion trap is to effectively pre-fractionate the sample prior to LC-ESI-MS/MS to achieve high signal-to-noise (Kielley \& Bradley, 1956; J. Marshall et al., 2004; Monika Tucholska et al., 2009).

\section{Error from random miss-correlation}

Near random correlation may result from fitting MS/MS spectra from peptides that show unexpected modifications, missed cleavages, and/or exopeptidase activity not accounted for in the MS/MS correlation parameters selected. The false positive identification that might result from modified, or non-tryptic, peptides may be modeled by the best fit of random spectra. Miscorrelations should be randomly distributed across the amino acid sequences in the library with a low frequency and so experimentally observed peptide-to-protein frequency might be compared to a null random model of noise or computer generated random spectra using the computationally efficient Chi-Square algorithm at the end of the correlation procedure (P. Zhu, P. Bowden, M. Tucholska, et al., 2011; Peihong Zhu et al., 2011). Near random correlation may result from fitting MS/MS spectra from peptides that show unexpected modifications, missed cleavages, and/or exopeptidase activity not accounted for in the MS/MS correlation parameters selected. A random spectra generator provides a portable metric that may be compared across conditions as a universal standard of type I error. The results from a linear ion trap mass spectrometer with correlation analysis under similar conditions $(\mathrm{z}=+2$ or +3 , with $\pm 3 \mathrm{~m} / \mathrm{z}$ for the 
precursor and $\pm 0.5 \mathrm{Da}$ for the fragments) may use the null random model of computer generated random spectra or source noise provided in the supplemental data to correct identifications.

\section{Human dust samples}

Dust contains proteins like keratins and actins and ankyrins that might contaminate buffers, reagents and solvents. The peptide to protein distributions of dust versus random spectra indicates the keratin peptides in dust digests are authentic peptide identifications that are distinguished from the null models of noise or random, i.e. control, distributions. The identification of Caspase 14 that is associated with the expression of keratin and terminal differentiation in skin cells leading to apoptosis was entirely consistent with the accurate identification of low abundance proteins (Denecker, Ovaere, Vandenabeele, \& Declercq, 2008). There is evidence that environmental dust samples contain human keratin (Fox, Castanha, Fox, Feigley, \& Salzberg, 2008) and some other human proteins in detectable amounts (An et al., 2013; Fang, Webster, \& Stapleton, 2015). Laboratory contaminants, specifically dust from human skin, were apparently not a large source of error in LC-ESI-MS/MS recordings of peptides in this study.

\section{Affinity purified IgG positive control}

Bulk human IgG contains a variable domain with some short regions of near random polypeptides that may serve as an internal control for the false positive identification of nearrandom peptides. The human bulk IgG sample is a powerful internal control for mis-correlation since the affinity purified molecule (Uhlen et al., 1984) is composed of both well-established 
heavy and light chain sequences that are in the protein library, and some regions of near-random sequence in the variable domain (Chin et al., 2015; Tonegawa, Steinberg, Dube, \& Bernardini, 1974). IgG may be affinity purified by protein A or protein $G$ chromatography (Uhlen et al., 1984). Bulk human IgG has conserved domains shared with other members of the IgG superfamily and might also be expected to present near random peptides from the variable domain that comprise a portion of the heavy and light chains (Tonegawa et al., 1974). The IgG standard showed correlations to know VDJ recombination borders and a high frequency of correlation to conserved sequences of the Ig superfamily, but the algorithms apparently did not correlate the random peptides (Tonegawa et al., 1974) of bulk IgG to other known human proteins, consistent with good correlation fidelity. The results indicated that the affinity purified human bulk IgG sample was highly purified and contained diverse and identifiable IgG structural variants as expected. The frequency of correlations to $\mathrm{IgG}$ and related members of the Ig Superfamily seems to indicate that the algorithms robustly identified the affinity purified heavy and light chains and were not easily susceptible to mis-correlations caused by large numbers of nearly random peptides from the variable domain (Tonegawa et al., 1974).

\section{Automatic computation of precursor data at the level of peptides, proteins and gene symbols}

The SQL/R system permits the automatic computation, transformation and graphical analysis of mean precursor intensity, standard error, p-value, normality, density and many other important statistical parameters between treatments at the level of peptides, proteins, accessions or gene symbols using all standard statistical tests such as Chi Square and ANOVA by the same R statistical system used in all other areas of science. The results show that random and 
independent sampling of precursor ions by LC-ESI-MS/MS leads to a low type I error of protein identity, Gaussian intensity distributions that frequently show less than $10 \%$ standard error, and Gaussian $[\mathrm{M}+\mathrm{H}]^{+}$and delta mass distributions that confirm the results that the results of LC-ESIMS/MS behave in a manner similar to most other biochemical and biophysical data. Thus, LCESI-MS/MS data should be analyzed using the same laws of statistics used in all other areas of science (A. Keller et al., 2002). The analysis of 300,000 synthetic peptides agrees with the use of random and noise MS/MS spectra that the fit of experimental MS/MS spectra to the predicted is excellent and shows a low error rate (Zolg et al., 2017). Since the masses of the amino acid residues in MS/MS spectra can be effectively identified by measurement to $0.5 \mathrm{Da}$ by a simple ion trap just as effectively as insensitive high resolution mass spectrometry, then there is no need for high mass resolution to identify unmodified tryptic peptides by their MS/MS spectra as confirmed by Western blots, siRNA, drugs, immune cyto staining or GFP fusions (J. C. Howard et al., 2016; Andy Jankowski, Peihong Zhu, \& John G. Marshall, 2008; M. Tucholska et al., 2010).

\section{Authentic myosin protein complex by STRING analysis}

Many peptides from the myosin standard were apparently correlated with high fidelity to known interacting proteins such as myosin binding protein $\mathrm{C}$ and actins that clearly indicated that the myosin standard was not pure. The empirical statistical model of Keller et al (A. Keller et al., 2002) assumed all correlations to peptides other than myosin, including actin, were erroneous, and therefore that LC-ESI-MS/MS of peptides with a simple ion trap suffered from high error rate. Robust and sensitive ion traps (Schwartz et al., 2002; Stafford, 1980) may not always resolve the isotopic forms ( $\mathrm{R} \geq 1,000$ to 3,000) leading to a concern that more than one peptide 
might be isolated resulting in mis-correlation of the MS/MS spectra. However, pre-separation of proteins or polypeptides by organic solvents, partition chromatography (including bio-specific or affinity separation), or differential centrifugation effectively enriches the sample in a sub-set of proteins that prevents co-elution of multiple tryptic peptides with similar $\mathrm{m} / \mathrm{z}$ values (Bowden et al., 2012; Angelica K Florentinus, Peter Bowden, Girish Sardana, et al., 2012; Monika Tucholska et al., 2009). In the case of myosin, the protein complex was purified by differential centrifugation (Lowey \& Risby, 1971) and so the probability of tryptic peptides from more than one protein with similar $\mathrm{m} / \mathrm{z}$ values eluting at the same time is dramatically reduced.

\section{Biotinylated ligand affinity receptor chromatography of the Fc Receptor with SA-agarose columns}

Here a more general method for monovalent IgG or aggregated IgG ligands that might bind individual or clusters of receptors at the nm scale was demonstrated using IgG, IgG-B and DTT cleavable biotin IgG-SS-B. Phagocytic receptors work cooperatively to recognize polyvalent ligands like IgG presented over the surface of a cell or microorganism on the order of microns across. As previously shown, it was possible to activate the polyvalent Fc receptor using the cognate ligand IgG presented on $2 \mu$ m polystyrene beads (Marshall et al., 2001) and thus capture phagocytic receptors from the surface of live cells as confirmed by drugs or siRNA (Jankowski et al., 2008) and subsequently identify or quantify the ligand specific proteins (Florentinus et al., 2011; Florentinus et al., 2012) in agreement with independent results. 


\section{Reagents and detection limit}

The SA-HRP and Dk anti-hIgG-HRP probes agreed that no more than 1-10 ng of IgG that might be detected and assuming a 1:1 stoichiometry between IgG and the Fc receptor(s) indicates that the affinity preparations eluted from live cells has no more than $\sim 1 \mathrm{ng}$ per microliter and so the $2 \mathrm{ml}$ volume of the eluant thus contain no more than about $2 \mu \mathrm{g}$ of $\operatorname{IgG}$ that should be sufficient to analyze a protein complex by LC-ESI-MS/MS.

\section{Background and non-specific binding}

There is a significant risk of background contamination in the affinity chromatography experiments here with the possibility of non-specific binding to the agarose support and at a lower affinity than expected binding to streptavidin. The background binding to agarose beads is high and many proteins adsorbed non-specifically. The non-specific binding must be especially taken into account in LC-ESI-MS/MS experiments that are at least one order of magnitude more sensitive than ECL or more. Under conditions where no non-specific binding is observed by dot blot or western blot significant binding may be observed by LC-ESI-MS/MS and so the background controls of the agarose support and the SA-agarose resin must be accounted for using the SQL database of control and treatment results.

\section{Affinity Treatments}

In this study the IgG, B-IgG and B-S-S-IgG were compared for ligand affinity chromatography of the Fc receptor(s) using over agarose and streptavidin-agarose. The Western blot and dot blot data indicated that homogenizing the cells with an excess of the biotinylated ligands eluted with SDS acted as an internal control that clearly demonstrate the IgG ligands bound to and were 
released from the agarose and SA-agarose and thus did not result in the anticipated affinity purification and release of the analytes for LC-ESI-MS/MS analysis. The use of $2 \mathrm{M} \mathrm{NH}_{4} \mathrm{OH}$ apparently resulted in a non-specific release of IgG-B and IgG-SS-B from the SA-agarose resin. Biotin has a tight association with streptavidin and a low off rate so under physiological conditions the interaction in essentially irreversible, but biotin is soluble in the stringent denaturing reagent $2 \mathrm{M} \mathrm{NH}_{4} \mathrm{OH}$. After pre-clearing the column with $2 \mathrm{M} \mathrm{NH} 4 \mathrm{OH}$, further elution with biotin dissolved in $2 \mathrm{M} \mathrm{NH}_{4} \mathrm{OH}$ apparently specifically released trace amounts of the high affinity FCGR1 isoform that should be present at the lowest stoichiometry in the complex and consistent with the stringency of the elution. There was no detectable IgG bound to streptavidin in the absence of biotinylation by dot blots or Western blots but unexpectedly, clearly detectable amounts of IgG were released from SA columns by DTT that may indicate IgG binds the SAagarose resin by an unknown mechanism and was released when the IgG is denatured by DTT. DTT specifically released the S-B-IgG and Fc receptors from the SA resin and seemed to convincingly demonstrate that $\mathrm{IgG}$ and Fc elutes from the resin by a mechanism sensitive to reduction. IgG glycoprotein that might bind agarose but is denatured by DTT that might result in elution.

\section{Fc surface receptor by IgG, B-IgG and B-S-S-IgG ligands and agarose chromatography}

The use of biotinylated ligand affinity chromatography with biotinylated ligands or cleavable biotinylated ligands may all provide sufficient material for identification by liquid chromatography and tandem mass spectrometry so long as control samples are also analyzed to account for non-specific binding. The approach of monomeric or polyvalent receptor ligands 
presented to live cells on the nano scale may have a broad application to a variety of soluble receptors crucial in human infection, disease or inflammation.

The good agreement between the ligand affinity methods and the expected proteins of the $\mathrm{Fc}$ receptor complex based on previous biochemical and genetic data confirms that not only were the expected $\mathrm{Fc}$ receptors themselves bound to the IgG ligand but many of their associated factors were also detected. However, the DTT, BME, $\mathrm{NH}_{4} \mathrm{OH}$, control beads, $\mathrm{Fc}$ receptors and bead digests can all be explained by non-specific binding protein complexes that are reduced by DTT and BME. The DTT and BME may be explained by a specific affinity chromatography of cell surface receptors (Frei et al., 2012; Frei et al., 2013). The release of proteins by the reducing agent DTT seems to indicate that proteins including Fc receptors bind the agarose as a protein complex that is released in part by reduction.

The presence of Fc receptors on the SA-agarose after the cleavage of the DTT spacer and on the beads alone without the IgG ligand seems to confirm that the observed receptors detected by the sensitive LC-ESI-MS/MS were bound to the agarose support by a chromatography mechanism other than affinity to the IgG ligand. All of the observations of SA-agarose beads may be explained by non-specific binding of protein complexes. The observation that known protein complexes eluted with DTT and BME may indicate that protein complexes bind non-specifically to the agarose support and require di-sulfide bridges to support protein interactions. The specific elution of IgG by DTT and mercaptoethanol suggests that the affinity chromatography was successful in that the ligand was recovered however the lack of FC receptors indicates either the 
FC targets were below a statistically relevant detection limit or there this an underlying mechanism that prevents the capture of membrane bound receptor complexes by this method.

\section{Ligand affinity receptor chromatography on micro or nano beads}

It appears that binding the receptor with a multivalent ligand complex on a dense nano support will permit the activation and accumulation of receptor complexes in a sufficient amount to provide a strong signal, while also providing a discrete location on the cell surface for imaging the accumulation, diffusion, phosphorylation, activation or other functional assays of the nanoscale receptor complex. The clustering of the $\mathrm{Fc}$ receptor by aggregated $\mathrm{IgG}$ on microbeads or nano beads may lead to intense cross activation that triggers complex accumulation and so we now proposed to compare the micro beads to nano beads that match the size scale of most endocytic and other receptors.

There may be interesting and highly interconnect networks between the comparisons of the peptides isolated by NHS-biotin and NHS-SS-biotin ligand affinity chromatography eluted with reducing agents including a large number of undefined GPCRs soluble receptor cleaved from the surface of the cell into the cell media.

\section{Ligand affinity versus ligands coupled with chromatography nano and micro particles}

It was apparent however that ligand coated silica nano beads showed a greater level of success compared to biotinylated ligands captured with SA- agarose. There was very low background binding on silica nano beads and so the ligand specific capture and Fc receptors could not result from non-specific binding to the support. To rule out this possibility, media was incubated with 
cells for four hours then removed and the used media without cells was incubated with the ligands over night. However, ligands incubated with media resulted in very low signal $\leq$ E4 signal strengths (not shown).

Many cell surface proteins may be recruited to patches of membrane that may be adsorb to nano beads and captured (John G Marshall et al., 2001). The low signals in the crude controls and media controls for nano silica indicate there was little contamination of the hydrophilic glasspolylysine-paraformaldehyde-IgG nano beads. Fc receptors were convincingly captured from the surface of live cells by the glass-polylysine-paraformaldehyde-IgG nano chromatography beads. In contrast to the SA-agarose beads, that showed a large background binding, the binding of Fc to the glass nano beads cannot be accounted for by background binding.

It is possible that the glass nano beads may capture membrane proteins not associated with Fc in the bulk adsorbed membrane. Thus, comparison of multiple ligands might rule out non-specific membrane proteins (Jeffrey C Howard et al., 2016). Activating and capturing cell surface receptors using glass nano beads may represent a tractable solution to the affinity chromatography of cell surface receptors. 


\section{CONCLUSION:}

\section{IgG ligand positive control}

The assumptions of the FDR method of setting error rates in LC-ESI-MS/MS by decoy library based on the empirical statistical model has led to a large type II error in proteomics and thus a large total error. Six independent lines of evidence, SDS-PAGE, Western blot, the p-value computed from X!TANDEM, the peptide-to-protein distribution of the test samples compared to random and source noise, and the myosin protein complex detected by STRING, all lead to the conclusion that many myosin related proteins were correctly identified with at least three best fit peptides and show a low type I error. If there was a concern for false positive identification, the proteins p-values generated by the goodness of fit of the experimental MS/MS spectra might be corrected by the bone fide FDR method of Benjamini and Hochberg (Benjamini. \& Hochberg, 1995). The simplest way to avoid type I error from noise or background contamination of LCESI-MS/MS with an ion trap is to collect high intensity MS and MS/MS spectra from highly enriched samples (P. Zhu, P. Bowden, M. Tucholska, et al., 2011; Peihong Zhu et al., 2011). The comparison of the peptide-to-protein distribution of authentic samples, such as IgG, myosin, or dust, to the null distributions of noise or random spectra by the Chi Square test may serve as a universal and portable metric to estimate the type I error of protein identification (Angelica $\mathrm{K}$ Florentinus, Peter Bowden, Girish Sardana, et al., 2012; Florentinus et al., 2011a; Monika Tucholska et al., 2009). Here it is unambiguously and clearly demonstrated for the first time using the classical comparison of purified protein standards IgG and myosin to null random models of noise or random MS/MS spectra that a linear ion trap is more than sufficient to sensitively identify and quantify the peptides from one Eukaryotic species with low measured 
levels of type I error in the identification and relative quantification of peptides and proteins. The results are completely consistent with those of synthetic peptides that show experimental MS/MS spectra exactly match the predicted spectra and so there is no need for decoy libraries or any novel statistical methods to fit MS/MS spectra to predicted spectra that show excellent crosscorrelation and goodness of fit to the predicted fragment sets (Bowden et al., 2009; P. Zhu, P. Bowden, M. Tucholska, et al., 2011; Peihong Zhu et al., 2011; Zolg et al., 2017). A simple, sensitive and robust linear ion trap is sufficient to distinguish the residues of the amino acids and insensitive, expensive high resolution mass spectrometers that have poor duty cycles do not perform the task any better in practice and so represent a waste of resources. Thus, unmodified tryptic peptides may be identified from MS/MS spectra and provide relative quantification of the precursors, from one species that may be performed with a simple, sensitive and robust linear ion trap that provides analytical error.

\section{SA-agarose affinity chromatography}

In agreement with previous experiments (Cutracases and others) the use of biotinylated ligands or biotinylated ligands with DTT cleavable spacer arms did not appear to isolated the Fc receptor complex by a specific ligand affinity chromatography mechanisms but seemed to result in the non-specific binding of cellular protein complexes to the chromatography support that confounded the separation of true interactions from background binding.

\section{Glass nano bead ligand affinitychromatography}

Here it is unambiguously and clearly demonstrated for the first time that a direct ligand affinity receptor chromatography using novel silica nanobeads achieved the isolation of an integral membrane receptor that represents a simple a robust general solution for animal cell surface 
receptors in the nano scale range that is a long standing and crucially important analytical challenge for biomedical and basic research.

The glass nano beads that show a low background binding, achieved the isolation of the integral Fc receptor from live cells. The novel silica-polylysine-paraformaldehyde-ligand nano beads might be applied to many different ligand receptors to determine the ligand specific complexes.

\section{Future works}

A comparison of the different ligands may be used to rule out non-specific proteins in the membrane. To confirm the identity of the IgG-Fc receptor interaction partners and co-receptors, live cell laser scanning confocal microscopy will be used to image the binding or recruitment of fluorescently labelled targets when activated by ligand coated micro and nano beads. Further replication of the apparently highly successful glass nano bead experiments and controls are required.

Single binding events between ligand coated beads and fluorescent targets may be used to measure protein accumulation and diffusional mobility using FRAP at the receptor complex. More specifically ligand dependent recruitment, binding and dissociation of receptors and coreceptors will be measured by Fluorescence Recovery after photobleaching with scanning laser confocal microscopy.

Ligand affinity chromatography is a highly selective way to capture and elute many ligandreceptors (A. K. Florentinus et al., 2012; Florentinus, Jankowski, Petrenko, Bowden, \& Marshall, 
2011b; Jeffrey C Howard et al., 2016; Andy Jankowski, Peihong Zhu, \& John G Marshall, 2008). In contrast, biotin bind so tightly that it is an excellent means to capture ligands but they cannot be selectively eluted by $\mathrm{pH}$, salt chaotropes or reducing agents. The DTT cleavable biotnylation reagent did show some selective elution of the $\operatorname{IgG}$ ligand but little or no selective elution of the Fc receptors.

There was a great deal of non-specific background binding by the agarose support and it remains possible that the Fc receptors bind and elute from the agarose support non-specifically. Presentation of the IgG ligand on the hydrophilic nano silica D-polylysine support showed very low background binding and effectively captured the $\mathrm{Fc}$ receptor from the surface of live cells

To better understand the underlying mechanism that prevents monomeric biotin tagged ligands from capturing membrane bound receptors after homogenization we plan to apply biotinylated ligands to live cells and measure the accessibility of a streptavidin-fluorescent probe before and after homogenization. siRNA will be used to confirm the funcational importance of the Fc receptors observed in the murine RAW 264.7 cell line. 


\section{REFERENCES}

Albrethsen, J., Bogebo, R., Olsen, J., Raskov, H., \& Gammeltoft, S. (2006). Preanalytical and analytical variation of surface-enhanced laser desorption-ionization time-of-flight mass spectrometry of human serum. Clin Chem Lab Med, 44(10), 1243-1252.

Allan, D., Auger, J., \& Crumpton, M. J. (1972). Purification of concanavalin A receptor from pig lymphocyte plasma membrane. Biochem J, 126(3), 6P.

An, S., Shen, C., Liu, X., Chen, L., Xu, X., Rong, M., . . Lai, R. (2013). Alpha-actinin is a new type of house dust mite allergen. PLoS ONE, 8(12), e81377. doi:10.1371/journal.pone.0081377

Baggerly, K. A., Morris, J. S., Wang, J., Gold, D., Xiao, L. C., \& Coombes, K. R. (2003). A comprehensive approach to the analysis of matrix-assisted laser desorption/ionizationtime of flight proteomics spectra from serum samples. Proteomics, 3(9), 1667-1672.

Banks, R. E. (2008). Preanalytical influences in clinical proteomic studies: raising awareness of fundamental issues in sample banking. Clin Chem, 54(1), 6-7.

Bayer, E. A., \& Wilchek, M. (1980). The use of the avidin-biotin complex as a tool in molecular biology. Methods Biochem Anal, 26, 1-45.

Benjamini., Y., \& Hochberg, Y. (1995). Controlling false discovery rate: A practical approach to multiple testing. Journal of the Royal Statistical Society, 57(1), 289-300.

Benovic, J. L., Shorr, R. G., Caron, M. G., \& Lefkowitz, R. J. (1984). Mammalian. beta. 2adrenergic receptor: purification and characterization. Biochemistry, 23(20), 4510-4518.

Bereman, M. S., Johnson, R., Bollinger, J., Boss, Y., Shulman, N., MacLean, B., . . MacCoss, M. J. (2014). Implementation of statistical process control for proteomic experiments via LC MS/MS. J Am Soc Mass Spectrom, 25(4), 581-587. doi:10.1007/s13361-013-0824-5

Boehm, A. M., Putz, S., Altenhofer, D., Sickmann, A., \& Falk, M. (2007). Precise protein quantification based on peptide quantification using iTRAQ. BMC Bioinformatics, 8 , 214. doi:10.1186/1471-2105-8-214

Bowden, P., Beavis, R., \& Marshall, J. (2009). Tandem mass spectrometry of human tryptic blood peptides calculated by a statistical algorithm and captured by a relational database with exploration by a general statistical analysis system. Journal of Proteomics, 73, 103111. doi:10.1016/j.jprot.2009.08.004

Bowden, P., Pendrak, V., Zhu, P., \& Marshall, J. G. (2010). Meta sequence analysis of human blood peptides and their parent proteins. Journal of Proteomics, 73, 1163-1175. doi:10.1016/j.jprot.2010.02.007

Bowden, P., Thavarajah, T., Zhu, P., McDonell, M., Thiele, H., \& Marshall, J. G. (2012). Quantitative statistical analysis of standard and human blood proteins from liquid chromatography, electrospray ionization, and tandem mass spectrometry. Journal of Proteome Research, 11, 2032-2047. doi:10.1021/pr2000013

Bruhns, P. (2012). Properties of mouse and human IgG receptors and their contribution to disease models. Blood, 119(24), 5640-5649.

Callesen, A. K., Vach, W., Jorgensen, P. E., Cold, S., Mogensen, O., Kruse, T. A., . . Madsen, J. S. (2008). Reproducibility of mass spectrometry based protein profiles for diagnosis of breast cancer across clinical studies: a systematic review. J Proteome Res, 7(4), 13951402. 
Cargile, B. J., Bundy, J. L., \& Stephenson, J. L., Jr. (2004). Potential for false positive identifications from large databases through tandem mass spectrometry. J Proteome Res, 3(5), 1082-1085.

Chin, S. T., Ignatius, J., Suraiya, S., Tye, G. J., Sarmiento, M. E., Acosta, A., . . Lim, T. S. (2015). Comparative study of IgA VH 3 gene usage in healthy TST(-) and TST(+) population exposed to tuberculosis: deep sequencing analysis. Immunology, 144(2), 302311. doi:10.1111/imm.12372

Cooper, B. (2012). The problem with peptide presumption and the downfall of target-decoy false discovery rates. Anal Chem, 84(22), 9663-9667. doi:10.1021/ac303051s

Cottrell, J. S., \& London, U. (1999). Probability-based protein identification by searching sequence databases using mass spectrometry data. Electrophoresis, 20(18), 3551-3567.

Craig, R., \& Beavis, R. C. (2003). A method for reducing the time required to match protein sequences with tandem mass spectra. Rapid Commun Mass Spectrom, 17(20), 2310-2316.

Craig, R., \& Beavis, R. C. (2004). TANDEM: matching proteins with tandem mass spectra. Bioinformatics, 20(9), 1466-1467.

Craig, R., \& Beavis, R. C. (2004). TANDEM: matching proteins with tandem mass spectra. Bioinformatics, 20(9), 1466-1467.

Cuatrecasas, P. (1972). Affinity chromatography and purification of the insulin receptor of liver cell membranes. Proc Natl Acad Sci U S A, 69(5), 1277-1281.

Cuatrecasas, P., \& Parikh, I. (1974). Affinity chromatography of insulin receptors. Methods Enzymol, 34, 653-670.

Cuatrecasas, P., Wilchek, M., \& Anfinsen, C. B. (1968). Selective enzyme purification by affinity chromatography. Proc Natl Acad Sci U S A, 61(2), 636-643.

de Noo, M. E., Tollenaar, R. A., Ozalp, A., Kuppen, P. J., Bladergroen, M. R., Eilers, P. H., \& Deelder, A. M. (2005). Reliability of human serum protein profiles generated with C8 magnetic beads assisted MALDI-TOF mass spectrometry. Anal Chem, 77(22), 72327241.

Denecker, G., Ovaere, P., Vandenabeele, P., \& Declercq, W. (2008). Caspase-14 reveals its secrets. J Cell Biol, 180(3), 451-458. doi:10.1083/jcb.200709098

Diamandis, E. P., \& Christopoulos, T. K. (1991). The biotin-(strept) avidin system: principles and applications in biotechnology. Clinical chemistry, 37(5), 625-636.

Dionne, R., Forest, J. C., Moutquin, J. M., De Grandpre, P., \& Masse, J. (1994). Electrophoretic method for separating small peptides in serum without extraction of macromolecules: application to the detection of preeclampsia. Clin Biochem, 27(2), 99-103.

Dobo, J., Major, B., Kekesi, K. A., Szabo, I., Megyeri, M., Hajela, K., . . Gal, P. (2011). Cleavage of kininogen and subsequent bradykinin release by the complement component: mannose-binding lectin-associated serine protease (MASP)-1. PLoS ONE, 6(5), e20036. doi:10.1371/journal.pone.0020036

Doi, E., Shibata, D., \& Matoba, T. (1981). Modified colorimetric ninhydrin methods for peptidase assay. Anal Biochem, 118(1), 173-184.

Eckel-Passow, J. E., Oberg, A. L., Therneau, T. M., \& Bergen, H. R., 3rd. (2009). An insight into high-resolution mass-spectrometry data. Biostatistics, 10(3), 481-500.

Elschenbroich, S., Kim, Y., Medin, J. A., \& Kislinger, T. (2010). Isolation of cell surface proteins for mass spectrometry-based proteomics. Expert review of proteomics, 7(1), 141154. 
Eng, J. K., McCormack, A. L., \& Yates, J. R. (1994). An approach to correlate tandem mass spectral data of peptides with amino acid sequences in a protein database. J Am Soc Mass Spectrom, 5(11), 976-989.

Fagerberg, L., Jonasson, K., von Heijne, G., Uhlén, M., \& Berglund, L. (2010). Prediction of the human membrane proteome. Proteomics, 10(6), 1141-1149.

Fang, M., Webster, T. F., \& Stapleton, H. M. (2015). Effect-Directed Analysis of Human Peroxisome Proliferator-Activated Nuclear Receptors (PPARgamma1) Ligands in Indoor Dust. Environ Sci Technol, 49(16), 10065-10073. doi:10.1021/acs.est.5b01524

Flannagan, R. S., Jaumouillé, V., \& Grinstein, S. (2012). The cell biology of phagocytosis. Annual Review of Pathology: Mechanisms of Disease, 7, 61-98.

Florentinus, A. K., Bowden, P., Barbisan, V., \& Marshall, J. (2012). Capture and qualitative analysis of the activated Fc receptor complex from live cells. Current protocols in protein science / editorial board, John E. Coligan ... [et al.], Chapter 19, Unit 19.22. doi:10.1002/0471140864.ps1922s67

Florentinus, A. K., Bowden, P., Sardana, G., Diamandis, E. P., \& Marshall, J. G. (2012). Identification and quantification of peptides and proteins secreted from prostate epithelial cells by unbiased liquid chromatography tandem mass spectrometry using goodness of fit and analysis of variance. Journal of Proteomics, 75, 1303-1317. doi:10.1016/j.jprot.2011.11.002

Florentinus, A. K., Jankowski, A., Petrenko, V., Bowden, P., \& Marshall, J. G. (2011). The Fc receptor-cytoskeleton complex from human neutrophils. Journal of Proteomics, 75, 450468. doi:10.1016/j.jprot.2011.08.011

Fox, K., Castanha, E., Fox, A., Feigley, C., \& Salzberg, D. (2008). Human K10 epithelial keratin is the most abundant protein in airborne dust of both occupied and unoccupied school rooms. J Environ Monit, 10(1), 55-59. doi:10.1039/b714802j

Frei, A. P., Jeon, O. Y., Kilcher, S., Moest, H., Henning, L. M., Jost, C., . . Wollscheid, B. (2012). Direct identification of ligand-receptor interactions on living cells and tissues. Nat Biotechnol, 30(10), 997-1001. doi:10.1038/nbt.2354

Frei, A. P., Moest, H., Novy, K., \& Wollscheid, B. (2013). Ligand-based receptor identification on living cells and tissues using TRICEPS. Nat Protoc, 8(7), 1321-1336. doi:10.1038/nprot.2013.072

Fung, K. W., Wright, D. W., Gor, J., Swann, M. J., \& Perkins, S. J. (2016). Domain structure of human complement $\mathrm{C} 4 \mathrm{~b}$ extends with increasing $\mathrm{NaCl}$ concentration: implications for its regulatory mechanism. Biochem J, 473(23), 4473-4491. doi:10.1042/BCJ20160744

Gast, M. C., van Gils, C. H., Wessels, L. F., Harris, N., Bonfrer, J. M., Rutgers, E. J., . . . Beijnen, J. H. (2009). Influence of sample storage duration on serum protein profiles assessed by surface-enhanced laser desorption/ionisation time-of-flight mass spectrometry (SELDI-TOF MS). Clin Chem Lab Med, 47(6), 694-705.

Geer, L. Y., Markey, S. P., Kowalak, J. A., Wagner, L., Xu, M., Maynard, D. M., . . Bryant, S. H. (2004). Open mass spectrometry search algorithm. J Proteome Res, 3(5), 958-964.

Geer, L. Y., Markey, S. P., Kowalak, J. A., Wagner, L., Xu, M., Maynard, D. M., . . Bryant, S. H. (2004). Open mass spectrometry search algorithm. J Proteome Res, 3(5), 958-964.

Gharahdaghi, F., Kirchner, M., Fernandez, J., \& Mische, S. M. (1996). Peptide-mass profiles of polyvinylidene difluoride-bound proteins by matrix-assisted laser desorption/ionization time-of-flight mass spectrometry in the presence of nonionic detergents. Anal Biochem, 233(1), 94-99. 
Ghosh, S., Gepstein, S., Heikkila, J. J., \& Dumbroff, E. B. (1988). Use of a scanning densitometer or an ELISA plate reader for measurement of nanogram amounts of protein in crude extracts from biological tissues. Anal Biochem, 169(2), 227-233.

Govorukhina, N. I., de Vries, M., Reijmers, T. H., Horvatovich, P., van der Zee, A. G., \& Bischoff, R. (2008). Influence of clotting time on the protein composition of serum samples based on LC-MS data. J Chromatogr B Analyt Technol Biomed Life Sci.

Haan, C., \& Behrmann, I. (2007). A cost effective non-commercial ECL-solution for Western blot detections yielding strong signals and low background. Journal of immunological methods, 318, 11-19. doi:10.1016/j.jim.2006.07.027

Helbig, A. O., Heck, A. J., \& Slijper, M. (2010). Exploring the membrane proteome-challenges and analytical strategies. J Proteomics, 73(5), 868-878.

Howard, J. C., Florentinus-Mefailoski, A., Bowden, P., Trimble, W., Grinstein, S., \& Marshall, J. G. (2016). OxLDL receptor chromatography from live human U937 cells identifies SYK (L) that regulates phagocytosis of oxLDL. Anal Biochem, 513, 7-20.

Howard, J. C., Florentinus-Mefailoski, A., Bowden, P., Trimble, W., Grinstein, S., \& Marshall, J. G. (2016). OxLDL receptor chromatography from live human U937 cells identifies SYK(L) that regulates phagocytosis of oxLDL. Anal Biochem. doi:10.1016/j.ab.2016.07.021

Humphries, J. D., Byron, A., Bass, M. D., Craig, S. E., Pinney, J. W., Knight, D., \& Humphries, M. J. (2009). Proteomic Analysis of Integrin-Associated Complexes Identifies RCC2 as a Dual Regulator of Rac1 and Arf6. Sci Signal, 2(87), ra51-ra51. doi:10.1126/scisignal.2000396

Jankowski, A., Zhu, P., \& Marshall, J. G. (2008). Capture of an activated receptor complex from the surface of live cells by affinity receptor chromatography. Analytical Biochemistry, 380, 235-248. doi:10.1016/j.ab.2008.05.047

Jankowski, A., Zhu, P., \& Marshall, J. G. (2008). Capture of an activated receptor complex from the surface of live cells by affinity receptor chromatography. Anal Biochem, 380(2), 235248. doi:10.1016/j.ab.2008.05.047

Jankowski, A., Zhu, P., \& Marshall, J. G. (2008). Capture of an activated receptor complex from the surface of live cells by affinity receptor chromatography. Anal Biochem, 380(2), 235248. doi:10.1016/j.ab.2008.05.047

Jin, Y., \& Manabe, T. (2009). Differences in protein distribution between human plasma preparations, EDTA-plasma and heparin-plasma, analyzed by non-denaturing micro-2DE and MALDI-MS PMF. Electrophoresis, 30(6), 931-938.

Kaisar, M., van Dullemen, L. F., Thezenas, M. L., Zeeshan Akhtar, M., Huang, H., Rendel, S., . . . Kessler, B. M. (2016). Plasma degradome affected by variable storage of human blood. Clin Proteomics, 13, 26. doi:10.1186/s 12014-016-9126-9

Karsan, A., Eigl, B. J., Flibotte, S., Gelmon, K., Switzer, P., Hassell, P., . . Veenstra, T. (2005). Analytical and preanalytical biases in serum proteomic pattern analysis for breast cancer diagnosis. Clin Chem, 51(8), 1525-1528.

Keller, A., Nesvizhskii, A. I., Kolker, E., \& Aebersold, R. (2002). Empirical statistical model to estimate the accuracy of peptide identifications made by MS/MS and database search. Anal Chem, 74(20), 5383-5392.

Keller, A., Nesvizhskii, A. I., Kolker, E., \& Aebersold, R. (2002). Empirical statistical model to estimate the accuracy of peptide identifications made by MS/MS and database search. Anal Chem, 74(20), 5383-5392. 
Keller, A., Purvine, S., Nesvizhskii, A. I., Stolyar, S., Goodlett, D. R., \& Kolker, E. (2002). Experimental protein mixture for validating tandem mass spectral analysis. Omics: A Journal of Integrative Biology, 6, 207-212. doi:10.1089/153623102760092805

Kielley, W. W., \& Bradley, L. B. (1956). The relationship between sulfhydryl groups and the activation of myosin adenosinetriphosphatase. J Biol Chem, 218(2), 653-659.

Kim, S., Gupta, N., \& Pevzner, P. A. (2008). Spectral probabilities and generating functions of tandem mass spectra: a strike against decoy databases. J Proteome Res, 7(8), 3354-3363.

Klett, R. P., Fulpius, B. W., Cooper, D., Smith, M., Reich, E., \& Possani, L. D. (1973). The acetylcholine receptor I. Purification and characterization of a macromolecule isolated from Electrophorus electricus. Journal of Biological Chemistry, 248(19), 6841-6853.

Koomen, J. M., Li, D., Xiao, L. C., Liu, T. C., Coombes, K. R., Abbruzzese, J., \& Kobayashi, R. (2005). Direct tandem mass spectrometry reveals limitations in protein profiling experiments for plasma biomarker discovery. J Proteome Res, 4(3), 972-981.

Krogh, A., Larsson, B., Von Heijne, G., \& Sonnhammer, E. L. (2001). Predicting transmembrane protein topology with a hidden Markov model: application to complete genomes. Journal of molecular biology, 305(3), 567-580.

Kulik, W., van Lenthe, H., Stet, F. S., Houtkooper, R. H., Kemp, H., Stone, J. E., . . Vaz, F. M. (2008). Bloodspot assay using HPLC-tandem mass spectrometry for detection of Barth syndrome. Clin Chem, 54(2), 371-378.

Langley, J. N. (1906). Croonian Lecture, 1906: On Nerve Endings and on Special Excitable Substances in Cells. Proceedings of the Royal Society of London. Series B, Containing Papers of a Biological Character, 170-194.

Lee, W. L., Cosio, G., Ireton, K., \& Grinstein, S. (2007). Role of CrkII in Fcy receptor-mediated phagocytosis. Journal of Biological Chemistry, 282(15), 11135-11143.

Lefkowitz, R. J. (1973). Isolated beta-adrenergic binding sites: a potential assay vehicle for catecholamines. Pharmacol Rev, 25(2), 259-268.

Lefkowitz, R. J. (2004). Historical review: a brief history and personal retrospective of seventransmembrane receptors. Trends Pharmacol Sci, 25(8), 413-422. doi:10.1016/j.tips.2004.06.006

LeWinter, M. M., \& Granzier, H. L. (2013). Titin is a major human disease gene. Circulation, 127(8), 938-944. doi:10.1161/CIRCULATIONAHA.112.139717

Li, J., Orlandi, R., White, C. N., Rosenzweig, J., Zhao, J., Seregni, E., . . Chan, D. W. (2005). Independent validation of candidate breast cancer serum biomarkers identified by mass spectrometry. Clin Chem, 51(12), 2229-2235.

Li, Y., Ozment, T., Wright, G. L., \& Peterson, J. M. (2016). Identification of Putative Receptors for the Novel Adipokine CTRP3 Using Ligand-Receptor Capture Technology. PloS one, 11(10), e0164593. doi:10.1371/journal.pone.0164593

Lodish, H., Berk, A., Zipursky, S. L., Matsudaira, P., Baltimore, D., \& Darnell, J. (2000). Molecular cell biology 4th edition. National Center for Biotechnology InformationÕs Bookshelf.

Lowey, S., \& Risby, D. (1971). Light chains from fast and slow muscle myosins. Nature, 234(5324), 81-85.

Marshall, J., Bowden, P., Schmit, J. C., \& Betsou, F. (2014). Creation of a federated database of blood proteins: a powerful new tool for finding and characterizing biomarkers in serum. Clin Proteomics, 11(1), 3. doi:10.1186/1559-0275-11-3 
Marshall, J., Jankowski, A., Furesz, S., Kireeva, I., Barker, L., Dombrovsky, M., . . Jackowski, G. (2004). Human serum proteins preseparated by electrophoresis or chromatography followed by tandem mass spectrometry. J Proteome Res, 3(3), 364-382.

Marshall, J., Kupchak, P., Zhu, W., Yantha, J., Vrees, T., Furesz, S., . . Jackowski, G. (2003). Processing of serum proteins underlies the mass spectral fingerprinting of myocardial infarction. Journal of Proteome Research, 2, 361-372.

Marshall, J. G., Booth, J. W., Stambolic, V., Mak, T., Balla, T., Schreiber, A. D., .. . Grinstein, S. (2001). Restricted accumulation of phosphatidylinositol 3-kinase products in a plasmalemmal subdomain during Fc gamma receptor-mediated phagocytosis. The Journal of Cell Biology, 153, 1369-1380.

Melo, S. A., Luecke, L. B., Kahlert, C., Fernandez, A. F., Gammon, S. T., Kaye, J., . . Kalluri, R. (2015). Glypican-1 identifies cancer exosomes and detects early pancreatic cancer. Nature, 523(7559), 177-182. doi:10.1038/nature14581

Mercier, C., Truntzer, C., Pecqueur, D., Gimeno, J. P., Belz, G., \& Roy, P. (2009). Mixed-model of ANOVA for measurement reproducibility in proteomics. J Proteomics, 72(6), 974981.

Metschnikoff, E. (1884). Ueber die beziehung der phagocyten zu milzbrandbacillen. Virchows Archiv, 97(3), 502-526.

Michael, I. P., Sotiropoulou, G., Pampalakis, G., Magklara, A., Ghosh, M., Wasney, G., \& Diamandis, E. P. (2005). Biochemical and enzymatic characterization of human kallikrein 5 (hK5), a novel serine protease potentially involved in cancer progression. $J$ Biol Chem, 280(15), 14628-14635. doi:10.1074/jbc.M408132200

Odin, J. A., Edberg, J. C., Painter, C. J., Kimberly, R. P., \& Unkeless, J. C. (1991). Regulation of phagocytosis and [Ca2+] i flux by distinct regions of an Fc receptor. science, 254(5039), 1785-1788.

Overington, J. P., Al-Lazikani, B., \& Hopkins, A. L. (2006). How many drug targets are there? Nat Rev Drug Discov, 5(12), 993-996. doi:10.1038/nrd2199

Parikh, I., Sica, V., Nola, E., Puca, G. A., \& Cuatrecasas, P. (1974). Affinity chromatography of estrogen receptors. Methods Enzymol, 34, 670-688.

Park SK, M. K. (1988). Random Number Generators: Good Ones Are Hard To Find. Communications of the ACM, 31(10), 1191-1201.

Perkins, D. N., Pappin, D. J., Creasy, D. M., \& Cottrell, J. S. (1999). Probability-based protein identification by searching sequence databases using mass spectrometry data. Electrophoresis, 20(18), 3551-3567.

Peterson, J. M., Seldin, M. M., Wei, Z., Aja, S., \& Wong, G. W. (2013). CTRP3 attenuates dietinduced hepatic steatosis by regulating triglyceride metabolism. Am J Physiol Gastrointest Liver Physiol, 305(3), G214-224. doi:10.1152/ajpgi.00102.2013

Pieper, U., Schlessinger, A., Kloppmann, E., Chang, G. A., Chou, J. J., Dumont, M. E., . . . Sali, A. (2013). Coordinating the impact of structural genomics on the human $\alpha$-helical transmembrane proteome. Nature structural \& molecular biology, 20(2), 135-138. doi: $10.1038 / \mathrm{nsmb} .2508$

Pilny, R., Bouchal, P., Borilova, S., Ceskova, P., Zaloudik, J., Vyzula, R., . . Valik, D. (2006). Surface-enhanced laser desorption ionization/time-of-flight mass spectrometry reveals significant artifacts in serum obtained from clot activator-containing collection devices. Clin Chem, 52(11), 2115-2116. 
Purves, D., Augustine, G., \& Fitzpatrick, D. (2001). et al., editors. Neuroscience. Sunderland (MA). In: Sinauer Associates.

Putnam, F. (1975). The plasma Proteins: Structure Function, and Genetic Control. Academic Press, New York, second ed.

Qin, S., Zhou, Y., Gray, L., Kusebauch, U., McEvoy, L., Antoine, D. J., . . . Hood, L. (2016). Identification of Blood Protein Biomarkers of Acute Liver Injury by Targeted Quantitative Proteomics in Acetaminophen and Carbon tetrachloride treated Mouse Models and Acetaminophen Overdose Patients. J Proteome Res. doi:10.1021/acs.jproteome.6b00547

Qu, Z. X., Odin, J., Glass, J. D., \& Unkeless, J. C. (1988). Expression and characterization of a truncated murine Fc gamma receptor. J Exp Med, 167(3), 1195-1210.

Rai, A. J., Gelfand, C. A., Haywood, B. C., Warunek, D. J., Yi, J., Schuchard, M. D., ... Chan, D. W. (2005). HUPO Plasma Proteome Project specimen collection and handling: towards the standardization of parameters for plasma proteome samples. Proteomics, 5(13), 3262-3277.

Sardesai, V. M., \& Provido, H. S. (1965). A Fluorometric Method for Determining the Tame Esterase (Tryptic) Activity of Plasma. J Lab Clin Med, 65, 1023-1029.

Schagger, H., \& von Jagow, G. (1987). Tricine-sodium dodecyl sulfate-polyacrylamide gel electrophoresis for the separation of proteins in the range from 1 to $100 \mathrm{kDa}$. Anal Biochem, 166(2), 368-379.

Schwartz, J. C., Senko, M. W., \& Syka, J. E. (2002). A two-dimensional quadrupole ion trap mass spectrometer. J Am Soc Mass Spectrom, 13(6), 659-669.

Semmes, O. J., Feng, Z., Adam, B. L., Banez, L. L., Bigbee, W. L., Campos, D., . . Zhu, L. (2005). Evaluation of serum protein profiling by surface-enhanced laser desorption/ionization time-of-flight mass spectrometry for the detection of prostate cancer: I. Assessment of platform reproducibility. Clin Chem, 51(1), 102-112.

Shi, T., Sun, X., Gao, Y., Fillmore, T. L., Schepmoes, A. A., Zhao, R., . . Qian, W. J. (2013). Targeted quantification of low $\mathrm{ng} / \mathrm{mL}$ level proteins in human serum without immunoaffinity depletion. J Proteome Res, 12(7), 3353-3361.

Shiu, R. P., \& Friesen, H. G. (1974). Solubilization and purification of a prolactin receptor from the rabbit mammary gland. J Biol Chem, 249(24), 7902-7911.

Sica, V., Nola, E., Parikh, I., Puca, G. A., \& Cuatrecasas, P. (1973). Purification of oestradiol receptors by affinity chromatography. Nat New Biol, 244(132), 36-39.

Sica, V., Parikh, I., Nola, E., Puca, G. A., \& Cuatrecasas, P. (1973). Affinity chromatography and the purification of estrogen receptors. J Biol Chem, 248(18), 6543-6558.

Sorace, J. M., \& Zhan, M. (2003). A data review and re-assessment of ovarian cancer serum proteomic profiling. BMC Bioinformatics, 4(1), 24.

Spencer, K. (2003). The influence of different sample collection types on the levels of markers used for Down's syndrome screening as measured by the Kryptor Immunosassay system. Ann Clin Biochem, 40(Pt 2), 166-168.

Stafford, G. C. (1980). Instrumental aspects of positive and negative ion chemical ionization mass spectrometry. Environ Health Perspect, 36, 85-88.

Staros, J. V. (1982). N-hydroxysulfosuccinimide active esters: bis(N-hydroxysulfosuccinimide) esters of two dicarboxylic acids are hydrophilic, membrane-impermeant, protein crosslinkers. Biochemistry, 21(17), 3950-3955. 
Stiles, G. L., Caron, M. G., \& Lefkowitz, R. J. (1984). Beta-adrenergic receptors: biochemical mechanisms of physiological regulation. Physiological reviews.

Thakur, S. S., Geiger, T., Chatterjee, B., Bandilla, P., Frohlich, F., Cox, J., \& Mann, M. (2011). Deep and highly sensitive proteome coverage by LC-MS/MS without prefractionation. Mol Cell Proteomics, 10(8), M110 003699. doi:10.1074/mcp.M110.003699

Tietz. (2001). Tietz Fundamentals of Clinical Chemistry (5th edition ed. Vol. 5th edition): Saunders.

Tonegawa, S., Steinberg, C., Dube, S., \& Bernardini, A. (1974). Evidence for somatic generation of antibody diversity. Proc Natl Acad Sci U S A, 71(10), 4027-4031.

Towbin, H., Staehelin, T., \& Gordon, J. (1979). Electrophoretic transfer of proteins from polyacrylamide gels to nitrocellulose sheets: procedure and some applications. Proc Natl Acad Sci U S A, 76(9), 4350-4354.

Tridandapani, S., Lyden, T. W., Smith, J. L., Carter, J. E., Coggeshall, K. M., \& Anderson, C. L. (2000). The adapter protein LAT enhances Fc $\gamma$ receptor-mediated signal transduction in myeloid cells. Journal of Biological Chemistry, 275(27), 20480-20487.

Tucholska, M., Bowden, P., Jacks, K., Zhu, P., Furesz, S., Dumbrovsky, M., \& Marshall, J. (2009). Human serum proteins fractionated by preparative partition chromatography prior to LC-ESI-MS/MS. Journal of Proteome Research, 8, 1143-1155. doi:10.1021/pr8005217

Tucholska, M., Florentinus, A., Williams, D., \& Marshall, J. G. (2010). The endogenous peptides of normal human serum extracted from the acetonitrile-insoluble precipitate using modified aqueous buffer with analysis by LC-ESI-Paul ion trap and Qq-TOF. $J$ Proteomics, 73(6), 1254-1269. doi:10.1016/j.jprot.2010.02.022

Tucholska, M., Scozzaro, S., Williams, D., Ackloo, S., Lock, C., Siu, K. W. M., . . Marshall, J. G. (2007). Endogenous peptides from biophysical and biochemical fractionation of serum analyzed by matrix-assisted laser desorption/ionization and electrospray ionization hybrid quadrupole time-of-flight. Analytical Biochemistry, 370, 228-245. doi:10.1016/j.ab.2007.07.029

Tuck, M. K., Chan, D. W., Chia, D., Godwin, A. K., Grizzle, W. E., Krueger, K. E., .. . Brenner, D. E. (2009). Standard operating procedures for serum and plasma collection: early detection research network consensus statement standard operating procedure integration working group. J Proteome Res, 8(1), 113-117.

Tzircotis, G., Braga, V. M., \& Caron, E. (2011). RhoG is required for both Fc $\gamma$ R-and CR3mediated phagocytosis. Journal of cell science, 124(17), 2897-2902.

Uhlen, M., Guss, B., Nilsson, B., Gatenbeck, S., Philipson, L., \& Lindberg, M. (1984). Complete sequence of the staphylococcal gene encoding protein A. A gene evolved through multiple duplications. J Biol Chem, 259(3), 1695-1702.

van den Broek, I., Romijn, F. P., Smit, N. P., van der Laarse, A., Drijfhout, J. W., van der Burgt, Y. E., \& Cobbaert, C. M. (2015). Quantifying protein measurands by peptide measurements: where do errors arise? J Proteome Res, 14(2), 928-942. doi:10.1021/pr5011179

van den Broek, I., Sparidans, R. W., Schellens, J. H., \& Beijnen, J. H. (2008). Liquid chromatography/tandem mass spectrometric method for the quantification of eight proteolytic fragments of ITIH4 with biomarker potential in human plasma and serum. Rapid Commun Mass Spectrom, 22(18), 2915-2928. 
van Winden, A. W., Gast, M. C., Beijnen, J. H., Rutgers, E. J., Grobbee, D. E., Peeters, P. H., \& van Gils, C. H. (2009). Validation of previously identified serum biomarkers for breast cancer with SELDI-TOF MS: a case control study. BMC Med Genomics, 2(1), 4.

Vergeynst, L., Van Langenhove, H., \& Demeestere, K. (2015). Balancing the false negative and positive rates in suspect screening with high-resolution Orbitrap mass spectrometry using multivariate statistics. Anal Chem, 87(4), 2170-2177. doi:10.1021/ac503426k

Volanakis, J. E. (1990). Participation of C3 and its ligands in complement activation. Curr Top Microbiol Immunol, 153, 1-21.

Von Jagow, G., Schägger, H., Riccio, P., Klingenberg, M., \& Kolb, H. (1977). b· c1 complex from beef heart: Hydrodynamic properties of the complex prepared by a refined hydroxyapatite chromatography in Triton X-100. Biochimica et Biophysica Acta (BBA)Bioenergetics, 462(3), 549-558.

von Mering, C., Jensen, L. J., Kuhn, M., Chaffron, S., Doerks, T., Kruger, B., . . Bork, P. (2007). STRING 7--recent developments in the integration and prediction of protein interactions. Nucleic Acids Res, 35(Database issue), D358-362.

von Mering, C., Jensen, L. J., Snel, B., Hooper, S. D., Krupp, M., Foglierini, M., . . Bork, P. (2005). STRING: known and predicted protein-protein associations, integrated and transferred across organisms. Nucleic Acids Res, 33(Database issue), D433-437.

Wallin, E., \& Heijne, G. V. (1998). Genome-wide analysis of integral membrane proteins from eubacterial, archaean, and eukaryotic organisms. Protein Science, 7(4), 1029-1038.

Weisman, H. F., Bartow, T., Leppo, M. K., Marsh, H. C., Jr., Carson, G. R., Concino, M. F., . . . Fearon, D. T. (1990). Soluble human complement receptor type 1: in vivo inhibitor of complement suppressing post-ischemic myocardial inflammation and necrosis. Science, 249(4965), 146-151.

West-Nielsen, M., Hogdall, E. V., Marchiori, E., Hogdall, C. K., Schou, C., \& Heegaard, N. H. (2005). Sample handling for mass spectrometric proteomic investigations of human sera. Anal Chem, 77(16), 5114-5123.

West-Norager, M., Kelstrup, C. D., Schou, C., Hogdall, E. V., Hogdall, C. K., \& Heegaard, N. H. (2007). Unravelling in vitro variables of major importance for the outcome of mass spectrometry-based serum proteomics. J Chromatogr B Analyt Technol Biomed Life Sci, 847(1), 30-37.

Wiita, A. P., Hsu, G. W., Lu, C. M., Esensten, J. H., \& Wells, J. A. (2014). Circulating proteolytic signatures of chemotherapy-induced cell death in humans discovered by Nterminal labeling. Proc Natl Acad Sci U S A, 111(21), 7594-7599. doi:10.1073/pnas.1405987111

Williams, D., Ackloo, S., Zhu, P., Bowden, P., Evans, K. R., Addison, C. L., . . Marshall, J. G. (2010). Precipitation and selective extraction of human serum endogenous peptides with analysis by quadrupole time-of-flight mass spectrometry reveals posttranslational modifications and low-abundance peptides. Analytical and bioanalytical chemistry, 396, 1223-1247. doi:10.1007/s00216-009-3345-0

Yang, X., Clifton, J., Huang, F., Kovac, S., Hixson, D. C., \& Josic, D. (2009). Proteomic analysis for process development and control of therapeutic protein separation from human plasma. Electrophoresis, 30(7), 1185-1193.

Yates, J. R., 3rd. (1998). Database searching using mass spectrometry data. Electrophoresis, 19(6), 893-900. 
Yates, J. R., 3rd, Eng, J. K., McCormack, A. L., \& Schieltz, D. (1995). Method to correlate tandem mass spectra of modified peptides to amino acid sequences in the protein database. Anal Chem, 67(8), 1426-1436.

Yi, J., Kim, C., \& Gelfand, C. A. (2007). Inhibition of intrinsic proteolytic activities moderates preanalytical variability and instability of human plasma. J Proteome Res, 6(5), 17681781.

Yi, J., Liu, Z., Craft, D., O'Mullan, P., Ju, G., \& Gelfand, C. A. (2008). Intrinsic peptidase activity causes a sequential multi-step reaction (SMSR) in digestion of human plasma peptides. J Proteome Res, 7(12), 5112-5118.

Zhang, R., Barker, L., Pinchev, D., Marshall, J., Rasamoelisolo, M., Smith, C., . . Jackowski, G. (2004). Mining biomarkers in human sera using proteomic tools. Proteomics, 4(1), 244256.

Zheng, X., Baker, H., Hancock, W. S., Fawaz, F., McCaman, M., \& Pungor, E., Jr. (2006). Proteomic analysis for the assessment of different lots of fetal bovine serum as a raw material for cell culture. Part IV. Application of proteomics to the manufacture of biological drugs. Biotechnol Prog, 22(5), 1294-1300.

Zhu, P., Bowden, P., Pendrak, V., Thiele, H., Zhang, D., Siu, M., . . Marshall, J. (2007). Comparison of protein expression lists from mass spectrometry of human blood fluids using exact peptide sequences versus BLAST. Clinical Proteomics, 2(3-4), 185-203.

Zhu, P., Bowden, P., Tucholska, M., \& Marshall, J. G. (2011). Chi-square comparison of tryptic peptide-to-protein distributions of tandem mass spectrometry from blood with those of random expectation. Anal Biochem, 409(2), 189-194.

Zhu, P., Bowden, P., Tucholska, M., Zhang, D., \& Marshall, J. G. (2011). Peptide-to-protein distribution versus a competition for significance to estimate error rate in blood protein identification. Analytical Biochemistry, 411, 241-253. doi:10.1016/j.ab.2010.12.003

Zhu, P., Bowden, P., Zhang, D., \& Marshall, J. G. (2011). Mass spectrometry of peptides and proteins from human blood. Mass Spectrom Rev, 30,(5), 685-732.

Zolg, D. P., Wilhelm, M., Schnatbaum, K., Zerweck, J., Knaute, T., Delanghe, B., ... Kuster, B. (2017). Building ProteomeTools based on a complete synthetic human proteome. Nat Methods, 14(3), 259-262. doi:10.1038/nmeth.4153 
SUPPLEMENT A 
Treatments and ESI-LC-MSMS runs for ligand affinity chromatography experiments

\begin{tabular}{|c|c|c|c|}
\hline File name & $\begin{array}{l}\text { TREATMENT } \\
\text { ID }\end{array}$ & $\begin{array}{l}\text { Records } \\
\text { Written }\end{array}$ & $\begin{array}{l}\text { Replicates } \\
\text { Per } \\
\text { Treatment }\end{array}$ \\
\hline \multicolumn{4}{|l|}{ DTT } \\
\hline \multicolumn{4}{|l|}{ Experiment 1} \\
\hline \multicolumn{4}{|l|}{ DDT } \\
\hline \multicolumn{4}{|l|}{ MODS } \\
\hline CcbD_FCBLARC_noBSA_MOUSE_NO_MODS & 78 & 72533 & 9 \\
\hline CsaD_FCBLARC_noBSA_MOUSE_NO_MODS & 90 & 53962 & 9 \\
\hline LsaD_FCBLARC_noBSA_MOUSE_NO_MODS & 123 & 59523 & 9 \\
\hline LBsaD_FCBLARC_noBSA_MOUSE_NO_MODS & 113 & 41770 & 9 \\
\hline LSBsaD_FCBLARC_noBSA_MOUSE_NO_MODS & 136 & 64059 & 9 \\
\hline \multicolumn{4}{|l|}{$\overline{\text { DTT }}$} \\
\hline \multicolumn{4}{|l|}{ Experiment 2} \\
\hline \multicolumn{4}{|l|}{ MODS } \\
\hline CcbD_BSA_MvsA_FcBLARC_BSA_Mono_Agg_MOUSE_MODS & 5 & 5022 & 1 \\
\hline CsaD_BSA_MvsA_FcBLARC_BSA_Mono_Agg_MOUSE_MODS & 17 & 4269 & 1 \\
\hline LBsaD_BSA_MvsA_FcBLARC_BSA_Mono_Agg_MOUSE_MODS & 40 & 6333 & 1 \\
\hline LSBsaD_BSA_MvsA_FcBLARC_BSA_Mono_Agg_MOUSE_MODS & 63 & 4267 & 1 \\
\hline LABsaD_BSA_MvsA_FcBLARC_BSA_Mono_Agg_MOUSE_MODS & 24 & 3534 & 1 \\
\hline LBAsaD_BSA_MvsA_FcBLARC_BSA_Mono_Agg_MOUSE_MODS & 32 & 4913 & 1 \\
\hline LASBsaD_BSA_MvsA_FcBLARC_BSA_Mono_Agg_MOUSE_MODS & 28 & 4262 & 1 \\
\hline LSBAsaD_BSA_MvsA_FcBLARC_BSA_Mono_Agg_MOUSE_MODS & 55 & 5399 & 1 \\
\hline \multicolumn{4}{|l|}{ DTT } \\
\hline \multicolumn{4}{|l|}{ Experiment 3} \\
\hline \multicolumn{4}{|l|}{ MODS } \\
\hline CcbDx10_BSA_FCBLARCX10_BSA_MOUSE_MODS & 7 & 8544 & 1 \\
\hline LBsaDx10_BSA_FCBLARCX10_BSA_MOUSE_MODS & 42 & 4454 & 1 \\
\hline LSBsaDx10_BSA_FCBLARCX10_BSA_MOUSE_MODS & 65 & 5184 & 1 \\
\hline \multicolumn{4}{|l|}{ Experiment4 (FcBLARC12) } \\
\hline \multicolumn{4}{|l|}{ MODS } \\
\hline C_NND_MOUSE_MODS_MODS & 0 & 16545 & 4 \\
\hline C_SND_MOUSE_MODS & 4 & 19389 & 4 \\
\hline C_SID_MOUSE_MODS & 3 & 18629 & 4 \\
\hline C_SBD_MOUSE_MODS_MODS & 2 & 15995 & 4 \\
\hline C_SSD_MOUSE_MODS & 6 & 14793 & 4 \\
\hline L_SID_MOUSE_MODS & 9 & 23462 & 4 \\
\hline L_SBD_MOUSE_MODS & 8 & 18838 & 4 \\
\hline
\end{tabular}




\begin{tabular}{|c|c|c|c|}
\hline L_SSD_MOUSE_MODS & 13 & 20432 & 5 \\
\hline L_SSD_D_MOUSE_MODS & 11 & 8166 & 2 \\
\hline L_SSD_O_MOUSE_MODS & 14 & 6516 & 2 \\
\hline L_SSD_XL_MOUSE_MODS & 15 & 5344 & 2 \\
\hline L_SSD_LX_MOUSE_MODS & 12 & 8150 & 2 \\
\hline LSSD_Ag_MOUSE_MODS & 17 & 10495 & 2 \\
\hline \multicolumn{4}{|l|}{ BME } \\
\hline \multicolumn{4}{|l|}{ Experiment 1} \\
\hline \multicolumn{4}{|l|}{ MODS } \\
\hline CcbM_FCBLARC_noBSA_MOUSE_NO_MODS & 81 & 58105 & 9 \\
\hline CsaM_FCBLARC_noBSA_MOUSE_NO_MODS & 92 & 64616 & 10 \\
\hline LsaM_FCBLARC_noBSA_MOUSE_NO_MODS & 124 & 56247 & 9 \\
\hline LBsaM_FCBLARC_noBSA_MOUSE_NO_MODS & 116 & 66136 & 9 \\
\hline LSBsaM_FCBLARC_noBSA_MOUSE_NO_MODS & 139 & 70719 & 9 \\
\hline \multicolumn{4}{|l|}{ BME } \\
\hline \multicolumn{4}{|l|}{ Experiment 2} \\
\hline \multicolumn{4}{|l|}{ MODS } \\
\hline CcbM_BSA_MvsA_FcBLARC_BSA_Mono_Agg_MOUSE_MODS & 8 & 6738 & 1 \\
\hline CsaM_BSA_MvsA_FcBLARC_BSA_Mono_Agg_MOUSE_MODS & 19 & 4865 & 1 \\
\hline LBsaM_BSA_MvsA_FcBLARC_BSA_Mono_Agg_MOUSE_MODS & 43 & 4706 & 1 \\
\hline LSBsaM_BSA_MvsA_FcBLARC_BSA_Mono_Agg_MOUSE_MODS & 66 & 3816 & 1 \\
\hline LABsaM_BSA_MvsA_FcBLARC_BSA_Mono_Agg_MOUSE_MODS & 25 & 3508 & 1 \\
\hline LBAsaM_BSA_MvsA_FcBLARC_BSA_Mono_Agg_MOUSE_MODS & 33 & 3560 & 1 \\
\hline LASBsaM_BSA_MvsA_FcBLARC_BSA_Mono_Agg_MOUSE_MODS & 29 & 4532 & 1 \\
\hline LSBAsaM_BSA_MvsA_FcBLARC_BSA_Mono_Agg_MOUSE_MODS & 56 & 2783 & 1 \\
\hline \multicolumn{4}{|l|}{ BME } \\
\hline \multicolumn{4}{|l|}{ Experiment 3} \\
\hline \multicolumn{4}{|l|}{ MODS } \\
\hline CcbMx10_BSA_FCBLARCX10_BSA_MOUSE_MODS & 10 & 2522 & 1 \\
\hline LBsaMx10_BSA_FCBLARCX10_BSA_MOUSE_MODS & 45 & 2707 & 1 \\
\hline LSBsaMx10_BSA_FCBLARCX10_BSA_MOUSE_NO_MODS & 140 & 3653 & 1 \\
\hline \multicolumn{4}{|l|}{ NH4OH } \\
\hline \multicolumn{4}{|l|}{ Experiment 1} \\
\hline \multicolumn{4}{|l|}{ MODS } \\
\hline CcbN_FCBLARC_noBSA_MOUSE_MODS & 12 & 64430 & 9 \\
\hline CsaN_FCBLARC_noBSA_MOUSE_MODS & 22 & 67820 & 9 \\
\hline LsaN_FCBLARC_noBSA_MOUSE_MODS & 53 & 53623 & 10 \\
\hline
\end{tabular}




\begin{tabular}{|c|c|c|c|}
\hline LBsaN_FCBLARC_noBSA_MOUSE_MODS & 47 & 39127 & 9 \\
\hline LSBsaN_FCBLARC_noBSA_MOUSE_MODS & 70 & 50736 & 10 \\
\hline \multicolumn{4}{|l|}{ NH4OH } \\
\hline \multicolumn{4}{|l|}{ Experiment 2} \\
\hline \multicolumn{4}{|l|}{ MODS } \\
\hline CcbN_BSA_MvsA_FcBLARC_BSA_Mono_Agg_MOUSE_NO_MODS & 83 & 2147 & 1 \\
\hline CsaN_BSA_MvsA_FcBLARC_BSA_Mono_Agg_MOUSE_NO_MODS & 93 & 2684 & 1 \\
\hline LBsaN_BSA_MvsA_FcBLARC_BSA_Mono_Agg_MOUSE_NO_MODS & 118 & 2394 & 1 \\
\hline LSBsaN_BSA_MvsA_FcBLARC_BSA_Mono_Agg_MOUSE_NO_MODS & 141 & 2146 & 1 \\
\hline LABsaN_BSA_MvsA_FcBLARC_BSA_Mono_Agg_MOUSE_NO_MODS & 98 & 2958 & 1 \\
\hline LBAsaN_BSA_MvsA_FcBLARC_BSA_Mono_Agg_MOUSE_NO_MODS & 106 & 2464 & 1 \\
\hline LASBsaN_BSA_MvsA_FcBLARC_BSA_Mono_Agg_MOUSE_NO_MODS & 102 & 2724 & 1 \\
\hline LSBAsaN_BSA_MvsA_FcBLARC_BSA_Mono_Agg_MOUSE_NO_MODS & 129 & 3408 & 1 \\
\hline \multicolumn{4}{|l|}{ NH4OH } \\
\hline \multicolumn{4}{|l|}{ Experiment 3} \\
\hline \multicolumn{4}{|l|}{ MODS } \\
\hline CcbNx10_BSA_FCBLARCX10_BSA_MOUSE_MODS & 13 & 2900 & 1 \\
\hline LBsaNx10_BSA_FCBLARCX10_BSA_MOUSE_MODS & 48 & 3486 & 1 \\
\hline LSBsaNx10_BSA_FCBLARCX10_BSA_MOUSE_MODS & 71 & 3175 & 1 \\
\hline \multicolumn{4}{|l|}{ NH4OH+B } \\
\hline \multicolumn{4}{|l|}{ Experiment 1} \\
\hline \multicolumn{4}{|l|}{ MODS } \\
\hline CcbB_FCBLARC_noBSA_MOUSE_MODS & 1 & 61879 & 9 \\
\hline CsaB_FCBLARC_noBSA_MOUSE_MODS & 15 & 74785 & 9 \\
\hline LBsaB_FCBLARC_noBSA_MOUSE_MODS & 36 & 74827 & 9 \\
\hline LsaB_FCBLARC_noBSA_MOUSE_MODS & 50 & 55860 & 9 \\
\hline LSBsaB_FCBLARC_noBSA_MOUSE_MODS & 59 & 73986 & 9 \\
\hline \multicolumn{4}{|l|}{ NH4OH+B } \\
\hline \multicolumn{4}{|l|}{ Experiment 2} \\
\hline \multicolumn{4}{|l|}{ MODS } \\
\hline CcbB_BSA_MvsA_FcBLARC_BSA_Mono_Agg_MOUSE_MODS & 0 & 3507 & 1 \\
\hline CsaB_BSA_MvsA_FcBLARC_BSA_Mono_Agg_MOUSE_MODS & 14 & 2526 & 1 \\
\hline LBsaB_BSA_MvsA_FcBLARC_BSA_Mono_Agg_MOUSE_MODS & 35 & 3304 & 1 \\
\hline LSBsaB_BSA_MvsA_FcBLARC_BSA_Mono_Agg_MOUSE_MODS & 58 & 3639 & 1 \\
\hline LBAsaB_BSA_MvsA_FcBLARC_BSA_Mono_Agg_MOUSE_MODS & 31 & 3177 & 1 \\
\hline LABsaB_BSA_MvsA_FcBLARC_BSA_Mono_Agg_MOUSE_MODS & 23 & 3773 & 1 \\
\hline LASBsaB_BSA_MvsA_FcBLARC_BSA_Mono_Agg_MOUSE_MODS & 27 & 3889 & 1 \\
\hline
\end{tabular}




\begin{tabular}{|c|c|c|c|}
\hline $\mathrm{NH} 4 \mathrm{OH}+\mathrm{B}$ & & & \\
\hline Experiment 3 & & & \\
\hline MODS & & & \\
\hline CcbBx10_BSA_FCBLARCX10_BSA_MOUSE_MODS & 4 & 3399 & 1 \\
\hline LBsaBx10_BSA_FCBLARCX10_BSA_MOUSE_MODS & 39 & 3089 & 1 \\
\hline LSBsaBx10_BSA_FCBLARCX10_BSA_MOUSE_MODS & 62 & 3111 & 1 \\
\hline BEADS & & & \\
\hline Experiment1 & & & \\
\hline MODS & & & \\
\hline CcbBD_FCBLARC_noBSA_MOUSE_MODS & 2 & 27874 & 3 \\
\hline CsaBD_FCBLARC_noBSA_MOUSE_MODS & 16 & 25084 & 3 \\
\hline LIsaBD_FCBLARC_noBSA_MOUSE_MODS & 49 & 27429 & 3 \\
\hline LBsaBD_FCBLARC_noBSA_MOUSE_MODS & 37 & 23528 & 3 \\
\hline LSBsaBD_FCBLARC_noBSA_MOUSE_MODS & 60 & 33357 & 3 \\
\hline Experiment 3 & & & \\
\hline MODS & & & \\
\hline CcbBdx10_BSA_FCBLARCX10_BSA_MOUSE_MODS & 3 & 3505 & 1 \\
\hline LBsaBDx10_BSA_FCBLARCX10_BSA_MOUSE_MODS & 38 & 2682 & 1 \\
\hline LSBsaBdx10_BSA_FCBLARCX10_BSA_MOUSE_MODS & 61 & 3886 & 1 \\
\hline Experiment 4 & & & \\
\hline MODS & & & \\
\hline C_NNZ_MOUSE_MODS_MODS & 1 & 37819 & 4 \\
\hline C_SNZ_MOUSE_MODS & 5 & 29610 & 4 \\
\hline C_SSZ_MOUSE_MODS & 7 & 4958 & 1 \\
\hline L_SIZ_MOUSE_MODS & 10 & 31133 & 4 \\
\hline L_SSZ_MOUSE_MODS & 16 & 28591 & 3 \\
\hline Experiment 5 & & & \\
\hline MODS & & & \\
\hline MelamineMicroIgG & & & 3 (78 runs) \\
\hline MelamineMicroCrudeCtrl & & & 1 (26 runs) \\
\hline MelamineMicroMediaCtrl & & & 1 (26 runs) \\
\hline PMMAmicroIgG & & & 1 (26 runs) \\
\hline GlassNanoIgG & & & 2 (52 runs) \\
\hline GlassNanoCrudeCtrl & & & 1 (26 runs) \\
\hline GlassNanoMediaCtrl & & & 1 (26 runs) \\
\hline & & & \\
\hline & & & \\
\hline & & & \\
\hline
\end{tabular}


SUPPLEMENT B 
SQL Query Code Used for grouping FC and IgG Tables:

SELECT

GeneSymbol,

IdentificationFilterMethod

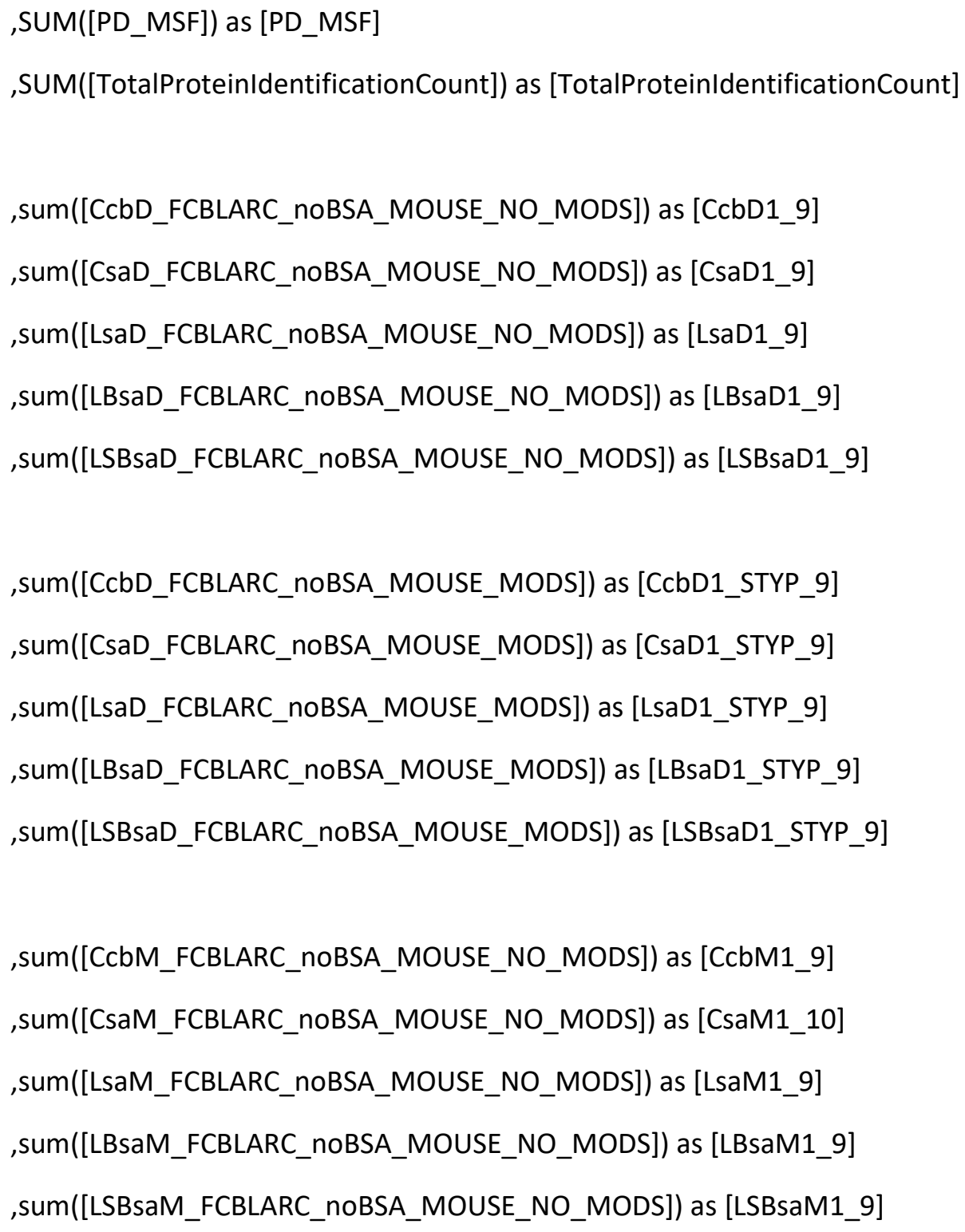


,sum([CcbM_FCBLARC_noBSA_MOUSE_MODS]) as [CcbM1_STYP_9]

,sum([CsaM_FCBLARC_noBSA_MOUSE_MODS]) as [CsaM1_STYP_10]

,sum([LsaM_FCBLARC_noBSA_MOUSE_MODS]) as [LsaM1_STYP_9]

,sum([LBsaM_FCBLARC_noBSA_MOUSE_MODS]) as [LBsaM1_STYP_9]

,sum([LSBsaM_FCBLARC_noBSA_MOUSE_MODS]) as [LSBsaM1_STYP_9]

,sum([CcbN_FCBLARC_noBSA_MOUSE_NO_MODS]) as [CcbN1_9]

,sum([CsaN_FCBLARC_noBSA_MOUSE_NO_MODS]) as [CsaN1_9]

,sum([LsaN_FCBLARC_noBSA_MOUSE_NO_MODS]) as [LsaN1_10]

,sum([LBsaN_FCBLARC_noBSA_MOUSE_NO_MODS]) as [LBsaN1_9]

,sum([LSBsaN_FCBLARC_noBSA_MOUSE_NO_MODS]) as [LSBsaN1_10]

,sum([CcbN_FCBLARC_noBSA_MOUSE_MODS]) as [CcbN1_STYP_9]

,sum([CsaN_FCBLARC_noBSA_MOUSE_MODS]) as [CsaN1_STYP_9]

,sum([LsaN_FCBLARC_noBSA_MOUSE_MODS]) as [LsaN1_STYP_10]

,sum([LBsaN_FCBLARC_noBSA_MOUSE_MODS]) as [LBsaN1_STYP_9]

,sum([LSBsaN_FCBLARC_noBSA_MOUSE_MODS]) as [LSBsaN1_STYP_10]

,sum([CcbB_FCBLARC_noBSA_MOUSE_NO_MODS]) as [CcbB1_9]

,sum([CsaB_FCBLARC_noBSA_MOUSE_NO_MODS]) as [CsaB1_9]

,sum([LsaB_FCBLARC_noBSA_MOUSE_NO_MODS]) as [LsaB1_9]

,sum([LBsaB_FCBLARC_noBSA_MOUSE_NO_MODS]) as [LBsaB1_9]

,sum([LSBsaB_FCBLARC_noBSA_MOUSE_NO_MODS]) as [LSBsaB_9]

,sum([CcbB_FCBLARC_noBSA_MOUSE_MODS]) as [CcbB1_STYP_9]

,sum([CsaB_FCBLARC_noBSA_MOUSE_MODS]) as [CsaB1_STYP_9]

,sum([LsaB_FCBLARC_noBSA_MOUSE_MODS]) as [LsaB1_STYP_9]

,sum([LBsaB_FCBLARC_noBSA_MOUSE_MODS]) as [LBsaB1_STYP_9] 
,sum([LSBsaB_FCBLARC_noBSA_MOUSE_MODS]) as [LSBsaB1_STYP_9]

,sum([CcbBD_FCBLARC_noBSA_MOUSE_NO_MODS]) as [CcbBD1_3]

,sum([CsaBD_FCBLARC_noBSA_MOUSE_NO_MODS]) as [CsaBD1_3]

,sum([LIsaBD_FCBLARC_noBSA_MOUSE_NO_MODS]) as [LsaBD1_3]

,sum([LBsaBD_FCBLARC_noBSA_MOUSE_NO_MODS]) as [LBsaBD1_3]

,sum([LSBsaBD_FCBLARC_noBSA_MOUSE_NO_MODS]) as [LSBsaBD1_3]

,sum([CcbBD_FCBLARC_noBSA_MOUSE_MODS]) as [CcbBD1_STYP_3]

,sum([CsaBD_FCBLARC_noBSA_MOUSE_MODS]) as [CsaBD1_STYP_3]

,sum([LIsaBD_FCBLARC_noBSA_MOUSE_MODS]) as [LsaBD1_STYP_3]

,sum([LBsaBD_FCBLARC_noBSA_MOUSE_MODS]) as [LBsaBD1_STYP_3]

,sum([LSBsaBD_FCBLARC_noBSA_MOUSE_MODS]) as [LSBsaBD1_STYP_3]

,sum([CcbDx10_BSA_FCBLARCX10_BSA_MOUSE_NO_MODS]) as [CcbD3_1]

,sum([LBsaDx10_BSA_FCBLARCX10_BSA_MOUSE_NO_MODS]) as [LBsaD3_1]

,sum([LSBsaDx10_BSA_FCBLARCX10_BSA_MOUSE_NO_MODS]) as [LSBsaD3_1]

,sum([CcbDx10_BSA_FCBLARCX10_BSA_MOUSE_MODS]) as [CcbD3_STYP_1]

,sum([LBsaDx10_BSA_FCBLARCX10_BSA_MOUSE_MODS]) as [LBsaD3_STYP_1]

,sum([LSBsaDx10_BSA_FCBLARCX10_BSA_MOUSE_MODS]) as [LSBsaD3_STYP_1]

,sum([CcbMx10_BSA_FCBLARCX10_BSA_MOUSE_NO_MODS]) as [CcbM3_1]

,sum([LBsaMx10_BSA_FCBLARCX10_BSA_MOUSE_NO_MODS]) as [LBsaM3_1]

,sum([LSBsaMx10_BSA_FCBLARCX10_BSA_MOUSE_NO_MODS]) as [LSBsaM3_1] 
,sum([CcbMx10_BSA_FCBLARCX10_BSA_MOUSE_MODS]) as [CcbM3_STYP_1] ,sum([LBsaMx10_BSA_FCBLARCX10_BSA_MOUSE_MODS]) as [LBsaM3_STYP_1] ,sum([LSBsaMx10_BSA_FCBLARCX10_BSA_MOUSE_MODS]) as [LSBsaM3_STYP_1]

,sum([CcbNx10_BSA_FCBLARCX10_BSA_MOUSE_NO_MODS]) as [CcbN3_1] ,sum([LBsaNx10_BSA_FCBLARCX10_BSA_MOUSE_NO_MODS]) as [LBsaN3_1] ,sum([LSBsaNx10_BSA_FCBLARCX10_BSA_MOUSE_NO_MODS]) as [LSBsaN3_1]

,sum([CcbNx10_BSA_FCBLARCX10_BSA_MOUSE_MODS]) as [CcbN3_STYP_1] ,sum([LBsaNx10_BSA_FCBLARCX10_BSA_MOUSE_MODS]) as [LBsaN3_STYP_1] ,sum([LSBsaNx10_BSA_FCBLARCX10_BSA_MOUSE_MODS]) as [LSBsaN3_STYP_1]

,sum([CcbBx10_BSA_FCBLARCX10_BSA_MOUSE_NO_MODS]) as [CcbB3_1] ,sum([LBsaBx10_BSA_FCBLARCX10_BSA_MOUSE_NO_MODS]) as [LBsaB3_1] ,sum([LSBsaBx10_BSA_FCBLARCX10_BSA_MOUSE_NO_MODS]) as [LSBsaB3_1] ,sum([CcbBx10_BSA_FCBLARCX10_BSA_MOUSE_MODS]) as [CcbB3_STYP_1] ,sum([LBsaBx10_BSA_FCBLARCX10_BSA_MOUSE_MODS]) as [LBsaB3_STYP_1] ,sum([LSBsaBx10_BSA_FCBLARCX10_BSA_MOUSE_MODS]) as [LSBsaB3_STYP_1]

,sum([CcbBdx10_BSA_FCBLARCX10_BSA_MOUSE_NO_MODS]) as [CcbBd3_1] ,sum([LBsaBDx10_BSA_FCBLARCX10_BSA_MOUSE_NO_MODS]) as [LBsaBD3_1] ,sum([LSBsaBdx10_BSA_FCBLARCX10_BSA_MOUSE_NO_MODS]) as [LSBsaBd3_1] ,sum([CcbBdx10_BSA_FCBLARCX10_BSA_MOUSE_MODS]) as [CcbBd3_STYP_1] 
,sum([LBsaBDx10_BSA_FCBLARCX10_BSA_MOUSE_MODS]) as [LBsaBD3_STYP_1] ,sum([LSBsaBdx10_BSA_FCBLARCX10_BSA_MOUSE_MODS]) as [LSBsaBd3_STYP_1]

,sum([CcbD_BSA_MvsA_FcBLARC_BSA_Mono_Agg_MOUSE_NO_MODS]) as [CcbD2_1] ,sum([CsaD_BSA_MvsA_FcBLARC_BSA_Mono_Agg_MOUSE_NO_MODS]) as [CsaD2_1] ,sum([LBsaD_BSA_MvsA_FcBLARC_BSA_Mono_Agg_MOUSE_NO_MODS]) as [LBsaD2_1] ,sum([LABsaD_BSA_MvsA_FcBLARC_BSA_Mono_Agg_MOUSE_NO_MODS]) as [LABsaD2_1] ,sum([LBAsaD_BSA_MvsA_FcBLARC_BSA_Mono_Agg_MOUSE_NO_MODS]) as [LBAsaD2_1] ,sum([LSBsaD_BSA_MvsA_FcBLARC_BSA_Mono_Agg_MOUSE_NO_MODS]) as [LSBsaD2_1] ,sum([LSBAsaD_BSA_MvsA_FcBLARC_BSA_Mono_Agg_MOUSE_NO_MODS]) as [LSBAsaD2_1] ,sum([LASBsaD_BSA_MvsA_FcBLARC_BSA_Mono_Agg_MOUSE_NO_MODS]) as [LASBsaD2_1]

,sum([CcbD_BSA_MvsA_FcBLARC_BSA_Mono_Agg_MOUSE_MODS]) as [CcbD2_STYP_1] ,sum([CsaD_BSA_MvsA_FcBLARC_BSA_Mono_Agg_MOUSE_MODS]) as [CsaD2_STYP_1] ,sum([LBsaD_BSA_MvsA_FcBLARC_BSA_Mono_Agg_MOUSE_MODS]) as [LBsaD2_STYP_1] ,sum([LABsaD_BSA_MvsA_FcBLARC_BSA_Mono_Agg_MOUSE_MODS]) as [LABsaD2_STYP_1] ,sum([LBAsaD_BSA_MvsA_FcBLARC_BSA_Mono_Agg_MOUSE_MODS]) as [LBAsaD2_STYP_1] ,sum([LSBsaD_BSA_MvsA_FcBLARC_BSA_Mono_Agg_MOUSE_MODS]) as [LSBsaD2_STYP_1] ,sum([LSBAsaD_BSA_MvsA_FcBLARC_BSA_Mono_Agg_MOUSE_MODS]) as [LSBAsaD2_STYP_1] ,sum([LASBsaD_BSA_MvsA_FcBLARC_BSA_Mono_Agg_MOUSE_MODS]) as [LASBsaD2_STYP_1]

,sum([CcbM_BSA_MvsA_FcBLARC_BSA_Mono_Agg_MOUSE_NO_MODS]) as [CcbM2_1] ,sum([CsaM_BSA_MvsA_FcBLARC_BSA_Mono_Agg_MOUSE_NO_MODS]) as [CsaM2_1] ,sum([LBsaM_BSA_MvsA_FcBLARC_BSA_Mono_Agg_MOUSE_NO_MODS]) as [LBsaM2_1] ,sum([LABsaM_BSA_MvsA_FcBLARC_BSA_Mono_Agg_MOUSE_NO_MODS]) as [LABsaM2_1] ,sum([LBAsaM_BSA_MvsA_FcBLARC_BSA_Mono_Agg_MOUSE_NO_MODS]) as [LBAsaM2_1] ,sum([LSBsaM_BSA_MvsA_FcBLARC_BSA_Mono_Agg_MOUSE_NO_MODS]) as [LSBsaM2_1] ,sum([LSBAsaM_BSA_MvsA_FcBLARC_BSA_Mono_Agg_MOUSE_NO_MODS]) as [LSBAsaM2_1] 
,sum([LASBsaM_BSA_MvsA_FcBLARC_BSA_Mono_Agg_MOUSE_NO_MODS]) as [LASBsaM2_1]

,sum([CcbM_BSA_MvsA_FcBLARC_BSA_Mono_Agg_MOUSE_MODS]) as [CcbM2_STYP_1]

,sum([CsaM_BSA_MvsA_FcBLARC_BSA_Mono_Agg_MOUSE_MODS]) as [CsaM2_STYP_1]

,sum([LBsaM_BSA_MvsA_FcBLARC_BSA_Mono_Agg_MOUSE_MODS]) as [LBsaM2_STYP_1]

,sum([LABsaM_BSA_MvsA_FcBLARC_BSA_Mono_Agg_MOUSE_MODS]) as [LABsaM2_STYP_1]

,sum([LBAsaM_BSA_MvsA_FcBLARC_BSA_Mono_Agg_MOUSE_MODS]) as [LBAsaM2_STYP_1]

,sum([LSBsaM_BSA_MvsA_FcBLARC_BSA_Mono_Agg_MOUSE_MODS]) as [LSBsaM2_STYP_1]

,sum([LSBAsaM_BSA_MvsA_FcBLARC_BSA_Mono_Agg_MOUSE_MODS]) as [LSBAsaM2_STYP_1]

,sum([LASBsaM_BSA_MvsA_FcBLARC_BSA_Mono_Agg_MOUSE_MODS]) as [LASBsaM2_STYP_1]

,sum([CcbN_BSA_MvsA_FcBLARC_BSA_Mono_Agg_MOUSE_NO_MODS]) as [CcbN2_1]

,sum([CsaN_BSA_MvsA_FcBLARC_BSA_Mono_Agg_MOUSE_NO_MODS]) as [CsaN2_1]

,sum([LBsaN_BSA_MvsA_FcBLARC_BSA_Mono_Agg_MOUSE_NO_MODS]) as [LBsaN2_1]

,sum([LABsaN_BSA_MvsA_FcBLARC_BSA_Mono_Agg_MOUSE_NO_MODS]) as [LABsaN2_1]

,sum([LBAsaN_BSA_MvsA_FcBLARC_BSA_Mono_Agg_MOUSE_NO_MODS]) as [LBAsaN2_1]

,sum([LSBsaN_BSA_MvsA_FcBLARC_BSA_Mono_Agg_MOUSE_NO_MODS]) as [LSBsaN2_1]

,sum([LSBAsaN_BSA_MvsA_FcBLARC_BSA_Mono_Agg_MOUSE_NO_MODS]) as [LSBAsaN2_1]

,sum([LASBsaN_BSA_MvsA_FcBLARC_BSA_Mono_Agg_MOUSE_NO_MODS]) as [LASBsaN2_1]

,sum([CcbN_BSA_MvsA_FcBLARC_BSA_Mono_Agg_MOUSE_MODS]) as [CcbN2_STYP_1]

,sum([CsaN_BSA_MvsA_FcBLARC_BSA_Mono_Agg_MOUSE_MODS]) as [CsaN2_STYP_1]

,sum([LBsaN_BSA_MvsA_FcBLARC_BSA_Mono_Agg_MOUSE_MODS]) as [LBsaN2_STYP_1]

,sum([LABsaN_BSA_MvsA_FcBLARC_BSA_Mono_Agg_MOUSE_MODS]) as [LABsaN2_STYP_1]

,sum([LBAsaN_BSA_MvsA_FcBLARC_BSA_Mono_Agg_MOUSE_MODS]) as [LBAsaN2_STYP_1]

,sum([LSBsaN_BSA_MvsA_FcBLARC_BSA_Mono_Agg_MOUSE_MODS]) as [LSBsaN2_STYP_1]

,sum([LSBAsaN_BSA_MvsA_FcBLARC_BSA_Mono_Agg_MOUSE_MODS]) as [LSBAsaN2_STYP_1]

,sum([LASBsaN_BSA_MvsA_FcBLARC_BSA_Mono_Agg_MOUSE_MODS]) as [LASBsaN2_STYP_1] 
,sum([CcbB_BSA_MvsA_FcBLARC_BSA_Mono_Agg_MOUSE_NO_MODS]) as [CcbB2_1] ,sum([CsaB_BSA_MvsA_FcBLARC_BSA_Mono_Agg_MOUSE_NO_MODS]) as [CsaB2_1] ,sum([LBsaB_BSA_MvsA_FcBLARC_BSA_Mono_Agg_MOUSE_NO_MODS]) as [LBsa2_1] ,sum([LABsaB_BSA_MvsA_FcBLARC_BSA_Mono_Agg_MOUSE_NO_MODS]) as [LABsaB2_1] ,sum([LBAsaB_BSA_MvsA_FcBLARC_BSA_Mono_Agg_MOUSE_NO_MODS]) as [LBAsaB2_1] ,sum([LSBsaB_BSA_MvsA_FcBLARC_BSA_Mono_Agg_MOUSE_NO_MODS]) as [LSBsaB2_1] ,sum([LSBAsaB_BSA_MvsA_FcBLARC_BSA_Mono_Agg_MOUSE_NO_MODS]) as [LSBAsaB2_1] ,sum([LASBsaB_BSA_MvsA_FcBLARC_BSA_Mono_Agg_MOUSE_NO_MODS]) as [LASBsaB2_1]

,sum([CcbB_BSA_MvsA_FcBLARC_BSA_Mono_Agg_MOUSE_MODS]) as [CcbB2_STYP_1] ,sum([CsaB_BSA_MvsA_FcBLARC_BSA_Mono_Agg_MOUSE_MODS]) as [CsaB2_STYP_1] ,sum([LBsaB_BSA_MvsA_FcBLARC_BSA_Mono_Agg_MOUSE_MODS]) as [LBsaB2_STYP_1] ,sum([LABsaB_BSA_MvsA_FcBLARC_BSA_Mono_Agg_MOUSE_MODS]) as [LABsaB2_STYP_1] ,sum([LBAsaB_BSA_MvsA_FcBLARC_BSA_Mono_Agg_MOUSE_MODS]) as [LBAsaB2_STYP_1] ,sum([LSBsaB_BSA_MvsA_FcBLARC_BSA_Mono_Agg_MOUSE_MODS]) as [LSBsaB2_STYP_1] ,sum([LSBAsaB_BSA_MvsA_FcBLARC_BSA_Mono_Agg_MOUSE_MODS]) as [LSBAsaB2_STYP_1] ,sum([LASBsaB_BSA_MvsA_FcBLARC_BSA_Mono_Agg_MOUSE_MODS]) as [LASBsaB2_STYP_1]

FROM [FcBlark_pass11_Mouse].[dbo].[DistinctProteinMatrix]

where

GeneSymbol like 'fcg\%' or GeneSymbol like 'Trim21\%' or GeneSymbol like 'fcrl\%'

Group by GeneSymbol, IdentificationFilterMethod

Order by IdentificationFilterMethod,GeneSymbol 
GeneSymbol,

IdentificationFilterMethod

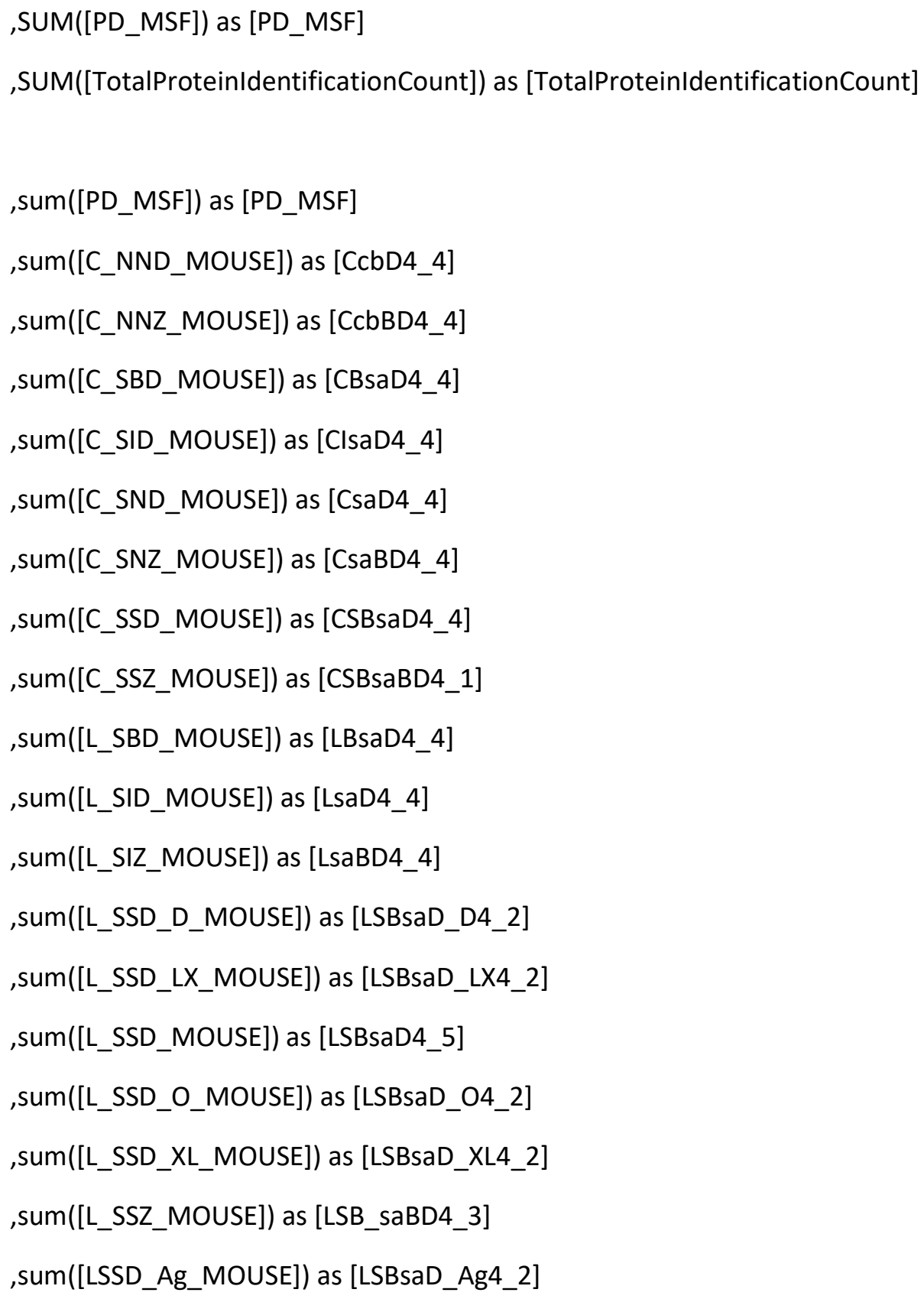


FROM [Fcbkark_mouse_pass12].[dbo].[DistinctProteinMatrix]

where

GeneSymbol like 'fcg\%' or GeneSymbol like 'Trim21\%' or GeneSymbol like 'fcrl\%'

Group by GeneSymbol, IdentificationFilterMethod

Order by IdentificationFilterMethod,GeneSymbol 
SUPPLEMENT C 
$\mathrm{R}$ code for the groupings of Fc tables

FcBLARC11_12\$a_CcbD <- with(FcBLARC11_12, (CcbD1_9 + CcbD2_1 + CcbD3_1 + CcbD4_4)/15*16)

FcBLARC11_12\$b_CcbD_STYP <- with(FcBLARC11_12, (CcbD1_STYP_9 + CcbD2_STYP_1 + CcbD3_STYP_1)/11*16)

FcBLARC11_12\$a_CcbM <- with(FcBLARC11_12, (CcbM1_9+ CcbM2_1+CcbM3_1)/11*16)

FcBLARC11_12\$b_CcbM_STYP <- with(FcBLARC11_12, (CcbM1_STYP_9+ CcbM2_STYP_1+ CcbM3_STYP_1)/11*16)

FcBLARC11_12\$a_CcbN <- with(FcBLARC11_12, (CcbN1_9+ CcbN2_1+ CcbN3_1/11*16))

FcBLARC11_12\$\$b_CcbN_STYP <- with(FcBLARC11_12, (CcbN1_STYP_9+ CcbN2_STYP_1+ CcbN3_STYP_1)/11*16)

FcBLARC11_12\$a_CcbB <- with(FcBLARC11_12, (CcbB1_9+ CcbB2_1+ CcbB3_1)/11*16)

FcBLARC11_12\$s_CcbB_STYP <- with(FcBLARC11_12, (CcbB1_STYP_9+ CcbB2_STYP_1+ CcbB3_STYP_1)/11*16)

FcBLARC11_12\$a_CcbBD <- with(FcBLARC11_12, (CcbBd3_1 + CcbBD1_3 + CcbBD4_4)/8*16)

FcBLARC11_12\$\$b_CcbBD_STYP <- with(FcBLARC11_12, (CcbBd3_STYP_1+ CcbBD1_STYP_3)/4*16)

FcBLARC11_12\$a_CsaD <- with(FcBLARC11_12, (CsaD1_9+ CsaD2_1+ CsaD4_4)/14*16)

FcBLARC11_12\$﹎_CsaD_STYP <- with(FcBLARC11_12, (CsaD1_9+ CsaD2_1+CsaD4_4)/14*16)

FcBLARC11_12\$a_CsaM <- with(FcBLARC11_12, (CsaM1_10 + CsaM2_1)/11*16)

FcBLARC11_12\$\$b_CsaM_STYP <- with(FcBLARC11_12, (CsaM1_STYP_10+CsaM2_STYP_1)/11*16)

FcBLARC11_12\$̇a_CsaB <- with(FcBLARC11_12, (CsaB1_9+CsaB2_1)/10*16)

FcBLARC11_12\$\$b_CsaB_STYP <- with(FcBLARC11_12, (CsaB1_STYP_9+ CsaB2_STYP_1)/10*16)

FcBLARC11_12\$̇a_CsaN <- with(FcBLARC11_12, (CsaN1_9+ CsaN2_1)/10*16)

FcBLARC11_12\$̧b_CsaN_STYP <- with(FcBLARC11_12, (CsaN1_STYP_9+ CsaN2_STYP_1)/10*16)

FcBLARC11_12\$a_CsaBD <- with(FcBLARC11_12, (CsaBD1_3+CsaBD4_4)/7*16) 
FcBLARC11_12\$b_CsaBD_STYP <- with(FcBLARC11_12, (CsaBD1_STYP_3)/3*16)

FcBLARC11_12\$a_LsaD <- with(FcBLARC11_12, (LsaD1_9+ LsaD4_4)/13*16)

FcBLARC11_12\$b_LsaD_STYP <- with(FcBLARC11_12, (LsaD1_STYP_9)/9*16)

FcBLARC11_12\$a_LsaM <- with(FcBLARC11_12, (LsaM1_9)/9*16)

FcBLARC11_12\$b_LsaM_STYP <- with(FcBLARC11_12, (LsaM1_STYP_9)/9*16)

FCBLARC11_12\$a_LsaN <- with(FcBLARC11_12, (LsaN1_10)/10*16)

FcBLARC11_12\$\$b_LsaN_STYP <- with(FcBLARC11_12, (LsaN1_STYP_10)/10*16)

FcBLARC11_12\$a_LsaB <- with(FcBLARC11_12, (LsaB1_9)/9*16)

FcBLARC11_12\$\$b_LsaB_STYP <- with(FcBLARC11_12, (LsaB1_STYP_9)/9*16)

FcBLARC11_12\$a_LsaBD <- with(FcBLARC11_12, (LsaBD1_3+ LsaBD4_4)/7*16)

FcBLARC11_12\$b_LsaBD_STYP <- with(FcBLARC11_12, (LsaBD1_STYP_3)/3*16)

FcBLARC11_12\$a_LBsaD <- with(FcBLARC11_12, (LBsaD1_9+ LBsaD2_1+ LBsaD3_1+ LBsaD4_4)/15*16)

FcBLARC11_12\$b_LBsaD_STYP <- with(FcBLARC11_12, (LBsaD1_STYP_9+ LBsaD2_STYP_1+ LBsaD3_STYP_1)/11*16)

FcBLARC11_12\$a_LBsaM <- with(FcBLARC11_12, (LBsaM1_9+ LBsaM2_1+ LBsaM3_1)/11*16)

FcBLARC11_12\$b_LBsaM_STYP <- with(FcBLARC11_12, (LBsaM1_STYP_9+ LBsaM2_STYP_1+ LBsaM3_STYP_1)/11*16)

FcBLARC11_12\$a_LBsaN <- with(FcBLARC11_12, (LBsaN1_9+ LBsaN2_1+ LBsaN3_1)/11*16)

FcBLARC11_12\$b_LBsaN_STYP <- with(FcBLARC11_12, (LBsaN1_STYP_9+ LBsaN2_STYP_1+ LBsaN3_STYP_1)/11*16)

FcBLARC11_12\$a_LBsaB <- with(FcBLARC11_12, (LBsaB1_9+ LBsa2_1+ LBsaB3_1)/11*16) 
FcBLARC11_12\$b_LBsaB_STYP <- with(FcBLARC11_12, (LBsaB1_STYP_9+ LBsaB2_STYP_1+ LBsaB3_STYP_1)/11*16)

FcBLARC11_12\$a_LBsaBD <- with(FcBLARC11_12, (LBsaBD1_3+ LBsaBD3_1)/4*16)

FcBLARC11_12\$b_LBsaBD_STYP <- with(FcBLARC11_12, (LSBsaBD1_STYP_3 + LSBsaBd3_STYP_1)/4*16)

FCBLARC11_12\$a_LSBsaD <- with(FcBLARC11_12, (LSBsaD1_9+ LSBsaD2_1+ LSBsaD3_1+ LSBsaD4_5)/16*16)

FcBLARC11_12\$b_LSBsaD_STYP <- with(FcBLARC11_12, (LSBsaD1_STYP_9+ LSBsaD2_STYP_1+ LSBsaD3_STYP_1)/11*16)

FcBLARC11_12\$a_LSBsaM <- with(FcBLARC11_12, (LSBsaM1_9+ LSBsaM2_1+ LSBsaM3_1)/11*16)

FcBLARC11_12\$b_LSBsaM_STYP <- with(FcBLARC11_12, (LSBsaM1_STYP_9+ LSBsaM2_STYP_1+ LSBsaM3_STYP_1)/11*16)

FcBLARC11_12\$a_LSBsaN <- with(FcBLARC11_12, (LSBsaN1_10+ LSBsaN2_1+ LSBsaN3_1)/12*16)

FcBLARC11_12\$\$b_LSBsaN_STYP <- with(FcBLARC11_12, (LSBsaN1_STYP_10+ LSBsaN2_STYP_1+ LSBsaN3_STYP_1)/12*16)

FcBLARC11_12\$a_LSBsaB <- with(FcBLARC11_12, (LSBsaB2_1+ LSBsaB3_1 + LSBsaB_9)/11*16)

FcBLARC11_12\$b_LSBsaB_STYP <- with(FcBLARC11_12, (LSBsaB1_STYP_9+ LSBsaB2_STYP_1+ LSBsaB3_STYP_1)/11*16)

FcBLARC11_12\$a_LSBsaBD <- with(FcBLARC11_12, (LSBsaBd3_1+ LSBsaBD1_3)/4*16)

FcBLARC11_12\$b_LSBsaBD_STYP <- with(FcBLARC11_12, (LSBsaBd3_STYP_1+ LSBsaBD1_STYP_3)/4*16)

FcBLARC11_12\$a_ClsaD <- with(FcBLARC11_12, (ClsaD4_4)/4*16)

FcBLARC11_12\$a_CBsaD <- with(FcBLARC11_12, (CBsaD4_4)/4*16) 
FcBLARC11_12\$a_CSBsaD <- with(FcBLARC11_12, (CSBsaBD4_1)/1*16)

FcBLARC11_12\$a_LABsaD <- with(FcBLARC11_12, (LABsaD2_1)/1*16)

FcBLARC11_12\$b_LABsaD_STYP <- with(FcBLARC11_12, (LABsaD2_STYP_1)/1*16)

FcBLARC11_12\$a_LABsaM <- with(FcBLARC11_12, (LABsaM2_1)/1*16)

FcBLARC11_12\$b_LABsaM_STYP <- with(FcBLARC11_12, (LABsaM2_STYP_1)/1*16)

FCBLARC11_12\$a_LABsaN <- with(FcBLARC11_12, (LABsaN2_1)/1*16)

FcBLARC11_12\$b_LABsaN_STYP <- with(FcBLARC11_12, (LABsaN2_STYP_1)/1*16)

FcBLARC11_12\$a_LABsaB <- with(FcBLARC11_12, (LABsaB2_1)/1*16)

FcBLARC11_12\$b_LABsaB_STYP <- with(FcBLARC11_12, (LABsaB2_STYP_1)/1*16)

FcBLARC11_12\$a_LASBsaD <- with(FcBLARC11_12, (LASBsaD2_1)/1*16)

FcBLARC11_12\$b_LASBsaD_STYP <- with(FcBLARC11_12, (LASBsaD2_STYP_1)/1*16)

FcBLARC11_12\$a_LASBsaM <- with(FcBLARC11_12, (LASBsaM2_1)/1*16)

FcBLARC11_12\$b_LASBsaM_STYP <- with(FcBLARC11_12, (LASBsaM2_STYP_1)/1*16)

FcBLARC11_12\$a_LASBsaN <- with(FcBLARC11_12, (LASBsaN2_1)/1*16)

FcBLARC11_12\$b_LASBsaN_STYP <- with(FcBLARC11_12, (LASBsaN2_STYP_1)/1*16)

FcBLARC11_12\$a_LASBsaB <- with(FcBLARC11_12, (LASBsaB2_1)*16)

FcBLARC11_12\$b_LASBsaB_STYP <- with(FcBLARC11_12, (LASBsaB2_STYP_1)*16)

FcBLARC11_12\$a_LBAsaD <- with(FcBLARC11_12, (LBAsaD2_1)*16)

FcBLARC11_12\$b_LBAsaD_STYP <- with(FcBLARC11_12, (LBAsaD2_STYP_1)*16) 
FcBLARC11_12\$a_LBAsaM <- with(FcBLARC11_12, (LBAsaM2_1)*16)

FcBLARC11_12\$b_LBAsaM_STYP <- with(FcBLARC11_12, (LBAsaM2_STYP_1)*16)

FcBLARC11_12\$a_LBAsaN <- with(FcBLARC11_12, (LBAsaN2_1)*16)

FcBLARC11_12\$b_LBAsaN_STYP <- with(FcBLARC11_12, (LBAsaN2_STYP_1)*16)

FcBLARC11_12\$a_LBAsaB <- with(FcBLARC11_12, (LBAsaB2_1)*16)

FcBLARC11_12\$b_LBAsaB_STYP <- with(FcBLARC11_12, (LBAsaB2_STYP_1)*16)

FCBLARC11_12\$a_LSBAsaD <- with(FcBLARC11_12, (LSBAsaD2_1)*16)

FcBLARC11_12\$b_LSBAsaD_STYP <- with(FcBLARC11_12, (LSBAsaD2_STYP_1)*16)

FcBLARC11_12\$a_LSBAsaM <- with(FcBLARC11_12, (LSBAsaM2_1)*16)

FcBLARC11_12\$b_LSBAsaM_STYP <- with(FcBLARC11_12, (LSBAsaM2_1)*16)

FCBLARC11_12\$a_LSBAsaN <- with(FcBLARC11_12, (LSBAsaN2_1)*16)

FcBLARC11_12\$b_LSBAsaN_STYP <- with(FcBLARC11_12, (LSBAsaN2_STYP_1)*16)

FcBLARC11_12\$a_LSBAsaB <- with(FcBLARC11_12, (LSBAsaB2_1)*16)

FcBLARC11_12\$b_LSBAsaB_STYP <- with(FcBLARC11_12, (LSBAsaB2_STYP_1)*16)

FcBLARC11_12\$a_LSBsaD_D <- with(FcBLARC11_12, (LSBsaD_D4_2)/2*16)

FcBLARC11_12\$a_LSBsaD_O <- with(FcBLARC11_12, (LSBsaD_O4_2)/2*16)

FcBLARC11_12\$a_LSBsaD_LX <- with(FcBLARC11_12, (LSBsaD_LX4_2)/2*16)

FcBLARC11_12\$a_LSBsaD_XL <- with(FcBLARC11_12, (LSBsaD_XL4_2)/2*16) 
FcBLARC11_12\$a_LSBsaD_Ag <- with(FcBLARC11_12, (LSBsaD_Ag4_2)/2*16)

$<$ ELUTION BUFFER>

FcBLARC11_12\$c_DDT <- with(FcBLARC11_12, a_CcbD+ a_CsaD+ a_LsaD + a_LBsaD + a_LSBsaD)

FcBLARC11_12\$c_BME <- with(FcBLARC11_12, a_CcbM+ a_CsaM + a_LsaM+ a_LBsaM+ a_LSBsaM)

FcBLARC11_12\$c_NH4OH <- with(FcBLARC11_12, a_CcbN+ a_CsaN+ a_LBsaN+ a_LsaN + a_LSBsaN)

FcBLARC11_12\$c_BIOTIN <- with(FcBLARC11_12, a_CcbB+ a_CsaB + a_LBsaB+ a_LsaB+ a_LSBsaB)

FCBLARC11_12\$c_BEADS <- with(FcBLARC11_12, a_CcbBD+ a_CsaBD+ a_LBsaBD+

a_LsaBD+ a_LSBsaBD)

$<$ ELUTION BUFFER STYP>

FcBLARC11_12\$c_DTT_STYP <- with(FcBLARC11_12, b_CcbD_STYP+ b_CsaD_STYP+

b_LBsaD_STYP+ b_LsaD_STYP+ b_LSBsaD_STYP)

FCBLARC11_12\$c_BME_STYP <- with(FcBLARC11_12, b_CcbM_STYP+ b_CsaM_STYP+

b_LBsaM_STYP + b_LSaM_STYP+ b_LSBsaM_STYP)

FcBLARC11_12\$c_NH4OH_STYP <- with(FcBLARC11_12, b_CcbN_STYP+ b_CsaN_STYP+ b_LBsaN_STYP+ b_LsaN_STYP+ b_LSBsaN_STYP)

FcBLARC11_12\$c_BIOTIN_STYP $<-$ with(FcBLARC11_12, b_CcbB_STYP+ b_CsaB_STYP+

b_LBsaB_STYP+ b_LsaB_STYP+ b_LSBsaB_STYP) 
FcBLARC11_12\$c_BEADS_STYP <- with(FcBLARC11_12, b_CcbBD_STYP+ b_CsaBD_STYP+ b_LBsaBD_STYP+ b_LsaBD_STYP+ b_LSBsaBD_STYP)

$<$ TREATMENT>

FcBLARC11_12\$d_Ccb <- with(FcBLARC11_12, a_CcbB+ a_CcbD+ a_CcbM+ a_CcbN+ a_CsaBD)

FcBLARC11_12\$d_Csa $<-$ with(FcBLARC11_12, a_CsaB+ a_CsaBD+ a_CsaD+ a_CsaM+ a_CsaN)

FcBLARC11_12\$d_Lsa <- with(FcBLARC11_12, a_LsaB+ a_LsaD+ a_LsaBD+ a_LsaM+ a_LsaN)

FcBLARC11_12\$d_LBsa <- with(FcBLARC11_12, a_LBsaB+ a_LBsaBD+ a_LBsaD+ a_LBsaM+ a_LBsaN)

FCBLARC11_12\$d_LSBsa <- with(FcBLARC11_12, a_LSBsaB+ a_LSBsaBD+ a_LSBsaD+ a_LSBsaN + a_LSBsaM)

<TREATMENT STYP>

FcBLARC11_12\$d_Ccb_STYP <- with(FcBLARC11_12, b_CcbB_STYP+ b_CcbBD_STYP+ b_CcbD_STYP+ b_CcbM_STYP+ b_CcbN_STYP)

FcBLARC11_12\$d_Csa_STYP <- with(FcBLARC11_12, b_CsaB_STYP+ b_CsaBD_STYP+ 
b_CsaD_STYP+ b_CsaM_STYP+ b_CsaN_STYP)

FcBLARC11_12\$d_Lsa_STYP <- with(FcBLARC11_12, b_LsaB_STYP+ b_LsaBD_STYP+

b_LsaD_STYP+ b_LsaM_STYP+ b_LsaN_STYP)

FcBLARC11_12\$d_LBsa_STYP <- with(FcBLARC11_12, b_LBsaB_STYP+ b_LBsaBD_STYP+

b_LBsaD_STYP+ b_LBsaM_STYP+ b_LBsaN_STYP)

FcBLARC11_12\$d_LSBsa_STYP <- with(FcBLARC11_12, b_LSBsaB_STYP+

b_LSBsaBD_STYP+ b_LSBsaD_STYP+ b_LSBsaM_STYP+ b_LSBsaN_STYP)

<AGGREGATION - no beads>

FcBLARC11_12\$e_Lsa <- with(FcBLARC11_12, a_LsaB+ a_LsaD + a_LsaM+

a_LsaN)

FCBLARC11_12\$e_LBsa <- with(FcBLARC11_12, a_LBsaB+ a_LBsaD+

a_LBsaM+ a_LBsaN)

FcBLARC11_12\$e_LSBsa $<-$ with(FcBLARC11_12, a_LSBsaB+ a_LSBsaD+

a_LSBsaN + a_LSBsaM)

FCBLARC11_12\$e_LABsa <- with(FcBLARC11_12, a_LABsaB+ a_LABsaD+ a_LABsaM+ a_LABsaN)

FCBLARC11_12\$e_LBAsa <- with(FcBLARC11_12, a_LBAsaB+ a_LBAsaD+ a_LBAsaM+ a_LBAsaN) 
FcBLARC11_12\$e_LASBsa <- with(FcBLARC11_12, a_LASBsaB+ a_LASBsaD+

a_LASBsaM+ a_LASBsaN)

FcBLARC11_12\$e_LSBAsa <- with(FcBLARC11_12, a_LSBAsaB + a_LSBAsaD+

a_LSBAsaM+a_LSBAsaN)

<AGGREGATION STYP>

FcBLARC11_12\$e_Lsa_STYP <- with(FcBLARC11_12, b_LsaB_STYP+ b_LsaD_STYP+ b_LsaM_STYP+ b_LsaN_STYP)

FcBLARC11_12\$e_LBsa_STYP <- with(FcBLARC11_12, b_LBsaB_STYP + b_LBsaD_STYP+ b_LBsaM_STYP+ b_LBsaN_STYP)

FcBLARC11_12\$e_LSBsa_STYP <- with(FcBLARC11_12, b_LSBsaB_STYP+ b_LSBsaD_STYP+ b_LSBsaM_STYP+ b_LSBsaN_STYP)

FcBLARC11_12\$e_LABsa_STYP <- with(FcBLARC11_12, b_LABsaB_STYP+

b_LABsaD_STYP+ b_LABsaM_STYP+ b_LABsaN_STYP)

FcBLARC11_12\$e_LBAsa_STYP <- with(FcBLARC11_12, b_LBAsaB_STYP+

b_LBAsaD_STYP+ b_LBAsaM_STYP+ b_LBAsaN_STYP)

FCBLARC11_12\$e_LSBAsa_STYP <- with(FcBLARC11_12, b_LSBAsaB_STYP+

b_LSBAsaD_STYP+ b_LSBAsaM_STYP+ b_LSBAsaN_STYP)

FcBLARC11_12\$e_LASBsa_STYP <- with(FcBLARC11_12, b_LASBsaB_STYP+

b_LASBsaD_STYP+ b_LASBSaM_STYP+ b_LASBsaN_STYP)

<Detergents Crosslinking Aggregation> 
FcBLARC11_12\$̧f_LSBsaD <- with(FcBLARC11_12, (LSBsaD1_9+ LSBsaD2_1+ LSBsaD3_1+ LSBsaD4_5)/16*16)

FcBLARC11_12\$f_LSBsaD_Ag $<-$ with(FcBLARC11_12, a_LSBsaD_Ag)

FcBLARC11_12\$̦f_LSBsaD_D <- with(FcBLARC11_12, a_LSBsaD_D)

FcBLARC11_12\$f_LSBsaD_O <- with(FcBLARC11_12, a_LSBsaD_O)

FcBLARC11_12\$̦f_LSBsaD_XL <- with(FcBLARC11_12, a_LSBsaD_XL)

FcBLARC11_12\$f_LSBsaD_LX <- with(FcBLARC11_12, a_LSBsaD_LX) 
SUPPLEMENT D 


\title{
Random and independent sampling of endogenous tryptic peptides from normal human EDTA plasma by liquid chromatography micro electrospray ionization and tandem mass spectrometry
}

${ }^{1}$ Jaimie Dufresne, ${ }^{1}$ Angelique Florentinus-Mefailoski, ${ }^{1}$ Juliet Ajambo, ${ }^{1}$ Ammara Ferwa, ${ }^{1}$ Peter Bowden, ${ }^{1,2}$ John Marshall

jaimie.dufresne@ryerson.ca, a3floren@ryerson.ca, juliet.ajambo@gmail.com, amaraferwa@gmail.com, pbowden@start.ca,4marshal@ ryerson.ca

${ }^{1}$ Ryerson University, 350 Victoria Street, Toronto, Ontario, Canada, M5B 2K3

${ }^{2}$ Integrated BioBank of Luxembourg, 6 r. Nicolas-Ernest Barblé 1210 Luxembourg

Running Title: ice versus room temperature plasma

\author{
Abbreviations \\ $\mathrm{ACN}$, acetonitrile \\ CBBR, Coomassie brilliant blue \\ SDS-PAGE, sodium dodecyl sulfate polyacrylamide gel electrophoresis
}




\section{ABSTRACT}

Normal human EDTA plasma samples were collected on ice, processed ice cold, and stored in a freezer at $-80^{\circ} \mathrm{C}$ prior to experiments. Plasma test samples from the $-80{ }^{\circ} \mathrm{C}$ freezer were thawed on ice or intentionally warmed to room temperature to measure protein content, the release of alcohol soluble amines and identify and quantify the plasma peptides released over time.

Endogenous peptides were collected over $\mathrm{C} 18$ and random and independently sampled by liquid chromatography micro electrospray ionization and tandem mass spectrometry (LC-ESI-MS/MS). Searching fully tryptic peptides by X!TANDEM returned the same set of proteins, but was more computationally efficient, than "no enzyme" correlations. Plasma samples maintained on ice, or ice with a cocktail of protease inhibitors, showed lower background amounts of plasma peptides compared to samples incubated at room temperature. Regression analysis indicated that warming plasma to room temperature, versus ice cold, resulted in a $\sim 2$-fold increase in the frequency of peptide identification that apparently reaches a steady state over hours to days of incubation at room temperature. X!TANDEM fit some 583,927 random and independently sampled MS/MS spectra that showed 48,019 correlations to a set of 5,855 peptides from 2068 proteins that reduced to 510 Gene Symbols with at least three peptides that had precursor intensity values greater than 1000 counts (E3). The type I error rate of the protein identification from the X!TANDEM algorithm combined was estimated to be low compared to a null model of computer generated random MS/MS spectra. The rigorous X!TANDEM algorithm showed a low Type I error of the primary structural identity and the relative intensity of human plasma proteins. 


\section{INTRODUCTION}

The analysis of proteins and peptides from human blood by liquid chromatography, electrospray ionization and tandem mass spectrometry (LC-ESI-MS/MS) will permit the diagnosis of diseases and evaluate the efficacy of therapeutic treatments (P. Zhu, P. Bowden, D. Zhang, et al., 2011). Both MALDI and electrospray ionization methods previously demonstrated that tryptic protease activities cleave peptides from blood proteins that differed between control and heart attack samples (J. Marshall et al., 2004; John Marshall et al., 2003). Some peptides observed in serum samples are known to be ex vivo artefacts that represent the steady-state balance of endo- versus exopeptidase action after sample collection (John Marshall et al., 2003; Williams et al., 2010). The peptides of blood are in a dynamic steady state that can be easily perturbed by sample incubation conditions (John Marshall et al., 2003; Williams et al., 2010). Artefacts introduced into the blood fluids after sampling and prior to freezing may be a major source of pre-analytical variation (Albrethsen, Bogebo, Olsen, Raskov, \& Gammeltoft, 2006; Banks, 2008; Callesen et al., 2008; de Noo et al., 2005; Gast et al., 2009; Govorukhina et al., 2008; Karsan et al., 2005; Koomen et al., 2005; J. Li et al., 2005; John Marshall et al., 2003; Pilny et al., 2006; Rai et al., 2005; Semmes et al., 2005; Spencer, 2003; Tuck et al., 2009; van Winden et al., 2009; WestNielsen et al., 2005; West-Norager et al., 2007). There is considerable variation in the peptides observed, and even the trends reported, in the study of degradation of blood proteins that may differ between groups, likely from the large variation that occurs immediately after sample collection (Albrethsen et al., 2006; Banks, 2008; Callesen et al., 2008; de Noo et al., 2005; Gast et al., 2009; Govorukhina et al., 2008; Karsan et al., 2005; Koomen et al., 2005; J. Li et al., 2005; John Marshall et al., 2003; Pilny et al., 2006; Rai et al., 2005; Semmes et al., 2005; Spencer, 2003; Tuck et al., 2009; van Winden et al., 2009; West-Nielsen et al., 2005; West-Norager et al., 
2007). One key contribution of this study is the collection of EDTA plasma directly onto ice for cold processing to establish a reliable baseline compared to plasma peptides at room temperature.

There are many potential sources of error in the identification and quantification of peptides and therefore proteins (van den Broek et al., 2015). Collecting rich MS/MS spectra at a high signalto- noise is the key to both reliable and sensitive identification and quantification of blood peptides and proteins with the X!TANDEM algorithm (P. Zhu, P. Bowden, M. Tucholska, et al., 2011; Peihong Zhu et al., 2011). It is necessary to pre-fractionate samples to prevent co-elution of peptides with similar $\mathrm{m} / \mathrm{z}$ values. Selective extraction from the solid phase with organic aqueous solvent is a simple means to enrich endogenous peptides from blood fluids (J. Marshall et al., 2004; John Marshall et al., 2003; Monika Tucholska et al., 2007; Williams et al., 2010). Robust and sensitive ion traps may have advantages in analysing low amounts of blood peptides and proteins that range across many orders of magnitude where signal-to-noise filters and statistical controls are employed to control type I error (Bowden et al., 2012; Angelica K Florentinus, Peter Bowden, Girish Sardana, et al., 2012; Florentinus et al., 2011a). The $\mathrm{X}$ !TANDEM algorithm is known to fit the MS/MS spectra from sensitive and robust ion traps with a high degree of statistical rigour using the default settings of $\pm 3 \mathrm{~m} / \mathrm{z}$ of the precursor and \pm 0.5 Da of the many fragments with up to three missed cleavages (R. Craig \& R. C. Beavis, 2004) with a low rate of type I error in the identification of peptides based on the goodness of fit of the MS/MS spectra (Bowden et al., 2009; Bowden et al., 2010; P. Zhu, P. Bowden, M. Tucholska, et al., 2011; Peihong Zhu et al., 2011). The proteins of human plasma contain many highly abundant proteins such as albumin, apolipoproteins, protease inhibitors and others that digest efficiently with the exogenous addition of trypsin and thus mask the detection of low 
abundance proteins by LC-ESI-MS/MS (P. Zhu, P. Bowden, D. Zhang, et al., 2011). Many of the well-known proteins of human blood (Putnam, 1975; Tietz, 2001) are cleaved by endogenous tryptic endopeptidases to release fully tryptic peptides that may be identified by collection over C18 followed by LC-ESI-MS/MS with an ion trap (J. Marshall et al., 2004). The peptides of blood fluids show good agreement on the detection of many unexpected cellular peptides by LCESI-MS/MS using both Qq-TOF and the sensitive ion trap (J. Marshall et al., 2004; John Marshall et al., 2003; M. Tucholska et al., 2010; Monika Tucholska et al., 2007; Williams et al., 2010). The secretion or release of cellular proteins into extracellular space may result in the preferential cleavage of the cellular proteins upon exposure to circulating protease activities (J. Marshall, Bowden, Schmit, \& Betsou, 2014). Here, the random and independent sampling of endogenous peptides from plasma collected and incubated on ice versus the same plasma incubated at room temperature shows that many plasma proteins are degraded over time by tryptic peptidases and so showed about a two-fold higher frequency of many tryptic peptides at room temperature compared to ice cold samples. A second key contribution of this paper is to show that the peptides from proteins expressed in tissues and cells may be identified and quantified by micro electrospray with LC-ESI-MS/MS of endogenous peptides was a simple linear ion trap (Schwartz et al., 2002) with low type I error rates by comparison to a null model of random MS/MS spectra or noise spectra and computing plasma protein p-values with X!TANDEM. 


\section{MATERIALS AND METHODS}

\section{Materials}

The Agilent 1100 HPLC (Santa Clara, CA, USA) was coupled to an XL LTQ linear ion trap mass spectrometer (Thermo Electron Corporation, Madison, WI, USA) for LC-ESI-MS/MS. The protease inhibitors, salts, buffers, Coomassie Blue and ninhydrin were obtained from Sigma Aldrich (St. Louis, MO,USA). The \#1 filter paper was obtained from Whatman (Maidstone, United Kingdom). The HPLC grade water and acetonitrile were obtained from Caledon Laboratories (Georgetown, Ontario, Canada).

\section{Plasma sample collection}

Human plasma was collected under a Comité National d'Ethique de Recherche (CNER) Protocol \#201107 "Biospecimen Research" at the Centre Hospitalier de Luxembourg. The plasma was collected in EDTA tubes (Becton Dickenson) that were rapidly inverted 5 times before packing in ice. The ice cold plasma was then separated from blood cells at 12,000 RCF for 20 minutes at $4{ }^{\circ} \mathrm{C}$ prior to aliquoting to $225 \mu 1$ samples on ice and randomly assigned to short term or long term experimental treatments. Plasma samples of $225 \mu \mathrm{l}$ were previously shown to be sufficient for peptide extraction (M. Tucholska et al., 2010; Monika Tucholska et al., 2007; Williams et al., 2010).

\section{Plasma sample treatments}

Random aliquots were maintained on ice, ice plus protease inhibitors, or incubated at room temperature for $\geq 96$ hours or more as indicated. Plasma samples permitted to degrade at room temperature for $1,4,12,24,48,72$ or $\geq 96$ hours alongside ice or ice plus inhibitor controls prior 
to random sampling and analysis. A total of 82 control plasma samples that were either frozen or never above ice cold (ALLICE), and 88 plasma samples that were incubated at room temperature for different lengths of time (ALLRT), were analyzed. The protease inhibitor treatment consisted of SIGMA Eukaryotic protease inhibitor cocktail plus 2 mM AEBSF, 2 mM PMSF, 2 mM EDTA, $2 \mathrm{mM}$ caproic acid, and $2 \mathrm{mM}$ benzamidine with the Sigma Eukaryotic protease inhibitor cocktail at 1/100 v/v. The Sigma Mammalian Protease inhibitor cocktail (contains at least: AEBSF, $104 \mathrm{mM}$, Aprotinin, $80 \mu \mathrm{M}$, Bestatin, 4 mM, E-64, 1.4 mM, Leupeptin, 2 mM, Pepstatin A, 1.5mM) was used at 1/100 (v/v). Plasma samples $(225 \mu \mathrm{l})$ from at least 10 different donors were tested at each time point and over the time course of degradation up to $72 \mathrm{~h}$. At the end of each time period, the samples were frozen, freeze dried and stored dried at $-80{ }^{\circ} \mathrm{C}$ until analysis.

\section{Protein assay, SDS-PAGE and free amine assays}

Protein content of the plasma samples was determined in the presence of SDS detergent by the Dumbroff method (S. Ghosh, S. Gepstein, J. J. Heikkila, \& E. B. Dumbroff, 1988) prior to separation by tricine SDS-PAGE followed by staining of the gel with Coomassie Brilliant Blue (Schagger \& von Jagow, 1987). The free amines were extracted in ethanol and measured using the Cd-ninhiydrin peptidase assay alongside glycine equivalents (Doi, Shibata, \& Matoba, 1981).

\section{Unbiased LC-ESI-MS/MS}

The mass spectrometer was cleaned, calibrated with the manufacturer's standard mixture, tuned with GluFib and Angiotensin and tested for sensitivity prior to each replicate block by infusion 
of a dilution series of GluFib and Angiotensin. The mass accuracy and sensitivity of the LC-ESIMS/MS system was tested with a tryptic digestion of a mixture of cytochrome C, glycogen phosphorylase B and alcohol dehydrogenase (Bowden et al., 2012). The plasma peptides were collected over a $\mathrm{C}_{18}$ preparative column with elution in $2 \mu \mathrm{l}$ of $5 \%$ formic acid and $65 \%$ acetonitrile and immediately diluted with $18 \mu 1$ of $5 \%$ formic acid for injections via a $20 \mu 1$ loop with a Rheodyne manual injector. A total of $\sim 5 \mu \mathrm{g}$ of extracted and purified peptides was injected for each analytical HPLC separation over a 300 micron ID column $(15 \mathrm{~cm})$ with inline filter frits. The peptides were ionized at $4.5 \mathrm{kV}$ via a micro electrospray ion source with $10 \mathrm{~L} \mathrm{~N}_{2}$ per minute with a transfer capillary temperature of $200{ }^{\circ} \mathrm{C}$ into a Thermo Electron Corporation LTQ ion trap mass spectrometer (Schwartz et al., 2002). The peptides were randomly and independently sampled without replacement as the peptides eluted from the HPLC column into the electrospray source from 350 to $2000 \mathrm{~m} / \mathrm{z}$.

\section{Peptide MS/MS spectra correlation analysis}

A federated library of 213,246 human proteins that differed by at least one amino acid was assembled from NCBI, Ensembl and Swiss Prot and made non-redundant using Structured Query Language (SQL). A physical filter of at least one thousand (E3) intensity counts for peptide parent ions was used to limit type I error (P. Zhu, P. Bowden, M. Tucholska, et al., 2011; Peihong Zhu et al., 2011). A sub-set of the data was analyzed by fully tryptic versus no enzyme specified to compare the sets of proteins identified. The MS and MS/MS spectra of all peptides recorded were correlated to the federated library set with fully tryptic enzyme specification, a charge state of $2^{+}$or $3^{+}$with up to three missed cleavages in SEQUEST (Yates, 1998; Yates et al., 1995), MASCOT (Perkins et al., 1999), OMSSA (L. Y. Geer et al., 2004) and X!TANDEM (R. 
Craig \& R. C. Beavis, 2004) with \pm 3 Da for the calculated peptide $[\mathrm{M}+\mathrm{H}]^{+}$and with the fragments within 0.5 Da with up to three missed cleavage sites (Bowden et al., 2009; Bowden et al., 2012; Angelica K Florentinus, Peter Bowden, Veronica Barbisan, et al., 2012; Angelica K Florentinus, Peter Bowden, Girish Sardana, et al., 2012; P. Zhu, P. Bowden, M. Tucholska, et al., 2011; Peihong Zhu et al., 2011). The data from SEQUEST, provided via the manufacturer's BIOWORKS algorithm, was further limited to the default setting of 0.05 maximum delta correlation of parent ions with maximum peptide mass set to $5000 \mathrm{Da}$ and peptide length set to $\geq$ 6 amino acids. The authentic results were compared to random mis-correlations from unexpected modifications using computer generated random MS/MS spectra or from noise spectra by blank runs with HPLC grade solvents over naïve columns.

\section{Computational analysis in SQL and statistical analysis with $\mathbf{R}$}

The combination of Chi Square and general linear models such as regression and ANOVA using a generic statistical analysis system are sufficient to provide a complete and satisfying statistical analysis of LC-ESI-MS/MS data (Bowden et al., 2012; Angelica K Florentinus, Peter Bowden, Veronica Barbisan, et al., 2012; Angelica K Florentinus, Peter Bowden, Girish Sardana, et al., 2012; Florentinus et al., 2011a). The resulting peptide and protein identifications together with the parent and fragment $\mathrm{m} / \mathrm{z}$ and intensity values were parsed into an SQL database (Bowden et al., 2009). The charge state with the best score (Filter 1) and the peptide sequence with the best score (Filter 2) were used to control type I error of identification: thus, the SQL database utilized a compound keytag for each MS-MS/MS event to ensure that only the best fit of each MS/MS spectra at only one charge state was accepted, and thus no MS/MS spectra was assigned to more than one peptide sequence. The peptide-to-protein counts of plasma samples were previously 
statistically analyzed in the generic statistical system S (John Marshall et al., 2003) or SAS (Bowden et al., 2009; Bowden et al., 2010; Bowden et al., 2012; Angelica K Florentinus, Peter Bowden, Veronica Barbisan, et al., 2012; Angelica K Florentinus, Peter Bowden, Girish Sardana, et al., 2012; P. Zhu, P. Bowden, M. Tucholska, et al., 2011; Peihong Zhu et al., 2011). However, in this study the data was analyzed using the generic open-source R statistical system (J. C. Howard et al., 2016). The R statistical system was also used to plot the peptide-to-protein distribution of authentic peptides compared to those of random spectra and to compute the cumulative $p$-value for proteins from the product of the observed peptides p-values (Bowden et al., 2009). 


\section{RESULTS}

The peptides of EDTA plasma collected directly onto ice were analyzed by C18 preparative chromatography followed by micro electrospray LC-ESI-MS/MS of samples incubated on ice and/or with protease inhibitors and showed about half of the endogenous tryptic peptides of samples incubated at room temperature, and thus many or most of the peptides observed in EDTA plasma over time are ex vivo artefacts.

\section{Protein assay}

The protein content of the blood samples in SDS-PAGE sample buffer were measured by the Dumbroff method (S. Ghosh et al., 1988) and was about $50 \mathrm{mg} / \mathrm{ml}$ but showed great variability within the randomly chosen samples. The total protein assays of the samples showed that there was a slight decline of about $10 \%$ in the protein content over the course of the 24 hours at room temperature (Supplemental Figure 1).

\section{Tricine SDS-PAGE}

Tricine SDS-PAGE showed no obvious protein degradation and no new bands were observed with time at room temperature (Supplemental Figure 2). The degradation of the plasma samples at room temperature for up to $72 \mathrm{hr}$ was not detectable by separation of $\sim 100 \mu \mathrm{g}$ of plasma proteins by tricine SDS-PAGE with staining by CBBR (John Marshall et al., 2003; R. Zhang et al., 2004). The major protein bands of plasma from SDS-PAGE appear to be stable with incubation at room temperature. 


\section{Free amine assay}

The modified colorimetric (Cd-ninhydrin) method to detect peptidase activity by the release of free amines is sensitive to micromole amounts of released free amines (Doi et al., 1981). The free amine assay showed that there is a rapid and statistically significant release of alcoholsoluble amines over time from 350 to about 410 micro molar soon after incubation at room temperature (Supplemental Figure 3).

\section{Random and independent sampling}

A random and independent sampling of endogenous peptides from EDTA plasma maintained on ice versus at room temperature was made without replacement as the peptides eluted from the C18 HPLC column in the LC-ESI-MS/MS system. The MS/MS spectra were then correlated to 158,072 federated protein sequences by the MASCOT, OMSSA, X!TANDEM and SEQUEST algorithms. The LC-ESI-MS/MS spectra and the results of the correlation algorithms were parsed into an SQL Server Database for statistical analysis with the generic statistical analysis system R (Bowden et al., 2009; Bowden et al., 2010; Zhu et al., 2007; P. Zhu, P. Bowden, M. Tucholska, et al., 2011; Peihong Zhu et al., 2011).

\section{Fully tryptic versus no enzyme correlation}

Comparing the set of proteins identified by fully tryptic correlation versus no enzyme correlation (all possible human peptide sequences) showed essentially complete agreement on the set of proteins identified (Fig. 1). The no-enzyme search returned a greater number of peptides to the same small group of proteins identified by tryptic correlation and did not identify many new proteins. 


\section{Filter by best charge state and peptide sequence}

The results of the LC-ESI-MS/MS from the fully tryptic correlation algorithms were parsed together into an SQL Server Database for statistical analysis with the R generic statistical analysis system (Bowden et al., 2009; Bowden et al., 2010; Zhu et al., 2007; P. Zhu, P. Bowden, M. Tucholska, et al., 2011; Peihong Zhu et al., 2011). A major source of error in the results of LC-ESI-MS/MS analysis was the re-use of MS/MS spectra for more than one protein by MASCOT, OMSSA, and SEQUEST while X!TANDEM (Table I). Filtering out all hits that were not the best charge state, i.e. $2+$ or $3+$, (Filter 1 ) and then accepting only the best peptide sequence at the best charge state (Filter 2) eliminates more than $80 \%$ of the potential peptide correlations (Table I). A total of 563,095 MS/MS spectra from parent ions $\geq$ E3 (1000) counts resulted in correlations to 729,533 peptides from a library of 158,072 proteins. A sum total of $3,788,530$ peptides were correlated by the algorithms together over all treatments. After selecting only the best fit in terms of charge state and peptide sequence where the parent protein showed at least three independent correlations, the number of peptides and proteins collapsed to 729,533 peptides from 82,276 protein accessions.

\section{Correlation algorithms}

From a total of MS/MS spectra of greater than 1000 arbitrary counts, MASCOT fitted just over 60 proteins, OMSSA fitted 74 proteins, X!TANDEM fitted over 2068 protein sequences, and SEQUEST fitted 78,929 proteins that differ by at least one amino acid from the federated library of all known protein forms with at least three peptides (Fig. 2). Essentially all of the peptides 
and proteins identified by X!TANDEM were also identified by SEQUEST. The peptides of normal human plasma extracted by $\mathrm{C} 18$ solid phase extraction and analyzed by micro electrospray are listed by accession number in the supplemental data. Plotting the best fit data in terms of the averaged peptides to proteins from MASCOT, OMSSA, X!TANDEM and SEQUEST shows that some proteins show more than a thousand independent best fit correlations (Fig. 3). Since X!TANDEM previously showed a low type I error rate for proteins from fully tryptic peptides (Bowden et al., 2012; Angelica K Florentinus, Peter Bowden, Girish Sardana, et al., 2012; P. Zhu, P. Bowden, M. Tucholska, et al., 2011; Peihong Zhu et al., 2011) and since the goodness of fit of MS/MS spectra also sensitively identified far more proteins than either MASCOT or OMSSA, X!TANDEM was selected for subsequent statistical analysis. X!TANDEM fit some 583,927 random and independently sampled MS/MS spectra that showed 48,019 correlations to a set of 5,855 peptides from 2068 proteins that reduced to 510 Gene Symbols with at least three peptides that had precursor intensity values greater than 1000 counts (E3). Thus under these conditions MASCOT and OMSSA showed few proteins compared to $\mathrm{X}$ !TANDEM, that is known to be reliable compared to random simulations, and thus showed a large type II error.

\section{Incubation treatments}

Collecting EDTA plasma samples directly onto ice, followed by incubation on ice \pm inhibitors, resulted in about 20,000 total peptides per LC-ESI-MS/MS run but also prevented the generation of endogenous plasma peptides for up to a week on ice especially if protease inhibitors were provided and so yields a stable baseline for degradation experiments. In contrast, samples that were collected on ice and then even briefly incubated at room temperature showed an increase to 
about 40,000 thousand peptides per run by $1 \mathrm{~h}$ at room temperature (Table II). The samples that were freeze dried and stored at room temperature or freeze dried and stored at $-20{ }^{\circ} \mathrm{C}$ both had about 20,000 thousand peptides identified per LC-ESI-MS/MS run similar to samples collected and stored briefly on ice. Samples preserved by freezing at $-80{ }^{\circ} \mathrm{C}$ or liquid nitrogen showed higher levels of peptides per run than ice or freeze drying (Table II).

\section{Analysis of ice versus room temperature samples}

Samples maintained on ice showed proteins with up to a hundred correlated peptides after filtering (i.e. peptides to proteins) (Fig. 4A) but samples at room temperature showed greater numbers of correlations of peptides to proteins (Fig. 4B). Taking the ratio of room temperature (ALLRT) versus ice samples (ALLICE) showed more than one hundred fold variation of individual proteins with incubation at room temperature (Fig. 5). The proteins that showed the largest release of peptides at room temperature compared to ice was complement chain 4B (C4B) and C3 (J. Marshall et al., 2004; John Marshall et al., 2003). Regression analysis revealed that most plasma proteins show on average a $\sim 2$-fold increase in sampling frequency at room temperature (Fig. 5).

\section{X!TANDEM analysis of endogenous tryptic peptides}

The results of the X!TANDEM algorithm were collected in SQL Server and analyzed compared to those of computer generated random MS/MS spectra or noise spectra (not shown) with the $\mathrm{R}$ statistical system that demonstrated a very low probability of false positive identification (Fig.

6). There was no relationship between log p-value and the peptide intensity for precursors $\geq 1000$ (E3) counts (Fig. 7A). The sorted p-values showed a linear relationship with an average $\mathrm{p}$ value 
of about 0.05 (Fig. 7B) that was normally distributed (Fig. 7C). Computing the cumulative pvalue for proteins with 3 or more peptides showed that the protein p-values ranged from E-3 to E-300 (Fig. 7D).

Most of the MS/MS spectra data was collected at values ranging from 350 to about $1700 \mathrm{~m} / \mathrm{z}$ (Fig. 8A). The $\log 10$ distribution of precursor intensity increased with mass (momentum) as expected from impact detection (Fig. 8B); About 24,000 precursors with intensity values ranging from E3 to E6 arbitrary detector counts were observed (Fig. 8C). The quantile plot of the $\log _{10}$ distribution of precursor intensity values shows that after $\log 10$ transformation that data approaches Gaussian normality (Fig. 8D). The calculated peptide $[\mathrm{M}+\mathrm{H}]^{+}$values ranged from 1000 to $5000 \mathrm{Da}$ (Fig. 8E). Moreover, the the quantile plot strongly indicated that peptide $[\mathrm{M}+\mathrm{H}]^{+}$was sampled from a Gaussian (i.e. normal) population (Fig. 8F). The peptide delta mass values ranged from $-2 \mathrm{Da}$ to $+4 \mathrm{Da}$ consistent with the known error of the instrument and the presence of natural isotopes (Fig. 8G). The quantile plot of the delta mass values was apparently Gaussian with a mean of +1 , i.e. showed a normal statistical distribution with about $68 \%$ of the peptides within $1 \mathrm{Da}$ of the mean predicted $[\mathrm{M}+\mathrm{H}]+$ while $95 \%$ were within $2 \mathrm{Da}$ of the mean consistent with the presence of natural isotopes. (Fig. $8 \mathrm{H}$ ). The relationship between delta $\mathrm{M}+\mathrm{H}$ versus peptide p-value was also apparently Gaussian (Fig. 8I). 


\section{DISSCUSSION}

The aim of this study was to perform the random and independent sampling of human plasma on ice versus room temperature to identify and quantify the peptides and proteins that were preferentially cleaved in normal human plasma during incubation at room temperature.

\section{Biochemical methods}

The lower total protein levels, and a concomitant increase in alcohol soluble amines, early in the incubation at room temperature were both consistent with the degradation of a pool of proteins in plasma with time. The consistent SDS-PAGE banding patterns showing no change in the major plasma proteins indicates that many of the well known plasma proteins are relatively stable. The degradation of fresh human plasma itself was not reflected by changes in the banding patterns that were readily detectable by SDS-PAGE and this clearly rules out the presence of general proteases that visibly act on the many major plasma proteins with brief periods at room temperature (John Marshall et al., 2003; R. Zhang et al., 2004). Moreover the stable banding pattern at room temperature observed here indicates the changes in SDS-PAGE banding patterns previously observed between different disease states were not merely due to differences in sample handling between normal and disease samples but rather must have resulted from the action of disease-specific protease activities (John Marshall et al., 2003; R. Zhang et al., 2004).

\section{Comparison of correlation parameters and algorithms}

The overwhelming agreement on the set of proteins identified between no enzyme specified (that shows a large degree of freedom and might fit the MS/MS spectra to any human peptide sequence), versus the proteins identified by fully tryptic peptides, is powerful evidence in favour 
of the veracity and low type I error of MS/MS correlation with an ion trap. The close agreement between no enzyme and fully tryptic correlations that both show the same small set of proteins with multiple peptides, while the vast majority of human protein sequences show no correlation, is entirely consistent with the high fidelity of MS/MS spectra matching to predicted fragmentation patterns as estimated from the agreement of blood proteins between groups (Bowden et al., 2010; Zhu et al., 2007), the computation of p-values from the fit of MS/MS spectra (Bowden et al., 2009) or from first principles using a null random model (Bowden et al., 2009; Bowden et al., 2012; Angelica K Florentinus, Peter Bowden, Girish Sardana, et al., 2012; Florentinus et al., 2011a; P. Zhu, P. Bowden, M. Tucholska, et al., 2011; Peihong Zhu et al., 2011) and recently confirmed by the elaborate synthesis of 300,000 peptides (Zolg et al., 2017). With respect to fully tryptic peptides, the MASCOT, OMSSA, X!TANDEM and SEQUEST algorithms all agreed on the identity of the most frequently sampled peptides of EDTA plasma indicating that all the algorithms can identify the major plasma peptides. In agreement with previous results (P. Zhu, P. Bowden, M. Tucholska, et al., 2011; Peihong Zhu et al., 2011), the endogenous tryptic peptides identified by X!TANDEM with at least three independent peptides also showed a low Type I (false positive) error rate after comparison to computer generated random (and noise) spectra consistent with the low cumulative protein p-values computed here. Conversely, MASCOT and OMSSA failed to identify many of the authentic peptides credibly detected by X!TANDEM and thus suffered from a high Type II (false negative) error rate. The failure of MASCOT and OMSSA to identify many proteins may result from the heuristic nature of these algorithms that rely on relative measures instead of accepting the best fit of the data based on correlation coefficient or goodness of fit. Thus MASCOT and OMSSA failed to meet the basic condition of providing the simplest model that explains all of the data. In contrast, 
SEQUEST that meets the obligate requirement to provide the simplest model that explains all data, frequently identified Titin and other giant proteins at a low frequency that likely results from the near random distribution of mis-correlations and so can be directly corrected by running noise or random MS/MS spectra simulations (Bowden et al., 2012; Angelica K Florentinus, Peter Bowden, Girish Sardana, et al., 2012; Florentinus et al., 2011a; P. Zhu, P. Bowden, M. Tucholska, et al., 2011; Peihong Zhu et al., 2011). Thus for C18 solid phase extraction of endogenous peptides and micro scale LC-ESI-MS/MS of EDTA plasma with a simple linear ion trap, X!TANDEM was the best choice of algorithm to fit the MS/MS spectra without correction by random or noise spectra (R. Craig \& R. C. Beavis, 2004).

\section{Baseline EDTA Plasma model}

Collecting EDTA plasma directly onto ice and processing ice cold may be a practical way to avoid cellular or biochemical reactions that might vary over time and thus create pre-analytical variation. Here a solid baseline was established for the EDTA plasma by incubating on ice or ice plus inhibitors over a time compared to plasma incubated at room temperature. Many proteins showed about a two fold variation between ice and room temperature samples consistent with the degradation of plasma proteins over time and sample handling conditions (Albrethsen et al., 2006; Banks, 2008; Callesen et al., 2008; de Noo et al., 2005; Gast et al., 2009; Govorukhina et al., 2008; Karsan et al., 2005; Koomen et al., 2005; J. Li et al., 2005; John Marshall et al., 2003;

Pilny et al., 2006; Rai et al., 2005; Semmes et al., 2005; Spencer, 2003; Tuck et al., 2009; van Winden et al., 2009; West-Nielsen et al., 2005; West-Norager et al., 2007). Collecting the EDTA plasma samples directly onto ice demonstrates that, in contrast to many reports, the complement peptides are almost non-observable in the baseline EDTA plasma but are almost 
instantaneously released at room temperature. From this study it is clear that EDTA plasma may be collected and processed ice cold to avoid the rapid degradation of a pool of proteins that commences rapidly upon incubation at room temperature.

\section{X!TANDEM analysis}

The tryptic peptides identified by X!TANDEM show a low type I error rate and are not heavily contaminated with mis-correlations to TTN or other giant proteins (Angelica K Florentinus, Peter Bowden, Girish Sardana, et al., 2012; Florentinus et al., 2011a; P. Zhu, P. Bowden, M. Tucholska, et al., 2011; Peihong Zhu et al., 2011). The results of the X!TANDEM algorithm showed that the protein identified by $\geq 3$ tryptic peptides with intensity values $\geq E 3$ counts with a linear 2D quadrupole ion trap show a negligible type I error in agreement with previous studies (Bowden et al., 2009; Bowden et al., 2010; Bowden et al., 2012; Angelica K Florentinus, Peter Bowden, Girish Sardana, et al., 2012; P. Zhu, P. Bowden, M. Tucholska, et al., 2011). The peptide fit by X!TANDEM reasonably explained the observed MS/MS fragmentation (Craig \& Beavis, 2003; R. Craig \& R. C. Beavis, 2004; John Marshall et al., 2003) and showed log intensity, $\log$ p-value and delta mass that were Gaussian and so were ideal for statistical analysis. From the computed p-values and the comparison to random spectra we can unambiguously conclude that plasma proteins identified with at least three peptides by X!TANDEM are statistically reliable enough for further study ( $\mathrm{E}-3$ to E-300). For simplicity, the peptides identified from SEQUEST that map to the proteins identified by X!TANDEM will reasonably meet the obligation to provide the simplest model to explain all data while avoiding type I error. 


\section{The cleavage products of EDTA plasma}

The comparison of samples on ice versus room temperature shows that thousands of proteins may be directly detected and monitored from plasma by solid phase extraction with $\mathrm{C}_{18}$ peptide collection and robust analysis with micro electrospray. The experiment demonstrates it is feasible to compare plasma samples across experimental treatments using the endogenous peptide frequencies without extensive chromatographic pre-separation of proteins. The complement proteins such as $\mathrm{C} 4$ and $\mathrm{C} 3$ are in high abundance in blood and designed to be rapidly processed in response to biochemical signals that might be propagated via the action of kininogen (Dobo et al., 2011; Michael et al., 2005). In contrast, fibrinogen peptides, that are abundant in serum (John Marshall et al., 2003), did not show the highly elevated levels of peptides in plasma at room temperature where no clotting occurs in agreement with the theory.

\section{Conclusion}

Independent protein assays, free amine assays and LC-ESI-MS/MS methods all agree that a pool of proteins start to degrade soon after incubation of plasma samples at room temperature and then slowly approach a steady state (John Marshall et al., 2003). The majority of blood peptides show a fairly robust two-fold increase in the random sampling frequency with incubation at room temperature indicating that most of the peptides observed were ex vivo artefacts. It was feasible to collect plasma samples from clinical subjects and identify the endogenous peptides, and thus proteins, released from the cells, tissue and organs of the body with simple C18 solid phase extraction for identification and quantification by LC-ESI-MS/MS with a linear ion trap. Here we show for the first time that random and independent sampling of endogenous blood peptides results in a population of thousands of peptides from hundreds of proteins that show intensity, 
calculated mass and delta mass values that are normally distributed and have a low type I error rate based on comparison to a null model of random spectra or noise and the fitting of the MS/MS spectra by the rigorous X!TANDEM algorithm that directly generates a p-value from the goodness of fit of the MS/MS spectra within 0.5 Da (Craig \& Beavis, 2003; R. Craig \& R. C. Beavis, 2004). The sampling frequency of peptides by LC-ESI-MS/MS may be used to detect variation over experimental treatments. Since the observed peptide frequency may vary by about two with respect to ice cold controls, it follows that in general, differences between disease states of experimental treatments would have to exceed the threshold of two-fold enrichment in order to rule out confounding effects from sample handling. EDTA plasma may be preserved, stored and transported freeze dried at room temperature that will have a positive economic impact on clinical trials. 


\section{EDITORIAL POLICY}

Ethics approval and consent to participate

Ethical approval and informed consent was obtained through the Comité National d'Ethique de Recherche (CNER) Protocol \#201107 "Biospecimen Research” at the Centre Hospitalier de Luxembourg.

Consent for publication

No material from any other publication was used in this publication

Availability of data and material

The raw data is provided in the supplemental materials

Competing interests

The authors declare no conflict of interest.

Funding

This research was paid for by the Fonds National de la Recherche, Mobility of Researcher award through Luxembourg Institute of Health (formerly CRP Sante) and the Integrated Biobank of Luxembourg to JGM.

Authors' contributions

Jaimie Dufresne calibrated, tuned and quality controlled the instruments and performed LC-ESIMS/MS analysis and edited the manuscript. 
Angelique Florentinus-Mefailoski performed the sample aliquoting and performed LC-ESI-

MS/MS analysis and edited the manuscript.

Juliet Ajambo performed LC-ESI-MS/MS analysis and edited the manuscript.

Ammara Ferwa performed SDS-PAGE \& CBBR staining, proteins, Free amine assays and LCESI-MS/MS

Peter Bowden collected the data into an SQL database for statistical analysis in R.

John Marshall planned the experiment, and performed the clinical plasma collection and sample treatments and storage experiments

Acknowledgment:

We gratefully acknowledge the review and critique of this manuscript by Dr. Fay Betsou and Dr. R.A. Phillips of IBBL. 


\section{REFERENCES}

1. Zhu, P., Bowden, P., Zhang, D., and Marshall, J. G. (2011) Mass spectrometry of peptides and proteins from human blood. Mass Spectrom Rev 30, 685-732

2. Marshall, J., Kupchak, P., Zhu, W., Yantha, J., Vrees, T., Furesz, S., Jacks, K., Smith, C., Kireeva, I., Zhang, R., Takahashi, M., Stanton, E., and Jackowski, G. (2003) Processing of serum proteins underlies the mass spectral fingerprinting of myocardial infarction. Journal of proteome research 2, 361-372

3. Marshall, J., Jankowski, A., Furesz, S., Kireeva, I., Barker, L., Dombrovsky, M., Zhu, W., Jacks, K., Ingratta, L., Bruin, J., Kristensen, E., Zhang, R., Stanton, E., Takahashi, M., and Jackowski, G. (2004) Human serum proteins preseparated by electrophoresis or chromatography followed by tandem mass spectrometry. J Proteome Res 3, 364-382

4. Williams, D., Ackloo, S., Zhu, P., Bowden, P., Evans, K. R., Addison, C. L., Lock, C., and Marshall, J. G. (2010) Precipitation and selective extraction of human serum endogenous peptides with analysis by quadrupole time-of-flight mass spectrometry reveals posttranslational modifications and low-abundance peptides. Analytical and bioanalytical chemistry 396, 1223-1247

5. Banks, R. E. (2008) Preanalytical influences in clinical proteomic studies: raising awareness of fundamental issues in sample banking. Clin Chem 54, 6-7

6. Tuck, M. K., Chan, D. W., Chia, D., Godwin, A. K., Grizzle, W. E., Krueger, K. E., Rom, W., Sanda, M., Sorbara, L., Stass, S., Wang, W., and Brenner, D. E. (2009) Standard operating procedures for serum and plasma collection: early detection research network consensus statement standard operating procedure integration working group. $J$ Proteome Res 8, 113-117

7. Karsan, A., Eigl, B. J., Flibotte, S., Gelmon, K., Switzer, P., Hassell, P., Harrison, D., Law, J., Hayes, M., Stillwell, M., Xiao, Z., Conrads, T. P., and Veenstra, T. (2005) Analytical and preanalytical biases in serum proteomic pattern analysis for breast cancer diagnosis. Clin Chem 51, 1525-1528

8. Semmes, O. J., Feng, Z., Adam, B. L., Banez, L. L., Bigbee, W. L., Campos, D., Cazares, L. H., Chan, D. W., Grizzle, W. E., Izbicka, E., Kagan, J., Malik, G., McLerran, D., Moul, J. W., Partin, A., Prasanna, P., Rosenzweig, J., Sokoll, L. J., Srivastava, S., Thompson, I., Welsh, M. J., White, N., Winget, M., Yasui, Y., Zhang, Z., and Zhu, L. (2005) Evaluation of serum protein profiling by surface-enhanced laser desorption/ionization time-of-flight mass spectrometry for the detection of prostate cancer: I. Assessment of platform reproducibility. Clin Chem 51, 102-112

9. de Noo, M. E., Tollenaar, R. A., Ozalp, A., Kuppen, P. J., Bladergroen, M. R., Eilers, P. H., and Deelder, A. M. (2005) Reliability of human serum protein profiles generated with C8 magnetic beads assisted MALDI-TOF mass spectrometry. Anal Chem 77, 7232-7241

10. Li, J., Orlandi, R., White, C. N., Rosenzweig, J., Zhao, J., Seregni, E., Morelli, D., Yu, Y., Meng, X. Y., Zhang, Z., Davidson, N. E., Fung, E. T., and Chan, D. W. (2005) Independent validation of candidate breast cancer serum biomarkers identified by mass spectrometry. Clin Chem 51, 2229-2235

11. Pilny, R., Bouchal, P., Borilova, S., Ceskova, P., Zaloudik, J., Vyzula, R., Vojtesek, B., and Valik, D. (2006) Surface-enhanced laser desorption ionization/time-of-flight mass spectrometry reveals significant artifacts in serum obtained from clot activator-containing collection devices. Clin Chem 52, 2115-2116 
12. Albrethsen, J., Bogebo, R., Olsen, J., Raskov, H., and Gammeltoft, S. (2006)

Preanalytical and analytical variation of surface-enhanced laser desorption-ionization time-of-flight mass spectrometry of human serum. Clin Chem Lab Med 44, 1243-1252

13. Koomen, J. M., Li, D., Xiao, L. C., Liu, T. C., Coombes, K. R., Abbruzzese, J., and Kobayashi, R. (2005) Direct tandem mass spectrometry reveals limitations in protein profiling experiments for plasma biomarker discovery. J Proteome Res 4, 972-981

14. Rai, A. J., Gelfand, C. A., Haywood, B. C., Warunek, D. J., Yi, J., Schuchard, M. D., Mehigh, R. J., Cockrill, S. L., Scott, G. B., Tammen, H., Schulz-Knappe, P., Speicher, D. W., Vitzthum, F., Haab, B. B., Siest, G., and Chan, D. W. (2005) HUPO Plasma Proteome Project specimen collection and handling: towards the standardization of parameters for plasma proteome samples. Proteomics 5, 3262-3277

15. Callesen, A. K., Vach, W., Jorgensen, P. E., Cold, S., Mogensen, O., Kruse, T. A., Jensen, O. N., and Madsen, J. S. (2008) Reproducibility of mass spectrometry based protein profiles for diagnosis of breast cancer across clinical studies: a systematic review. J Proteome Res 7, 1395-1402

16. van Winden, A. W., Gast, M. C., Beijnen, J. H., Rutgers, E. J., Grobbee, D. E., Peeters, P. H., and van Gils, C. H. (2009) Validation of previously identified serum biomarkers for breast cancer with SELDI-TOF MS: a case control study. BMC Med Genomics 2, 4

17. Spencer, K. (2003) The influence of different sample collection types on the levels of markers used for Down's syndrome screening as measured by the Kryptor Immunosassay system. Ann Clin Biochem 40, 166-168

18. Govorukhina, N. I., de Vries, M., Reijmers, T. H., Horvatovich, P., van der Zee, A. G., and Bischoff, R. (2008) Influence of clotting time on the protein composition of serum samples based on LC-MS data. J Chromatogr B Analyt Technol Biomed Life Sci

19. Gast, M. C., van Gils, C. H., Wessels, L. F., Harris, N., Bonfrer, J. M., Rutgers, E. J., Schellens, J. H., and Beijnen, J. H. (2009) Influence of sample storage duration on serum protein profiles assessed by surface-enhanced laser desorption/ionisation time-of-flight mass spectrometry (SELDI-TOF MS). Clin Chem Lab Med 47, 694-705

20. West-Nielsen, M., Hogdall, E. V., Marchiori, E., Hogdall, C. K., Schou, C., and Heegaard, N. H. (2005) Sample handling for mass spectrometric proteomic investigations of human sera. Anal Chem 77, 5114-5123

21. West-Norager, M., Kelstrup, C. D., Schou, C., Hogdall, E. V., Hogdall, C. K., and Heegaard, N. H. (2007) Unravelling in vitro variables of major importance for the outcome of mass spectrometry-based serum proteomics. J Chromatogr B Analyt Technol Biomed Life Sci 847, 30-37

22. van den Broek, I., Romijn, F. P., Smit, N. P., van der Laarse, A., Drijfhout, J. W., van der Burgt, Y. E., and Cobbaert, C. M. (2015) Quantifying protein measurands by peptide measurements: where do errors arise? J Proteome Res 14, 928-942

23. Zhu, P., Bowden, P., Tucholska, M., and Marshall, J. G. (2011) Chi-square comparison of tryptic peptide-to-protein distributions of tandem mass spectrometry from blood with those of random expectation. Anal Biochem 409, 189-194

24. Zhu, P., Bowden, P., Tucholska, M., Zhang, D., and Marshall, J. G. (2011) Peptide-toprotein distribution versus a competition for significance to estimate error rate in blood protein identification. Analytical biochemistry 411, 241-253

25. Tucholska, M., Scozzaro, S., Williams, D., Ackloo, S., Lock, C., Siu, K. W. M., Evans, K. R., and Marshall, J. G. (2007) Endogenous peptides from biophysical and biochemical 
fractionation of serum analyzed by matrix-assisted laser desorption/ionization and electrospray ionization hybrid quadrupole time-of-flight. Analytical biochemistry 370, 228-245

26. Bowden, P., Thavarajah, T., Zhu, P., McDonell, M., Thiele, H., and Marshall, J. G. (2012) Quantitative statistical analysis of standard and human blood proteins from liquid chromatography, electrospray ionization, and tandem mass spectrometry. Journal of proteome research 11, 2032-2047

27. Florentinus, A. K., Bowden, P., Sardana, G., Diamandis, E. P., and Marshall, J. G. (2012) Identification and quantification of peptides and proteins secreted from prostate epithelial cells by unbiased liquid chromatography tandem mass spectrometry using goodness of fit and analysis of variance. Journal of proteomics 75, 1303-1317

28. Florentinus, A. K., Jankowski, A., Petrenko, V., Bowden, P., and Marshall, J. G. (2011) The Fc receptor-cytoskeleton complex from human neutrophils. Journal of proteomics 75, 450-468

29. Craig, R., and Beavis, R. C. (2004) TANDEM: matching proteins with tandem mass spectra. Bioinformatics 20, 1466-1467

30. Bowden, P., Beavis, R., and Marshall, J. (2009) Tandem mass spectrometry of human tryptic blood peptides calculated by a statistical algorithm and captured by a relational database with exploration by a general statistical analysis system. Journal of proteomics 73, 103-111

31. Bowden, P., Pendrak, V., Zhu, P., and Marshall, J. G. (2010) Meta sequence analysis of human blood peptides and their parent proteins. Journal of proteomics 73, 1163-1175

32. Putnam, F. (1975) The plasma Proteins: Structure Function, and Genetic Control. Academic Press, New York second ed

33. Tietz. (2001) Tietz Fundamentals of Clinical Chemistry, 5th edition ed., Saunders

34. Tucholska, M., Florentinus, A., Williams, D., and Marshall, J. G. (2010) The endogenous peptides of normal human serum extracted from the acetonitrile-insoluble precipitate using modified aqueous buffer with analysis by LC-ESI-Paul ion trap and Qq-TOF. $J$ Proteomics 73, 1254-1269

35. Marshall, J., Bowden, P., Schmit, J. C., and Betsou, F. (2014) Creation of a federated database of blood proteins: a powerful new tool for finding and characterizing biomarkers in serum. Clin Proteomics 11, 3

36. Schwartz, J. C., Senko, M. W., and Syka, J. E. (2002) A two-dimensional quadrupole ion trap mass spectrometer. J Am Soc Mass Spectrom 13, 659-669.

37. Ghosh, S., Gepstein, S., Heikkila, J. J., and Dumbroff, E. B. (1988) Use of a scanning densitometer or an ELISA plate reader for measurement of nanogram amounts of protein in crude extracts from biological tissues. Anal Biochem 169, 227-233

38. Schagger, H., and von Jagow, G. (1987) Tricine-sodium dodecyl sulfate-polyacrylamide gel electrophoresis for the separation of proteins in the range from 1 to $100 \mathrm{kDa}$. Anal Biochem 166, 368-379.

39. Doi, E., Shibata, D., and Matoba, T. (1981) Modified colorimetric ninhydrin methods for peptidase assay. Anal Biochem 118, 173-184

40. Yates, J. R., 3rd, Eng, J. K., McCormack, A. L., and Schieltz, D. (1995) Method to correlate tandem mass spectra of modified peptides to amino acid sequences in the protein database. Anal Chem 67, 1426-1436. 
41. Yates, J. R., 3rd. (1998) Database searching using mass spectrometry data. Electrophoresis 19, 893-900.

42. Perkins, D. N., Pappin, D. J., Creasy, D. M., and Cottrell, J. S. (1999) Probability-based protein identification by searching sequence databases using mass spectrometry data. Electrophoresis 20, 3551-3567

43. Geer, L. Y., Markey, S. P., Kowalak, J. A., Wagner, L., Xu, M., Maynard, D. M., Yang, X., Shi, W., and Bryant, S. H. (2004) Open mass spectrometry search algorithm. $J$ Proteome Res 3, 958-964

44. Florentinus, A. K., Bowden, P., Barbisan, V., and Marshall, J. (2012) Capture and qualitative analysis of the activated Fc receptor complex from live cells. Current protocols in protein science / editorial board, John E. Coligan ... [et al.] Chapter 19, Unit 19.22

45. Howard, J. C., Florentinus-Mefailoski, A., Bowden, P., Trimble, W., Grinstein, S., and Marshall, J. G. (2016) OxLDL receptor chromatography from live human U937 cells identifies SYK(L) that regulates phagocytosis of oxLDL. Anal Biochem

46. Zhang, R., Barker, L., Pinchev, D., Marshall, J., Rasamoelisolo, M., Smith, C., Kupchak, P., Kireeva, I., Ingratta, L., and Jackowski, G. (2004) Mining biomarkers in human sera using proteomic tools. Proteomics 4, 244-256

47. Zhu, P., Bowden, P., Pendrak, V., Thiele, H., Zhang, D., Siu, M., Diamandis, E. P., and Marshall, J. (2007) Comparison of protein expression lists from mass spectrometry of human blood fluids using exact peptide sequences versus BLAST. Clinical Proteomics 2, 185-203

48. Zolg, D. P., Wilhelm, M., Schnatbaum, K., Zerweck, J., Knaute, T., Delanghe, B., Bailey, D. J., Gessulat, S., Ehrlich, H. C., Weininger, M., Yu, P., Schlegl, J., Kramer, K., Schmidt, T., Kusebauch, U., Deutsch, E. W., Aebersold, R., Moritz, R. L., Wenschuh, H., Moehring, T., Aiche, S., Huhmer, A., Reimer, U., and Kuster, B. (2017) Building ProteomeTools based on a complete synthetic human proteome. Nat Methods 14, 259262

49. Craig, R., and Beavis, R. C. (2003) A method for reducing the time required to match protein sequences with tandem mass spectra. Rapid Commun Mass Spectrom 17, 23102316

50. Dobo, J., Major, B., Kekesi, K. A., Szabo, I., Megyeri, M., Hajela, K., Juhasz, G., Zavodszky, P., and Gal, P. (2011) Cleavage of kininogen and subsequent bradykinin release by the complement component: mannose-binding lectin-associated serine protease (MASP)-1. PLoS One 6, e20036

51. Michael, I. P., Sotiropoulou, G., Pampalakis, G., Magklara, A., Ghosh, M., Wasney, G., and Diamandis, E. P. (2005) Biochemical and enzymatic characterization of human kallikrein 5 (hK5), a novel serine protease potentially involved in cancer progression. $J$ Biol Chem 280, 14628-14635 


\section{TABLES}

Table I. The summary of the total number of peptide correlations and the number of protein accession numbers detected from the degraded human EDTA plasma in an SQL Server Database with respect to filter conditions. The results of 170 samples collected and maintained ice cold or preserved (82) versus samples incubated at room temperature for for up to 72 hours (88) prior to C18 solid phase extraction and micro LC-ESI-MS/MS with a linear ion trap are shown.

Table II. The sum peptide-to-protein corrections per LC-ESI-MS/MS run from the MASCOT, OMSSA, X!TANDEM and SEQUEST algorithms over the experimental treatments of preserved and degraded human EDTA plasma. The redundant correlations made by the sum of all four algorithms to any human protein are shown. Abbreviations: FD- $20^{\circ} \mathrm{C}$, Freeze Dried and stored in an electric freezer at $-20^{\circ} \mathrm{Celsius}$ for 1 year; $-80^{\circ} \mathrm{C}$, fresh plasma stored in an electric freezer at $-80^{\circ} \mathrm{C}$ for 1 year; $\mathrm{LN}$, fresh plasma stored in liquid nitrogen for 1 year; FDRT, freeze dried and stored at room temperature for 1 year; ICE_INHIB, fresh plasma stored on ice with a cocktail of protease inhibitors; ICE, fresh plasma stored on ice; RT, room temperature. 


\section{FIGURES}

Figure 1. The comparison of the fully tryptic peptide correlation with up to three missed cleavage sites versus no enzyme specification. All proteins with at least 1 peptide correlation under either condition were plotted. The results indicate that the same set of proteins are correlated with no enzyme versus trypsin but that more peptides per protein are detected with the computationally more intense no enzyme parameter.

Figure 2. The comparison of the MASCOT, OMSSA, X!TANDEM and SEQUEST algorithms for the identification of endogenous tryptic peptides from human plasma on ice and incubated at room temperature combined.

Figure 3. The total peptide to protein counts for all algorithms and all treatments. A total of 563,095 MS/MS spectra were obtained from LC-ESI-MS/MS analysis of 170 samples x 90 minute HPLC runs over C18 300 micron x150 mm were computed.

Figure 4. The log peptide to protein count of the preserved and degraded peptides of human EDTA plasma. Panels: A, the peptide to protein counts of plasma maintained frozen or on ice; $\mathrm{B}$, the peptide to protein distribution of plasma incubated at room temperature.

Figure 5. Regression analysis of the peptide-to-protein counts from samples incubated at room temperature (ALLRT) versus samples maintained on ice or frozen (ALLICE). Residual standard error: 2.945 on 22853 degrees of freedom, Adjusted R-squared: 0.3103, F-statistic: $1.028 \mathrm{e}+04$ on 1 and $22853 \mathrm{DF}, \mathrm{p}$-value <2.2e-16. To avoid infinity values 1 was added to each value (p1).

Figure 6. Estimation of type I error rate in the proteins identified by X!TANDEM from endogenous peptides of normal human plasma (NHP) versus random spectra (Log Random). The corrected mean peptide-to-protein counts of the first 200 protein accessions with at least three peptides from X!TANDEM are shown. Note the peptide-to-protein distribution of real spectra exceeds that of random spectra by $\geq$ order of magnitude at all values and the Chi Square test indicates that the probability that the experimental data is the same as the null random model is essentially zero $(\mathrm{p}<0.0001)$. that is a formal estimate of the type I error with respect to a null random model for the whole experiment using the classical statistical approach of the Chi Square test. The result indicates that the X!TANDEM algorithm that fits MS/MS spectra to predicted peptide spectra shows an acceptable type I error rate over the whole experiment.

Figure 7. The confidence of endogenous tryptic peptides and proteins identified by X!TANDEM from human plasma on ice and incubated at room temperature. The X!TANDEM algorithm was used to assign the probability of type I error to peptides and thus the cumulative probability of type I error of proteins with 3 or more independent peptide correlations. Panels: A, scatter plot of peptide $\log _{10}$ P-value versus $\log _{10}$ precursor intensity; B, sorted Peptide p-values versus observations; C, Peptide p-value quantile plot; $\mathrm{D}$, cumulative protein $\mathrm{p}$-value per protein accession.

Figure 8. The distributions of the endogenous peptides from proteins with three or more independent correlations to the same protein accession by X!TANDEM. Panels: A, the scatter plot of $\log _{10}$ precursor intensity values versus precursor $\mathrm{m} / \mathrm{z} ; \mathrm{B}$, the scatter plot of the $\log _{10}$ 
distribution of precursor intensity values versus peptide mass $[\mathrm{M}+\mathrm{H}]^{+} ; \mathrm{C}$, the sorted distribution of $\log _{10}$ intensity values; D, the quantile plot of precursor $\log _{10}$ intensity values; $\mathrm{E}$, the sorted distribution of calculated peptide mass $[\mathrm{M}+\mathrm{H}]^{+} ; \mathrm{F}$, the quantile plot of peptide $[\mathrm{M}+\mathrm{H}]^{+} ; \mathrm{G}$, the sorted distribution of the peptide delta mass; $\mathrm{H}$, the quantile plot of the delta mass; $\mathrm{I}$, the relationship between delta $\mathrm{M}+\mathrm{H}$ versus peptide $\log _{10} \mathrm{p}$-value.

Supplemental Figure 1. Total protein analysis by the Dumbroff method of non-covalent binding to filter paper followed by washing of non-proteinaceous components with methanol prior to staining with $0.1 \mathrm{~g} \mathrm{CBBR}_{250}$ in $40 \%$ methanol and $10 \%$ acetic acid

Supplemental Figure 2. The stability of EDTA plasma as demonstrated by electrophoresis and staining with Coomassie Brilliant Blue. EDTA Plasma samples incubated at room temperature for the time indicated were prepared over QA resin for tricine SDS-PAGE. Panels: A, CBBR staining of plasma after preparation over QA resin Molecular markers 250, 150, 100, 75, 50, 37, 25, $15 \mathrm{kDa}$. (T5, T6, T7, T8, T9 were left at room Temperature the most. The tricine SDSPAGE gel shown is representative of 4 technical replicates.

Supplemental Figure 3. The release of ethanol-soluble free amines from human EDTA plasma over time at room temperature. Dried plasma $(225 \mu \mathrm{L})$ was extracted with $250 \mu \mathrm{L}$ or absolute ethanol prior to reaction with Cd-ninhydrin alongside glycine standards. 


\section{TABLES}

Table I. The summary of the total number of peptide correlations and the number of protein accession numbers detected from the degraded human EDTA plasma in an SQL Server Database with respect to filter conditions. The results of 170 samples collected and maintained ice cold or preserved (82) versus samples incubated at room temperature for for up to 72 hours (88) prior to C18 solid phase extraction and micro LC-ESI-MS/MS with a linear ion trap are shown.

Filter

All

Filter 0

Filter 1

Filter 2

Filter 2

$\geq 3$ peptides

Per Accession

\section{Total Correlations Number of Protein \\ Accessions}

401,960

$3,788,530$

150,678

833,029

125,641

793,309

125,641

729,533

81,276 
Table II. The sum peptide-to-protein corrections per LC-ESI-MS/MS run from the MASCOT, OMSSA, X!TANDEM and SEQUEST algorithms over the experimental treatments of preserved and degraded human EDTA plasma. The redundant correlations made by the sum of all four algorithms to any human protein are shown. Abbreviations: FD- $20^{\circ} \mathrm{C}$, Freeze Dried and stored in an electric freezer at $-20^{\circ} \mathrm{Celsius}$ for 1 year; $-80^{\circ} \mathrm{C}$, fresh plasma stored in an electric freezer at $-80^{\circ} \mathrm{C}$ for 1 year; LN, fresh plasma stored in liquid nitrogen for 1 year; FDRT, freeze dried and stored at room temperature for 1 year; ICE_INHIB, fresh plasma stored on ice with a cocktail of protease inhibitors; ICE, fresh plasma stored on ice; RT, room temperature.

\begin{tabular}{|c|c|c|c|}
\hline TreatmentName & Sum.Correlations I & $\mathrm{N}$ & CorrelationsPerRun \\
\hline $\mathrm{FD}-20^{\circ} \mathrm{C}$ & 346290 & 12 & 28858 \\
\hline$-80^{\circ} \mathrm{C}$ & 254832 & 7 & 36405 \\
\hline LN & 401209 & 11 & 36474 \\
\hline FDRT & 280050 & 12 & 23338 \\
\hline ICE_INHIB_1 hr & 42197 & 2 & 21099 \\
\hline ICE_INHIB_4 hr & 44270 & 2 & 22135 \\
\hline ICE_INHIB_8 hr & 90814 & 4 & 22704 \\
\hline ICE_INHIB_24 hr & 102271 & 4 & 25568 \\
\hline ICE_INHIB_48 hr & 80709 & 4 & 20177 \\
\hline ICE_INHIB_72 hr & 77590 & 3 & 25863 \\
\hline ICE_1 hr & 49197 & 2 & 24599 \\
\hline ICE_4 hr & 74232 & 3 & 24744 \\
\hline ICE_8 hr & 66377 & 2 & 33189 \\
\hline ICE_24 hr & 115975 & 4 & 28994 \\
\hline ICE_48 hr & 107433 & 5 & 21487 \\
\hline ICE_72 hr & 113023 & 3 & 37674 \\
\hline RT_1 hr & 538873 & 12 & 44906 \\
\hline RT_4 hr & 351457 & 12 & 29288 \\
\hline RT_8 hr & 327895 & 10 & 32790 \\
\hline RT_12 hr & 435866 & 12 & 36322 \\
\hline RT_24 hr & 507283 & 12 & 42274 \\
\hline RT_48 hr & 349692 & 10 & 34969 \\
\hline RT_72 hr & 403881 & 11 & 36716 \\
\hline RT_96 hr & 227777 & 6 & 37963 \\
\hline
\end{tabular}




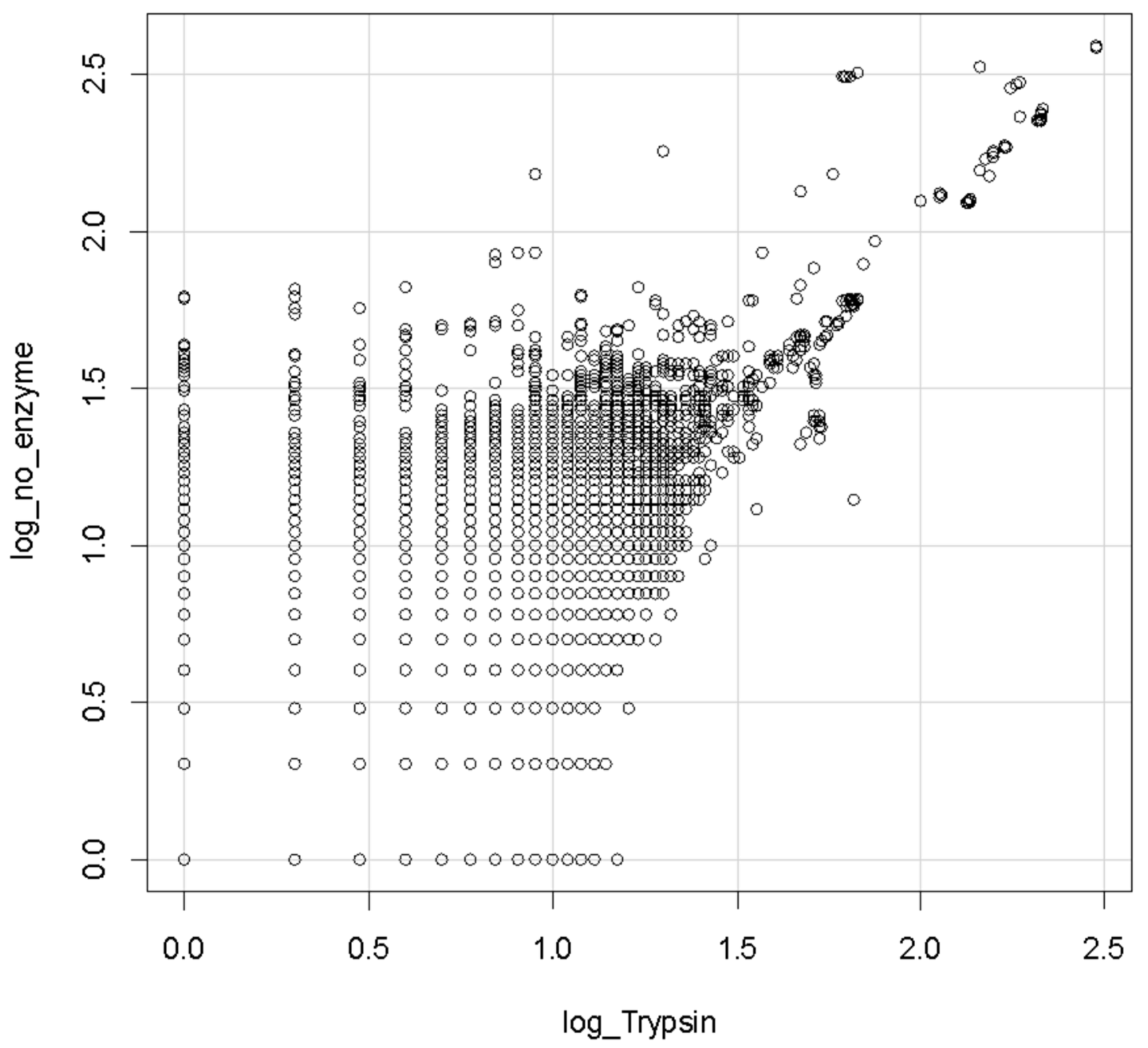

Figure 1. The comparison of the fully tryptic peptide correlation with up to three missed cleavage sites versus no enzyme specification. All proteins with at least 1 peptide correlation under either condition were plotted. The results indicate that the same set of proteins are correlated with no enzyme versus trypsin but that more peptides per protein are detected with the computationally more intense no enzyme parameter. 

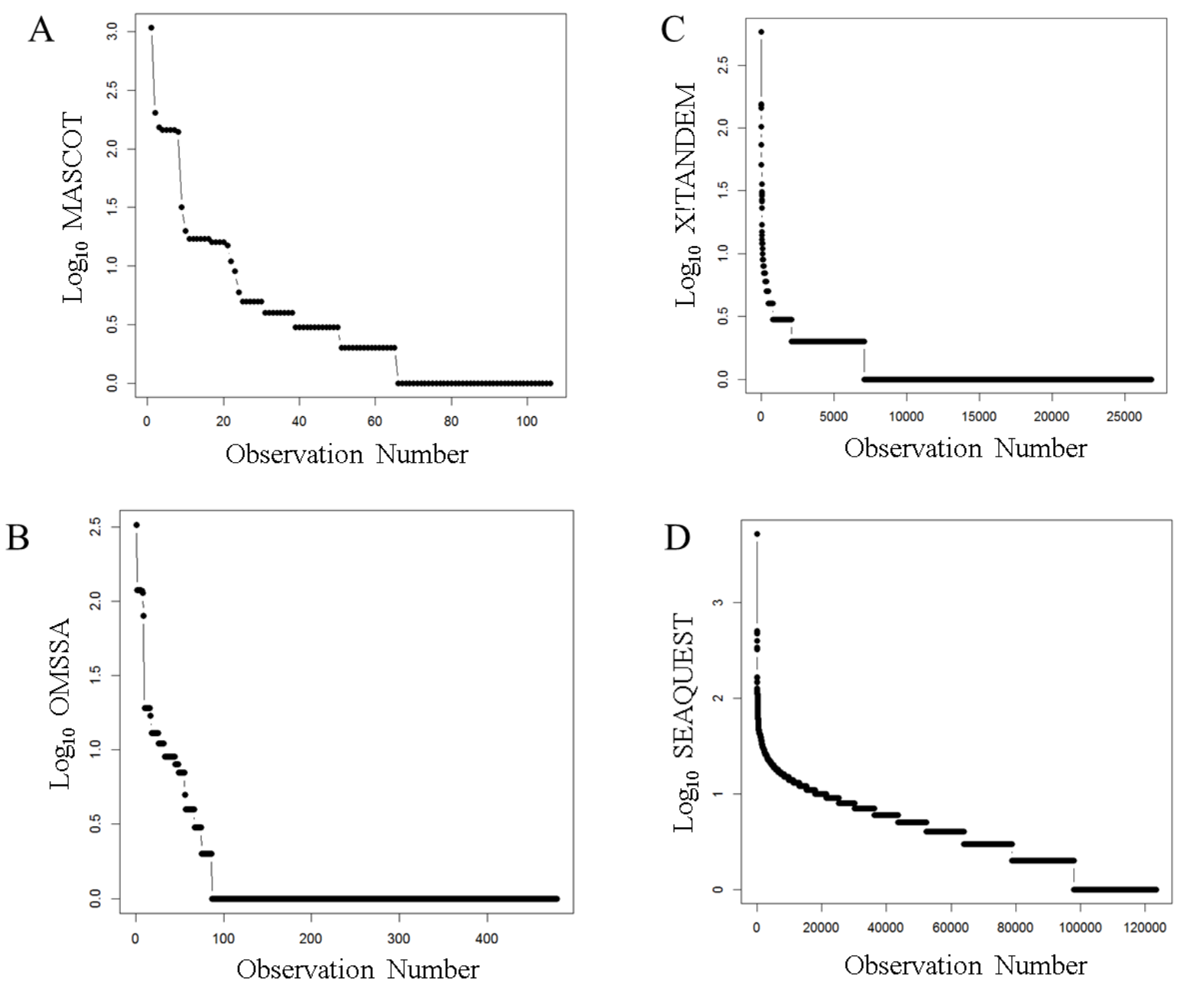

Figure 2. The comparison of the MASCOT, OMSSA, X!TANDEM and SEQUEST algorithms for the identification of endogenous tryptic peptides from human plasma on ice and incubated at room temperature combined. 


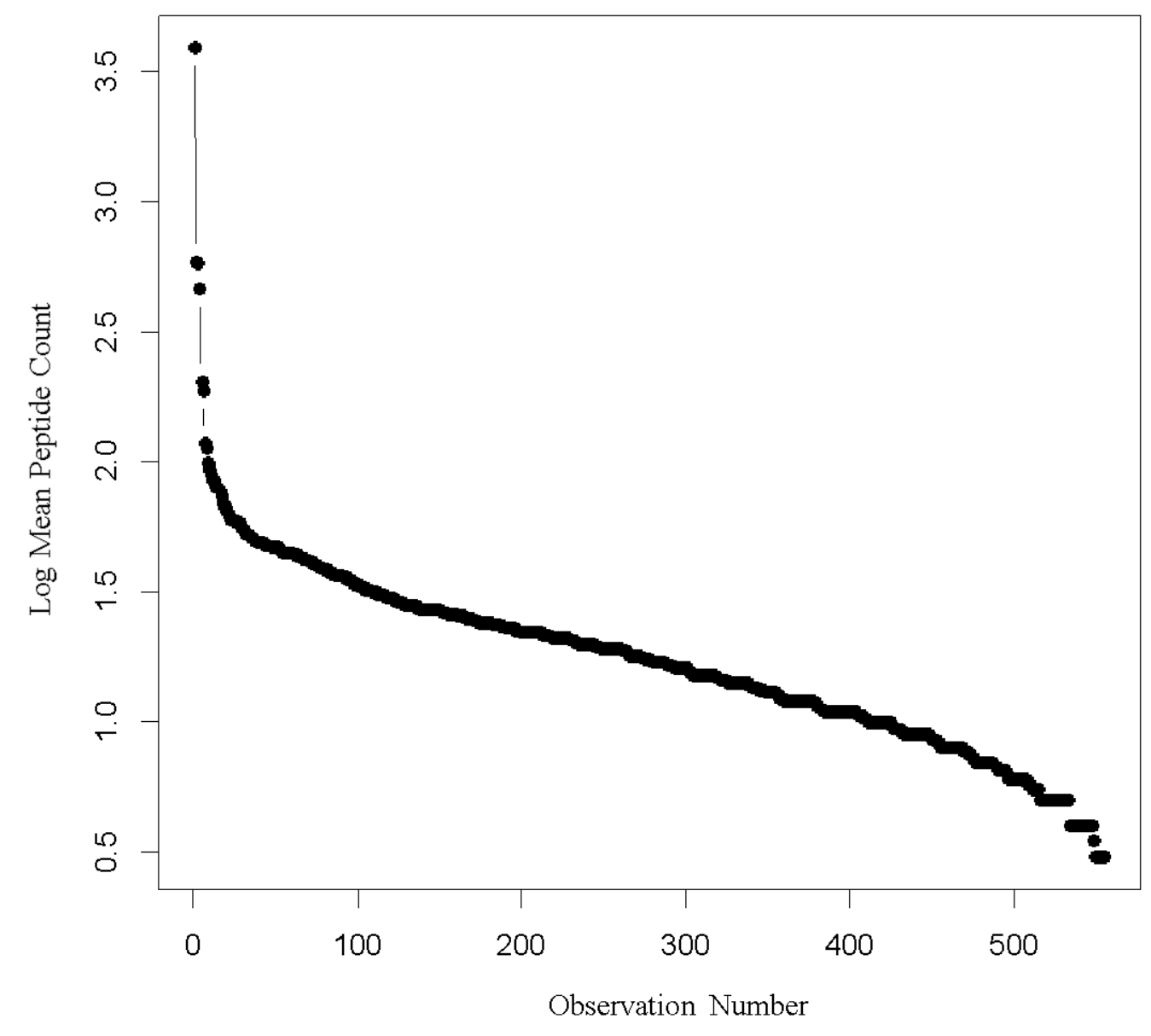

Figure 3. The total peptide to protein counts for all algorithms and all treatments. A total of 563,095 MS/MS spectra were obtained from LC-ESI-MS/MS analysis of 170 samples x 90 minute HPLC runs over C18 300 micron x150 mm were computed. 

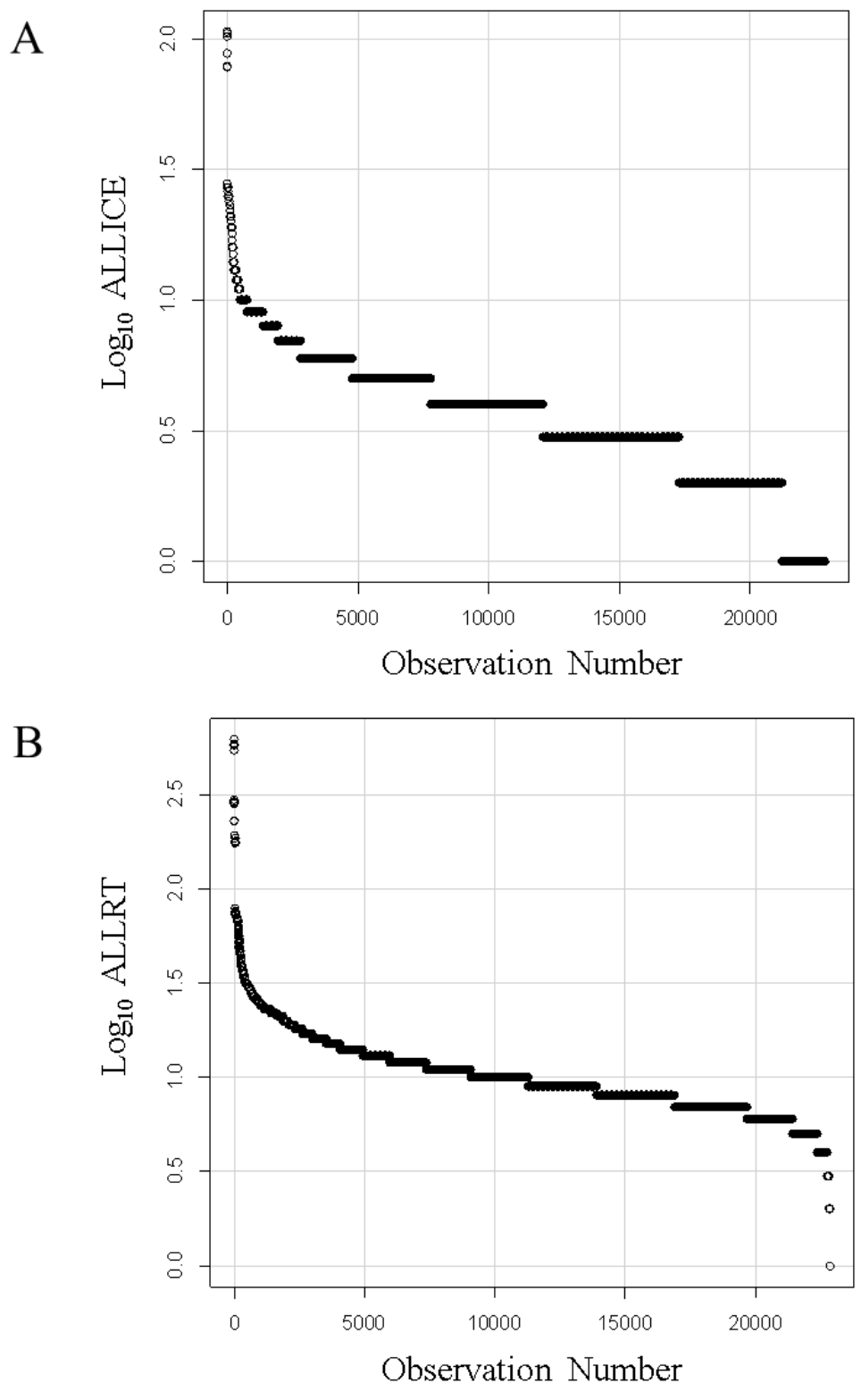

Figure 4. The log peptide to protein count of the preserved and degraded peptides of human EDTA plasma. Panels: A, the peptide to protein counts of plasma maintained frozen or on ice; $\mathrm{B}$, the peptide to protein distribution of plasma incubated at room temperature. 


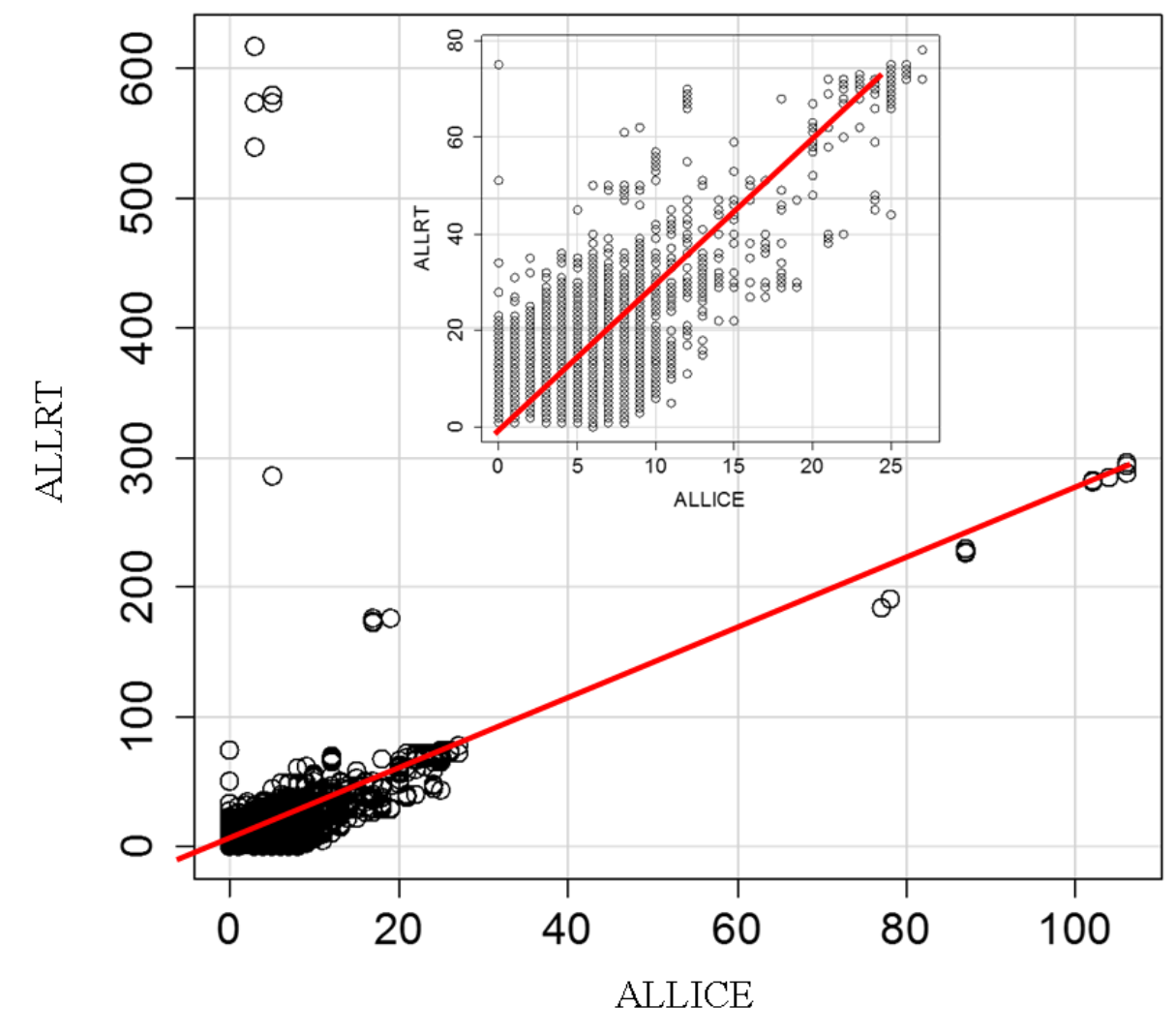

Figure 5. Regression analysis of the peptide-to-protein counts from samples incubated at room temperature (ALLRT) versus samples maintained on ice or frozen (ALLICE). Residual standard error: 2.945 on 22853 degrees of freedom, Adjusted R-squared: 0.3103, F-statistic: 1.028e+04 on 1 and $22853 \mathrm{DF}, \mathrm{p}$-value $<2.2 \mathrm{e}-16$. To avoid infinity values 1 was added to each value (p1). 


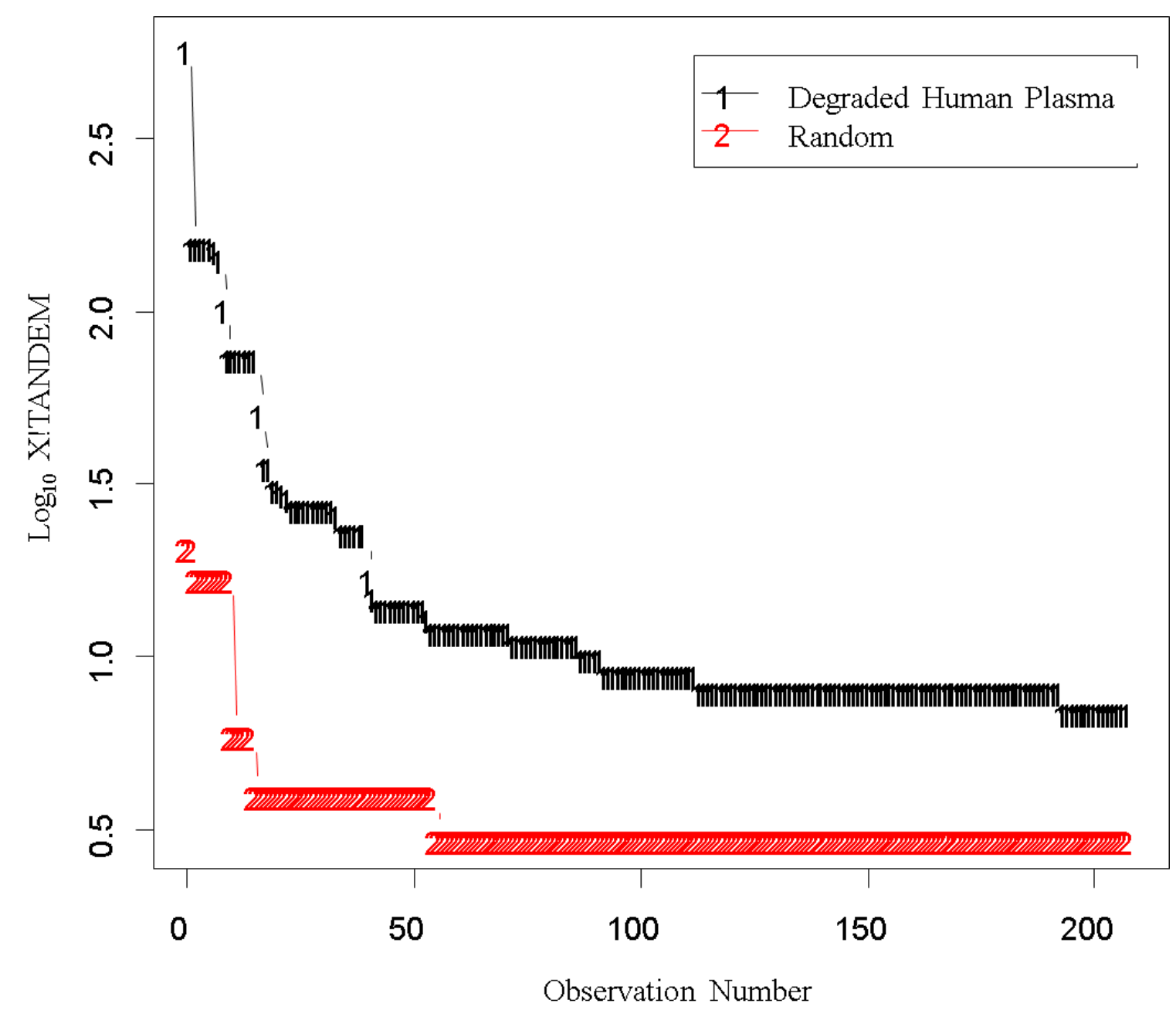

Figure 6. The frequency authentic endogenous tryptic peptides from 583,927 MS/MS spectra collected from EDTA plasma versus an equal number of random MS/MS spectra for the estimation of type I error rate in the proteins identified by X!TANDEM. The corrected mean peptide-to-protein counts of the first 200 protein accessions with at least three peptides from $X$ !TANDEM are shown. Note the peptide-to-protein distribution of real spectra exceeds that of random spectra by $\geq$ order of magnitude at all values and the Chi Square test indicates that the probability that the experimental data is the same as the null random model is essentially zero $(\mathrm{p}<0.0001)$ and is a formal estimate of the type I error with respect to a null random model for the whole experiment using the classical statistical approach. The result indicates that the $\mathrm{X}$ !TANDEM algorithm that fits MS/MS spectra to predicted peptide spectra shows an acceptable type I error rate over the whole experiment. 

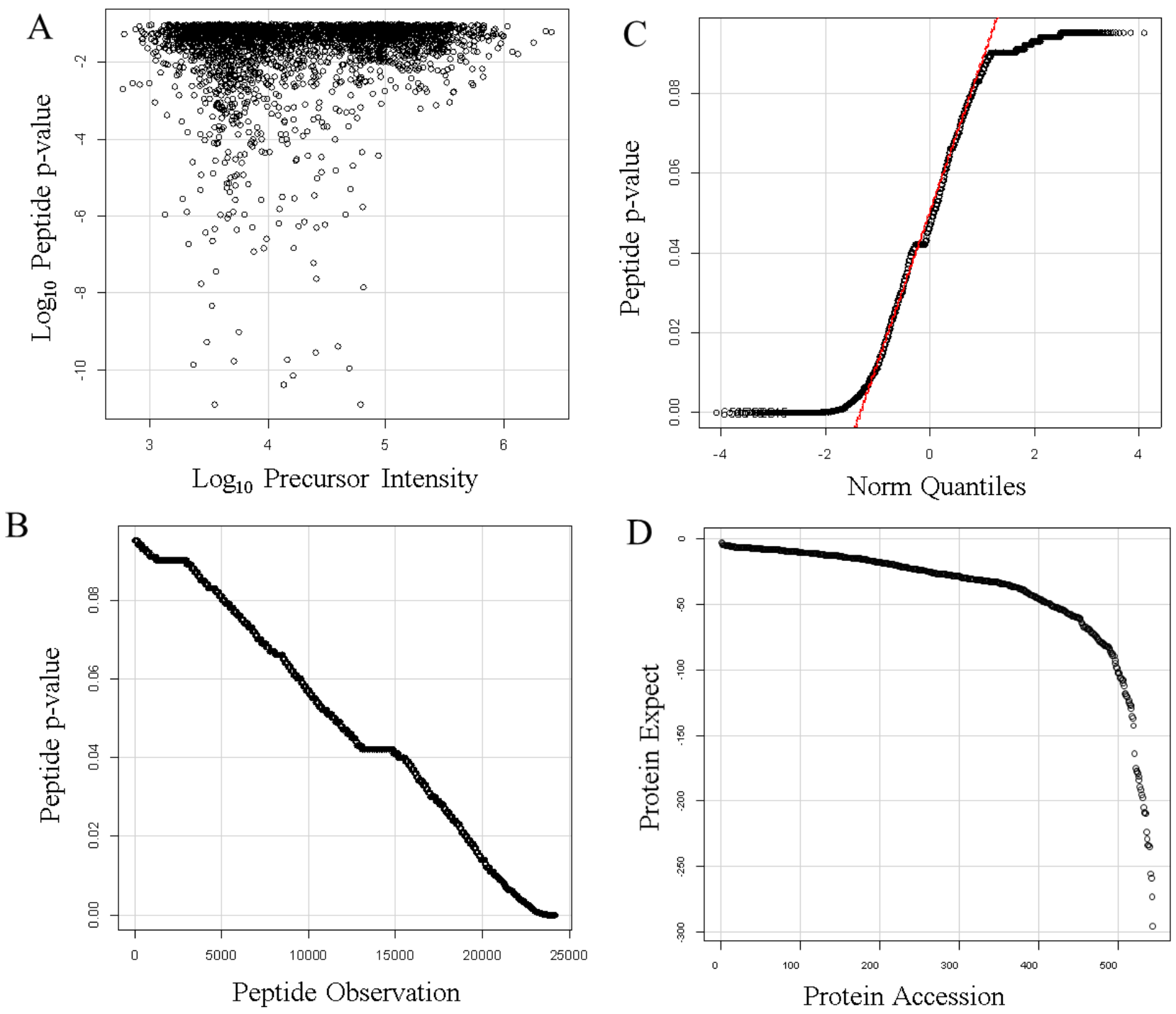

Figure 7. The confidence of endogenous tryptic peptides and proteins identified by X!TANDEM from human plasma on ice and incubated at room temperature. The X!TANDEM algorithm was used to assign the probability of type I error to peptides and thus the cumulative probability of type I error of proteins with 3 or more independent peptide correlations. Panels: A, scatter plot of peptide $\log _{10}$ P-value versus $\log _{10}$ precursor intensity; B, sorted Peptide p-values versus observations; C, Peptide p-value quantile plot; $\mathrm{D}$, cumulative protein $\mathrm{p}$-value per protein accession. 
A

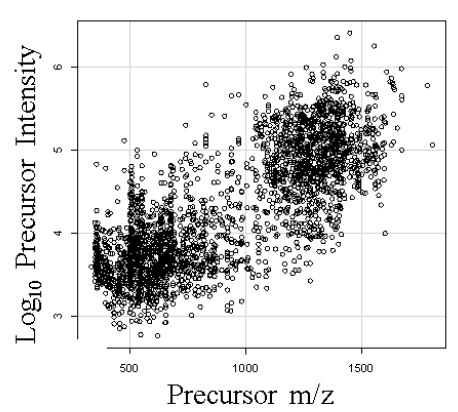

$\mathrm{B}$

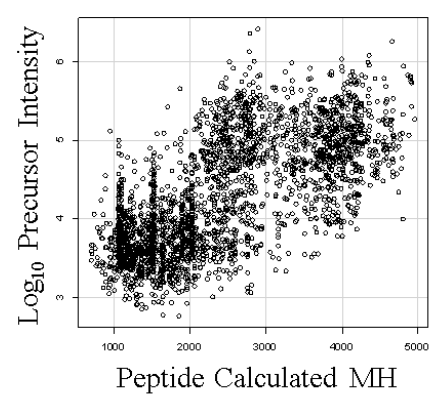

C

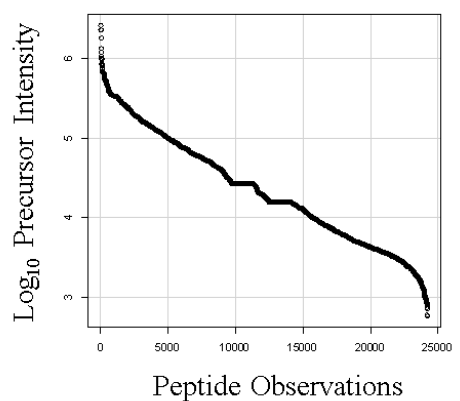

$\mathrm{D}$

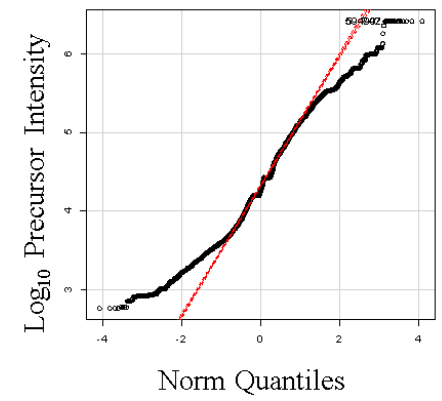

$\mathrm{E}$

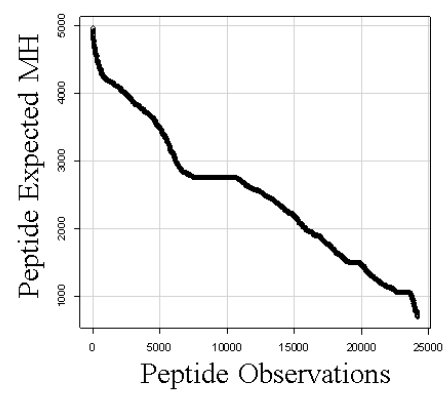

F

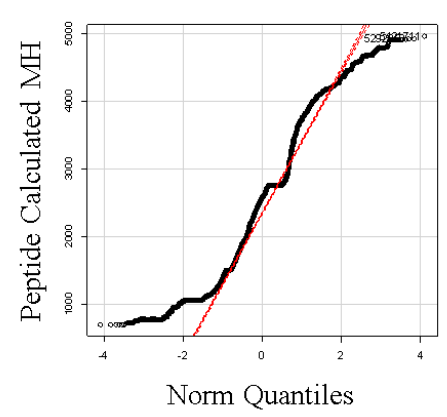

G

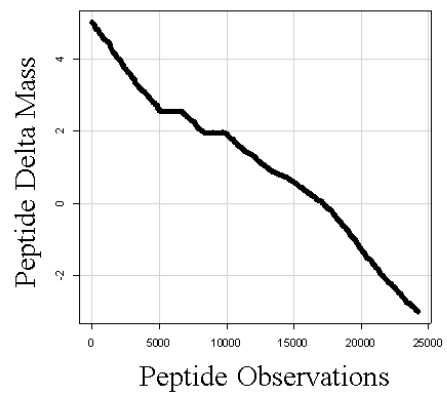

$\mathrm{H}$

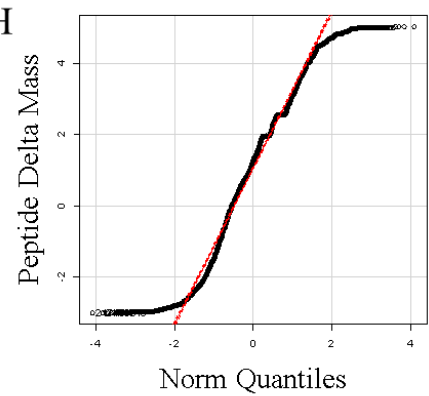

I

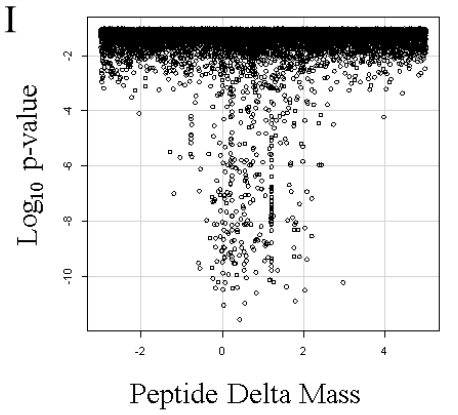

Figure 8. The distributions of the endogenous peptides from proteins with three or more independent correlations to the same protein accession by X!TANDEM. Panels: A, the scatter plot of $\log _{10}$ precursor intensity values versus precursor $\mathrm{m} / \mathrm{z} ; \mathrm{B}$, the scatter plot of the $\log _{10}$ distribution of precursor intensity values versus peptide mass $[\mathrm{M}+\mathrm{H}]^{+} ; \mathrm{C}$, the sorted distribution of $\log _{10}$ intensity values; D, the quantile plot of precursor $\log _{10}$ intensity values; $\mathrm{E}$, the sorted distribution of calculated peptide mass $[\mathrm{M}+\mathrm{H}]^{+} ; \mathrm{F}$, the quantile plot of peptide $[\mathrm{M}+\mathrm{H}]^{+} ; \mathrm{G}$, the sorted distribution of the peptide delta mass; $\mathrm{H}$, the quantile plot of the delta mass; $\mathrm{I}$, the relationship between delta $\mathrm{M}+\mathrm{H}$ versus peptide $\log _{10} \mathrm{p}$-value. 


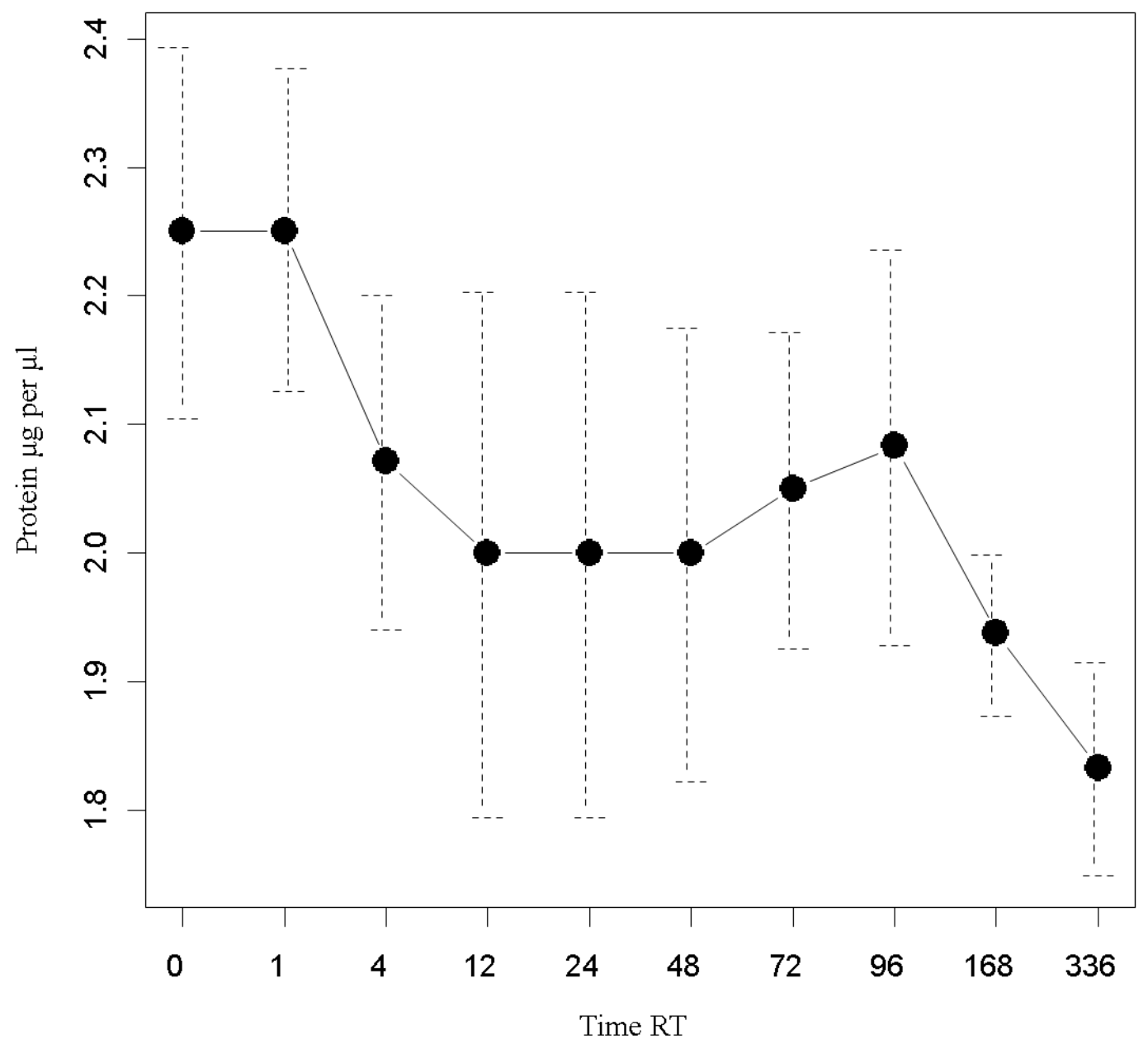

Supplemental Figure 1. Total protein analysis by the Dumbroff method of non-covalent binding to filter paper followed by washing of non-proteinaceous components with methanol prior to staining with $0.1 \mathrm{~g} \mathrm{CBBR}_{250}$ in $40 \%$ methanol and $10 \%$ acetic acid 


\section{Hours at Room Temperature}

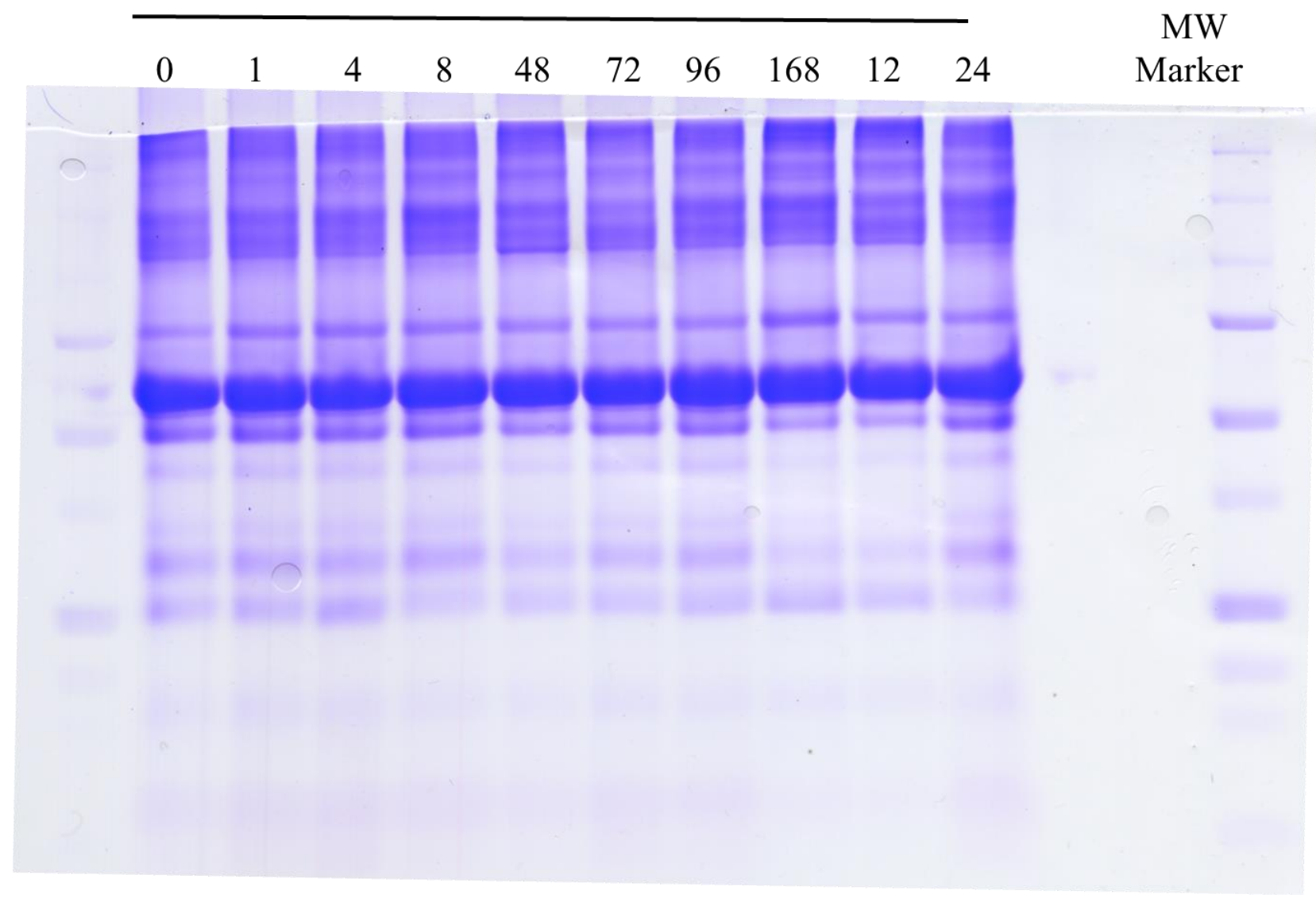

Supplemental Figure 2. The stability of EDTA plasma as demonstrated by electrophoresis and staining with Coomassie Brilliant Blue. EDTA Plasma samples incubated at room temperature for the time indicated were prepared over QA resin for tricine SDS-PAGE. Panels: A, CBBR staining of plasma after preparation over QA resin Molecular markers 250, 150, 100, 75, 50, 37, $25,15 \mathrm{kDa}$. (T5, T6, T7, T8, T9 were left at room Temperature the most. The tricine SDSPAGE gel shown is representative of 4 technical replicates. 


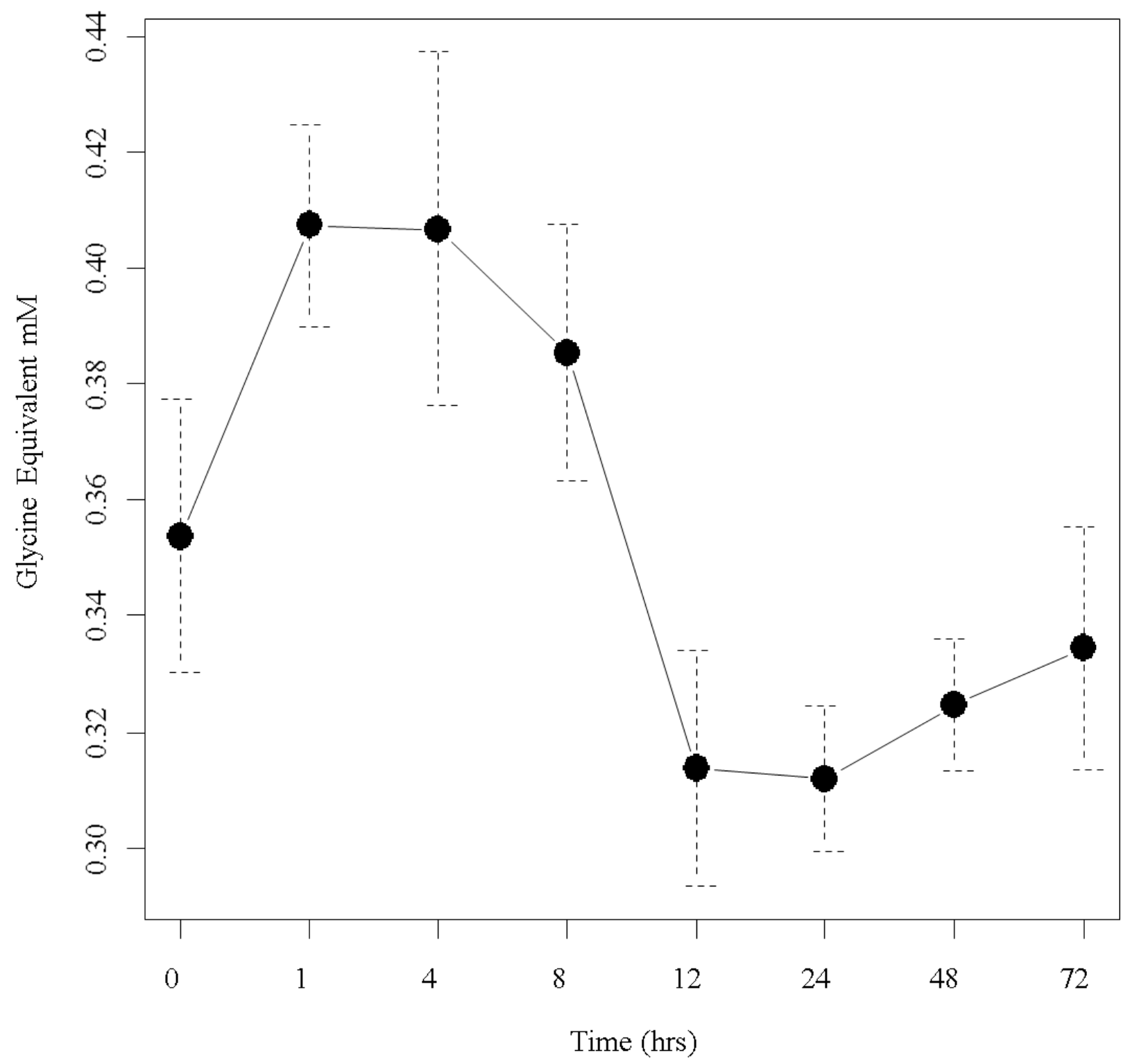

Supplemental Figure 3. The release of ethanol-soluble free amines from human EDTA plasma over time at room temperature. Dried plasma $(225 \mu \mathrm{L})$ was extracted with $250 \mu \mathrm{L}$ or absolute ethanol prior to reaction with Cd-ninhydrin alongside glycine standards. 
SUPPLEMENT E 


\section{Freeze-dried plasma proteins are stable at room temperature for at least one year}

Jaimie Dufresne ${ }^{1}$, Trung Hoang ${ }^{1}$, Juliet Ajambo ${ }^{1}$, Angelique Florentinus-Mefailoski ${ }^{1}$, Peter Bowden $^{1}$, John Marshall ${ }^{1,2}$

jaimie.dufresne@ryerson.ca, thhoang@ryerson.ca, juliet.ajambo@gmail.com,

a3floren@ ryerson.ca, pbowden@ start.ca, 4marshal@ ryerson.ca

${ }^{1}$ Ryerson University, 350 Victoria Street, Toronto, Ontario, Canada, M5B 2K3

${ }^{2}$ Integrated BioBank of Luxembourg, 6 r. Nicolas-Ernest Barblé 1210 Luxembourg

Running Title: NGFKSHALQLNNR cleaved from C4B at room temperature

Acknowledgment: This research was paid for by the Fonds National de la Recherche, Mobility of Researcher award through Luxembourg Institute of Health (formerly CRP Sante) and a direct grant from the Integrated Biobank of Luxembourg to JGM. We gratefully acknowledge the review and critique of this manuscript by Dr. Fay Betsou and Dr. R.A. Phillips of IBBL.

\footnotetext{
Abbreviations

AEBSF, 4-benzenesulfonyl fluoride hydrochloride

$\mathrm{ACN}$, acetonitrile

C4B, complement component 4B

C4B-peptide, NGFKSHALQLNNR

ICE, fresh EDTA plasma stored on wet ice

ICE_INHIB, fresh EDTA plasma stored on wet ice with protease inhibitors

RT, fresh EDTA plasma stored at room temperature

$\mathrm{LN}_{2}$, fresh EDTA plasma stored in Liquid Nitrogen

FD, Freeze Dried EDTA plasma

FD-20 ${ }^{\circ} \mathrm{C}$, freeze dried EDTA plasma stored in an electric freezer at $-20^{\circ} \mathrm{C}$

FDRT, EDTA plasma freeze dried and stored in a desiccator at room temperature

PMSF, phenylmethane sulfonyl fluoride

PVDF, Polyvinylidene fluoride
} 


\section{ABSTRACT}

Thirty human EDTA plasma samples from male and female subjects ranging in age from 24 to 74 years were collected on ice, processed ice cold and stored frozen at $-80^{\circ} \mathrm{C}$, in liquid nitrogen (LN2), or freeze dried and stored at room temperature in a desiccator (FDRT) or freeze dried and stored at $-20^{\circ} \mathrm{C}$ for 1 year (FD-20). In a separate experiment, EDTA plasma samples were collected onto ice, processed ice cold and maintained on ice \pm protease inhibitors versus incubated at room temperature for up to 96 hours. Random and independent sampling by liquid chromatography and tandem mass spectrometry (LC-ESI-MS/MS), as correlated by the MASCOT, OMSSA, X!TANDEM and SEQUEST algorithms, showed that tryptic peptides from complement component 4B (C4B) were rapidly released in plasma at room temperature.

Random sampling by LC-ESI-MS/MS showed that peptides from C4B were undetectable on ice, but peptides were cleaved from the mature C4B protein including NGFKSHALQLNNR within as little as $1 \mathrm{hr}$ at room temperature. The frequency and intensity of precursors within $\pm 3 \mathrm{~m} / \mathrm{z}$ of the C4B peptide NGFKSHALQLNNR was confirmed by automated targeted analysis where the precursors from MS/MS spectra that correlated to the target sequence were analyzed in SQL/R. The C4B preproprotein was processed at the $\mathrm{N}$ terminus to release the mature chain that was cleaved on the carboxyl side of the isoprene $\mathrm{C} 2$ domain within a polar $\mathrm{C}$ terminal sequence of the mature C4B protein, to reveal the thioester reaction site, consistent with LC-ESI-MS/MS and Western blot. Random sampling showed that proteolytic peptides from complement component C4B were rarely observed with long term storage at $-80^{\circ} \mathrm{C}$ in a freezer or in liquid nitrogen (LN2), freeze drying with storage at $-20^{\circ} \mathrm{C}\left(\mathrm{FD}-20^{\circ} \mathrm{C}\right)$ or freeze drying and storage at room temperature in a desiccator (FDRT). Plasma samples maintained at room temperature (RT) showed at least 10- to 100-fold greater frequency of peptide correlation to C4B and measured 
peptide intensity compared to samples on ice for up to 72 hours or stored at $-80^{\circ} \mathrm{C}, \mathrm{LN} 2$, FDRT or $\mathrm{FD}-20^{\circ} \mathrm{C}$ for up to a year. 


\section{INTRODUCTION}

The proteins and endogenous peptides of human plasma samples may be purified by partition chromatography with identification and quantification by liquid chromatography, electrospray ionization and tandem mass spectrometry (LC-ESI-MS/MS) (J. Marshall et al., 2004; John Marshall et al., 2003). Peptides from blood proteins might facilitate the diagnosis of diseases and the evaluation of the efficacy of therapeutic treatments for individual patients (Melo et al., 2015; Qin et al., 2016; Wiita, Hsu, Lu, Esensten, \& Wells, 2014; P. Zhu, P. Bowden, D. Zhang, et al., 2011). Plasma expresses a weak tryptic protease activity that may slowly degrade the sample proteins over time thus releasing endogenous peptides that may be unrelated to the disease process (Sardesai \& Provido, 1965). The proteolytic activation of the complement system is an important mediator of the acute inflammatory response and humoral immunity (Volanakis, 1990; Weisman et al., 1990). C4B is cleaved to expose a thioester group that permits covalent modification of target macromolecules (Fung, Wright, Gor, Swann, \& Perkins, 2016). It has been shown that levels of C4B peptides may be associated with the sampling conditions (Albrethsen et al., 2006; Banks, 2008; Callesen et al., 2008; de Noo et al., 2005; Gast et al., 2009; Govorukhina et al., 2008; Jin \& Manabe, 2009; Kaisar et al., 2016; Karsan et al., 2005; Koomen et al., 2005; J. Li et al., 2005; John Marshall et al., 2003; Pilny et al., 2006; Rai et al., 2005; Semmes et al., 2005; Spencer, 2003; Tuck et al., 2009; van Winden et al., 2009; West-Nielsen et al., 2005; West-Norager et al., 2007). The steady state balance of ex vivo endo-proteinase and exopeptidase activity may change over time and result in a large variation in the blood peptides observed (John Marshall et al., 2003; Williams et al., 2010). Pre-analytical variation in the time the serum or plasma sample remains at room temperature before aliquoting and freezing may be a source of bias in subsequent mass spectrometric measurements (Albrethsen et al., 2006; Banks, 
2008; Callesen et al., 2008; de Noo et al., 2005; Govorukhina et al., 2008; Karsan et al., 2005; Koomen et al., 2005; J. Li et al., 2005; Pilny et al., 2006; Rai et al., 2005; Semmes et al., 2005; Spencer, 2003; Tuck et al., 2009; van Winden et al., 2009). The evidence to date indicates that variation in handling immediately after sample collection and prior to centrifugation is the largest source of variation in blood samples (Gast et al., 2009; John Marshall et al., 2003; Rai et al., 2005; West-Nielsen et al., 2005; West-Norager et al., 2007). To prevent degradation, the sample should be kept on ice during sample handling (van den Broek, Sparidans, Schellens, \& Beijnen, 2008). Adding serine centered endo-peptidase inhibitors like PMSF or AEBSF (Yi, Kim, \& Gelfand, 2007; Yi et al., 2008) to blood fluids in order to preserve the proteins will result in alterations of endogenous peptides (Gast et al., 2009; John Marshall et al., 2003; Williams et al., 2010; Yang et al., 2009; Yi et al., 2007; Zheng et al., 2006). Alternatively, it may be possible to quench ex vivo reactions and store blood samples by freeze drying (P. Zhu, P. Bowden, D. Zhang, et al., 2011), or rapid drying on filter paper (Kulik et al., 2008) or PVDF (Gharahdaghi, Kirchner, Fernandez, \& Mische, 1996). Sensitive and reproducible methods to isolate the cleaved peptides from human plasma have been compared and showed C18 solid phase extraction was a reliable method (M. Tucholska et al., 2010; Monika Tucholska et al., 2007). C18 solid phase extraction of peptides was used to establish that peptides from C4B are released into plasma at room temperature. Here release of the C4B-peptide (NGFKSHALQLNNRQIR) in human plasma was compared over storage and incubation conditions by random and independent sampling with LC-ESI-MS/MS. 
The experiments showed that a plasma sample remaining at room temperature undergoes polypeptide degradation compared to ice cold, frozen samples or freeze dried samples stored at room temperature. Proteolytic degradation of plasma at room temperature resulted in the production of peptides containing the C4B-peptide sequence. Here release of the C4B-peptide (NGFKSHALQLNNRQIR) in human plasma was compared over storage and incubation conditions by random and independent sampling with LC-ESI-MS/MS as confirmed by automated targeted analysis. The C4B peptides showed a low frequency of detection and low ion intensity values in samples collected on ice \pm protease inhibitors, but showed a sharp increase at room temperature and remained strongly detectable for days on the laboratory bench. Plasma samples temporarily stored on ice \pm protease inhibitors, stored at $80^{\circ} \mathrm{C}$ in or liquid nitrogen, or freeze dried and stored at $-20^{\circ} \mathrm{C}$ or at room temperature, all show low levels of C4B-peptide, compared to plasma samples incubated at room temperature for a few hours or days. Here, three methods, random and independent sampling, automatic-targeted quantification, and Western blot all showed that processing of C4B reflects the proteolytic degradation of plasma at room temperature. An additional aim of the experiment was to determine if blood cells are required for the processing of plasma proteins. 


\section{MATERIALS AND METHODS}

\section{Materials}

The freeze dryer was from Labconco (Kansas City, MO, USA) and the $30 \mathrm{~L}$ pump was from Edwards (Sanborn, NY, USA). The Agilent 1100 HPLC (Santa Clara, CA, USA) for LC-ESIMS/MS mass spectrometry was coupled to an LTQ XL linear ion trap mass spectrometer from the Thermo Electron Corporation (Waltham, MA, USA). The HPLC grade water and acetonitrile were obtained from Caledon Laboratories (Georgetown, Ontario, Canada). The C4B antibody (PA1-9534, Lot no. QB1980891) and the Pierce EZ Link NHS biotinyation kit was obtained from Thermo Fisher Scientific (Waltham, MA, USA). The streptavidin-HRP conjugate was obtained from Jackson ImmunoResearch (West Grove, PA, USA).

\section{Sample collection}

Human plasma was collected under a protocol approved by the Comité National d'Ethique de Recherche (CNER) Protocol \#201107 “Biospecimen Research” at the Centre Hospitalier de Luxembourg. EDTA blood samples from 30 male and female healthy subjects, ranging in age from 24 to 74 were collected immediately onto ice, centrifuged at $2000 \mathrm{xg}$ for 20 minutes at $4^{\circ} \mathrm{C}$ and plasma was aliquoted in $225 \mu \mathrm{l}$ volumes on ice, and briefly held at $-80 \mathrm{C}$ prior to randomly assigning to short-term or long-term experimental storage treatments. Short term storage experiments were ice (ICE) or ice plus protease inhibitors (ICE-INH) or room temperature (RT) for up to $96 \mathrm{~h}$ as indicated. Long term storage conditions included $-80^{\circ} \mathrm{C}$ or liquid nitrogen, (LN2), or freeze drying (FD) followed by storage at $-20^{\circ} \mathrm{C}\left(\mathrm{FD}-20^{\circ} \mathrm{C}\right)$ or room temperature (FDRT). Freeze dried samples were first frozen to $-80^{\circ} \mathrm{C}$ and then rapidly placed in a Labconco rotary sample speedvac with the condenser maintained at $-90{ }^{\circ} \mathrm{C}$ with a $30 \mathrm{~L}$ per 
minute pump (a vacuum strong enough to rapidly freeze water) for 24 hours before rapidly resealing the vials. There was no heat applied, and no organic solvents or salts were added, and the samples remained frozen under strong vacuum during the drying by means of a $-90^{\circ} \mathrm{C}$ condenser and thus were lyophilized, i.e. freeze dried.

\section{Short term storage experiment}

Plasma aliquots $(225 \mu \mathrm{l})$ from the thirty donors were thawed on ice and then randomly assigned to incubation at room temperature or on ice for varying times. The room temperature (RT) and control samples on ice \pm protease inhibitors (ICE \& ICE-INH) were incubated for various times, up to 72 hrs. The protease inhibitors AEBSF, PMSF, benzamidine $\mathrm{HCl}$, and caproic acid were used at $2 \mathrm{mM}$ each with the Sigma Eukaryotic protease inhibitor cocktail at 1/100 (v/v). The Sigma Mammalian Protease inhibitor cocktail contains at least: AEBSF, 104 mM, Aprotinin, 80 $\mu \mathrm{M}$, Bestatin, $4 \mathrm{mM}$, E-64, $1.4 \mathrm{mM}$, Leupeptin, $2 \mathrm{mM}$, Pepstatin A, 1.5mM used at 1/100 (v/v). Plasma samples $(225 \mu \mathrm{l})$ from at least 10 different donors were tested at each time point and over the time course of degradation up to $72 \mathrm{hrs}$. At the end of each time period, the samples were frozen, freeze dried and stored dried at $-80^{\circ} \mathrm{C}$ until analysis.

\section{Long term storage experiment}

Plasma aliquots from the 30 donors $(225 \mu \mathrm{l})$ were randomly assigned to a $-80^{\circ} \mathrm{C}$ freezer $\left(-80^{\circ} \mathrm{C}\right)$, liquid nitrogen (LN2), freeze dried and stored in a desiccator at room temperature (FDRT) or freeze dried and stored at $-20^{\circ} \mathrm{C}\left(\mathrm{FD}-20^{\circ} \mathrm{C}\right)$ until analysis.

\section{Random and independent sampling by LC-ESI-MS/MS}


Plasma samples of $25 \mu \mathrm{l}$ were dissolved in $225 \mu \mathrm{l}$ of ice cold $5 \%$ formic acid prior to collection of the peptides over a preparative, ZipTip, C18 column (Monika Tucholska et al., 2007). The 2 $\mu \mathrm{L}$ elution volume was aspirated and ejected across the C18 resin bed carefully 5 times to avoid permitting air bubbles into the resin bed. Collected peptides were eluted off the ZipTip in $2 \mu \mathrm{l}$ of acidified $65 \%$ acetonitrile and immediately diluted with $18 \mu \mathrm{l}$ of $5 \%$ formic acid and injected for analytical HPLC separation over a $15 \mathrm{~cm}$ x 300 micron ID column coupled to an electrospray source for the LTQ XL linear ion trap mass spectrometer (Thermo Electron Corporation). A federated library of human proteins was assembled from NCBI, Ensembl and Swiss-Prot and made non-redundant using Structured Query Language (SQL) (Bowden et al., 2009; Bowden et al., 2010). The experimental MS and MS/MS spectra of peptides recorded were correlated to predicted spectra from the federated library at a charge state of $2+$ and $3+$ to identify fully tryptic peptides using the X!TANDEM (R. Craig \& R. C. Beavis, 2004), OMSSA(L. Y. Geer et al., 2004), MASCOT (Perkins et al., 1999) and SEQUEST algorithms (Yates, 1998; Yates et al., 1995) set within $\pm 3 \mathrm{~m} / \mathrm{z}$ in the precursor mass and within $\pm 0.5 \mathrm{Da}$ in the fragment mass with up to three missed cleavages (P. Zhu, P. Bowden, M. Tucholska, et al., 2011; Peihong Zhu et al., 2011), as proteins may be only partially digested by proteases. Only the best fit peptide to each MS/MS spectra in terms of charge state or amino acid sequence was accepted.

\section{Automated targeted LC-ESI-MS/MS}

The LC-ESI-MS/MS was repeated with targeted analysis to monitor the C4B-peptide (NGFKSHALQLNNR) released from complement component 4B. The LC-ESI-MS/MS analysis by micro electrospray was repeated but instead of random and independent sampling, the LTQ 
ion trap was set to monitor NGFKSHALQLNNR [with a monoisotopic mass 1497.78 and so at +

2 charge $[\mathrm{M}+2 \mathrm{H}]^{2+}$ then $(1497.78+2) / 2=749.5 \mathrm{~m} / \mathrm{z}$ and at +3 charge $[\mathrm{M}+3 \mathrm{H}]^{3+}$ then

$(1497.78+3) / 3=500.26 \mathrm{~m} / \mathrm{z}]$ with $\pm 3 \mathrm{~m} / \mathrm{z}$. The MS/MS spectra from the targeted LC-MS/MS of the C4B-peptide was confirmed by searching the results against a protein library that only contained the target sequence NGFKSHALQLNNR from C4B using SEQUEST as described above, but with no cut off at 1000 counts, in order to increase sensitivity. The frequency and intensity of precursors within $\pm 3 \mathrm{~m} / \mathrm{z}$ of the C4B peptide NGFKSHALQLNNR were confirmed by automated targeted analysis where only the MS/MS spectra that correlated to the target sequence was stored in SQL for statistical analysis R.

\section{Western blot}

A total of $2 \mu \mathrm{L}$ of EDTA plasma was dissolved in $25 \mu \mathrm{L}$ of $2 \times$ SDS-PAGE sample-buffer and boiled for 10 minutes. The EDTA plasma samples were separated over $9 \%$ acrylamide discontinuous tris gels with a pH 6.8 stacking gel and a pH 8.8 separating gel in tricine tank buffer at 100 volts (Schagger \& von Jagow, 1987). The samples were transferred to PVDF (that was pre-wetted in methanol) at 100 volts in Towbin buffer (Towbin, Staehelin, \& Gordon, 1979). The transfer was confirmed by staining with CBBR in 50\% methanol, the position of the molecular weight markers was marked in pencil and the blots erased in pure methanol prior to equilibrating in PBST and Western blot with 1/500 (v/v) biotinylated primary antibody. The binding of the primary antibody was detected with 1/10,000 (v/v) streptavidin HRP conjugate using enhanced chemiluminescence (Haan \& Behrmann, 2007). 


\section{Statistical analysis}

The precursor ion intensity values that were correlated to C4B tryptic peptides by SEQUEST, together with the parent and fragment $\mathrm{m} / \mathrm{z}$ and intensity values, that were automatically parsed into an SQL database (Bowden et al., 2009). The peptide intensity and frequency values were statistically analyzed using classical statistical approaches such as Chi Square and ANOVA for each protein (Baggerly et al., 2003; Eckel-Passow et al., 2009; Angelica K Florentinus, Peter Bowden, Girish Sardana, et al., 2012; Florentinus et al., 2011a; John Marshall et al., 2003; Sorace \& Zhan, 2003). The peptide intensity values were $\log _{10}$ transformed to approximate a normal distribution and then analyzed by ANOVA prior to the Tukey-Kramer Honestly Significant Difference (HSD) and plotted using the R Statistical Analysis System (Bowden et al., 2012; Angelica K Florentinus, Peter Bowden, Girish Sardana, et al., 2012; Florentinus et al., 2011a). The Chi Square analysis of the frequency versus intensity histogram for the various treatments was performed and graphed using the generic open-source R statistical system. 


\section{RESULTS}

\section{Random and independent sampling of all peptides from all proteins}

The tryptic peptides in human EDTA plasma were analyzed by random and independent sampling of the 5 highest intensity peptides eluting at any moment from the HPLC-ESI-MS/MS system. The MS/MS spectra were correlated to the tryptic peptides of the human proteome in a federated protein library with up to three missed cleavages that revealed the processing of complement component 4B (C4B) (Table I, Fig. 1). Random and independent sampling of C4B from ice cold or room temperature EDTA plasma by LC-ESI-MS/MS with correlation by MASCOT, OMSSA, X!TANDEM and SEQUEST showed p-values for individual MS/MS spectra that ranged from $\mathrm{p}<0.1$ (E-1) to $\mathrm{p}<\mathrm{E}-150$ (Fig. 1). Plasma samples that were maintained on ice over time showed very little cleavage of C4B in contrast to samples incubated at room temperature that showed the cleavage of the C4B peptides (Table II, Fig. 2). The C4B peptides NGFKSHALQLNNR and GLEEELQFSLGSK were among the most commonly observed in agreement with previous results (Kaisar et al., 2016). The release of peptides from complement C4B was readily detected in room temperature samples by unbiased LC-ESI-MS/MS (Table II, Fig. 2). The C4B peptides were almost undetectable at time zero (ICE), but were clearly detectable in as little as $1 \mathrm{hr}$ or more at room temperature. After incubation at room temperature for 4 to 8 hrs the most characteristic peptide from C4B (NGFKSHALQLNNR) was observed with greater frequency that was apparently significant by the Chi Square test (Fig. 3) that indicated a low probability $(<0.0001)$ that the room temperature samples were the same as the control. The intensity distribution of the C4B-peptide approached normality as assessed by quantile plots (Fig. 4A). The C4B-peptide intensity values were typically less than 1000 
intensity counts at time zero (ICE). The C4B-peptide showed detectable intensity values after 1 hr with a mean of just above 1000 intensity counts, that increased by almost an order of magnitude over 4 to $8 \mathrm{~h}$ at room temperature (RT).

\section{Automatic-Targeted LC-ESI-MS/MS Analysis}

Random and independent sampling showed a large increase in C4B detection frequency and intensity in plasma with time at room temperature but has greater sampling error compared to targeted measurement of C4B peptides. The MS/MS spectra from peptides with precursors within $\pm 3 \mathrm{~m} / \mathrm{z}$ of the predicted $2^{+}$or $3^{+} \mathrm{m} / \mathrm{z}$ values were automatically searched against the C4B-peptide NGFKSHALQLNNR using the SEQUEST algorithm. The correlated peptide count and precursor intensity values were automatically collected in SQL Server for the automatic transformation, computation of means, normality, intensity differences by ANOVA and frequency differences by Chi Square using the R statistical analysis system (Fig. 5). The fully automated targeted analysis of the C4B peptide NGFKSHALQLNNR using SQL and R showed a $\log _{10}$ intensity distribution of the peptide that approached Gaussian normality (Fig. 5A). Comparing baseline plasma samples versus those incubated for $1 \mathrm{hr}$ or $72 \mathrm{hrs}$ at RT confirmed a sharp increase in average peptide intensity values from time 0 to 1 or $72 \mathrm{hrs}$ at room temperature by ANOVA followed by the Tukey Kramer test (Fig. 5B). The

NGFKSHALQLNNR peptide counts from all precursors increased dramatically from time 0 (on ice) to thousands or even tens of thousands of counts by as little as $1 \mathrm{hr}$ and up to $72 \mathrm{hr}$ at room temperature (Fig. 5C). 


\section{Primary structural analysis of C4B}

Peptides consistent with the cleavage of the $\mathrm{C} 4 \mathrm{~B}$ preproprotein to yield the mature chain were observed (Fig. 6). The most commonly detected cleavage site on the mature C4B chain was on the carboxyl side of the isoprene $\mathrm{C} 2$ domain shared with alpha 2 macroglobulin as reflected by LC-ESI-MS/MS. A frequent C4B cleavage site was within the polar C terminal sequence ${ }^{1337}{ }^{\text {RNGFKSHALQLNNRQIRGLEEELQFSLGSKINVK }}{ }^{1370}$ (NP_001002029.3) that contained the most commonly observed peptides NGFKSHALQLNNR and GLEEELQFSLGSK (Fig. 6).

\section{Western blot against the mature $\mathrm{C} 4 \mathrm{~B}$ protein}

Samples of EDTA plasma were resolved by tricine SDS-PAGE and blotted to PVDF supports for Western analysis. The Western blot showed that the antibody recognizes the processed form of mature C4B as expected. Western analysis strongly detected the parent C4B protein in close agreement with the predicted relative mass of $84,163 \mathrm{Da}$ and its major cleavage product at 71,539 $\mathrm{kD}$ based on the annotation found at NCBI NP_001002029.3 (Fig. 7). The cleavage of the c-terminal portion of the $\mathrm{C} 4 \mathrm{~B}$ chain should yield a protein of about $63 \mathrm{kDa}$ as observed (Fig. 7).

\section{Plasma sample long-term storage by freezing and freeze-drying}

Plasma was protected from degradation, as measured by the release of C4B-peptide, by freeze drying followed by 1 year storage at room temperature (FDRT), freeze drying followed by 1 year storage at $-20^{\circ} \mathrm{C}\left(\mathrm{FD}-20^{\circ} \mathrm{C}\right), 1$ year freezing at $-80^{\circ} \mathrm{C}$ or 1 year freezing in liquid nitrogen $\left(\mathrm{LN}_{2}\right)$ (Fig. 8). Furthermore, there were no signs of proteolytic degradation after short-term 
storage of plasma samples on ice \pm protease inhibitors, for up to 3 days (Fig. 8). The Chi Square test showed a low probability $(<0.0001)$ that the degraded samples were the same as the control.

\section{DISCUSSION}

This study regarded the cleavage of the C4B protein at room temperature compared to ice cold, frozen, or freeze dried plasma samples by random and independent sampling, or automatic targeted quantification by LC-ESI-MS/MS, and SDS-PAGE with Western blot. All three analytical methods show a similar trend of little detectable C4B-peptide cleavage in ice cold, frozen or freeze dried plasma samples. Cleavage of C4B rapidly became apparent after incubation for 1 to $4 \mathrm{hrs}$ at room temperature using sensitive LC-ESI-MS/MS.

\section{Random and Independent sampling}

Without any pre-conceived notion of what proteins or peptides might be the best for measuring sample degradation, random and independent sampling was used to compare all possible human tryptic peptides across all sample storage conditions. The counting of peptides to proteins across treatments with SQL/R was a simple means to identify the proteins in human plasma that degrade with incubation at room temperature. The C4B-peptide levels may serve as a marker of sample degradation based on the $\geq 10$-fold increase in the frequency of detection or peptide intensity over time at room temperature. We conclude that the use of random and independent sampling of peptide frequency and/or intensity values may serve as a means to quantify the cleavage of C4B. In randomly and independently sampled experiments the intensity values at time zero for the C4B-peptide NGFKSHALQLNNR were apparently below the noise cut off used in this statistical experiment but the peptide was detectable by $1 \mathrm{hr}$ at room temperature and showed a marked increase in both frequency and intensity by $8 \mathrm{hrs}$. The large differences in the 
peptides frequency and intensity over time indicated that the cleavage of the peptide NGFKSHALQLNNR from complement C4B may serve as a measure of the degradation of the sample due to endopeptidase activity at room temperature after sample collection (J. Marshall et al., 2004; John Marshall et al., 2003). Random and independent sampling using unbiased LCESI-MS/MS is a costly and time consuming approach that is necessary to make unbiased discoveries of candidate markers but is not an efficient means to assay an individual protein.

\section{Automatic-targeted analysis of Complement 4B NGFKSHALQLNNR}

Automatic targeted analysis showed that the C4B-peptide (NGFKSHALQLNNR) was readily detected in the ice control and degraded plasma samples and increased by about $\geq 10$ fold by $1 \mathrm{hr}$ at room temperature. After $\log _{10}$ transformation, the C4B-peptide intensity was Gaussian and could be used to provide relative quantification and statistical analysis using ANOVA. The automatic-targeted quantification of the precursor peptide ion of the C4B-peptide is a promising step towards creating a quantitative assay for the quality control of EDTA plasma samples. In this approach a low resolution, yet robust and sensitive, ion trap is set to monitor the precursor window where automatic computations based on the fit of the MS/MS spectra to the target peptide sequence resolves the intended precursor from any ions with similar $\mathrm{m} / \mathrm{z}$ values.

\section{Complementary sampling strategy}

Random and independent sampling from a totally random experimental design is the statistical gold standard for avoiding false discovery. Pooling representative samples, or taking ratios of isotopic or isobaric tags may reduce or eliminate measured biological and sampling error leading to false discovery. Moreover, making a ratio of isotopic or isobaric 
peptides, such as ICAT ratios, lacks independence and tends to multiply the error in the two samples (denominator error $\mathbf{x}$ numerator error) leading to reduced statistical power. Hence the random and independent sampling of separate biological samples is an appropriate strategy to discover real differences between populations that provides a realistic assessment of population variance. In a complementary approach, the automatic targeted method captured biological variation but avoided sampling error by a high frequency sampling of the targeted analyte that shows that C4B intensity levels increased within $1 \mathrm{hr}$ at room temperature and remained elevated and increased for at least 72 hours that showed little sampling error. Only the intensity and frequency values of precursors within $\pm 3 \mathrm{~m} / \mathrm{z}$ that yielded MS/MS spectra automatically correlated to the target peptide by the SEQUEST algorithm were accepted in SQL. Thus the fit of the MS/MS spectra ensured the validity of the precursors accepted into the dataset for automated statistical analysis in $R$. The combination of random and independent sampling, together with automatic targeted confirmation, as demonstrated for the first time here, is a sensitive and practical approach to biomarker discovery in human plasma that avoids false discovery and yet automatically provides confirmatory measurements with low technical error.

Room temperature versus ice, frozen or freeze-dried samples Storing samples on ice was an effective means to prevent proteolytic degradation for up to three days. The use of C4B-peptide counting and precursor intensity versus frequency analysis clearly indicated that freeze drying and storage at room temperature, or freeze drying and storage at -20 ${ }^{\circ} \mathrm{C}$ were just as effective in preventing sample degradation as freezing at $-80^{\circ} \mathrm{C}$ or liquid nitrogen for up to one year. However degradation of complement C4B commences very rapidly upon 
thawing and does not reflect the stability of most plasma proteins and so unless the samples are collected on ice, freeze dried and reconstituted on ice \pm protease inhibitors some degradation of C4B is likely unavoidable.

\section{Conclusion}

Three different methods, random and independent sampling, automatic-targeted analysis, and Western blot all agreed that the cleavage of $\mathrm{C} 4 \mathrm{~B}$ gives a good indication of the sample incubation at RT. The time that a clinical plasma sample remains at room temperature was a key factor in the cleavage of C4B. The simplest explanation of the observations here is that a tryptic protease activity in human plasma acts ex vivo at room temperature to cleave a proportion of the total pool of complement $\mathrm{C} 4 \mathrm{~B}$ and this processing may be directly detectable by sensitive LCESI-MS/MS or immunological methods. We conclude that the complement proteins, that are part of a proteolytic cascade, are especially sensitive to ex vivo degradation of EDTA plasma during sample warming. The peptide NGFKSHALQLNNR from complement 4B showed a rapid increase in the frequency and intensity of detection after warming to room temperature and increased with hours of incubation and then remained elevated over the subsequent days. Thus, after all blood cells are removed from plasma on ice, $\mathrm{C} 4 \mathrm{~B}$ peptide levels remained low, but yet subsequently increased with time at room temperature entirely in the absence of white blood cells. All of the results are consistent with the expression of endogenous complement fragments that represents a balance of endoproteinase activity versus exopeptidase activity. The C4B peptide assays indicates that the highly soluble proteins in human plasma may be

preserved by freeze drying and entirely reconstituted upon the addition of water and so freeze 
drying is an attractive option for sample preservation that would also permit robust and reliable shipment of samples at low cost. 


\section{EDITORIAL POLICY}

\section{Ethics approval and consent to participate}

Ethical approval and informed consent was obtained through the Comité National d'Ethique de Recherche (CNER) Protocol \#201107 “Biospecimen Research” at the Centre Hospitalier de Luxembourg.

\section{Consent for publication}

No material from any other publication was used in this publication

\section{Availability of data and material}

The raw data is provided in the supplemental materials or companion publications

\section{Competing interests}

The authors declare no conflict of interest.

\section{Funding}

This research was paid for by the Fonds National de la Recherche, Mobility of Researcher award through Luxembourg Institute of Health (formerly CRP Sante) and the Integrated Biobank of Luxembourg to JGM. 


\section{Authors' contributions}

Jaimie Dufresne calibrated, tuned and quality controlled the instruments and performed LC-ESIMS/MS analysis.

Trung Hoang performed LC-ESI-MS/MS analysis

Angelique Florentinus-Mefailoski performed the sample aliquoting and performed LC-ESIMS/MS analysis and edited the manuscript.

Juliet Ajambo performed LC-ESI-MS/MS analysis and helped to edit the manuscript.

Ammara Ferwa performed protein assays and SDS-PAGE

Peter Bowden collected the data into an SQL database for statistical analysis in R.

John Marshall planned the experiment, and performed the clinical plasma collection and sample treatments and storage experiments

\section{Acknowledgment:}

We gratefully acknowledge the review and critique of this manuscript by Dr. Fay Betsou and Dr. R.A. Phillips of IBBL. 


\section{REFERENCES}

Albrethsen, J., Bogebo, R., Olsen, J., Raskov, H., \& Gammeltoft, S. (2006). Preanalytical and analytical variation of surface-enhanced laser desorption-ionization time-of-flight mass spectrometry of human serum. Clin Chem Lab Med, 44(10), 1243-1252.

Allan, D., Auger, J., \& Crumpton, M. J. (1972). Purification of concanavalin A receptor from pig lymphocyte plasma membrane. Biochem J, 126(3), 6P.

An, S., Shen, C., Liu, X., Chen, L., Xu, X., Rong, M., . . Lai, R. (2013). Alpha-actinin is a new type of house dust mite allergen. PLOS ONE, 8(12), e81377. doi:10.1371/journal.pone.0081377

Baggerly, K. A., Morris, J. S., Wang, J., Gold, D., Xiao, L. C., \& Coombes, K. R. (2003). A comprehensive approach to the analysis of matrix-assisted laser desorption/ionization-time of flight proteomics spectra from serum samples. Proteomics, 3(9), 1667-1672.

Banks, R. E. (2008). Preanalytical influences in clinical proteomic studies: raising awareness of fundamental issues in sample banking. Clin Chem, 54(1), 6-7.

Bayer, E. A., \& Wilchek, M. (1980). The use of the avidin-biotin complex as a tool in molecular biology. Methods Biochem Anal, 26, 1-45.

Beekman, J. M., van der Linden, J. A., van de Winkel, J. G., \& Leusen, J. H. (2008). Fc $\gamma R$ R (CD64) resides constitutively in lipid rafts. Immunol Lett, 116(2), 149-155.

Ben-Chetrit, E., Chan, E., Sullivan, K. F., \& Tan, E. M. (1988). A 52-kD protein is a novel component of the SS-A/Ro antigenic particle. Journal of Experimental Medicine, 167(5), 1560-1571.

Benjamini., Y., \& Hochberg, Y. (1995). Controlling false discovery rate: A practical approach to multiple testing. Journal of the Royal Statistical Society, 57(1), 289-300.

Benovic, J. L., Shorr, R. G., Caron, M. G., \& Lefkowitz, R. J. (1984). Mammalian. beta. 2-adrenergic receptor: purification and characterization. Biochemistry, 23(20), 4510-4518.

Bereman, M. S., Johnson, R., Bollinger, J., Boss, Y., Shulman, N., MacLean, B., . . MacCoss, M. J. (2014). Implementation of statistical process control for proteomic experiments via LC MS/MS. $J$ Am Soc Mass Spectrom, 25(4), 581-587. doi:10.1007/s13361-013-0824-5

Boehm, A. M., Putz, S., Altenhofer, D., Sickmann, A., \& Falk, M. (2007). Precise protein quantification based on peptide quantification using iTRAQ. BMC Bioinformatics, 8, 214. doi:10.1186/14712105-8-214

Bowden, P., Beavis, R., \& Marshall, J. (2009). Tandem mass spectrometry of human tryptic blood peptides calculated by a statistical algorithm and captured by a relational database with exploration by a general statistical analysis system. Journal of Proteomics, 73, 103-111. doi:10.1016/j.jprot.2009.08.004

Bowden, P., Pendrak, V., Zhu, P., \& Marshall, J. G. (2010). Meta sequence analysis of human blood peptides and their parent proteins. Journal of Proteomics, 73, 1163-1175. doi:10.1016/j.jprot.2010.02.007

Bowden, P., Thavarajah, T., Zhu, P., McDonell, M., Thiele, H., \& Marshall, J. G. (2012). Quantitative statistical analysis of standard and human blood proteins from liquid chromatography, electrospray ionization, and tandem mass spectrometry. Journal of Proteome Research, 11, 20322047. doi:10.1021/pr2000013

Bruhns, P. (2012). Properties of mouse and human IgG receptors and their contribution to disease models. Blood, 119(24), 5640-5649.

Callesen, A. K., Vach, W., Jorgensen, P. E., Cold, S., Mogensen, O., Kruse, T. A., . . Madsen, J. S. (2008). Reproducibility of mass spectrometry based protein profiles for diagnosis of breast cancer across clinical studies: a systematic review. J Proteome Res, 7(4), 1395-1402.

Cargile, B. J., Bundy, J. L., \& Stephenson, J. L., Jr. (2004). Potential for false positive identifications from large databases through tandem mass spectrometry. J Proteome Res, 3(5), 1082-1085. 
Chin, S. T., Ignatius, J., Suraiya, S., Tye, G. J., Sarmiento, M. E., Acosta, A., . . Lim, T. S. (2015). Comparative study of IgA VH 3 gene usage in healthy TST(-) and TST(+) population exposed to tuberculosis: deep sequencing analysis. Immunology, 144(2), 302-311. doi:10.1111/imm.12372

Cooper, B. (2012). The problem with peptide presumption and the downfall of target-decoy false discovery rates. Anal Chem, 84(22), 9663-9667. doi:10.1021/ac303051s

Corbett-Nelson, E. F., Mason, D., Marshall, J. G., Collette, Y., \& Grinstein, S. (2006). Signalingdependent immobilization of acylated proteins in the inner monolayer of the plasma membrane. $J$ Cell Biol, 174(2), 255-265.

Cottrell, J. S., \& London, U. (1999). Probability-based protein identification by searching sequence databases using mass spectrometry data. Electrophoresis, 20(18), 3551-3567.

Craig, R., \& Beavis, R. C. (2003). A method for reducing the time required to match protein sequences with tandem mass spectra. Rapid Commun Mass Spectrom, 17(20), 2310-2316.

Craig, R., \& Beavis, R. C. (2004). TANDEM: matching proteins with tandem mass spectra. Bioinformatics, 20(9), 1466-1467.

Craig, R., \& Beavis, R. C. (2004). TANDEM: matching proteins with tandem mass spectra. Bioinformatics, 20(9), 1466-1467.

Crowley, M. T., Costello, P. S., Fitzer-Attas, C. J., Turner, M., Meng, F., Lowell, C., . . DeFranco, A. L. (1997). A critical role for Syk in signal transduction and phagocytosis mediated by Fcgamma receptors on macrophages. J Exp Med, 186(7), 1027-1039.

Cuatrecasas, P. (1972). Affinity chromatography and purification of the insulin receptor of liver cell membranes. Proc Natl Acad Sci U S A, 69(5), 1277-1281.

Cuatrecasas, P., \& Parikh, I. (1974). Affinity chromatography of insulin receptors. Methods Enzymol, 34, 653-670.

Cuatrecasas, P., Wilchek, M., \& Anfinsen, C. B. (1968). Selective enzyme purification by affinity chromatography. Proc Natl Acad Sci U S A, 61(2), 636-643.

de Noo, M. E., Tollenaar, R. A., Ozalp, A., Kuppen, P. J., Bladergroen, M. R., Eilers, P. H., \& Deelder, A. M. (2005). Reliability of human serum protein profiles generated with C8 magnetic beads assisted MALDI-TOF mass spectrometry. Anal Chem, 77(22), 7232-7241.

Denecker, G., Ovaere, P., Vandenabeele, P., \& Declercq, W. (2008). Caspase-14 reveals its secrets. J Cell Biol, 180(3), 451-458. doi:10.1083/jcb.200709098

Desjardins, M., \& Griffiths, G. (2003). Phagocytosis: latex leads the way. Current opinion in cell biology, 15(4), 498-503.

Diamandis, E. P., \& Christopoulos, T. K. (1991). The biotin-(strept) avidin system: principles and applications in biotechnology. Clinical chemistry, 37(5), 625-636.

Dionne, R., Forest, J. C., Moutquin, J. M., De Grandpre, P., \& Masse, J. (1994). Electrophoretic method for separating small peptides in serum without extraction of macromolecules: application to the detection of preeclampsia. Clin Biochem, 27(2), 99-103.

Dobo, J., Major, B., Kekesi, K. A., Szabo, I., Megyeri, M., Hajela, K., . . Gal, P. (2011). Cleavage of kininogen and subsequent bradykinin release by the complement component: mannose-binding lectin-associated serine protease (MASP)-1. PLOS ONE, 6(5), e20036. doi:10.1371/journal.pone.0020036

Doi, E., Shibata, D., \& Matoba, T. (1981). Modified colorimetric ninhydrin methods for peptidase assay. Anal Biochem, 118(1), 173-184.

Eckel-Passow, J. E., Oberg, A. L., Therneau, T. M., \& Bergen, H. R., 3rd. (2009). An insight into highresolution mass-spectrometry data. Biostatistics, 10(3), 481-500.

Elschenbroich, S., Kim, Y., Medin, J. A., \& Kislinger, T. (2010). Isolation of cell surface proteins for mass spectrometry-based proteomics. Expert review of proteomics, 7(1), 141-154.

Eng, J. K., McCormack, A. L., \& Yates, J. R. (1994). An approach to correlate tandem mass spectral data of peptides with amino acid sequences in a protein database. J Am Soc Mass Spectrom, 5(11), 976-989. 
Fagerberg, L., Jonasson, K., von Heijne, G., Uhlén, M., \& Berglund, L. (2010). Prediction of the human membrane proteome. Proteomics, 10(6), 1141-1149.

Fang, M., Webster, T. F., \& Stapleton, H. M. (2015). Effect-Directed Analysis of Human Peroxisome Proliferator-Activated Nuclear Receptors (PPARgamma1) Ligands in Indoor Dust. Environ Sci Technol, 49(16), 10065-10073. doi:10.1021/acs.est.5b01524

Flannagan, R. S., Jaumouillé, V., \& Grinstein, S. (2012). The cell biology of phagocytosis. Annual Review of Pathology: Mechanisms of Disease, 7, 61-98.

Florentinus, A. K., Bowden, P., Barbisan, V., \& Marshall, J. (2012). Capture and qualitative analysis of the activated Fc receptor complex from live cells. Current protocols in protein science / editorial board, John E. Coligan ... [et al.], Chapter 19, Unit 19.22. doi:10.1002/0471140864.ps1922s67

Florentinus, A. K., Bowden, P., Barbisan, V., \& Marshall, J. (2012). Capture and qualitative analysis of the activated Fc receptor complex from live cells. Curr Protoc Protein Sci, Chapter 19, Unit 19 22. doi:10.1002/0471140864.ps1922s67

Florentinus, A. K., Bowden, P., Sardana, G., Diamandis, E. P., \& Marshall, J. G. (2012). Identification and quantification of peptides and proteins secreted from prostate epithelial cells by unbiased liquid chromatography tandem mass spectrometry using goodness of fit and analysis of variance. Journal of Proteomics, 75, 1303-1317. doi:10.1016/j.jprot.2011.11.002

Florentinus, A. K., Jankowski, A., Petrenko, V., Bowden, P., \& Marshall, J. G. (2011). The Fc receptorcytoskeleton complex from human neutrophils. Journal of Proteomics, 75, 450-468. doi:10.1016/j.jprot.2011.08.011

Fox, K., Castanha, E., Fox, A., Feigley, C., \& Salzberg, D. (2008). Human K10 epithelial keratin is the most abundant protein in airborne dust of both occupied and unoccupied school rooms. J Environ Monit, 10(1), 55-59. doi:10.1039/b714802j

Frei, A. P., Jeon, O. Y., Kilcher, S., Moest, H., Henning, L. M., Jost, C., . . Wollscheid, B. (2012). Direct identification of ligand-receptor interactions on living cells and tissues. Nat Biotechnol, 30(10), 997-1001. doi:10.1038/nbt.2354

Frei, A. P., Moest, H., Novy, K., \& Wollscheid, B. (2013). Ligand-based receptor identification on living cells and tissues using TRICEPS. Nat Protoc, 8(7), 1321-1336. doi:10.1038/nprot.2013.072

Fung, K. W., Wright, D. W., Gor, J., Swann, M. J., \& Perkins, S. J. (2016). Domain structure of human complement $\mathrm{C} 4 \mathrm{~b}$ extends with increasing $\mathrm{NaCl}$ concentration: implications for its regulatory mechanism. Biochem J, 473(23), 4473-4491. doi:10.1042/BCJ20160744

Gagnon, E., Duclos, S., Rondeau, C., Chevet, E., Cameron, P. H., Steele-Mortimer, O., . . Desjardins, M. (2002). Endoplasmic reticulum-mediated phagocytosis is a mechanism of entry into macrophages. Cell, 110(1), 119-131.

Gast, M. C., van Gils, C. H., Wessels, L. F., Harris, N., Bonfrer, J. M., Rutgers, E. J., . . Beijnen, J. H. (2009). Influence of sample storage duration on serum protein profiles assessed by surfaceenhanced laser desorption/ionisation time-of-flight mass spectrometry (SELDI-TOF MS). Clin Chem Lab Med, 47(6), 694-705.

Geer, L. Y., Markey, S. P., Kowalak, J. A., Wagner, L., Xu, M., Maynard, D. M., . . Bryant, S. H. (2004). Open mass spectrometry search algorithm. J Proteome Res, 3(5), 958-964.

Geer, L. Y., Markey, S. P., Kowalak, J. A., Wagner, L., Xu, M., Maynard, D. M., . . Bryant, S. H. (2004). Open mass spectrometry search algorithm. J Proteome Res, 3(5), 958-964.

Gharahdaghi, F., Kirchner, M., Fernandez, J., \& Mische, S. M. (1996). Peptide-mass profiles of polyvinylidene difluoride-bound proteins by matrix-assisted laser desorption/ionization time-offlight mass spectrometry in the presence of nonionic detergents. Anal Biochem, 233(1), 94-99.

Ghosh, S., Gepstein, S., Heikkila, J. J., \& Dumbroff, E. B. (1988). Use of a scanning densitometer or an ELISA plate reader for measurement of nanogram amounts of protein in crude extracts from biological tissues. Anal Biochem, 169(2), 227-233.

Govorukhina, N. I., de Vries, M., Reijmers, T. H., Horvatovich, P., van der Zee, A. G., \& Bischoff, R. (2008). Influence of clotting time on the protein composition of serum samples based on LC-MS data. J Chromatogr B Analyt Technol Biomed Life Sci. 
Haan, C., \& Behrmann, I. (2007). A cost effective non-commercial ECL-solution for Western blot detections yielding strong signals and low background. Journal of immunological methods, 318, 11-19. doi:10.1016/j.jim.2006.07.027

Helbig, A. O., Heck, A. J., \& Slijper, M. (2010). Exploring the membrane proteome-challenges and analytical strategies. J Proteomics, 73(5), 868-878.

Howard, J. C., Florentinus-Mefailoski, A., Bowden, P., Trimble, W., Grinstein, S., \& Marshall, J. G. (2016). OxLDL receptor chromatography from live human U937 cells identifies SYK (L) that regulates phagocytosis of oxLDL. Anal Biochem, 513, 7-20.

Howard, J. C., Florentinus-Mefailoski, A., Bowden, P., Trimble, W., Grinstein, S., \& Marshall, J. G. (2016). OxLDL receptor chromatography from live human U937 cells identifies SYK(L) that regulates phagocytosis of oxLDL. Anal Biochem. doi:10.1016/j.ab.2016.07.021

Humphries, J. D., Byron, A., Bass, M. D., Craig, S. E., Pinney, J. W., Knight, D., \& Humphries, M. J. (2009). Proteomic Analysis of Integrin-Associated Complexes Identifies RCC2 as a Dual Regulator of Rac1 and Arf6. Sci Signal, 2(87), ra51-ra51. doi:10.1126/scisignal.2000396

Indik, Z. K., Park, J. G., Hunter, S., \& Schreiber, A. D. (1995). The molecular dissection of Fc gamma receptor mediated phagocytosis. Blood, 86(12), 4389-4399.

Inoue, M., Kinne, R., Tran, T., Biempica, L., \& Arias, I. (1983). Rat liver canalicular membrane vesicles. Isolation and topological characterization. Journal of Biological Chemistry, 258(8), 5183-5188.

Jankowski, A., Zhu, P., \& Marshall, J. G. (2008). Capture of an activated receptor complex from the surface of live cells by affinity receptor chromatography. Analytical Biochemistry, 380, 235-248. doi:10.1016/j.ab.2008.05.047

Jankowski, A., Zhu, P., \& Marshall, J. G. (2008). Capture of an activated receptor complex from the surface of live cells by affinity receptor chromatography. Anal Biochem, 380(2), 235-248. doi:10.1016/j.ab.2008.05.047

Jankowski, A., Zhu, P., \& Marshall, J. G. (2008). Capture of an activated receptor complex from the surface of live cells by affinity receptor chromatography. Anal Biochem, 380(2), 235-248. doi:10.1016/j.ab.2008.05.047

Jin, Y., \& Manabe, T. (2009). Differences in protein distribution between human plasma preparations, EDTA-plasma and heparin-plasma, analyzed by non-denaturing micro-2-DE and MALDI-MS PMF. Electrophoresis, 30(6), 931-938.

Kaisar, M., van Dullemen, L. F., Thezenas, M. L., Zeeshan Akhtar, M., Huang, H., Rendel, S., . . . Kessler, B. M. (2016). Plasma degradome affected by variable storage of human blood. Clin Proteomics, 13, 26. doi:10.1186/s12014-016-9126-9

Karsan, A., Eigl, B. J., Flibotte, S., Gelmon, K., Switzer, P., Hassell, P., . . Veenstra, T. (2005). Analytical and preanalytical biases in serum proteomic pattern analysis for breast cancer diagnosis. Clin Chem, 51(8), 1525-1528.

Keeble, A. H., Khan, Z., Forster, A., \& James, L. C. (2008). TRIM21 is an IgG receptor that is structurally, thermodynamically, and kinetically conserved. Proceedings of the national academy of sciences of the United States of America, 105(16), 6045-6050. doi:10.1073/pnas.0800159105

Keller, A., Nesvizhskii, A. I., Kolker, E., \& Aebersold, R. (2002). Empirical statistical model to estimate the accuracy of peptide identifications made by MS/MS and database search. Anal Chem, 74(20), 5383-5392.

Keller, A., Nesvizhskii, A. I., Kolker, E., \& Aebersold, R. (2002). Empirical statistical model to estimate the accuracy of peptide identifications made by MS/MS and database search. Anal Chem, 74(20), 5383-5392.

Keller, A., Purvine, S., Nesvizhskii, A. I., Stolyar, S., Goodlett, D. R., \& Kolker, E. (2002). Experimental protein mixture for validating tandem mass spectral analysis. Omics: A Journal of Integrative Biology, 6, 207-212. doi:10.1089/153623102760092805

Kielley, W. W., \& Bradley, L. B. (1956). The relationship between sulfhydryl groups and the activation of myosin adenosinetriphosphatase. J Biol Chem, 218(2), 653-659. 
Kikuchi, H., Goto, Y., \& Hamaguchi, K. (1986). Reduction of the buried intrachain disulfide bond of the constant fragment of the immunoglobulin light chain: global unfolding under physiological conditions. Biochemistry, 25(8), 2009-2013.

Kim, S., Gupta, N., \& Pevzner, P. A. (2008). Spectral probabilities and generating functions of tandem mass spectra: a strike against decoy databases. J Proteome Res, 7(8), 3354-3363.

Klett, R. P., Fulpius, B. W., Cooper, D., Smith, M., Reich, E., \& Possani, L. D. (1973). The acetylcholine receptor I. Purification and characterization of a macromolecule isolated from Electrophorus electricus. Journal of Biological Chemistry, 248(19), 6841-6853.

Koomen, J. M., Li, D., Xiao, L. C., Liu, T. C., Coombes, K. R., Abbruzzese, J., \& Kobayashi, R. (2005). Direct tandem mass spectrometry reveals limitations in protein profiling experiments for plasma biomarker discovery. J Proteome Res, 4(3), 972-981.

Krogh, A., Larsson, B., Von Heijne, G., \& Sonnhammer, E. L. (2001). Predicting transmembrane protein topology with a hidden Markov model: application to complete genomes. Journal of molecular biology, 305(3), 567-580.

Kulik, W., van Lenthe, H., Stet, F. S., Houtkooper, R. H., Kemp, H., Stone, J. E., . . Vaz, F. M. (2008). Bloodspot assay using HPLC-tandem mass spectrometry for detection of Barth syndrome. Clin Chem, 54(2), 371-378.

Lagerstrom, M. C., \& Schioth, H. B. (2008). Structural diversity of G protein-coupled receptors and significance for drug discovery. Nat Rev Drug Discov, 7(4), 339-357. doi:10.1038/nrd2518

Langley, J. N. (1906). Croonian Lecture, 1906: On Nerve Endings and on Special Excitable Substances in Cells. Proceedings of the Royal Society of London. Series B, Containing Papers of a Biological Character, 170-194.

Lee, W. L., Cosio, G., Ireton, K., \& Grinstein, S. (2007). Role of CrkII in Fc $\gamma$ receptor-mediated phagocytosis. Journal of Biological Chemistry, 282(15), 11135-11143.

Lefkowitz, R. J. (1973). Isolated beta-adrenergic binding sites: a potential assay vehicle for catecholamines. Pharmacol Rev, 25(2), 259-268.

Lefkowitz, R. J. (2004). Historical review: a brief history and personal retrospective of seventransmembrane receptors. Trends Pharmacol Sci, 25(8), 413-422. doi:10.1016/j.tips.2004.06.006

Lerm, M., Brodin, V. P., Ruishalme, I., Stendahl, O., \& Sarndahl, E. (2007). Inactivation of Cdc42 is necessary for depolymerization of phagosomal F-actin and subsequent phagosomal maturation. $J$ Immunol, 178(11), 7357-7365.

LeWinter, M. M., \& Granzier, H. L. (2013). Titin is a major human disease gene. Circulation, 127(8), 938-944. doi:10.1161/CIRCULATIONAHA.112.139717

Li, J., Orlandi, R., White, C. N., Rosenzweig, J., Zhao, J., Seregni, E., . . Chan, D. W. (2005). Independent validation of candidate breast cancer serum biomarkers identified by mass spectrometry. Clin Chem, 51(12), 2229-2235.

Li, Y., Ozment, T., Wright, G. L., \& Peterson, J. M. (2016). Identification of Putative Receptors for the Novel Adipokine CTRP3 Using Ligand-Receptor Capture Technology. PloS one, 11(10), e0164593. doi:10.1371/journal.pone.0164593

Lodish, H., Berk, A., Zipursky, S. L., Matsudaira, P., Baltimore, D., \& Darnell, J. (2000). Molecular cell biology 4th edition. National Center for Biotechnology InformationÕs Bookshelf.

Lowey, S., \& Risby, D. (1971). Light chains from fast and slow muscle myosins. Nature, 234(5324), 8185.

MacDonald, R. G., Pfeffer, S. R., Coussens, L., Tepper, M. A., Brocklebank, C. M., Mole, J. E., .. . Ullrich, A. (1988). A single receptor binds both insulin-like growth factor II and mannose-6phosphate. science, 239(4844), 1134-1137.

Makino, S., Reynolds, J. A., \& Tanford, C. (1973). The binding of deoxycholate and Triton X-100 to proteins. Journal of Biological Chemistry, 248(14), 4926-4932.

Marshall, J., Bowden, P., Schmit, J. C., \& Betsou, F. (2014). Creation of a federated database of blood proteins: a powerful new tool for finding and characterizing biomarkers in serum. Clin Proteomics, 11(1), 3. doi:10.1186/1559-0275-11-3 
Marshall, J., Jankowski, A., Furesz, S., Kireeva, I., Barker, L., Dombrovsky, M., . . Jackowski, G. (2004). Human serum proteins preseparated by electrophoresis or chromatography followed by tandem mass spectrometry. J Proteome Res, 3(3), 364-382.

Marshall, J., Kupchak, P., Zhu, W., Yantha, J., Vrees, T., Furesz, S., . . Jackowski, G. (2003). Processing of serum proteins underlies the mass spectral fingerprinting of myocardial infarction. Journal of Proteome Research, 2, 361-372.

Marshall, J. G., Booth, J. W., Stambolic, V., Mak, T., Balla, T., Schreiber, A. D., . . Grinstein, S. (2001). Restricted accumulation of phosphatidylinositol 3-kinase products in a plasmalemmal subdomain during Fc gamma receptor-mediated phagocytosis. The Journal of Cell Biology, 153, 1369-1380.

Marshall, J. G., Booth, J. W., Stambolic, V., Mak, T., Balla, T., Schreiber, A. D., . . Grinstein, S. (2001). Restricted accumulation of phosphatidylinositol 3-kinase products in a plasmalemmal subdomain during Fc $\gamma$ receptor-mediated phagocytosis. The Journal of cell biology, 153(7), 1369-1380.

Melo, S. A., Luecke, L. B., Kahlert, C., Fernandez, A. F., Gammon, S. T., Kaye, J., . . Kalluri, R. (2015). Glypican-1 identifies cancer exosomes and detects early pancreatic cancer. Nature, 523(7559), 177-182. doi:10.1038/nature14581

Mercier, C., Truntzer, C., Pecqueur, D., Gimeno, J. P., Belz, G., \& Roy, P. (2009). Mixed-model of ANOVA for measurement reproducibility in proteomics. J Proteomics, 72(6), 974-981.

Metschnikoff, E. (1884). Ueber die beziehung der phagocyten zu milzbrandbacillen. Virchows Archiv, 97(3), 502-526.

Michael, I. P., Sotiropoulou, G., Pampalakis, G., Magklara, A., Ghosh, M., Wasney, G., \& Diamandis, E. P. (2005). Biochemical and enzymatic characterization of human kallikrein 5 (hK5), a novel serine protease potentially involved in cancer progression. J Biol Chem, 280(15), 14628-14635. doi:10.1074/jbc.M408132200

Milstein, C. (1966). The disulphide bridges of immunoglobulin kappa-chains. Biochem. J, 101, 338-351.

Moutsopoulos, H. M., Skopouli, F. N., Sarras, A., Tsampoulas, C., Mavridis, A., Constantopoulos, S., \& Maddison, P. (1985). Anti-Ro (SSA) positive rheumatoid arthritis (RA): a clinicoserological group of patients with high incidence of D-penicillamine side effects. Ann Rheum Dis, 44(4), 215-219.

Odin, J. A., Edberg, J. C., Painter, C. J., Kimberly, R. P., \& Unkeless, J. C. (1991). Regulation of phagocytosis and [Ca2+] i flux by distinct regions of an Fc receptor. science, 254(5039), 17851788.

Overington, J. P., Al-Lazikani, B., \& Hopkins, A. L. (2006). How many drug targets are there? Nat Rev Drug Discov, 5(12), 993-996. doi:10.1038/nrd2199

Parikh, I., Sica, V., Nola, E., Puca, G. A., \& Cuatrecasas, P. (1974). Affinity chromatography of estrogen receptors. Methods Enzymol, 34, 670-688.

Park SK, M. K. (1988). Random Number Generators: Good Ones Are Hard To Find. Communications of the ACM, 31(10), 1191-1201.

Perkins, D. N., Pappin, D. J., Creasy, D. M., \& Cottrell, J. S. (1999). Probability-based protein identification by searching sequence databases using mass spectrometry data. Electrophoresis, 20(18), 3551-3567.

Peterson, J. M., Seldin, M. M., Wei, Z., Aja, S., \& Wong, G. W. (2013). CTRP3 attenuates diet-induced hepatic steatosis by regulating triglyceride metabolism. Am J Physiol Gastrointest Liver Physiol, 305(3), G214-224. doi:10.1152/ajpgi.00102.2013

Pieper, U., Schlessinger, A., Kloppmann, E., Chang, G. A., Chou, J. J., Dumont, M. E., . . Sali, A. (2013). Coordinating the impact of structural genomics on the human $\alpha$-helical transmembrane proteome. Nature structural \& molecular biology, 20(2), 135-138. doi:10.1038/nsmb.2508

Pilny, R., Bouchal, P., Borilova, S., Ceskova, P., Zaloudik, J., Vyzula, R., . . . Valik, D. (2006). Surfaceenhanced laser desorption ionization/time-of-flight mass spectrometry reveals significant artifacts in serum obtained from clot activator-containing collection devices. Clin Chem, 52(11), 21152116. 
Purves, D., Augustine, G., \& Fitzpatrick, D. (2001). et al., editors. Neuroscience. Sunderland (MA). In: Sinauer Associates.

Putnam, F. (1975). The plasma Proteins: Structure Function, and Genetic Control. Academic Press, New York, second ed.

Qin, S., Zhou, Y., Gray, L., Kusebauch, U., McEvoy, L., Antoine, D. J., . . Hood, L. (2016). Identification of Blood Protein Biomarkers of Acute Liver Injury by Targeted Quantitative Proteomics in Acetaminophen and Carbon tetrachloride treated Mouse Models and Acetaminophen Overdose Patients. J Proteome Res. doi:10.1021/acs.jproteome.6b00547

Qu, Z. X., Odin, J., Glass, J. D., \& Unkeless, J. C. (1988). Expression and characterization of a truncated murine Fc gamma receptor. J Exp Med, 167(3), 1195-1210.

Rabinovitch, M. (1995). Professional and non-professional phagocytes: an introduction. Trends Cell Biol, $5(3), 85-87$.

Rai, A. J., Gelfand, C. A., Haywood, B. C., Warunek, D. J., Yi, J., Schuchard, M. D., . . Chan, D. W. (2005). HUPO Plasma Proteome Project specimen collection and handling: towards the standardization of parameters for plasma proteome samples. Proteomics, 5(13), 3262-3277.

Ravetch, J. V., \& Kinet, J.-P. (1991). Fc receptors. Annual review of immunology, 9(1), 457-492.

Sardesai, V. M., \& Provido, H. S. (1965). A Fluorometric Method for Determining the Tame Esterase (Tryptic) Activity of Plasma. J Lab Clin Med, 65, 1023-1029.

Schagger, H., \& von Jagow, G. (1987). Tricine-sodium dodecyl sulfate-polyacrylamide gel electrophoresis for the separation of proteins in the range from 1 to $100 \mathrm{kDa}$. Anal Biochem, 166(2), 368-379.

Schwartz, J. C., Senko, M. W., \& Syka, J. E. (2002). A two-dimensional quadrupole ion trap mass spectrometer. J Am Soc Mass Spectrom, 13(6), 659-669.

Semmes, O. J., Feng, Z., Adam, B. L., Banez, L. L., Bigbee, W. L., Campos, D., . . Zhu, L. (2005). Evaluation of serum protein profiling by surface-enhanced laser desorption/ionization time-offlight mass spectrometry for the detection of prostate cancer: I. Assessment of platform reproducibility. Clin Chem, 51(1), 102-112.

Shi, T., Sun, X., Gao, Y., Fillmore, T. L., Schepmoes, A. A., Zhao, R., . . Qian, W. J. (2013). Targeted quantification of low $\mathrm{ng} / \mathrm{mL}$ level proteins in human serum without immunoaffinity depletion. $J$ Proteome Res, 12(7), 3353-3361.

Shiu, R. P., \& Friesen, H. G. (1974). Solubilization and purification of a prolactin receptor from the rabbit mammary gland. Journal of Biological Chemistry, 249(24), 7902-7911.

Sica, V., Nola, E., Parikh, I., Puca, G. A., \& Cuatrecasas, P. (1973). Purification of oestradiol receptors by affinity chromatography. Nat New Biol, 244(132), 36-39.

Sica, V., Parikh, I., Nola, E., Puca, G. A., \& Cuatrecasas, P. (1973). Affinity chromatography and the purification of estrogen receptors. J Biol Chem, 248(18), 6543-6558.

Sorace, J. M., \& Zhan, M. (2003). A data review and re-assessment of ovarian cancer serum proteomic profiling. BMC Bioinformatics, 4(1), 24.

Spencer, K. (2003). The influence of different sample collection types on the levels of markers used for Down's syndrome screening as measured by the Kryptor Immunosassay system. Ann Clin Biochem, 40(Pt 2), 166-168.

Stafford, G. C. (1980). Instrumental aspects of positive and negative ion chemical ionization mass spectrometry. Environ Health Perspect, 36, 85-88.

Staros, J. V. (1982). N-hydroxysulfosuccinimide active esters: bis(N-hydroxysulfosuccinimide) esters of two dicarboxylic acids are hydrophilic, membrane-impermeant, protein cross-linkers. Biochemistry, 21(17), 3950-3955.

Stiles, G. L., Caron, M. G., \& Lefkowitz, R. J. (1984). Beta-adrenergic receptors: biochemical mechanisms of physiological regulation. Physiological reviews.

Thakur, S. S., Geiger, T., Chatterjee, B., Bandilla, P., Frohlich, F., Cox, J., \& Mann, M. (2011). Deep and highly sensitive proteome coverage by LC-MS/MS without prefractionation. Mol Cell Proteomics, 10(8), M110 003699. doi:10.1074/mcp.M110.003699 
Tietz. (2001). Tietz Fundamentals of Clinical Chemistry (5th edition ed. Vol. 5th edition): Saunders. Tonegawa, S., Steinberg, C., Dube, S., \& Bernardini, A. (1974). Evidence for somatic generation of antibody diversity. Proc Natl Acad Sci U S A, 71(10), 4027-4031.

Towbin, H., Staehelin, T., \& Gordon, J. (1979). Electrophoretic transfer of proteins from polyacrylamide gels to nitrocellulose sheets: procedure and some applications. Proc Natl Acad Sci U S A, 76(9), 4350-4354.

Tridandapani, S., Lyden, T. W., Smith, J. L., Carter, J. E., Coggeshall, K. M., \& Anderson, C. L. (2000). The adapter protein LAT enhances Fc $\gamma$ receptor-mediated signal transduction in myeloid cells. Journal of Biological Chemistry, 275(27), 20480-20487.

Tucholska, M., Bowden, P., Jacks, K., Zhu, P., Furesz, S., Dumbrovsky, M., \& Marshall, J. (2009). Human serum proteins fractionated by preparative partition chromatography prior to LC-ESIMS/MS. Journal of Proteome Research, 8, 1143-1155. doi:10.1021/pr8005217

Tucholska, M., Florentinus, A., Williams, D., \& Marshall, J. G. (2010). The endogenous peptides of normal human serum extracted from the acetonitrile-insoluble precipitate using modified aqueous buffer with analysis by LC-ESI-Paul ion trap and Qq-TOF. J Proteomics, 73(6), 1254-1269. doi:10.1016/j.jprot.2010.02.022

Tucholska, M., Scozzaro, S., Williams, D., Ackloo, S., Lock, C., Siu, K. W. M., . . Marshall, J. G. (2007). Endogenous peptides from biophysical and biochemical fractionation of serum analyzed by matrix-assisted laser desorption/ionization and electrospray ionization hybrid quadrupole timeof-flight. Analytical Biochemistry, 370, 228-245. doi:10.1016/j.ab.2007.07.029

Tuck, M. K., Chan, D. W., Chia, D., Godwin, A. K., Grizzle, W. E., Krueger, K. E., . . Brenner, D. E. (2009). Standard operating procedures for serum and plasma collection: early detection research network consensus statement standard operating procedure integration working group. $J$ Proteome Res, 8(1), 113-117.

Tzircotis, G., Braga, V. M., \& Caron, E. (2011). RhoG is required for both Fc $\gamma$ R-and CR3-mediated phagocytosis. Journal of cell science, 124(17), 2897-2902.

Uhlen, M., Guss, B., Nilsson, B., Gatenbeck, S., Philipson, L., \& Lindberg, M. (1984). Complete sequence of the staphylococcal gene encoding protein A. A gene evolved through multiple duplications. J Biol Chem, 259(3), 1695-1702.

van den Broek, I., Romijn, F. P., Smit, N. P., van der Laarse, A., Drijfhout, J. W., van der Burgt, Y. E., \& Cobbaert, C. M. (2015). Quantifying protein measurands by peptide measurements: where do errors arise? J Proteome Res, 14(2), 928-942. doi:10.1021/pr5011179

van den Broek, I., Sparidans, R. W., Schellens, J. H., \& Beijnen, J. H. (2008). Liquid chromatography/tandem mass spectrometric method for the quantification of eight proteolytic fragments of ITIH4 with biomarker potential in human plasma and serum. Rapid Commun Mass Spectrom, 22(18), 2915-2928.

van Winden, A. W., Gast, M. C., Beijnen, J. H., Rutgers, E. J., Grobbee, D. E., Peeters, P. H., \& van Gils, C. H. (2009). Validation of previously identified serum biomarkers for breast cancer with SELDITOF MS: a case control study. BMC Med Genomics, 2(1), 4.

Vance, D. T., Dufresne, J., Florentinus-Mefailoski, A., Tucholska, M., Trimble, W., Grinstein, S., \& Marshall, J. G. (2016). A phagocytosis assay for oxidized low-density lipoprotein versus immunoglobulin G-coated microbeads in human U937 macrophages. Anal Biochem, 500, 24-34. doi:10.1016/j.ab.2016.01.007

Vergeynst, L., Van Langenhove, H., \& Demeestere, K. (2015). Balancing the false negative and positive rates in suspect screening with high-resolution Orbitrap mass spectrometry using multivariate statistics. Anal Chem, 87(4), 2170-2177. doi:10.1021/ac503426k

Volanakis, J. E. (1990). Participation of C3 and its ligands in complement activation. Curr Top Microbiol Immunol, 153, 1-21.

Von Jagow, G., Schägger, H., Riccio, P., Klingenberg, M., \& Kolb, H. (1977). b· c1 complex from beef heart: Hydrodynamic properties of the complex prepared by a refined hydroxyapatite 
chromatography in Triton X-100. Biochimica et Biophysica Acta (BBA)-Bioenergetics, 462(3), 549-558.

von Mering, C., Jensen, L. J., Kuhn, M., Chaffron, S., Doerks, T., Kruger, B., . . Bork, P. (2007).

STRING 7--recent developments in the integration and prediction of protein interactions. Nucleic Acids Res, 35(Database issue), D358-362.

von Mering, C., Jensen, L. J., Snel, B., Hooper, S. D., Krupp, M., Foglierini, M., . . Bork, P. (2005). STRING: known and predicted protein-protein associations, integrated and transferred across organisms. Nucleic Acids Res, 33(Database issue), D433-437.

Wallin, E., \& Heijne, G. V. (1998). Genome-wide analysis of integral membrane proteins from eubacterial, archaean, and eukaryotic organisms. Protein Science, 7(4), 1029-1038.

Weisman, H. F., Bartow, T., Leppo, M. K., Marsh, H. C., Jr., Carson, G. R., Concino, M. F., ... Fearon, D. T. (1990). Soluble human complement receptor type 1: in vivo inhibitor of complement suppressing post-ischemic myocardial inflammation and necrosis. Science, 249(4965), 146-151.

West-Nielsen, M., Hogdall, E. V., Marchiori, E., Hogdall, C. K., Schou, C., \& Heegaard, N. H. (2005). Sample handling for mass spectrometric proteomic investigations of human sera. Anal Chem, 77(16), 5114-5123.

West-Norager, M., Kelstrup, C. D., Schou, C., Hogdall, E. V., Hogdall, C. K., \& Heegaard, N. H. (2007). Unravelling in vitro variables of major importance for the outcome of mass spectrometry-based serum proteomics. J Chromatogr B Analyt Technol Biomed Life Sci, 847(1), 30-37.

Wiita, A. P., Hsu, G. W., Lu, C. M., Esensten, J. H., \& Wells, J. A. (2014). Circulating proteolytic signatures of chemotherapy-induced cell death in humans discovered by N-terminal labeling. Proc Natl Acad Sci U S A, 111(21), 7594-7599. doi:10.1073/pnas.1405987111

Williams, D., Ackloo, S., Zhu, P., Bowden, P., Evans, K. R., Addison, C. L., . . Marshall, J. G. (2010). Precipitation and selective extraction of human serum endogenous peptides with analysis by quadrupole time-of-flight mass spectrometry reveals posttranslational modifications and lowabundance peptides. Analytical and bioanalytical chemistry, 396, 1223-1247. doi:10.1007/s00216-009-3345-0

Yang, X., Clifton, J., Huang, F., Kovac, S., Hixson, D. C., \& Josic, D. (2009). Proteomic analysis for process development and control of therapeutic protein separation from human plasma. Electrophoresis, 30(7), 1185-1193.

Yates, J. R., 3rd. (1998). Database searching using mass spectrometry data. Electrophoresis, 19(6), 893900.

Yates, J. R., 3rd, Eng, J. K., McCormack, A. L., \& Schieltz, D. (1995). Method to correlate tandem mass spectra of modified peptides to amino acid sequences in the protein database. Anal Chem, 67(8), 1426-1436.

Yi, J., Kim, C., \& Gelfand, C. A. (2007). Inhibition of intrinsic proteolytic activities moderates preanalytical variability and instability of human plasma. J Proteome Res, 6(5), 1768-1781.

Yi, J., Liu, Z., Craft, D., O'Mullan, P., Ju, G., \& Gelfand, C. A. (2008). Intrinsic peptidase activity causes a sequential multi-step reaction (SMSR) in digestion of human plasma peptides. $J$ Proteome Res, 7(12), 5112-5118.

Zhang, R., Barker, L., Pinchev, D., Marshall, J., Rasamoelisolo, M., Smith, C., . . Jackowski, G. (2004). Mining biomarkers in human sera using proteomic tools. Proteomics, 4(1), 244-256.

Zheng, X., Baker, H., Hancock, W. S., Fawaz, F., McCaman, M., \& Pungor, E., Jr. (2006). Proteomic analysis for the assessment of different lots of fetal bovine serum as a raw material for cell culture. Part IV. Application of proteomics to the manufacture of biological drugs. Biotechnol Prog, 22(5), 1294-1300.

Zhu, P., Bowden, P., Pendrak, V., Thiele, H., Zhang, D., Siu, M., . . Marshall, J. (2007). Comparison of protein expression lists from mass spectrometry of human blood fluids using exact peptide sequences versus BLAST. Clinical Proteomics, 2(3-4), 185-203. 
Zhu, P., Bowden, P., Tucholska, M., \& Marshall, J. G. (2011). Chi-square comparison of tryptic peptideto-protein distributions of tandem mass spectrometry from blood with those of random expectation. Anal Biochem, 409(2), 189-194.

Zhu, P., Bowden, P., Tucholska, M., Zhang, D., \& Marshall, J. G. (2011). Peptide-to-protein distribution versus a competition for significance to estimate error rate in blood protein identification. Analytical Biochemistry, 411, 241-253. doi:10.1016/j.ab.2010.12.003

Zhu, P., Bowden, P., Zhang, D., \& Marshall, J. G. (2011). Mass spectrometry of peptides and proteins from human blood. Mass Spectrom Rev, 30,(5), 685-732.

Zolg, D. P., Wilhelm, M., Schnatbaum, K., Zerweck, J., Knaute, T., Delanghe, B., . . Kuster, B. (2017). Building ProteomeTools based on a complete synthetic human proteome. Nat Methods, 14(3), 259-262. doi:10.1038/nmeth.4153 
Table I. The peptide counts to complement C4B protein sequences from correlation analysis of the MS/MS peptides detected in human plasma. The MS/MS spectra were correlated to charge (z) of $2+$ and $3+$ with up to three missed cleavages of fully tryptic peptides with precursor ions within $\pm 3 \mathrm{~m} / \mathrm{z}$ and MS/MS fragments matched within $0.5 \mathrm{Da}$. The data were filtered by possible charge states (Filter 1) and amino acid sequences (Filter 2) to ensure that only the single best fit of the MS/MS spectra was accepted. The sum p-value or $\mathrm{X}_{\text {corr }}$ (a function of cross correlation) value for filter 2 is listed. The cumulative p-values for all C4B data with filter 2 ranged from E2492 from OMSSA, E-1129 for X!TANDEM and E-2610 from MASCOT (where E-2 is considered significant) while SEQUEST showed a sum $\mathrm{X}_{\text {Corr }}$ of 1192 (where 2.5 to 3.75 is considered significant). The sum total peptide correlation counts from preserved samples, control samples and time course samples from both random sampling and target analysis in this study.

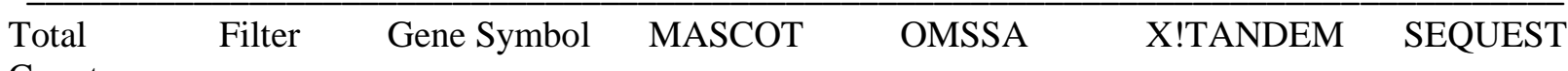
Count

\begin{tabular}{rrrrrrr}
\hline 972 & 0 & $C 4 B$ & 145 & 248 & 311 & 268 \\
857 & 1 & $C 4 B$ & 145 & 238 & 308 & 166 \\
583 & 2 & $C 4 B$ & 145 & 119 & 155 & 164 \\
\hline & & & & & & \\
\hline
\end{tabular}


Table II. The endogenous peptides of C4B detected by LC-ESI-MS/MS of samples where any peptide correlation to C4B by X!TANDEM was accepted. The most commonly detected discrete peptide sequences were NGFKSHALQLNNR and GLEEELQFSLGSK.

\begin{tabular}{|c|c|c|c|}
\hline Peptide Sequence & $\begin{array}{l}\text { Log10 mean } \\
\text { Intensity }\end{array}$ & $\begin{array}{l}\text { STND } \\
\text { ERR }\end{array}$ & $\mathrm{N}$ \\
\hline EELQFSLGSK & 3.59 & 0.03 & 4 \\
\hline GFKSHALQLNNR & 4.22 & 0.00 & 2 \\
\hline GLEEELQFSLGSK & 3.59 & 0.19 & 18 \\
\hline GLEEELQFSLGSKINVK & 3.66 & 0.38 & 12 \\
\hline GLEEELQFSLGSKINVKVGGNSK & 3.56 & 0.00 & 2 \\
\hline HALQLNNR & 3.70 & 0.06 & 2 \\
\hline NGFKSHALQLNN & 3.85 & 0.22 & 14 \\
\hline NGFKSHALQLNNR & 3.79 & 0.32 & 156 \\
\hline NGFKSHALQLNNRQI & 3.78 & 0.09 & $\epsilon$ \\
\hline NGFKSHALQLNNRQIR & 3.79 & 0.26 & 56 \\
\hline QFSLGSKINVK & 2.99 & 0.00 & 2 \\
\hline SHALQLNNR & 3.72 & 0.19 & 20 \\
\hline SHALQLNNRQIR & 3.87 & 0.18 & 10 \\
\hline STQDTVIALDALSAYWIASHTTEERGLNVTLSSTGR & 5.52 & 0.00 & 2 \\
\hline TLEIPGNSDPNMIPDGDFNSYVR & 3.53 & 0.11 & 4 \\
\hline VTASDPLDTLGSEGALSPGGVASLLRLPRGCGEQTMIYLAPTLAASR & 4.78 & 0.00 & 1 \\
\hline
\end{tabular}



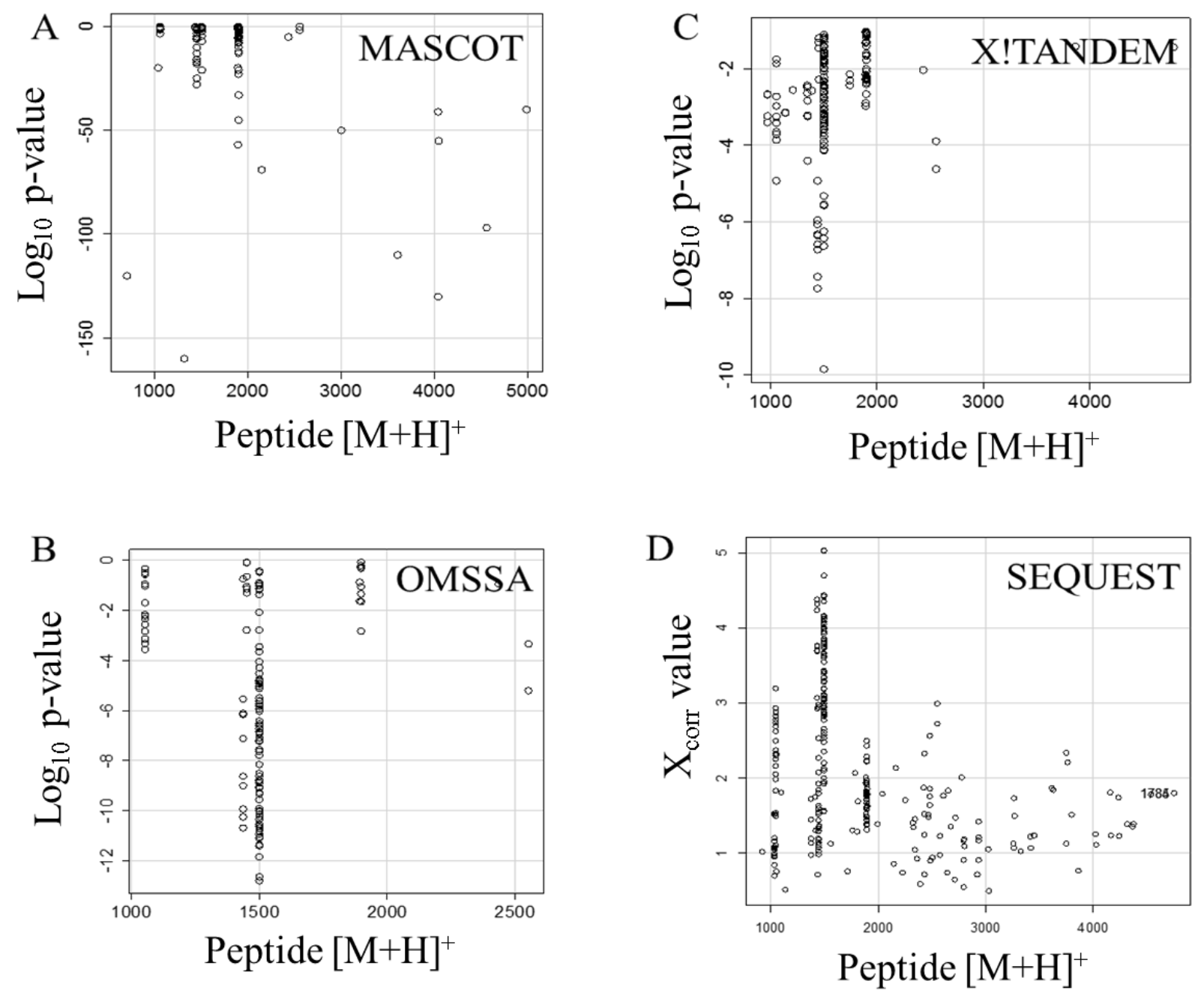

Figure 1. The distribution of $\mathrm{p}$-values (or $\mathrm{X}_{\text {corr }}$ values) versus the peptide expected $[\mathrm{M}+\mathrm{H}]^{+}$of $\mathrm{C} 4 \mathrm{~B}$ from the random and independent sampling degraded plasma. The $\log _{10}$ expectation values (p-values) from MASCOT, OMSSA, X!TANDEM and (or $\mathrm{X}_{\text {corr }}$ values) SEQUEST algorithms are plotted against peptide $[\mathrm{M}+\mathrm{H}]^{+}$values. Panels: A, MASCOT a heuristic probability-based MOWSE score algorithm; B, OMSSA a heuristic probability algorithm; C, X!TANDEM a goodness of fit algorithm; D, SEQUEST a modified Pearson cross correlation algorithm ( $\mathrm{X}_{\text {corr }}$ ). C4B was the most commonly observed protein by many independent peptides in room temperature samples. 


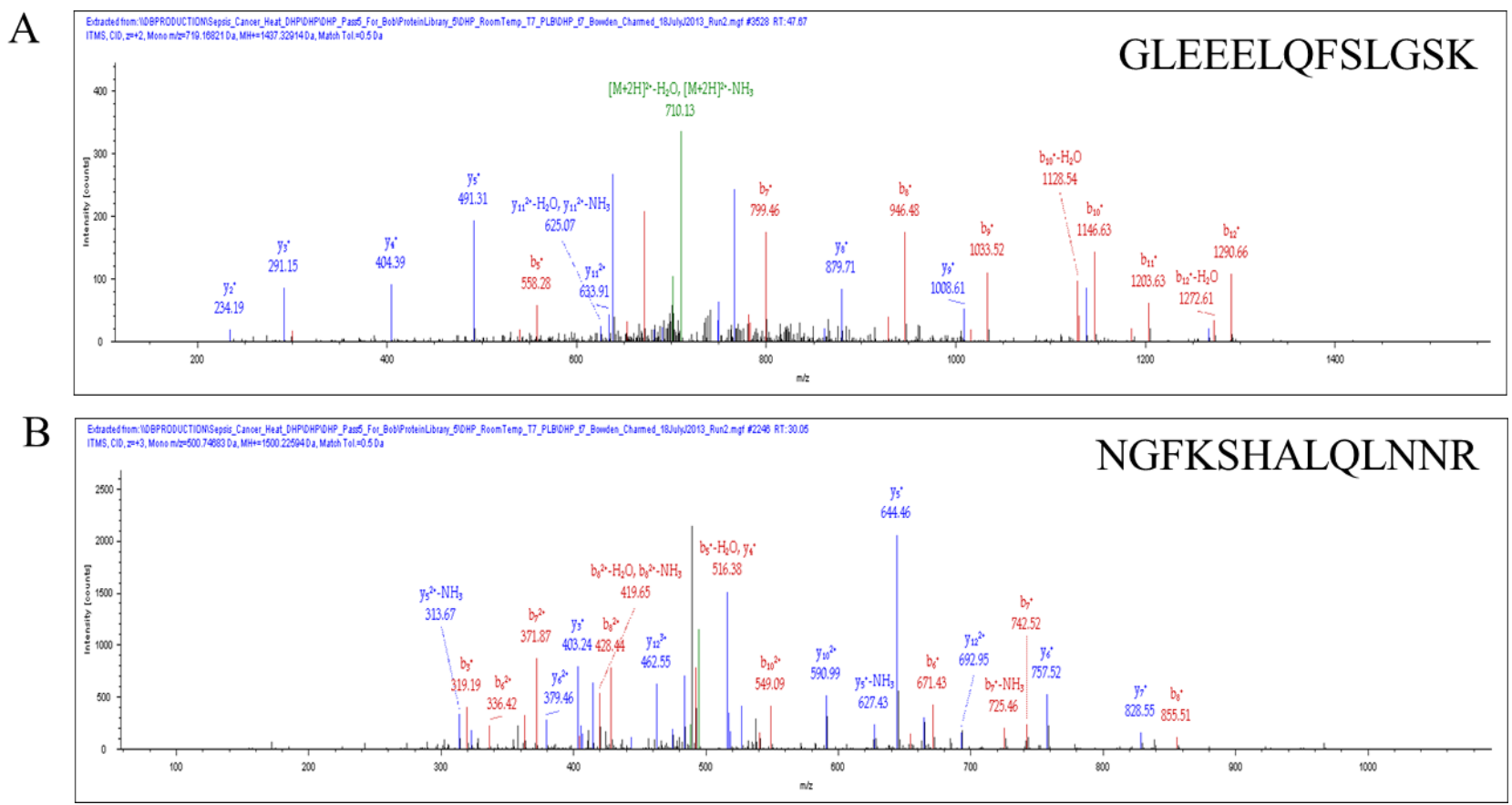

Figure 2. Typical examples of the MS/MS spectra of unique peptides from complement C4B. Panels: A, MS/MS spectra that correlated to the fully tryptic amino acid sequence GLEEELQFSLGSK with $[\mathrm{M}+\mathrm{H}]^{+} 1437.33$ and a $\mathrm{z}$ value of $2+; \mathrm{B}, \mathrm{MS} / \mathrm{MS}$ spectra that correlated to the fully tryptic amino acid sequence NGFKSHALQLNNR with $[\mathrm{M}+\mathrm{H}]^{+} 1500.23$ and a $\mathrm{z}$ value of $3+$. The MS/MS fragment ions are labelled exactly as provided by the Proteome Discoverer system that used the SEQUEST algorithm provided by Thermo Fisher. The MS/MS spectra were obtained from random and independent sampling of all precursor ions during unbiased LC-ESI-MS/MS. 

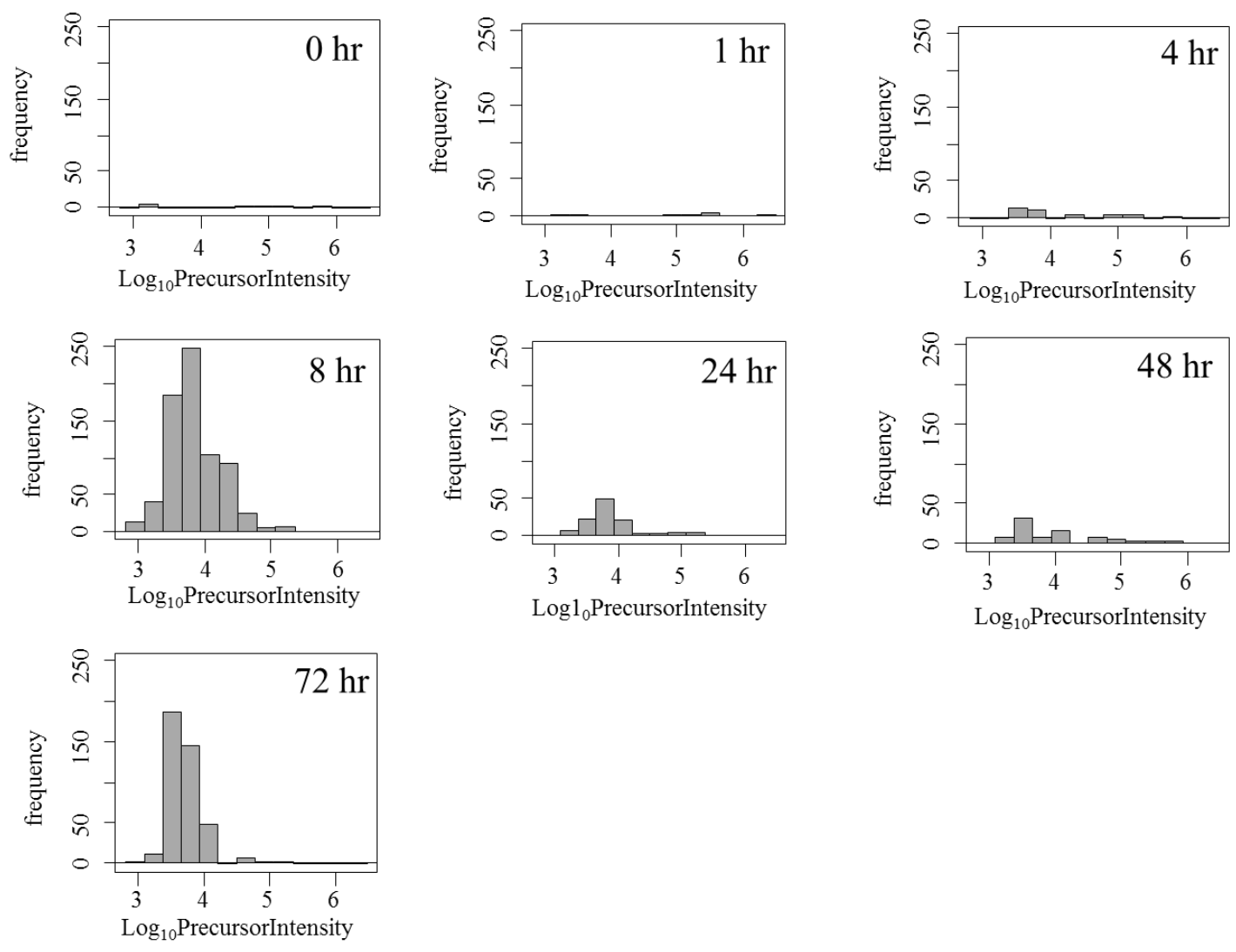

Figure 3. The histogram of $\log _{10}$ precursor intensity of the C4B-peptide (NGFKSHALQLNNR) by random and independent sampling over incubation of EDTA plasma on ice versus room temperature over time. The precursor ion intensity filter of 1000 counts was imposed before examining the precursor with the fitting algorithms. Random and independent sampling showed a 10 to 100-fold increase in C4B-peptide detection with incubation at room temperature. 

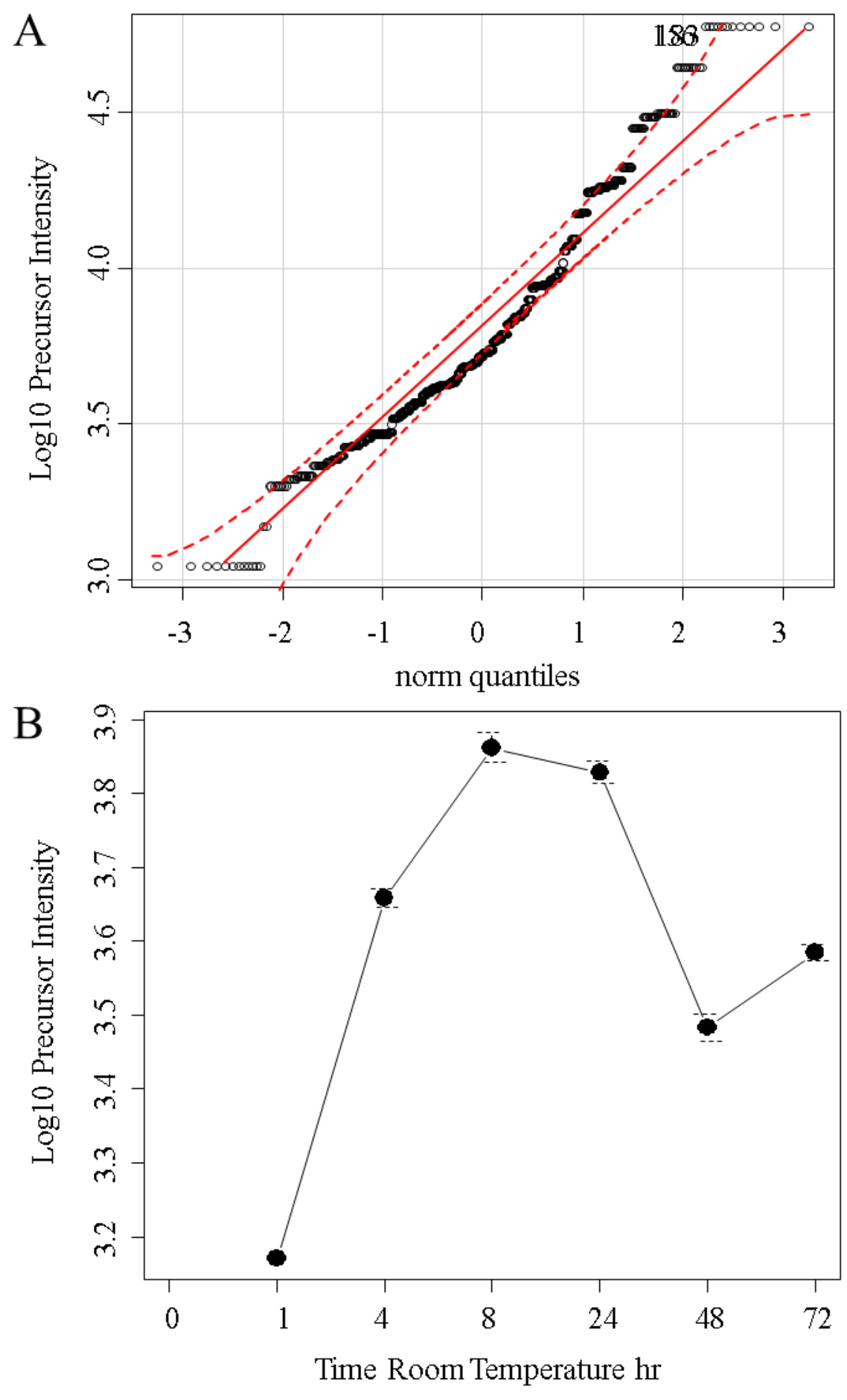

Figure 4. The quantification of C4B-peptide (NGFKSHALQLNNR) by random and independent sampling by LC-ESI-MS/MS. Panels: A, the quantile plot of the randomly sampled C4B peptides; $\mathrm{B}$, the intensity values of the randomly sampled C4B-peptide $\log _{10}$ intensity value (arbitrary counts) over time. The probability that the time points were the same by ANOVA was $\mathrm{p} \leq 0.001$. The time point 0 refers to samples maintained on ice. 

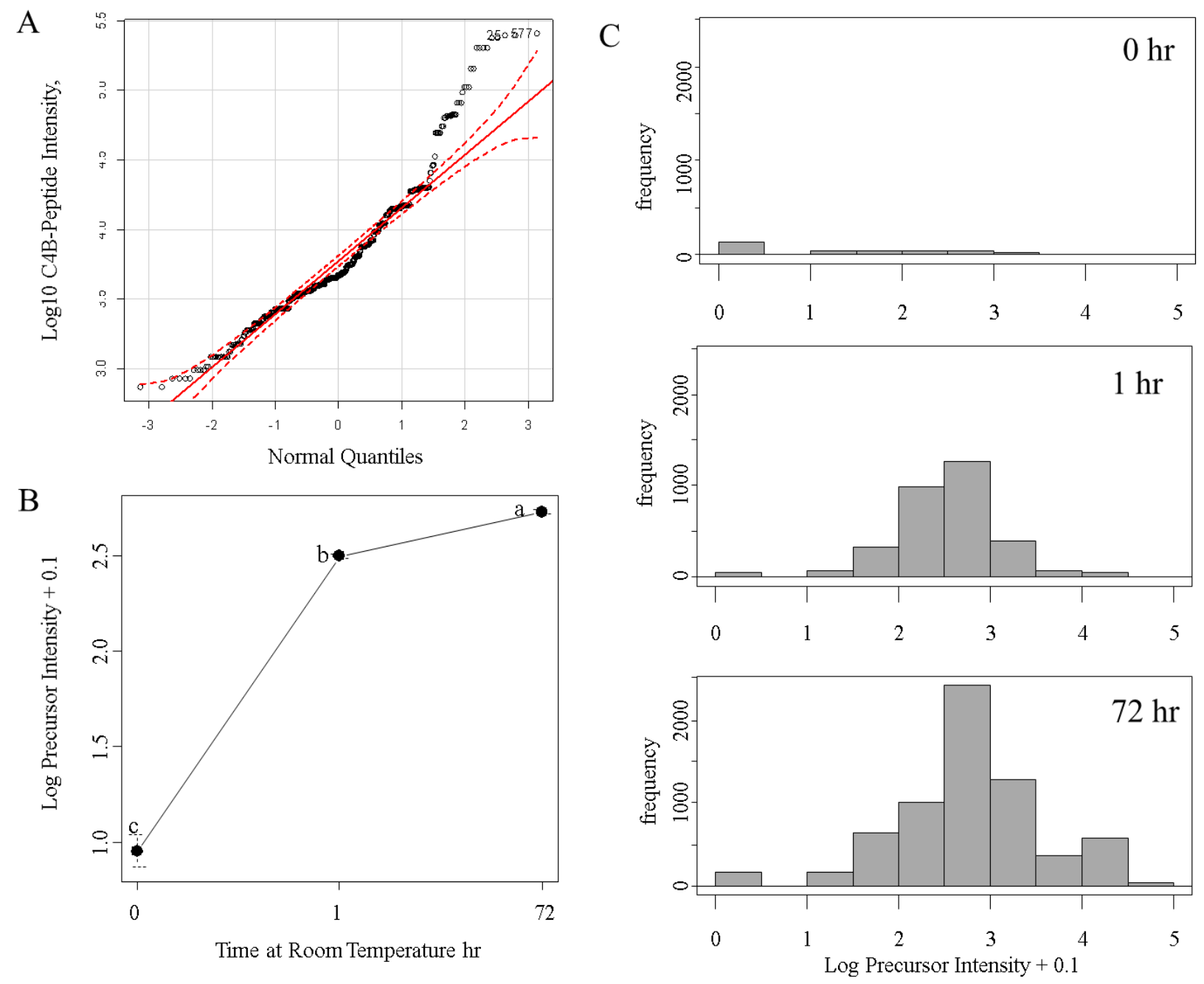

Figure 5. The automatic-targeted analysis of the complement C4B-peptide (NGFKSHALQLNNR) at time 0,1 hour and $72 \mathrm{~h}$ incubation at room temperature. Panels: A, the normality of the $\log _{10}$ transformed intensity data as assessed by quantile plot; B, the mean and standard deviation of the targeted precursor intensity value (different letters indicate a significant difference by the Tukey Kramer Honestly Significant Difference Test); C, the histogram showing the frequency of observing peptides in each $\log _{10}$ intensity bin. Automatic targeted sampling showed a 100-fold increase in frequency and about a 10- to 100-fold increase in C4B-peptide intensity with incubation at room temperature. 


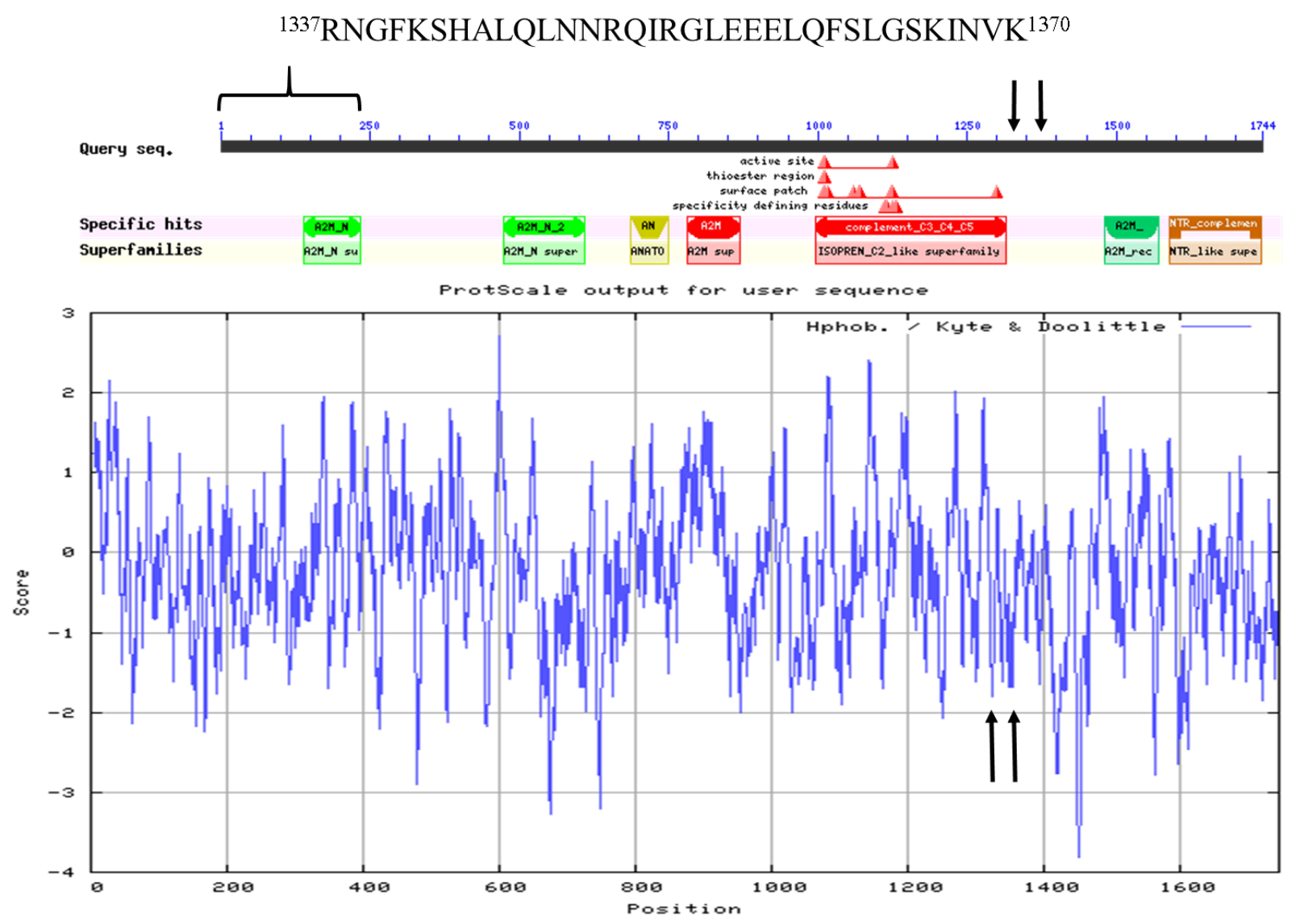

Figure 6. The location of the most common peptide sequences that were cleaved within the complement C4B protein from the protein accession NP_001002029.3 The peptide sequence NGFKSHALQLNNRQIR shows no significant relationship to any other protein and contained $31 \%$ of all observed peptides from C4B. The peptide sequence GLEEELQFSLGSKINVK accounted for $11 \%$ of all peptides. The arrows show the locations of the main peptide cleavage sites on the carboxyl side of the isoprene $\mathrm{C} 2$ domain at a site within a sequence of basic, acid and polar amino acids. The bracket shows a section of the preproprotein that is also cleaved upon warming to room temperature producing the mature chain detectable by Western blot. The C4B amino acid sequence ${ }^{1337}$ RNGFKSHALQLNNRQIRGLEEELQFSLGSKINVK ${ }^{1370}$ on the carboxyl side of the isoprene C2-like superfamily domain was the most frequent site of cleavage (see arrows). The sub-sequence ${ }^{1137}$ NGFKSHALQLNNR ${ }^{1352}$ is located just to the carboxyl terminal side of a local hydrophilic maximum and flanks the evolutionarily conserved isoprene C2 sequence shared by the innate defense proteins complement 4A/B and alpha 2 macroglobulin. The cleavage sites of the peptide(s) NGFKSHALQLNNRQIR are flanked on the amino side by short stretches of hydrophilic amino acids, the released peptide includes asparagine and glutamine, and there is a stretch of three glutamic acid residues and glutamine adjacent on the carboxyl side of the cleavage site. 


\section{A $\quad \begin{array}{llllllllllll}1 & 2 & 3 & 4 & 5 & 6 & 7 & 8 & 9 & 10\end{array}$}

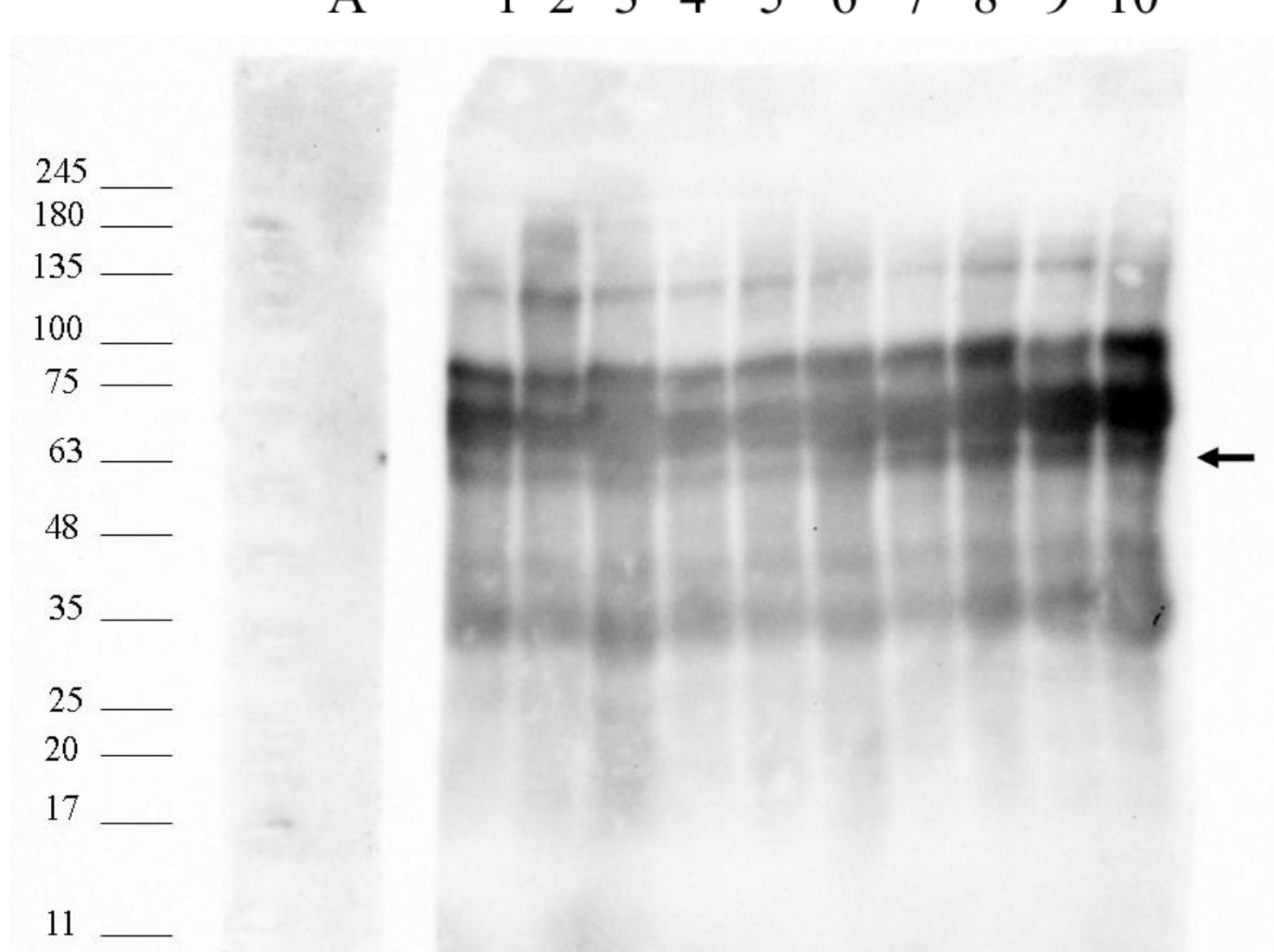

Figure 7. Western blot against the mature C4B protein chain in human EDTA plasma over the time course of degradation at room temperature. The monoclonal antibody against $\mathrm{C} 4 \mathrm{~B}$ was biotinylated and detected with Streptavidin-HRP. The arrow indicates the expected C4B peptide after proteolysis. Lanes: MW, Molecular weight marker as indicated; A, Streptavidin HRP alone; 1, time 0; 2, 1 hr; 3, 1 hr; 4, 4hr; 5, 8hr; 6, 24 hr; 7, 36 hr; 8, 48 hr; 9, 72, hr; 10, 96 hr. Molecular weight markers for a 9\% Tricine SDS-PAGE gel are shown in kilodaltons (kDa). The Western blot analysis showed the increased formation of mature $\mathrm{C} 4 \mathrm{~B}$ with time and the development of an additional band with time at room temperature confirming the proteolytic processing of $\mathrm{C} 4 \mathrm{~B}$. Complement $\mathrm{C} 4$ is expressed as a high molecular mass preproprotein that reacts poorly with the antibody raised against the mature protein that exits in blood as a $79 \mathrm{kDa}$ mature chain in close agreement with the most intense band in the Western blot that appears roughly in line with the $75 \mathrm{kDa}$ molecular mass marker. Proteolytic cleavage of the C-terminal domain from C4B starting at NGFKSHALQLNNR to the carboxyl terminus of the C4B protein should yield a $63 \mathrm{kDa}$ protein (see arrow). Control blots stained with Coomassie Blue confirmed equal loading of all lanes (see supplemental). These data confirm the finding shown in Table II and Figure 2. The manufacturer (THERMO) indicates the antibody recognizes an $88 \mathrm{kDa}, 75$ $\mathrm{kDa}$ and $33 \mathrm{kDa}$ form of $\mathrm{C} 4 \mathrm{~B}$ in good agreement. 

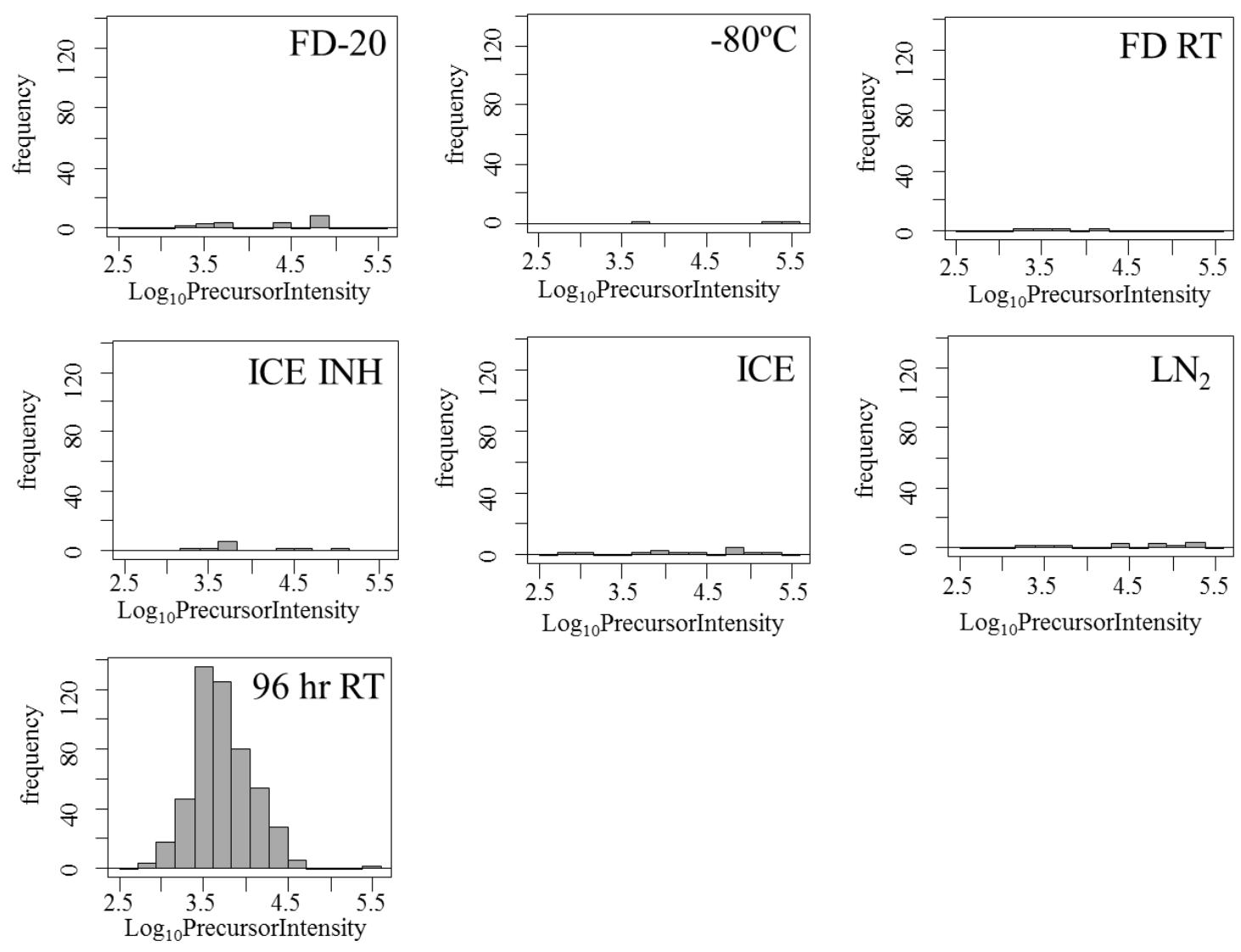

Figure 8. The histogram showing a comparison of the preservation methods of ice \pm protease inhibitors, freezing versus freeze drying alongside a positive control for sample degradation as illustrated by C4B-peptide (NGFKSHALQLNNR) by random and independent sampling by LCESI-MS/MS. Storage treatments: freeze drying and storage at room temperature (FDRT); freeze drying and storage at -20 (FD-20); freezing storage at $-80^{\circ} \mathrm{C}(-80)$; or liquid nitrogen (LN2); versus plasma degraded for 96 hours at room temperature $(96 \mathrm{hr}$ RT). The histogram shows the frequency of peptides in each $\log _{10}$ intensity bin. The precursor ion intensity filter of 1000 counts was imposed before examining the data in $\mathrm{R}$. 


\section{$\begin{array}{lllllllllll}\mathrm{kDa} & 1 & 2 & 3 & 4 & 5 & 6 & 7 & 8 & 9 & 10\end{array}$}

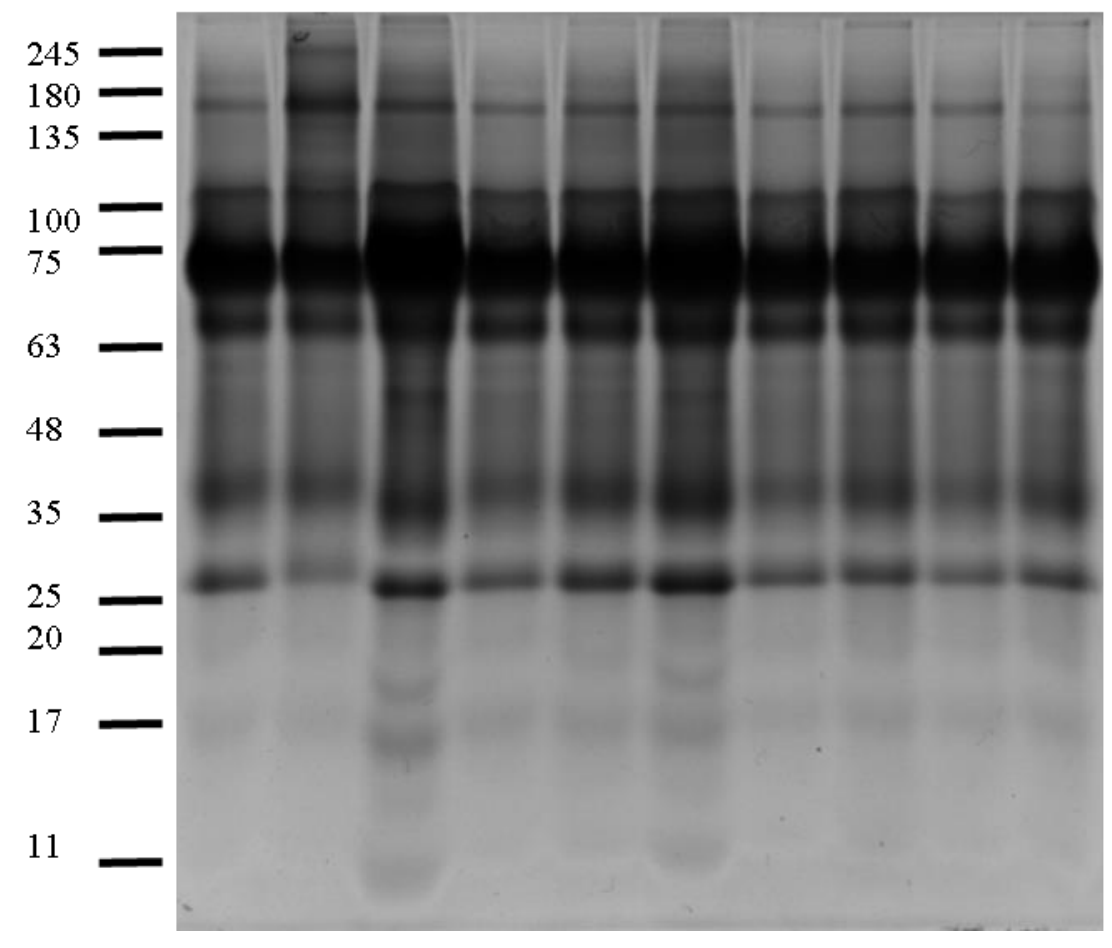

Supplemental Figure 1. A replicate CBBR stained gel to confirm the equal loading of the Western blot shown in Figure 8 of the main paper. (see legend of Figure 8 for details). 


\section{LEGENDS}

Table I. The peptide counts to complement $\mathrm{C} 4 \mathrm{~B}$ protein sequences from correlation analysis of the MS/MS peptides detected in human plasma. The MS/MS spectra were correlated to charge (z) of +2 or +3 with up to three missed cleavages of fully tryptic peptides with precursor ions within $\pm 3 \mathrm{~m} / \mathrm{z}$ and MS/MS fragments matched within $0.5 \mathrm{Da}$. The data were filtered to ensure no MS/MS spectra was assigned to any other peptides sequence at any other charge state. The sum p-value or Xcorr (a function of cross correlation) value for filter 2 is listed. The cumulative $P$ values for all C4B data with filter 2 ranged from E-2492 from OMSSA, E-1755 for $\mathrm{X}$ !TANDEM and E-2684 from MASCOT (where E-2 is considered significant) while SEQUEST showed a sum XCorr of 1192 (where 2.5 to 3.75 is considered significant). The sum total peptide correlation counts from preserved samples, control samples and time course samples from both random sampling and target analysis in this study.

Table II. The endogenous peptides of C4B detected by LC-ESI-MS/MS of samples where any peptide correlation to C4B by X!TANDEM was accepted. The most commonly detected discrete peptide sequences were NGFKSHALQLNNR and GLEEELQFSLGSK.

Figure 1. The distribution of p-values (or $\mathrm{X}_{\text {corr }}$ values) versus the peptide expected $[\mathrm{M}+\mathrm{H}]^{+}$of $\mathrm{C} 4 \mathrm{~B}$ from the random and independent sampling degraded plasma. The $\log _{10}$ expectation values (p-values) from MASCOT, OMSSA, X!TANDEM and (or $\mathrm{X}_{\text {corr }}$ values) SEQUEST algorithms are plotted against peptide $[\mathrm{M}+\mathrm{H}]^{+}$values. Panels: A, MASCOT a heuristic probability-based MOWSE score algorithm; B, OMSSA a heuristic probability algorithm; C, X!TANDEM a goodness of fit algorithm; D, SEQUEST a modified Pearson cross correlation algorithm ( $\left.\mathrm{X}_{\text {corr }}\right)$. C4B was the most commonly observed protein by many independent peptides in room temperature samples.

Figure 2. Typical examples of the MS/MS spectra of unique peptides from complement C4B. Panels: A, MS/MS spectra that correlated to the fully tryptic amino acid sequence GLEEELQFSLGSK with $[\mathrm{M}+\mathrm{H}]+1437.33$ and a $\mathrm{z}$ value of $2+$; $\mathrm{B}$, MS/MS spectra that correlated to the fully tryptic amino acid sequence NGFKSHALQLNNR with $[\mathrm{M}+\mathrm{H}]+1500.23$ and a $\mathrm{z}$ value of $3+$. The MS/MS fragment ions are labelled exactly as provided by the Proteome Discoverer system that used the SEQUEST algorithm provided by Thermo Fisher. The MS/MS spectra were obtained from random and independent sampling of all precursor ions during unbiased LC-ESI-MS/MS.

Figure 3. The histogram of $\log 10$ precursor intensity of the C4B-peptide (NGFKSHALQLNNR) by random and independent sampling over incubation of EDTA plasma on ice versus room temperature over time. The precursor ion intensity filter of 1000 counts was imposed before 
examining the precursor with the fitting algorithms. Random and independent sampling showed a 10 to 100-fold increase in C4B-peptide detection with incubation at room temperature.

Figure 4. The quantification of C4B-peptide (NGFKSHALQLNNR) by random and independent sampling by LC-ESI-MS/MS. Panels: A, the quantile plot of the randomly sampled C4B peptides; B, the intensity values of the randomly sampled C4B-peptide log 10 intensity value (arbitrary counts) over time. The probability that the time points were the same by ANOVA was $\mathrm{p} \leq 0.001$. The time point 0 refers to samples maintained on ice.

Figure 5. The automatic-targeted analysis of the complement C4B-peptide (NGFKSHALQLNNR) at time 0,1 hour and $72 \mathrm{~h}$ incubation at room temperature. Panels: A, the normality of the $\log 10$ transformed intensity data as assessed by quantile plot; $\mathrm{B}$, the mean and standard deviation of the targeted precursor intensity value (different letters indicate a significant difference by the Tukey Kramer Honestly Significant Difference Test); C, the histogram showing the frequency of observing peptides in each $\log 10$ intensity bin. Automatic targeted sampling showed a 100-fold increase in frequency and about a 10- to 100-fold increase in C4B-peptide intensity with incubation at room temperature.

Figure 6. The location of the most common peptide sequences that were cleaved within the complement C4B protein from the protein accession NP_001002029.3 The peptide sequence NGFKSHALQLNNRQIR shows no significant relationship to any other protein and contained $31 \%$ of all observed peptides from C4B. The peptide sequence GLEEELQFSLGSKINVK accounted for $11 \%$ of all peptides. The arrows show the locations of the main peptide cleavage sites on the carboxyl side of the isoprene $\mathrm{C} 2$ domain at a site within a sequence of basic, acid and polar amino acids. The bracket shows a section of the preproprotein that is also cleaved upon warming to room temperature producing the mature chain detectable by Western blot. The C4B amino acid sequence 1337RNGFKSHALQLNNRQIRGLEEELQFSLGSKINVK1370 on the carboxyl side of the isoprene C2-like superfamily domain was the most frequent site of cleavage (see arrows). The sub-sequence 1137NGFKSHALQLNNR1352 is located just to the carboxyl terminal side of a local hydrophilic maximum and flanks the evolutionarily conserved isoprene $\mathrm{C} 2$ sequence shared by the innate defense proteins complement 4A/B and alpha 2 macroglobulin. The cleavage sites of the peptide(s) NGFKSHALQLNNRQIR are flanked on the amino side by short stretches of hydrophilic amino acids, the released peptide includes asparagine and glutamine, and there is a stretch of three glutamic acid residues and glutamine adjacent on the carboxyl side of the cleavage site.

Figure 7. Western blot against the mature C4B protein chain in human EDTA plasma over the time course of degradation at room temperature. The monoclonal antibody against $\mathrm{C} 4 \mathrm{~B}$ was biotinylated and detected with Streptavidin-HRP. The arrow indicates the expected C4B peptide after proteolysis. Lanes: MW, Molecular weight marker as indicated; A, Streptavidin HRP alone; 1, time 0; 2, $1 \mathrm{hr} ; 3,1 \mathrm{hr}$; 4, 4hr; 5, 8hr; 6, 24 hr; 7, $36 \mathrm{hr} ; 8,48 \mathrm{hr}$; 9, 72, hr; 10, $96 \mathrm{hr}$. Molecular weight markers for a 9\% Tricine SDS-PAGE gel are shown in kilodaltons (kDa). The Western blot analysis showed the increased formation of mature $\mathrm{C} 4 \mathrm{~B}$ with time and the development of an additional band with time at room temperature confirming the proteolytic processing of $\mathrm{C} 4 \mathrm{~B}$. Complement $\mathrm{C} 4$ is expressed as a high molecular mass preproprotein that 
reacts poorly with the antibody raised against the mature protein that exits in blood as a $79 \mathrm{kDa}$ mature chain in close agreement with the most intense band in the Western blot that appears roughly in line with the $75 \mathrm{kDa}$ molecular mass marker. Proteolytic cleavage of the C-terminal domain from C4B starting at NGFKSHALQLNNR to the carboxyl terminus of the C4B protein should yield a $63 \mathrm{kDa}$ protein (see arrow). Control blots stained with Coomassie Blue confirmed equal loading of all lanes (see supplemental). These data confirm the finding shown in Table II and Figure 2. The manufacturer (THERMO) indicates the antibody recognizes an $88 \mathrm{kDa}, 75$ $\mathrm{kDa}$ and $33 \mathrm{kDa}$ form of $\mathrm{C} 4 \mathrm{~B}$ in good agreement.

Figure 8. The histogram showing a comparison of the preservation methods of ice \pm protease inhibitors, freezing versus freeze drying alongside a positive control for sample degradation as illustrated by C4B-peptide (NGFKSHALQLNNR) by random and independent sampling by LCESI-MS/MS. Storage treatments: freeze drying and storage at room temperature (FDRT); freeze drying and storage at -20 (FD-20); frozen storage at $-80^{\circ} \mathrm{C}$; Frozen storage in liquid nitrogen (LN2); versus plasma degraded for 96 hours at room temperature ( $96 \mathrm{hr} \mathrm{RT}$ ). The histogram shows the frequency of peptides in each $\log 10$ intensity bin. The precursor ion intensity filter of 1000 counts was imposed before examining the data in $\mathrm{R}$.

Supplemental Figure 1. A replicate CBBR stained gel to confirm the equal loading of the Western blot shown in Figure 8 of the main paper. (see legend of Figure 8 for details). 
SUPPLEMENT G 
The endogenous tryptic peptides of EDTA plasma from Alzheimer's Dementia, Heart Attack, Sepsis, Ovarian Cancer, Breast Cancer, and Multiple Sclerosis and institution/study matched controls

Jaimie Dufresne, Thanusi Thavarajah, Angelique Florentinus, Zhuo Zhen Chen, Monika

Tucholska, Tenzin Norzin, Morla Phan, Margaret Truc Ho, Pete Bowden, John G Marshall

Department of Chemistry and Biology, Ryerson University, Toronto, ON M5B 2K3

Running Title:

John G. Marshall,

Department of Chemistry and Biology,

Faculty of Science,

Ryerson University,

350 Victoria St., Toronto,

ON, Canada,

Tel.: 416-979-5000 x4219;

Fax: 416-979-5044;

E-mail: 4marshal@ ryerson.ca

\section{ABSTRACT}


In order to assess the specific variation in endogenous tryptic peptides across institution/study and/or disease state, the EDTA plasma of Alzheimer's Dementia (AD), Breast cancer (BC), Heart Attack artery \& venous (MIA, MIV), Ovarian Cancer (OC) Sepsis (SP) and Multiple Sclerosis (MS) and the corresponding institution-matched controls were compared. The peptides were quantitatively precipitated in acetonitrile and then selectively extracted from the pellet with a step gradient of increasing water. The step fractions were collected over preparative $\mathrm{C} 18$ and analyzed on a new $\mathrm{C} 18$ analytical column for each patient to prevent cross contamination. The peptide-to-protein distribution of the precursor ions of greater than 1000 counts were confirmed against a null random model of noise or computer generated random spectra. There was significant variation between EDTA plasma provided by the different study/institution. Peptides from many well-established acute phase markers such as amyloids, haptoglobin, complements, fibrinogens, hemopexin, antitrypsin, alpha 2 macroblobulin, and others showed variation in terms of frequency and/or intensity summed over all of the disease states $(n \geq 60)$ versus all of the controls $(\mathrm{n} \geq 60$ ) by the Chi Square test. There was significant disease-specific variation in peptides or phosphopeptides from cellular proteins, membrane proteins, Nucleic acid binding proteins, signaling factors, metabolic enzymes and others including uncharacterized factors between disease states $(n \geq 10)$ versus the matched institution/study control $(n \geq 10)$ the Chi Square test. The $\log 10$ precursor intensity data was normally distributed and there were significant differences in intensity values between samples from different institution or disease treatments. 


\section{INTRODUCTION}

Many non-specific or so called "acute phase" proteins have been detected to increase by the analysis of blood fluids by mass spectrometry such as amyloids, haptoglobin, alpha 1 antitrypsin, clusterin, (ApoJ), complement components, heat shock, fibrinogens, hemopexin, alpha 2 macroglobulin and others that may be of limited diagnostic value (Ye et al., 2003; R. Zhang et al., 2004). It will be necessary to compare the results of many diseases and controls together under the same conditions to ensure the reported proteins are specific to only one disease. With optimal sample preparation low abundance proteins of $\leq 1 \mathrm{ng} / \mathrm{ml}$ and low abundance factors like insulin like growth factor may be detected in blood samples by mass spectrometry (Monika Tucholska et al., 2009; Monika Tucholska et al., 2007). In order to avoid cross-contamination, preparative and analytical separations of polypeptides should be performed with single-use,

disposable apparatus. A practical way to estimate the variation that results from institution/study verses disease(s) is to compare the frequency and intensity of peptides of phosphopeptides from many disease and normal plasma from each different institution/study under the same conditions. The pre-analytical variation between institution/study and/or disease was analyzed by comparing samples from different sources by Chi Square and ANOVA. In addition, to control for differences in sample handing and pre-clinical variation, EDTA plasma was collected on ice and then purposefully degraded at room temperature over time to reveal normal sample degradation products with time and these were also discounted. After discarding proteins observed to vary between all diseases versus all controls there apparently remained significant variation attributable to disease. Previously it was established that precipitating all of the polypeptides with $90 \%$ ACN followed by step-wise extraction of the peptides with mixtures of organic solvent 
and water was the optimal method to sensitively detected peptides from blood (Declan Williams, 2007; M. Tucholska et al., 2010; Monika Tucholska et al., 2007; Williams et al., 2010).

\section{Discovery of Biomarkers by Mass Spectrometry}

Recently mass spectrometry has been used for purposes as diverse as looking for plasma proteome biomarkers of inflammation were investigated in school children in Nepal (S. E. Lee et al., 2015), chemical warfare in snails (Safavi-Hemami et al., 2015) or to quantify angiotensin using magnetic epitope-imprinted polymers (L. Tan et al., 2015). The polypeptides of human plasma or serum may have many biomedical applications (Govorun \& Ivanov, 2011; Yatskin et al., 2009). Insulin-like peptide 3 levels were monitored in healthy peripubertal girls over time (Hagen et al., 2015). Enrichment of the low-molecular-weight fraction of plasma identified neuropeptide-Y as a prostate cancer biomarker polypeptide (Ueda et al., 2013). The application of LC-ESI-MS/MS to peptide hormones in athletics is increasing (Esposito, Deventer, Geldof, \& Van Eenoo, 2015; Vogel et al., 2014). LC-ESI-MS/MS was used to analyze regulatory peptides such as glargine, insulin like growth factors and others (Thomas et al., 2015; Xu et al., 2014). Mass spectrometry methods detected and quantified the pyridoxamine or adenosine products of ELISA in female plasma alongside blind controls and were found to be superior to both colorimetric and fluorescent ELISA and reached yoctomole sensisitivty (Florentinus-Mefailoski \& Marshall, 2014, 2016; Florentinus-Mefailoski, Safi, \& Marshall, 2014; FlorentinusMefailoski, Soosaipillai, Dufresne, Diamandis, \& Marshall, 2015; Xu et al., 2014). Plasma natriuretic peptide levels were measured by mass spectrometry and predicted the risk of cardiovascular events and death (T. J. Wang et al., 2004). Proteins involved in cell adhesion, immune response, coagulation process and metabolism including collagen I dynamically 
changed from colorectal cancer stages I to IV, with maximum expression in stage II, as detected in serum by MS analysis (Zou et al., 2013). Circulating proteolytic signatures of chemotherapyinduced cell death in humans were discovered by $\mathrm{N}$-terminal derivatization and differential mass spectrometry (Wiita et al., 2014). Osteopontin and latent-TGF $\beta$ binding-protein 2 were potential diagnostic markers for HBV-related hepatocellular carcinoma (da Costa et al., 2015). Ovarian ascites fluid may be a source of novel blood peptides that might have diagnostic applications (Bery, Leung, Smith, Diamandis, \& Kulasingam, 2014). A quantitative mass spectrometric immunoassay for the chemokine RANTES and its variants was developed (Trenchevska et al., 2015). Evidence was provided that Tumor necrosis factor receptor superfamily member 6B (TNFRSF6B) and Growth Differentiation Factor 15 (GDF15) may serve as biomarkers of colon cancer (Lancrajan et al., 2015). Differential activity of endo and exopeptidase, including changes in Bradykinin, was reflected over the progression of colorectal cancer in plasma samples (Bedin et al., 2015). Protein assays of circulating proteins such a leptin, renin, interleukin-1 receptor antagonist [IL-1ra], hepatocyte growth factor, fatty acid-binding protein 4, and tissue plasminogen activator [t-PA]) cathepsin D might be used to detect insulin resistance (IR), the precursor of type 2 diabetes (Nowak et al., 2016). Proteins or RNA may be packaged in exosomes and it appears that supramolecular complexes from cells may survive in circulation (Bery et al., 2014; Karagiannis et al., 2014; J. Marshall et al., 2014). Circulating immune complexes containing thrombospondin-1 (TSP-1) and platelet factor 4 (PF4) were serum markers of rheumatoid arthritis (Ohyama et al., 2011). Kawasaki disease (KD), an acute febrile vasculitis, was associated with changes in interleukin-17F, sCD40L, E-selectin, CCL23 (myeloid progenitor inhibitory factor 1) and CXCL10 (IFN- $\gamma$-inducible protein) was observed to be both a biomarker and mediator of the pathology (Ko et al., 2015). Elevated levels of 14-3-3 proteins 
(Angelica K Florentinus, Peter Bowden, Girish Sardana, et al., 2012), serotonin, gamma enolase and pyruvate kinase were identified in clinical samples from patients diagnosed with colorectal cancer (Dowling et al., 2015). Peripheral $\alpha$-defensins 1 and 2 were elevated in Alzheimer's disease (Watt et al., 2015). Circulating FABP4 was a prognostic biomarker in patients with acute coronary syndrome but was not found in asymptomatic individuals (Reiser et al., 2015). Serum angiotensin II concentration was a strong predictor of breast cancer mortality following breast surgery (Boccardo et al., 2015; Schulz, Jankowski, Zidek, \& Jankowski, 2014). Haptoglobin has been suggested as a biomarker of many diseases including lung squamous cell carcinoma by serum proteome and peptidome profiling (Okano et al., 2016; Ye et al., 2003). Peptidomic analysis showed Domain of Unknown Function (DUF4588), perhaps from intrinsic factor-B12 receptor precursor, might be used for renal clear cell carcinoma diagnosis (Huang et al., 2014). A panel of biglycan, annexin-A6, myeloperoxidase, and protein S100-A9 was used for the detection of esophageal adenocarcinoma (Zaidi et al., 2014). The analysis of circulating extracellular vesicles identified CD44 as a prognostic serum biomarker of myeloma (Harshman et al., 2016). Mycobacterium tuberculosis peptides were detected in the exosomes of patients with active and latent M. tuberculosis infection (Kruh-Garcia et al., 2014). Autophagy-related gene16L2, a potential serum biomarker of multiple sclerosis evaluated by bead-based proteomic technology (L. Yin et al., 2014). Depletion chromatography and 2D LC of peptides, followed by targeted proteomics that to date can consistently assay 34 proteins to as low as 100 picomolar concentration range, that was a large improvement over previous attempts (Abbatiello et al., 2015). Semaphorin $7 A$ and ala- $\beta$-his-dipeptidase were validated as biomarkers associated with the conversion from clinically isolated syndrome to multiple sclerosis (Canto et al., 2014). Lowmolecular-mass secretome profiling identifies $\mathrm{C}-\mathrm{C}$ motif chemokine 5 as a potential plasma 
biomarker and therapeutic target for nasopharyngeal carcinoma (Lin et al., 2013). Blood plasma IgG Fc glycans are significantly altered in Alzheimer's disease and progressive mild cognitive impairment (Lundstrom et al., 2014). Annexin II was observed to be a novel secretory biomarker for breast cancer (Jeon et al., 2013). Trypanosome proteins were identified in plasma from African sleeping sickness patients infected with Trypanosoma brucei rhodesiense (Eyford, Ahmad, Enyaru, Carr, \& Pearson, 2013). Potential biomarkers for minimal residual disease assessment were examined in adult acute myeloid leukemia was made by serum peptidome profiling (Bai et al., 2013). Kininogen-1 was identified as a serum biomarker for the early detection of advanced colorectal adenoma and colorectal cancer (J. Wang et al., 2013). The peptides of bacteria may be directly detectable in human serum using mass spectrometry (Cheung et al., 2015). It may be possible to detect the presence of infectious agents either via the host response or from the presence of the pathogen's own proteins (Fragnoud, YuguerosMarcos, Pachot, \& Bedin, 2012; Haverland, Villeneuve, Ciborowski, \& Fox, 2016; Kruh-Garcia et al., 2014). Blood-based protein biomarkers for diagnosis of Alzheimer disease including VCAM1, ILGFBP2, Carcinoembryonic antigen, CD40, and macrophage inflammatory protein alpha 1 and SOD were discovered by screening with a panel of immunoassays (Doecke et al., 2012). Blood cells themselves may act as important biomarkers of immunological or infectious disease and these might also be quantified by mass spectrometry (Chiu, Ling, Chiang, Lin, \& Huang, 2015). Multiple biomarkers were observed for the early detection of type 2 diabetes, including insulin, leptin, and adiponectin (Umeno et al., 2015). Significant variation in blood proteins were discovered followed by a longitudinal evaluation of prostate cancer that showed variation between patients over time (Tonry et al., 2015). Beta 2 microglobulin induced epithelial-mesenchymal transition in human renal proximal tubule epithelial cells in vitro (A. 
Zhang, Wang, Yang, Shi, \& Gan, 2015). The drug olanzapine that is used to treat psychotic disorders such as schizophrenia or premenstrual syndrome had an effect on serum protein phosphorylation patterns (Jaros et al., 2015). ApoA-I/HDL-C levels were inversely associated with abdominal aortic aneurysm progression (Burillo et al., 2015). Levels of apolipoprotein E, haemoglobin $\alpha-2$, and peroxiredoxin 2 and other plasma proteins were gender-specific plasma biomarkers in patients with Anderson-Fabry disease (Hollander et al., 2015). Endogenous angiotensin II levels were absolutely quantified in human plasma using ESI-LC-MS/MS (Schulz et al., 2014). The metabolism of therapeutic peptides was investigated by tagging with stable ${ }^{127} I$ and using inductively-coupled plasma mass spectrometry (Lim, Cao, Qiu, Silva, \& Evans, 2015). CRP and LRG1 were predictive biomarkers of survival for non-small cell lung cancer patients undergoing radical radiotherapy (Walker et al., 2015). APOE, DBP and AGT predicted response to vascular endothelial growth factor inhibitor and chemotherapy in metastatic colorectal cancer (Martin et al., 2014). Mass spectrometry-based serum and plasma peptidome profiling predicted treatment outcome in patients with solid malignancies (Labots et al., 2014). LC-MS/MS was used to measure plasma vancomycin and compared to immunoassays (Oyaert et al., 2015). Human kallikrein-related peptidases in were assayed in biological fluids by targeted mass spectrometry (Karakosta, Soosaipillai, Diamandis, Batruch, \& Drabovich, 2016). Levels of creactive protein (CRP) and angiopoietin-like 3 (ANGPTL3) were associated with depression using iTRAQ identification of human peripheral serum proteins (Q. Wang et al., 2016). The apolipoprotein family, C-reactive protein, gender-related proteins and more than 40 FDAapproved biomarkers were reproducibly quantified (CV <20\% with label-free quantification) within a 1,000-protein, quantitative plasma proteome from a finger prick (Geyer et al., 2016). Apolipoprotein D, apolipoprotein B, vitamin D-binding protein, ceruloplasmin, hornerin, and 
profilin 1 distinguished drug-free patients with major depressive disorder (M. Y. Lee et al., 2016). Evidence of a role for cytochrome P450 system in Niemann-Pick Type C Disease was revealed by mass spectrometry (Nicoli et al., 2016). Secreted protein acidic and rich in cysteine (SPARK) was identified as a prognostic biomarker in malignant pleural mesothelioma (Kao et al., 2016). B-Type Natriuretic Peptide (BNP) and BNP Precursor (proBNP) were differentially cleaved by neprilysin (Semenov \& Katrukha, 2016). Protein $Z$ was identified as a putative novel biomarker for early detection of ovarian cancer (Russell et al., 2016). Enzymes and structural proteins were revealed by auto immune probing with sera from patients with cholangiocarcinoma against tumor versus adjacent normal tissue (Mustafa et al., 2016). A novel cystine transporter in the renal proximal tubule was identified as a missing partner of cystinuria-related plasma membrane protein rBAT/SLC3A1 (Nagamori et al., 2016). Changes in acute-phase proteins such as Haptoglobin, Hemopexin, C-reactive protein, Serum Fibrinogen, amyloid A-2, Lipopolysaccharide binding protein, Albumin, Afamin, Retinol-binding protein 4, cytochrome P450, Ankyrin-1, and Pulmonary surfactant associated protein B were associated with sepsis (Malmstrom et al., 2016). Comparative mass spectrometric and immunoassay-based proteome analysis in serum of Duchenne muscular dystrophy patients revealed titin, myosin, and carbonic anhydrase I, vitamin D binding protein fibulin-1 (FBLN1) and gelsolin (GSN) as potential biomarkers (Oonk et al., 2016). Proteomic biomarkers of nutrition were examined 1000 human plasma samples with mass spectrometry (Cominetti et al., 2016). Serum Apolipoproteins (APO) A-I, B, C-I, C-II, C-III, and E phenotyping was made with an automated multiplex LC-MS/MS Assay (van den Broek et al., 2016). Parathyroid Hormone-Related Peptide may be measured by LC-ESI-MS/MS (Kushnir et al., 2016). Infectious Microorganisms were rapidly identified in clinical Samples by MALDI-TOF MS Analysis (Nakanishi, 2015). Borrelia burgdorferi 
membrane proteins were identified in human serum for the detection of bacterial infection (Cheung et al., 2015). Characterisation of serum transthyretin by electrospray ionisation-ion mobility mass spectrometry was applied to familial amyloidotic polyneuropathy type I (FAP-I) (Pont, Benavente, Vilaseca, Gimenez, \& Sanz-Nebot, 2015). Proglucagon-derived peptides by were analyzed by immunoaffinity chromatography and tandem mass spectrometry for a food tolerance test. (A. Y. Lee et al., 2016). Heat shock 71 (HSC71) was identified as novel serum biomarker for renal cell carcinoma (Y. Zhang, Cai, Yu, \& Li, 2015). Glycoproteins were identified as diagnostic biomarker candidates for esophageal adenocarcinoma (Shah et al., 2015). Fucosylation of serum proteins was analyzed in hepatocellular carcinoma (H. Yin et al., 2015). Vitamin D-binding Protein concentration was quantified by mass spectrometry (Hoofnagle, Eckfeldt, \& Lutsey, 2015). Serum peptides were analyzed for the identification of potential biomarkers in cervical intraepithelial neoplasia patients (Y. Liu et al., 2015). Serum proteins were analyzed for the differential expression of proteins for pulmonary tuberculosis (C. Li et al., 2015). Colorectal cancer diagnosis was based on circulating SERPINA3, CP, CRC, TIMP1, PON1, and LRG1 (Surinova et al., 2015). LYVE-1, REG1A, and TFF1 comprised a biomarker panel for early detection of pancreatic adenocarcinoma (Radon et al., 2015). Analysis of plasma by LC-ESI-MS/MS improved risk prediction for renal disease in patients with hypertension or type 2 diabetes (Pena et al., 2015). Degradation and stabilization of peptide hormones such as GLP-1, GIP, glucagon, and OXM was quantified in human blood specimens (Yi, Warunek, \& Craft, 2015). High-resolution epitope mapping of ADAMTS13 binding epitope for three human monoclonal autoantibodies using deuterium exchange revealed the pathogenic mechanism and a possible therapy for autoimmune TTP syndrome (Casina et al., 2015). A top-down LC/MS+ method assessed actin isoforms as a potential cardiac disease marker (Y. C. Chen et al., 2015). 
SAA1 and SAA2 were quantified in lung cancer plasma using the isotype-specific PRM assays (Y. J. Kim, Gallien, et al., 2015). Lysozyme C and leucine-rich alpha-2 glycoprotein were observed with high-resolution plasma proteome analysis (Glorieux et al., 2015). An autoimmune signature was associated with the development of triple-negative breast cancer that reflects disease pathogenesis (Katayama et al., 2015). Analysis of plasma glycoproteins was correlated to the prognosis in sepsis and was linked to distinct proteins in common pathways (DeCoux et al., 2015). Serum proteomic profiling reveals fragments of MYOM3 as potential biomarkers for monitoring the outcome of therapeutic interventions in muscular dystrophies (Rouillon et al., 2015). Mutations of epidermal growth factor receptor (EGFR) were made using using serum proteomic profiling to predicted tumor response in patients with tage IIIB or IV non-small-cell lung cancer without the ethically invasive collection of patient DNA (L. Yang et al., 2015). Microparticles released from Mycobacterium tuberculosis-infected human macrophages contain increased levels of the type I interferon inducible proteins including ISG15 (Hare et al., 2015). Characterization of endogenously circulating IGFBP-4 fragments may be novel biomarkers for cardiac risk assessment (Konev et al., 2015). A cluster of proteins implicated in kidney disease were increased in high-density lipoprotein isolated from hemodialysis subjects (Shao et al., 2015). The discovery of over one hundred proteins, and MRM assays of 41 proteins, were employed for longitudinal evaluation of candidate protein biomarkers, for disease recurrence in prostate cancer (Tonry et al., 2015). Stromal cell-derived factor 1 (SDF-1 $\alpha$ ) and HSA fragments were examined in myelodysplastic syndromes (Frobel et al., 2015). Inter-alpha-trypsin inhibitor heavy chain H4 (ITIH4), Mannose-binding protein C (MBL2), sex hormone-binding globulin (SHBG), insulin-like growth factor-binding protein 2 (IGFBP2), serum amyloid A protein (SAA1), Orosomucoid 1 (ORM1) and extracellular 
superoxide dismutase [Cu-Zn] (SOD3) were discovered as biomarkers and MRM multiple reaction monitoring assays specifically examined ITIH4 and SAA1 proteins in serum from gastric cancer patients (Subbannayya et al., 2015). Aldosterone-regulated sodium reabsorption, immune cell migration, renin-angiotensin, Th17 dependent immunity, notch signaling, remyelination and vitamin digestion and absorption were associated with specific and shared pathways for multiple sclerosis clinical subtypes (Avsar et al., 2015). Multimarker proteomic profiling for the prediction of cardiovascular mortality in patients with chronic heart failure was made with MALDI analysis of un-identified peaks (Lemesle et al., 2015). Clusterin glycopeptide variant characterization revealed a significant site-specific glycan changes in the plasma of clear cell renal cell carcinoma patients (Gbormittah et al., 2015). Leptin levels were negatively correlated with 2-arachidonoylglycerol in the cerebrospinal fluid of patients with osteoarthritis (Nicholson et al., 2015). Circulating serum-derived microparticles provided novel proteomic biomarkers of spontaneous preterm birth (Ezrin et al., 2015). Von Willdebrand factor (VWF) and ADAMTS13 and S100A7 were identified with endothelial dysfunction in the serum of acute ischemic stroke patients by the iTRAQ-Based LC-MS Approach (Sharma et al., 2015). The proteins LUM, GSN, TIMP1, transglutaminase 2 (TGM2), Stratifin (SFN), and prolinehydroxylated $\alpha$-fibrinogen were analyzed by SRM in pancreatic cancer plasma (Pan, Brentnall, \& Chen, 2015). Apolipoprotein C-II (APOC2), apolipoprotein C-III (APOC3), heparin cofactor 2 (SERPIND1), kininogen-1 (KNG1), phosphatidylinositol-glycan-specific phospholipase D (GPLD1), vitamin K-dependent protein Z (PROZ) increased while multimerin-1 (MMRN1), prostaglandin-H2 D-isomerase (PTGDS), vascular cell adhesion protein 1 (VCAM1), complement and component $\mathrm{C} 1 \mathrm{q}$ receptor (CD93), and vasorin (VASN) were observed to decrease in LC-MS/MS analysis of serum of hepatocellular carcinoma (Tsai et al., 2015). 
Autoantibodies to plasminogen were identified in plasma of patients with prostate cancer (Goufman, Iakovlev, Tikhonova, \& Lokshin, 2015). Platelet glycoprotein V (GP5) ficolin-2 (FCN2), thrombospondin-1 (THBS1) S100-A9 (S100A9) platelet basic protein (PPBP), insulinlike growth factor-binding protein 3 (IGFBP3), cholinesterase (BCHE), lipopolysaccharidebinding protein (LBP), leucine-rich alpha-2-glycoprotein (LRG1), corticosteroid-binding globulin (CBG), and inter-alpha-trypsin inhibitor heavy chain H4 (ITIH4) were related to multiple sclerosis disease progression (Tremlett et al., 2015). Analysis of core-fucosylated glycopeptides revealed voltage-dependent calcium channel subunit alpha-2/delta-1 (CACNA2D1), complement C1q tumor necrosis factor-related protein 3 (C1QTNF3), receptortype tyrosine-protein phosphatase eta (PTPRJ), macrophage mannose receptor 1 (MRC1), Receptor for complement C3Dd also called complement receptor type 2 (CR2), lysyl oxidase homologue 3 (LOXL3), alpha-1-antichymotrypsin (SERPINA3) as markers of pancreatic cancer in serum using mass spectrometry (Z. Tan et al., 2015). Adipocyte enhancer-binding protein 1 (AEBP1), Four And A Half LIM Domains 1 (FHL1) and Follistatin Like 1 (FSTL1) were identified as early markers of myocardial injury (Keshishian et al., 2015). Paraoxonase-3 (PON3) is depleted from the high-density lipoproteins of autoimmune disease patients with subclinical atherosclerosis (Marsillach et al., 2015). Neurofilament medium polypeptide (NEFM) protein concentration was increased in CSF and serum samples from patients with brain injury (Martinez-Morillo et al., 2015). SERPINA1 and ENOSF1 were potential serum biomarkers for gastric cancer (J. Yang et al., 2015). ATP Synthase Subunit $\beta$ (ATP5/6) was a novel biomarker of renal mitochondrial dysfunction in acute kidney injury (Whitaker et al., 2015). Serotransferrin (TF), complement C3c (C3), hemopexin (HPX), zinc-2-alpha glycoprotein (ZAG1), apolipoprotein A1 (Apo-A1), haptoglobin (HAP), and alpha-1-B- 
glycoprotein (A1BG) were observed to correlated with Plasmodium infection (Y. Chen et al., 2015). Malarial infection with Plasmodium knowlesi show immunogenicity to serotransferrin (TF) and hemopexin (HPX), but haptoglobin (HP) antigenicity was only observed with Plasmodium vivax infection (Y. Chen et al., 2015). Glucagon(3-29), [pGlu](3) (3-29), (19-29), (20-29) and (21-29) were identified plasma under typical laboratory sample handling conditions (Howard, Kay, Tan, Minnion, \& Creaser, 2015). Ubiquitin COOH-terminal hydrolase 1 (UCHL1) showed potential as biomarker for destruction of pancreatic beta cells (Brackeva et al., 2015). Cystatin B (CYTB) may be a potential diagnostic biomarker in ovarian clear cell carcinoma (Takaya et al., 2015). Afamin (AFAM), components of the membrane attack complex adiponectin (ADIPO), insulin-like growth factor-binding protein 2 (IGFBP2), serum amyloid protein A, and C-reactive protein distinguished children developing type 1 diabetes in a cohort with HLA-conferred susceptibility (Moulder et al., 2015). Peptides from Mycobacterium tuberculosis were identified in tuberculosis lung samples a including 34 amino acid peptide named PKAp that stimulated antigen-specific IFN- $\gamma$ secretion, T-cell proliferation, cytokine expression, and a cytotoxic activity human tuberculosis Granulomas (Yu et al., 2015).

Measurement of dabigatran plasma concentrations were by calibrated thrombin clotting time in comparison to LC-MS/MS in human volunteers on dialysis (Schmohl, Gansser, Moschetti, \& Stangier, 2015). Verification of the biomarker candidate zyxin for non-small-cell lung cancer was made by a targeted proteomics approach and confirmed by ELISA (Y. J. Kim, Sertamo, et al., 2015). Serum hepcidin-25 levels were linked with high mortality in patients with nonHodgkin lymphoma (Hara, Ando, Tsuchiya, \& Nitta, 2015). Apolipoproteins including A-IV, Complement C3, and fibrinogen alpha, beta and gamma showed variation across lean, type II diabetes and a very low calorie diet (Sleddering et al., 2014). Profliling of unknown serum peaks 
by carboxy methyl cellulose chromatography and MALDI TOF was applied to large B-cell lymphoma (W. Xu et al., 2015). Peripheral $\alpha$-defensins 1 and 2 are elevated in Alzheimer's disease (Watt et al., 2015). Serum protein S100A9, SOD3, and MMP9 were shown to be new diagnostic biomarkers for pulmonary tuberculosis by iTRAQ-coupled two-dimensional LCMS/MS (D. Xu et al., 2015). Complement C4 (C4) and paraoxonase/arylesterase 1 (PON1) were found to be significantly altered when comparing the serum proteome from Multiple Myeloma patients with varying degrees of bone disease (Dowling et al., 2014). Fucosylated hemopexin and complement factor $\mathrm{H}(\mathrm{CFH})$ were quantified in the plasma of patients with liver disease (Benicky, Sanda, Pompach, Wu, \& Goldman, 2014). The pattern of unidentified peaks were examined by MALDI as potential serum biomarkers for glioblastoma (Popescu et al., 2014). Together human $\alpha 1$-Antitrypsin (SERPINA1) secretory leukocyte protease inhibitor (SLPI) Apolipoprotein A IV (APOA4), vitamin D binding protein (VDBP) ) significantly discriminated malignant from benign casesof ovarian cancer but was not as good as CA125 for diagnostic accuracy (Timms et al., 2014). Lack of a $5.9 \mathrm{kDa}$ peptide C-terminal fragment of fibrinogen $\alpha$ chain precedes fibrosis progression in patients with liver disease (Marfa et al., 2014). Serum amyloid A (APP) and clusterin (CLU) were examined as potential predictive biomarkers for severe hand, foot and mouth disease by 2D-DIGE proteomics analysis (J. Liu et al., 2014). Alpha-1-acid glycoprotein 1 (ORM2), alpha-1-antitrypsin (SERPINA1), serotransferrin (TF), haptoglobin (HP), alpha-2-HS-glycoprotein (AHSG), and vitamin D-binding protein (GC) were compared in serum from patients with cervical intraepithelial neoplasia and cervical cancer (Boichenko et al., 2014). Apolipoprotein C-I (APOC1) was a potential Wilms' tumor marker after excluding inflammatory factors (J. Zhang et al., 2014). The proteins BAZ2A, CDK13, DAPK1, DST, EXOSC3, INHBE, KAT2B, KIF20B, SMC1B, and SPAG5 in serum were 
observed to be promising for stratifying patients with pancreatic cancer (Ansari et al., 2015). Melanotransferrin (TRFM) was established as a serological marker of colorectal cancer by secretome analysis and quantitative proteomics (Dus-Szachniewicz et al., 2015; Shin et al., 2014). The epitopes of human apolipoprotein A-I (APOA1) recognized by autoantibodies present in patients with cardiovascular diseases have been established (Teixeira et al., 2014). Complement regulators $\mathrm{C} 1$ inhibitor and factor $\mathrm{H}$, fibronectin, ceruloplasmin, and vitamin $\mathrm{D}$ binding protein apolipoprotein AIV, B-100, and H were significantly decreased in plasma protein profiling of mild cognitive impairment or Alzheimer's disease across two independent cohorts (Muenchhoff et al., 2015). Increased sialyl-Lewis X on ceruloplasmin may be a serum marker of pancreatic cancer (Balmana et al., 2015). Elevated levels of 14-3-3 proteins, serotonin, gamma enolase and pyruvate kinase identified in clinical samples from patients diagnosed with colorectal cancer (Dowling et al., 2015). DEAD-Box Helicase 39A (DDX39A) was discovered as a potential biomarker for unfavorable neuroblastoma using a proteomic approach (Otake et al., 2016). Glypican-1 was identified in cancer exosomes and detects early pancreatic cancer (Melo et al., 2015). Some FDA approved serum tumor markers are glycoproteins, such as CEA (carcinoembryonic antigen), PSA (prostate specific antigen), CA125, CA19-9 (Tian \& Zhang, 2010).

\section{Optimization of Sample Extraction}

Previously the use of precipitation and selective extraction of the pellet (SPE) was shown to be superior to precipitation and analysis of the ACN supernatant (Chertov et al., 2004), ultrafiltration, (Tirumalai et al., 2003) albumin depletion chromatography (R. Pieper et al., 2003) or C18 partition chromatography alone (J. Marshall et al., 2004). Here the extension of this 
technique with a step gradient of acetonitrile/water (ACN/H2O). Analysis of the ACN/water stepwise gradient by LC-ESI-MS/MS showed a high signal to noise ratio.

\section{Contamination, chemical and physical noise and random correlation}

In a separate study, the sources of error in discovery including chemical and physical noise, contamination, and random or stochastic effects of MS/MS correlation were directly addressed using blank solvent gradient runs, the tryptic digestion of laboratory dust, and random MS/MS spectra. Any proteins identified by these three sources of error including keratins, titin, spectrins, distrophins, nebulin, microtubule cross linking factor, obscurin, ankyrins and others were noted.

\section{Sample handling and pre-analytical variation}

In a separate study, in order to control for sample handling and pre-analytical variation EDTA plasma samples were collected on ice and frozen or purposefully degraded over time. Proteins

that were degraded in the controls at room temperature including complement $\mathrm{C} 4 \mathrm{~B}$ and $\mathrm{C} 3$ were noted.

Here, the endogenous tryptic peptides from human EDTA plasma are compared between human heart attack, sepsis, Azheimer's dementia, multiple sclerosis, breast cancer and ovarian cancer and their respective normal EDTA human plasma from six different institutions. Here the random and independent sampling of endogenous tryptic peptides from EDTA plasma by the optimal extraction method analyzed with ANOVA and Chi-Square provide a complete and satisfying statistical analysis (Bowden et al., 2012; Angelica K Florentinus, Peter Bowden, 
Girish Sardana, et al., 2012; Florentinus et al., 2011a) that reveal general acute phase response proteins as well as disease specific polypeptides. 


\section{MATERIALS AND METHODS}

\section{Materials}

The disease and matched control human EDTA plasma was obtained from multiple clinical locations: Heart attack (St Joseph's Hospital of McMaster University), Ovarian and Breast Cancer (Ontario Tumor bank, Mt Sinai Hospital Toronto and Ottawa Hospital), Sepsis (St Michaels Hospital Toronto), Alzheimer's and Multiple Sclerosis (Hospital Zentral, Amsterdam), EDTA plasma collected on ice and room temperature degraded (IBBL Luxembourg). C18 zip tips were obtained from Millipore (Bedford, MA), C18 HPLC resin was from Agilent (Zorbax 300 SB-C18 5-micron). Solvents were obtained from Caledon Laboratories (Georgetown, Ontario, Canada). All other salts and reagents were obtained from Sigma-Aldrich-Fluka (St Louis, MO) except where indicated.

\section{Sample Preparation}

Disposable plastic $1.5 \mathrm{ml}$ sample tubes and plastic pipette tips were used to handle samples. The $200 \mu 1$ of EDTA plasma samples were precipitated with $90 \%$ acetonitrile that contains few peptides followed by the selective extraction of the pellet using a step gradient to achieve selectivity across sub-fractions and thus greater sensitivity (Monika Tucholska et al., 2007). Human EDTA plasma samples $(200 \mu \mathrm{l})$ were precipitated with 9 volumes of acetonitrile. The acetonitrile suspension was separated with a centrifuge at $12,000 \mathrm{RCF}$ for 15 minutes. The acetonitrile supernatant was collected, transferred to a fresh sample tube and dried in a rotary lyophilizer for comparison to the other methods. The organic precipitate (pellet) that contains a much larger total amount of endogenous polypeptides (Monika Tucholska et al., 2009) was manually re-suspending using a step gradient of increasing water content to yield 10 fractions 
from the most hydrophilic $90 \% \mathrm{ACN}$ supernatant to $10 \% \mathrm{ACN}$, followed by $100 \% \mathrm{H}_{2} 0$, and then $5 \%$ formic acid. The extract was clarified with a centrifuge at 12,000 RCF for 2 minutes. The filtrate sample was dried under vacuum in a rotary lyophillizer and stored at $-80^{\circ} \mathrm{C}$. The dried sample was re-dissolved in 5\% formic acid for (SDS-PAGE or) preparative C18 chromatography.

\section{Preparative C18 chromatography}

The peptides of EDTA plasma precipitated in ACN and then solid phase extracted (SPE) from the pellet in a step-gradient were then collected over $\mathrm{C} 18$ preparative partition chromatography. Solid phase extraction with C18 for LC-ESI-MS/MS were performed as previously described (Declan Williams, 2007; J. Marshall et al., 2004; John Marshall et al., 2003; Monika Tucholska et al., 2009; Monika Tucholska et al., 2007). The C18 chromatography resin (zip tip) was wet with $65 \%$ acetonitrile before equilibration in water with 5\% acetonitrile and 5\% formic acid. The plasma extract was dissolved in $200 \mu 1$ of 5\% Acetonitrile and 5\% formic acid in water. The resin was washed with at least five volumes of the same binding buffer. The resin was eluted with one column volume of $65 \%$ acetonitrile in $5 \%$ formic acid. The preparative resin was discarded after a single use.

\section{LC-ESI-MS/MS}

The statistical validity of the LC-ESI-ion trap for non-human protein standards and plasma was demonstrated prior to collecting data (Bowden et al., 2012; P. Zhu, P. Bowden, M. Tucholska, et al., 2011; Peihong Zhu et al., 2011). The traps were cleaned and tested for sensitivity and accuracy between each patient. In order to entirely prevent any possibility of cross 
contamination, a new analytical column was struck and quality controlled with a mixture of three non-human protein standards (Bowden et al., 2012) prior to recording each patient's peptides. The stepwise extractions were collected and desalted over $\mathrm{C} 18$ preparative micro columns were eluted in $2 \mu \mathrm{L}$ of $65 \% \mathrm{ACN}$ and $5 \%$ formic acid (see above). The $2 \mu \mathrm{L}$ eluants of the gradient steps individually collected by preparative $\mathrm{C} 18$ reversed phase were diluted ten-fold with 5\% formic acid in water and 5\% acetonitrile loaded manually into a $20 \mu \mathrm{l}$ metal sample loop before injecting onto the analytical column via a Rhodynne injector. Endogenous peptide samples were analyzed over a discontinuous gradient at a flow rate of $\sim 10$ microlitres per minute generated with an Agilent 1100 series capillary pump and split upstream of the column during recording to about $\sim 200 \mathrm{nl}$ per minute. The separation was performed with a C18 $(150 \mathrm{~mm} \times 0.3 \mathrm{~mm})$ fritted capillary column. The acetonitrile profile was started at 5\%, ramped to $12 \%$ after 5 minutes and then increased to $65 \%$ over $\sim 30$ minutes, remained at $65 \%$ for 5 minutes, decreased to $50 \%$ for 15 minutes and then declined to a final proportion of 5\% prior to injection of the next step fraction from the same patient. The nano HPLC effluent was analyzed by ESI ionization with detection by MS and fragmentation by MS/MS with a linear quadrupole ion trap (Schwartz et al., 2002). The device was set to collect the precursor for up to 200 milli second prior to MS/MS fragmentation with up to four fragmentations per precursor ion that were averaged. At least 1020 individual independent control $(\mathrm{n} \geq 10)$ and disease $(\mathrm{n} \geq 10)$ samples from each institution/study (McMaster, St Michael, Mt Sinai, Hospital Zentraal, ICBH) were precipitated, fractionated over a step gradient and collected over $\mathrm{C} 18$ for manual injection. The entire process was performed on the same set of three identical ion traps that were statistically similar. 


\section{Correlation Analysis}

Correlation analysis of ion trap data was performed with a goodness of fit test using MASCOT (Perkins et al., 1999), OMSSA (L. Y. Geer et al., 2004), X!TANDEM (R. Craig \& R. C. Beavis, 2004) and SEQUEST (Yates et al., 1995) algorithms to match tandem mass spectra to peptide sequences from the Homo sapiens RefSeq Library obtained September 2015. Endogenous peptides were searched only as fully tryptic peptides or phosphopeptides on separate servers for each algorithm and the results combined in SQL Server. The ion trap data was analyzed within $\pm 3 \mathrm{~m} / \mathrm{z}$ from precursors peptides considered from 300 to $2000 \mathrm{~m} / \mathrm{z}$ with a tolerance of $0.5 \mathrm{Da}$ error in the fragments (R. Craig \& R. C. Beavis, 2004). Only the single best fit peptide from the MS/MS at charge states of +2 versus +3 were accepted with additional acetylation or oxidation of methionine and with possible loss of water or ammonia or the addition of phosphate. The resulting accession numbers, actual and estimated masses, correlated peptide sequences, peptide and protein scores, resulting protein sequences and other associated data were captured and assembled together in an SQL Server relational database (Bowden et al., 2009).

\section{Data sorting, transformation and visualization}

At least 10-20 individual independent control $(\mathrm{n} \geq 10)$ and disease ( $\mathrm{n} \geq 10)$ samples were separated into 10 sub-fractions from each institution/study (McMaster, St Michael, Mt Sinai, Hospital Zentraal, ICBH) were randomly and independently sampled by the linear quadrupole ion trap that provided the precursor ion intensity values of the MS/MS spectra. The MS/MS spectra were correlated to specific tryptic peptide sequence by the X!TANDEM, OMSSA, MASCOT and SEQUEST data from the SQL database was plotted using the Statistical Analysis System (SAS) (Bowden et al., 2009). The MS and MS/MS spectra together with the results of the correlation 
algorithms were parsed into and SQL Server that was analyzed by the generic R statistical analysis system (Bowden et al., 2009; Bowden et al., 2010; Bowden et al., 2012; Angelica K Florentinus, Peter Bowden, Girish Sardana, et al., 2012; Florentinus et al., 2011a). The peptide to protein correlation frequency counts for each gene symbol were summed for all diseases versus all controls to spot non-specific acute phase markers, versus those specific for one disease versus its institution/study matched control and the average of all 6 diseases versus institution/study controls was determined with the Chi Square distribution using equations i:

i) $\quad(\text { Disease-Control })^{\wedge} 2 /($ Control+1)

The intensity data was $\log 10$ transformed, tested for normality and analyzed across institution/study and diseases verses controls by means, standard errors and ANOVA. 


\section{RESULTS}

\section{LC-ESI-MS/MS}

The pool of endogenous tryptic (TRYP) or tryptic phosphopeptides (STYP) were selectively extracted and compared to the control by random and independent sampling without replacement by solid phase extraction followed by liquid chromatography, electrospray ionization and tandem mass spectrometry (LC-ESI-MS/MS). Some 15,043,678 MS/MS spectra of greater than 1000 intensity counts from 2881 LC-ESI-MS/MS runs were computed together in SQL server for analysis with the $\mathrm{R}$ generic statistical analysis system in a matrix of disease and control ratios that reveals the set of blood peptides specific to each disease state. Nearly every one of the $\sim 157,000$ protein in the federated library of human proteins from RefSEQ, ENSEMBL and SwissProt were detected at least once under the stringent filter condition of best charge stated and peptide sequence fit (Fig. 1).

\section{The sum of proteins from all diseases versus all controls compared by chi square}

There is a great deal of variation between the 6 sets of control samples from cancer, heart attack, Alzheimer's, Multiple Sclerosis and Sepsis and reference controls. Some of the variation likely results from the institution/study source of the sample. However, the presence of so many disease and controls from matched institutions recorded together under the same conditions together with the Chi Square test can reveal many important facets of the data set that lead to strong conclusions. Comparing all the disease results to all the controls reveals summed over all 6 studies revealed a large set of acute phase proteins previously observed in many different disease states that clearly shows the disease specific variation was large compared to 
institution/study error or the biological and sampling error for many hundreds or even thousands of proteins (Fig. 2). Peptides from haptogloblin, Serpin, complements, fibrinogens, hemopexin, antitrypsin, amyloids, apolipoproteins and others were significantly increased in the disease samples compared to the controls showing Chi Square values approaching up to 50,000. A large number of molecules like amyloids or haptoglobin were observed that showed significant values $(\chi 2 \geq 25)$ in the institution/study-specific disease versus control comparison but that were also significant when all diseases were summed and compared to all controls and so these proteins are acute phase or non-specific markers of poor health. The list of proteins that were observed to significantly increase in the sum of all disease compared to the sum of all the controls revealed a set of non-specific or acute phase markers of disease (Table I). However some disease-specific proteins showed such intense expression that they may be detected even against the background of all other diseases by Chi Square (Table I).

\section{The proteins of disease versus normal control compared by chi square}

Variation may also be due to disease-specific molecules but these are confounded by patient biological error and LC-ESI-MS/MS sampling error which is known to be large in the analysis of blood and so no computation strategy could resolve these errors with only 10-20 samples of each disease and control. Two ways to estimate the disease-specific molecules is to use the Chi Square test for each disease versus its institution/study matched control, and the average of all six institution/study controls, that should avoid error due to differences in sample handling between institutions. Comparing each disease to its corresponding controls, and the average of all 6 study/institution controls, provided a computationally simple means to detected disease specific molecules while avoiding both the variation between institutions and proteins shared 
across all diseases (Fig. 2). In contrast, there were peptides or phosphopeptides from many proteins that were specifically associated with one disease $\left(\chi^{2} \geq 25\right)$ but where the Chi square value from the sum of all diseases versus all controls was not large and so these may represent disease specific markers, biological variation or sampling error and will required independent targeted analysis to reach definitive conclusions (Table I). There was significant diseasespecific variation in peptides or phosphopeptides from cellular proteins, membrane proteins, Nucleic acid binding proteins, signaling factors, metabolic enzymes and others including uncharacterized factors between disease states $(n \geq 10)$ versus the matched institution/study control ( $\mathrm{n} \geq 10)$ the Chi Square test.

\section{STRING Analysis}

In computationally independent method to ensure the proteins detected as specific to each disease are not just the result of some random process we analyzed the distribution of the known protein-protein interactions and the distribution of the cellular location, molecular function and biological processes of the protein identified from endogenous peptides with respect to a random sampling of the human genome. There were many protein interactions apparent between the proteins computed to be specific to diseases (Fig. 3). All of the diseases showed statistically significant enrichment of proteins interactions and Gene Ontology terms that were consistent with structural and functional relationships between the proteins identified in each disease compared to a random sampling of the human genome (Table II).

\section{Normality of the $\log 10$ precursor intensity values and ANOVA across treatments}


A set of almost $\sim 800$ gene symbols that showed Chi Square $\left(\chi^{2}\right)$ values of $\geq 25$, that roughly corresponds to 5 fold or greater difference between the disease and the appropriate control were observed. The peptides and precursor intensity values of the set of Gene Symbols that were observed to vary by Chi Square were collected together and analyzed in R (a descendent of S) that was used previously to analyze MALDI data (John Marshall et al., 2003). The Gene Symbols that were observed to vary between disease and controls showed precursor intensity values that ranged from E3 to E7 counts and the standard error of the these peptides $\log _{10}$ transformation of the precursor intensity approximated the normal distribution as assessed by the quantile plot (Fig. 4). The mean precursor intensity was compared across treatments that showed strong differences in average peptide intensity values as judged by ANOVA followed by the Tukey Kramer test. The ANOVA analysis showed very significant variation between institutions 


\section{DISCUSSION}

\section{Strategy of examining endogenous tryptic peptides and phosphopeptides}

The use of step wise organic solvent gradients for the selective extraction of tryptic peptides or phosphopeptides resulted in MS/MS correlations to protein that showed a variation across specific disease versus control samples. It was possible to fractionate the blood fluids using differential solubility in a salt step gradient over multiple partition chromatography resins, to reveal endogenous tryptic peptides and phosphopeptides from thousands of blood proteins.

\section{Fully tryptic peptides between the sums of all diseases versus all controls}

The major peptides of human blood fluids have been compared using multivariate approaches to look for patterns (Ardekani, Liotta, \& Petricoin, 2002; Petricoin et al., 2002) but multivariate analysis was shown to provide no additional benefit over traditional statistical methods such as ANOVA (Baggerly et al., 2003; Eckel-Passow et al., 2009; John Marshall et al., 2003). The ANOVA analysis indicated a significant effect of institution/study and disease on precursor intensity. All of the diseased from the six institutions/studies were combined and then compared to the union of all controls by Chi Square that revealed disease specific variation that cannot be due to sampling error between institutions. Many of the proteins that were significantly increased in disease compared to the 6 sets of controls were merely acute phase or other proteins that are elevated in more than one diseases including amyloids, complements, haptoglobin, $\operatorname{IgG}$ chains, IITI, anti-trypsin, alpha 2 macroglobulin, fibrinopeptides fibrinogens, hemopexin, apolipoproteins. The proteins that were observed to vary between the disease and controls were not the $\mathrm{C} 4 \mathrm{~B}$ or $\mathrm{C} 3$ proteins observed to degrade at room temperature and were not the same as chemical or physical noise, random spectra or laboratory contaminants. The results here with 
Haptoglobin in Ovarian Cancer agree with previous results [2] that some proteins detected from tryptic peptides in serum or plasma by ESI represent an increase in the circulating levels of that protein. While large increases in amyloids or other acute phase proteins were observed, the greater representation of these acute phase response proteins is not likely to be highly specific to one disease.

\section{Fully tryptic peptides between each disease versus control}

In an independent method to avoid the confounding effect of between institution/study variation, each disease was compared only to its specifically matched control, and the average of all 6 Institution/study controls, by Chi analysis. The fractionation of human EDTA blood plasma followed by liquid chromatography and tandem mass spectrometry with a linear quadrupole ion trap showed that fully tryptic endogenous peptides showed striking differences the frequency of some protein, peptides or phospho- peptides between disease and control states including peptides or phosphopeptides from cellular proteins, membrane proteins, Nucleic acid binding proteins, signaling factors, metabolic enzymes and others including uncharacterized factors between disease states ( $\mathrm{n} \geq 10$ ) versus the matched institution/study control ( $\mathrm{n} \geq 10)$ the Chi Square test that may show some relation to the disease processes. However, an increase in the proteins corresponding to the peptides discovered here would have to be established on a case by case basis using an immunological or targeted analysis of the parent protein. It remains possible that specific phosphorylation of acute phase or other common blood proteins might provide some greater utility than increases in these proteins alone (Williams et al., 2010; P. Zhang et al., 2012). 


\section{STRING analysis}

The results of the network analysis by STRING indicated that the peptides and proteins detected were not merely a random selection of the proteins from the human genome but seemed to show statistically significant protein-protein interactions and showed significant enrichment of proteins associated with specific cellular components, biological processes, molecular functions. The significant results from STRING analysis seemed to indicate that at least some of the results could not have resulted from differences in sampling error between disease and controls samples.

\section{Potential Utility of the Disease Specific peptides and proteins}

Specific proteins were detected from endogenous peptides from each disease versus its corresponding control. The Differences in tryptic peptides and phosphopeptides between randomly sampled disease and controls samples may represent sampling error. Additionally, some of the proteins identified may like TTN, NEB, SYNE1, MACF1, OBSC ANK1 and others are likely the results of random mis-correlations from un expected modifications or the peptide or the recording of background noise. Where real differences may exist in endogenous tryptic peptides or tryptic phosphopeptides between control and disease these may reflect a lower stability of the parent proteins in the presence of circulating proteases in the EDTA plamsa and do not necessarily reflect an increase in the concentration of the parent proteins in circulation. However its is possible the the increased in the endogenous peptides itself may act as a diagnostic in some cases. We cannot rule out that at least some of the parent proteins from endogenous peptides detected more specifically in one disease may reflect and increased concentration of the parent protein. Organic extraction of peptides and/or partition chromatography of the parent proteins overs ion exchange of heparin resins prior to digestion 
LC-ESI-MS/MS (J. Marshall et al., 2004; Monika Tucholska et al., 2009) might provide for the targeted confirmation of the peptides and proteins revealed here from independent samples. 


\section{REFERENCES}

Abbatiello, S. E., Schilling, B., Mani, D. R., Zimmerman, L. J., Hall, S. C., MacLean, B., . . Carr, S. A. (2015). Large-Scale Interlaboratory Study to Develop, Analytically Validate and Apply Highly Multiplexed, Quantitative Peptide Assays to Measure Cancer-Relevant Proteins in Plasma. Mol Cell Proteomics, 14(9), 2357-2374. doi:10.1074/mcp.M114.047050

Albrethsen, J., Bogebo, R., Olsen, J., Raskov, H., \& Gammeltoft, S. (2006). Preanalytical and analytical variation of surface-enhanced laser desorption-ionization time-of-flight mass spectrometry of human serum. Clin Chem Lab Med, 44(10), 1243-1252.

Allan, D., Auger, J., \& Crumpton, M. J. (1972). Purification of concanavalin A receptor from pig lymphocyte plasma membrane. Biochem J, 126(3), 6P.

An, S., Shen, C., Liu, X., Chen, L., Xu, X., Rong, M., . . Lai, R. (2013). Alpha-actinin is a new type of house dust mite allergen. PLOS ONE, 8(12), e81377. doi:10.1371/journal.pone.0081377

Anderson, C. L., Shen, L., Eicher, D. M., Wewers, M. D., \& Gill, J. K. (1990). Phagocytosis mediated by three distinct Fc gamma receptor classes on human leukocytes. Journal of Experimental Medicine, 171(4), 1333-1345.

Ansari, D., Andersson, R., Bauden, M. P., Andersson, B., Connolly, J. B., Welinder, C., . . Marko-Varga, G. (2015). Protein deep sequencing applied to biobank samples from patients with pancreatic cancer. J Cancer Res Clin Oncol, 141(2), 369-380. doi:10.1007/s00432-014-1817-x

Ardekani, A. M., Liotta, L. A., \& Petricoin, E. F., 3rd. (2002). Clinical potential of proteomics in the diagnosis of ovarian cancer. Expert Rev Mol Diagn, 2(4), 312-320.

Avsar, T., Durasi, I. M., Uygunoglu, U., Tutuncu, M., Demirci, N. O., Saip, S., . . Tahir Turanli, E. (2015). CSF Proteomics Identifies Specific and Shared Pathways for Multiple Sclerosis Clinical Subtypes. PLOS ONE, 10(5), e0122045. doi:10.1371/journal.pone.0122045

Baggerly, K. A., Morris, J. S., Wang, J., Gold, D., Xiao, L. C., \& Coombes, K. R. (2003). A comprehensive approach to the analysis of matrix-assisted laser desorption/ionization-time of flight proteomics spectra from serum samples. Proteomics, 3(9), 1667-1672.

Bai, J., He, A., Zhang, W., Huang, C., Yang, J., Yang, Y., . . Zhang, Y. (2013). Potential biomarkers for adult acute myeloid leukemia minimal residual disease assessment searched by serum peptidome profiling. Proteome Sci, 11, 39. doi:10.1186/1477-5956-11-39

Balmana, M., Sarrats, A., Llop, E., Barrabes, S., Saldova, R., Ferri, M. J., . . Peracaula, R. (2015). Identification of potential pancreatic cancer serum markers: Increased sialyl-Lewis $X$ on ceruloplasmin. Clin Chim Acta, 442, 56-62. doi:10.1016/j.cca.2015.01.007

Banks, R. E. (2008). Preanalytical influences in clinical proteomic studies: raising awareness of fundamental issues in sample banking. Clin Chem, 54(1), 6-7.

Bayer, E. A., \& Wilchek, M. (1980). The use of the avidin-biotin complex as a tool in molecular biology. Methods Biochem Anal, 26, 1-45.

Bedin, C., Crotti, S., Ragazzi, E., Pucciarelli, S., Agatea, L., Tasciotti, E., . . Agostini, M. (2015). Alterations of the Plasma Peptidome Profiling in Colorectal Cancer Progression. J Cell Physiol. doi:10.1002/jcp.25196

Ben-Chetrit, E., Chan, E., Sullivan, K. F., \& Tan, E. M. (1988). A 52-kD protein is a novel component of the SS-A/Ro antigenic particle. Journal of Experimental Medicine, 167(5), 1560-1571.

Benicky, J., Sanda, M., Pompach, P., Wu, J., \& Goldman, R. (2014). Quantification of fucosylated hemopexin and complement factor $\mathrm{H}$ in plasma of patients with liver disease. Anal Chem, 86(21), 10716-10723. doi:10.1021/ac502727s

Benjamini., Y., \& Hochberg, Y. (1995). Controlling false discovery rate: A practical approach to multiple testing. Journal of the Royal Statistical Society, 57(1), 289-300. 
Benovic, J. L., Shorr, R. G., Caron, M. G., \& Lefkowitz, R. J. (1984). Mammalian. beta. 2-adrenergic receptor: purification and characterization. Biochemistry, 23(20), 4510-4518.

Bereman, M. S., Johnson, R., Bollinger, J., Boss, Y., Shulman, N., MacLean, B., . . MacCoss, M. J. (2014). Implementation of statistical process control for proteomic experiments via LC MS/MS. J Am SoC Mass Spectrom, 25(4), 581-587. doi:10.1007/s13361-013-0824-5

Bery, A., Leung, F., Smith, C. R., Diamandis, E. P., \& Kulasingam, V. (2014). Deciphering the ovarian cancer ascites fluid peptidome. Clin Proteomics, 11(1), 13. doi:10.1186/1559-0275-11-13

Boccardo, F., Rubagotti, A., Nuzzo, P. V., Argellati, F., Savarino, G., Romano, P., . . Profumo, A. (2015). Matrix-assisted laser desorption/ionisation (MALDI) TOF analysis identifies serum angiotensin II concentrations as a strong predictor of all-cause and breast cancer (BCa)-specific mortality following breast surgery. Int J Cancer, 137(10), 2394-2402. doi:10.1002/ijc.29609

Boehm, A. M., Putz, S., Altenhofer, D., Sickmann, A., \& Falk, M. (2007). Precise protein quantification based on peptide quantification using iTRAQ. BMC Bioinformatics, 8, 214. doi:10.1186/14712105-8-214

Boichenko, A. P., Govorukhina, N., Klip, H. G., van der Zee, A. G., Guzel, C., Luider, T. M., \& Bischoff, R. (2014). A panel of regulated proteins in serum from patients with cervical intraepithelial neoplasia and cervical cancer. J Proteome Res, 13(11), 4995-5007. doi:10.1021/pr500601w

Bolland, S., \& Ravetch, J. V. (1999). Inhibitory pathways triggered by ITIM-containing receptors. Advances in immunology, 72, 149-177.

Bowden, P., Beavis, R., \& Marshall, J. (2009). Tandem mass spectrometry of human tryptic blood peptides calculated by a statistical algorithm and captured by a relational database with exploration by a general statistical analysis system. Journal of Proteomics, 73, 103-111. doi:10.1016/j.jprot.2009.08.004

Bowden, P., Pendrak, V., Zhu, P., \& Marshall, J. G. (2010). Meta sequence analysis of human blood peptides and their parent proteins. Journal of Proteomics, 73, 1163-1175. doi:10.1016/j.jprot.2010.02.007

Bowden, P., Thavarajah, T., Zhu, P., McDonell, M., Thiele, H., \& Marshall, J. G. (2012). Quantitative statistical analysis of standard and human blood proteins from liquid chromatography, electrospray ionization, and tandem mass spectrometry. Journal of Proteome Research, 11, 2032-2047. doi:10.1021/pr2000013

Brackeva, B., De Punt, V., Kramer, G., Costa, O., Verhaeghen, K., Stange, G., . . Martens, G. A. (2015). Potential of UCHL1 as biomarker for destruction of pancreatic beta cells. J Proteomics, 117, 156167. doi:10.1016/j.jprot.2015.01.009

Bruhns, P. (2012). Properties of mouse and human IgG receptors and their contribution to disease models. Blood, 119(24), 5640-5649.

Burillo, E., Lindholt, J. S., Molina-Sanchez, P., Jorge, I., Martinez-Pinna, R., Blanco-Colio, L. M., ... MartinVentura, J. L. (2015). ApoA-I/HDL-C levels are inversely associated with abdominal aortic aneurysm progression. Thromb Haemost, 113(6), 1335-1346. doi:10.1160/TH14-10-0874

Callesen, A. K., Vach, W., Jorgensen, P. E., Cold, S., Mogensen, O., Kruse, T. A., . . Madsen, J. S. (2008). Reproducibility of mass spectrometry based protein profiles for diagnosis of breast cancer across clinical studies: a systematic review. J Proteome Res, 7(4), 1395-1402.

Canto, E., Tintore, M., Villar, L. M., Borras, E., Alvarez-Cermeno, J. C., Chiva, C., . . Comabella, M. (2014). Validation of semaphorin 7A and ala-beta-his-dipeptidase as biomarkers associated with the conversion from clinically isolated syndrome to multiple sclerosis. J Neuroinflammation, 11, 181. doi:10.1186/s12974-014-0181-8

Cargile, B. J., Bundy, J. L., \& Stephenson, J. L., Jr. (2004). Potential for false positive identifications from large databases through tandem mass spectrometry. J Proteome Res, 3(5), 1082-1085. 
Casina, V. C., Hu, W., Mao, J. H., Lu, R. N., Hanby, H. A., Pickens, B., . . Zheng, X. L. (2015). Highresolution epitope mapping by HX MS reveals the pathogenic mechanism and a possible therapy for autoimmune TTP syndrome. Proc Natl Acad Sci U S A, 112(31), 9620-9625. doi:10.1073/pnas.1512561112

Chen, Y., Chan, C. K., Kerishnan, J. P., Lau, Y. L., Wong, Y. L., \& Gopinath, S. C. (2015). Identification of circulating biomarkers in sera of Plasmodium knowlesi-infected malaria patients--comparison against Plasmodium vivax infection. BMC Infect Dis, 15, 49. doi:10.1186/s12879-015-0786-2

Chen, Y. C., Ayaz-Guner, S., Peng, Y., Lane, N. M., Locher, M. R., Kohmoto, T., . . Ge, Y. (2015). Effective top-down LC/MS+ method for assessing actin isoforms as a potential cardiac disease marker. Anal Chem, 87(16), 8399-8406. doi:10.1021/acs.analchem.5b01745

Chertov, O., Biragyn, A., Kwak, L. W., Simpson, J. T., Boronina, T., Hoang, V. M., . . Fisher, R. J. (2004). Organic solvent extraction of proteins and peptides from serum as an effective sample preparation for detection and identification of biomarkers by mass spectrometry. Proteomics, 4(4), 1195-1203.

Cheung, C. S., Anderson, K. W., Benitez, K. Y., Soloski, M. J., Aucott, J. N., Phinney, K. W., \& Turko, I. V. (2015). Quantification of Borrelia burgdorferi Membrane Proteins in Human Serum: A New Concept for Detection of Bacterial Infection. Anal Chem, 87(22), 11383-11388. doi:10.1021/acs.analchem.5b02803

Chin, S. T., Ignatius, J., Suraiya, S., Tye, G. J., Sarmiento, M. E., Acosta, A., . . Lim, T. S. (2015). Comparative study of IgA VH 3 gene usage in healthy TST(-) and TST(+) population exposed to tuberculosis: deep sequencing analysis. Immunology, 144(2), 302-311. doi:10.1111/imm.12372

Chiu, W. J., Ling, T. K., Chiang, H. P., Lin, H. J., \& Huang, C. C. (2015). Monitoring Cluster lons Derived from Aptamer-Modified Gold Nanofilms under Laser Desorption/Ionization for the Detection of Circulating Tumor Cells. ACS Appl Mater Interfaces, 7(16), 8622-8630. doi:10.1021/acsami.5b00731

Cominetti, O., Nunez Galindo, A., Corthesy, J., Oller Moreno, S., Irincheeva, I., Valsesia, A., . .. Dayon, L. (2016). Proteomic Biomarker Discovery in 1000 Human Plasma Samples with Mass Spectrometry. J Proteome Res, 15(2), 389-399. doi:10.1021/acs.jproteome.5b00901

Cooper, B. (2012). The problem with peptide presumption and the downfall of target-decoy false discovery rates. Anal Chem, 84(22), 9663-9667. doi:10.1021/ac303051s

Corbett-Nelson, E. F., Mason, D., Marshall, J. G., Collette, Y., \& Grinstein, S. (2006). Signaling-dependent immobilization of acylated proteins in the inner monolayer of the plasma membrane. J Cell Biol, 174(2), 255-265.

Cottrell, J. S., \& London, U. (1999). Probability-based protein identification by searching sequence databases using mass spectrometry data. Electrophoresis, 20(18), 3551-3567.

Craig, R., \& Beavis, R. C. (2003). A method for reducing the time required to match protein sequences with tandem mass spectra. Rapid Commun Mass Spectrom, 17(20), 2310-2316.

Craig, R., \& Beavis, R. C. (2004). TANDEM: matching proteins with tandem mass spectra. Bioinformatics, 20(9), 1466-1467.

Craig, R., \& Beavis, R. C. (2004). TANDEM: matching proteins with tandem mass spectra. Bioinformatics, 20(9), 1466-1467.

Creutz, C. E., \& Harrison, J. R. (1984). Clathrin light chains and secretory vesicle binding proteins are distinct. Nature, 308(5955), 208-210.

Crowley, M. T., Costello, P. S., Fitzer-Attas, C. J., Turner, M., Meng, F., Lowell, C., . . DeFranco, A. L. (1997). A critical role for Syk in signal transduction and phagocytosis mediated by Fcgamma receptors on macrophages. J Exp Med, 186(7), 1027-1039.

Cuatrecasas, P. (1972). Affinity chromatography and purification of the insulin receptor of liver cell membranes. Proc Natl Acad Sci U S A, 69(5), 1277-1281. 
Cuatrecasas, P., \& Parikh, I. (1974). Affinity chromatography of insulin receptors. Methods Enzymol, 34, 653-670.

Cuatrecasas, P., Wilchek, M., \& Anfinsen, C. B. (1968). Selective enzyme purification by affinity chromatography. Proc Natl Acad Sci U S A, 61(2), 636-643.

da Costa, A. N., Plymoth, A., Santos-Silva, D., Ortiz-Cuaran, S., Camey, S., Guilloreau, P., ... Hainaut, P. (2015). Osteopontin and latent-TGF beta binding-protein 2 as potential diagnostic markers for HBV-related hepatocellular carcinoma. Int J Cancer, 136(1), 172-181. doi:10.1002/ijc.28953

Daëron, M. (1997). Fc receptor biology. Annual review of immunology, 15(1), 203-234.

de Noo, M. E., Tollenaar, R. A., Ozalp, A., Kuppen, P. J., Bladergroen, M. R., Eilers, P. H., \& Deelder, A. M. (2005). Reliability of human serum protein profiles generated with C8 magnetic beads assisted MALDI-TOF mass spectrometry. Anal Chem, 77(22), 7232-7241.

Declan Williams, P. Z., Peter Bowden, Catherine Stacey, Mike McDonell, Paul Kowalski, Jane Marie Kowalski, Ken Evans, Eleftherios P Diamandis, K.W. Michael Siu, John Marshall. (2007). Comparison of Methods to Examine the Endogenous Peptides of Fetal Calf Serum Clinical Proteomics. Clinical Proteomics, 2(1), 67-89.

DeCoux, A., Tian, Y., DeLeon-Pennell, K. Y., Nguyen, N. T., de Castro Bras, L. E., Flynn, E. R., ... Lindsey, M. L. (2015). Plasma Glycoproteomics Reveals Sepsis Outcomes Linked to Distinct Proteins in Common Pathways. Crit Care Med, 43(10), 2049-2058. doi:10.1097/CCM.0000000000001134

Denecker, G., Ovaere, P., Vandenabeele, P., \& Declercq, W. (2008). Caspase-14 reveals its secrets. J Cell Biol, 180(3), 451-458. doi:10.1083/jcb.200709098

Desjardins, M., \& Griffiths, G. (2003). Phagocytosis: latex leads the way. Current opinion in cell biology, 15(4), 498-503.

Diamandis, E. P., \& Christopoulos, T. K. (1991). The biotin-(strept) avidin system: principles and applications in biotechnology. Clinical chemistry, 37(5), 625-636.

Dionne, R., Forest, J. C., Moutquin, J. M., De Grandpre, P., \& Masse, J. (1994). Electrophoretic method for separating small peptides in serum without extraction of macromolecules: application to the detection of preeclampsia. Clin Biochem, 27(2), 99-103.

Dobo, J., Major, B., Kekesi, K. A., Szabo, I., Megyeri, M., Hajela, K., ... Gal, P. (2011). Cleavage of kininogen and subsequent bradykinin release by the complement component: mannose-binding lectin-associated serine protease (MASP)-1. PLoS ONE, 6(5), e20036. doi:10.1371/journal.pone.0020036

Doecke, J. D., Laws, S. M., Faux, N. G., Wilson, W., Burnham, S. C., Lam, C. P., . . Lifestyle Research, G. (2012). Blood-based protein biomarkers for diagnosis of Alzheimer disease. Arch Neurol, 69(10), 1318-1325. doi:10.1001/archneurol.2012.1282

Doi, E., Shibata, D., \& Matoba, T. (1981). Modified colorimetric ninhydrin methods for peptidase assay. Anal Biochem, 118(1), 173-184.

Dowling, P., Hayes, C., Ting, K. R., Hameed, A., Meiller, J., Mitsiades, C., . . O'Gorman, P. (2014). Identification of proteins found to be significantly altered when comparing the serum proteome from Multiple Myeloma patients with varying degrees of bone disease. BMC Genomics, 15, 904. doi:10.1186/1471-2164-15-904

Dowling, P., Hughes, D. J., Larkin, A. M., Meiller, J., Henry, M., Meleady, P., . . Clynes, M. (2015). Elevated levels of 14-3-3 proteins, serotonin, gamma enolase and pyruvate kinase identified in clinical samples from patients diagnosed with colorectal cancer. Clin Chim Acta, 441, 133-141. doi:10.1016/j.cca.2014.12.005

Dus-Szachniewicz, K., Ostasiewicz, P., Wozniak, M., Kolodziej, P., Wisniewski, J. R., \& Ziolkowski, P. (2015). Pattern of Melanotransferrin Expression in Human Colorectal Tissues: An Immunohistochemical Study on Potential Clinical Application. Anticancer Res, 35(12), 65516561. 
Eckel-Passow, J. E., Oberg, A. L., Therneau, T. M., \& Bergen, H. R., 3rd. (2009). An insight into highresolution mass-spectrometry data. Biostatistics, 10(3), 481-500.

Elschenbroich, S., Kim, Y., Medin, J. A., \& Kislinger, T. (2010). Isolation of cell surface proteins for mass spectrometry-based proteomics. Expert review of proteomics, 7(1), 141-154.

Eng, J. K., McCormack, A. L., \& Yates, J. R. (1994). An approach to correlate tandem mass spectral data of peptides with amino acid sequences in a protein database. J Am Soc Mass Spectrom, 5(11), 976989.

Esposito, S., Deventer, K., Geldof, L., \& Van Eenoo, P. (2015). In vitro models for metabolic studies of small peptide hormones in sport drug testing. J Pept Sci, 21(1), 1-9. doi:10.1002/psc.2710

Eyford, B. A., Ahmad, R., Enyaru, J. C., Carr, S. A., \& Pearson, T. W. (2013). Identification of Trypanosome proteins in plasma from African sleeping sickness patients infected with T. b. rhodesiense. PLoS ONE, 8(8), e71463. doi:10.1371/journal.pone.0071463

Ezrin, A. M., Brohman, B., Willmot, J., Baxter, S., Moore, K., Luther, M., . . Sibai, B. (2015). Circulating serum-derived microparticles provide novel proteomic biomarkers of spontaneous preterm birth. Am J Perinatol, 32(6), 605-614. doi:10.1055/s-0035-1547322

Fagerberg, L., Jonasson, K., von Heijne, G., Uhlén, M., \& Berglund, L. (2010). Prediction of the human membrane proteome. Proteomics, 10(6), 1141-1149.

Fang, M., Webster, T. F., \& Stapleton, H. M. (2015). Effect-Directed Analysis of Human Peroxisome Proliferator-Activated Nuclear Receptors (PPARgamma1) Ligands in Indoor Dust. Environ Sci Technol, 49(16), 10065-10073. doi:10.1021/acs.est.5b01524

Ferrante, A., Beard, L. J., \& Feldman, R. G. (1990). IgG subclass distribution of antibodies to bacterial and viral antigens. The Pediatric infectious disease journal, 9(8), 516-524.

Flannagan, R. S., Jaumouille, V., \& Grinstein, S. (2012). The cell biology of phagocytosis. Annu Rev Pathol, 7, 61-98. doi:10.1146/annurev-pathol-011811-132445

Florentinus-Mefailoski, A., \& Marshall, J. G. (2014). A pyridoxamine-5-phosphate ELIMSA substrate for linear absolute quantification of alkaline phosphatase to the yoctomole range applied to prostate specific antigen. Anal Chem. doi:10.1021/ac502572a

Florentinus-Mefailoski, A., \& Marshall, J. G. (2016). Linear quantification of a streptavidin-alkaline phosphatase probe for enzyme-linked immuno mass spectrometric assay. Anal Biochem, 503, 50-55. doi:10.1016/j.ab.2016.02.014

Florentinus-Mefailoski, A., Safi, F., \& Marshall, J. G. (2014). Enzyme Linked Immuno Mass Spectrometric Assay (ELIMSA). J Proteomics, 96, 343-352. doi:10.1016/j.jprot.2013.11.022

Florentinus-Mefailoski, A., Soosaipillai, A., Dufresne, J., Diamandis, E. P., \& Marshall, J. G. (2015). An enzyme-linked immuno-mass spectrometric assay with the substrate adenosine monophosphate. Anal Bioanal Chem, 407(4), 1119-1130. doi:10.1007/s00216-014-8323-5

Florentinus, A. K., Bowden, P., Barbisan, V., \& Marshall, J. (2012). Capture and qualitative analysis of the activated Fc receptor complex from live cells. Curr Protoc Protein Sci, Chapter 19, Unit 1922. doi:10.1002/0471140864.ps1922s67

Florentinus, A. K., Bowden, P., Barbisan, V., \& Marshall, J. (2012). Capture and qualitative analysis of the activated Fc receptor complex from live cells. Current protocols in protein science / editorial board, John E. Coligan ... [et al.], Chapter 19, Unit 19.22. doi:10.1002/0471140864.ps1922s67

Florentinus, A. K., Bowden, P., Sardana, G., Diamandis, E. P., \& Marshall, J. G. (2012). Identification and quantification of peptides and proteins secreted from prostate epithelial cells by unbiased liquid chromatography tandem mass spectrometry using goodness of fit and analysis of variance. Journal of Proteomics, 75, 1303-1317. doi:10.1016/j.jprot.2011.11.002

Florentinus, A. K., Jankowski, A., Petrenko, V., Bowden, P., \& Marshall, J. G. (2011a). The Fc receptorcytoskeleton complex from human neutrophils. Journal of Proteomics, 75, 450-468. doi:10.1016/j.jprot.2011.08.011 
Florentinus, A. K., Jankowski, A., Petrenko, V., Bowden, P., \& Marshall, J. G. (2011b). The Fc receptorcytoskeleton complex from human neutrophils. J Proteomics, 75(2), 450-468.

doi:10.1016/j.jprot.2011.08.011

Fox, K., Castanha, E., Fox, A., Feigley, C., \& Salzberg, D. (2008). Human K10 epithelial keratin is the most abundant protein in airborne dust of both occupied and unoccupied school rooms. J Environ Monit, 10(1), 55-59. doi:10.1039/b714802j

Fragnoud, R., Yugueros-Marcos, J., Pachot, A., \& Bedin, F. (2012). Isotope Coded Protein Labeling analysis of plasma specimens from acute severe dengue fever patients. Proteome Sci, 10(1), 60. doi:10.1186/1477-5956-10-60

Frei, A. P., Jeon, O. Y., Kilcher, S., Moest, H., Henning, L. M., Jost, C., . . Wollscheid, B. (2012). Direct identification of ligand-receptor interactions on living cells and tissues. Nat Biotechnol, 30(10), 997-1001. doi:10.1038/nbt.2354

Frei, A. P., Moest, H., Novy, K., \& Wollscheid, B. (2013). Ligand-based receptor identification on living cells and tissues using TRICEPS. Nat Protoc, 8(7), 1321-1336. doi:10.1038/nprot.2013.072

Freychet, P., Roth, J., \& Neville, D. M. (1971). Insulin Receptors in the Liver: Specific Binding of [(125)I]Insulin to the Plasma Membrane and Its Relation to Insulin Bioactivity. Proceedings of the national academy of sciences of the United States of America, 68(8), 1833-1837.

Frobel, J., Hartwig, S., Jourdain, S., Fischer, J. C., Zilkens, C., Kundgen, A., . . Lehr, S. (2015). Deep serum discoveries: SDF-1alpha and HSA fragments in myelodysplastic syndromes. Am J Hematol, 90(9), E185-187. doi:10.1002/ajh.24070

Fung, K. W., Wright, D. W., Gor, J., Swann, M. J., \& Perkins, S. J. (2016). Domain structure of human complement $\mathrm{C} 4 \mathrm{~b}$ extends with increasing $\mathrm{NaCl}$ concentration: implications for its regulatory mechanism. Biochem J, 473(23), 4473-4491. doi:10.1042/BCJ20160744

Gagnon, E., Duclos, S., Rondeau, C., Chevet, E., Cameron, P. H., Steele-Mortimer, O., . . Desjardins, M. (2002). Endoplasmic reticulum-mediated phagocytosis is a mechanism of entry into macrophages. Cell, 110(1), 119-131.

Gast, M. C., van Gils, C. H., Wessels, L. F., Harris, N., Bonfrer, J. M., Rutgers, E. J., . . Beijnen, J. H. (2009). Influence of sample storage duration on serum protein profiles assessed by surface-enhanced laser desorption/ionisation time-of-flight mass spectrometry (SELDI-TOF MS). Clin Chem Lab Med, 47(6), 694-705.

Gbormittah, F. O., Bones, J., Hincapie, M., Tousi, F., Hancock, W. S., \& Iliopoulos, O. (2015). Clusterin glycopeptide variant characterization reveals significant site-specific glycan changes in the plasma of clear cell renal cell carcinoma. J Proteome Res, 14(6), 2425-2436. doi:10.1021/pr501104j

Geer, L. Y., Markey, S. P., Kowalak, J. A., Wagner, L., Xu, M., Maynard, D. M., . . Bryant, S. H. (2004). Open mass spectrometry search algorithm. J Proteome Res, 3(5), 958-964.

Geer, L. Y., Markey, S. P., Kowalak, J. A., Wagner, L., Xu, M., Maynard, D. M., . . Bryant, S. H. (2004). Open mass spectrometry search algorithm. J Proteome Res, 3(5), 958-964.

Geyer, P. E., Kulak, N. A., Pichler, G., Holdt, L. M., Teupser, D., \& Mann, M. (2016). Plasma Proteome Profiling to Assess Human Health and Disease. Cell Syst, 2(3), 185-195. doi:10.1016/j.cels.2016.02.015

Gharahdaghi, F., Kirchner, M., Fernandez, J., \& Mische, S. M. (1996). Peptide-mass profiles of polyvinylidene difluoride-bound proteins by matrix-assisted laser desorption/ionization time-offlight mass spectrometry in the presence of nonionic detergents. Anal Biochem, 233(1), 94-99.

Ghosh, S., Gepstein, S., Heikkila, J. J., \& Dumbroff, E. B. (1988). Use of a scanning densitometer or an ELISA plate reader for measurement of nanogram amounts of protein in crude extracts from biological tissues. Anal Biochem, 169(2), 227-233. 
Ghosh, S., Gepstein, S., Heikkila, J. J., \& Dumbroff, E. B. (1988). Use of a scanning densitometer or an ELISA plate reader for measurement of nanogram amounts of protein in crude extracts from biological tissues. Anal Biochem, 169(2), 227-233.

Glorieux, G., Mullen, W., Duranton, F., Filip, S., Gayrard, N., Husi, H., . . Klein, J. (2015). New insights in molecular mechanisms involved in chronic kidney disease using high-resolution plasma proteome analysis. Nephrol Dial Transplant, 30(11), 1842-1852. doi:10.1093/ndt/gfv254

Goufman, E. I., lakovlev, V. N., Tikhonova, N. B., \& Lokshin, A. E. (2015). Quantification of autoantibodies to plasminogen in plasma of patients with cancer. Cancer Biomark, 15(3), 281-287. doi:10.3233/CBM-150469

Govorukhina, N. I., de Vries, M., Reijmers, T. H., Horvatovich, P., van der Zee, A. G., \& Bischoff, R. (2008). Influence of clotting time on the protein composition of serum samples based on LC-MS data. J Chromatogr B Analyt Technol Biomed Life Sci.

Govorun, V. M., \& Ivanov, V. T. (2011). [Proteomics and peptidomics in fundamental and applied medical studies]. Bioorg Khim, 37(2), 199-215.

Haan, C., \& Behrmann, I. (2007). A cost effective non-commercial ECL-solution for Western blot detections yielding strong signals and low background. Journal of immunological methods, 318, 11-19. doi:10.1016/j.jim.2006.07.027

Hagen, C. P., Mieritz, M. G., Nielsen, J. E., Anand-Ivell, R., Ivell, R., \& Juul, A. (2015). Longitudinal assessment of circulating insulin-like peptide 3 levels in healthy peripubertal girls. Fertil Steril, 103(3), 780-786 e781. doi:10.1016/j.fertnstert.2014.11.014

Hara, M., Ando, M., Tsuchiya, K., \& Nitta, K. (2015). Serum hepcidin-25 level linked with high mortality in patients with non-Hodgkin lymphoma. Ann Hematol, 94(4), 603-608. doi:10.1007/s00277-0142255-1

Hare, N. J., Chan, B., Chan, E., Kaufman, K. L., Britton, W. J., \& Saunders, B. M. (2015). Microparticles released from Mycobacterium tuberculosis-infected human macrophages contain increased levels of the type I interferon inducible proteins including ISG15. Proteomics, 15(17), 3020-3029. doi:10.1002/pmic.201400610

Harris, L. J., Skaletsky, E., \& McPherson, A. (1998). Crystallographic structure of an intact IgG1 monoclonal antibody. Journal of molecular biology, 275(5), 861-872.

Harshman, S. W., Canella, A., Ciarlariello, P. D., Agarwal, K., Branson, O. E., Rocci, A., . . Pichiorri, F. (2016). Proteomic characterization of circulating extracellular vesicles identifies novel serum myeloma associated markers. J Proteomics, 136, 89-98. doi:10.1016/j.jprot.2015.12.016

Haverland, N. A., Villeneuve, L. M., Ciborowski, P., \& Fox, H. S. (2016). The Proteomic Characterization of Plasma or Serum from HIV-Infected Patients. Methods Mol Biol, 1354, 293-310. doi:10.1007/978-1-4939-3046-3_20

Heinrich, J., Pilch, P. F., \& Czech, M. P. (1980). Purification of the adipocyte insulin receptor by immunoaffinity chromatography. J Biol Chem, 255(4), 1732-1737.

Helbig, A. O., Heck, A. J., \& Slijper, M. (2010). Exploring the membrane proteome-challenges and analytical strategies. J Proteomics, 73(5), 868-878.

Hollander, Z., Dai, D. L., Putko, B. N., Yogasundaram, H., Wilson-McManus, J. E., Thompson, R. B., . . Oudit, G. Y. (2015). Gender-specific plasma proteomic biomarkers in patients with AndersonFabry disease. Eur J Heart Fail, 17(3), 291-300. doi:10.1002/ejhf.230

Hoofnagle, A. N., Eckfeldt, J. H., \& Lutsey, P. L. (2015). Vitamin D-Binding Protein Concentrations Quantified by Mass Spectrometry. N Engl J Med, 373(15), 1480-1482. doi:10.1056/NEJMc1502602

Howard, J. C., Florentinus-Mefailoski, A., Bowden, P., Trimble, W., Grinstein, S., \& Marshall, J. G. (2016). OxLDL receptor chromatography from live human U937 cells identifies SYK (L) that regulates phagocytosis of oxLDL. Anal Biochem, 513, 7-20. 
Howard, J. C., Florentinus-Mefailoski, A., Bowden, P., Trimble, W., Grinstein, S., \& Marshall, J. G. (2016). OxLDL receptor chromatography from live human U937 cells identifies SYK(L) that regulates phagocytosis of oxLDL. Anal Biochem. doi:10.1016/j.ab.2016.07.021

Howard, J. W., Kay, R. G., Tan, T., Minnion, J., \& Creaser, C. S. (2015). Identification of plasma protease derived metabolites of glucagon and their formation under typical laboratory sample handling conditions. Rapid Commun Mass Spectrom, 29(2), 171-181. doi:10.1002/rcm.7090

Huang, Z., Zhang, S., Hang, W., Chen, Y., Zheng, J., Li, W., . . Yan, X. (2014). Liquid chromatography-mass spectrometry based serum peptidomic approach for renal clear cell carcinoma diagnosis. $J$ Pharm Biomed Anal, 100, 175-183. doi:10.1016/j.jpba.2014.07.028

Humphries, J. D., Byron, A., Bass, M. D., Craig, S. E., Pinney, J. W., Knight, D., \& Humphries, M. J. (2009). Proteomic Analysis of Integrin-Associated Complexes Identifies RCC2 as a Dual Regulator of Rac1 and Arf6. Sci Signal, 2(87), ra51-ra51. doi:10.1126/scisignal.2000396

Indik, Z. K., Park, J. G., Hunter, S., \& Schreiber, A. D. (1995). The molecular dissection of Fc gamma receptor mediated phagocytosis. Blood, 86(12), 4389-4399.

Jankowski, A., Zhu, P., \& Marshall, J. G. (2008). Capture of an activated receptor complex from the surface of live cells by affinity receptor chromatography. Analytical Biochemistry, 380, 235-248. doi:10.1016/j.ab.2008.05.047

Jankowski, A., Zhu, P., \& Marshall, J. G. (2008). Capture of an activated receptor complex from the surface of live cells by affinity receptor chromatography. Anal Biochem, 380(2), 235-248. doi:10.1016/j.ab.2008.05.047

Jankowski, A., Zhu, P., \& Marshall, J. G. (2008). Capture of an activated receptor complex from the surface of live cells by affinity receptor chromatography. Anal Biochem, 380(2), 235-248. doi:10.1016/j.ab.2008.05.047

Jaros, J. A., Rahmoune, H., Wesseling, H., Leweke, F. M., Ozcan, S., Guest, P. C., \& Bahn, S. (2015). Effects of olanzapine on serum protein phosphorylation patterns in patients with schizophrenia. Proteomics Clin Appl, 9(9-10), 907-916. doi:10.1002/prca.201400148

Jeon, Y. R., Kim, S. Y., Lee, E. J., Kim, Y. N., Noh, D. Y., Park, S. Y., \& Moon, A. (2013). Identification of annexin II as a novel secretory biomarker for breast cancer. Proteomics, 13(21), 3145-3156. doi:10.1002/pmic.201300127

Jin, Y., \& Manabe, T. (2009). Differences in protein distribution between human plasma preparations, EDTA-plasma and heparin-plasma, analyzed by non-denaturing micro-2-DE and MALDI-MS PMF. Electrophoresis, 30(6), 931-938.

Joshi, T., Butchar, J. P., \& Tridandapani, S. (2006). Fcy receptor signaling in phagocytes. International journal of hematology, 84(3), 210-216.

Kaisar, M., van Dullemen, L. F., Thezenas, M. L., Zeeshan Akhtar, M., Huang, H., Rendel, S., ... Kessler, B. M. (2016). Plasma degradome affected by variable storage of human blood. Clin Proteomics, 13, 26. doi:10.1186/s12014-016-9126-9

Kao, S. C., Kirschner, M. B., Cooper, W. A., Tran, T., Burgers, S., Wright, C., . . Reid, G. (2016). A proteomics-based approach identifies secreted protein acidic and rich in cysteine as a prognostic biomarker in malignant pleural mesothelioma. Br J Cancer, 114(5), 524-531. doi:10.1038/bjc.2015.470

Karagiannis, G. S., Pavlou, M. P., Saraon, P., Musrap, N., Xie, A., Batruch, I., . . Diamandis, E. P. (2014). In-depth proteomic delineation of the colorectal cancer exoproteome: Mechanistic insight and identification of potential biomarkers. J Proteomics, 103, 121-136. doi:10.1016/j.jprot.2014.03.018

Karakosta, T. D., Soosaipillai, A., Diamandis, E. P., Batruch, I., \& Drabovich, A. P. (2016). Quantification of Human Kallikrein-Related Peptidases in Biological Fluids by Multiplatform Targeted Mass Spectrometry Assays. Mol Cell Proteomics, 15(9), 2863-2876. doi:10.1074/mcp.M115.057695 
Karsan, A., Eigl, B. J., Flibotte, S., Gelmon, K., Switzer, P., Hassell, P., . . Veenstra, T. (2005). Analytical and preanalytical biases in serum proteomic pattern analysis for breast cancer diagnosis. Clin Chem, 51(8), 1525-1528.

Katayama, H., Boldt, C., Ladd, J. J., Johnson, M. M., Chao, T., Capello, M., ... Hanash, S. (2015). An Autoimmune Response Signature Associated with the Development of Triple-Negative Breast Cancer Reflects Disease Pathogenesis. Cancer Res, 75(16), 3246-3254. doi:10.1158/00085472.CAN-15-0248

Keeble, A. H., Khan, Z., Forster, A., \& James, L. C. (2008). TRIM21 is an IgG receptor that is structurally, thermodynamically, and kinetically conserved. Proceedings of the national academy of sciences of the United States of America, 105(16), 6045-6050. doi:10.1073/pnas.0800159105

Keller, A., Nesvizhskii, A. I., Kolker, E., \& Aebersold, R. (2002). Empirical statistical model to estimate the accuracy of peptide identifications made by MS/MS and database search. Anal Chem, 74(20), 5383-5392.

Keller, A., Nesvizhskii, A. I., Kolker, E., \& Aebersold, R. (2002). Empirical statistical model to estimate the accuracy of peptide identifications made by MS/MS and database search. Anal Chem, 74(20), 5383-5392.

Keller, A., Purvine, S., Nesvizhskii, A. I., Stolyar, S., Goodlett, D. R., \& Kolker, E. (2002). Experimental protein mixture for validating tandem mass spectral analysis. Omics: A Journal of Integrative Biology, 6, 207-212. doi:10.1089/153623102760092805

Keshishian, H., Burgess, M. W., Gillette, M. A., Mertins, P., Clauser, K. R., Mani, D. R., . . Carr, S. A. (2015). Multiplexed, Quantitative Workflow for Sensitive Biomarker Discovery in Plasma Yields Novel Candidates for Early Myocardial Injury. Mol Cell Proteomics, 14(9), 2375-2393. doi:10.1074/mcp.M114.046813

Kielley, W. W., \& Bradley, L. B. (1956). The relationship between sulfhydryl groups and the activation of myosin adenosinetriphosphatase. J Biol Chem, 218(2), 653-659.

Kikuchi, H., Goto, Y., \& Hamaguchi, K. (1986). Reduction of the buried intrachain disulfide bond of the constant fragment of the immunoglobulin light chain: global unfolding under physiological conditions. Biochemistry, 25(8), 2009-2013.

Kim, S., Gupta, N., \& Pevzner, P. A. (2008). Spectral probabilities and generating functions of tandem mass spectra: a strike against decoy databases. J Proteome Res, 7(8), 3354-3363.

Kim, Y. J., Gallien, S., El-Khoury, V., Goswami, P., Sertamo, K., Schlesser, M., . . Domon, B. (2015). Quantification of SAA1 and SAA2 in lung cancer plasma using the isotype-specific PRM assays. Proteomics, 15(18), 3116-3125. doi:10.1002/pmic.201400382

Kim, Y. J., Sertamo, K., Pierrard, M. A., Mesmin, C., Kim, S. Y., Schlesser, M., . . Domon, B. (2015). Verification of the biomarker candidates for non-small-cell lung cancer using a targeted proteomics approach. J Proteome Res, 14(3), 1412-1419. doi:10.1021/pr5010828

Klett, R. P., Fulpius, B. W., Cooper, D., Smith, M., Reich, E., \& Possani, L. D. (1973). The acetylcholine receptor I. Purification and characterization of a macromolecule isolated from Electrophorus electricus. Journal of Biological Chemistry, 248(19), 6841-6853.

Ko, T. M., Kuo, H. C., Chang, J. S., Chen, S. P., Liu, Y. M., Chen, H. W., . . Chen, Y. T. (2015). CXCL10/IP-10 is a biomarker and mediator for Kawasaki disease. Circ Res, 116(5), 876-883. doi:10.1161/CIRCRESAHA.116.305834

Konev, A. A., Smolyanova, T. I., Kharitonov, A. V., Serebryanaya, D. V., Kozlovsky, S. V., Kara, A. N., .. . Postnikov, A. B. (2015). Characterization of endogenously circulating IGFBP-4 fragments-Novel biomarkers for cardiac risk assessment. Clin Biochem, 48(12), 774-780.

doi:10.1016/j.clinbiochem.2015.05.010 
Kono, T., \& Barham, F. W. (1971). The relationship between the insulin-binding capacity of fat cells and the cellular response to insulin studies with intact and trypsin-treated fat cells. Journal of Biological Chemistry, 246(20), 6210-6216.

Koomen, J. M., Li, D., Xiao, L. C., Liu, T. C., Coombes, K. R., Abbruzzese, J., \& Kobayashi, R. (2005). Direct tandem mass spectrometry reveals limitations in protein profiling experiments for plasma biomarker discovery. J Proteome Res, 4(3), 972-981.

Krogh, A., Larsson, B., Von Heijne, G., \& Sonnhammer, E. L. (2001). Predicting transmembrane protein topology with a hidden Markov model: application to complete genomes. Journal of molecular biology, 305(3), 567-580.

Kruh-Garcia, N. A., Wolfe, L. M., Chaisson, L. H., Worodria, W. O., Nahid, P., Schorey, J. S., ... Dobos, K. M. (2014). Detection of Mycobacterium tuberculosis peptides in the exosomes of patients with active and latent $M$. tuberculosis infection using MRM-MS. PLOS ONE, 9(7), e103811. doi:10.1371/journal.pone.0103811

Kulik, W., van Lenthe, H., Stet, F. S., Houtkooper, R. H., Kemp, H., Stone, J. E., . . Vaz, F. M. (2008). Bloodspot assay using HPLC-tandem mass spectrometry for detection of Barth syndrome. Clin Chem, 54(2), 371-378.

Kushnir, M. M., Rockwood, A. L., Strathmann, F. G., Frank, E. L., Straseski, J. A., \& Meikle, A. W. (2016). LC-MS/MS Measurement of Parathyroid Hormone-Related Peptide. Clin Chem, 62(1), 218-226. doi:10.1373/clinchem.2015.244012

Labots, M., Schutte, L. M., van der Mijn, J. C., Pham, T. V., Jimenez, C. R., \& Verheul, H. M. (2014). Mass spectrometry-based serum and plasma peptidome profiling for prediction of treatment outcome in patients with solid malignancies. Oncologist, 19(10), 1028-1039. doi:10.1634/theoncologist.2014-0101

Lagerstrom, M. C., \& Schioth, H. B. (2008). Structural diversity of G protein-coupled receptors and significance for drug discovery. Nat Rev Drug Discov, 7(4), 339-357. doi:10.1038/nrd2518

Lancrajan, I., Schneider-Stock, R., Naschberger, E., Schellerer, V. S., Sturzl, M., \& Enz, R. (2015). Absolute quantification of DcR3 and GDF15 from human serum by LC-ESI MS. J Cell Mol Med, 19(7), 16561671. doi:10.1111/jcmm.12540

Langley, J. N. (1906). Croonian Lecture, 1906: On Nerve Endings and on Special Excitable Substances in Cells. Proceedings of the Royal Society of London. Series B, Containing Papers of a Biological Character, 170-194.

Lee, A. Y., Chappell, D. L., Bak, M. J., Judo, M., Liang, L., Churakova, T., . . Laterza, O. F. (2016). Multiplexed Quantification of Proglucagon-Derived Peptides by Immunoaffinity Enrichment and Tandem Mass Spectrometry after a Meal Tolerance Test. Clin Chem, 62(1), 227-235. doi:10.1373/clinchem.2015.244251

Lee, M. Y., Kim, E. Y., Kim, S. H., Cho, K. C., Ha, K., Kim, K. P., \& Ahn, Y. M. (2016). Discovery of serum protein biomarkers in drug-free patients with major depressive disorder. Prog Neuropsychopharmacol Biol Psychiatry, 69, 60-68. doi:10.1016/j.pnpbp.2016.04.009

Lee, S. E., West, K. P., Jr., Cole, R. N., Schulze, K. J., Christian, P., Wu, L. S., . . Ruczinski, I. (2015). Plasma Proteome Biomarkers of Inflammation in School Aged Children in Nepal. PLOS ONE, 10(12), e0144279. doi:10.1371/journal.pone.0144279

Lee, W. L., Cosio, G., Ireton, K., \& Grinstein, S. (2007). Role of CrkIl in Fcy receptor-mediated phagocytosis. Journal of Biological Chemistry, 282(15), 11135-11143.

Lefkowitz, R. J. (1973). Isolated beta-adrenergic binding sites: a potential assay vehicle for catecholamines. Pharmacol Rev, 25(2), 259-268.

Lefkowitz, R. J. (2004). Historical review: a brief history and personal retrospective of seventransmembrane receptors. Trends Pharmacol Sci, 25(8), 413-422. doi:10.1016/j.tips.2004.06.006 
Lemesle, G., Maury, F., Beseme, O., Ovart, L., Amouyel, P., Lamblin, N., . . Pinet, F. (2015). Multimarker proteomic profiling for the prediction of cardiovascular mortality in patients with chronic heart failure. PLOS ONE, 10(4), e0119265. doi:10.1371/journal.pone.0119265

Lerm, M., Brodin, V. P., Ruishalme, I., Stendahl, O., \& Sarndahl, E. (2007). Inactivation of Cdc42 is necessary for depolymerization of phagosomal F-actin and subsequent phagosomal maturation. J Immunol, 178(11), 7357-7365.

LeWinter, M. M., \& Granzier, H. L. (2013). Titin is a major human disease gene. Circulation, 127(8), 938944. doi:10.1161/CIRCULATIONAHA.112.139717

Li, C., He, X., Li, H., Zhou, Y., Zang, N., Hu, S., . . He, M. (2015). Discovery and verification of serum differential expression proteins for pulmonary tuberculosis. Tuberculosis (Edinb), 95(5), 547-554. doi:10.1016/j.tube.2015.06.001

Li, J., Orlandi, R., White, C. N., Rosenzweig, J., Zhao, J., Seregni, E., . . Chan, D. W. (2005). Independent validation of candidate breast cancer serum biomarkers identified by mass spectrometry. Clin Chem, 51(12), 2229-2235.

Li, Y., Ozment, T., Wright, G. L., \& Peterson, J. M. (2016). Identification of Putative Receptors for the Novel Adipokine CTRP3 Using Ligand-Receptor Capture Technology. PloS one, 11(10), e0164593. doi:10.1371/journal.pone.0164593

Lim, H. K., Cao, Y., Qiu, X., Silva, J., \& Evans, D. C. (2015). A nonradioactive approach to investigate the metabolism of therapeutic peptides by tagging with $127 \mathrm{i}$ and using inductively-coupled plasma mass spectrometry analysis. Drug Metab Dispos, 43(1), 17-26. doi:10.1124/dmd.114.059774

Lin, S. J., Chang, K. P., Hsu, C. W., Chi, L. M., Chien, K. Y., Liang, Y., . . Yu, J. S. (2013). Low-molecularmass secretome profiling identifies $\mathrm{C}$ - $\mathrm{C}$ motif chemokine 5 as a potential plasma biomarker and therapeutic target for nasopharyngeal carcinoma. J Proteomics, 94, 186-201. doi:10.1016/j.jprot.2013.09.013

Liu, J., Huang, P., He, Y., Hong, W. X., Ren, X., Yang, X., . . Cheng, J. (2014). Serum amyloid A and clusterin as potential predictive biomarkers for severe hand, foot and mouth disease by 2D-DIGE proteomics analysis. PLOS ONE, 9(9), e108816. doi:10.1371/journal.pone.0108816

Liu, Y., Wei, F., Wang, F., Li, C., Meng, G., Duan, H., .. . Zhang, W. (2015). Serum peptidome profiling analysis for the identification of potential biomarkers in cervical intraepithelial neoplasia patients. Biochem Biophys Res Commun, 465(3), 476-480. doi:10.1016/j.bbrc.2015.08.042

Lodish, H., Berk, A., Zipursky, S. L., Matsudaira, P., Baltimore, D., \& Darnell, J. (2000). Molecular cell biology 4th edition. National Center for Biotechnology InformationÕs Bookshelf.

Lowey, S., \& Risby, D. (1971). Light chains from fast and slow muscle myosins. Nature, 234(5324), 81-85.

Lundstrom, S. L., Yang, H., Lyutvinskiy, Y., Rutishauser, D., Herukka, S. K., Soininen, H., \& Zubarev, R. A. (2014). Blood plasma IgG Fc glycans are significantly altered in Alzheimer's disease and progressive mild cognitive impairment. J Alzheimers Dis, 38(3), 567-579. doi:10.3233/JAD131088

Makino, S., Reynolds, J. A., \& Tanford, C. (1973). The binding of deoxycholate and Triton X-100 to proteins. Journal of Biological Chemistry, 248(14), 4926-4932.

Malmstrom, E., Kilsgard, O., Hauri, S., Smeds, E., Herwald, H., Malmstrom, L., \& Malmstrom, J. (2016). Large-scale inference of protein tissue origin in gram-positive sepsis plasma using quantitative targeted proteomics. Nat Commun, 7, 10261. doi:10.1038/ncomms10261

Marfa, S., Crespo, G., Reichenbach, V., Forns, X., Casals, G., Morales-Ruiz, M., . . Jimenez, W. (2014). Lack of a $5.9 \mathrm{kDa}$ peptide C-terminal fragment of fibrinogen alpha chain precedes fibrosis progression in patients with liver disease. PLOS ONE, 9(10), e109254.

doi:10.1371/journal.pone.0109254 
Marshall, J., Bowden, P., Schmit, J. C., \& Betsou, F. (2014). Creation of a federated database of blood proteins: a powerful new tool for finding and characterizing biomarkers in serum. Clin Proteomics, 11(1), 3. doi:10.1186/1559-0275-11-3

Marshall, J., Jankowski, A., Furesz, S., Kireeva, I., Barker, L., Dombrovsky, M., . . Jackowski, G. (2004). Human serum proteins preseparated by electrophoresis or chromatography followed by tandem mass spectrometry. J Proteome Res, 3(3), 364-382.

Marshall, J., Kupchak, P., Zhu, W., Yantha, J., Vrees, T., Furesz, S., . . Jackowski, G. (2003). Processing of serum proteins underlies the mass spectral fingerprinting of myocardial infarction. Journal of Proteome Research, 2, 361-372.

Marshall, J. G., Booth, J. W., Stambolic, V., Mak, T., Balla, T., Schreiber, A. D., . . Grinstein, S. (2001). Restricted accumulation of phosphatidylinositol 3-kinase products in a plasmalemmal subdomain during Fc gamma receptor-mediated phagocytosis. The Journal of Cell Biology, 153, 1369-1380.

Marshall, J. G., Booth, J. W., Stambolic, V., Mak, T., Balla, T., Schreiber, A. D., . . Grinstein, S. (2001). Restricted accumulation of phosphatidylinositol 3-kinase products in a plasmalemmal subdomain during Fcy receptor-mediated phagocytosis. The Journal of cell biology, 153(7), 13691380.

Marsillach, J., Becker, J. O., Vaisar, T., Hahn, B. H., Brunzell, J. D., Furlong, C. E., . . Group, D. E. R. (2015). Paraoxonase-3 is depleted from the high-density lipoproteins of autoimmune disease patients with subclinical atherosclerosis. J Proteome Res, 14(5), 2046-2054. doi:10.1021/pr5011586

Martin, P., Noonan, S., Mullen, M. P., Scaife, C., Tosetto, M., Nolan, B., . . O'Sullivan, J. (2014). Predicting response to vascular endothelial growth factor inhibitor and chemotherapy in metastatic colorectal cancer. BMC Cancer, 14, 887. doi:10.1186/1471-2407-14-887

Martinez-Morillo, E., Childs, C., Garcia, B. P., Alvarez Menendez, F. V., Romaschin, A. D., Cervellin, G., . . . Diamandis, E. P. (2015). Neurofilament medium polypeptide (NFM) protein concentration is increased in CSF and serum samples from patients with brain injury. Clin Chem Lab Med, 53(10), 1575-1584. doi:10.1515/cclm-2014-0908

Melo, S. A., Luecke, L. B., Kahlert, C., Fernandez, A. F., Gammon, S. T., Kaye, J., . . Kalluri, R. (2015). Glypican-1 identifies cancer exosomes and detects early pancreatic cancer. Nature, 523(7559), 177-182. doi:10.1038/nature14581

Mercier, C., Truntzer, C., Pecqueur, D., Gimeno, J. P., Belz, G., \& Roy, P. (2009). Mixed-model of ANOVA for measurement reproducibility in proteomics. J Proteomics, 72(6), 974-981.

Metschnikoff, E. (1884). Ueber die beziehung der phagocyten zu milzbrandbacillen. Virchows Archiv, 97(3), 502-526.

Michael, I. P., Sotiropoulou, G., Pampalakis, G., Magklara, A., Ghosh, M., Wasney, G., \& Diamandis, E. P. (2005). Biochemical and enzymatic characterization of human kallikrein 5 (hK5), a novel serine protease potentially involved in cancer progression. J Biol Chem, 280(15), 14628-14635. doi:10.1074/jbc.M408132200

Milstein, C. (1966). The disulphide bridges of immunoglobulin kappa-chains. Biochem. J, 101, 338-351.

Moulder, R., Bhosale, S. D., Erkkila, T., Laajala, E., Salmi, J., Nguyen, E. V., . . Lahesmaa, R. (2015). Serum proteomes distinguish children developing type 1 diabetes in a cohort with HLA-conferred susceptibility. Diabetes, 64(6), 2265-2278. doi:10.2337/db14-0983

Moutsopoulos, H. M., Skopouli, F. N., Sarras, A., Tsampoulas, C., Mavridis, A., Constantopoulos, S., \& Maddison, P. (1985). Anti-Ro (SSA) positive rheumatoid arthritis (RA): a clinicoserological group of patients with high incidence of D-penicillamine side effects. Ann Rheum Dis, 44(4), 215-219.

Muenchhoff, J., Poljak, A., Song, F., Raftery, M., Brodaty, H., Duncan, M., . . Sachdev, P. S. (2015). Plasma protein profiling of mild cognitive impairment and Alzheimer's disease across two independent cohorts. J Alzheimers Dis, 43(4), 1355-1373. doi:10.3233/JAD-141266 
Mustafa, M. Z., Nguyen, V. H., Le Naour, F., De Martin, E., Beleoken, E., Guettier, C., . . Ballot, E. (2016). Autoantibody signatures defined by serological proteome analysis in sera from patients with cholangiocarcinoma. J Transl Med, 14, 17. doi:10.1186/s12967-015-0751-2

Nagamori, S., Wiriyasermkul, P., Guarch, M. E., Okuyama, H., Nakagomi, S., Tadagaki, K., . . Kanai, Y. (2016). Novel cystine transporter in renal proximal tubule identified as a missing partner of cystinuria-related plasma membrane protein rBAT/SLC3A1. Proc Natl Acad Sci U S A, 113(3), 775-780. doi:10.1073/pnas.1519959113

Nakanishi, T. (2015). [Rapid Identification of Infectious Microorganisms in Clinical Samples by MALDITOF MS Analysis]. Rinsho Byori, 63(4), 465-471.

Nicholson, J., Azim, S., Rebecchi, M. J., Galbavy, W., Feng, T., Reinsel, R., . . Kaczocha, M. (2015). Leptin levels are negatively correlated with 2-arachidonoylglycerol in the cerebrospinal fluid of patients with osteoarthritis. PLOS ONE, 10(4), e0123132. doi:10.1371/journal.pone.0123132

Nicoli, E. R., Al Eisa, N., Cluzeau, C. V., Wassif, C. A., Gray, J., Burkert, K. R., . . Platt, F. M. (2016). Defective Cytochrome P450-Catalysed Drug Metabolism in Niemann-Pick Type C Disease. PLoS ONE, 11(3), e0152007. doi:10.1371/journal.pone.0152007

Nowak, C., Sundstrom, J., Gustafsson, S., Giedraitis, V., Lind, L., Ingelsson, E., \& Fall, T. (2016). Protein Biomarkers for Insulin Resistance and Type 2 Diabetes Risk in Two Large Community Cohorts. Diabetes, 65(1), 276-284. doi:10.2337/db15-0881

Odin, J. A., Edberg, J. C., Painter, C. J., Kimberly, R. P., \& Unkeless, J. C. (1991). Regulation of phagocytosis and [Ca2+] i flux by distinct regions of an Fc receptor. science, 254(5039), 17851788.

Ohyama, K., Ueki, Y., Kawakami, A., Kishikawa, N., Tamai, M., Osaki, M., . . Kuroda, N. (2011). Immune complexome analysis of serum and its application in screening for immune complex antigens in rheumatoid arthritis. Clin Chem, 57(6), 905-909. doi:10.1373/clinchem.2010.157776

Okano, T., Seike, M., Kuribayashi, H., Soeno, C., Ishii, T., Kida, K., \& Gemma, A. (2016). Identification of haptoglobin peptide as a novel serum biomarker for lung squamous cell carcinoma by serum proteome and peptidome profiling. Int J Oncol, 48(3), 945-952. doi:10.3892/ijo.2016.3330

Oonk, S., Spitali, P., Hiller, M., Switzar, L., Dalebout, H., Calissano, M., . . van der Burgt, Y. E. (2016). Comparative mass spectrometric and immunoassay-based proteome analysis in serum of Duchenne muscular dystrophy patients. Proteomics Clin Appl, 10(3), 290-299. doi:10.1002/prca.201500044

Otake, K., Uchida, K., Ide, S., Kobayashi, Y., Kobayashi, I., \& Kusunoki, M. (2016). Identification of DDX39A as a Potential Biomarker for Unfavorable Neuroblastoma Using a Proteomic Approach. Pediatr Blood Cancer, 63(2), 221-227. doi:10.1002/pbc.25778

Overington, J. P., Al-Lazikani, B., \& Hopkins, A. L. (2006). How many drug targets are there? Nat Rev Drug Discov, 5(12), 993-996. doi:10.1038/nrd2199

Oyaert, M., Peersman, N., Kieffer, D., Deiteren, K., Smits, A., Allegaert, K., . . Pauwels, S. (2015). Novel LC-MS/MS method for plasma vancomycin: comparison with immunoassays and clinical impact. Clin Chim Acta, 441, 63-70. doi:10.1016/j.cca.2014.12.012

Pan, S., Brentnall, T. A., \& Chen, R. (2015). Proteomics analysis of bodily fluids in pancreatic cancer. Proteomics, 15(15), 2705-2715. doi:10.1002/pmic.201400476

Parikh, I., Sica, V., Nola, E., Puca, G. A., \& Cuatrecasas, P. (1974). Affinity chromatography of estrogen receptors. Methods Enzymol, 34, 670-688.

Park SK, M. K. (1988). Random Number Generators: Good Ones Are Hard To Find. Communications of the ACM, 31(10), 1191-1201.

Pena, M. J., Jankowski, J., Heinze, G., Kohl, M., Heinzel, A., Bakker, S. J., . . Jankowski, V. (2015). Plasma proteomics classifiers improve risk prediction for renal disease in patients with hypertension or type 2 diabetes. J Hypertens, 33(10), 2123-2132. doi:10.1097/HJH.0000000000000685 
Perkins, D. N., Pappin, D. J., Creasy, D. M., \& Cottrell, J. S. (1999). Probability-based protein identification by searching sequence databases using mass spectrometry data. Electrophoresis, 20(18), 35513567.

Peterson, J. M., Seldin, M. M., Wei, Z., Aja, S., \& Wong, G. W. (2013). CTRP3 attenuates diet-induced hepatic steatosis by regulating triglyceride metabolism. Am J Physiol Gastrointest Liver Physiol, 305(3), G214-224. doi:10.1152/ajpgi.00102.2013

Petricoin, E. F., Ardekani, A. M., Hitt, B. A., Levine, P. J., Fusaro, V. A., Steinberg, S. M., . . Liotta, L. A. (2002). Use of proteomic patterns in serum to identify ovarian cancer. Lancet, 359(9306), 572577.

Pieper, R., Gatlin, C. L., Makusky, A. J., Russo, P. S., Schatz, C. R., Miller, S. S., . . Steiner, S. (2003). The human serum proteome: display of nearly 3700 chromatographically separated protein spots on two-dimensional electrophoresis gels and identification of 325 distinct proteins. Proteomics, 3(7), 1345-1364.

Pieper, U., Schlessinger, A., Kloppmann, E., Chang, G. A., Chou, J. J., Dumont, M. E., . . Sali, A. (2013). Coordinating the impact of structural genomics on the human $\alpha$-helical transmembrane proteome. Nature structural \& molecular biology, 20(2), 135-138. doi:10.1038/nsmb.2508

Pilny, R., Bouchal, P., Borilova, S., Ceskova, P., Zaloudik, J., Vyzula, R., . . Valik, D. (2006). Surfaceenhanced laser desorption ionization/time-of-flight mass spectrometry reveals significant artifacts in serum obtained from clot activator-containing collection devices. Clin Chem, 52(11), 2115-2116.

Pont, L., Benavente, F., Vilaseca, M., Gimenez, E., \& Sanz-Nebot, V. (2015). Characterisation of serum transthyretin by electrospray ionisation-ion mobility mass spectrometry: Application to familial amyloidotic polyneuropathy type I (FAP-I). Talanta, 144, 1216-1224. doi:10.1016/j.talanta.2015.07.079

Popescu, I. D., Codrici, E., Albulescu, L., Mihai, S., Enciu, A. M., Albulescu, R., \& Tanase, C. P. (2014). Potential serum biomarkers for glioblastoma diagnostic assessed by proteomic approaches. Proteome Sci, 12(1), 47. doi:10.1186/s12953-014-0047-0

Purves, D., Augustine, G., \& Fitzpatrick, D. (2001). et al., editors. Neuroscience. Sunderland (MA). In: Sinauer Associates.

Putnam, F. (1975). The plasma Proteins: Structure Function, and Genetic Control. Academic Press, New York, second ed.

Qin, S., Zhou, Y., Gray, L., Kusebauch, U., McEvoy, L., Antoine, D. J., . . Hood, L. (2016). Identification of Blood Protein Biomarkers of Acute Liver Injury by Targeted Quantitative Proteomics in Acetaminophen and Carbon tetrachloride treated Mouse Models and Acetaminophen Overdose Patients. J Proteome Res. doi:10.1021/acs.jproteome.6b00547

Qu, Z. X., Odin, J., Glass, J. D., \& Unkeless, J. C. (1988). Expression and characterization of a truncated murine Fc gamma receptor. J Exp Med, 167(3), 1195-1210.

Rabinovitch, M. (1995). Professional and non-professional phagocytes: an introduction. Trends Cell Biol, 5(3), 85-87.

Radon, T. P., Massat, N. J., Jones, R., Alrawashdeh, W., Dumartin, L., Ennis, D., . . Crnogorac-Jurcevic, T. (2015). Identification of a Three-Biomarker Panel in Urine for Early Detection of Pancreatic Adenocarcinoma. Clin Cancer Res, 21(15), 3512-3521. doi:10.1158/1078-0432.CCR-14-2467

Rai, A. J., Gelfand, C. A., Haywood, B. C., Warunek, D. J., Yi, J., Schuchard, M. D., . . Chan, D. W. (2005). HUPO Plasma Proteome Project specimen collection and handling: towards the standardization of parameters for plasma proteome samples. Proteomics, 5(13), 3262-3277.

Ravetch, J. V., \& Kinet, J.-P. (1991). Fc receptors. Annual review of immunology, 9(1), 457-492.

Reiser, H., Klingenberg, R., Hof, D., Cooksley-Decasper, S., Fuchs, N., Akhmedov, A., . . Gawinecka, J. (2015). Circulating FABP4 is a prognostic biomarker in patients with acute coronary syndrome 
but not in asymptomatic individuals. Arterioscler Thromb Vasc Biol, 35(8), 1872-1879. doi:10.1161/ATVBAHA.115.305365

Rouillon, J., Poupiot, J., Zocevic, A., Amor, F., Leger, T., Garcia, C., . . Svinartchouk, F. (2015). Serum proteomic profiling reveals fragments of MYOM3 as potential biomarkers for monitoring the outcome of therapeutic interventions in muscular dystrophies. Hum Mol Genet, 24(17), 49164932. doi:10.1093/hmg/ddv214

Russell, M. R., Walker, M. J., Williamson, A. J., Gentry-Maharaj, A., Ryan, A., Kalsi, J., . . Graham, R. L. (2016). Protein Z: A putative novel biomarker for early detection of ovarian cancer. Int J Cancer, 138(12), 2984-2992. doi:10.1002/ijc.30020

Safavi-Hemami, H., Gajewiak, J., Karanth, S., Robinson, S. D., Ueberheide, B., Douglass, A. D., ... Olivera, B. M. (2015). Specialized insulin is used for chemical warfare by fish-hunting cone snails. Proc Natl Acad Sci U S A, 112(6), 1743-1748. doi:10.1073/pnas.1423857112

Sardesai, V. M., \& Provido, H. S. (1965). A Fluorometric Method for Determining the Tame Esterase (Tryptic) Activity of Plasma. J Lab Clin Med, 65, 1023-1029.

Savage, M., Mattson, G., Desai, S., Nielander, G., Morgensen, S., \& Conklin, E. (1994). Avidin-biotin chemistry: a handbook, 2 edn (Rockford, Pierce Chemical Company).

Schagger, H., \& von Jagow, G. (1987). Tricine-sodium dodecyl sulfate-polyacrylamide gel electrophoresis for the separation of proteins in the range from 1 to $100 \mathrm{kDa}$. Anal Biochem, 166(2), 368-379.

Schmohl, M., Gansser, D., Moschetti, V., \& Stangier, J. (2015). Measurement of dabigatran plasma concentrations by calibrated thrombin clotting time in comparison to LC-MS/MS in human volunteers on dialysis. Thromb Res, 135(3), 532-536. doi:10.1016/j.thromres.2014.12.021

Schulz, A., Jankowski, J., Zidek, W., \& Jankowski, V. (2014). Absolute quantification of endogenous angiotensin II levels in human plasma using ESI-LC-MS/MS. Clin Proteomics, 11(1), 37. doi:10.1186/1559-0275-11-37

Schwartz, J. C., Senko, M. W., \& Syka, J. E. (2002). A two-dimensional quadrupole ion trap mass spectrometer. J Am Soc Mass Spectrom, 13(6), 659-669.

Semenov, A. G., \& Katrukha, A. G. (2016). Different Susceptibility of B-Type Natriuretic Peptide (BNP) and BNP Precursor (proBNP) to Cleavage by Neprilysin: The N-Terminal Part Does Matter. Clin Chem, 62(4), 617-622. doi:10.1373/clinchem.2016.254524

Semmes, O. J., Feng, Z., Adam, B. L., Banez, L. L., Bigbee, W. L., Campos, D., .. . Zhu, L. (2005). Evaluation of serum protein profiling by surface-enhanced laser desorption/ionization time-of-flight mass spectrometry for the detection of prostate cancer: I. Assessment of platform reproducibility. Clin Chem, 51(1), 102-112.

Shah, A. K., Cao, K. A., Choi, E., Chen, D., Gautier, B., Nancarrow, D., . . Hill, M. M. (2015). Serum Glycoprotein Biomarker Discovery and Qualification Pipeline Reveals Novel Diagnostic Biomarker Candidates for Esophageal Adenocarcinoma. Mol Cell Proteomics, 14(11), 3023-3039. doi:10.1074/mcp.M115.050922

Shao, B., de Boer, I., Tang, C., Mayer, P. S., Zelnick, L., Afkarian, M., . . Himmelfarb, J. (2015). A Cluster of Proteins Implicated in Kidney Disease Is Increased in High-Density Lipoprotein Isolated from Hemodialysis Subjects. J Proteome Res, 14(7), 2792-2806. doi:10.1021/acs.jproteome.5b00060

Sharma, R., Gowda, H., Chavan, S., Advani, J., Kelkar, D., Kumar, G. S., . . Christopher, R. (2015). Proteomic Signature of Endothelial Dysfunction Identified in the Serum of Acute Ischemic Stroke Patients by the iTRAQ-Based LC-MS Approach. J Proteome Res, 14(6), 2466-2479. doi:10.1021/pr501324n

Shi, T., Sun, X., Gao, Y., Fillmore, T. L., Schepmoes, A. A., Zhao, R., . . Qian, W. J. (2013). Targeted quantification of low $\mathrm{ng} / \mathrm{mL}$ level proteins in human serum without immunoaffinity depletion. $J$ Proteome Res, 12(7), 3353-3361. 
Shin, J., Kim, H. J., Kim, G., Song, M., Woo, S. J., Lee, S. T., . . Lee, C. (2014). Discovery of melanotransferrin as a serological marker of colorectal cancer by secretome analysis and quantitative proteomics. J Proteome Res, 13(11), 4919-4931. doi:10.1021/pr500790f

Shiu, R. P., \& Friesen, H. G. (1974). Solubilization and purification of a prolactin receptor from the rabbit mammary gland. Journal of Biological Chemistry, 249(24), 7902-7911.

Sica, V., Nola, E., Parikh, I., Puca, G. A., \& Cuatrecasas, P. (1973). Purification of oestradiol receptors by affinity chromatography. Nat New Biol, 244(132), 36-39.

Sica, V., Parikh, I., Nola, E., Puca, G. A., \& Cuatrecasas, P. (1973). Affinity chromatography and the purification of estrogen receptors. J Biol Chem, 248(18), 6543-6558.

Sleddering, M. A., Markvoort, A. J., Dharuri, H. K., Jeyakar, S., Snel, M., Juhasz, P., . . Van Dijk, K. W. (2014). Proteomic analysis in type 2 diabetes patients before and after a very low calorie diet reveals potential disease state and intervention specific biomarkers. PLOS ONE, 9(11), e112835. doi:10.1371/journal.pone.0112835

Sondermann, P., Huber, R., \& Jacob, U. (1999). Crystal structure of the soluble form of the human Fcyreceptor IIb: a new member of the immunoglobulin superfamily at $1.7 \AA$ resolution. Embo $j$, 18(5), 1095-1103.

Sorace, J. M., \& Zhan, M. (2003). A data review and re-assessment of ovarian cancer serum proteomic profiling. BMC Bioinformatics, 4(1), 24.

Spencer, K. (2003). The influence of different sample collection types on the levels of markers used for Down's syndrome screening as measured by the Kryptor Immunosassay system. Ann Clin Biochem, 40(Pt 2), 166-168.

Stafford, G. C. (1980). Instrumental aspects of positive and negative ion chemical ionization mass spectrometry. Environ Health Perspect, 36, 85-88.

Staros, J. V. (1982). N-hydroxysulfosuccinimide active esters: bis(N-hydroxysulfosuccinimide) esters of two dicarboxylic acids are hydrophilic, membrane-impermeant, protein cross-linkers. Biochemistry, 21(17), 3950-3955.

Stiles, G. L., Caron, M. G., \& Lefkowitz, R. J. (1984). Beta-adrenergic receptors: biochemical mechanisms of physiological regulation. Physiological reviews.

Subbannayya, Y., Mir, S. A., Renuse, S., Manda, S. S., Pinto, S. M., Puttamallesh, V. N., . . Gowda, H. (2015). Identification of differentially expressed serum proteins in gastric adenocarcinoma. $J$ Proteomics, 127(Pt A), 80-88. doi:10.1016/j.jprot.2015.04.021

Surinova, S., Choi, M., Tao, S., Schuffler, P. J., Chang, C. Y., Clough, T., . . Aebersold, R. (2015). Prediction of colorectal cancer diagnosis based on circulating plasma proteins. EMBO Mol Med, 7(9), 11661178. doi:10.15252/emmm.201404873

Takaya, A., Peng, W. X., Ishino, K., Kudo, M., Yamamoto, T., Wada, R., . . Naito, Z. (2015). Cystatin B as a potential diagnostic biomarker in ovarian clear cell carcinoma. Int J Oncol, 46(4), 1573-1581. doi:10.3892/ijo.2015.2858

Tan, L., Yu, Z., Zhou, X., Xing, D., Luo, X., Peng, R., \& Tang, Y. (2015). Antibody-free ultra-high performance liquid chromatography/tandem mass spectrometry measurement of angiotensin I and II using magnetic epitope-imprinted polymers. J Chromatogr A, 1411, 69-76. doi:10.1016/j.chroma.2015.07.114

Tan, Z., Yin, H., Nie, S., Lin, Z., Zhu, J., Ruffin, M. T., . . Lubman, D. M. (2015). Large-scale identification of core-fucosylated glycopeptide sites in pancreatic cancer serum using mass spectrometry. $J$ Proteome Res, 14(4), 1968-1978. doi:10.1021/acs.jproteome.5b00068

Teixeira, P. C., Ducret, A., Ferber, P., Gaertner, H., Hartley, O., Pagano, S., . . Cutler, P. (2014). Definition of human apolipoprotein A-I epitopes recognized by autoantibodies present in patients with cardiovascular diseases. J Biol Chem, 289(41), 28249-28259. doi:10.1074/jbc.M114.589002 
Thakur, S. S., Geiger, T., Chatterjee, B., Bandilla, P., Frohlich, F., Cox, J., \& Mann, M. (2011). Deep and highly sensitive proteome coverage by LC-MS/MS without prefractionation. Mol Cell Proteomics, 10(8), M110 003699. doi:10.1074/mcp.M110.003699

Thomas, A., Walpurgis, K., Tretzel, L., Brinkkotter, P., Fichant, E., Delahaut, P., . . Thevis, M. (2015). Expanded test method for peptides $>2 \mathrm{kDa}$ employing immunoaffinity purification and LCHRMS/MS. Drug Test Anal, 7(11-12), 990-998. doi:10.1002/dta.1868

Tian, Y., \& Zhang, H. (2010). Glycoproteomics and clinical applications. Proteomics Clin Appl, 4(2), 124132. doi:10.1002/prca.200900161

Tietz. (2001). Tietz Fundamentals of Clinical Chemistry (5th edition ed. Vol. 5th edition): Saunders.

Timms, J. F., Arslan-Low, E., Kabir, M., Worthington, J., Camuzeaux, S., Sinclair, J., . . . Jacobs, I. (2014). Discovery of serum biomarkers of ovarian cancer using complementary proteomic profiling strategies. Proteomics Clin Appl, 8(11-12), 982-993. doi:10.1002/prca.201400063

Tirumalai, R. S., Chan, K. C., Prieto, D. A., Issaq, H. J., Conrads, T. P., \& Veenstra, T. D. (2003). Characterization of the low molecular weight human serum proteome. Mol Cell Proteomics, 2(10), 1096-1103.

Titus, J. A., Perez, P., Kaubisch, A., Garrido, M. A., \& Segal, D. M. (1987). Human K/natural killer cells targeted with hetero-cross-linked antibodies specifically lyse tumor cells in vitro and prevent tumor growth in vivo. The Journal of Immunology, 139(9), 3153-3158.

Tonegawa, S., Steinberg, C., Dube, S., \& Bernardini, A. (1974). Evidence for somatic generation of antibody diversity. Proc Natl Acad Sci U S A, 71(10), 4027-4031.

Tonry, C. L., Doherty, D., O'Shea, C., Morrissey, B., Staunton, L., Flatley, B., . . Pennington, S. R. (2015). Discovery and Longitudinal Evaluation of Candidate Protein Biomarkers for Disease Recurrence in Prostate Cancer. J Proteome Res, 14(7), 2769-2783. doi:10.1021/acs.jproteome.5b00041

Towbin, H., Staehelin, T., \& Gordon, J. (1979). Electrophoretic transfer of proteins from polyacrylamide gels to nitrocellulose sheets: procedure and some applications. Proc Natl Acad Sci U SA, 76(9), 4350-4354.

Tremlett, H., Dai, D. L., Hollander, Z., Kapanen, A., Aziz, T., Wilson-McManus, J. E., . . Cohen Freue, G. V. (2015). Serum proteomics in multiple sclerosis disease progression. J Proteomics, 118, 2-11. doi:10.1016/j.jprot.2015.02.018

Trenchevska, O., Sherma, N. D., Oran, P. E., Reaven, P. D., Nelson, R. W., \& Nedelkov, D. (2015). Quantitative mass spectrometric immunoassay for the chemokine RANTES and its variants. J Proteomics, 116, 15-23. doi:10.1016/j.jprot.2014.12.011

Tridandapani, S., Lyden, T. W., Smith, J. L., Carter, J. E., Coggeshall, K. M., \& Anderson, C. L. (2000). The adapter protein LAT enhances Fcy receptor-mediated signal transduction in myeloid cells. Journal of Biological Chemistry, 275(27), 20480-20487.

Tsai, T. H., Song, E., Zhu, R., Di Poto, C., Wang, M., Luo, Y., . . Ressom, H. W. (2015). LC-MS/MS-based serum proteomics for identification of candidate biomarkers for hepatocellular carcinoma. Proteomics, 15(13), 2369-2381. doi:10.1002/pmic.201400364

Tucholska, M., Bowden, P., Jacks, K., Zhu, P., Furesz, S., Dumbrovsky, M., \& Marshall, J. (2009). Human serum proteins fractionated by preparative partition chromatography prior to LC-ESI-MS/MS. Journal of Proteome Research, 8, 1143-1155. doi:10.1021/pr8005217

Tucholska, M., Florentinus, A., Williams, D., \& Marshall, J. G. (2010). The endogenous peptides of normal human serum extracted from the acetonitrile-insoluble precipitate using modified aqueous buffer with analysis by LC-ESI-Paul ion trap and Qq-TOF. J Proteomics, 73(6), 1254-1269. doi:10.1016/j.jprot.2010.02.022

Tucholska, M., Scozzaro, S., Williams, D., Ackloo, S., Lock, C., Siu, K. W. M., . . Marshall, J. G. (2007). Endogenous peptides from biophysical and biochemical fractionation of serum analyzed by 
matrix-assisted laser desorption/ionization and electrospray ionization hybrid quadrupole timeof-flight. Analytical Biochemistry, 370, 228-245. doi:10.1016/j.ab.2007.07.029

Tuck, M. K., Chan, D. W., Chia, D., Godwin, A. K., Grizzle, W. E., Krueger, K. E., . . Brenner, D. E. (2009). Standard operating procedures for serum and plasma collection: early detection research network consensus statement standard operating procedure integration working group. $J$ Proteome Res, 8(1), 113-117.

Tzircotis, G., Braga, V. M., \& Caron, E. (2011). RhoG is required for both FcyR-and CR3-mediated phagocytosis. Journal of cell science, 124(17), 2897-2902.

Ueda, K., Tatsuguchi, A., Saichi, N., Toyama, A., Tamura, K., Furihata, M., ... Nakagawa, H. (2013). Plasma low-molecular-weight proteome profiling identified neuropeptide- $Y$ as a prostate cancer biomarker polypeptide. J Proteome Res, 12(10), 4497-4506. doi:10.1021/pr400547s

Uhlen, M., Guss, B., Nilsson, B., Gatenbeck, S., Philipson, L., \& Lindberg, M. (1984). Complete sequence of the staphylococcal gene encoding protein A. A gene evolved through multiple duplications. $J$ Biol Chem, 259(3), 1695-1702.

Umeno, A., Yoshino, K., Hashimoto, Y., Shichiri, M., Kataoka, M., \& Yoshida, Y. (2015). Multi-Biomarkers for Early Detection of Type 2 Diabetes, Including 10- and 12-(Z,E)-Hydroxyoctadecadienoic Acids, Insulin, Leptin, and Adiponectin. PLOS ONE, 10(7), e0130971. doi:10.1371/journal.pone.0130971

van den Broek, I., Romijn, F. P., Nouta, J., van der Laarse, A., Drijfhout, J. W., Smit, N. P., . . Cobbaert, C. M. (2016). Automated Multiplex LC-MS/MS Assay for Quantifying Serum Apolipoproteins A-I, B, C-I, C-II, C-III, and E with Qualitative Apolipoprotein E Phenotyping. Clin Chem, 62(1), 188-197. doi:10.1373/clinchem.2015.246702

van den Broek, I., Romijn, F. P., Smit, N. P., van der Laarse, A., Drijfhout, J. W., van der Burgt, Y. E., \& Cobbaert, C. M. (2015). Quantifying protein measurands by peptide measurements: where do errors arise? J Proteome Res, 14(2), 928-942. doi:10.1021/pr5011179

van den Broek, I., Sparidans, R. W., Schellens, J. H., \& Beijnen, J. H. (2008). Liquid chromatography/tandem mass spectrometric method for the quantification of eight proteolytic fragments of ITIH4 with biomarker potential in human plasma and serum. Rapid Commun Mass Spectrom, 22(18), 2915-2928.

van Winden, A. W., Gast, M. C., Beijnen, J. H., Rutgers, E. J., Grobbee, D. E., Peeters, P. H., \& van Gils, C. H. (2009). Validation of previously identified serum biomarkers for breast cancer with SELDI-TOF MS: a case control study. BMC Med Genomics, 2(1), 4.

Vance, D. T., Dufresne, J., Florentinus-Mefailoski, A., Tucholska, M., Trimble, W., Grinstein, S., \& Marshall, J. G. (2016). A phagocytosis assay for oxidized low-density lipoprotein versus immunoglobulin G-coated microbeads in human U937 macrophages. Anal Biochem, 500, 24-34. doi:10.1016/j.ab.2016.01.007

Vergeynst, L., Van Langenhove, H., \& Demeestere, K. (2015). Balancing the false negative and positive rates in suspect screening with high-resolution Orbitrap mass spectrometry using multivariate statistics. Anal Chem, 87(4), 2170-2177. doi:10.1021/ac503426k

Vogel, M., Blobel, M., Thomas, A., Walpurgis, K., Schanzer, W., Reichel, C., \& Thevis, M. (2014). Isolation, enrichment, and analysis of erythropoietins in anti-doping analysis by receptor-coated magnetic beads and liquid chromatography-mass spectrometry. Anal Chem, 86(24), 12014-12021. doi:10.1021/ac5024765

Volanakis, J. E. (1990). Participation of C3 and its ligands in complement activation. Curr Top Microbiol Immunol, 153, 1-21.

Von Jagow, G., Schägger, H., Riccio, P., Klingenberg, M., \& Kolb, H. (1977). b· c1 complex from beef heart: Hydrodynamic properties of the complex prepared by a refined hydroxyapatite chromatography in Triton X-100. Biochimica et Biophysica Acta (BBA)-Bioenergetics, 462(3), 549558. 
von Mering, C., Jensen, L. J., Kuhn, M., Chaffron, S., Doerks, T., Kruger, B., . . Bork, P. (2007). STRING 7-recent developments in the integration and prediction of protein interactions. Nucleic Acids Res, 35(Database issue), D358-362.

von Mering, C., Jensen, L. J., Snel, B., Hooper, S. D., Krupp, M., Foglierini, M., . . Bork, P. (2005). STRING: known and predicted protein-protein associations, integrated and transferred across organisms. Nucleic Acids Res, 33(Database issue), D433-437.

Walker, M. J., Zhou, C., Backen, A., Pernemalm, M., Williamson, A. J., Priest, L. J., ... Whetton, A. D. (2015). Discovery and Validation of Predictive Biomarkers of Survival for Non-small Cell Lung Cancer Patients Undergoing Radical Radiotherapy: Two Proteins With Predictive Value. EBioMedicine, 2(8), 841-850. doi:10.1016/j.ebiom.2015.06.013

Wallin, E., \& Heijne, G. V. (1998). Genome-wide analysis of integral membrane proteins from eubacterial, archaean, and eukaryotic organisms. Protein Science, 7(4), 1029-1038.

Wang, J., Wang, X., Lin, S., Chen, C., Wang, C., Ma, Q., \& Jiang, B. (2013). Identification of kininogen-1 as a serum biomarker for the early detection of advanced colorectal adenoma and colorectal cancer. PLOS ONE, 8(7), e70519. doi:10.1371/journal.pone.0070519

Wang, Q., Su, X., Jiang, X., Dong, X., Fan, Y., Zhang, J., . . . Wei, T. (2016). iTRAQ technology-based identification of human peripheral serum proteins associated with depression. Neuroscience, 330, 291-325. doi:10.1016/j.neuroscience.2016.05.055

Wang, T. J., Larson, M. G., Levy, D., Benjamin, E. J., Leip, E. P., Omland, T., . . Vasan, R. S. (2004). Plasma natriuretic peptide levels and the risk of cardiovascular events and death. N Engl J Med, 350(7), 655-663. doi:10.1056/NEJMoa031994

Watt, A. D., Perez, K. A., Ang, C. S., O'Donnell, P., Rembach, A., Pertile, K. K., . . Barnham, K. J. (2015). Peripheral alpha-defensins 1 and 2 are elevated in Alzheimer's disease. J Alzheimers Dis, 44(4), 1131-1143. doi:10.3233/JAD-142286

Weisman, H. F., Bartow, T., Leppo, M. K., Marsh, H. C., Jr., Carson, G. R., Concino, M. F., . . Fearon, D. T. (1990). Soluble human complement receptor type 1: in vivo inhibitor of complement suppressing post-ischemic myocardial inflammation and necrosis. Science, 249(4965), 146-151.

West-Nielsen, M., Hogdall, E. V., Marchiori, E., Hogdall, C. K., Schou, C., \& Heegaard, N. H. (2005). Sample handling for mass spectrometric proteomic investigations of human sera. Anal Chem, 77(16), 5114-5123.

West-Norager, M., Kelstrup, C. D., Schou, C., Hogdall, E. V., Hogdall, C. K., \& Heegaard, N. H. (2007). Unravelling in vitro variables of major importance for the outcome of mass spectrometry-based serum proteomics. J Chromatogr B Analyt Technol Biomed Life Sci, 847(1), 30-37.

Whitaker, R. M., Korrapati, M. C., Stallons, L. J., Jesinkey, S. R., Arthur, J. M., Beeson, C. C., . . Schnellmann, R. G. (2015). Urinary ATP Synthase Subunit beta Is a Novel Biomarker of Renal Mitochondrial Dysfunction in Acute Kidney Injury. Toxicol Sci, 145(1), 108-117. doi:10.1093/toxsci/kfv038

Wiita, A. P., Hsu, G. W., Lu, C. M., Esensten, J. H., \& Wells, J. A. (2014). Circulating proteolytic signatures of chemotherapy-induced cell death in humans discovered by N-terminal labeling. Proc Natl Acad Sci U S A, 111(21), 7594-7599. doi:10.1073/pnas.1405987111

Williams, D., Ackloo, S., Zhu, P., Bowden, P., Evans, K. R., Addison, C. L., . . Marshall, J. G. (2010). Precipitation and selective extraction of human serum endogenous peptides with analysis by quadrupole time-of-flight mass spectrometry reveals posttranslational modifications and lowabundance peptides. Analytical and bioanalytical chemistry, 396, 1223-1247. doi:10.1007/s00216-009-3345-0

Xu, D., Li, Y., Li, X., Wei, L. L., Pan, Z., Jiang, T. T., .. . Li, J. C. (2015). Serum protein S100A9, SOD3, and MMP9 as new diagnostic biomarkers for pulmonary tuberculosis by iTRAQ-coupled twodimensional LC-MS/MS. Proteomics, 15(1), 58-67. doi:10.1002/pmic.201400366 
Xu, W., Hu, Y., He, X., Li, J., Pan, T., Liu, H., ... Chen, Y. (2015). Serum profiling by mass spectrometry combined with bioinformatics for the biomarkers discovery in diffuse large B-cell lymphoma. Tumour Biol, 36(3), 2193-2199. doi:10.1007/s13277-014-2830-z

Xu, Y., Prohn, M., Cai, X., Crutchlow, M., Shankar, S. S., Bateman, K., \& Woolf, E. J. (2014). Direct comparison of radioimmunoassay and LC-MS/MS for PK assessment of insulin glargine in clinical development. Bioanalysis, 6(24), 3311-3323. doi:10.4155/bio.14.219

Yang, J., Xiong, X., Wang, X., Guo, B., He, K., \& Huang, C. (2015). Identification of peptide regions of SERPINA1 and ENOSF1 and their protein expression as potential serum biomarkers for gastric cancer. Tumour Biol, 36(7), 5109-5118. doi:10.1007/s13277-015-3163-2

Yang, L., Tang, C., Xu, B., Wang, W., Li, J., Li, X., . . Liu, X. (2015). Classification of Epidermal Growth Factor Receptor Gene Mutation Status Using Serum Proteomic Profiling Predicts Tumor Response in Patients with Stage IIIB or IV Non-Small-Cell Lung Cancer. PLOS ONE, 10(6), e0128970. doi:10.1371/journal.pone.0128970

Yang, X., Clifton, J., Huang, F., Kovac, S., Hixson, D. C., \& Josic, D. (2009). Proteomic analysis for process development and control of therapeutic protein separation from human plasma. Electrophoresis, 30(7), 1185-1193.

Yates, J. R., 3rd. (1998). Database searching using mass spectrometry data. Electrophoresis, 19(6), 893900.

Yates, J. R., 3rd, Eng, J. K., McCormack, A. L., \& Schieltz, D. (1995). Method to correlate tandem mass spectra of modified peptides to amino acid sequences in the protein database. Anal Chem, 67(8), 1426-1436.

Yatskin, O. N., Sazonova, O. V., Khachin, D. P., Blishchenko, E. Y., Karelin, A. A., \& Ivanov, V. T. (2009). Isolation of peptides from rat tissues: peptidomics vs. degradomics. Adv Exp Med Biol, 611, 399400.

Ye, B., Cramer, D. W., Skates, S. J., Gygi, S. P., Pratomo, V., Fu, L., ... Mok, S. C. (2003). Haptoglobinalpha subunit as potential serum biomarker in ovarian cancer: identification and characterization using proteomic profiling and mass spectrometry. Clin Cancer Res, 9(8), 29042911.

Yi, J., Kim, C., \& Gelfand, C. A. (2007). Inhibition of intrinsic proteolytic activities moderates preanalytical variability and instability of human plasma. J Proteome Res, 6(5), 1768-1781.

Yi, J., Liu, Z., Craft, D., O'Mullan, P., Ju, G., \& Gelfand, C. A. (2008). Intrinsic peptidase activity causes a sequential multi-step reaction (SMSR) in digestion of human plasma peptides. J Proteome Res, 7(12), 5112-5118.

Yi, J., Warunek, D., \& Craft, D. (2015). Degradation and Stabilization of Peptide Hormones in Human Blood Specimens. PLOS ONE, 10(7), e0134427. doi:10.1371/journal.pone.0134427

Yin, H., Tan, Z., Wu, J., Zhu, J., Shedden, K. A., Marrero, J., \& Lubman, D. M. (2015). Mass-Selected SiteSpecific Core-Fucosylation of Serum Proteins in Hepatocellular Carcinoma. J Proteome Res, 14(11), 4876-4884. doi:10.1021/acs.jproteome.5b00718

Yin, L., Liu, J., Dong, H., Xu, E., Qiao, Y., Wang, L., . . Geng, X. (2014). Autophagy-related gene16L2, a potential serum biomarker of multiple sclerosis evaluated by bead-based proteomic technology. Neurosci Lett, 562, 34-38. doi:10.1016/j.neulet.2013.12.070

Young, J., Ko, S. S., \& Cohn, Z. A. (1984). The increase in intracellular free calcium associated with IgG gamma 2b/gamma $1 \mathrm{Fc}$ receptor-ligand interactions: role in phagocytosis. Proceedings of the National Academy of Sciences, 81(17), 5430-5434.

Yu, Y., Jin, D., Hu, S., Zhang, Y., Zheng, X., Zheng, J., . . . Jin, Q. (2015). A novel tuberculosis antigen identified from human tuberculosis granulomas. Mol Cell Proteomics, 14(4), 1093-1103. doi:10.1074/mcp.M114.045237 
Zaidi, A. H., Gopalakrishnan, V., Kasi, P. M., Zeng, X., Malhotra, U., Balasubramanian, J., ... Jobe, B. A. (2014). Evaluation of a 4-protein serum biomarker panel-biglycan, annexin-A6, myeloperoxidase, and protein S100-A9 (B-AMP)-for the detection of esophageal adenocarcinoma. Cancer, 120(24), 3902-3913. doi:10.1002/cncr.28963

Zhang, A., Wang, B., Yang, M., Shi, H., \& Gan, W. (2015). beta2-microglobulin induces epithelialmesenchymal transition in human renal proximal tubule epithelial cells in vitro. BMC Nephrol, 16, 60. doi:10.1186/s12882-015-0057-x

Zhang, J., Guo, F., Wang, L., Zhao, W., Zhang, D., Yang, H., ... Wang, J. (2014). Identification of apolipoprotein C-I as a potential Wilms' tumor marker after excluding inflammatory factors. Int $J$ Mol Sci, 15(9), 16186-16195. doi:10.3390/ijms150916186

Zhang, P., Kirk, J. A., Ji, W., dos Remedios, C. G., Kass, D. A., Van Eyk, J. E., \& Murphy, A. M. (2012). Multiple reaction monitoring to identify site-specific troponin I phosphorylated residues in the failing human heart. Circulation, 126(15), 1828-1837. doi:10.1161/CIRCULATIONAHA.112.096388

Zhang, R., Barker, L., Pinchev, D., Marshall, J., Rasamoelisolo, M., Smith, C., . . Jackowski, G. (2004). Mining biomarkers in human sera using proteomic tools. Proteomics, 4(1), 244-256.

Zhang, Y., Cai, Y., Yu, H., \& Li, H. (2015). iTRAQ-Based Quantitative Proteomic Analysis Identified HSC71 as a Novel Serum Biomarker for Renal Cell Carcinoma. Biomed Res Int, 2015, 802153. doi:10.1155/2015/802153

Zheng, X., Baker, H., Hancock, W. S., Fawaz, F., McCaman, M., \& Pungor, E., Jr. (2006). Proteomic analysis for the assessment of different lots of fetal bovine serum as a raw material for cell culture. Part IV. Application of proteomics to the manufacture of biological drugs. Biotechnol Prog, 22(5), 1294-1300.

Zhu, P., Bowden, P., Pendrak, V., Thiele, H., Zhang, D., Siu, M., . . Marshall, J. (2007). Comparison of protein expression lists from mass spectrometry of human blood fluids using exact peptide sequences versus BLAST. Clinical Proteomics, 2(3-4), 185-203.

Zhu, P., Bowden, P., Tucholska, M., \& Marshall, J. G. (2011). Chi-square comparison of tryptic peptide-toprotein distributions of tandem mass spectrometry from blood with those of random expectation. Anal Biochem, 409(2), 189-194.

Zhu, P., Bowden, P., Tucholska, M., Zhang, D., \& Marshall, J. G. (2011). Peptide-to-protein distribution versus a competition for significance to estimate error rate in blood protein identification. Analytical Biochemistry, 411, 241-253. doi:10.1016/j.ab.2010.12.003

Zhu, P., Bowden, P., Zhang, D., \& Marshall, J. G. (2011). Mass spectrometry of peptides and proteins from human blood. Mass Spectrom Rev, 30,(5), 685-732.

Zolg, D. P., Wilhelm, M., Schnatbaum, K., Zerweck, J., Knaute, T., Delanghe, B., .. Kuster, B. (2017). Building ProteomeTools based on a complete synthetic human proteome. Nat Methods, 14(3), 259-262. doi:10.1038/nmeth.4153

Zou, X., Feng, B., Dong, T., Yan, G., Tan, B., Shen, H., . . Zhang, Y. (2013). Up-regulation of type I collagen during tumorigenesis of colorectal cancer revealed by quantitative proteomic analysis. $J$ Proteomics, 94, 473-485. doi:10.1016/j.jprot.2013.10.020 


\section{TABLES}

Table I. Proteins with tryptic (TRYP) or phosphor (STYP) peptides specific to each disease with the criteria of a Chi Square of at least 9.

\begin{tabular}{|c|c|}
\hline \multicolumn{2}{|l|}{ ALL Diseases TRP } \\
\hline GeneSymbol & DescriptionDatabase \\
\hline $\mathrm{HP}$ & Haptoglobin \\
\hline HPR & Haptoglobin-related protein \\
\hline SERPINA1 & Alpha-1-antitrypsin \\
\hline A1AT & PRO2275 \\
\hline FGA & fibrinogen alpha chain isoform alpha preproprotein \\
\hline APOA1 & Apolipoprotein A-I \\
\hline APOA1 & Truncated apolipoprotein A-I \\
\hline HP & Haptoglobin (Fragment) \\
\hline LTBP4 & Latent-transforming growth factor beta-binding protein 4 \\
\hline C3 & Complement C3 \\
\hline \multirow[t]{2}{*}{ ZNF254 } & zinc finger protein 254 isoform a \\
\hline & cDNA FL76837, highly similar to Homo sapiens zinc finger protein 539 (ZNF539), mRNA \\
\hline CPEB1 & Cytoplasmic polyadenylation element-binding protein 1 (Fragment) \\
\hline ZNF91 & Zinc finger protein 91 \\
\hline CPEB1 & Cytoplasmic polyadenylation element-binding protein 1 \\
\hline C19orf82 & Uncharacterized protein C19orf82 \\
\hline \multirow[t]{2}{*}{ PTPDC1 } & protein tyrosine phosphatase domain-containing protein 1 isoform 3 \\
\hline & $\begin{array}{l}\text { cDNA FL75103, highly similar to Homo sapiens protein tyrosine phosphatase domain } \\
\text { containing } 1 \text { (PTPDC1), transcript variant 1, mRNA }\end{array}$ \\
\hline CHD6 & Chromodomain-helicase-DNA-binding protein 6 \\
\hline C8orf34 & uncharacterized protein C8orf34 isoform 2 \\
\hline C8orf34 & PREDICTED: uncharacterized protein C8orf34 isoform X1 \\
\hline CHD6 & PREDICTED: chromodomain-helicase-DNA-binding protein 6 isoform $\times 2$ \\
\hline $\mathrm{A} 2 \mathrm{M}$ & PREDICTED: alpha-2-macroglobulin isoform X1 \\
\hline \multirow[t]{2}{*}{ DKFZp779G1236 } & Putative uncharacterized protein DKFZp779G1236 \\
\hline & cDNA FL54364, highly similar to Hypoxia-inducible factor 1 alpha \\
\hline OCLN & Occludin \\
\hline \multirow[t]{3}{*}{ PCLO } & Protein piccolo \\
\hline & $\begin{array}{l}\text { cDNA FL44011 fis, clone TESTI4024420, highly similar to Rattus norvegicus presynaptic } \\
\text { cytomatrix protein (Pclo) }\end{array}$ \\
\hline & $\begin{array}{l}\text { cDNA, FL92729, highly similar to Homo sapiens hypoxia-inducible factor } 1 \text {, alpha subunit } \\
\text { (basic helix-loop-helix transcription factor) (HIF1A), mRNA }\end{array}$ \\
\hline HIF1A & Hypoxia-inducible factor 1-alpha \\
\hline TUBGCP6 & Gamma-tubulin complex component 6 \\
\hline
\end{tabular}


HIF1A

HIF-1A

TUBGCP6

QSER1

APOE

QSER1

LOC101929927

TMC3

FAM90A2P

DISC1

FBL

TMC3

IGHE

DCHS2

RUNDC1

$\mathrm{PCDHJ}$

ZNF562

$\mathrm{CFI}$

All Disease STYP

GeneSymbol

HP

FGA

C8orf34

APOA1

C3

KNOP1

LOC102723665

HIF-1A

WEE1

TUBGCP6

SPATA13

WEE1

ERCC6-PGBD3

CSMD3

QSER1
Hypoxia-inducible factor 1, alpha subunit (Basic helix-loop-helix transcription factor), isoform CRA_a

Hypoxia-inducible factor1alpha

gamma-tubulin complex component 6

PREDICTED: glutamine and serine-rich protein 1 isoform $X 2$

Apolipoprotein E

Glutamine and serine-rich protein 1

CDNA: FL21924 fis, clone HEP04086

cDNA FL433914 fis, clone TESTI4011161, weakly similar to Proline-rich protein 12

Endozepine-like protein type 2 mutant

PREDICTED: putative protein FAM90A16P/FAM90A17P-like

Transmembrane channel-like protein

cDNA FL53361, highly similar to Zinc finger protein 562

Putative protein FAM90A2P

Tyrosine kinase

ALB protein

disrupted in schizophrenia 1 protein isoform $\mathrm{h}$

rRNA 2'-O-methyltransferase fibrillarin (Fragment)

Transmembrane channel-like protein 3

Ig epsilon chain $\mathrm{C}$ region

CDNA FL25952 fis, clone SYN00911

Protocadherin-23

PREDICTED: RUN domain-containing protein 1 isoform X1

Protocadherin protein (Fragment)

Zinc finger protein 562

cDNA FL58124, highly similar to Complement factor I (EC 3.4.21.45)

\section{DescriptionDatabase}

Haptoglobin

fibrinogen alpha chain isoform alpha preproprotein

uncharacterized protein C8orf34 isoform 1

Apolipoprotein A-I

Complement C3

PREDICTED: lysine-rich nucleolar protein 1 isoform X1

PREDICTED: uncharacterized protein LOC284861-like

Hypoxia-inducible factor1alpha

WEE1 homolog (S. pombe)

gamma-tubulin complex component 6

spermatogenesis-associated protein 13 isoform 1

PREDICTED: wee1-like protein kinase isoform X1

PiggyBac transposable element-derived protein 3

CUB and sushi domain-containing protein 3 isoform 3

Glutamine and serine-rich protein 1 


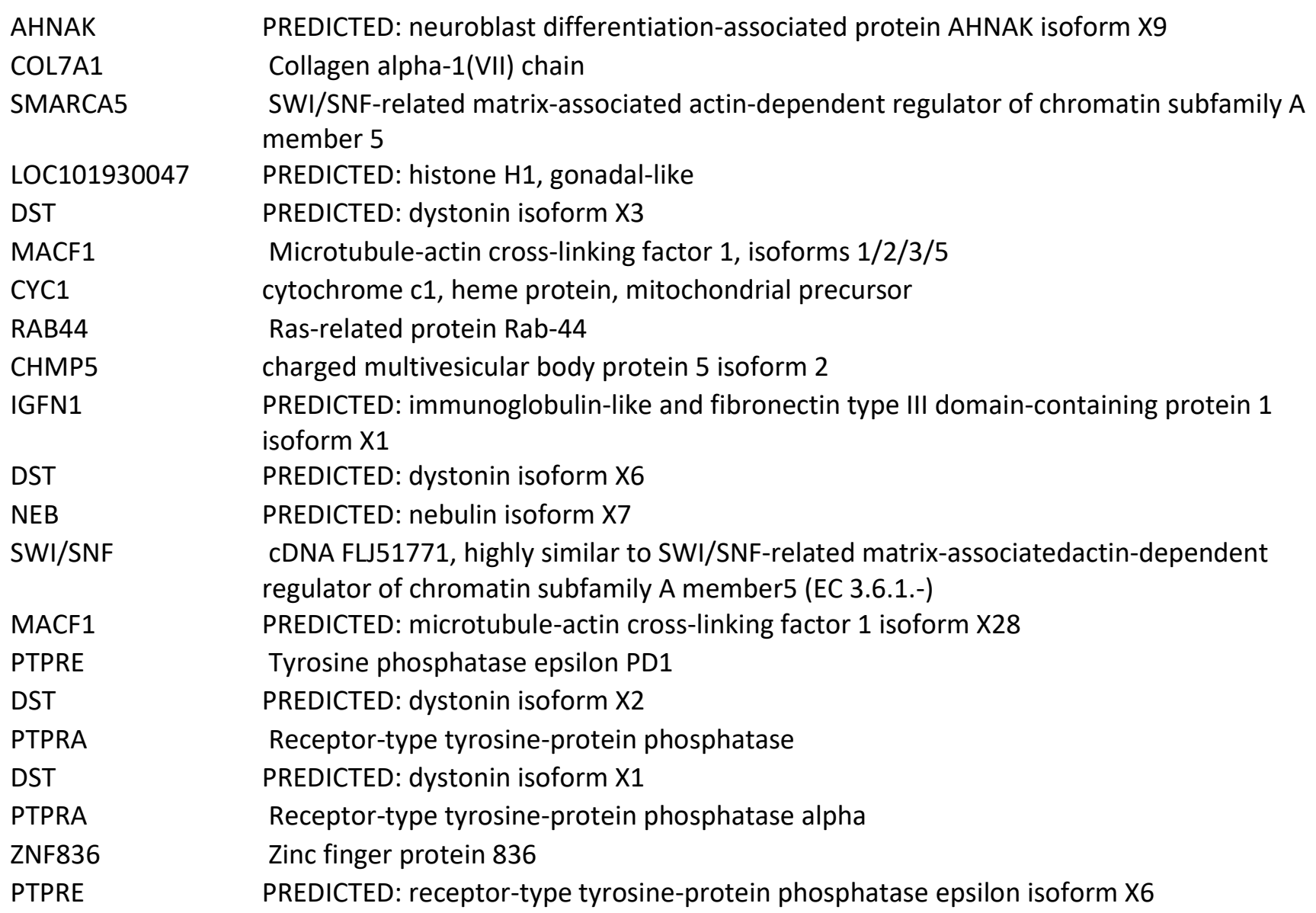

PREDICTED: neuroblast differentiation-associated protein AHNAK isoform X9 Collagen alpha-1(VII) chain SWI/SNF-related matrix-associated actin-dependent regulator of chromatin subfamily A member 5

PREDICTED: histone $\mathrm{H} 1$, gonadal-like PREDICTED: dystonin isoform X3

Microtubule-actin cross-linking factor 1 , isoforms $1 / 2 / 3 / 5$

cytochrome c1, heme protein, mitochondrial precursor

Ras-related protein Rab-44

charged multivesicular body protein 5 isoform 2

PREDICTED: immunoglobulin-like and fibronectin type III domain-containing protein 1 isoform X1

PREDICTED: dystonin isoform $X 6$

PREDICTED: nebulin isoform $X 7$

cDNA FL51771, highly similar to SWI/SNF-related matrix-associatedactin-dependent regulator of chromatin subfamily A member5 (EC 3.6.1.-)

PREDICTED: microtubule-actin cross-linking factor 1 isoform X28

Tyrosine phosphatase epsilon PD1

PREDICTED: dystonin isoform $\mathrm{X} 2$

Receptor-type tyrosine-protein phosphatase

PREDICTED: dystonin isoform X1

Receptor-type tyrosine-protein phosphatase alpha

Zinc finger protein 836

PREDICTED: receptor-type tyrosine-protein phosphatase epsilon isoform X6

\section{DescriptionDatabase}

Latent-transforming growth factor beta-binding protein 4

Cytoplasmic polyadenylation element-binding protein 1 (Fragment)

uncharacterized protein C8orf34 isoform 1

Uncharacterized protein C19orf82

protein tyrosine phosphatase domain-containing protein 1 isoform 1

PREDICTED: protein piccolo isoform X1

Apolipoprotein A-I

Occludin

Putative RNA-binding protein 15

cDNA FL54364, highly similar to Hypoxia-inducible factor 1 alpha

Endozepine-like protein type 2 mutant

Putative uncharacterized protein DKFZp779G1236

cDNA FL44011 fis, clone TESTI4024420, highly similar to Rattus norvegicus presynaptic cytomatrix protein (Pclo)

TUBGCP6 protein

Protein bassoon

Apolipoprotein E (Fragment) 
DNAH17

IGHE

BC STYP

GeneSymbol

PCDHGA5

LTBP4

C8orf34

KNOP1

DCHS2

LOC101060770

LOC102723665

HMMR

ZFAND1

CYC1

ZFAND1

WEE1

LMO2

HIF1A

OC TRYP

GeneSymbol

HP

SERPINA1

ZNF91

ZNF254

CHD6

QSER1

HPX

APOA1

AHNAK2

GPC6

DNAH8

OC STYP

GeneSymbol dynein heavy chain 17 , axonemal

Ig epsilon chain $\mathrm{C}$ region

cDNA FL53361, highly similar to Zinc finger protein 562

DescriptionDatabase

Protocadherin gamma-A5

Latent-transforming growth factor beta-binding protein 4

uncharacterized protein C8orf34 isoform 1

PREDICTED: Iysine-rich nucleolar protein 1 isoform X1

Protocadherin-23

PREDICTED: oleosin-B4-like

PREDICTED: uncharacterized protein LOC284861-like

cDNA FL57968, highly similar to Zinc finger protein 438

Hyaluronan-mediated motility receptor (Fragment)

AN1-type zinc finger protein 1

cytochrome $\mathrm{c} 1$, heme protein, mitochondrial precursor

AN1-type zinc finger protein 1

WEE1 homolog (S. pombe)

cDNA FL55752, highly similar to Hypoxia-inducible factor 1 alpha

PREDICTED: rhombotin-2 isoform X1

Hypoxia-inducible factor 1, alpha subunit (Basic helix-loop-helix transcription factor), isoform CRA_a

DescriptionDatabase

haptoglobin isoform 2 preproprotein

Alpha-1-antitrypsin

Zinc finger protein 91

zinc finger protein 254 isoform a

cDNA FL76837, highly similar to Homo sapiens zinc finger protein 539 (ZNF539), mRNA

CDNA FL58216, highly similar to Zinc finger protein 539

PREDICTED: chromodomain-helicase-DNA-binding protein 6 isoform X3

PREDICTED: glutamine and serine-rich protein 1 isoform $X 2$

cDNA: FL21924 fis, clone HEP04086

HPX protein

Apolipoprotein A-I

PREDICTED: protein AHNAK2 isoform X1

Glypican-6

PREDICTED: dynein heavy chain 8, axonemal isoform X1 


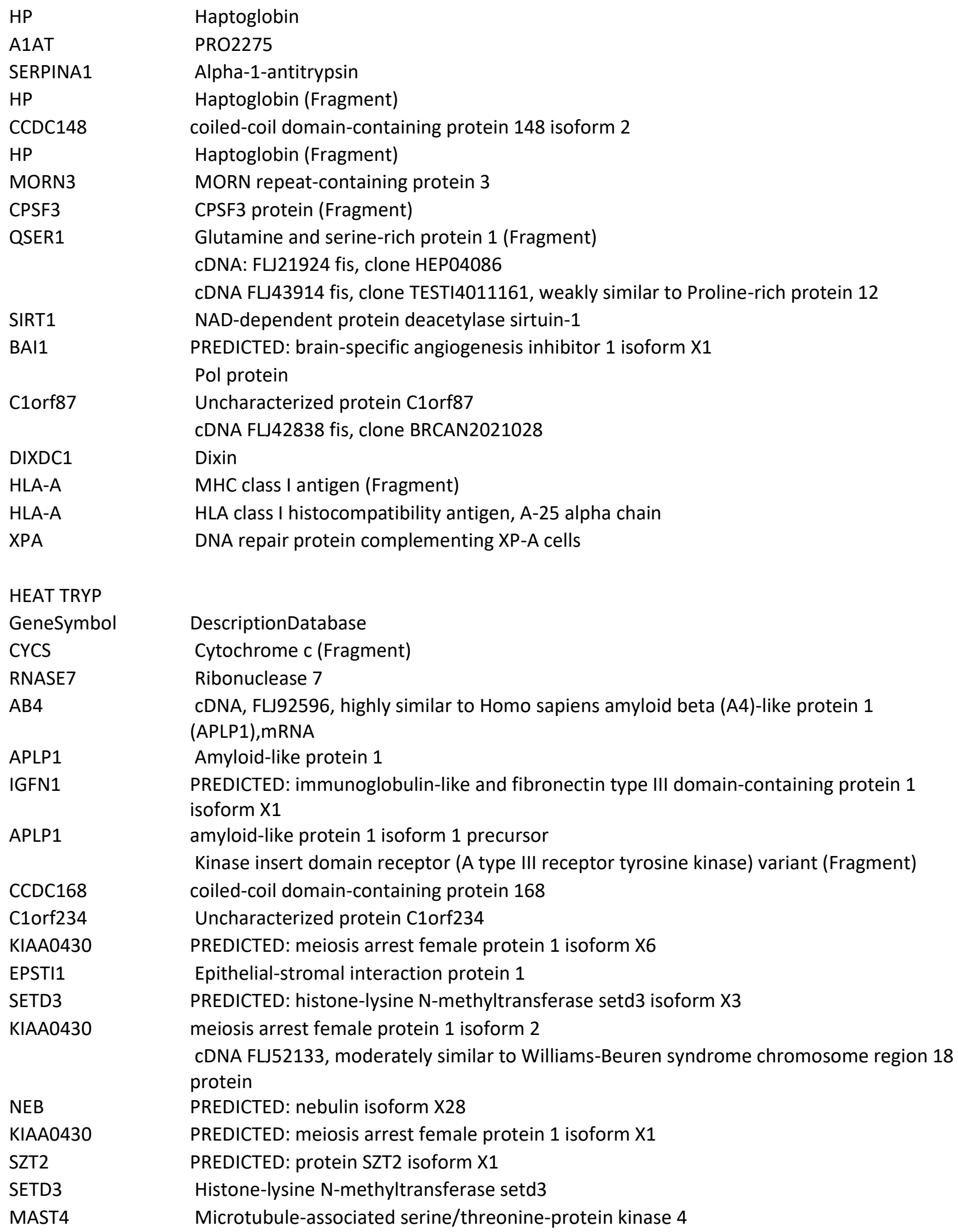

\section{DescriptionDatabase}

Cytochrome c (Fragment)

Ribonuclease 7

CDNA, FL92596, highly similar to Homo sapiens amyloid beta (A4)-like protein 1 (APLP1),mRNA

Amyloid-like protein 1

PREDICTED: immunoglobulin-like and fibronectin type III domain-containing protein 1 isoform X1

amyloid-like protein 1 isoform 1 precursor

Kinase insert domain receptor (A type III receptor tyrosine kinase) variant (Fragment)

coiled-coil domain-containing protein 168

Uncharacterized protein C1orf234

PREDICTED: meiosis arrest female protein 1 isoform X6

Epithelial-stromal interaction protein 1

PREDICTED: histone-lysine $\mathrm{N}$-methyltransferase setd3 isoform X3

meiosis arrest female protein 1 isoform 2

cDNA FL52133, moderately similar to Williams-Beuren syndrome chromosome region 18 protein

PREDICTED: nebulin isoform $\mathrm{X} 28$

PREDICTED: meiosis arrest female protein 1 isoform $X 1$

PREDICTED: protein SZT2 isoform X1

Histone-lysine $\mathrm{N}$-methyltransferase setd3

Microtubule-associated serine/threonine-protein kinase 4 
EPSTI1

MAST4

KIAA1529

KIAA0430

FOXM1

ASPM

FLJ00404

LOC101928841

MACF1

UNC13D

AARS

TMEM218

DIP2B

PCLO

UBR4

DNAH10

OBSL1

AARS

DST

UBR4

HEAT STYP

GeneSymbol

CYCS

EPSTI1

COL6A3

KMT2A

CACNA1I

HTR3E

TCOF1

PRR14L

SLCO2B1

RGS12

RPL4

SETX

PRR14L

SLCO2B1

COL6A3

SETX

CCAR1

SEC61A2

ANKRD36B

CROCC
PREDICTED: epithelial-stromal interaction protein 1 isoform $\mathrm{X} 3$

PREDICTED: microtubule-associated serine/threonine-protein kinase 4 isoform $X 2$

KIAA1529 protein (Fragment)

PREDICTED: meiosis arrest female protein 1 isoform $\mathrm{X} 3$

forkhead box protein M1 isoform 1

Abnormal spindle-like microcephaly-associated protein

FLJ00404 protein (Fragment)

PREDICTED: filaggrin-like isoform X1

PREDICTED: microtubule-actin cross-linking factor 1 isoform $\mathrm{X} 26$

PREDICTED: protein unc-13 homolog $D$ isoform $X 2$

PREDICTED: alanine--tRNA ligase, cytoplasmic isoform X1

Transmembrane protein 218

Disco-interacting protein 2 homolog B

protein piccolo isoform 2

PREDICTED: E3 ubiquitin-protein ligase UBR4 isoform X6

Dynein heavy chain 10, axonemal

PREDICTED: obscurin-like protein 1 isoform X12

Alanine--tRNA ligase, cytoplasmic

PREDICTED: dystonin isoform X1

PREDICTED: E3 ubiquitin-protein ligase UBR4 isoform X7

\section{DescriptionDatabase \\ Cytochrome c (Fragment)}

Epithelial-stromal interaction protein 1

PREDICTED: collagen alpha-3(VI) chain isoform X3

histone-lysine $\mathrm{N}$-methyltransferase $2 \mathrm{~A}$ isoform 1 precursor

Voltage-dependent T-type calcium channel subunit alpha-1I

Ribosomal protein L4 variant (Fragment)

5-hydroxytryptamine receptor $3 \mathrm{E}$ isoform a

treacle protein isoform $\mathrm{f}$

Protein PRR14L

solute carrier organic anion transporter family member 2B1 isoform 2

Regulator of G-protein signaling 12

cDNA FL50996, highly similar to 60S ribosomal protein L4

Probable helicase senataxin

PREDICTED: protein PRR14L isoform X4

Solute carrier organic anion transporter family member 2B1

Collagen alpha-3(VI) chain (Fragment)

PREDICTED: probable helicase senataxin isoform X1

cell division cycle and apoptosis regulator protein 1 isoform $b$ protein transport protein Sec61 subunit alpha isoform 2 isoform $b$

PREDICTED: ankyrin repeat domain-containing protein 36B isoform X12

Rootletin (Fragment) 
COL24A1

STK38L

PHLDB1

FAM53C

ANKRD36B

PHLDB1

KMT2A

PHLDB1

CACNA1I

PHLDB1

MS TRYP

GeneSymbol

FGA

CHD4

$\mathrm{CHD} 3$

TNRC18

IKBKB

DAPK2

IKBKB

IKBKB

SYNE1

GCN1L1

PRIC295

SPEN

ZFHX4

PLEC

COL7A1

ZFHX4

REV3L

LOC728763

C19orf68

MS STYP

GeneSymbol

FGA

IKBKB

GCN1L1

GRASP

PRIC295
Collagen alpha-1(XXIV) chain

Serine/threonine-protein kinase 38-like

PREDICTED: pleckstrin homology-like domain family B member 1 isoform X22

Protein FAM53C (Fragment)

PREDICTED: ankyrin repeat domain-containing protein 36B isoform $\mathrm{X} 10$

PREDICTED: pleckstrin homology-like domain family $B$ member 1 isoform $X 21$

PREDICTED: histone-lysine N-methyltransferase 2A isoform X1

PREDICTED: pleckstrin homology-like domain family $B$ member 1 isoform X18

PREDICTED: voltage-dependent T-type calcium channel subunit alpha-1I isoform X1

PREDICTED: pleckstrin homology-like domain family B member 1 isoform X19

\section{DescriptionDatabase}

fibrinogen alpha chain isoform alpha preproprotein

Chromodomain-helicase-DNA-binding protein 4

PREDICTED: chromodomain-helicase-DNA-binding protein 3 isoform X12

Trinucleotide repeat-containing gene 18 protein

IKBKB protein

Death-associated protein kinase 2

Inhibitor of kappa light polypeptide gene enhancer in B-cells, kinase beta, isoform CRA_b

Inhibitor of nuclear factor kappa-B kinase subunit beta

cDNA FL77419

Nesprin-1

translational activator GCN1

Peroxisome proliferator activated receptor interacting complex protein

Msx2-interacting protein

Zinc finger homeobox protein 4

PREDICTED: plectin isoform $X 2$

Collagen alpha-1(VII) chain

zinc finger homeobox protein 4

DNA polymerase zeta catalytic subunit

PREDICTED: rootletin-like

PREDICTED: uncharacterized protein C19orf68 isoform X2

\section{DescriptionDatabase}

fibrinogen alpha chain isoform alpha preproprotein

Inhibitor of kappa light polypeptide gene enhancer in B-cells, kinase beta, isoform CRA_b CDNA FL77419

Translational activator GCN1

General receptor for phosphoinositides 1-associated scaffold protein (Fragment)

Peroxisome proliferator activated receptor interacting complex protein 


$\begin{array}{ll}\text { RAB21 } & \text { Ras-related protein Rab-21 } \\ \text { PDZD2 } & \text { PREDICTED: PDZ domain-containing protein 2 isoform X6 } \\ \text { SPEN } & \text { MsX2-interacting protein } \\ \text { MYH10 } & \text { PREDICTED: myosin-10 isoform X2 } \\ \text { PDZD2 } & \text { PREDICTED: PDZ domain-containing protein 2 isoform X8 } \\ \text { ZFHX4 } & \text { Zinc finger homeobox protein } 4 \\ \text { MYH10 } & \text { Myosin-10 } \\ \text { ZNF541 } & \text { PREDICTED: zinc finger protein 541 isoform X2 } \\ \text { MYH10 } & \text { MYH10 protein } \\ \text { BCORL1 } & \text { PREDICTED: BCL-6 corepressor-like protein 1 isoform X5 } \\ \text { KIF1C } & \text { Kinesin-like protein KIF1C } \\ \text { RP1 } & \text { Oxygen-regulated protein 1 } \\ \text { KIF1C } & \text { Kinesin-like protein KIF1C } \\ \text { ANKRD18B } & \text { ankyrin repeat domain-containing protein 18B } \\ \text { MYH10 } & \text { PREDICTED: myosin-10 isoform X3 } \\ \text { DPH1 } & \text { Diphthamide biosynthesis protein } 1 \text { (Fragment) } \\ \text { ERCC6-PGBD3 } & \text { PiggyBac transposable element-derived protein 3 } \\ \text { hCG_32740 } & \text { HCG32740, isoform CRA_b } \\ \text { ZMYND8 } & \text { protein kinase C-binding protein } 1 \text { isoform j } \\ & \text { CDNA FL55380, highly similar to Protein kinase C-binding protein 1 } \\ \text { DIP2C } & \text { PREDICTED: disco-interacting protein 2 homolog C isoform X3 } \\ \text { BCOR-RARA } & \text { BCL6 corepressor/retinoic acid receptor alpha fusion protein } \\ \text { ZNF541 } & \text { PREDICTED: zinc finger protein 541 isoform X5 } \\ \text { ANKRD18B } & \text { Ankyrin repeat domain-containing protein 18B } \\ \text { PRKCBP1 } & \text { Protein kinase C binding protein 1 } \\ \text { ZMYND8 } & \text { protein kinase C-binding protein } 1 \text { isoform c } \\ \text { DDX41 } & \text { DEAD (Asp-Glu-Ala-Asp) box polypeptide 41, isoform CRA_a } \\ \end{array}$

AD TRYP

$\begin{array}{ll}\text { GeneSymbol } & \text { DescriptionDatabase } \\ \text { SPEG } & \text { PREDICTED: striated muscle preferentially expressed protein kinase isoform X2 } \\ \text { TSPEAR } & \text { Thrombospondin-type laminin G domain and EAR repeat-containing protein } \\ \text { C1orf234 } & \text { Uncharacterized protein C1orf234 } \\ \text { SPEG } & \text { PREDICTED: striated muscle preferentially expressed protein kinase isoform X12 } \\ \text { DENND5A } & \text { DENN domain-containing protein } 5 \mathrm{~A} \text { isoform } 2 \\ \text { PEX26intG231T } & \text { Pex26pintG231T } \\ \text { PLEC } & \text { PREDICTED: plectin isoform X3 } \\ \text { DNAH1 } & \text { PREDICTED: dynein heavy chain 1, axonemal isoform X1 } \\ \text { MISP } & \text { PREDICTED: mitotic interactor and substrate of PLK1 isoform X1 } \\ \text { DISC1 } & \text { Disrupted in schizophrenia } 1 \text { isoform } 12 \\ \text { CDH13 } & \text { PREDICTED: cadherin-13 isoform X1 } \\ & \text { CDNA FL53422, highly similar to TATA-binding protein-associated factor } 2 \mathrm{~N}\end{array}$


Histone $\mathrm{H} 4$ gene (Fragment)

\begin{tabular}{|c|c|}
\hline AD STYP & \\
\hline GeneSymbol & DescriptionDatabase \\
\hline PEX26intG231T & Pex26pintG231T \\
\hline DENND5A & DENN domain-containing protein $5 \mathrm{~A}$ \\
\hline PTPN13 & Protein tyrosine phosphatase, non-receptor type 13 isoform 2 variant (Fragment) \\
\hline DAPK2 & Death-associated protein kinase 2 \\
\hline & Histone H4 gene (Fragment) \\
\hline KIF7 & PREDICTED: kinesin-like protein KIF7 isoform X3 \\
\hline C2CD4B & C2 calcium-dependent domain-containing protein 4B \\
\hline RBM17 & Splicing factor 45 \\
\hline PROSC & PREDICTED: proline synthase co-transcribed bacterial homolog protein isoform X1 \\
\hline NKIRAS2 & NF-kappa-B inhibitor-interacting Ras-like protein 2 \\
\hline COL4A2 & Collagen alpha-2(IV) chain \\
\hline XIRP1 & xin actin-binding repeat-containing protein 1 isoform 2 \\
\hline BRCA1 & Breast cancer type 1 susceptibility protein \\
\hline RBM17 & Splicing factor 45 (Fragment) \\
\hline BRCA1 & $\begin{array}{l}\text { cDNA FL58524, highly similar to Homo sapiens breast cancer } 1 \text {, early onset (BRCA1), } \\
\text { transcript variant BRCA1-delta 11b, mRNA }\end{array}$ \\
\hline PPHLN1 & periphilin-1 isoform 5 \\
\hline AHNAK2 & Protein AHNAK2 \\
\hline LAMA2 & laminin subunit alpha-2 isoform b precursor \\
\hline PDCD7 & Programmed cell death protein 7 \\
\hline PPHLN1 & periphilin-1 isoform 4 \\
\hline GOLGA3 & PREDICTED: Golgin subfamily A member 3 isoform X6 \\
\hline EPPK1 & epiplakin \\
\hline LOC440563 & Heterogeneous nuclear ribonucleoprotein C-like \\
\hline NOTCH4 & Notch 4 intracellular domain \\
\hline KIF7 & KIF7 protein \\
\hline TRPM4 & PREDICTED: transient receptor potential cation channel subfamily $\mathrm{M}$ member 4 isoform $\mathrm{X} 5$ \\
\hline RBM17 & Splicing factor 45 (Fragment) \\
\hline LRP4 & Low-density lipoprotein receptor-related protein 4 \\
\hline
\end{tabular}

SEPSIS TRYP

GeneSymbol

SAA1

TAF15

SAA2

MACF1

LRP2

\section{DescriptionDatabase}

Serum amyloid A protein

TATA-binding protein-associated factor 2N (Fragment)

Serum amyloid A-2 protein

Microtubule-actin cross-linking factor 1 , isoforms $1 / 2 / 3 / 5$

Putative uncharacterized protein LRP2 (Fragment) 


$\begin{array}{ll}\text { DNAH11 } & \text { Dynein, axonemal, heavy chain 11 } \\ \text { CCL16 } & \text { C-C motif chemokine 16 } \\ \text { RPS6KL1 } & \text { PREDICTED: ribosomal protein S6 kinase-like 1 isoform X10 } \\ \text { SUPT6H } & \text { PREDICTED: transcription elongation factor SPT6 isoform X3 } \\ \text { GPR98 } & \text { G-protein coupled receptor } 98 \\ \text { VPS13C } & \text { vacuolar protein sorting-associated protein 13C isoform 1A } \\ \text { LRBA } & \text { Lipopolysaccharide-responsive and beige-like anchor protein } \\ \text { BCLAF1 } & \text { Bcl-2-associated transcription factor 1 } \\ \text { NCAPD3 } & \text { Condensin-2 complex subunit D3 } \\ \text { HUWE1 } & \text { PREDICTED: E3 ubiquitin-protein ligase HUWE1 isoform X4 } \\ & \\ \text { SEPSIS STYP } & \\ \text { GeneSymbol } & \text { DescriptionDatabase } \\ \text { SYNE2 } & \text { PREDICTED: nesprin-2 isoform X1 } \\ \text { TROPH } & \text { Polytrophin } \\ \text { SAA2 } & \text { Serum amyloid A-2 protein } \\ \text { SAA1 } & \text { Serum amyloid A-1 protein } \\ \text { USP26 } & \text { Ubiquitin carboxyl-terminal hydrolase } \\ & \text { CDNA, FL96511 } \\ \text { SYDE1 } & \text { Rho GTPase-activating protein SYDE1 } \\ \text { USP26 } & \text { Ubiquitin carboxyl-terminal hydrolase } \\ \text { ITPR2 } & \text { Inositol 1,4,5-trisphosphate receptor type 2 } \\ \text { MORC2 } & \text { PREDICTED: MORC family CW-type zinc finger protein 2 isoform X2 } \\ \text { SYDE1 } & \text { F02569_2 } \\ \text { DNAH2 } & \text { Rho GTPase-activating protein SYDE1 } \\ \text { ASPM } & \text { Dynein heavy chain 2, axonemal } \\ \text { CKAP5 } & \text { Abnormal spindle-like microcephaly-associated protein } \\ \text { HES6 } & \text { Cytoskeleton-associated protein } 5 \text { isoform b } \\ \text { EVPL } & \text { Envoplakin } \\ & \end{array}$


Table II. The summary of STRING analysis with respect to a random sampling of the human Genome.

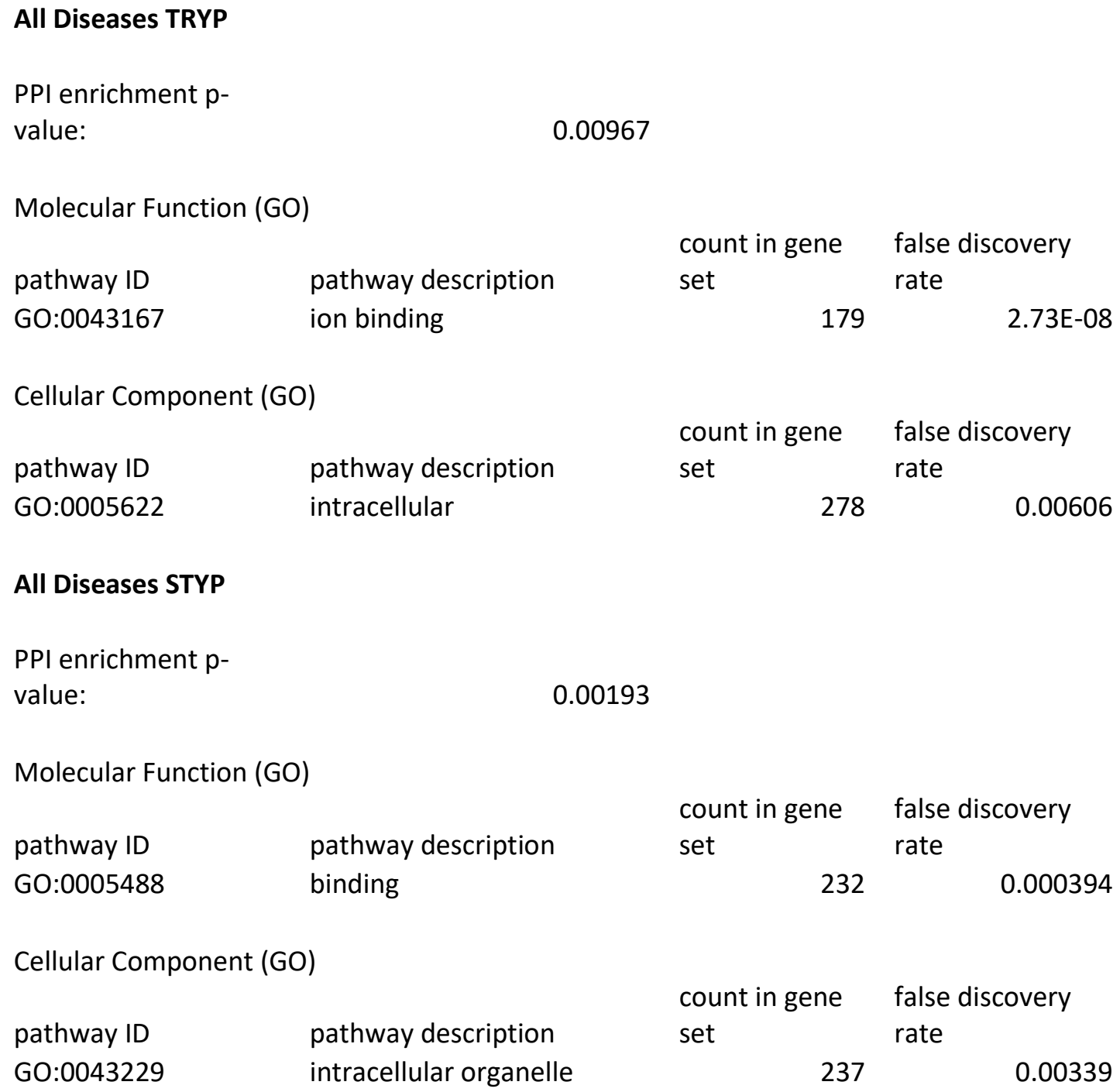

\section{All Diseases STYP}

PPI enrichment $p$ -

value:

Molecular Function (GO)

pathway ID

GO:0005488

pathway description binding

Cellular Component (GO)

pathway ID

GO:0043229

pathway description

intracellular organelle

$$
\begin{aligned}
& \text { count in gene false discovery } \\
& 232 \quad 0.000394
\end{aligned}
$$

count in gene false discovery set rate

$237 \quad 0.00339$

\section{Breast Cancer TRYP}

PPI enrichment $p$ -

value:

0

Biological Process

(GO)

$\begin{array}{ll}\begin{array}{ll}\text { count in gene false discovery } \\ \text { set }\end{array} & \text { rate }\end{array}$

pathway ID

pathway description

177

4.71E-10

multicellular organismal development 
Molecular Function (GO)

pathway ID

GO:0005488

Cellular Component (GO)

pathway ID

GO:0005856

pathway description

cytoskeleton

binding count in gene false discovery

set

rate

372

$2.78 \mathrm{E}-14$

count in gene false discovery

set

rate

123

5.64E-18

\section{Breast Cancer STYP}

PPI enrichment $p$ -

value:

\subsection{E-07}

Biological Process

(GO)

pathway ID

GO:0030198

pathway description

count in gene

set

false discovery

rate

extracellular matrix organization

Molecular Function (GO)

pathway ID

pathway description

GO:0005198

structural molecule activity

count in gene

set

Cellular Component (GO)

pathway ID

pathway description

GO:0098644 count in gene

set false discovery

rate

$24 \quad 1.26 \mathrm{E}-05$

18

9.03E-05

false discovery

rate

8

\section{Ovarian Cancer TRYP}

PPI enrichment $p$ -

value:

Biological Process

(GO)

pathway ID

pathway description

count in gene

set

false discovery

rate

platelet degranulation

8

0.000693

GO:0002576

count in gene

set

false discovery

rate

pathway description

48

0.0231 


\begin{tabular}{|c|c|c|c|}
\hline GO:0005515 & protein binding & & \\
\hline \multicolumn{2}{|l|}{ Cellular Component (GO) } & $\begin{array}{l}\text { count in gene } \\
\text { set }\end{array}$ & $\begin{array}{l}\text { false discovery } \\
\text { rate }\end{array}$ \\
\hline pathway ID & pathway description & 16 & 5.34E-15 \\
\hline GO:0072562 & blood microparticle & & \\
\hline KEGG Pathways & & $\begin{array}{l}\text { count in gene } \\
\text { set }\end{array}$ & $\begin{array}{l}\text { false discovery } \\
\text { rate }\end{array}$ \\
\hline pathway ID & pathway description & 7 & 5.48E-05 \\
\hline 4610 & Complement and coa & cascades & \\
\hline
\end{tabular}

\section{Ovarian Cancer STYP}

PPI enrichment $p$ -

value:

6.49E-09

Biological Process

(GO)

count in gene false discovery

set

rate

pathway ID

pathway description

5

0.0113

GO:0006953

acute-phase response

Cellular Component (GO)

pathway ID

GO:0072562

pathway description

blood microparticle

count in gene false discovery

set

rate

$9 \quad 5.22 \mathrm{E}-06$

count in gene false discovery

KEGG Pathways

set

rate

pathway ID

pathway description

6

0.000343

4610 Complement and coagulation cascades

PFAM Protein

count in gene

false discovery

Domains

set

rate

pathway ID

pathway description

3

0.00148

PF06623

MHC_I C-terminus

INTERPRO Protein Domains and Features

count in gene

set

false discovery

rate

pathway description

3

0.00316

MHC class I, alpha chain, C-terminal

\section{Multiple Sclerosis TRYP}




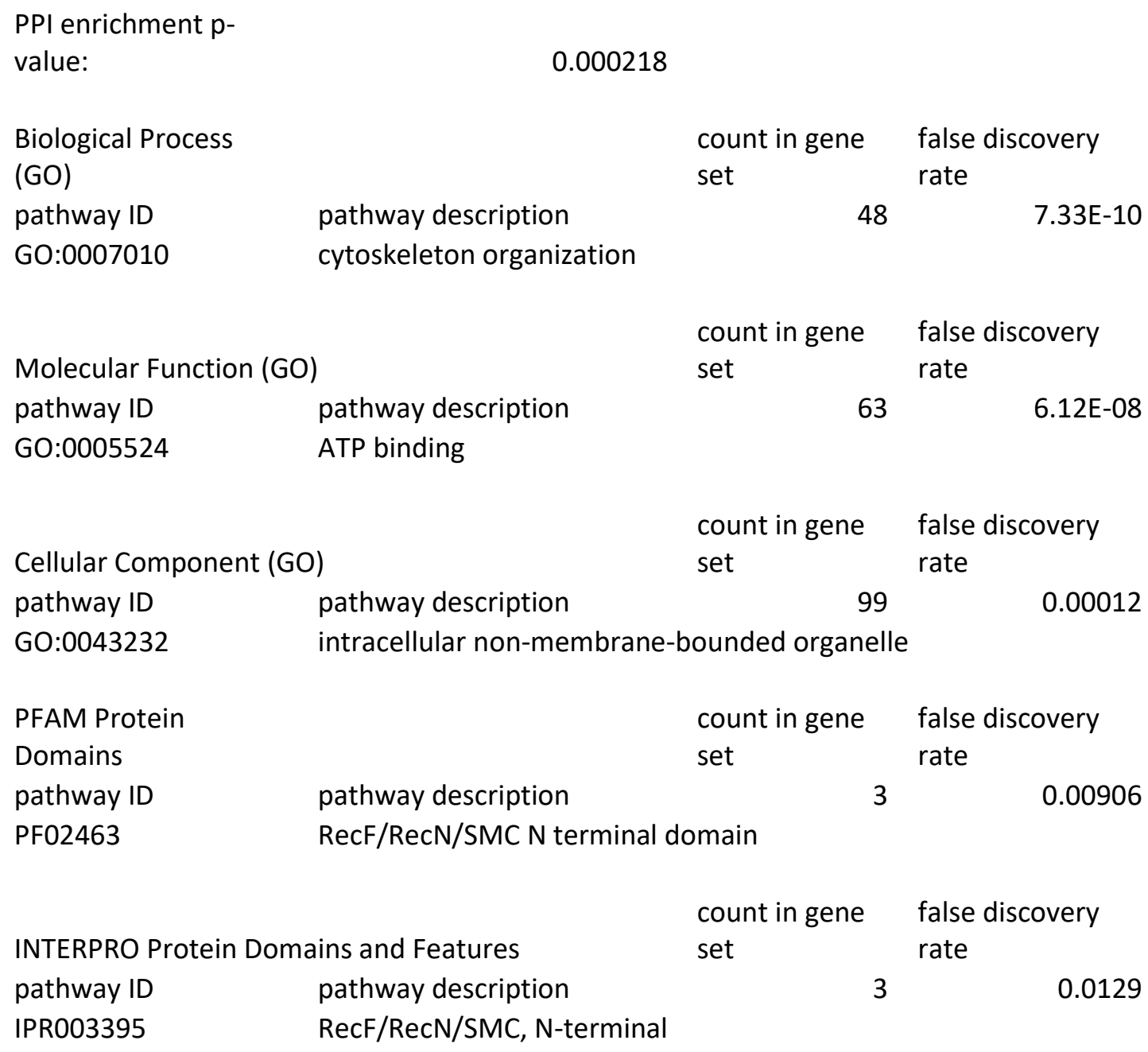

\section{Multiple Sclerosis \\ STYP}

PPI enrichment $p$ -

value:

0.000221

Biological Process

(GO)

pathway ID

GO:0006417

pathway description

regulation of translation

$$
\begin{aligned}
& \text { count in gene false discovery } \\
& \text { set rate } \\
& 23 \quad 0.00216
\end{aligned}
$$

Molecular Function (GO)

pathway ID

pathway description

GO:0043167

ion binding

\begin{tabular}{lrr}
$\begin{array}{l}\text { count in gene } \\
\text { set }\end{array}$ & \multicolumn{2}{l}{$\begin{array}{l}\text { false discovery } \\
\text { rate }\end{array}$} \\
& 214 & $2.01 \mathrm{E}-07$
\end{tabular}


Cellular Component (GO)

pathway ID

GO:0005737

pathway description

cytoplasm $\begin{array}{ll}\text { count in gene false discovery } \\ \text { set } & \text { rate }\end{array}$

291

0.000116

\section{Alzheimer's Dementia TRYP \\ PPI enrichment $p$ - \\ value: \\ 0.000121 \\ Biological Process \\ (GO) \\ pathway ID \\ pathway description \\ GO:0006996 \\ organelle organization
count in gene false discovery
set
rate
$64 \quad 0.0261$ \\ Molecular Function (GO) \\ pathway ID \\ GO:0016887 \\ pathway description \\ ATPase activity
count in gene false discovery
set
rate
$19 \quad 0.00246$ \\ count in gene false discovery \\ set rate \\ pathway ID \\ GO:0005654 \\ pathway description \\ nucleoplasm}

\section{Alzheimer's Dementia STYP}

PPI enrichment $p$ -

value:

Biological Process

(GO)

pathway ID

GO:1902589

Molecular Function (GO)

pathway ID

GO:0000166 pathway description

nucleotide binding count in gene false discovery

set

rate

59

7.64E-06

single-organism organelle organization

count in gene

set

false discovery

rate

0.000273 


\begin{tabular}{|c|c|c|c|}
\hline Cellular Component (GC & & $\begin{array}{l}\text { count in gene } \\
\text { set }\end{array}$ & $\begin{array}{l}\text { false discovery } \\
\text { rate }\end{array}$ \\
\hline pathway ID & pathway description & 84 & 1.25E-05 \\
\hline GO:0043232 & intracellular non-men & pounded organelle & \\
\hline KEGG Pathways & & $\begin{array}{l}\text { count in gene } \\
\text { set }\end{array}$ & $\begin{array}{l}\text { false discovery } \\
\text { rate }\end{array}$ \\
\hline pathway ID 4510 & $\begin{array}{l}\text { pathway description } \\
\text { Focal adhesion }\end{array}$ & 11 & 0.0425 \\
\hline
\end{tabular}

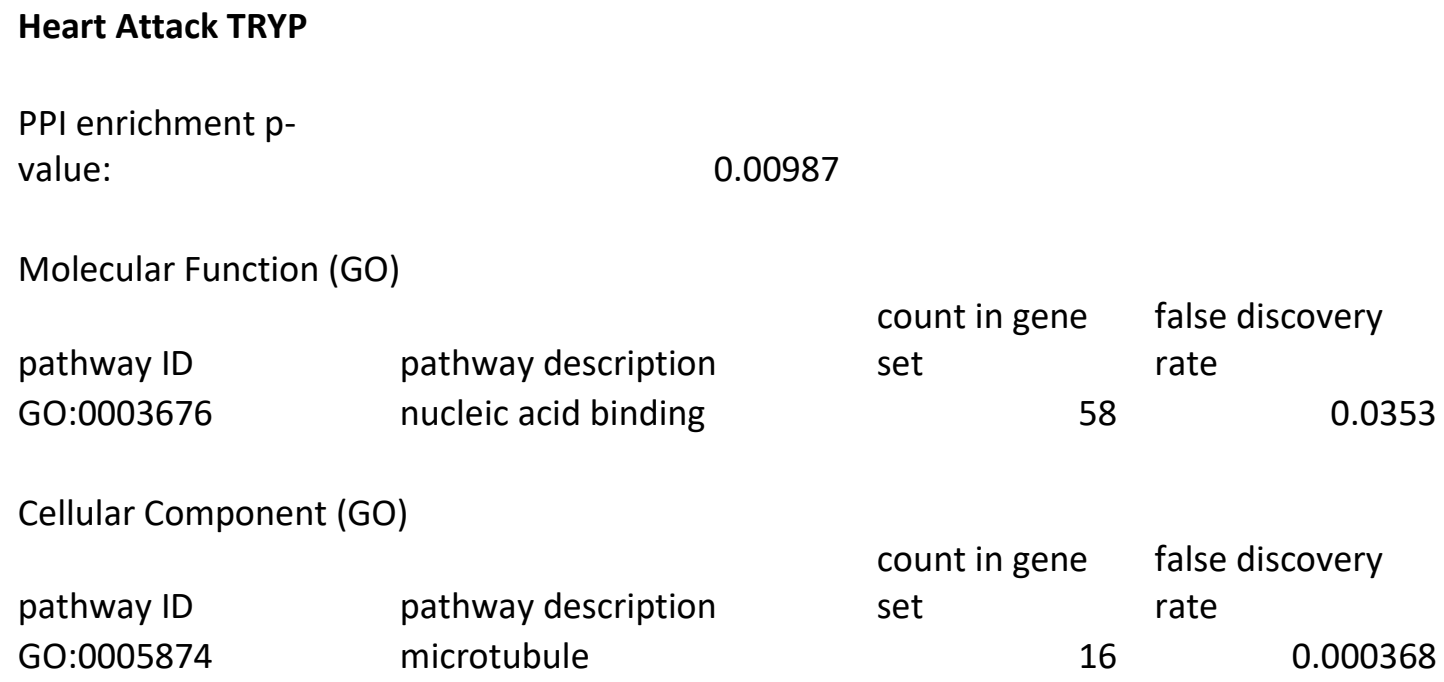

\section{Heart Attack STYP}

PPI enrichment $p$ -

value:

Biological Process

(GO)

pathway ID

pathway description

count in gene

set

false discovery

microtubule-based process

23

0.000743

Cellular Component (GO)

pathway ID

GO:0043234

pathway description

protein complex

\begin{tabular}{lrr}
$\begin{array}{l}\text { count in gene } \\
\text { set }\end{array}$ & \multicolumn{2}{l}{$\begin{array}{l}\text { false discovery } \\
\text { rate }\end{array}$} \\
& 88 & \\
& & $5.18 \mathrm{E}-06$
\end{tabular}

KEGG Pathways 
pathway ID

pathway description

4512 ECM-receptor interaction count in gene false discovery

set

rate

7

0.0398

\section{Sepsis TRYP}

PPI enrichment $p$ -

value:

0.000152

Biological Process

(GO)

pathway ID

pathway description

count in gene

false discovery

microtubule-based

GO:0007018

movement

set

rate

9

0.014

Molecular Function (GO)

pathway ID

GO:0003777

pathway description

microtubule motor activity

Cellular Component (GO)

pathway ID

GO:0044430

pathway description

cytoskeletal part
0.0543

count in gene

set

false discovery

rate

24

0.000516

\section{Sepsis STYP}

PPI enrichment $p$ -

value:

count in gene

set

false discovery

rate

7

0.0012

Cellular Component (GO)

pathway ID

GO:0005737

pathway description

cytoplasm $\begin{array}{ll}\text { count in gene false discovery } \\ \text { set } & \text { rate }\end{array}$

82

0.000835

\section{FIGURES}




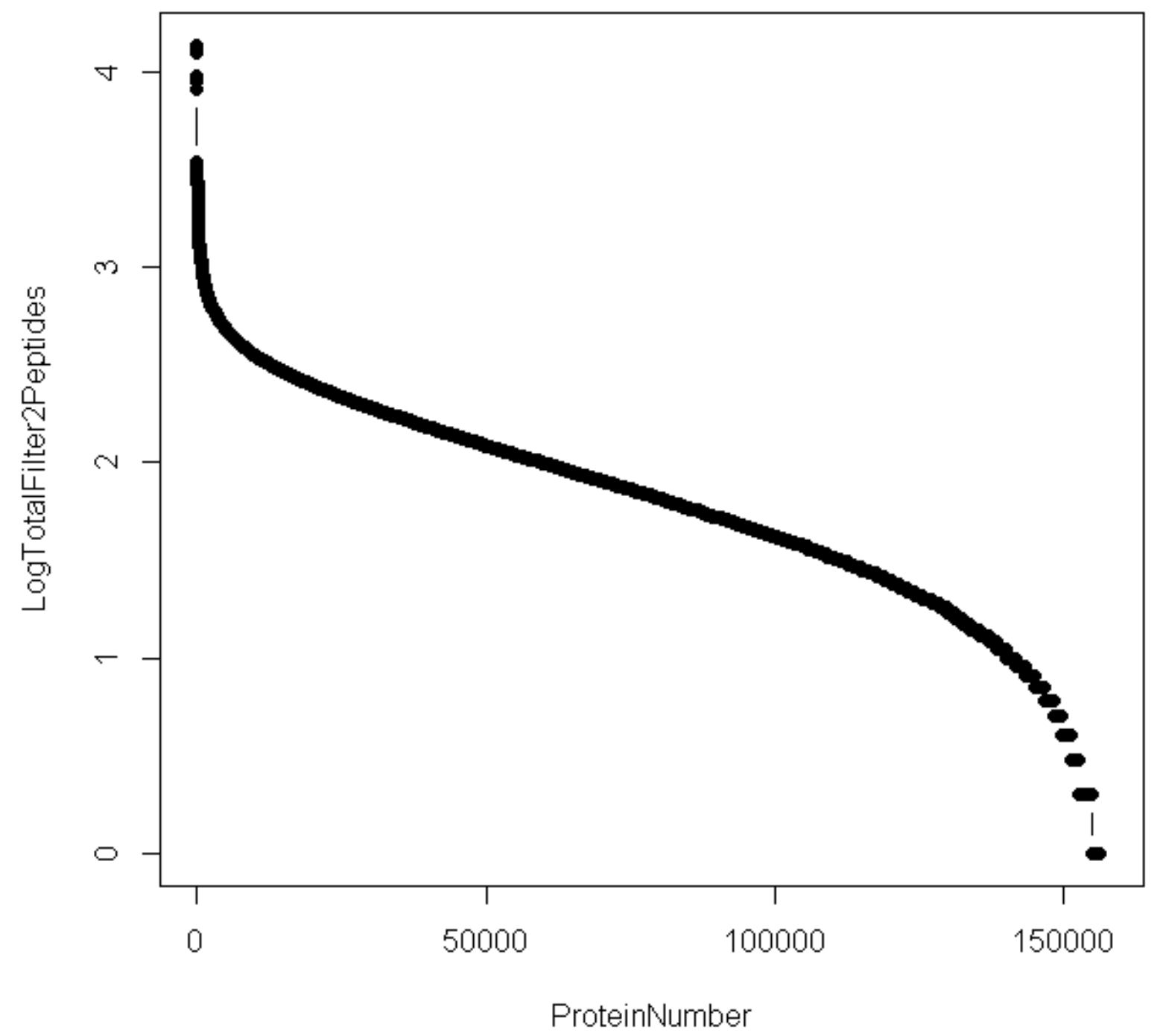

Figure 1. The human proteins identified by endogenous tryptic peptides and/or phosphopeptides using Filter 2 where precursor intensity is greater than E3 $(1,000)$ arbitrary detector counts. 

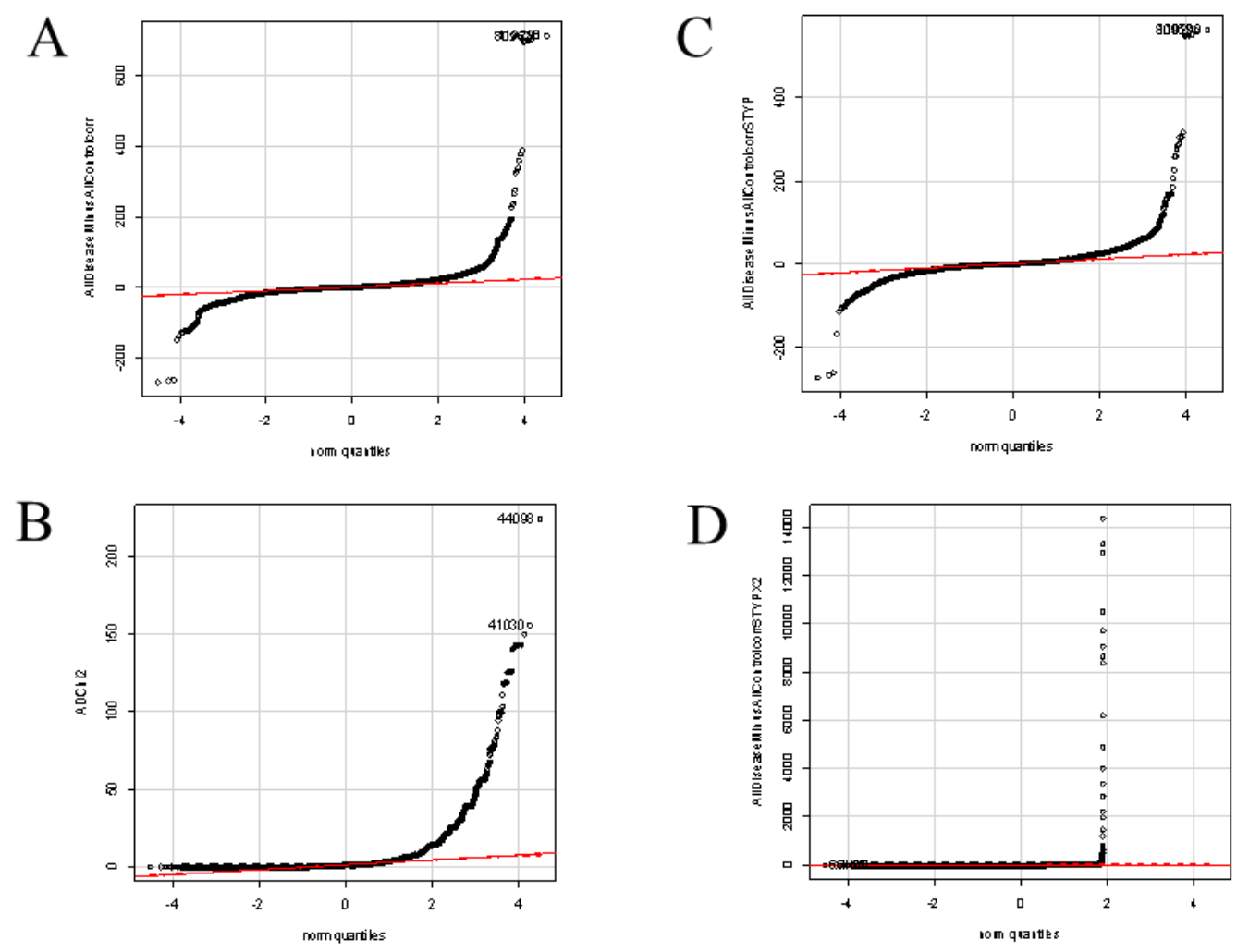

All Disease vs All controls 

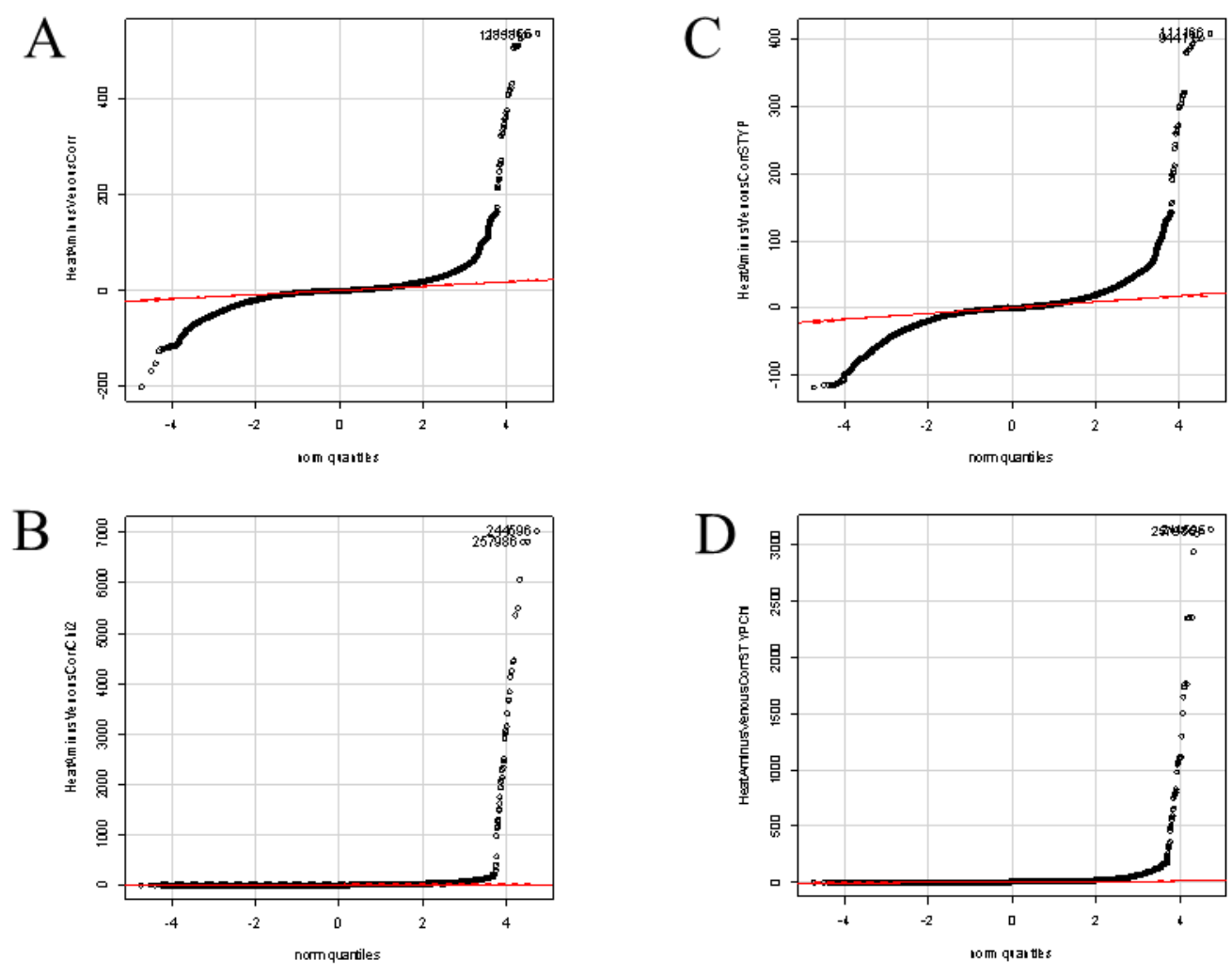

Heart Attack 

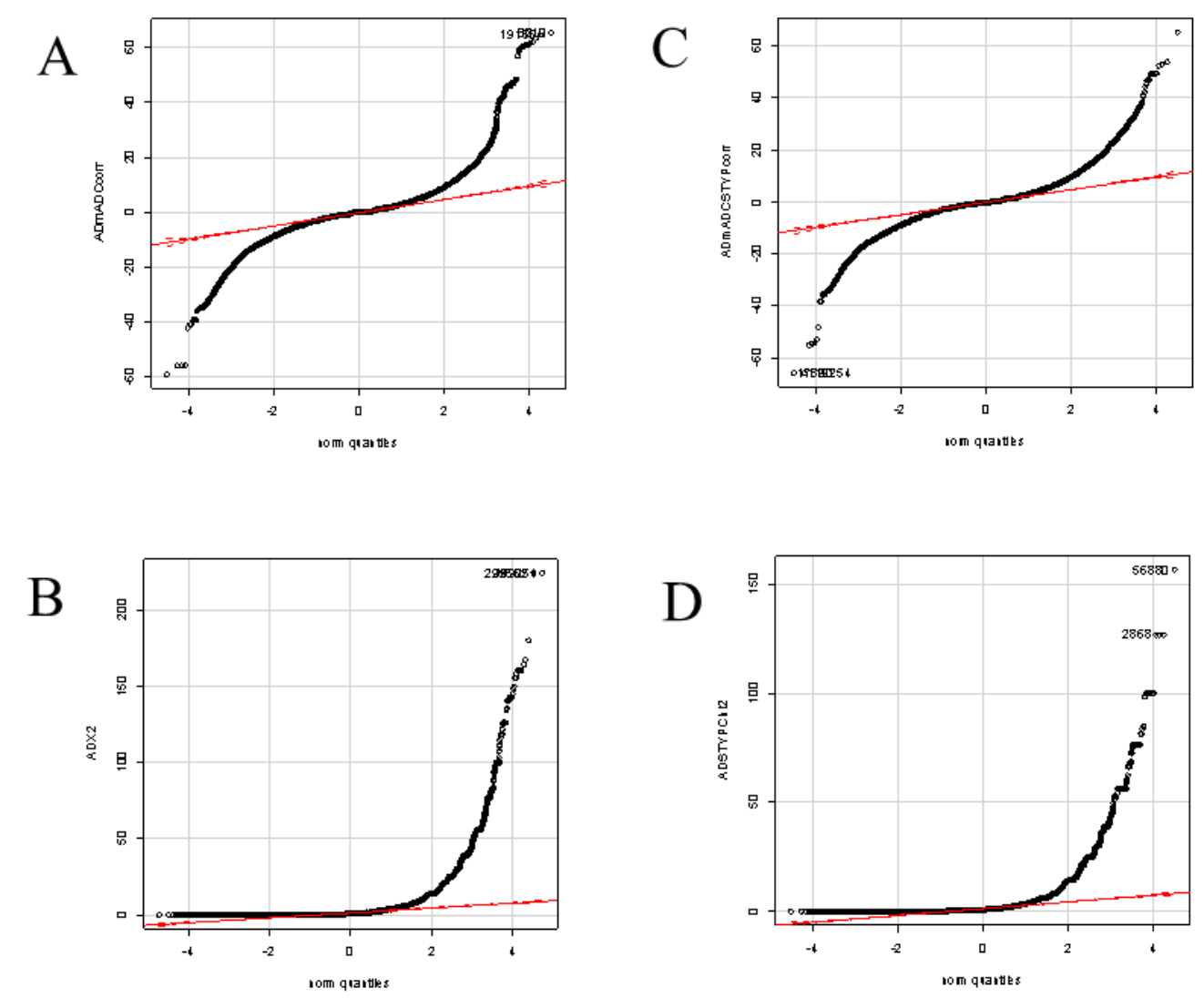

Alzheimer's Dementia 

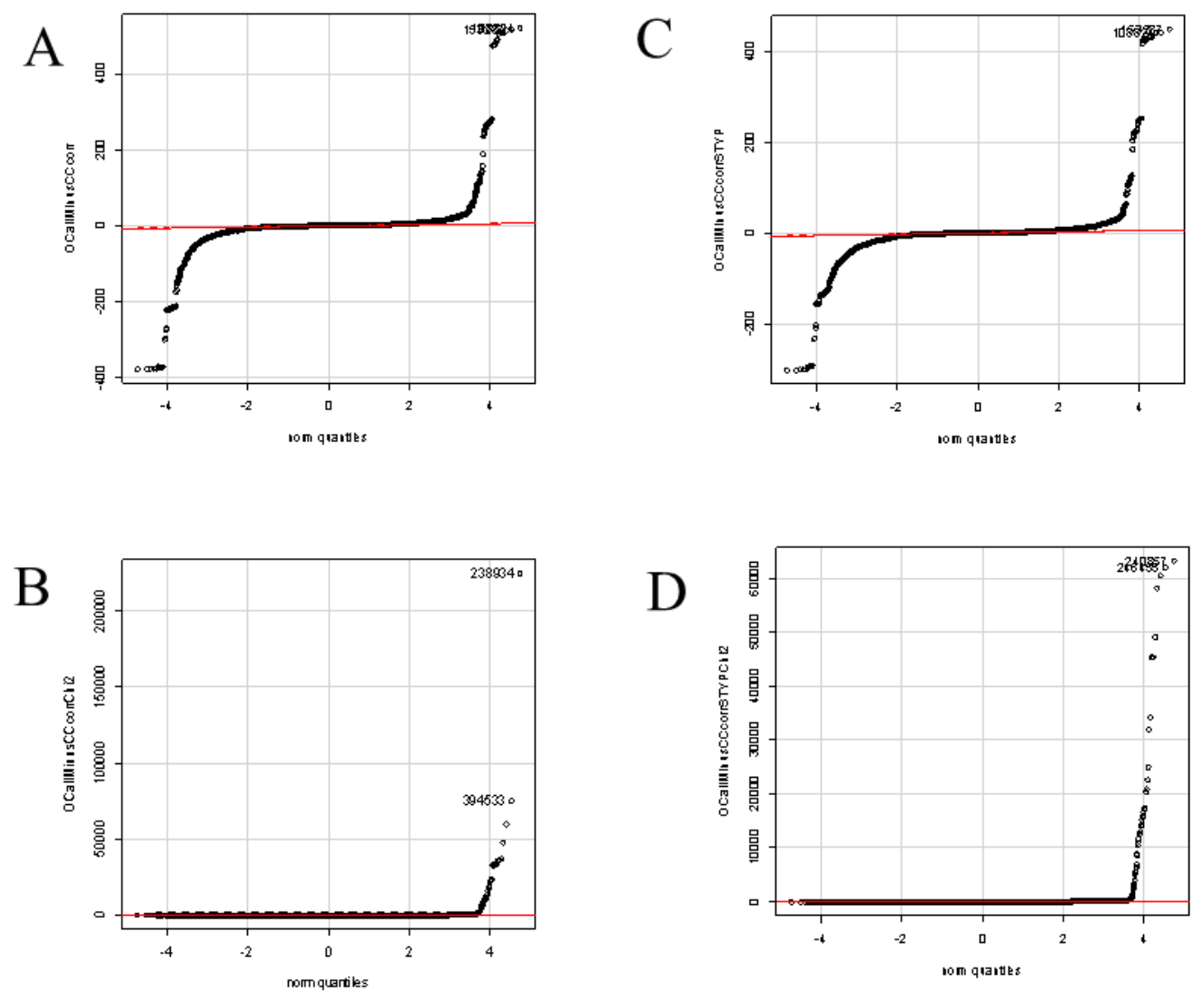

Ovarian Cancer 
A

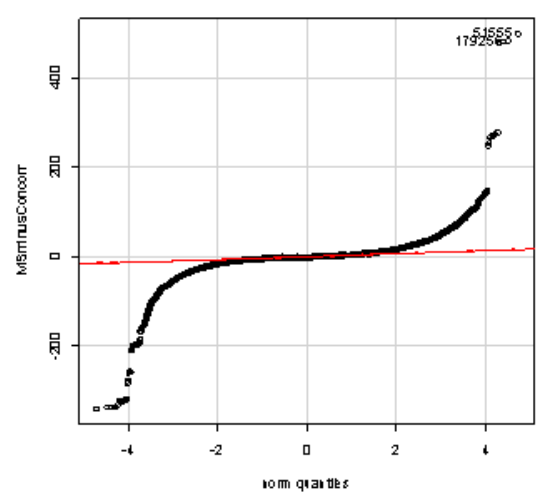

B

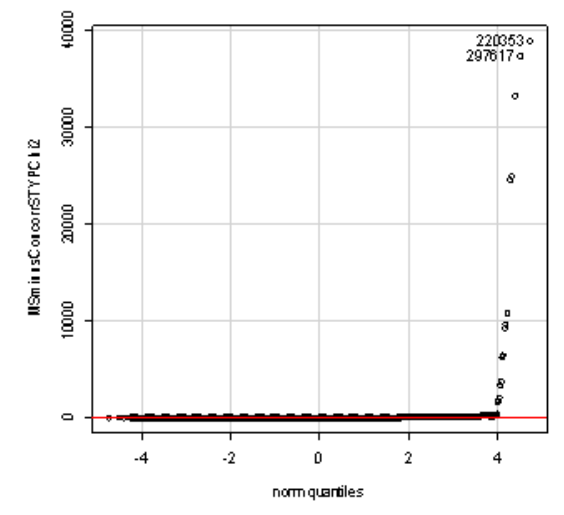

C

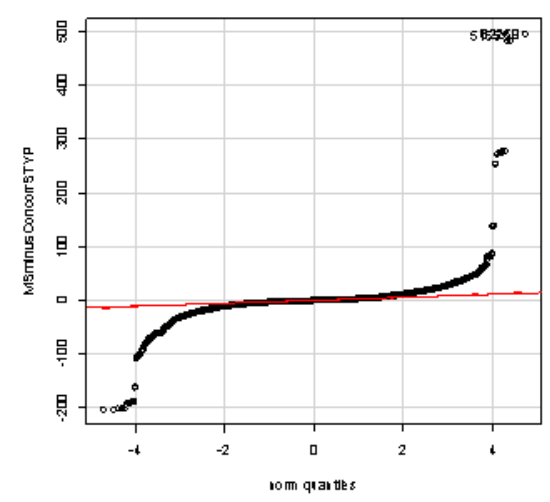

D

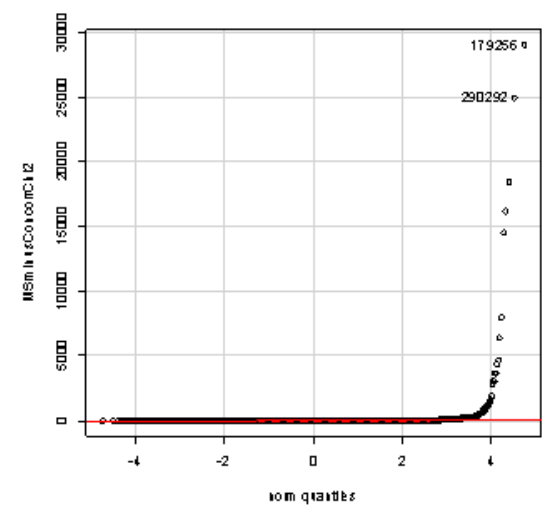

Multiple Sclerosis 

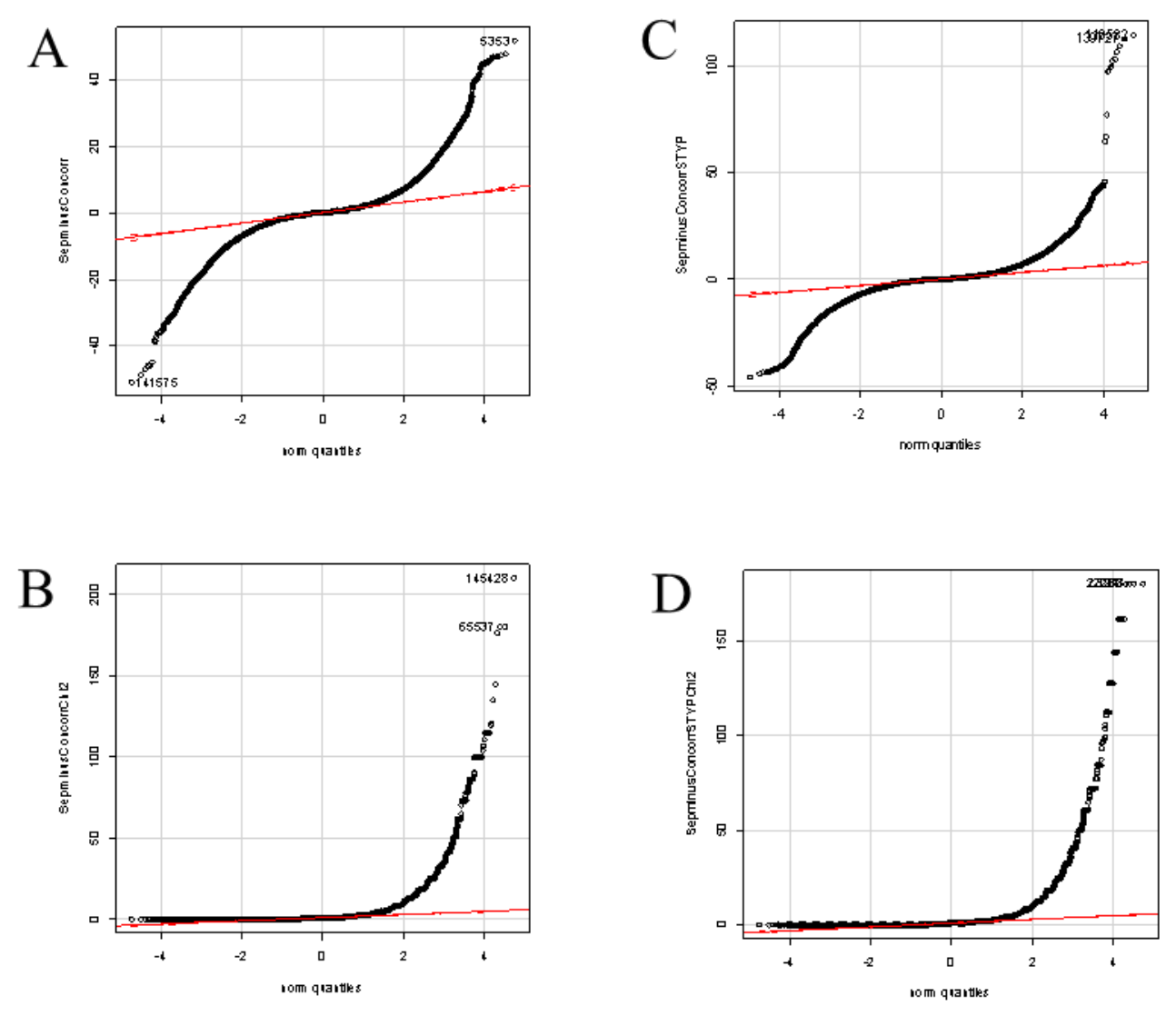

Sepsis 

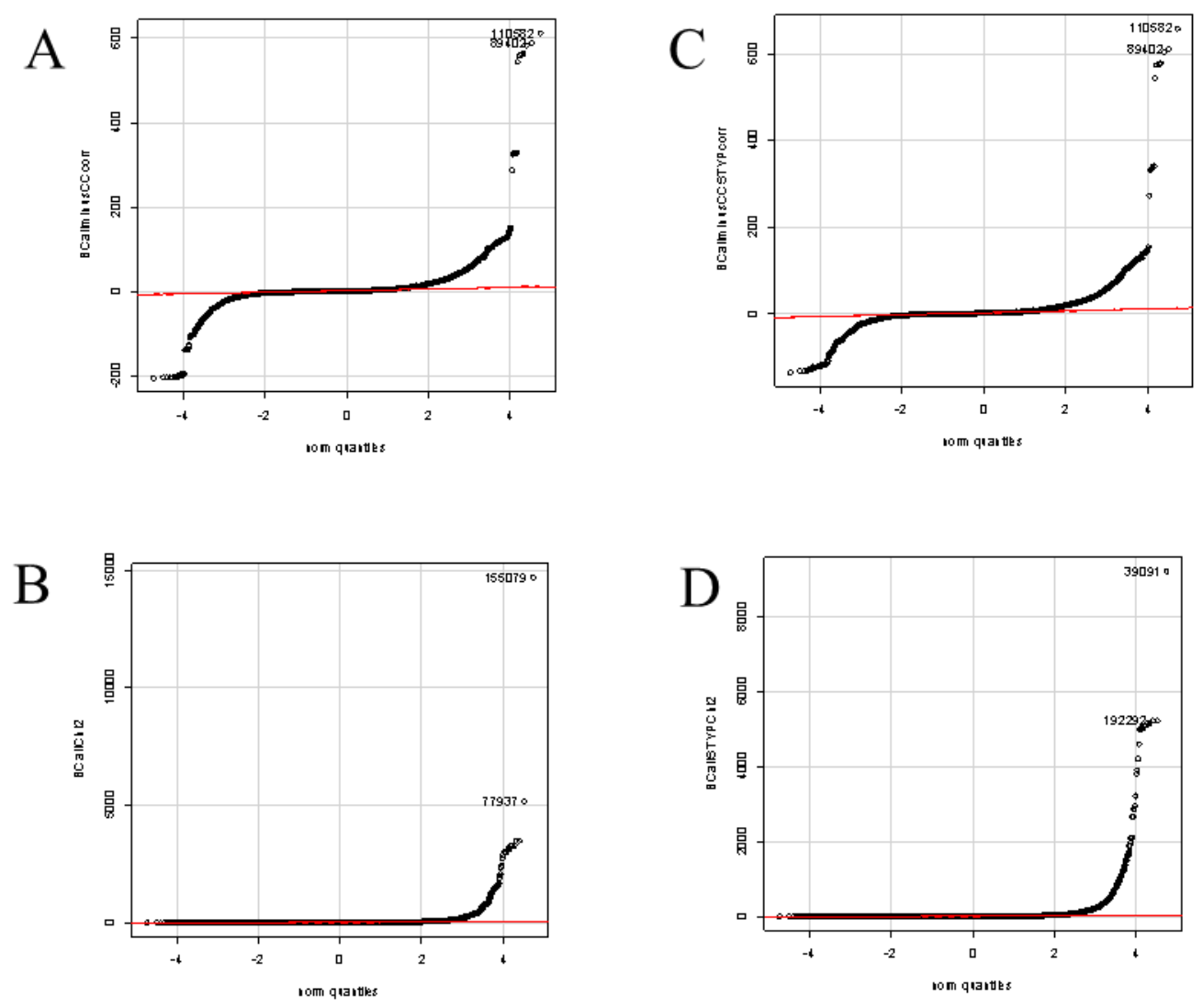

Breast Cancer

Figure 2. Quantile plots of the corrected difference and Chi Square values of the non-specific and disease versus control data from each institution as indicated. Figure 4, The difference of each diseases state $(n \geq 10)$ versus each of the controls $(n \geq 10)$ using the Quantile plot that tended to zero (see red line). 


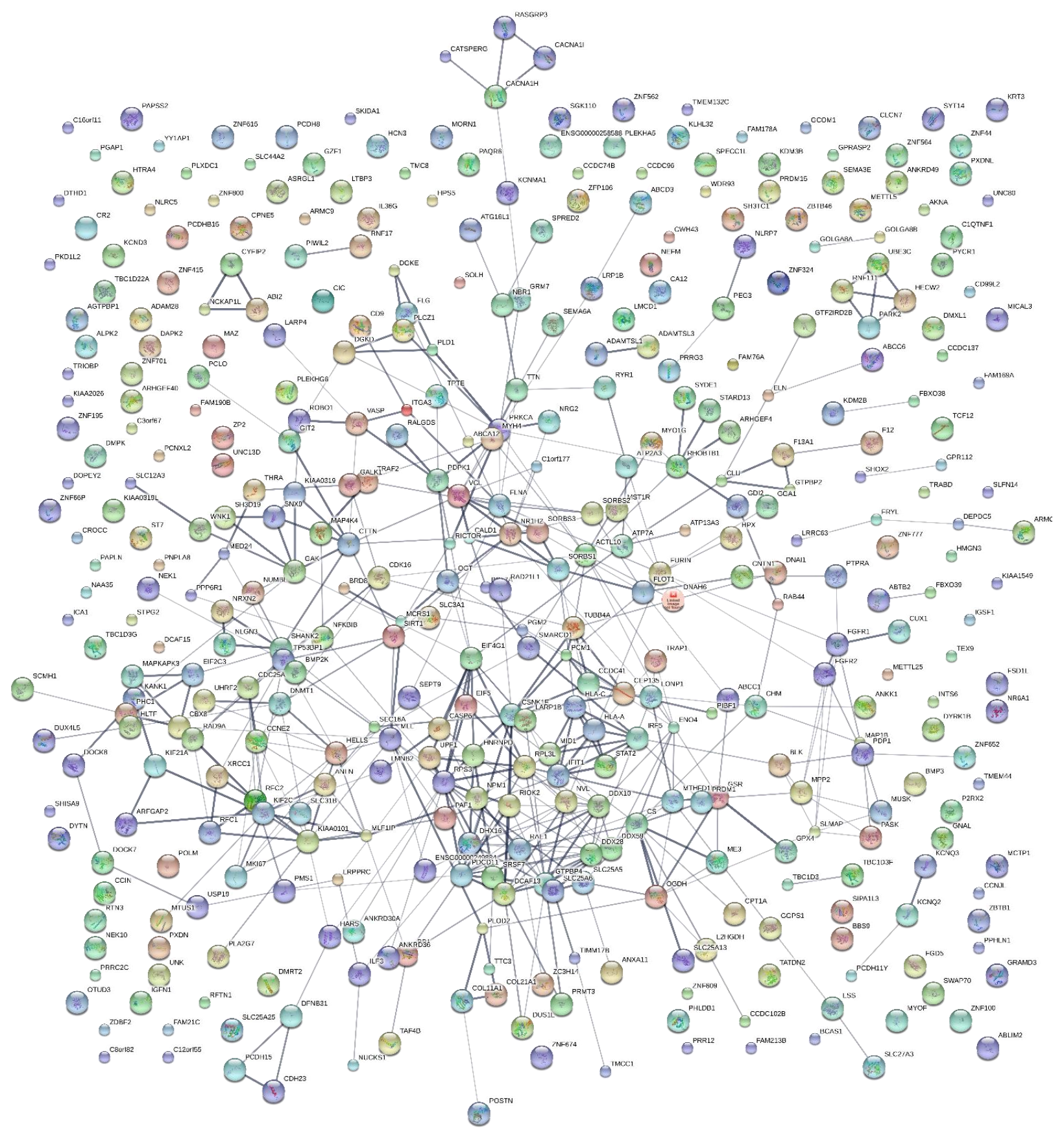

Non Specific Delta gt 15 X2 gt 9 


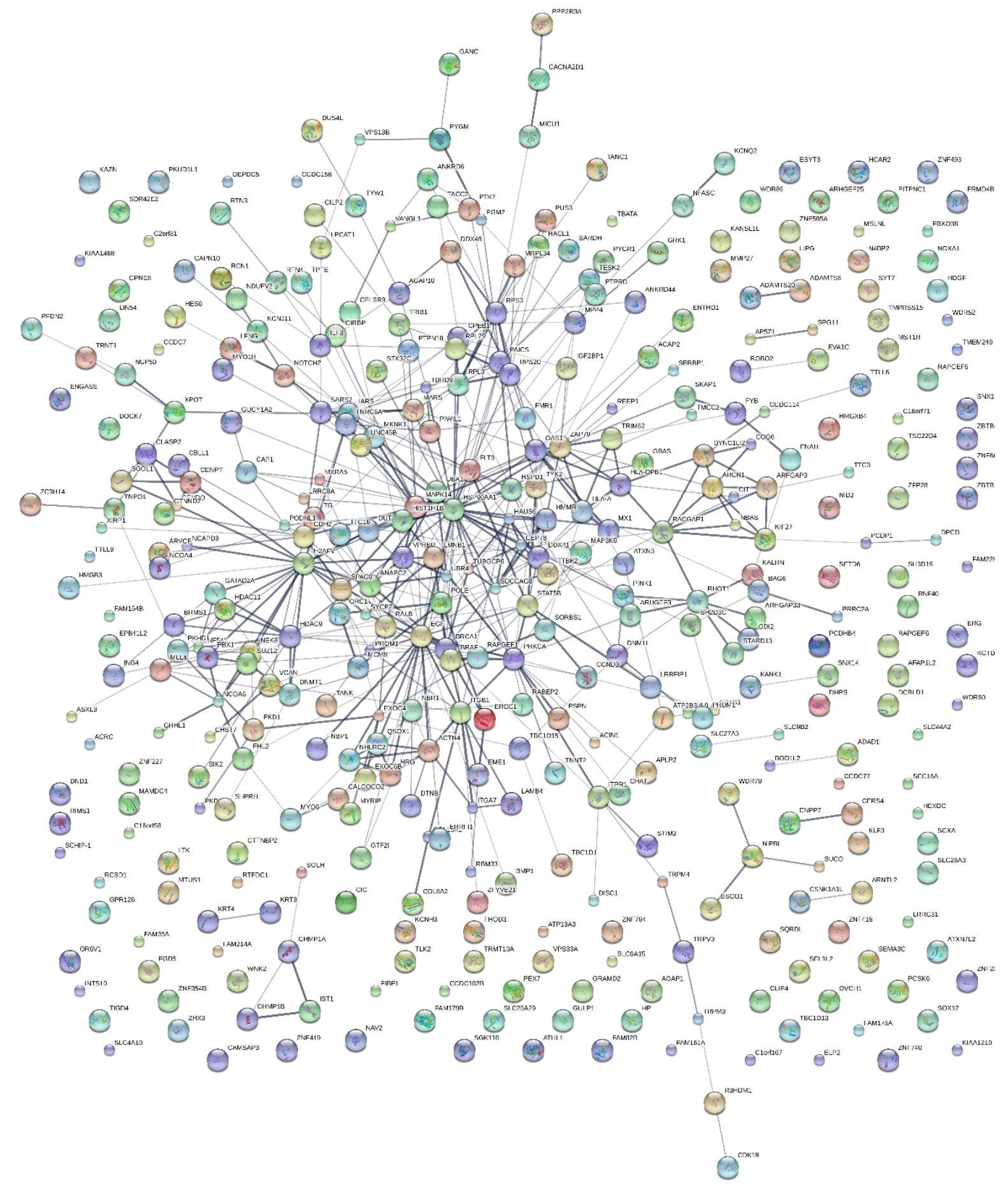

Non Specific STYP Delta gt 15 X2 gt 9 


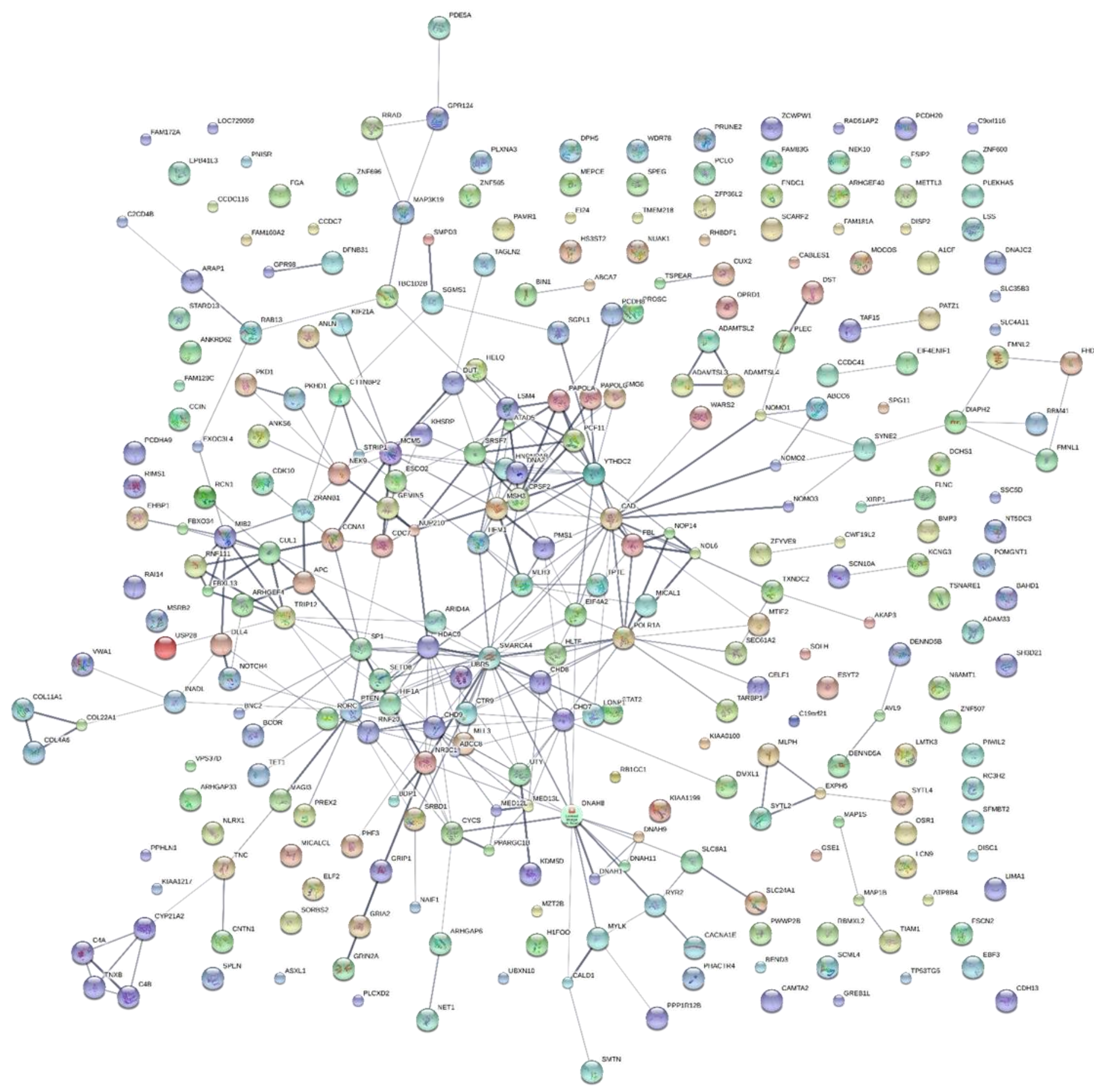

Alzheimer's TRYP Delta gt 15 X2 gt 9 


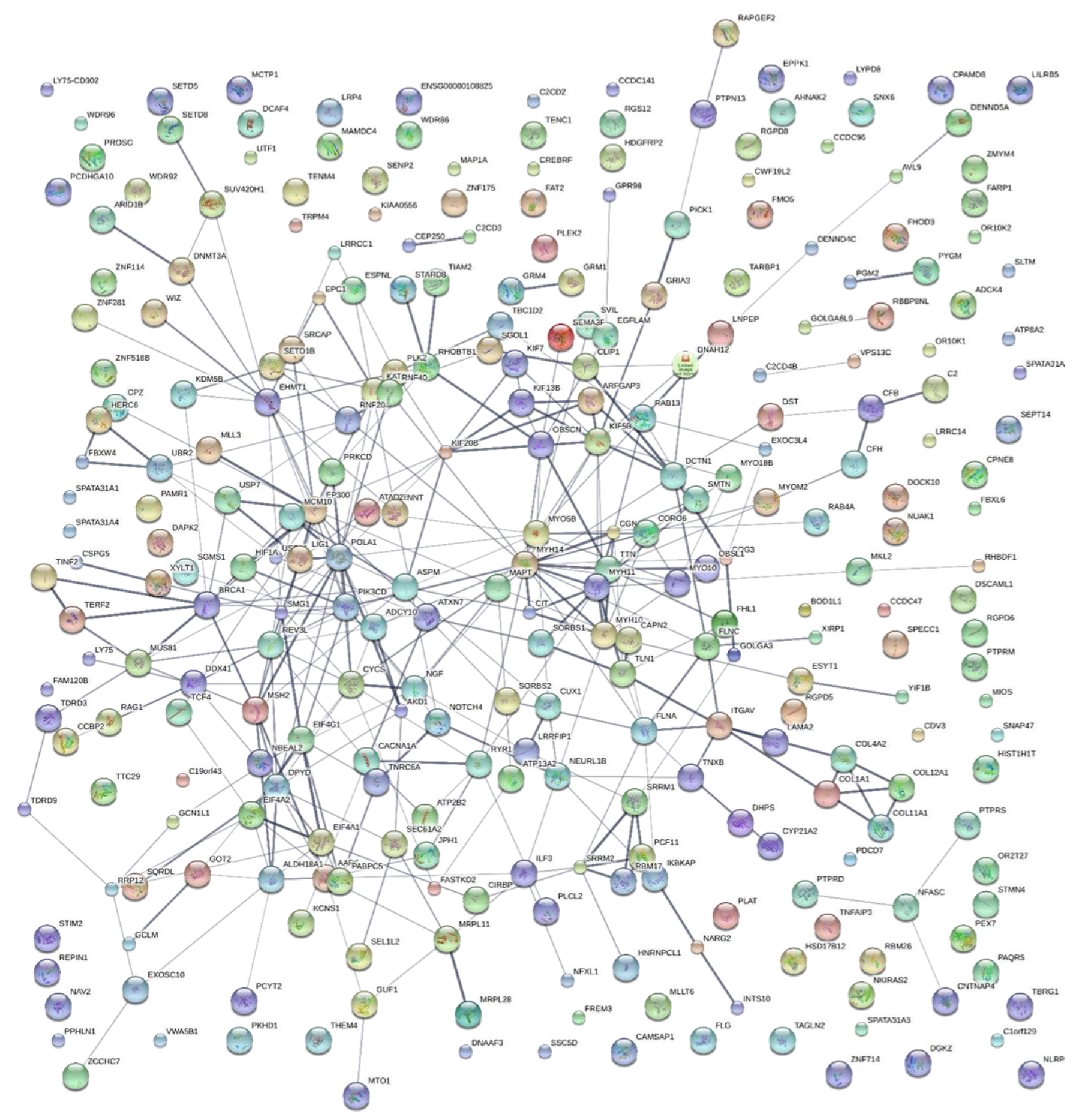

Alzheimer's STYP gt 15 X2 gt 9 


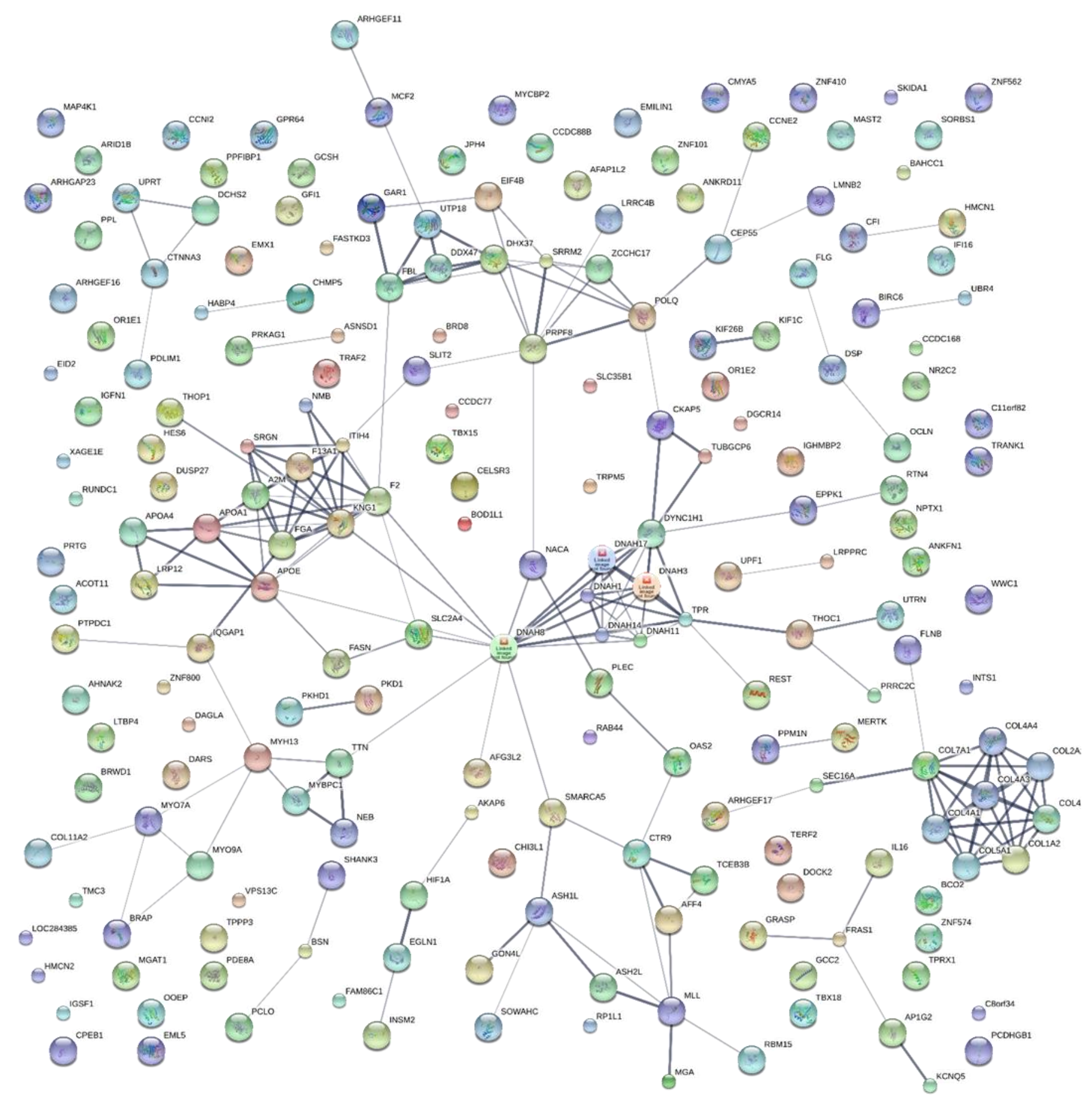

Breast Cancer Delta gt 15 X2 gt 9 


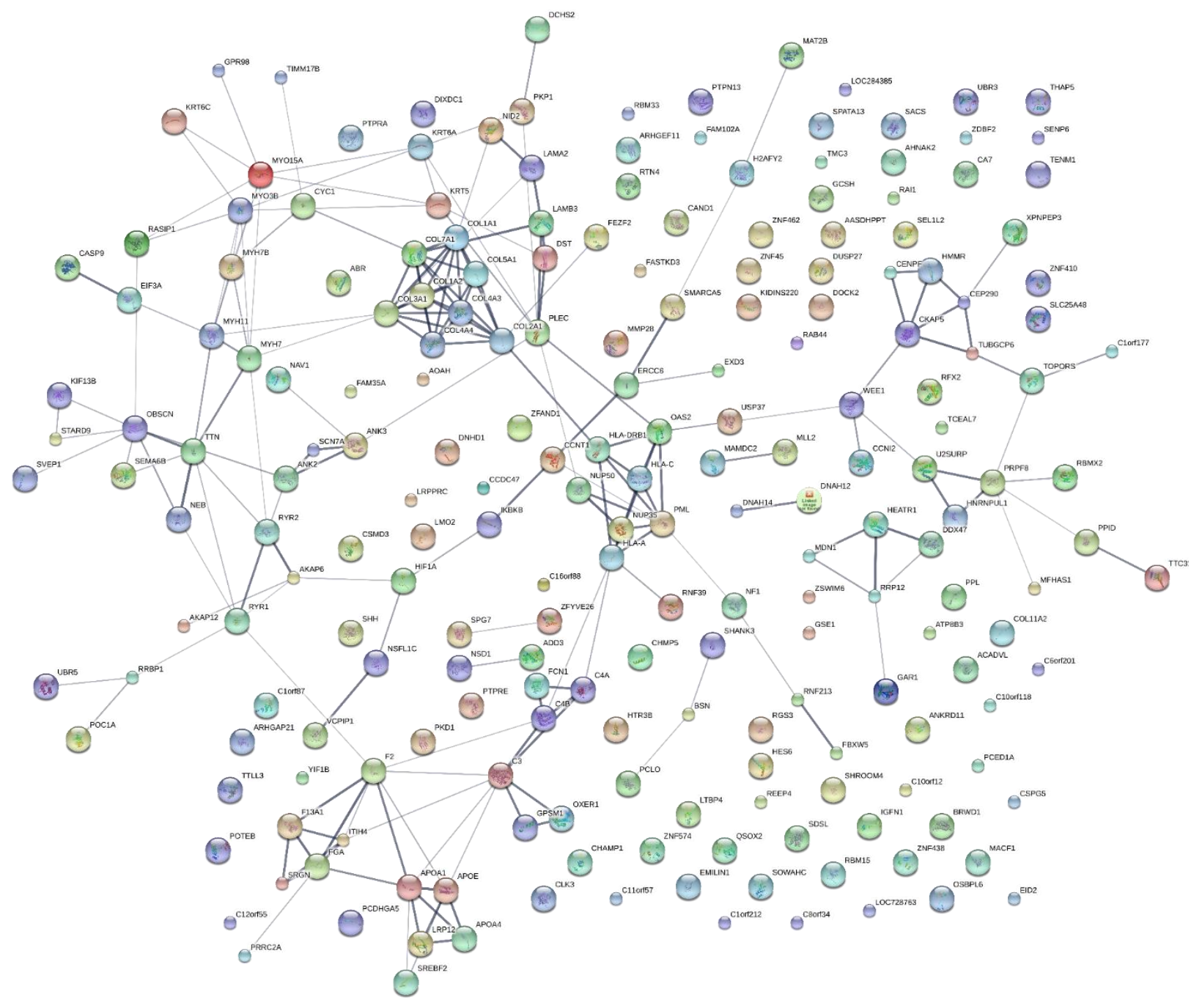

Breast Cancer STYP Delta gt 15 X2 gt 9 


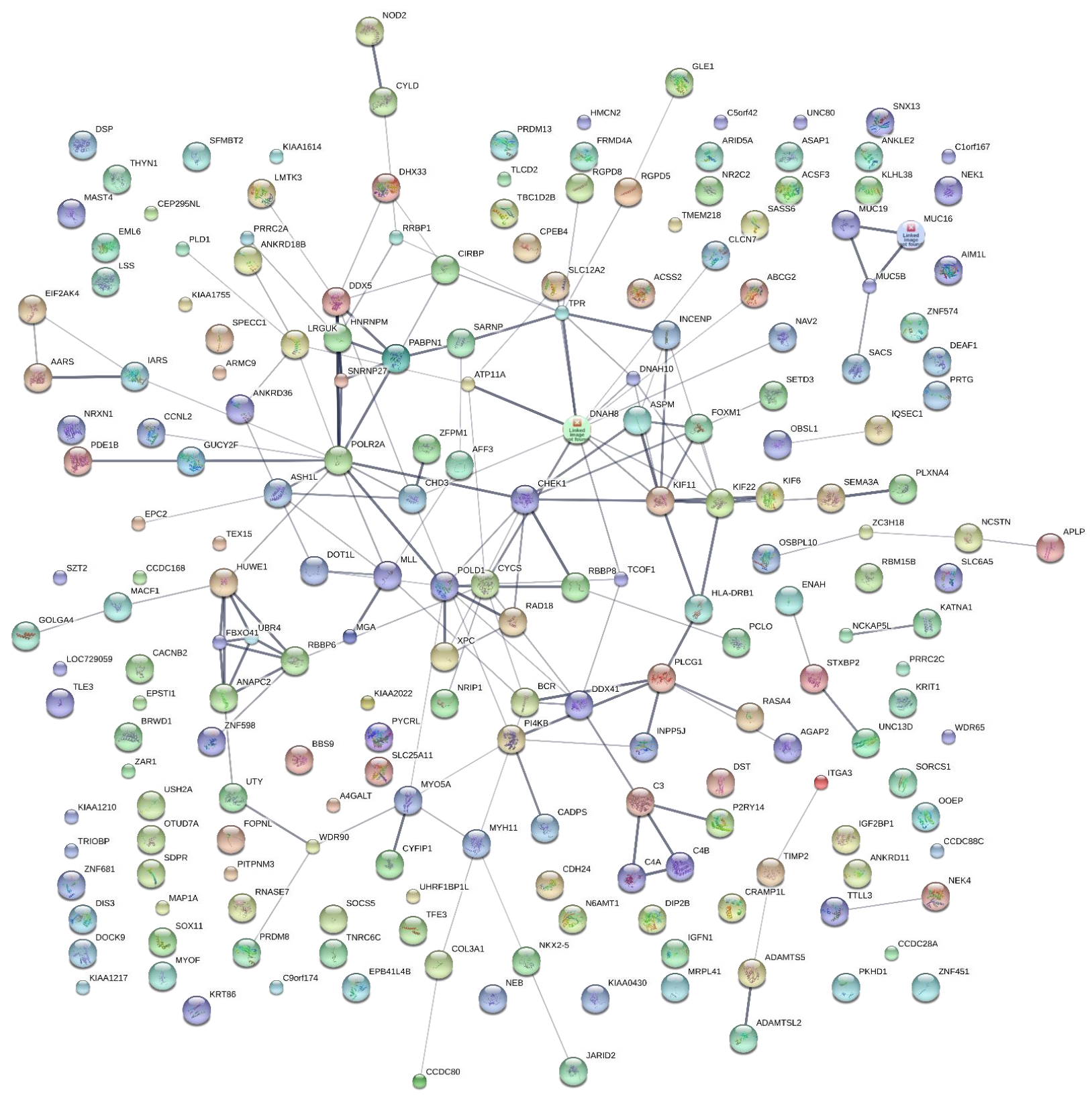

Heart Attack Delta gt 25 X2 gt 9 


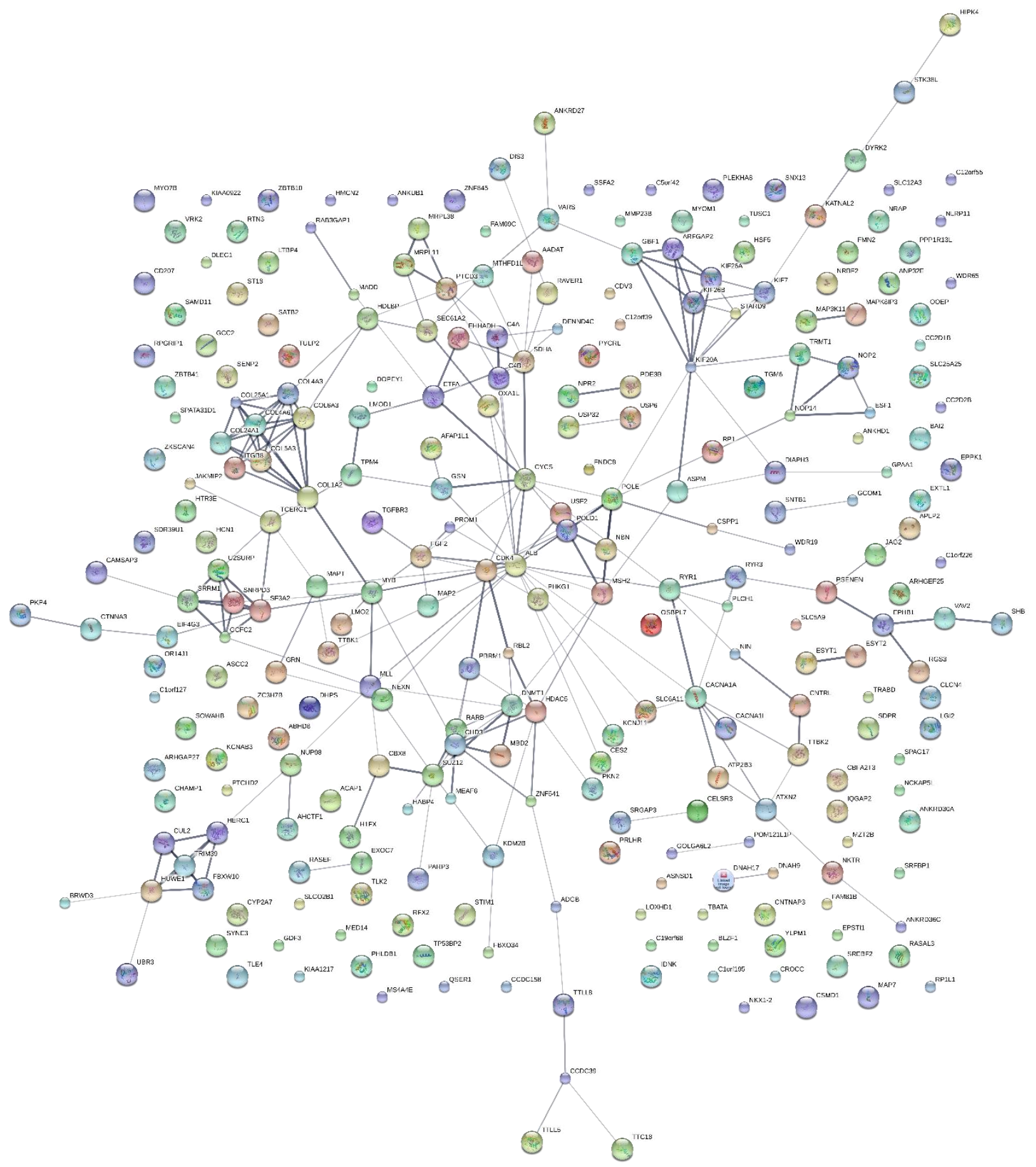

Heart Attack STYP Delta gt 25 X2 gt 9 


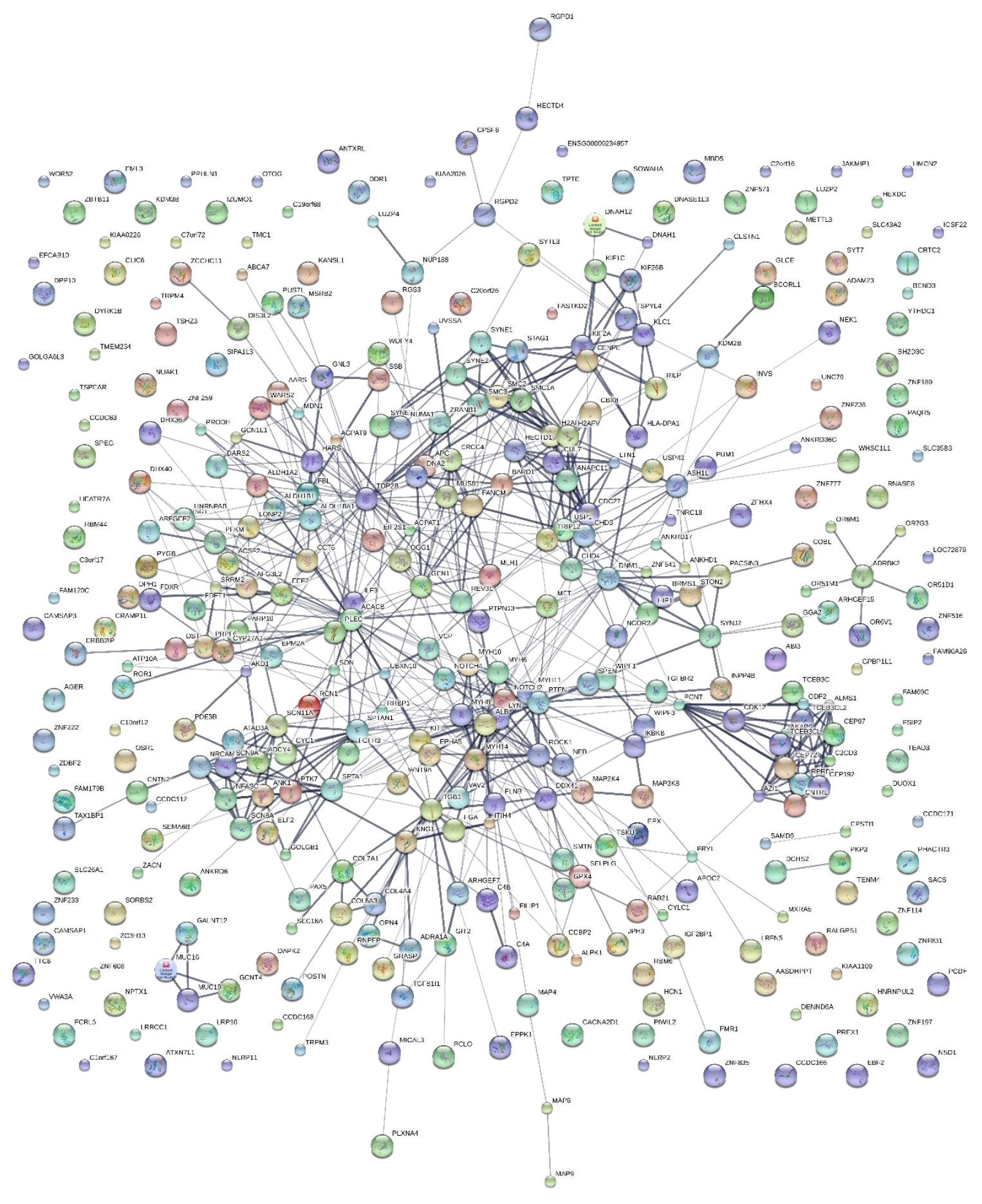

Multiple Sclerosis Delta gt 15 X2 gt 9 


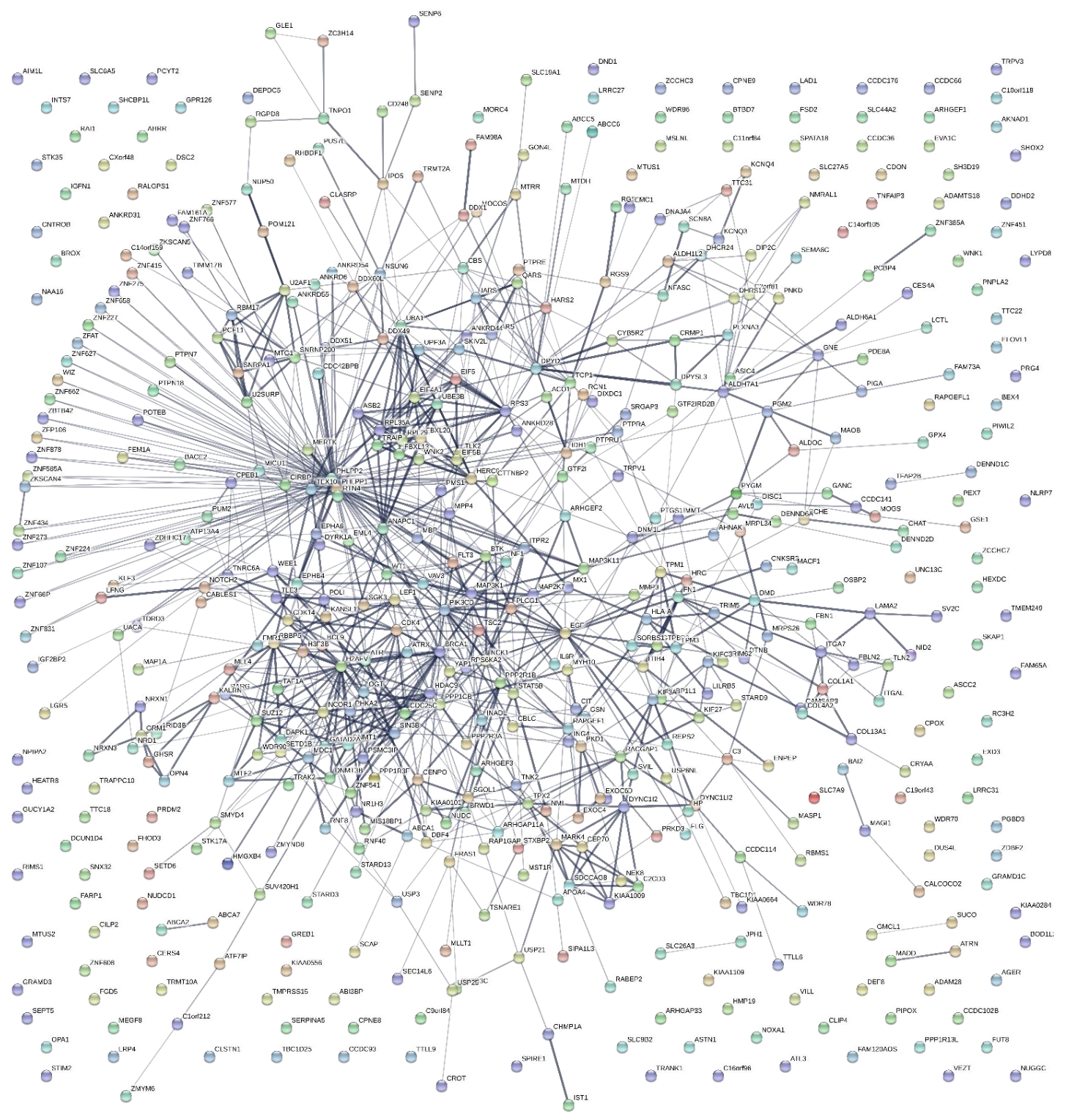

Multiple Sclerosis STYP, Delta gt 15, X2 gt 9 


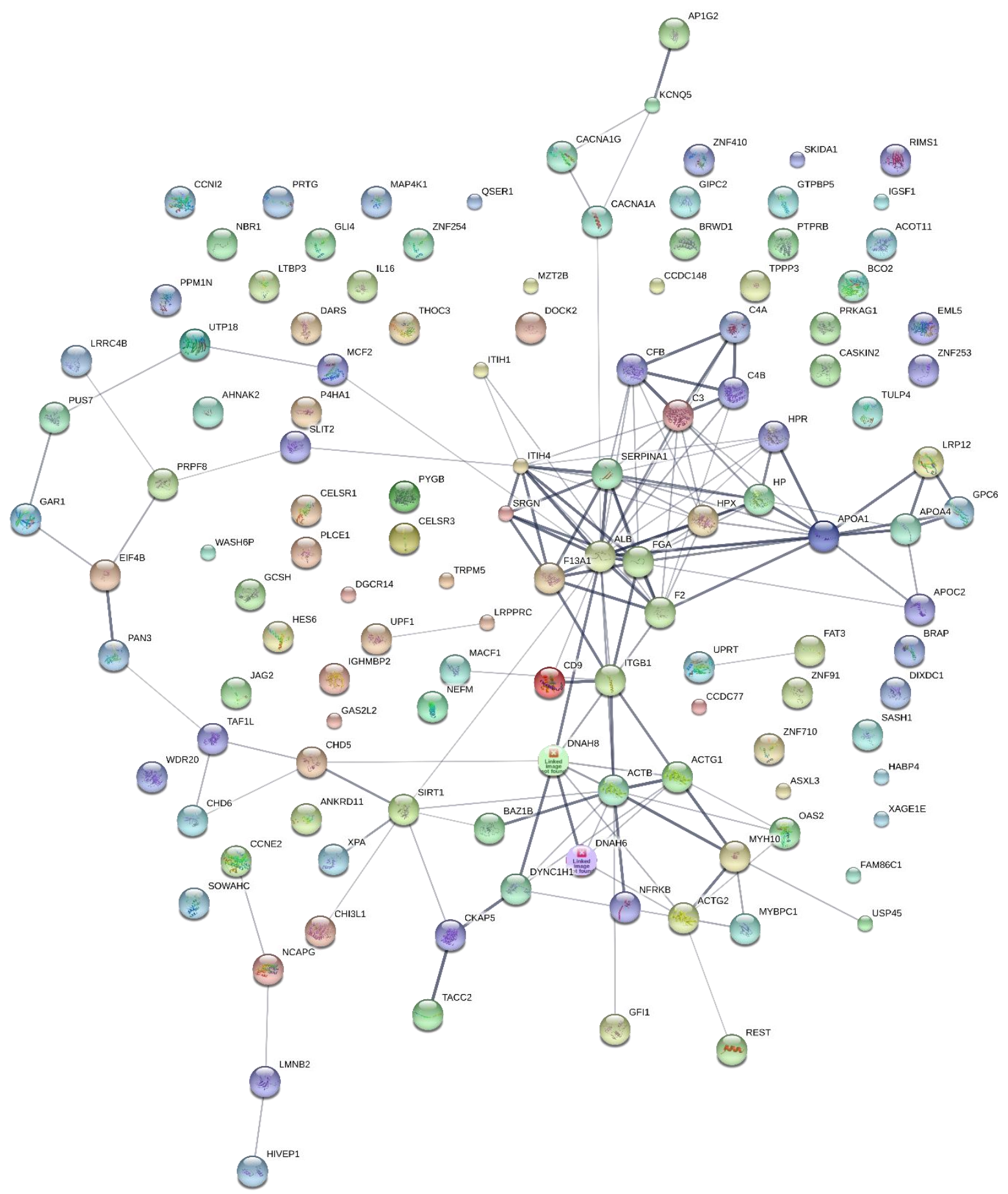

Ovarian Cancer Delta gt 10 X2 gt 9 


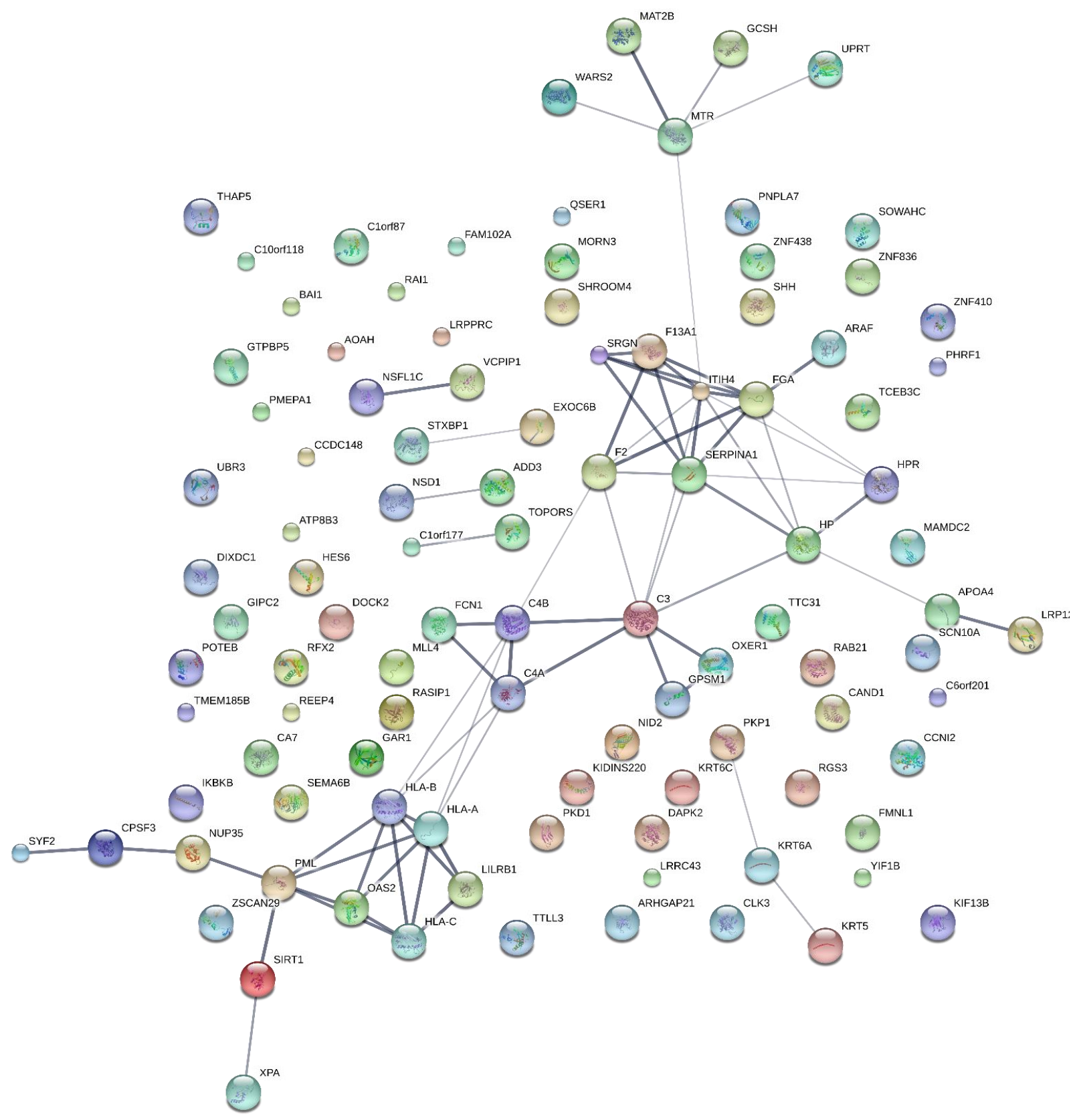

Ovarian Cancer STYP, Delta gt 15, X2 gt 9 


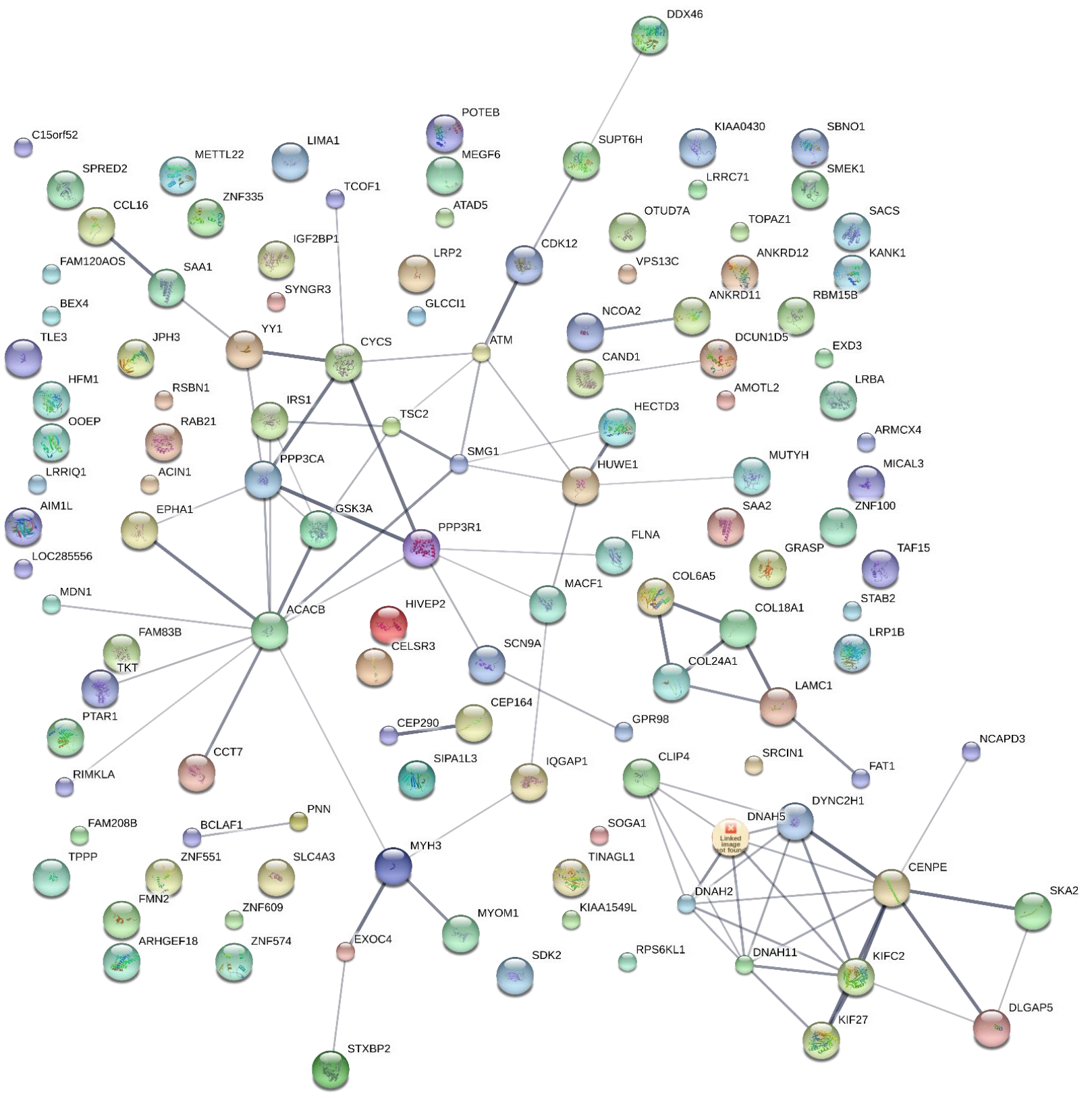

Sepsis Delta gt 10 X2 gt 5 


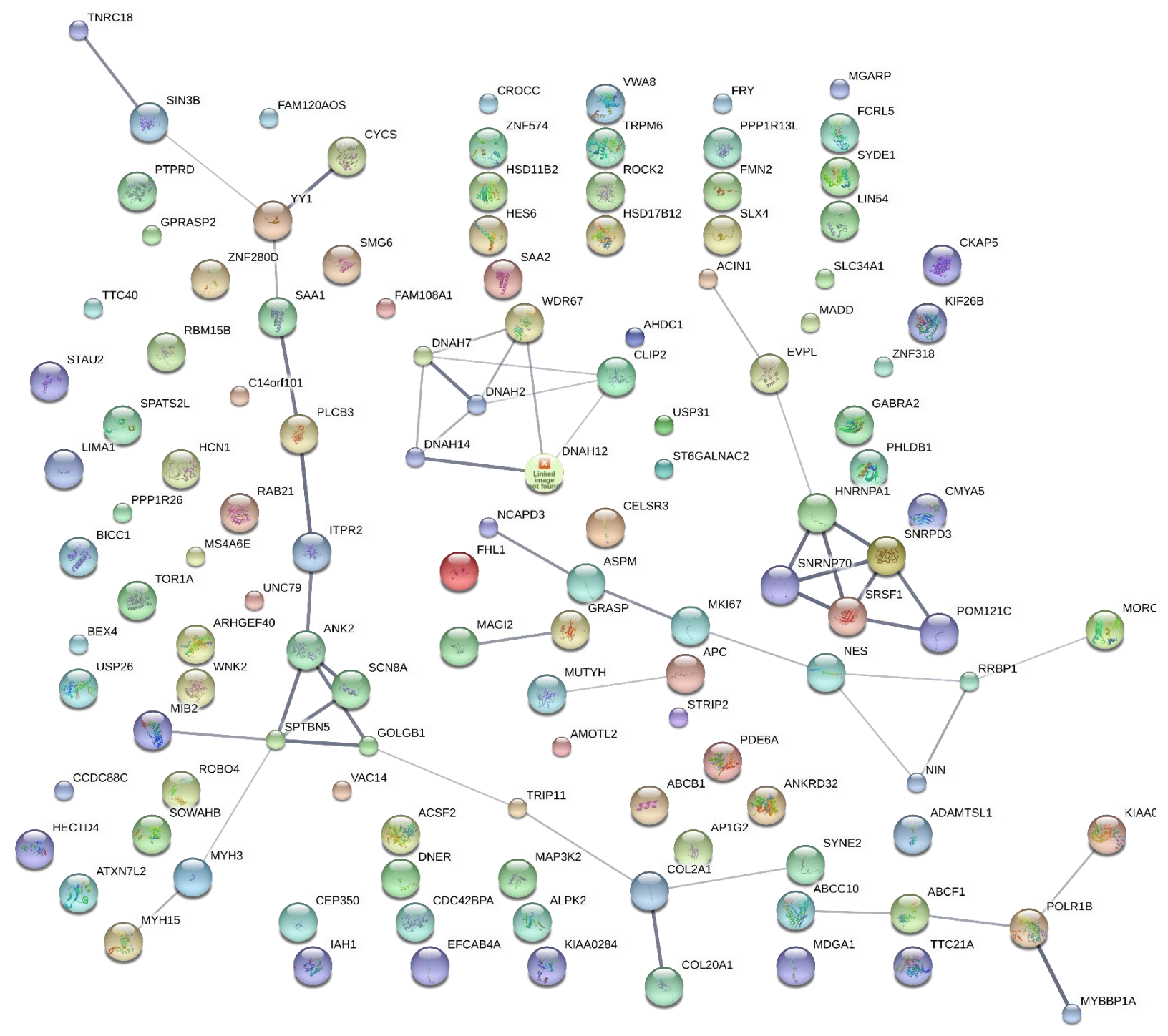

Sepsis STYP, Delta gt 10, X2 gt 5

Figure 3. STRING The difference of each diseases state $(n \geq 10)$ versus the matching controls under the Difference and Chi Square parameters indicated per protein observation. 
A

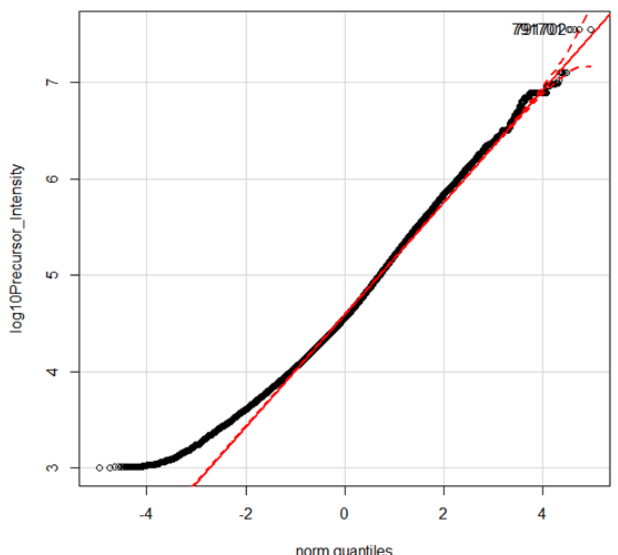

$\mathrm{B}$

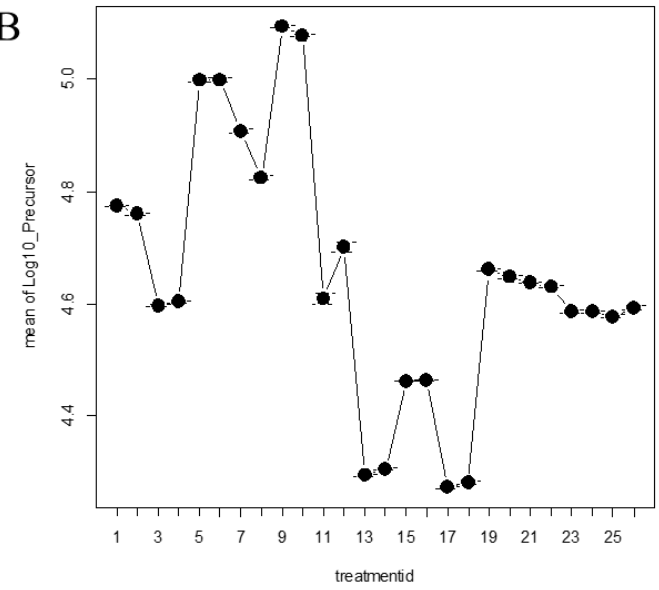

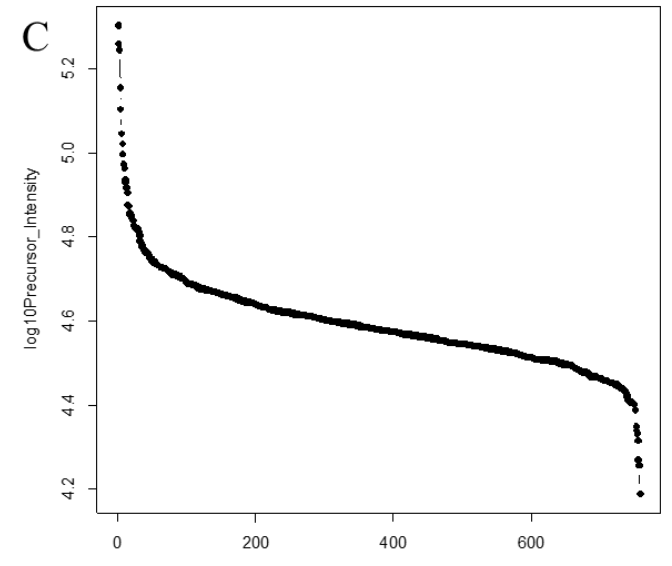

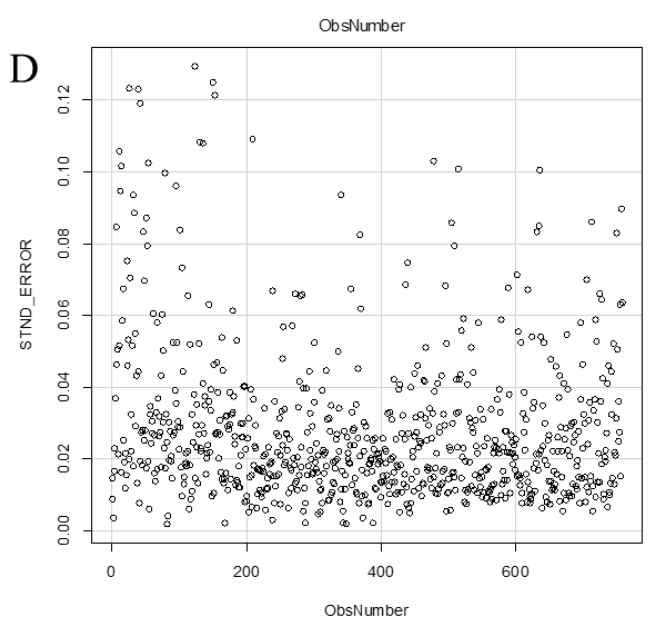

Figure 4. The normality of $\log 10$ transformed precursor ions followed by ANOVA of the controls and disease samples from the different Institution/study of the Gene Symbols that showed Chi Square $(\chi 2 \geq 25)$ versus the matched control. Panels: A, the quantile plot of Log 10 precursor intensity; B, the mean and standard error of the precursor intensity values (the letter indicate differences by Tukey Kramer; C, the sorted intensity values of the Gene Symbols; D, the standard error of the mean of the gene symbols. The analysis of $\log 10$ transformed precursor ion intensity values by ANOVA indicated there was significant variation in the intensity values between the institution/study as well as variation between specific control sample sets. 